ORNL/TM-2013/188

\title{
National Weatherization Assistance Program Characterization - Describing the Pre-ARRA Program
}

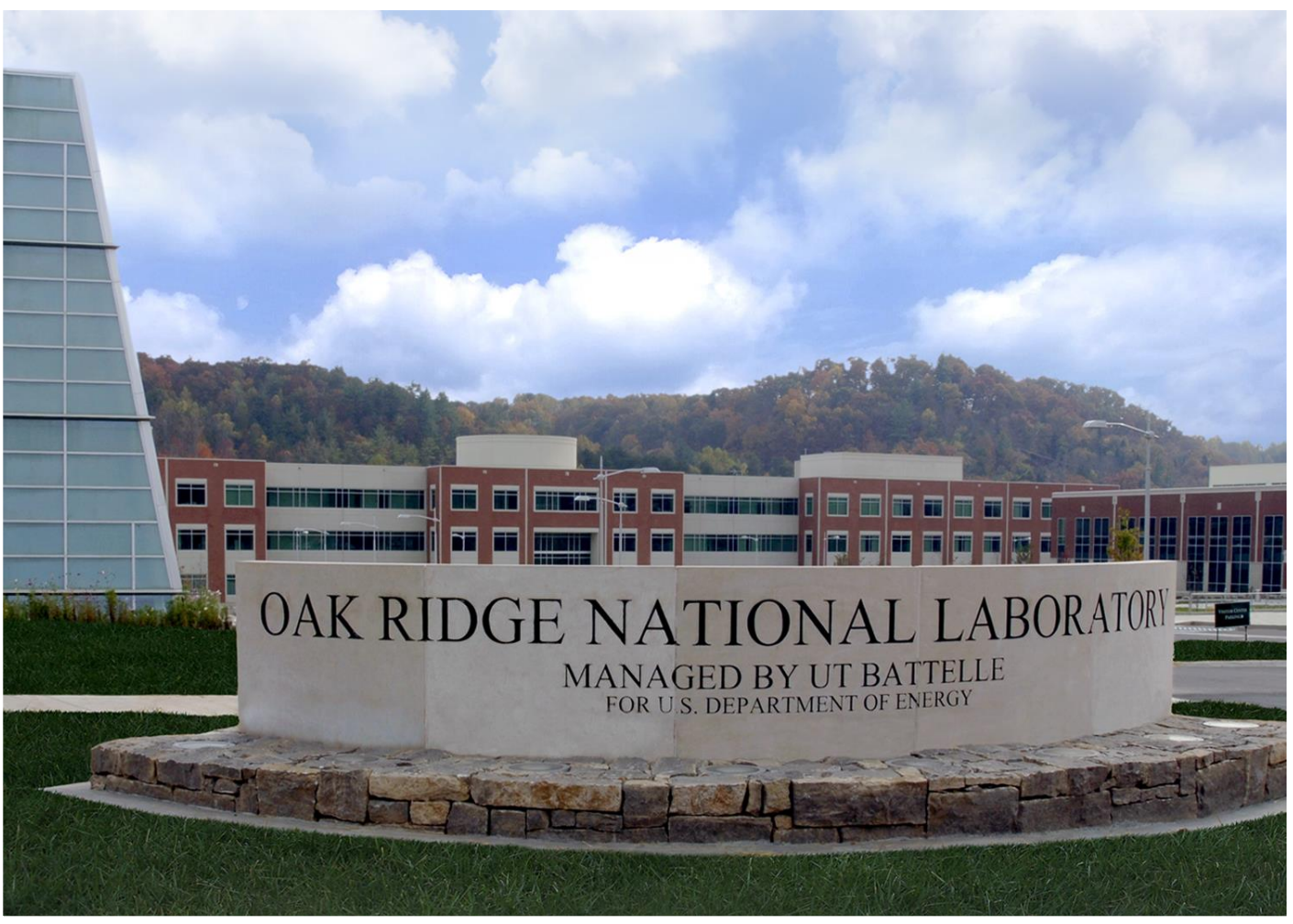

Ingo Bensch Ashleigh Keene Claire Cowan Karen Koski

September 2014 


\section{DOCUMENT AVAILABILITY}

Reports produced after January 1, 1996, are generally available free via US Department of Energy (DOE) SciTech Connect.

Website http://www.osti.gov/scitech/

Reports produced before January 1, 1996, may be purchased by members of the public from the following source:

National Technical Information Service

5285 Port Royal Road

Springfield, VA 22161

Telephone 703-605-6000 (1-800-553-6847)

TDD 703-487-4639

Fax 703-605-6900

E-mail info@ntis.gov

Website http://www.ntis.gov/help/ordermethods.aspx

Reports are available to DOE employees, DOE contractors, Energy Technology Data Exchange representatives, and International Nuclear Information System representatives from the following source:

Office of Scientific and Technical Information

PO Box 62

Oak Ridge, TN 37831

Telephone 865-576-8401

Fax 865-576-5728

E-mail reports@osti.gov

Website http://www.osti.gov/contact.html

This report was prepared as an account of work sponsored by an agency of the United States Government. Neither the United States Government nor any agency thereof, nor any of their employees, makes any warranty, express or implied, or assumes any legal liability or responsibility for the accuracy, completeness, or usefulness of any information, apparatus, product, or process disclosed, or represents that its use would not infringe privately owned rights. Reference herein to any specific commercial product, process, or service by trade name, trademark, manufacturer, or otherwise, does not necessarily constitute or imply its endorsement, recommendation, or favoring by the United States Government or any agency thereof. The views and opinions of authors expressed herein do not necessarily state or reflect those of the United States Government or any agency thereof. 
Environmental Sciences Division

\section{NATIONAL WEATHERIZATION ASSISTANCE PROGRAM CHARACTERIZATION - DESCRIBING THE PRE-ARRA PROGRAM}

Ingo Bensch, Energy Center of Wisconsin Ashleigh Keene, Energy Center of Wisconsin Claire Cowan, Energy Center of Wisconsin Karen Koski, Energy Center of Wisconsin

September 2014

$$
\text { Prepared by }
$$

OAK RIDGE NATIONAL LABORATORY

Oak Ridge, Tennessee 37831-6283

managed by

UT-BATTELLE, LLC

for the

US DEPARTMENT OF ENERGY

under contract DE-AC05-00OR22725 



\section{CONTENTS}

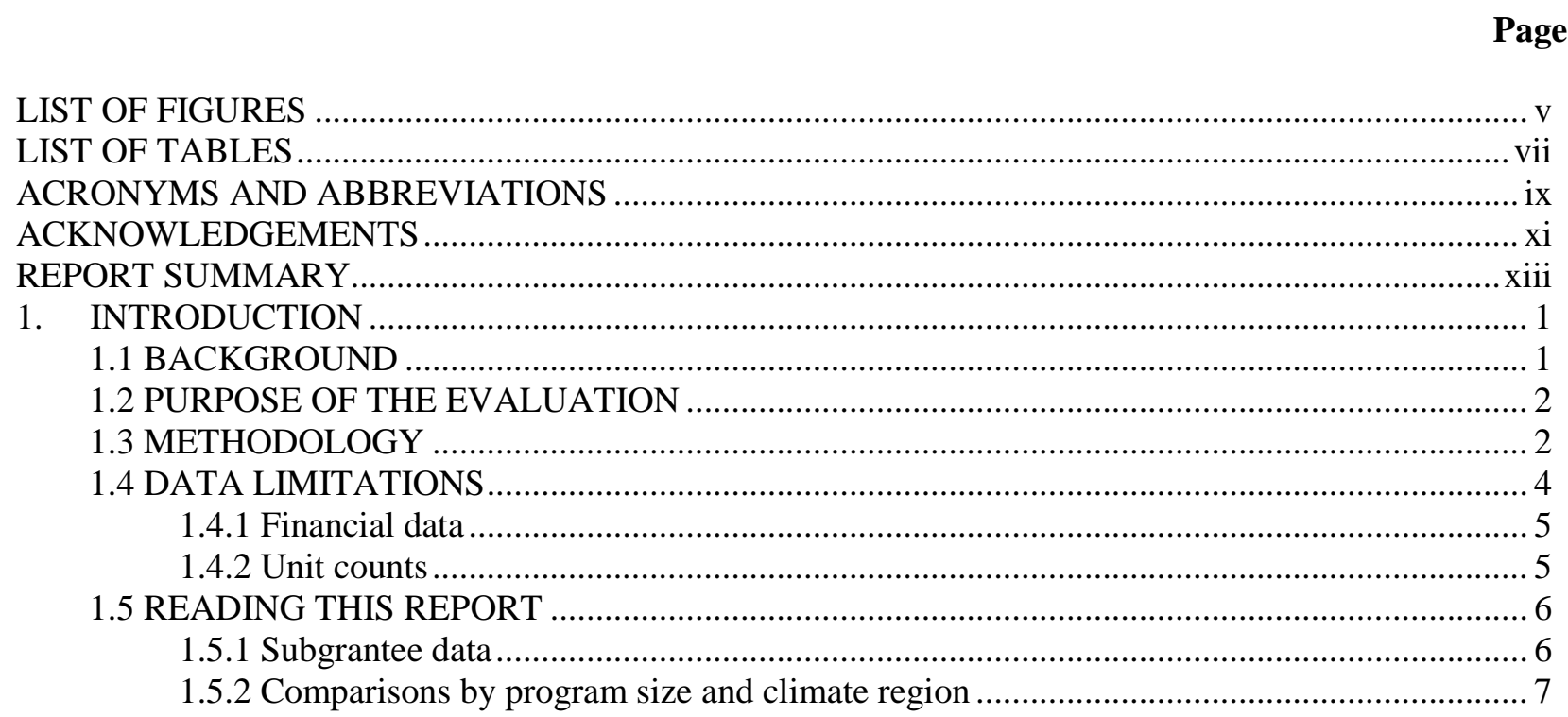

2. HOW ARE WEATHERIZATION AGENCIES STRUCTURED AND FUNDED TO DO

THEIR WORK?

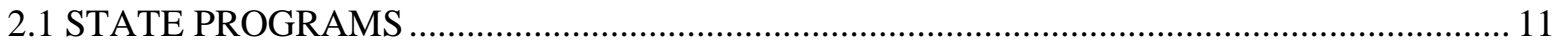

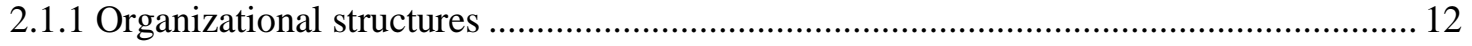

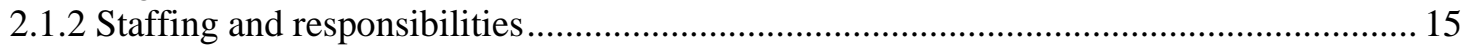

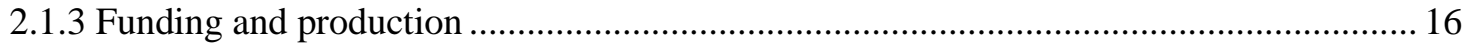

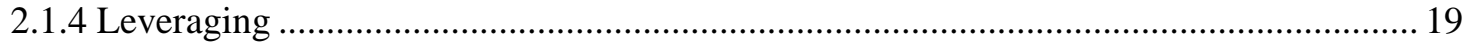

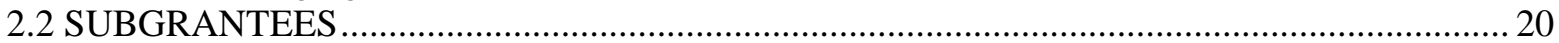

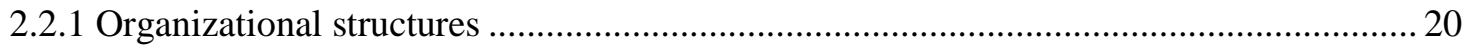

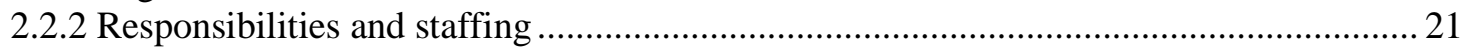

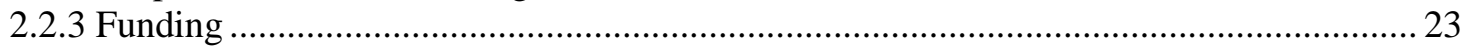

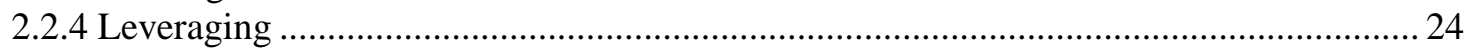

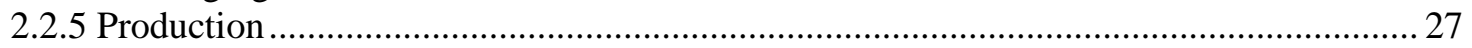

2.3 SUPPORT FROM THE DEPARTMENT OF ENERGY AND OTHERS ................................. 28

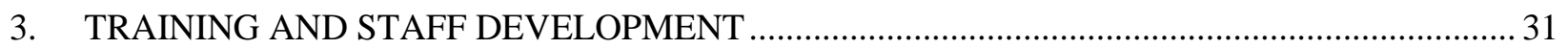

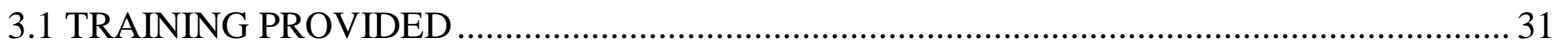

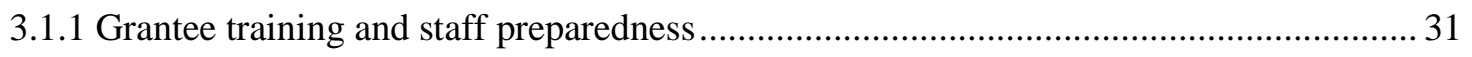

3.1.2 Staff preparedness and training in subgrantees ......................................................... 37

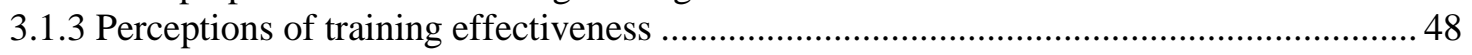

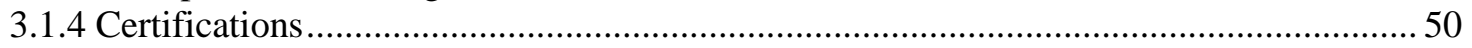

4. HOW WEATHERIZATION SERVICES ARE DELIVERED …............................................ 55

4.1 DISTRIBUTION OF EFFORT AMONG THE COMPONENTS OF WEATHERIZATION ..... 55

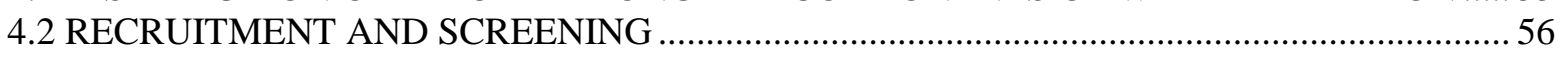

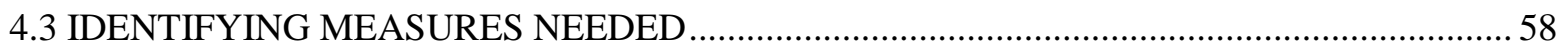

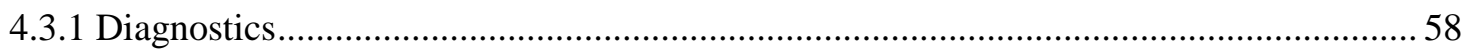

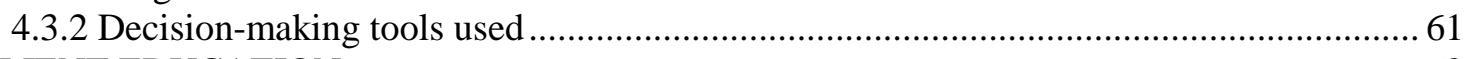

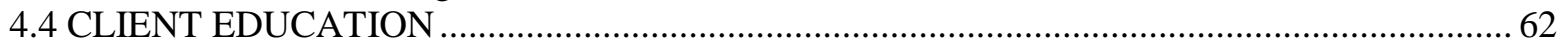

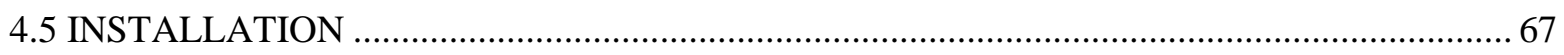

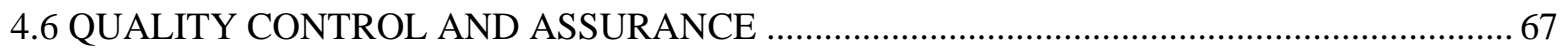

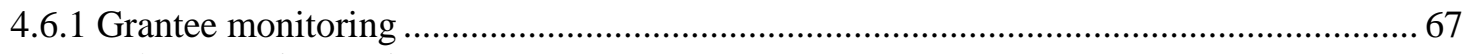

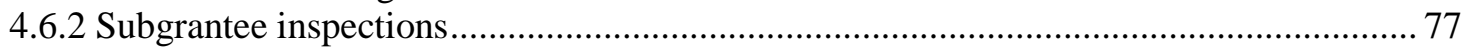


5. WHOM DO WEATHERIZATION AGENCIES SERVE WITH WHAT SERVICES?................... 81

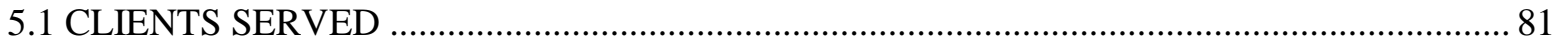

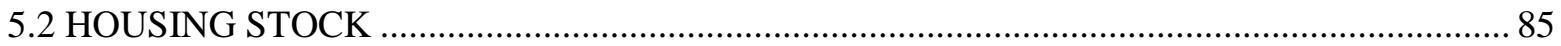

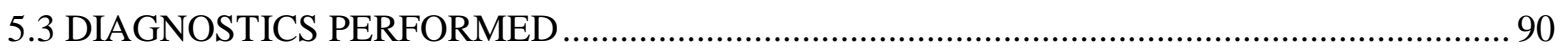

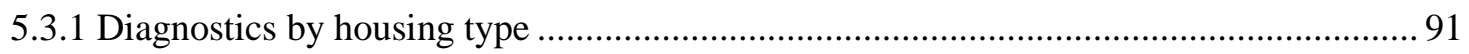

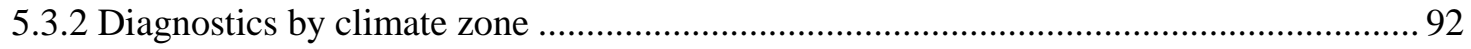

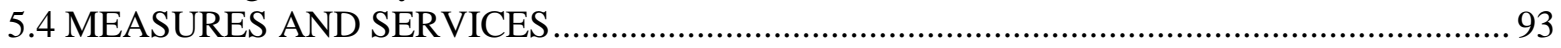

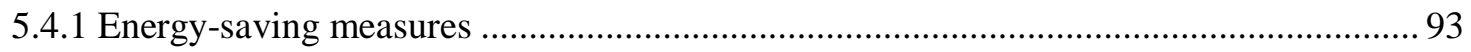

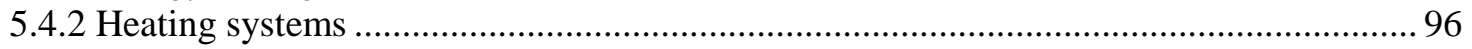

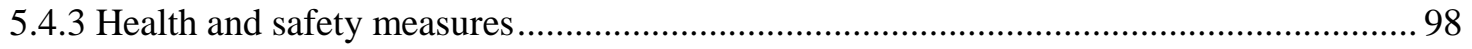

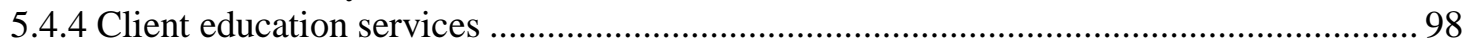

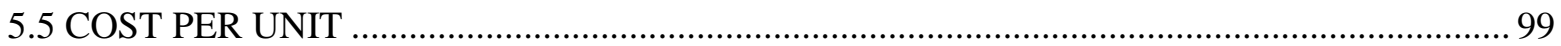

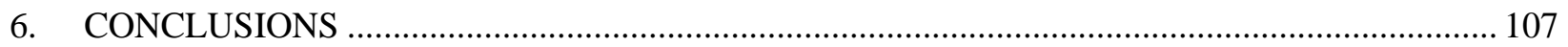

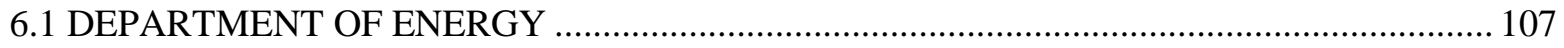

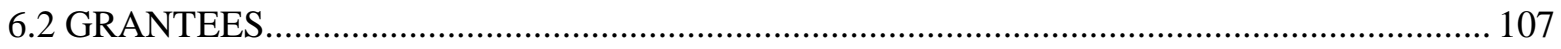

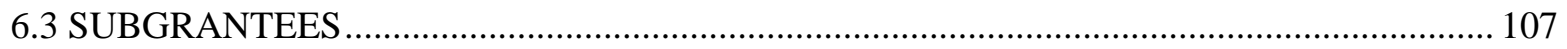

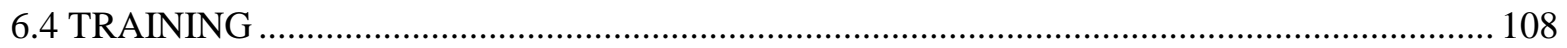

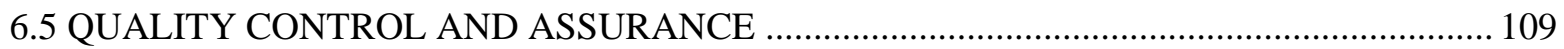

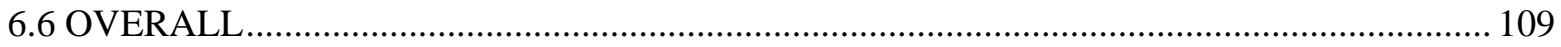

APPENDIX A: ALL STATES AGENCIES INFORMATION SURVEY …...................................... A-1

APPENDIX B: ALL AGENCIES OVERVIEW DATA FORM .....................................................

APPENDIX C: ALL STATES PROGRAM INFORMATION SURVEY …....................................... B-1

APPENDIX D: ALL AGENCIES PROGRAM INFORMATION SURVEY ........................................ D-1

APPENDIX E: SAMPLED AGENCIES DETAILED PROGRAM INFORMATION SURVEY ........... E-1

APPENDIX F: BUILDING \& HOUSING UNIT INFORMATION SURVEYS....................................F-1 


\section{LIST OF FIGURES}

Figure $\quad$ Page

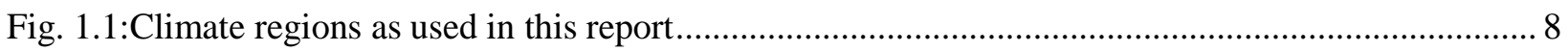

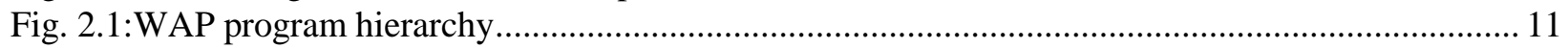

Fig. 2.2:WAP office proximity to governor's office (by program size) ................................................. 13

Fig. 2.3:WAP office proximity to governor's office (by climate region) .............................................. 14

Fig. 2.4:Turnover in weatherization director position during 1999-2008 (by program size) .................... 15

Fig. 2.5:Turnover in weatherization director position during 1999-2008 (by climate region) ................... 15

Fig. 2.6: Weatherization funding structure for Program Year 2008 ....................................................... 17

Fig. 2.7:Differences in rules between DOE funds and other sources ..................................................... 18

Fig. 2.8:Importance of leveraged resources by program size ............................................................ 19

Fig. 2.9:Grantee success in attracting leveraged funds (by resources allocated) .................................... 20

Fig. 2.10:Layers of supervision between program director and crews in subgrantees ............................ 21

Fig. 2.11:Turnover in subgrantees by functional category (Program Years 2005 through 2007) ${ }^{+}$............. 23

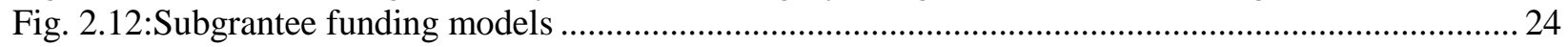

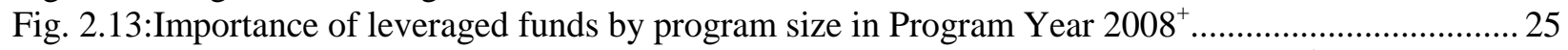

Fig. 2.14:Success of subgrantee efforts to acquire leveraged funds in Program Year $2008^{+}$................... 26

Fig. 2.15: Flexibility in federal program rules as perceived by grantee program directors ....................... 29

Fig. 2.16:Grantee perceptions of DOE support in selected areas .......................................................... 30

Fig. 2.17:Importance of improvement in DOE support in selected areas ................................................. 30

Fig. 3.1: Grantee assessment of staff knowledge on technical weatherization topics in PY08 .................. 32

Fig. 3.2:Grantee assessment of staff knowledge on technical weatherization topics, by housing type ....... 33

Fig. 3.3: Grantee assessment of staff knowledge on technical/administrative topics in PY08 ................... 34

Fig. 3.4: Grantee assessment of staff knowledge on health and safety topics in PY08 ............................ 35

Fig. 3.5: Grantee training for its staff - most common topics ............................................................... 37

Fig. 3.6: Subgrantee assessment of staff knowledge on technical weatherization topics in PY08 ${ }^{+}$............ 39

Fig. 3.7: Subgrantee assessment of staff knowledge on technical weatherization topics, by housing

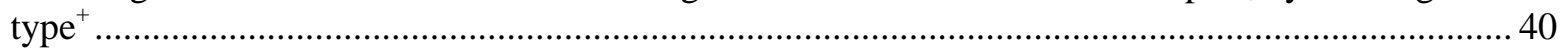

Fig. 3.8: Subgrantee assessment of staff knowledge on technical/administrative topics in $\mathrm{PY}_{0} 8^{+}$............ 41

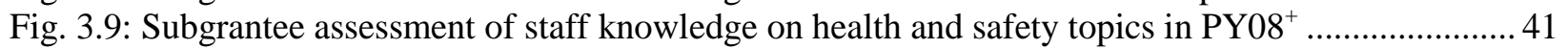

Fig. 3.10: Training received by subgrantee staff by functional category ${ }^{+}$............................................ 43

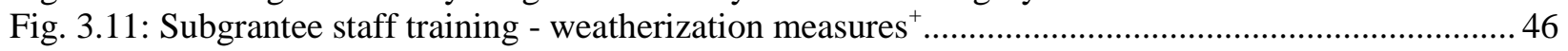

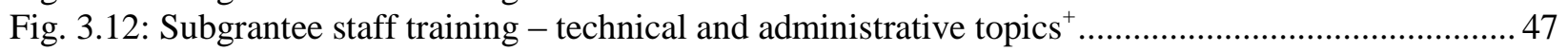

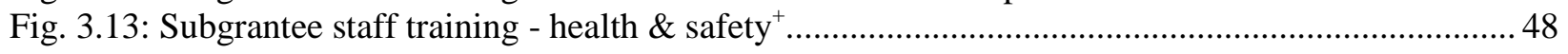

Fig. 3.14: Technical certification requirements for grantee trainers in PY2008 .................................... 51

Fig. 3.15: Comparison of technical certification requirements for key grantee roles in PY2008 ...............52

Fig. 3.16: Comparison of technical certification requirements for key subgrantee roles in PY $2008^{+} \ldots \ldots . . .54$

Fig. 4.1: Marketing for weatherization services by subgrantees ${ }^{+}$........................................................ 56

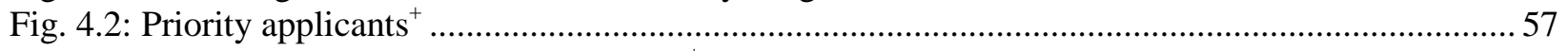

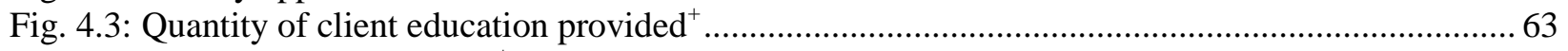

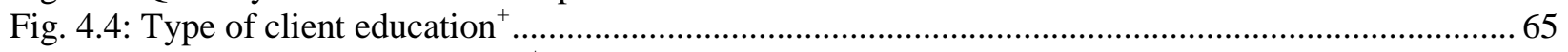

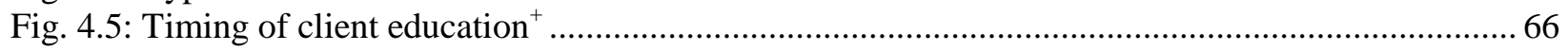

Fig. 4.6: Proportion of staff time (FTEs) spent on quality control and assurance activities ...................... 68

Fig. 4.7: Proportion of staff time spent on quality control and assurance by program size ....................... 69

Fig. 4.8: Proportion of staff time spent on quality control and assurance by climate type ........................69

Fig. 4.9: Frequency of most common inspection types by program size ................................................. 72

Fig. 4.10: Frequency of grantee administrative monitoring visits to subgrantees ................................... 73 
Fig. 4.11: Frequency of grantee administrative monitoring visits to subgrantees (by program size) ......... 74

Fig. 4.12: Frequency of grantee administrative monitoring visits to subgrantees (by climate region)....... 74

Fig. 4.13: Occurrences of administrative problems per grantee (by climate region) ................................. 75

Fig. 4 14: Corrective actions taken by grantees in response to subgrantee administrative problems ......... 75

Fig. 4.15: Portion of grantees that made training program changes in response to subgrantee administrative problems (by program size)

Fig. 4.16: Grantee training program changes in response to subgrantee administrative problems (by climate region) .77

Fig. 4.17: Post-weatherization inspection components. .79

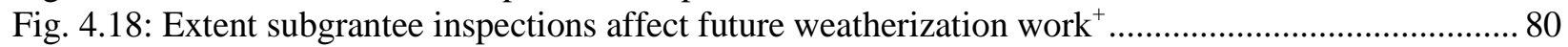

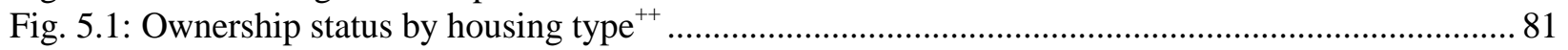

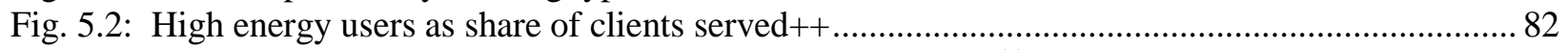

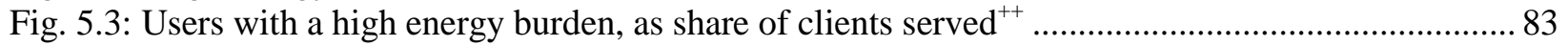

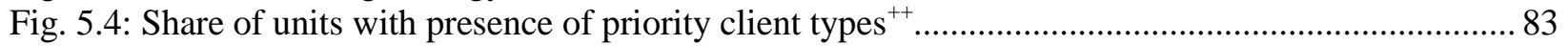

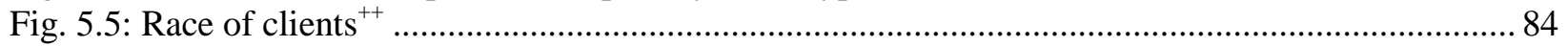

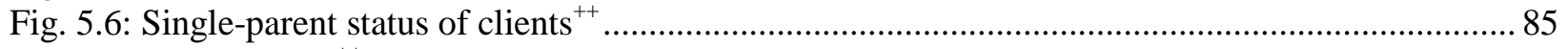

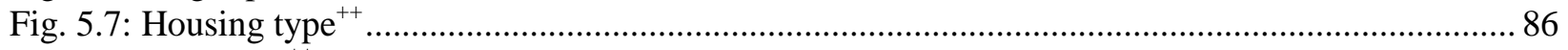

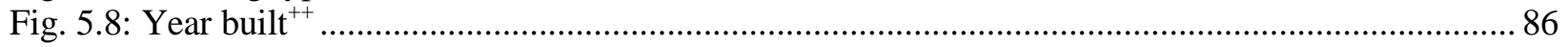

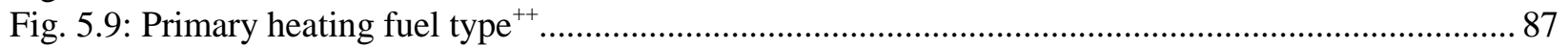

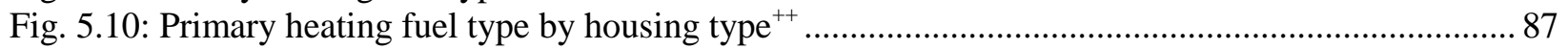

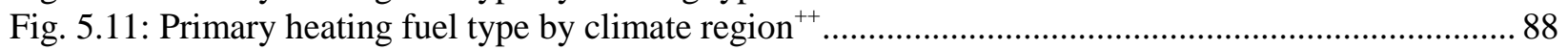

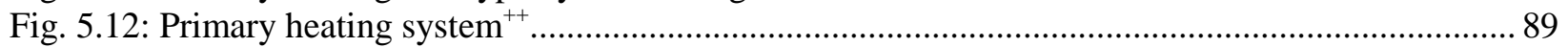

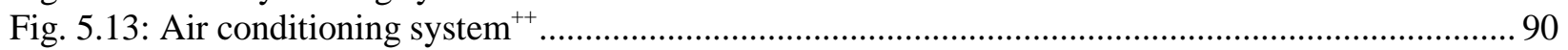

Fig. 5.14: Percent of weatherized units receiving at least one inspection from diagnostic category ${ }^{++}$....... 91

Fig. 5.15:Percent of weatherized units receiving at least one inspection from diagnostic category, by

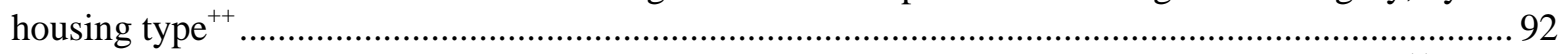

Fig. 5.16:Percent of weatherized units receiving at least one inspection from diagnostic category ${ }^{++}$....... 93

Fig. 5.17:Percent of weatherized units receiving work in various measure categories ${ }^{++} \ldots \ldots \ldots \ldots \ldots \ldots \ldots \ldots . . . . . . . . . . . . .94$

Fig. 5.18: Percent of weatherized units receiving at least one measure from broader category, by

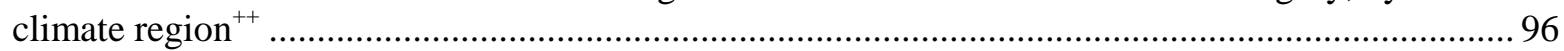

Fig. 5.19: Percent of weatherized units receiving 10 most common health and safety measures ${ }^{++}$........... 98

Fig. 5.20: Percent of weatherized units receiving client education measures ${ }^{++}$...................................... 99

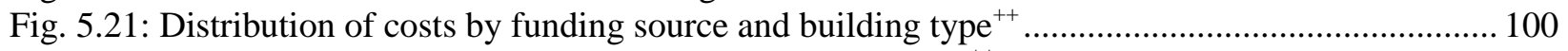

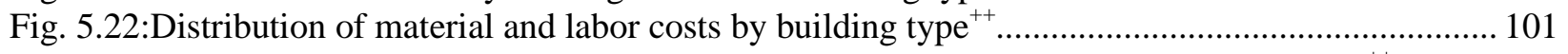

Fig. 5.23: Distribution of labor costs among in-house crews and contractors, by building type ${ }^{++} \ldots \ldots \ldots . . . .103$

Fig. 5.24: Project cost distribution among energy-saving measures, health \& safety, and incidental

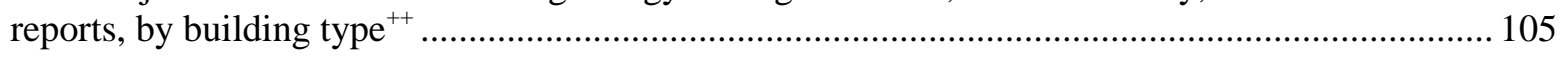




\section{LIST OF TABLES}

Table

Page

Table 1.1: Instruments used in grantee and subgrantee data collection .................................................. 3

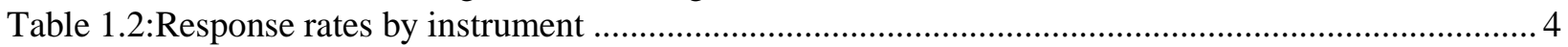

Table 1.3:Sampling uncertainties for agency-level data from sampled subgrantees ................................ 7

Table 1.4:Sampling uncertainties for housing-unit data from sampled subgrantees ............................... 7

Table 1.5 Funding ranges for grantees and subgrantees by program size............................................. 9

Table 2.1: Mean network size and funding per subgrantee by climate region........................................ 12

Table 2.2: Mean network size and funding per subgrantee by program size ........................................... 12

Table 2.3: Grantee support functions in Program Year 2008 - in FTE staff ............................................ 16

Table 2.4: Program Year 2008 units weatherized by WAP subgrantees* .............................................. 18

Table 2.5: Subgrantee staffing by function in Program Year 2008 ....................................................... 22

Table 2.6: Other programs that cooperated with subgrantee weatherization programs $(n=396)^{+} \ldots \ldots \ldots \ldots \ldots .27$

Table 2.7: Duration on waitlists in PY08 by program size (for subgrantees with waitlists)...................... 28

Table 2.8: Duration on waitlists in PY08 by climate region (for subgrantees with waitlists) .................... 28

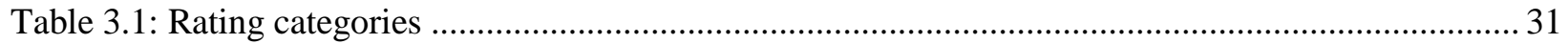

Table 3.2: Training supported by grantee weatherization programs ........................................................ 36

Table 3.3: Perceptions of grantees and subgrantees on training/preparedness in key topic areas by

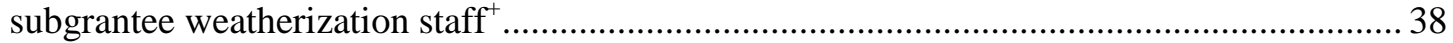

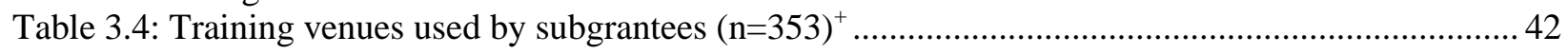

Table 3.5: Training quality by venue - grantee assessment.................................................................... 49

Table 3.6: Training quality by venue - subgrantee assessment …............................................................ 49

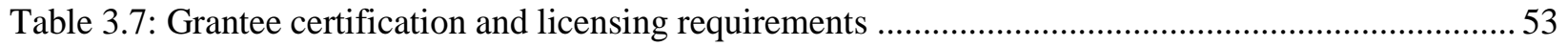

Table 4.1: Distribution of subgrantees' expenditures by category .........................................................5 55

Table 4.2: Diagnostic procedures performed by subgrantees in Program Year $2008^{+}$............................ 59

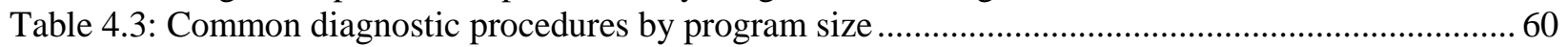

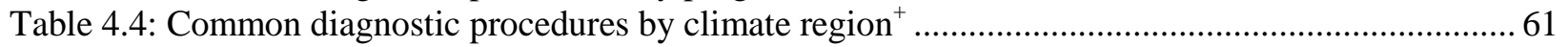

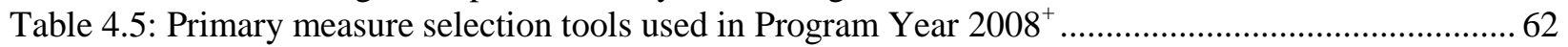

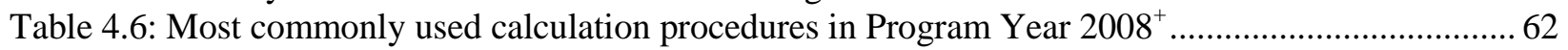

Table 4.7: Topics covered as part of client education in more than half of weatherization

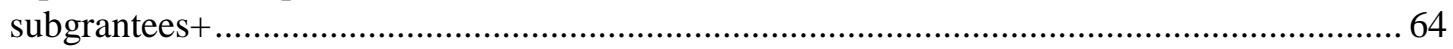

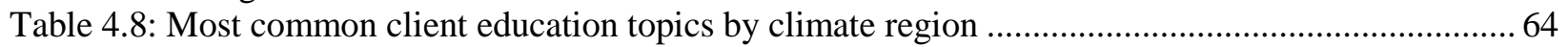

Table 4.9: Perceived effectiveness and level of investment needed for various client education

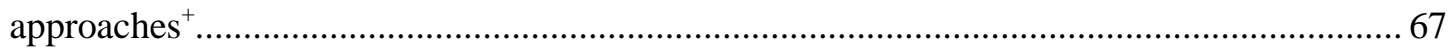

Table 4.10: Post-weatherization inspections performed by grantees ................................................. 71

Table 4.11:Post-weatherization inspections performed by subgrantees .............................................. 78

Table 5.1: Percent of weatherized units receiving at least one inspection from diagnostic category ${ }^{++}$...... 90

Table 5.2: Percent of weatherized units receiving at least one inspection from diagnostic category ${ }^{++}$...... 91

Table 5.3: Percent of weatherized units receiving work in various measure categories ${ }^{++}$....................... 94

Table 5.4: Percent of weatherized units receiving at least one measure from broader category, by

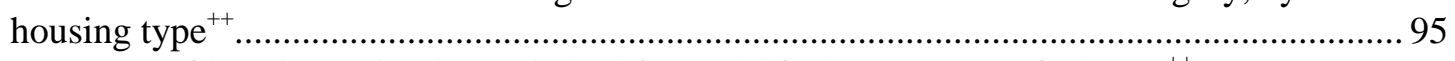

Table 5.5: Percentage of housing units that switched from old fuel type to new fuel type ${ }^{++}$................... 97

Table 5.6:Percentage of housing units that switched from old heating system to new heating

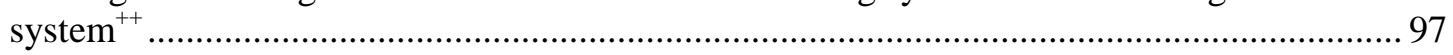

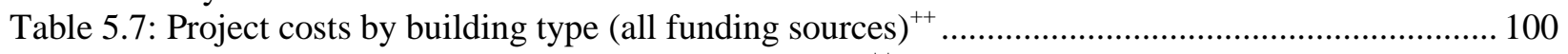

Table 5.8:Typical project cost breakdown for single family ${ }^{++}$............................................................ 101 
Table 5.9: Subgrantee cost allocation for labor costs (in-house crew or contractor), by building type $^{++}$ 


\section{ACRONYMS AND ABBREVIATIONS}

$\begin{array}{ll}\text { ARRA } & \text { American Recovery and Reinvestment Act } \\ \text { DOE } & \text { U.S. Department of Energy } \\ \text { FTE } & \text { Full-time Equivalent } \\ \text { HVAC } & \text { Heating, Ventilation and Air Conditioning } \\ \text { LIHEAP } & \text { Low Income Home Energy Assistance Program } \\ \text { NCAF } & \text { National Community Action Foundation } \\ \text { NASCSP } & \text { National Association for State Community Services Program } \\ \text { OMB } & \text { Office of Management and Budget } \\ \text { ORNL } & \text { Oak Ridge National Laboratory } \\ \text { PVE } & \text { Petroleum Violation Escrow } \\ \text { PY } & \text { Program Year } \\ \text { WAP } & \text { Weatherization Assistance Program } \\ \text { WAPTAC } & \text { Weatherization Assistance Program Technical Assistance Center }\end{array}$





\title{
ACKNOWLEDGEMENTS
}

The work presented in this report was funded by the U.S. Department of Energy's (DOE) Office of Weatherization and Intergovernmental Programs (OWIP.)

This report summarizes the findings from the Program Characterization Study of the Weatherization Assistance Program (WAP) Evaluation. In this study, the evaluation team contacted 51 WAP grantees and 877 WAP subgrantees in all 50 states and the District of Columbia to collect information to characterize WAP in Program Year 2008. The study included two grantee surveys and two subgrantee surveys. In addition, the subgrantees furnished service delivery information for 14,496 clients.

The original design for the study was developed by staff from the Oak Ridge National Laboratory (ORNL) as one component of the National Evaluation of the Weatherization Assistance Program. (National Evaluation of the Weatherization Assistance Program: Preliminary Evaluation Plan for Program 2006 - ORNL/CON-498). As part of the evaluation plan development the design team consulted with and received feedback the Network Planning Committee (41 individuals from the weatherization network).

ORNL contracted with the research team of APPRISE Incorporated, the Energy Center of Wisconsin, Michael Blasnik and Associates, and Dalhoff Associates LLC to conduct the National Evaluation. The Energy Center of Wisconsin took primary responsibility for the design and implementation of this study. The members of the Evaluation Team who contributed to the design and implementation of this survey included:

\author{
The Energy Center of Wisconsin \\ Ingo Bensch \\ Claire Cowan \\ Sharon Flores \\ LaShanta Goodwin \\ Ashleigh Keene \\ Steve Kihm \\ Karen Koski \\ Jeannette LeZaks \\ Melanie Lord \\ Andy Mendyk \\ Scott Pigg \\ Benjamin Rickelman \\ Jaimie Rule \\ Nick Sayen \\ Cheryl Schmidt \\ Erin Vallicelli
}

\section{APPRISE Incorporated}

David Carroll

Regina Yang

Chisoo Kim

Michael Blasnik and Associates

Michael Blasnik 


\section{Dalhoff Associates LLC \\ Greg Dalhoff}

The Dieringer Research Group, Inc. prepared the on-line survey instrument and data collection forms and delivered a data file for this study.

This study could only be completed with the cooperation and contributions of the grantees and subgrantees who responded to the surveys and data collection forms.

Finally, we would like to acknowledge the assistance and guidance of the Oak Ridge National Laboratory, Department of Energy Project Officers, and Department of Energy Headquarters Staff.

Ingo Bensch

Claire Cowan 


\section{REPORT SUMMARY}

The Department of Energy's (DOE) Weatherization Assistance Program (WAP) has supported energy efficiency improvements to the homes of low-income households in the United States since 1976. The program provides grants, guidance, and other support to grantees: weatherization programs administered by each of the 50 states, the District of Columbia and some Native American tribes. Although there have been studies of some grantee-administered weatherization programs, the overall effectiveness of the national weatherization program has not been formally evaluated since Program Year 1989. Since that time, the program has evolved significantly, with an increased focus on baseload electric usage, continued evolution of diagnostic tools, new guidelines and best practices for heating-related measures, and adjustments in program rules. More recently, the program has also adjusted to large, temporary funding increases and changes in federal rules spurred by the American Recovery and Reinvestment Act (ARRA).

Because the WAP of today is dramatically different from the one evaluated in 1989, DOE determined to undertake a new comprehensive evaluation of the national program. This new national evaluation is managed by Oak Ridge National Laboratory (ORNL). Under a competitive solicitation process, ORNL selected APPRISE, Inc., Blasnik \& Associates, Dalhoff Associates and the Energy Center of Wisconsin (ECW) to conduct the evaluation. The national evaluation comprises two independent evaluations. The first evaluation — of which this report is a part-focuses on Program Year 2008 (PY08). The second evaluation focuses on the ARRA-funded years of 2009 through 2011.

This report, together with its companion — the Eligible Population Study—addresses specific program characterization goals established for the greater evaluation. ECW led grantee and subgrantee data collection efforts, administering surveys to 51 grantees and 851 of the approximately 900 subgrantees that were slated to receive DOE weatherization funds in PY08. In all, seven different data collection instruments were used to gather the needed data - two instruments for grantees and five for subgrantees. See Table 1.1 for a list of these survey instruments. These surveys were used to determine, among other things:

- Structure and funding of weatherization programs

- Training and staff development of service providers

- How weatherization services are delivered

- Clients served

\section{Results}

The national WAP is delivered through a varied network of state offices (grantees) that run statewide weatherization programs and local agencies (subgrantees) that weatherize homes of eligible program clients. DOE provides a substantial amount of funding to grantees for their respective statewide programs, as well as program guidance and rules governing the use of these funds.

\section{Funding}

In PY08, DOE made available \$227.2 million in program grants to 51 grantees (all 50 states plus the District of Columbia) for their use in administering their respective statewide programs. In turn, the grantees distributed funding to a network of approximately 900 subgrantees. In PY08, grantees allocated an average of $\$ 240,000$ in DOE funds to subgrantees.

While DOE funding is an important source of funding for low-income weatherization, it is not the only source. Grantees and subgrantees use a mixture of funding sources to pay for weatherization program activities. Grantees, particularly those with larger programs, reported that leveraging DOE funds was important to their program funding. For 21 of the grantees, leveraged funds accounted for half or more of their total program funding. Altogether, grantees received nearly $\$ 720$ million to support their 
weatherization programs in PY08. Of that amount, grantee weatherization offices spent about $\$ 42$ million on their activities and passed along $\$ 677$ million to subgrantees for their work. Fig. 2.6 illustrates the aggregated national funding scenario as reported to us by the grantees and subgrantees.

\section{Structure and staff development}

As described above, a varied network of organizations deliver the Weatherization Assistance Program. At the grantee level, the program resides within state government, often in a state department of social services or housing. On average, grantees have eight full-time equivalent (FTE) positions for a total of about 400 state-level staff administering the 51 weatherization programs in the states and the District of Columbia.

Subgrantees tend to be locally-based nonprofit organizations, although some are county or local government agencies. Some subgrantees focus only on weatherization while others provide a variety of social services.

The Weatherization Assistance Program focuses substantial attention on training and staff development at both the federal and state levels. Nine percent of DOE funds spent in PY08 plus another three percent in non-DOE funds were allocated to training and technical assistance. Based on self-reports from grantees and subgrantees, staff are well trained in the areas in which they require particular knowledge to perform their jobs.

\section{How services are provided}

The full weatherization process involves a multi-step sequence that comprises client intake, home audit, weatherization (installation of measures), client education and post-weatherization inspection. To understand the relative effort expended on these various activities at the local level, we broke them out by spending category. In PY08, subgrantees spent approximately 70 percent of their weatherization funds on the installation of measures, 12 percent on program management, 10 percent on health and safety measures, 7 percent on audits and inspections, and 1 percent on training and technical assistance.

The home audit is the first step in identifying the measures that provide cost-effective energy efficiency improvements and that address health and safety concerns. Subgrantees use some fairly sophisticated tools to identify heat loss, system efficiency, and other sources of energy waste.

Most subgrantees perform a range of procedures to test air infiltration and the safety and performance of space and water heating systems. These diagnostic procedures include (among others):

- Blower door (used by $99 \%$ of subgrantees)

- Flue gas analysis (used by $83 \%$ of subgrantees)

- Draft/spillage (used by $77 \%$ of subgrantees)

- Duct pressure pan measurements (used by $59 \%$ of subgrantees)

- $\mathrm{CO}$ testing (done by a majority of subgrantees in some form)

Subgrantees use the diagnostic results to select the measures to be installed or implemented on the home being weatherized. Tools used to select these measures include:

- a priority list (used by $52 \%$ of subgrantees)

- a calculation procedure (used by $33 \%$ of subgrantees)

- combination of a priority list and a calculation procedure (used by $12 \%$ of subgrantees) 
Subgrantees using a calculation procedure as their primary decision-making tool overwhelmingly used Weatherization Assistant (53\% of subgrantees).

\section{Clients served}

WAP serves low-income families. In PY08 DOE rules allowed households at or below 150 percent of the federal poverty level or 60 percent of the state's median income to qualify for weatherization assistance. Additionally, some particularly vulnerable groups receive priority at the federal or grantee level. Clients that receive special priority - either nationally or regionally - include households with elderly members, disabled residents, or children. Clients with high energy expenditures or burdens also receive special consideration.

A snapshot of PY08 WAP clients shows that:

- Approximately three-fourths own the home in which they live (primarily site-built single-family home or mobile home)

- Approximately one-third can be classified as either high energy users or as having a high energy burden (for those clients in states with a defined threshold for high energy users or high energy burden)

- Nearly half (45 percent) of the households have elderly residents, 38 percent have a resident with a qualifying disability and 30 percent have children living at home

- Approximately half of the households with children are single-parent households

- Approximately half identify themselves as white, 16 percent as black, 5 percent as Hispanic and less than 4 percent as American Indian/Alaskan Native, Asian or Native Hawaiian/Pacific Islander (for the three-fourths of PY08 clients for which racial identity data are available)

\section{Production}

In PY08 WAP subgrantees responding to our data request weatherized:

- $\quad 54,121$ single-family units (site-built)

- 5,920 small multifamily (2-4) units

- 11,058 large multifamily (5+) units

- 14,998 mobile homes

Nationally, subgrantees spent on average $\$ 3,500$ per unit across building types. Of this spending, just less than $\$ 2,000$ came from DOE funds.

Most homes weatherized during PY08 received at least one pressure diagnostic ( 83 percent), such as a blower door test, zonal pressure analysis or duct pressure measurements, among others. Similarly, most homes received diagnostic measurements or inspection pertaining to the water-heating system and/or space-heating system ( 82 percent, each).

Energy-saving measures constitute 63 percent of all installations among single-family homes and 61 percent among mobile homes. On average, energy-saving measures make up the largest portion (67 percent) of total measure installations among homes in multifamily buildings.

Finally, weatherization programs across the country tend to install some common measures fairly universally. Nearly all PY08 weatherized homes (91 percent) received some form of air sealing. The next most common types of improvements were insulation ( 75 percent) and 'other baseload applications' such as lighting, refrigerators, and other energy-consuming equipment that is not part of the Heating, Ventilation and Air Conditioning (HVAC) system (69 percent). 



\section{INTRODUCTION}

This report is part of a national evaluation of the Weatherization Assistance Program (WAP) being managed by Oak Ridge National Laboratory (ORNL) on behalf of the U.S. Department of Energy (DOE). The pages that follow describe the nationwide WAP network and weatherization activities during Program Year 2008 (PY08). ${ }^{1}$ Other reports being issued as part of the national evaluation will address indoor air quality in weatherized homes; energy savings and non-energy benefits attributable to weatherization activities in electrically and natural gas-heated homes, dwellings heated with delivered fuels, and multifamily buildings heated with fuel oil; a process evaluation; and a series of case studies. In addition, a population eligibility report complements this characterization report by describing the broader population context in which the weatherization program operates.

\subsection{BACKGROUND}

DOE's WAP has supported energy efficiency improvements to the homes of low-income households in the United States since 1976. The program provides grants, guidance, and other support to grantees: weatherization programs administered by each of the 50 states, the District of Columbia and some Native American tribes. ${ }^{2}$ (For the sake of convenience - and because the District of Columbia functions much like a state program - we will refer to 51 grantees in this report.) The grantees, in turn, oversee a network of local weatherization agencies (subgrantees): community action agencies, nonprofit organizations, and local government agencies that are eligible to receive weatherization funding from DOE. These weatherization agencies qualify eligible households, assess their homes' energy efficiency opportunities, install energy-saving measures, and inspect the work. The work performed includes air sealing, insulation upgrades, furnace replacements, and other dwelling-specific measures found to be cost-effective, as well as home improvements needed to ensure the health and safety of household occupants. The work is done at no cost to the eligible participants. The Weatherization Assistance Program Technical Assistance Center (WAPTAC) reports that over 6.7 million households have been served through this program since its inception. ${ }^{3}$

In PY08, DOE made available \$227.2 million ${ }^{4}$ in program grants to all 51 grantees for their use in administering their respective statewide programs. In turn, the grantees distributed funding to a network of approximately 900 subgrantees. These funds were used to weatherize nearly 98,000 units ${ }^{5}$ that year. In addition, as reported below, many grantees and subgrantees supplemented the DOE funds with other funding sources for use on both the housing units weatherized as part of the DOE program and for weatherization that is performed outside the program.

\footnotetext{
${ }^{1}$ References to program years in this report are consistent with definitions used by DOE, which names program years according to the year in which the funding period begins. Some states name program years according to the year in which the funding period ends. DOE's Program Year 2008 is referred to as Program Year 2009 in those states.

${ }^{2}$ The program also provides funding for weatherization in some U.S. territories and to two Native American tribal governments. The territories are not included in our analysis. Two Native American tribes appear to have functioned as both grantees and subgrantees. They had a direct funding relationship with DOE, but did local weatherization work. We classified them as subgrantees for the purposes of our analysis.

${ }^{3}$ Reported at the following url on April 11, 2012:

www.waptac.org/data/files/website_docs/public_information/combined\%20know\%20the\%20facts_talking\%20point s.pdf.

${ }^{4}$ As reported in Weatherization Program Notice (WPN) 08-2

${ }^{5}$ Our exact control total was 97,965 units, as reported by grantees to DOE and tracked in the department's WinSAGA system.
} 
Although there have been studies of some grantee-administered weatherization programs, the overall effectiveness of the national weatherization program has not been formally evaluated since Program Year 1989. The program has evolved significantly since the last national evaluation was conducted, with an increased focus on baseload electric usage, continued evolution of diagnostic tools, new guidelines and best practices for heating-related measures, and adjustments in program rules. More recently, the program has also adjusted to large, temporary funding increases and changes in federal rules spurred by the American Recovery and Reinvestment Act (ARRA).

Consequently, ORNL is managing two independent evaluations of the national weatherization program on behalf of DOE. The first evaluation - of which this report is a part - focuses on PY08, which was the last year before substantial ARRA funding became available to the national weatherization network. The second evaluation focuses on the ARRA-funded years of 2009 through 2011.

\subsection{PURPOSE OF THE EVALUATION}

The purposes of the overall evaluation - and the collection of reports stemming from this work - are to (1) provide a comprehensive review of program performance, (2) enable DOE to make any necessary improvements and guide the direction of the program into the next decade, and (3) provide information of interest to potential funders in order to support leveraging activities. With a subsequent evaluation of the ARRA-era weatherization program to follow, this evaluation effort also provides a baseline against which ARRA results can be compared.

This report, together with its companion - the Eligible Population Study - addresses specific program characterization goals established for the greater evaluation. These goals are to characterize the following elements of the weatherization program:

- Low-income population eligible for and in need of the program

- Segment of the eligible population served by the program,

- Housing units and clients served by the program

- Weatherization and other services performed by the program

- Program expenditures and funding sources

\subsection{METHODOLOGY}

ORNL solicited input from the weatherization community in developing the evaluation plan and survey instruments. Forty-one people served on the Network Planning Committee which was comprised of representatives from grantees, subgrantees, DOE headquarters and regional offices, training centers and advocacy organizations. Input from the Network Planning Committee informed the goals and research priorities of the evaluation. Members provided feedback on draft survey instruments. The Office of Management and Budget (OMB) also reviewed the evaluation plan and survey instruments and solicited feedback during a public comment period publicized in the Federal Register.

ORNL used a competitive solicitation to select a team of independent energy program evaluators to conduct the evaluation: APPRISE, Inc., Blasnik \& Associates, Dalhoff Associates and the Energy Center of Wisconsin. The Energy Center led grantee and subgrantee data collection efforts, administering the surveys that collected the data used in this program characterization report.

This characterization report is based on self-reports by all 51 grantees and 851 of the 904 subgrantees that were slated to receive DOE weatherization funds in PY08. The evaluation team used seven different data collection instruments to gather the needed data - two instruments for grantees and five for subgrantees. We asked all 51 grantees to complete two state-level instruments and all 904 subgrantees to complete two 
agency-level instruments. We concentrated the bulk of the subgrantee data collection effort on a representative sample of $400 \mathrm{PY} 08$ agencies, which were asked to complete three additional instruments.

Table 1.1 lists the survey instruments and data forms ${ }^{6}$ used and the types of respondents to which each instrument pertained. The instruments are attached as Appendices A through $\mathrm{H}$.

Table 1.1: Instruments used in grantee and subgrantee data collection

\begin{tabular}{|c|c|c|c|c|}
\hline Instrument name & Label & $\begin{array}{c}\text { State } \\
\text { programs }\end{array}$ & $\begin{array}{l}\text { Sampled local } \\
\text { agencies }\end{array}$ & $\begin{array}{l}\text { Non-sampled } \\
\text { local agencies }\end{array}$ \\
\hline All States Agencies Information Survey & DF1 & $\mathrm{X}$ & & \\
\hline All Agencies Overview Data Form & DF10 & & $\mathrm{X}$ & $\mathrm{X}$ \\
\hline All States Program Information Survey & S1 & $\mathrm{X}$ & & \\
\hline All Agencies Program Information Survey & S2 & & $\mathrm{X}$ & $\mathrm{X}$ \\
\hline Sampled Agencies Detailed Program Information Survey & S3 & & $\mathrm{X}$ & \\
\hline $\begin{array}{l}\text { Sampled Agencies Electric and Gas Bills Information Data } \\
\text { Form }\end{array}$ & $\mathrm{DF} 4 \mathrm{a} / \mathrm{b}$ & & $\mathrm{X}$ & \\
\hline Housing Unit \& Building Information Surveys & $\mathrm{DF} 2 / 3$ & & $\mathrm{X}$ & \\
\hline
\end{tabular}

ORNL sampled 400 subgrantees from a list of 904 agencies that were slated to receive DOE funds in PY08. ORNL stratified the sample by state and allocated the subgrantee sample to states in proportion to the PY08 planned allocation of WAP funds, with a minimum of one subgrantee sampled per state.

Subgrantees were sampled within state (without replacement) with probability proportional to size, where the measure of size was the PY08 planned WAP allocation, with minimum and maximum measures of size of $\$ 10,000$ and $\$ 450,000$, respectively. Two very large subgrantees were sampled with certainty.

The evaluation team used a case manager approach to collect data from grantees and subgrantees. A team of five case managers and two outreach coordinators managed evaluation-related communications and data requests for grantees and subgrantees. Each case manager was assigned a portfolio of states and served as the designated point of contact for the grantee and subgrantees in the state. The case managers worked with their assigned grantees and subgrantees to facilitate and overcome barriers to their participation in the evaluation.

The data collection effort began with the DF1 survey for grantees in May 2010. Survey data collection for grantees continued through February 2011. Survey data collection for subgrantees began in June 2010 and was finished for nonsampled subgrantees in early December. Survey data collection for sampled subgrantees continued through July 2011.

The evaluation team made surveys available in multiple modes to allow flexibility for respondents. All surveys except DF4 were available in a secure online system developed and administered by the Dieringer Research Group, Inc. Survey instruments were also made available in Microsoft Word or PDF format. Survey responses were accepted via email, mail, fax and over the phone. The DF4 survey for sampled subgrantees was administered in Microsoft Excel, with files transferred over a secure file-sharing website to protect personal information about WAP participants.

In addition, to alleviate some of the response burden for sampled subgrantees, we partially precompleted the data forms that requested unit-level data (DF4 and DF2/3) wherever this was feasible using

\footnotetext{
${ }^{6}$ Survey instruments (marked with an ' $\mathrm{S}$ ' in their shortened names) addressed quantitative and qualitative information about program operations and administration at an aggregate level; data forms (marked with a 'DF') collected detailed information about specific weatherized units or subgrantees.
} 
information from state-level databases. In all, we migrated some data for sampled subgrantees in 18 states.

Overall participation rates ranged from 97 percent for subgrantees to 100 percent for grantees. All grantees completed two data requests, giving us a 100 percent response rate at the state level. Of the 904 subgrantees that appeared slated to receive DOE funds in PY08, 877 actually received an allocation to weatherize units and were still part of the weatherization network by the time data collection began in the spring of 2010. Of these 877 subgrantees, 852 completed at least one instrument for a participation rate of 97 percent. Similarly, among the sampled subgrantees, 396 of the 400 agencies identified in the sample actually received an allocation to weatherize units and were still part of the weatherization network when we attempted to contact them. Three hundred eighty-four of the sampled subgrantees completed at least one instrument. However, it should be noted that not every participating subgrantee completed every survey or data request, and respondents did not always answer all of the applicable questions, so response rates to individual questions are somewhat lower.

Table 1.2 lists instrument-by-instrument response rates.

Table 1.2:Response rates by instrument

\begin{tabular}{|c|c|c|}
\hline Instrument name & Label & Response Rate \\
\hline All States Agencies Information Survey & DF1 & $100 \%$ \\
\hline All States Program Information Survey & S1 & $100 \%$ \\
\hline All Agencies Overview Data Form & DF10 & $97 \%$ \\
\hline All Agencies Program Information Survey & $\mathrm{S} 2$ & $93 \%$ \\
\hline Sampled Agencies Detailed Program Information Survey & S3 & $90 \%$ \\
\hline Sampled Agencies Electric and Gas Bills Information Data Form & $\mathrm{DF} 4 \mathrm{a} / \mathrm{b}$ & $93 \%$ \\
\hline Housing Unit \& Building Information Surveys & DF2/3 & $94 \%$ \\
\hline
\end{tabular}

\subsection{DATA LIMITATIONS}

The data presented in this report are based almost exclusively on self-reports by grantees and subgrantees. While we believe that respondents answered the questions we posed in good faith, several factors impinge on the reliability and validity of the data we received. These factors include:

- Loss of institutional memory: Several respondents indicated that no one currently associated with their weatherization program was part of the organization during the program year addressed by this study.

- Respondent fatigue: Several of the instruments were lengthy, requiring several days of respondent time to complete at a time that many agencies were being audited, included in other studies, or otherwise being asked to respond to various inquiries while working toward aggressive production goals. This combination of circumstances may have limited the amount of attention respondents paid to any one question.

- Inconsistent interpretation of question meaning: Some questions could be interpreted in multiple ways. While we provided mouse-over guidance for some of the questions and clarified questions whenever asked, we suspect that respondents may have interpreted some questions in different ways than intended without seeking clarification from us.

- Recordkeeping: Some respondents keep records - especially financial records - in ways that do not lend themselves to answering specific questions in our instruments. In particular, we heard from financial staff that they do not track data in a way that maps easily to the financial matrices we included in two of the surveys. 
Two areas of particular challenge included financial data and unit counts.

\subsubsection{Financial data}

We included financial questions on several instruments and asked for funding information to be broken out in a matrix that did not always match the way grantees and subgrantees keep their records. The data we received generally matched what we would expect based on other published data, such as the National Association for State Community Services Program (NASCSP) annual funding survey and initial DOE allocations contained in the department's WinSAGA database. However, there were substantial inconsistencies in detailed numbers - both within responses from individual grantees or subgrantees and across respondents (grantee reports compared to the sum of subgrantee reports) - and some inconsistencies between aggregate amounts reported to us and published funding reports available elsewhere.

We followed up with selected grantees to understand the reasons for these apparent discrepancies and to correct obvious reporting errors. These follow-ups suggest that discrepancies among reported financial values were due to multiple factors, including:

- Adjustments to funding distribution and timing of expenditures after states submitted data to the NASCSP funding survey (one of the external data sources we used as a cross-check).

- Inconsistencies in whether funds related to weatherization, such as emergency furnace repair and replacement, were included as weatherization funding.

- Inconsistencies in whether funds coordinated by a grantee but disseminated directly from utilities to subgrantees are counted in state totals.

- Errors in funding amounts reported to us.

- Uses of funds in ways that do not have a clear home in the reporting structure we defined.

- Uncertainty by some subgrantees about the mix of funding sources that make up the allocations they receive from their grantee.

We corrected obvious errors at the direction of grantees, replaced some subgrantee reports with granteeprovided values, and used funding allocations in place of missing values for non-responding subgrantees. Nevertheless, reconciling all differences would have gone beyond the scope of our effort and respondents' abilities to answer questions. As a result, readers should keep in mind that financial data reported here may not match other data sources.

Unless otherwise noted, this report presents the funding amounts reported to us by grantees and subgrantees. A few aggregate values were drawn from other sources, such as initial DOE allocations, in order to anchor our discussion of the overall program size to official funding amounts. In those cases, we identified the external source of those data.

\subsubsection{Unit counts}

We found similar apparent discrepancies with reported numbers of housing units weatherized under the DOE program. Depending on their funding sources, local agencies can weatherize homes as part of the national WAP program or outside the confines of the program. We sought to obtain unit counts by housing type for both DOE and non-DOE projects.

For some grantees and subgrantees, we obtained different unit counts for PY08 from the department's WinSAGA database, state-maintained databases of weatherization projects, and completed units reported by subgrantees on two different instruments. It appears that there is some ambiguity about which projects should be reported as DOE units and differences in how grantees allocate total units to the WAP program. 
Furthermore, subgrantees do not consistently know which, or how many, of their projects the grantee reported as DOE units to the department.

As with financial data, unless otherwise noted, the results presented in this report draw from the responses we received from grantees and subgrantees plus whatever data we were able to extract from statewide databases provided to us by some grantees. These data may not match official department production numbers.

A few aggregate values were drawn from other sources, such as official DOE totals from the WinSAGA database, in order to anchor our discussion to official measures of overall program activity. In those cases, we identified the external source of those data.

\subsection{READING THIS REPORT}

We hope the readers will find this report straightforward to read. The following information may be helpful to those readers wishing to understand what is behind the data more fully.

\subsubsection{Subgrantee data}

Subgrantee data presented in this report draw from some questionnaires completed by the full set of respondents and others completed only by sampled subgrantees. Sampled subgrantee data are weighted to represent the full population of subgrantees in PY08. Because our response rates were high, we did not correct for non-response.

Table 1.3 displays approximate error margins due to sampling uncertainties for agency-level data drawn from the Sampled Agencies Detailed Program Survey; tables and figures to which these sampling errors apply are marked with $\mathrm{a}^{+}$in the table or figure title. Table 1.4 displays error margins for housing-unit data drawn from the Housing Unit and Building Information Surveys; tables and figures to which these sampling errors apply are marked with $\mathrm{a}^{++}$in the table or figure title. These error margins are at a 90 percent confidence level. Margins of error are highest for the hot climate zones because the number of sampled and responding agencies from those areas was lower than for the cooler climate regions. There is no sampling uncertainty for grantee-level data or subgrantee data taken from the "all agencies" survey and data form. 
Table 1.3:Sampling uncertainties for agency-level data from sampled subgrantees

\begin{tabular}{|c|c|}
\hline Agency Group & $\begin{array}{c}\text { Approximate margin of } \\
\text { error }\end{array}$ \\
\hline Overall & $+/-6 \%$ \\
\hline \multicolumn{2}{|c|}{ By program size } \\
\hline small & $+/-21 \%$ \\
\hline medium & $+/-11 \%$ \\
\hline large & $+/-7 \%$ \\
\hline very cold & $+/-9 \%$ \\
\hline cold & $+/-7 \%$ \\
\hline moderate & $+/-16 \%$ \\
\hline hot-humid & $+/-20 \%$ \\
\hline hot-dry & $+/-26 \%$ \\
\hline
\end{tabular}

Table 1.4:Sampling uncertainties for housing-unit data from sampled subgrantees

\begin{tabular}{|c|c|}
\hline $\begin{array}{c}\text { Agency Group } \\
\text { Approximate margin of } \\
\text { error }\end{array}$ \\
\hline Overall & $+/-1$ to $4 \%$ \\
\hline \multicolumn{2}{|c|}{ By program size } \\
\hline small & $+/-4$ to $12 \%$ \\
\hline medium & $+/-1$ to $6 \%$ \\
\hline large & $+/-2$ to $7 \%$ \\
\hline very cold & $+/-1$ to $8 \%$ \\
\hline cold & $+/-1$ to $5 \%$ \\
\hline moderate & $+/-3$ to $8 \%$ \\
\hline hot-humid & $+/-6$ to $16 \%$ \\
\hline hot-dry & $+/-13$ to $29 \%$ \\
\hline
\end{tabular}

\subsubsection{Comparisons by program size and climate region}

Throughout the report, we compare relevant responses from grantees and subgrantees representing different climate regions and differently sized programs.

\footnotetext{
${ }^{7}$ Margins of error are shown as ranges because they will differ depending on the degree to which there is variance between subgrantees and between weatherized units within subgrantees.
} 
Fig. 1.1 illustrates the way we defined five climate regions, which are based in large part on the climate zones recognized by DOE's Building America program except that states are uniquely assigned to a single zone. Each state was assigned to a climate region based on estimates of the heating and cooling degree days for the major population centers. ${ }^{8}$ Among temperate climates, there is also a distinction between humid and dry regions. We did not subdivide states with varied climates within their borders because WAP policies and procedures are implemented at the state-level and because an important part of our data collection was state-based.

Climate-based comparisons of weatherization programs allow readers to see differences in policies and procedures among parts of the country that are heavily heating or cooling-dominated and those that fall in-between. Similarly, size-based comparisons show differences between larger and smaller programs.

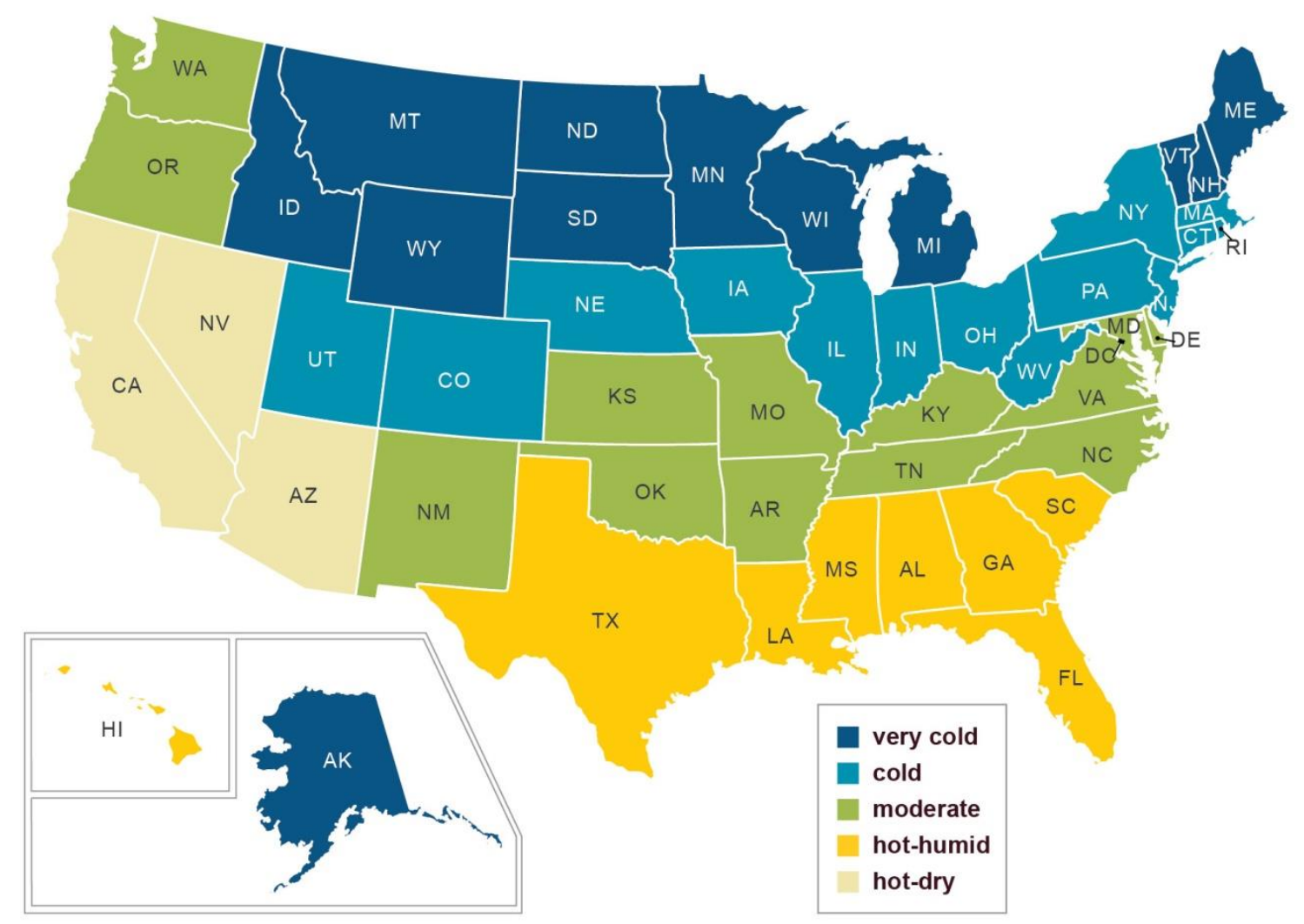

Fig. 1.1:Climate regions as used in this report

We divided grantees and subgrantees by weatherization program size. We defined grantees into quartiles of total reported weatherization funding from all sources for PY08. In this report, we will refer to the grantees that fell into the quartile with the greatest funding as large, those in the quartile with the least funding as small, and those in between as medium-sized. We followed the same approach for subgrantees. Table 1.5 shows the funding ranges for each of these groupings.

\footnotetext{
${ }^{8}$ This resulted in some allocations of states to climate regions that may seem counterintuitive. For example, while most of Nevada is quite cold, the main population center of Las Vegas has only 2276 heating degree days. By comparison, one might think of New Mexico as warmer, but the main population center of Albuquerque has 4281 heating degree days.
} 
Table 1.5 Funding ranges for grantees and subgrantees by program size

\begin{tabular}{|c|r|r|r|r|r|}
\hline \multirow{2}{*}{ Program size } & \multirow{2}{*}{ \% of agencies } & \multicolumn{2}{|c|}{ Grantees } & \multicolumn{2}{|c|}{ Subgrantees } \\
\cline { 3 - 6 } & & \multicolumn{1}{|c|}{ lower limit } & \multicolumn{1}{c|}{ upper limit } & \multicolumn{1}{c|}{ lower limit } & upper limit \\
\hline small & $25 \%$ & $\$ 0$ & $\$ 4,237,100$ & $\$ 271,533$ \\
\hline medium & $50 \%$ & $\$ 4,237,101$ & $\$ 15,526,000$ & $\$ 271,534$ & $\$ 939,782$ \\
\hline large & $25 \%$ & $\$ 15,526,001$ & no limit & $\$ 939,783$ & no limit \\
\hline
\end{tabular}





\section{HOW ARE WEATHERIZATION AGENCIES STRUCTURED AND FUNDED TO DO THEIR WORK?}

Low-income weatherization consists of a varied network of state offices (grantees) that run statewide weatherization programs and local agencies (subgrantees) that weatherize homes of eligible program clients. DOE provides a substantial amount of funding to state offices for their respective statewide programs, as well as program guidance and rules that govern the use of the DOE funds. Fig. 2.1 illustrates this three-layer structure. Many grantees and subgrantees also obtain and use other funding sources for weatherization work, some of which is used alongside DOE funds on the same homes and some of which funds a unique set of homes for projects that need not adhere to the rules of the DOE program. This chapter describes this network of weatherization-related organizations. For convenience, we will refer to the 50 states and the District of Columbia as the 51 state weatherization programs.

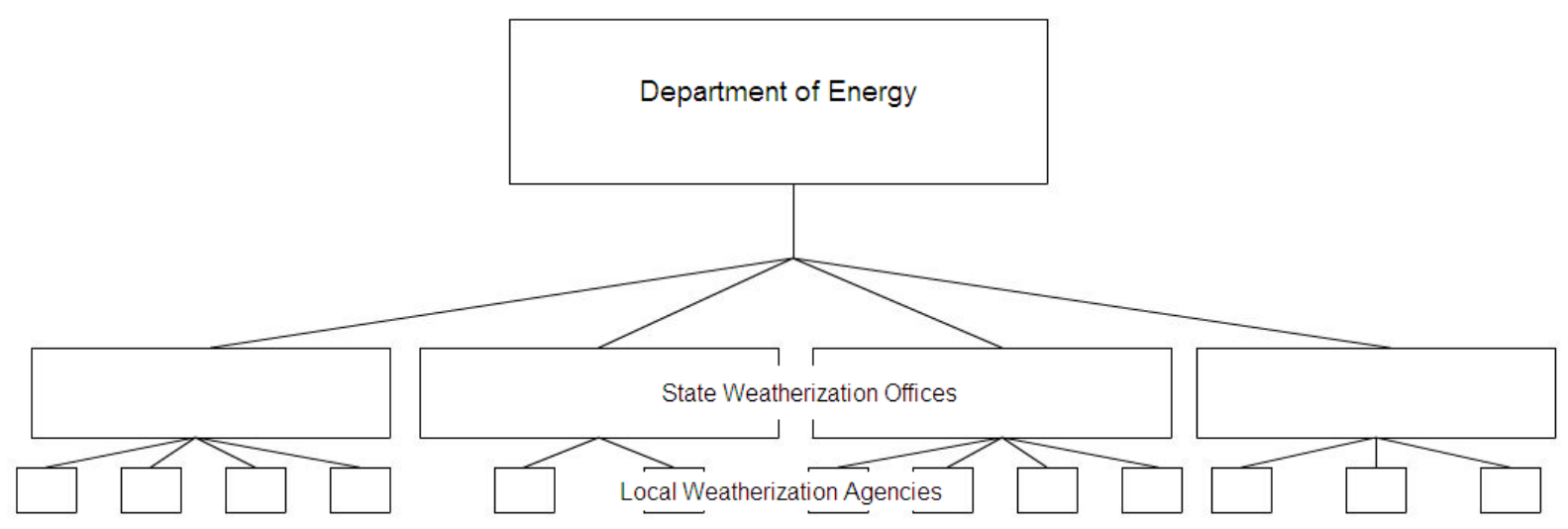

Fig. 2.1:WAP program hierarchy

\subsection{STATE PROGRAMS}

State programs consist of state administrative and technical staff (grantees) and an in-state network of local weatherization agencies (subgrantees). The state offices are all part of state government, but housed in a variety of departments. They oversee and administer the weatherization program within their jurisdiction.

Each grantee's network of subgrantees coordinates and completes weatherization of eligible clients' homes at the local level. The typical grantee subcontracted with about 17 local agencies in PY08 and allocated an average of $\$ 240,000$ in DOE funds to subgrantees. (See below for a more thorough discussion of funding allocations and non-DOE funding sources.) However, the number of subgrantees and funding allocations varied substantially, ranging from two to 64 subgrantees per state and $\$ 6,000$ to $\$ 6,000,000$ per local agency.

Interestingly, grantees in colder climates use a smaller network of local agencies and allocate more DOE funds per agency than those in warmer climates. Table 2.1 shows the mean number of subgrantees and DOE allocation per agency in PY08 by climate region. 
Table 2.1: Mean network size and funding per subgrantee by climate region

\begin{tabular}{|c|c|c|}
\hline Climate region & $\begin{array}{c}\text { Mean number of } \\
\text { subgrantees per state }\end{array}$ & $\begin{array}{c}\text { Mean DOE allocation per } \\
\text { subgrantee }\end{array}$ \\
\hline very cold & 12 & $\$ 320,000$ \\
\hline cold & 23 & $\$ 290,000$ \\
\hline moderate & 16 & $\$ 200,000$ \\
\hline hot-humid & 17 & $\$ 160,000$ \\
\hline hot-dry & 18 & $\$ 130,000$ \\
\hline all combined & 17 & $\$ 240,000$ \\
\hline
\end{tabular}

Larger grantees (those with more total weatherization funding from all sources) tend to distribute their DOE funds across larger networks of subgrantees, but keep the average allocations per agency at relatively similar levels as smaller grantees. Table 2.2 shows the mean number of subgrantees and DOE allocations per subgrantee by program size.

Table 2.2: Mean network size and funding per subgrantee by program size

\begin{tabular}{|l|c|c|}
\hline $\begin{array}{l}\text { State Program } \\
\text { Size }\end{array}$ & Mean \# of subgrantees & $\begin{array}{c}\text { Mean DOE allocation per } \\
\text { subgrantee }\end{array}$ \\
\hline Small & 8 & $\$ 240,000$ \\
\hline Medium & 16 & $\$ 220,000$ \\
\hline Large & 32 & $\$ 330,000$ \\
\hline
\end{tabular}

\subsubsection{Organizational structures}

At the grantee level, the weatherization program is often administered by a state department of social services or housing. Thirty-three of these state program offices also administer the Low Income Home Energy Assistance Program (LIHEAP), which provides payments to eligible households' energy providers to offset some of their energy costs and can fund some weatherization activities as well. ${ }^{9}$ While weatherization and energy assistance are the largest low income energy programs in most states, the offices that administer the weatherization program also run other programs, including:

- Community Service Block Grants (10 states)

- HOME Investment Partnerships Program (9 states)

- Community Development Block Grants (5 states)

- Emergency Shelter Grant Program (5 states)

- (Unspecified) tax credits (5 states)

- Public housing (4 states)

- Weatherization Plus / Rehabilitation Program (4 states)

The placement of the weatherization program within the hierarchy of the state's executive branch varies widely. In nearly half of the states $(47 \%)$, the program is only one or two layers removed from the governor's office, which we categorized as close proximity to political decision-making. In most of the remaining states (another 47\%), the weatherization program is located between three and five layers away from the governor's office. We labeled these as being a medium distance removed from political

\footnotetext{
${ }^{9}$ The other 17 states administer LIHEAP through a different agency than the one responsible for WAP administration.
} 
decision-making. The remaining few states have weatherization programs that are located even further away from the governor's office-six layers or more.

There is no clear pattern for how proximity to the governor's office varies with program size. As shown in Fig. 2.2, more of the medium-sized programs were placed close to the governor's office in PY08, while small and large programs tended to be further removed.

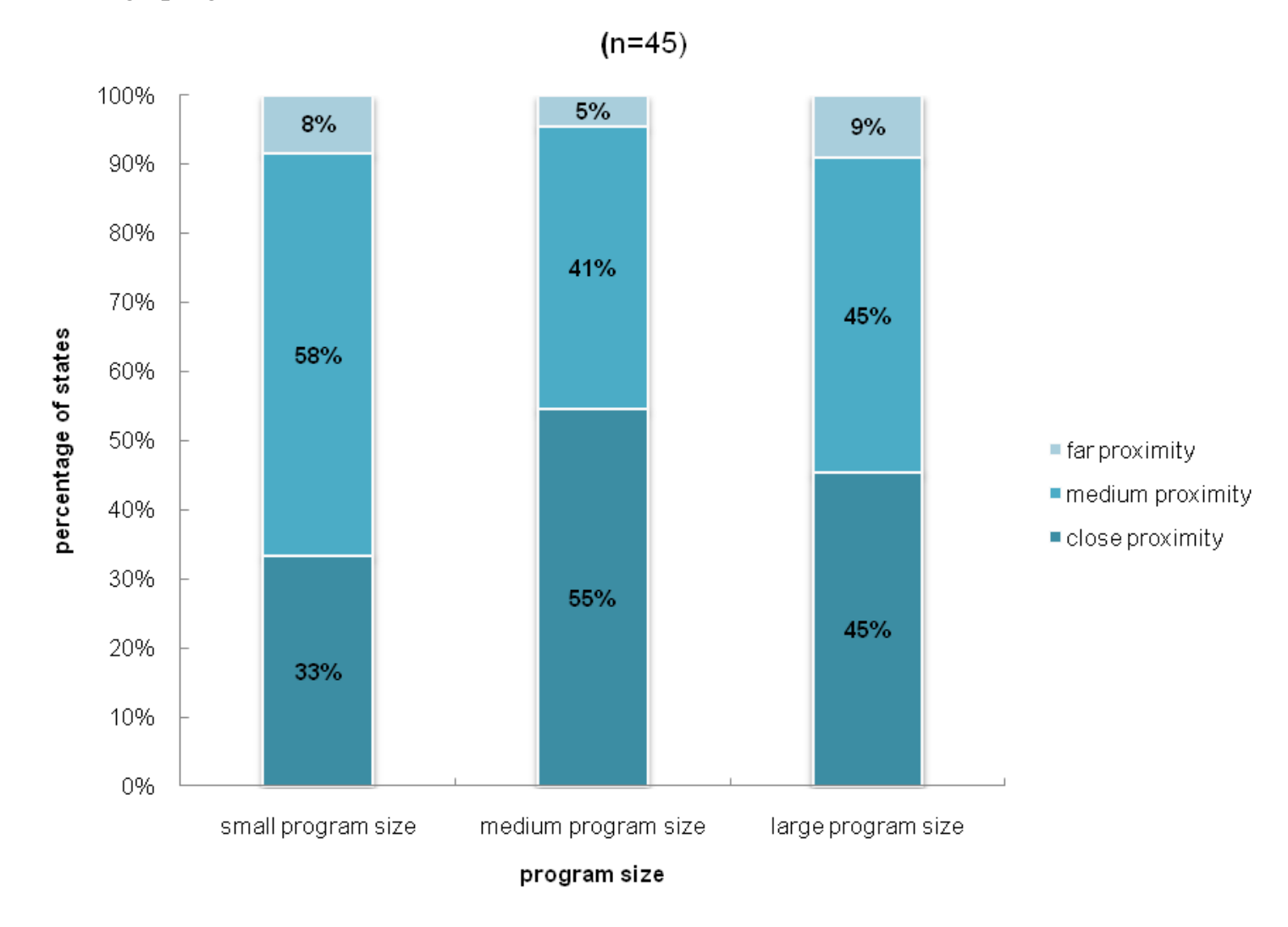

Fig. 2.2:WAP office proximity to governor's office (by program size)

The coldest climate states tend to place their weatherization programs further from the governor's office while the warmer states maintain fewer layers between the weatherization program and the governor's office. As Table 2.3 illustrates, only 30 percent of very cold climate states had their weatherization offices placed one or two layers from the governor's office in PY08, while the other climate regions had between 43 and 67 percent of their weatherization offices within one or two layers from the governor's office. 


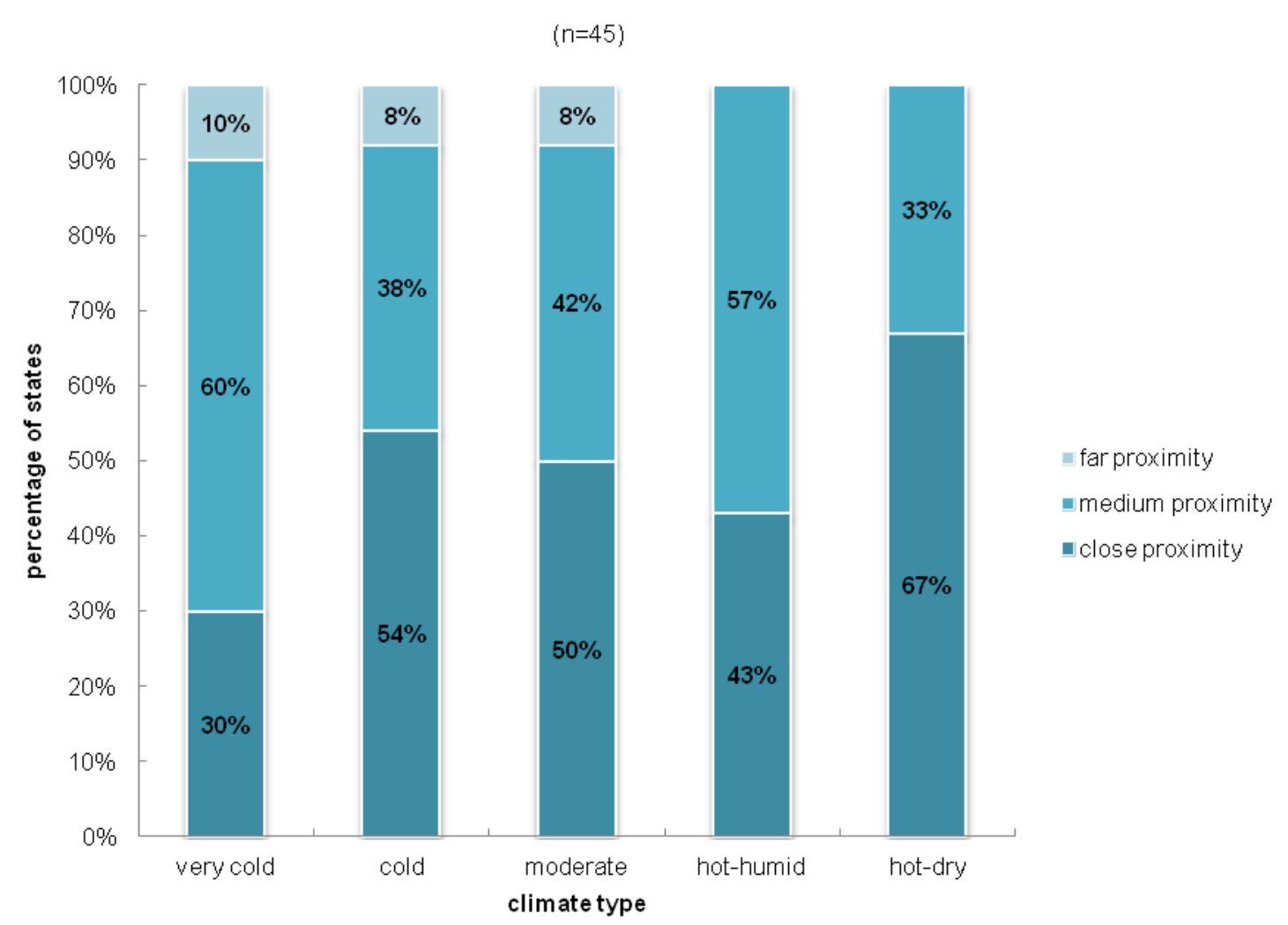

Fig. 2.3:WAP office proximity to governor's office (by climate region)

Weatherization offices are run by the grantee's weatherization director. In a majority of states (82\%), the weatherization directors were civil servants in PY08 with political appointees accounting for the remainder (18\%). Political appointees were slightly more common among small programs, where 25 percent of weatherization directors were political appointees. No grantee programs were run by elected officials.

The average (mean) tenure of the weatherization directors active in PY08 was eight years, but their experience in that position ranged from less than a year to 30 years. Thirty-six percent had been in that position for more than 10 years.

Generally, there was moderate turnover in weatherization program leadership during the ten years prior to PY08. The majority of grantees (57\%) were directed by two or three individuals during that ten-year period. Thirty-three percent of grantees had low turnover, maintaining the same weatherization director for the entire decade, and ten percent experienced high turnover, having been directed by four or more individuals during that time.

Turnover was somewhat greater among small programs than larger ones, as shown in Fig. 2.4, and sporadic differences by climate region, as shown in Fig. 2.5. 


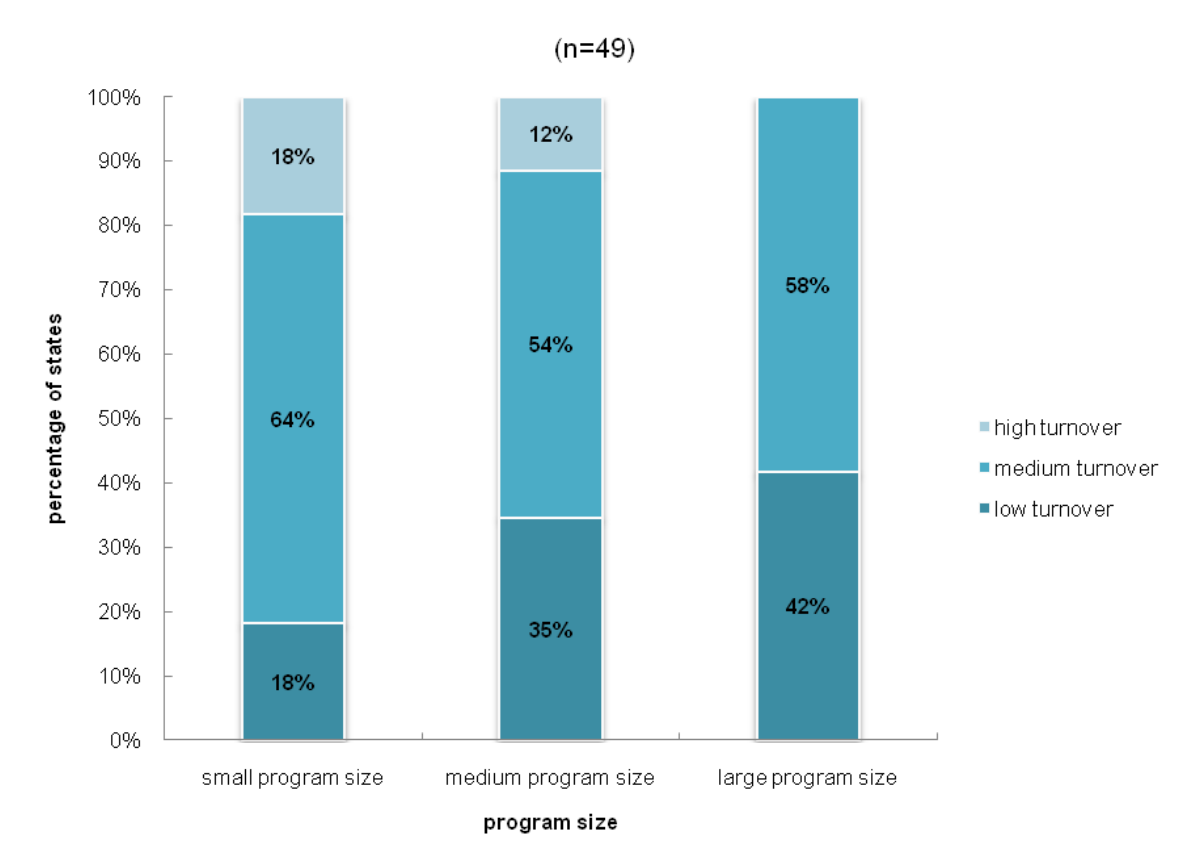

Fig. 2.4:Turnover in weatherization director position during 1999-2008 (by program size)

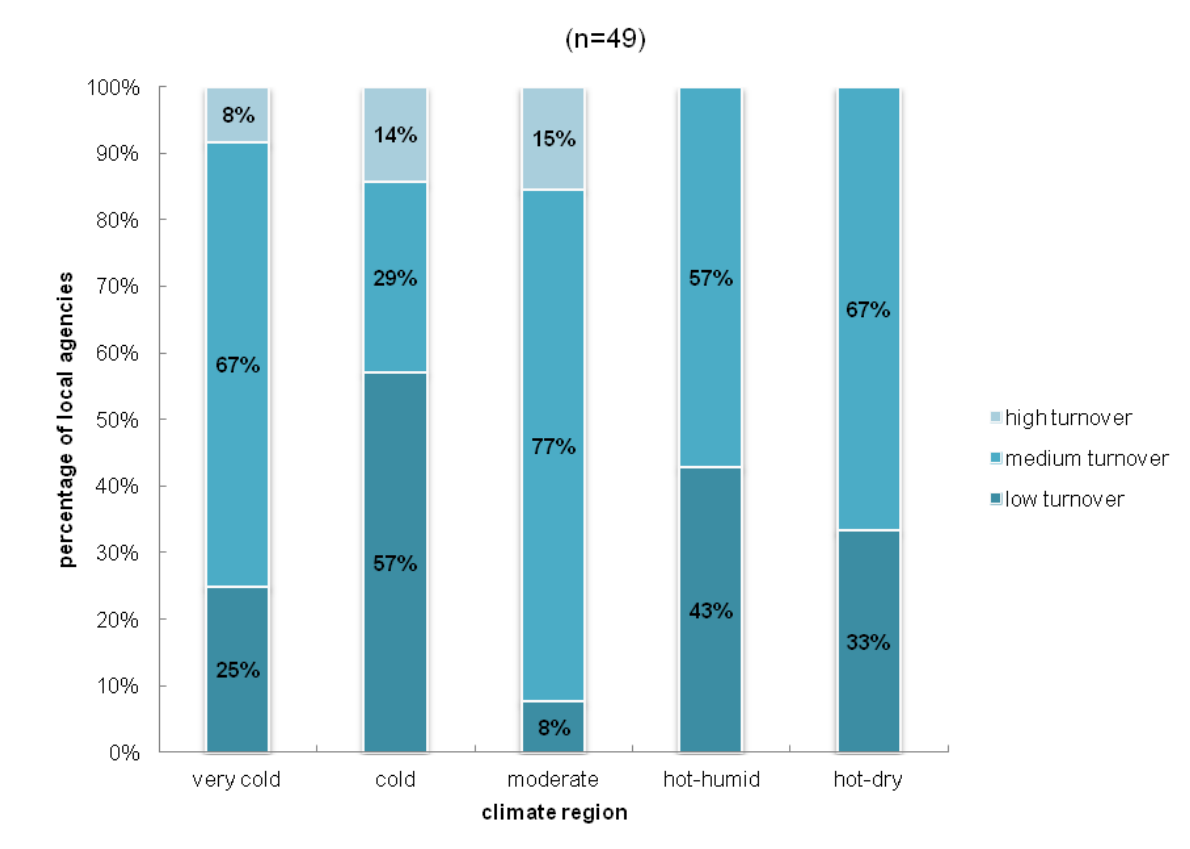

Fig. 2.5:Turnover in weatherization director position during 1999-2008 (by climate region)

\subsubsection{Staffing and responsibilities}

On average, grantees have eight full-time equivalent (FTE) positions for a total of about 400 state-level staff10 administering the 51 weatherization programs in the states and the District of Columbia. These positions serve several supporting functions needed to run the statewide programs. As one would expect

\footnotetext{
${ }^{10}$ These 400 full-time equivalent positions are distributed among approximately 500 individuals.
} 
with any program, there is some management and administration - usually two to three positions - to handle program and staff oversight, grant management, program reports to DOE, and accountability to the state agency within which the program is housed.

Most of the remaining positions at the grantee level are designed to ensure effective implementation of weatherization by the subgrantees. One of these functions - agency monitoring - tracks subgrantee performance and thereby provides a quality assurance function. Staffing levels for agency monitoring tends to vary with program size, ranging from one or two people in small programs to four or more in large programs. The other supporting function - training and technical assistance-provides direct and indirect assistance to subgrantees on technical matters related to weatherization. This function comprises another one to six positions, depending on program size, but can be as high as 14 positions.

Table 2.3 below summarizes grantee staffing by function and program size. Not surprisingly, the average number of FTE staff increases with increasing program size across each support function except for the "other" category. Small programs reported a large number of staff in the "other" category, perhaps because staff from small programs perform multiple support functions.

Table 2.3: Grantee support functions in Program Year 2008-in FTE staff

\begin{tabular}{|c|c|c|c|}
\hline Support function & $\begin{array}{c}\text { Grantees with small } \\
\text { programs } \\
(\text { pop }=12)\end{array}$ & $\begin{array}{c}\text { Grantees with mid-sized } \\
\text { programs } \\
(\text { pop }=26)\end{array}$ & $\begin{array}{c}\text { Grantees with large } \\
\text { programs } \\
(\text { pop }=12)\end{array}$ \\
\hline $\begin{array}{l}\text { Management \& } \\
\text { administration }\end{array}$ & $\begin{array}{l}\text { Mean: } 1.89 \\
\text { Range: } .25 \text { to } 5 \text { FTE } \\
\mathrm{n}=7\end{array}$ & $\begin{array}{l}\text { Mean: } 2.43 \\
\text { Range: } .5 \text { to } 12 \text { FTE } \\
n=23\end{array}$ & $\begin{array}{l}\text { Mean: } 3.56 \\
\text { Range: } 1 \text { to } 7 \text { FTE } \\
\mathrm{n}=10\end{array}$ \\
\hline Agency monitoring & $\begin{array}{l}\text { Mean: } 1.35 \\
\text { Range: } .1 \text { to } 5 \text { FTE } \\
\mathrm{n}=6\end{array}$ & $\begin{array}{l}\text { Mean: } 2.58 \\
\text { Range: } .1 \text { to } 18 \text { FTE } \\
\mathrm{n}=21\end{array}$ & $\begin{array}{l}\text { Mean: } 4.30 \\
\text { Range: } .5 \text { to } 7 \text { FTE } \\
\mathrm{n}=10\end{array}$ \\
\hline $\begin{array}{l}\text { Training \& technical } \\
\text { assistance }\end{array}$ & $\begin{array}{l}\text { Mean: } 1.00 \\
\text { Range: } .5 \text { to } 2 \text { FTE } \\
\mathrm{n}=5\end{array}$ & $\begin{array}{l}\text { Mean: } 1.55 \\
\text { Range: } .3 \text { to } 6 \text { FTE } \\
\mathrm{n}=19\end{array}$ & $\begin{array}{l}\text { Mean: } 5.74 \\
\text { Range: } .5 \text { to } 14 \text { FTE } \\
\mathrm{n}=10\end{array}$ \\
\hline Other & $\begin{array}{l}\text { Mean: } 4.00 \\
\text { Range: } 4 \text { to } 4 \text { FTE } \\
n=1\end{array}$ & $\begin{array}{l}\text { Mean: } 0.49 \\
\text { Range: } 0 \text { to } 1 \text { FTE } \\
n=7\end{array}$ & $\begin{array}{l}\text { Mean: } 2.5 \\
\text { Range: } 0 \text { to } 6.8 \text { FTE } \\
n=4\end{array}$ \\
\hline
\end{tabular}

\subsubsection{Funding and production}

The Weatherization Assistance Program provides an important source of funding for low-income weatherization, but it is not the only source. Grantees and subgrantees use a mixture of funding sources to pay for weatherization program activities. While the funding scenarios vary greatly from state to state, Fig. 2.6 illustrates the aggregated national funding scenario as reported to us by the grantees and subgrantees.

Altogether, grantees received nearly $\$ 720$ million to support their weatherization programs in PY08. Of that amount, grantee weatherization offices spent about $\$ 42$ million on their activities and passed along $\$ 677$ million to subgrantees for their work. The DOE WAP program and LIHEAP comprise the two main funding sources for the grantees, accounting for 77 percent of the funds, but some grantees also receive substantial support from state funding sources, state public benefit programs, and utility support. In addition, some state and utility funds go directly to local agencies and are not counted in these amounts. 
We estimate that funds provided directly to subgrantees from other sources totaled at least $\$ 76$ million in PY08. Of this amount, utility funds provided directly accounted for $\$ 46$ million and state funds provided independently of the state weatherization office accounted for $\$ 30$ million. This estimate is probably on the conservative side because we counted only funds reported to us by subgrantees whose state offices do not also distribute funds of that type. ${ }^{11}$ Funds received by subgrantees who did not report their funding mix to us are not included here. ${ }^{12}$ Furthermore, funds from state and utility sources reported by subgrantees are also not included in our estimate of direct funding if the grantee reported providing such funding to its network of subgrantees.

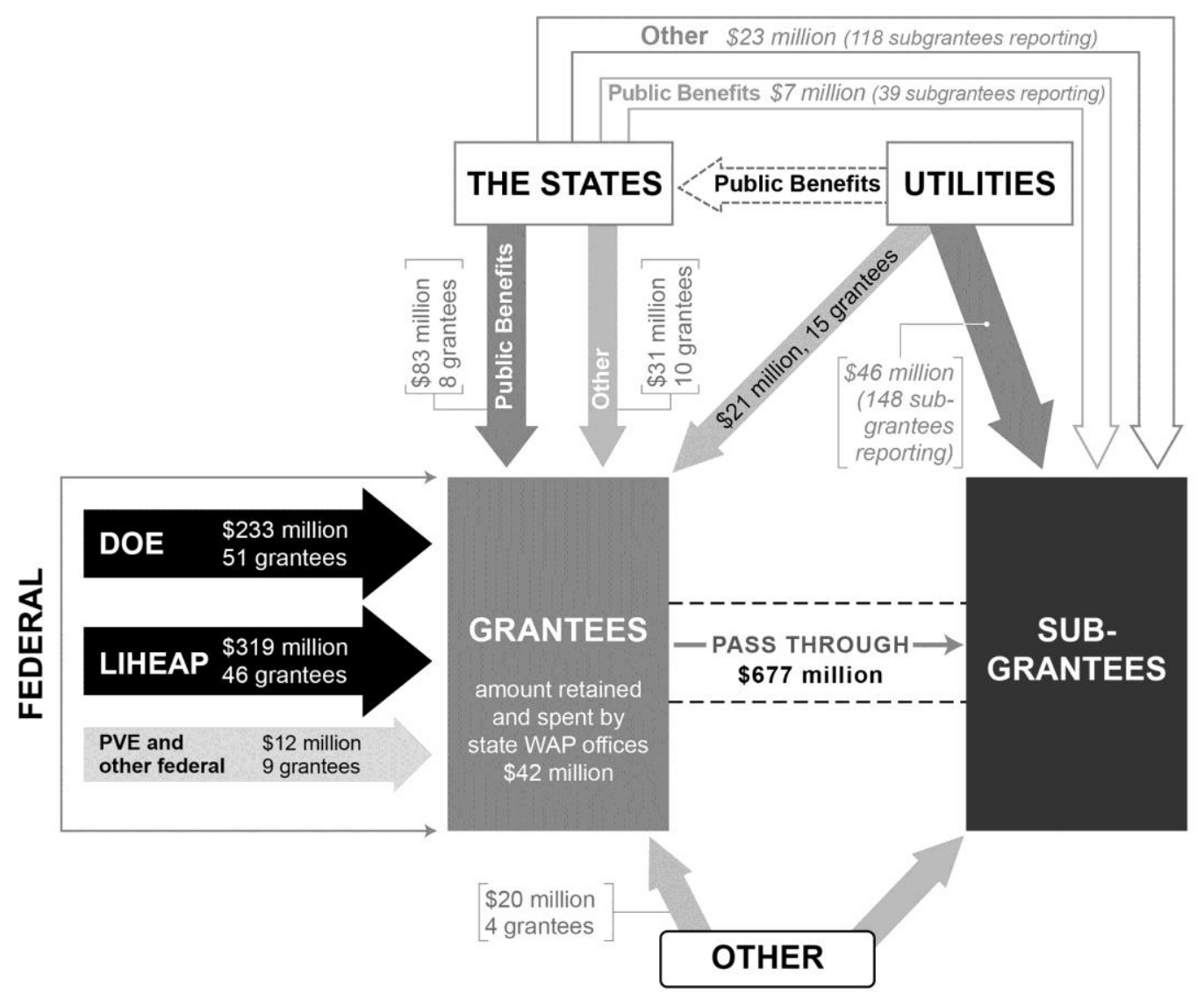

Fig. 2.6: Weatherization funding structure for Program Year 2008

Based on official DOE unit counts and subgrantees reports, we estimate that subgrantees weatherized approximately 180,000 units in PY08. Slightly more than half of these units are generally considered to be DOE units, meaning that they were funded entirely or partially using DOE funds and, therefore, were required to adhere to DOE program rules and standards. The remaining weatherization projects included both non-comprehensive and comprehensive weatherization projects, some of which also adhered to DOE

\footnotetext{
11 Hence, utility or state funds given directly to subgrantees are excluded if the grantee in that jurisdiction also distributes utility funds or the same kind of state funds to subgrantees. It is possible, however, that some subgrantees received such funds from both the grantee and the funding source directly. We needed to make this simplifying assumption due to limitations in the data available.

${ }^{12}$ Our analysis includes direct allocations of utility funds to 148 subgrantees, state public benefit funds to 39 subgrantees, and other state funds to 118 subgrantees. We estimate that additional funds may have been provided directly to the following numbers of subgrantees who did not provide funding data in sufficient detail for our analysis: utility - up to 40 ; state public benefits - up to 28; other state funds - up to 52 .
} 
standards. Table 2.4 shows the overall distribution of weatherization projects completed by the subgrantee weatherization network by building type and DOE funding status in PY08.

Table 2.4: Program Year 2008 units weatherized by WAP subgrantees*

\begin{tabular}{|l|c|c|}
\hline Type of Structure & $\begin{array}{c}\text { Number of units weatherized as } \\
\text { part of the DOE program }\end{array}$ & $\begin{array}{c}\text { Number of units weatherized } \\
\text { outside the DOE program }\end{array}$ \\
\hline single-family & 62,835 & 49,897 \\
\hline small multifamily (2-4 units) & 17,047 & 6,231 \\
\hline large multifamily (5+ units) & 17,754 & 16,416 \\
\hline mobile homes & 329 & 10,394 \\
\hline shelter & no data \\
\hline
\end{tabular}
* DOE units shown in the table are based on DOE's WinSAGA database, while nonDOE units are based on subgrantee reports. NonDOE units exclude projects
completed by subgrantees that no longer exist or did not respond to our data requests.

DOE rules are being applied to large shares of the funds received from LIHEAP and state public benefits funds as summarized in Fig. 2.7.

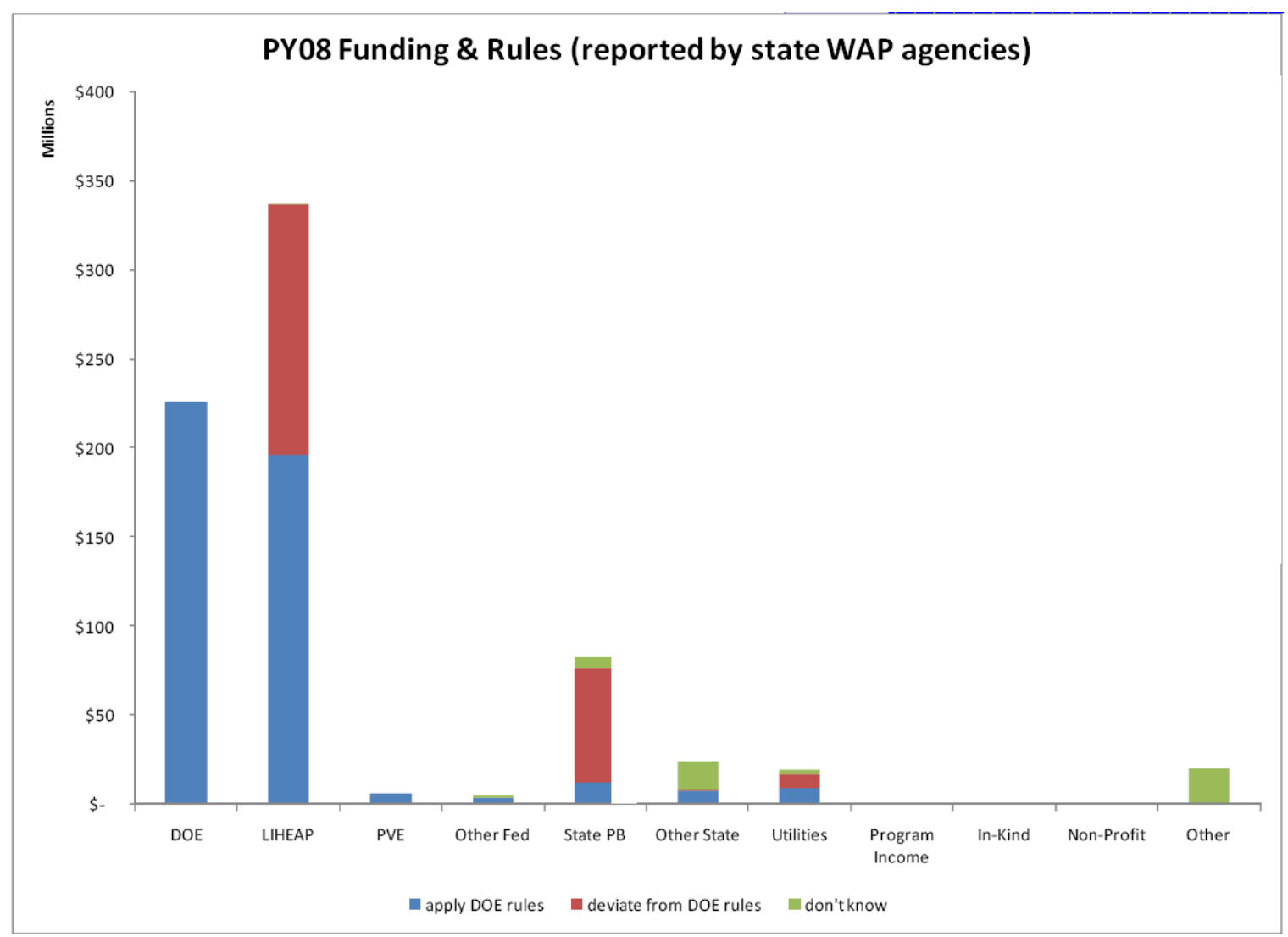

Fig. 2.7:Differences in rules between DOE funds and other sources

There were a number of departures from DOE rules in the LIHEAP rules. The differences involved measures that were not allowed per DOE rules, including allowances for repair work such as furnace repair/replacement, freezer replacement, roof replacement and reweatherization. If there was a crisis situation, LIHEAP would allow for more flexibility and installed heating equipment. Also, LIHEAP did 
not have to meet SIR values and the funds could be used to address health and safety measures found in the home. In terms of costs, unit expenditure average was higher for LIHEAP, with higher allowable costs for health and safety related repairs.

When comparing the State Public Benefits funds and DOE sources, a number of differences were also noted between the rules governing the expenditures of funds. Like LIHEAP, there were differences in the allowable measures, such as roof and heating system replacements, and reweatherization eligibility. The State Public Benefits funds allowed for higher income eligibility limits.

\subsubsection{Leveraging}

Grantees reported that leveraging DOE funds was important to their program funding. Eighty-two percent of grantees indicated that leveraging was important to their program. As noted in Fig. 2.6 above, nearly all grantees received leveraged (non-DOE) funds. Indeed, for 21 of the grantees, leveraged funds accounted for half or more of their total program funding. Not surprisingly, as shown in Fig. 2.8, grantees with larger programs found leveraging to be more important than smaller programs that are less likely to rely on leveraged funds.

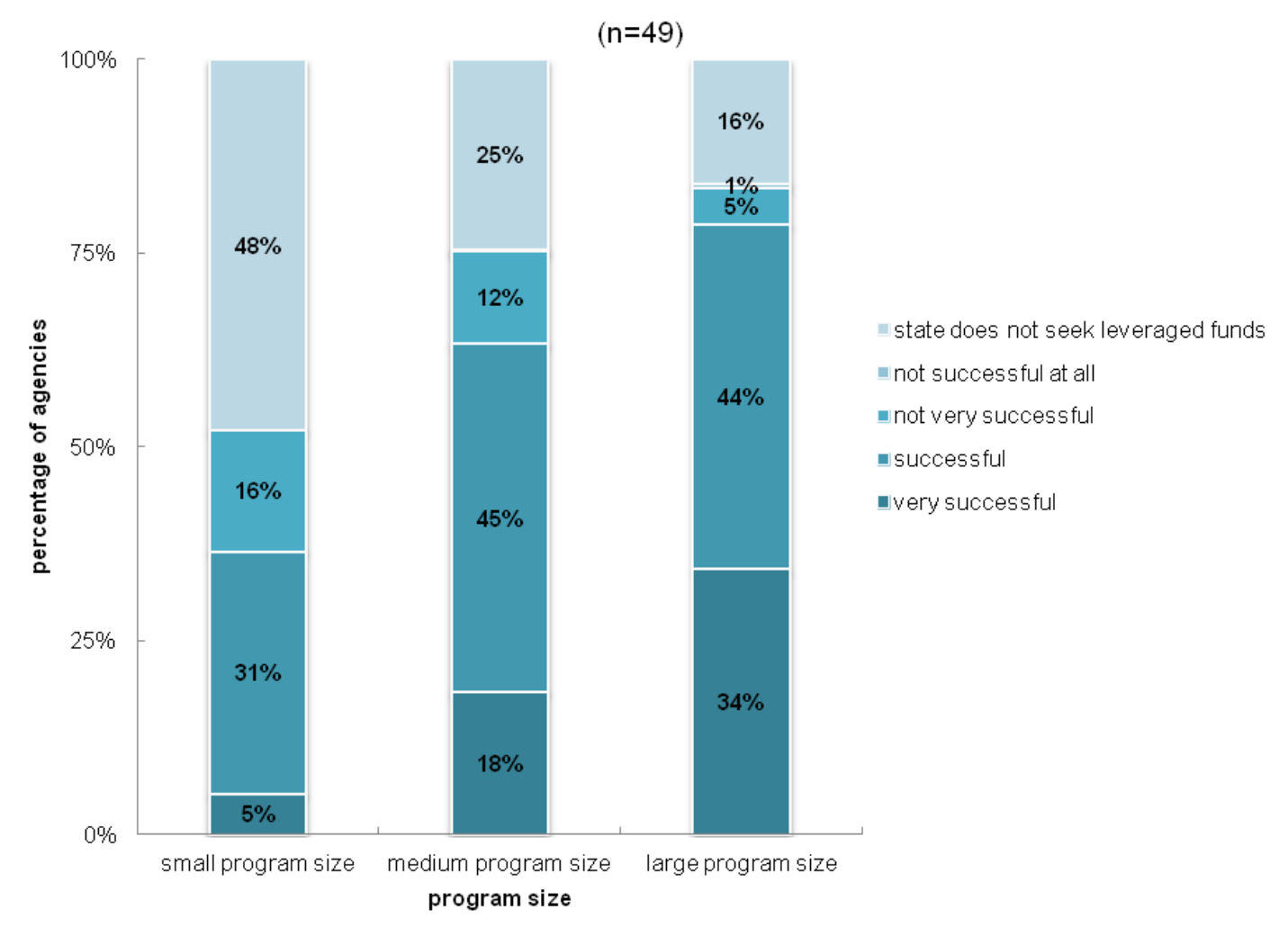

Fig. 2.8:Importance of leveraged resources by program size

Most grantees seek leveraged funds - only 14 percent of responding grantees indicated that they do notbut only a minority -29 percent of grantees — set aside funds to advocate for leveraged resources. Those 
that set aside funds feel that they were no more successful in acquiring leveraged funds than those who did not allocate financial resources to pursue leveraged resources (see Fig. 2.9).

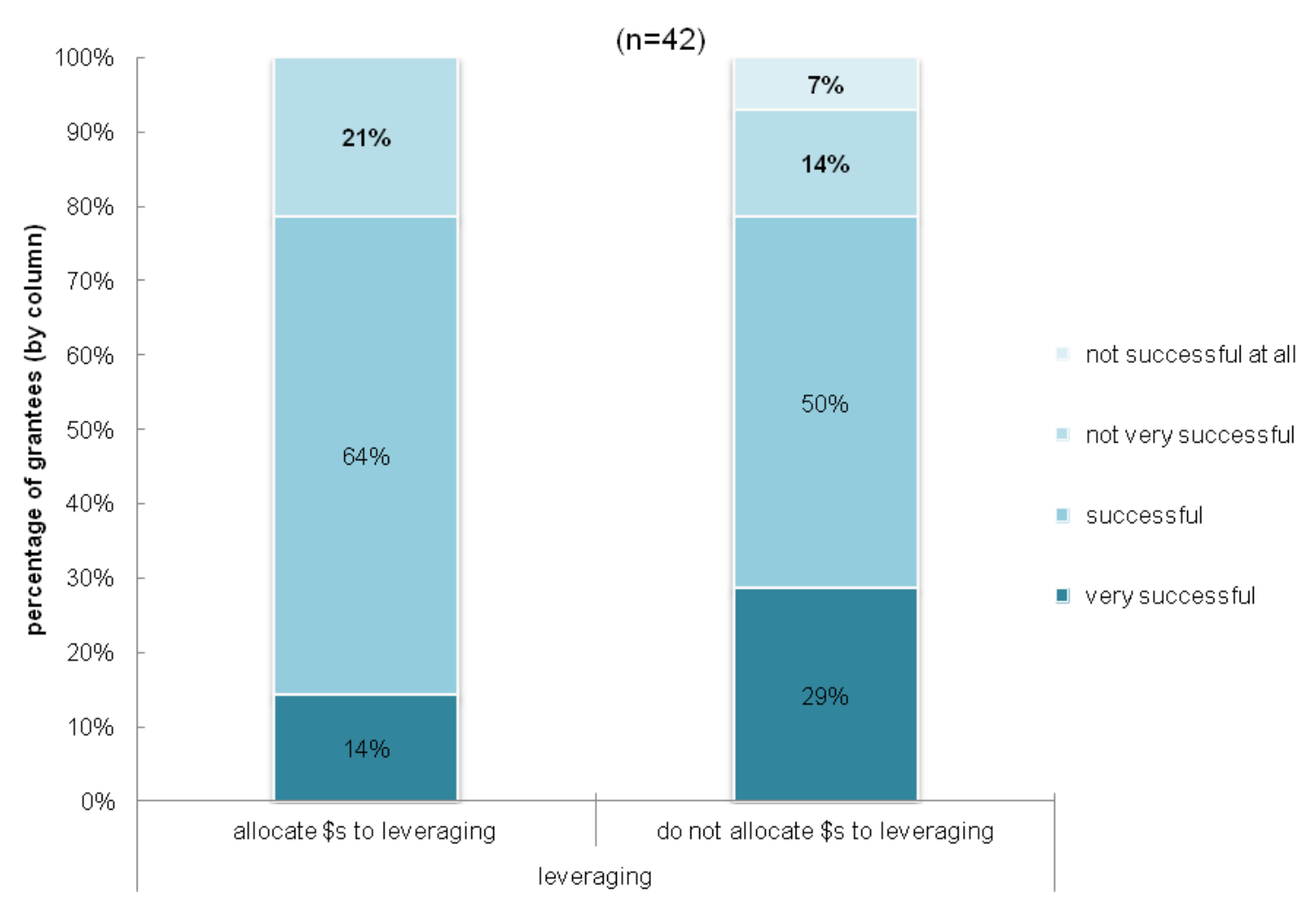

Fig. 2.9:Grantee success in attracting leveraged funds (by resources allocated)

Barriers grantees reported to greater success in acquiring leveraged resources included availability and competing interests for funding, lack of political interest, and insufficient staffing.

Some grantees (19\%) reported having modified their program in some way in the three years prior to PY08 to facilitate spending and reporting on leveraged funds.

\subsection{SUBGRANTEES}

Clients see and experience the weatherization program through their interactions with the subgrantee serving their area. In PY08, about 900 organizations nationwide were slated to receive DOE funds for their weatherization activities. This section describes their organizational structure, staffing levels and staff roles, funding and production.

\subsubsection{Organizational structures}

Most subgrantees are locally based nonprofit organizations. Eighty-seven percent of respondents selfidentified into that category, while five percent were county government agencies and another four percent were local government agencies. A handful of subgrantees are Native American tribes and the remainder are other assorted types of entities. 
Some weatherization subgrantees focus only on weatherization while others have weatherization programs alongside a variety of other social service functions. In both cases, someone functions as the weatherization program director. On average, the program directors serving in PY08 had been in that position for 11 years (mean; median $=8$ years). Seventeen percent of subgrantee programs were being directed by someone who had been in that position for a year or less, while another 17 percent of program directors had 20 or more years of experience on the job.

Generally, there was light turnover in the ten years before PY08: a large majority of subgrantees (80\%) were directed by one or two individuals during that period. Half of the subgrantees maintained the same weatherization director for the entire decade, and seven percent were directed by four or more individuals during that time.

Most subgrantees have a relatively flat organizational hierarchy. As shown in Fig. 2.10, three quarters of agencies have at most one layer of management or supervision between the weatherization program director and the weatherization crews that install measures in client homes.

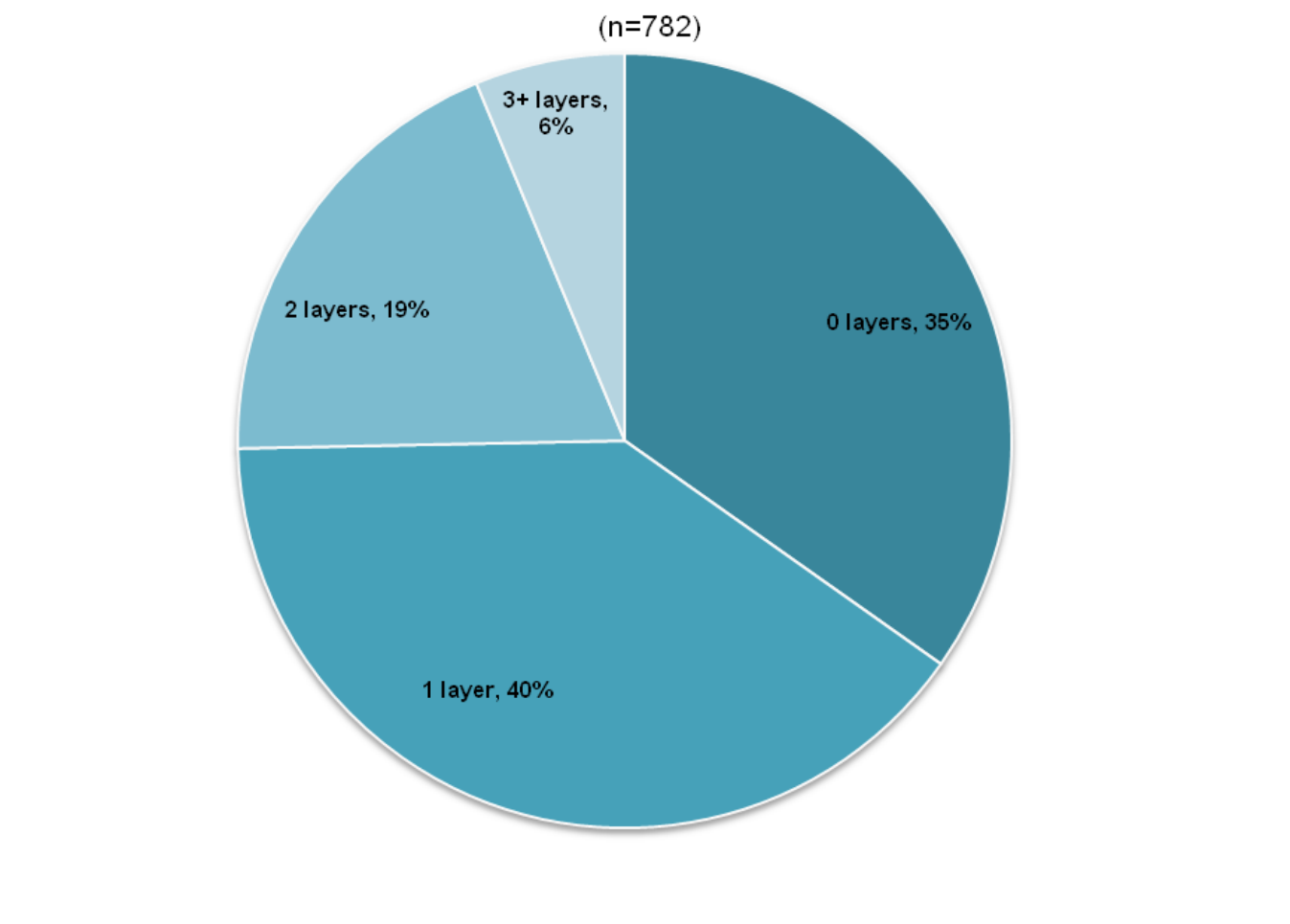

Fig. 2.10:Layers of supervision between program director and crews in subgrantees

\subsubsection{Responsibilities and staffing}

The functional responsibilities of subgrantee weatherization agency staff can be divided into three main categories:

- Program management and administration: Includes office functions such as program management, client intake, outreach, and all administrative tasks. ${ }^{13}$

- Auditing/inspection: Entails the field work needed to determine what measures would costeffectively increase a home's energy efficiency and what other work needs to be done for health

\footnotetext{
${ }^{13}$ Note that our survey questions focused on weatherization program activities and staff, so we use the term administration to refer generally to activities within the weatherization programs run by local organizations, even if there is additional administrative oversight at the executive level of the organization.
} 
and safety reasons. It also includes the post-inspection visit to ensure the weatherization work was performed to standards.

- Weatherization: Entails the installation of all specified energy efficiency and health and safety measures.

We estimate there were about 7,600 people employed by subgrantee weatherization agencies in PY08. Table 2.5 further divides this workforce total by job function and program size. It appears that a typical, mid-sized weatherization program has a staff of three staff that manage and administer the program, two auditors/inspectors, three weatherization installers, and one person assigned to other responsibilities. The mean number of staff in each functional category increases with program size. Please note that these are total individuals. We were not able to reliably estimate FTE positions because of the high non-response rate on the pertinent question in our survey.

Table 2.5: Subgrantee staffing by function in Program Year 2008

\begin{tabular}{|l|l|l|l|}
\hline Function & \multicolumn{1}{|c|}{$\begin{array}{c}\text { Subgrantees with small* } \\
\text { programs } \\
(\mathbf{n = 1 8 3 )}\end{array}$} & \multicolumn{1}{|c|}{$\begin{array}{c}\text { Subgrantees with mid- } \\
\text { sized programs } \\
(\mathbf{n}=\mathbf{4 1 3})\end{array}$} & $\begin{array}{c}\text { Subgrantees with large } \\
\text { programs } \\
(\mathbf{n}=\mathbf{1 6 9})\end{array}$ \\
\hline $\begin{array}{l}\text { Program management / } \\
\text { administration }\end{array}$ & $\begin{array}{l}\text { Mean: } 2 \\
\text { Total: } 350\end{array}$ & $\begin{array}{l}\text { Mean: } 3 \\
\text { Total: } 1,230\end{array}$ & $\begin{array}{l}\text { Mean: } 5 \\
\text { Total: } 870\end{array}$ \\
\hline Auditing / inspection & Mean: 1 & Mean: 2 & Mean: 4 \\
& Total: 230 & Total: 730 & Mean: 8 \\
\hline Weatherization & Mean: 2 & Mean: 3 & Total: 1,350 \\
(in-house only) & Total: 350 & Mean: 1,250 & Mean: 2 \\
Other & Mean: $<1$ & Total: 300 & 270 \\
\hline
\end{tabular}

The work of the weatherization crews is a staff-intensive function, particularly for large subgrantees. Some agencies maintain in-house crews while others contract out for the installations entirely or for particular types of work, such as heating system replacements and repair. (See the cost section for additional insights about the use of contractors.)

Turnover among subgrantee agency staff had been low in the three years leading to PY08. As shown in Fig. 2.11, the majority of subgrantees reported no turnover in management/administration (72\%), auditing and inspections (71\%), and weatherization (57\%). Where there was turnover, it tended to be light and rarely involved a complete exchange of applicable staff. 


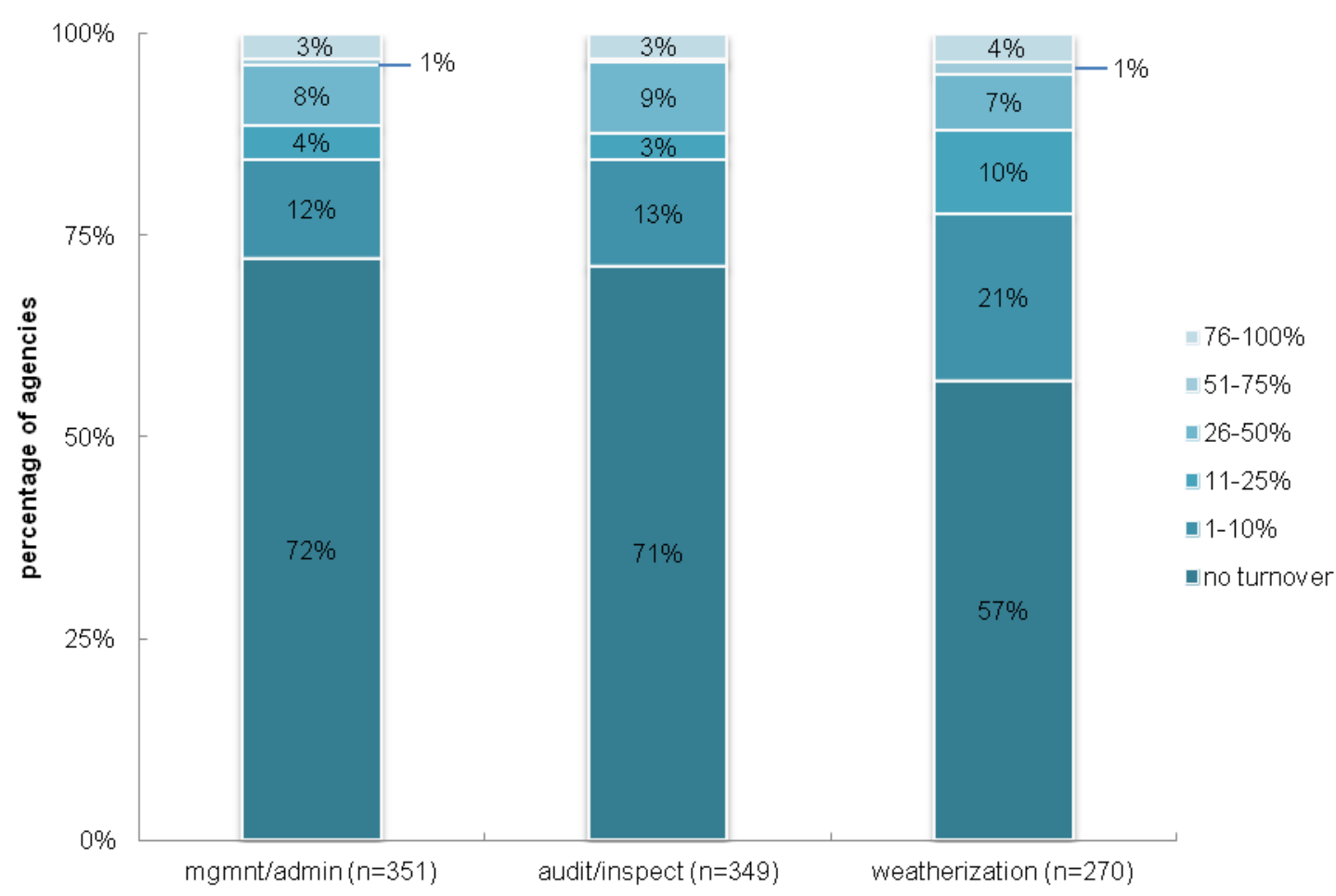

Fig. 2.11:Turnover in subgrantees by functional category (Program Years 2005 through 2007) ${ }^{+}$

\subsubsection{Funding}

Fig. 2.6 and Table 2.4 above already depicted the funding flows and source for weatherization in PY08 and the units weatherized by subgrantees in that year.

Any individual subgrantee's funding structure will look different than shown in the figure, however. Some subgrantees rely heavily or exclusively on whatever funds the grantee provides, while others have forged their own relationships with funding sources - primarily utilities and other programs within state government.

Fig. 2.12 illustrates various subgrantee funding models. As shown, 126 subgrantees (16\% of those from whom we had sufficient data for this analysis) reported to us that they used only DOE funds in PY08 and 278 subgrantees $(35 \%)$ relied almost exclusively on a combination of DOE and LIHEAP funds they received from the state office. In contrast, 391 subgrantees (49\%) reported the use of non-DOE/LIHEAP funds for at least 10 percent of their funding - 141 of these (18\% of reporting agencies) drew less than half of their funding from DOE and LIHEAP (typical mainstays of weatherization).

Subgrantees for individual grantees do have a tendency to follow similar funding models. For 23 of the grantees, three quarters or more of the subgrantees followed the same funding model. Among 10 grantees, all subgrantees used the same model. 


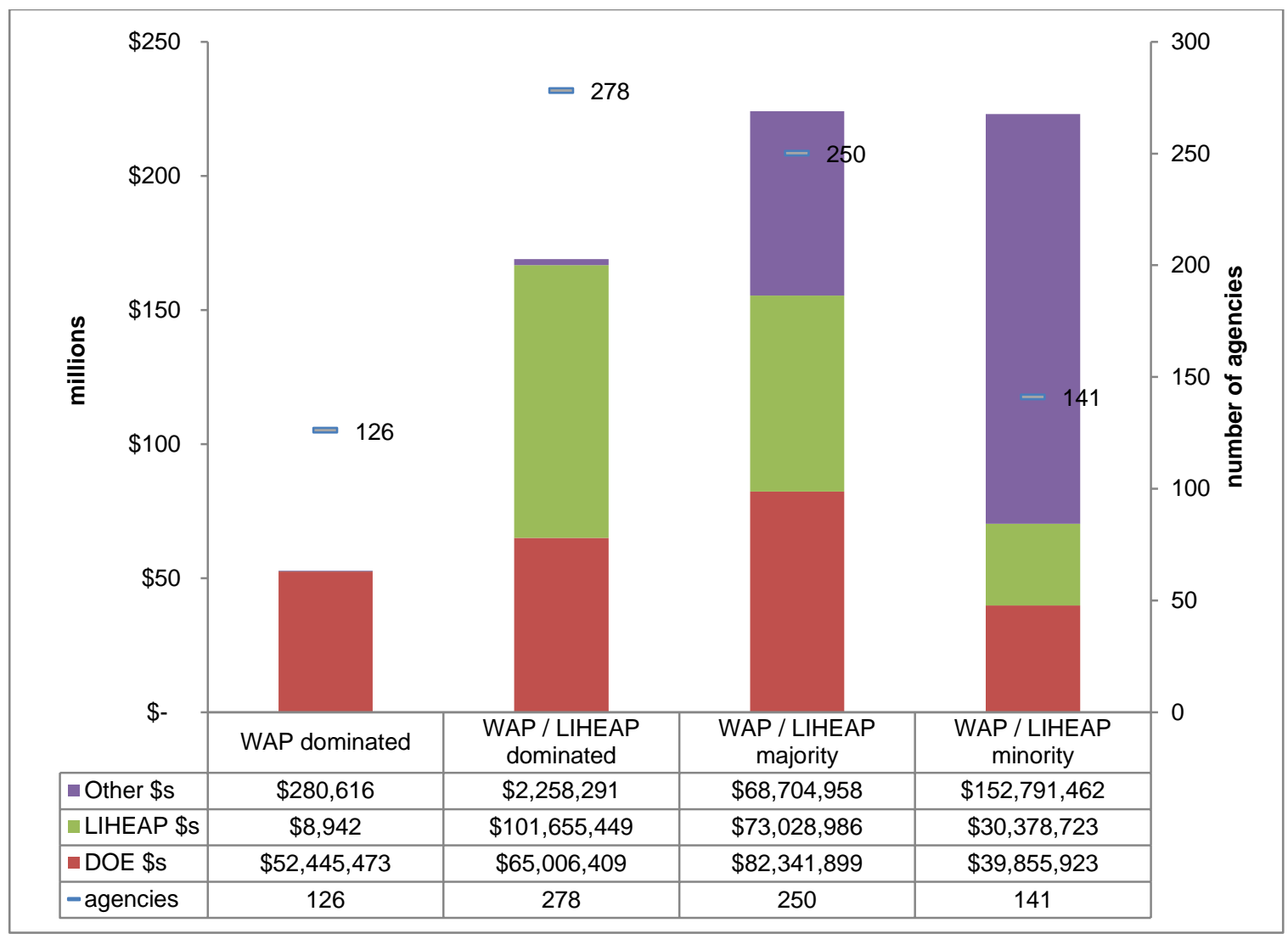

Fig. 2.12:Subgrantee funding models

\subsubsection{Leveraging}

Leveraging, the contribution of weatherization funds from sources other than DOE, is an important aspect of the program. As shown in Fig. 2.6, the DOE WAP program represented approximately 32 percent of the grantee-administered weatherization funding in PY08. LIHEAP was the largest funding source, representing 44 percent of the funding total reported for that program year. State funding - ratepayerfunded public benefits funds and other state programs - represented 15 percent of the total. Direct funding to subgrantees appears to expand the total by at least 11 percent.

Seventy nine percent of subgrantees reported that leveraged funds were important or very important to their PY08 weatherization efforts. As 
illustrates the importance of leveraging increases with program size. Eighty-eight percent of large programs reported that leveraging was important or very important, compared with 64 percent of small programs. Despite the importance of leveraging, however, only 13 percent of subgrantees reported that they set aside funding to advocate for leveraged resources in PY08. This percentage was slightly higher in large subgrantees (15 percent) than in small ones (11 percent).

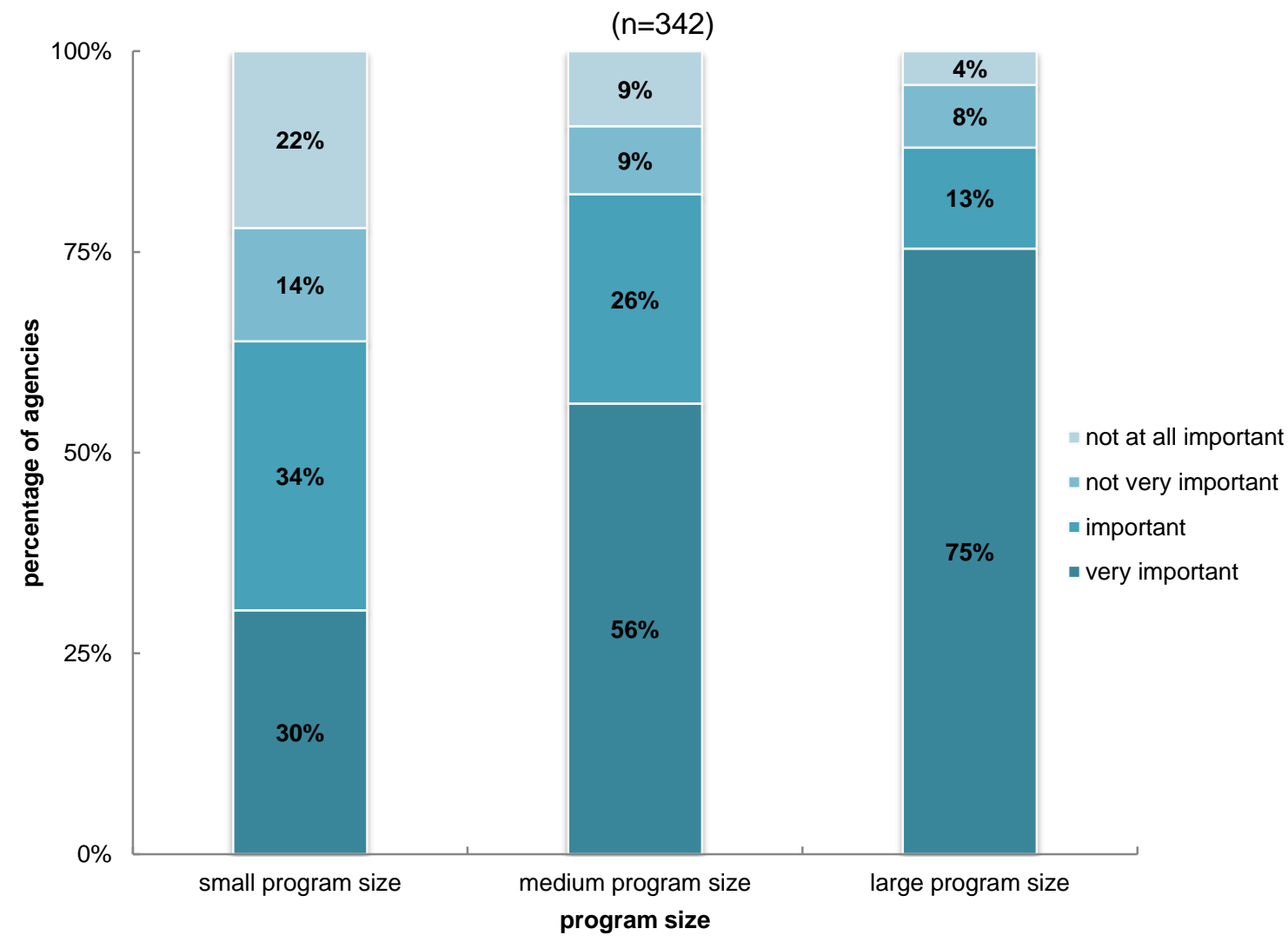

Fig. 2.13:Importance of leveraged funds by program size in Program Year $\mathbf{2 0 0 8}^{+}$

We asked subgrantees to rate the success of their efforts to acquire leveraged funds in PY08. Overall, 60 percent of agencies rated their efforts as successful or very successful. Twenty-eight percent reported that seeking leveraged funds is not part of their state's weatherization program. As shown in Fig. 2.14 success ratings improved with increasing program size. Seventy-eight percent of large subgrantees rated their leveraging efforts as successful or very successful compared with 36 percent of small agencies. 


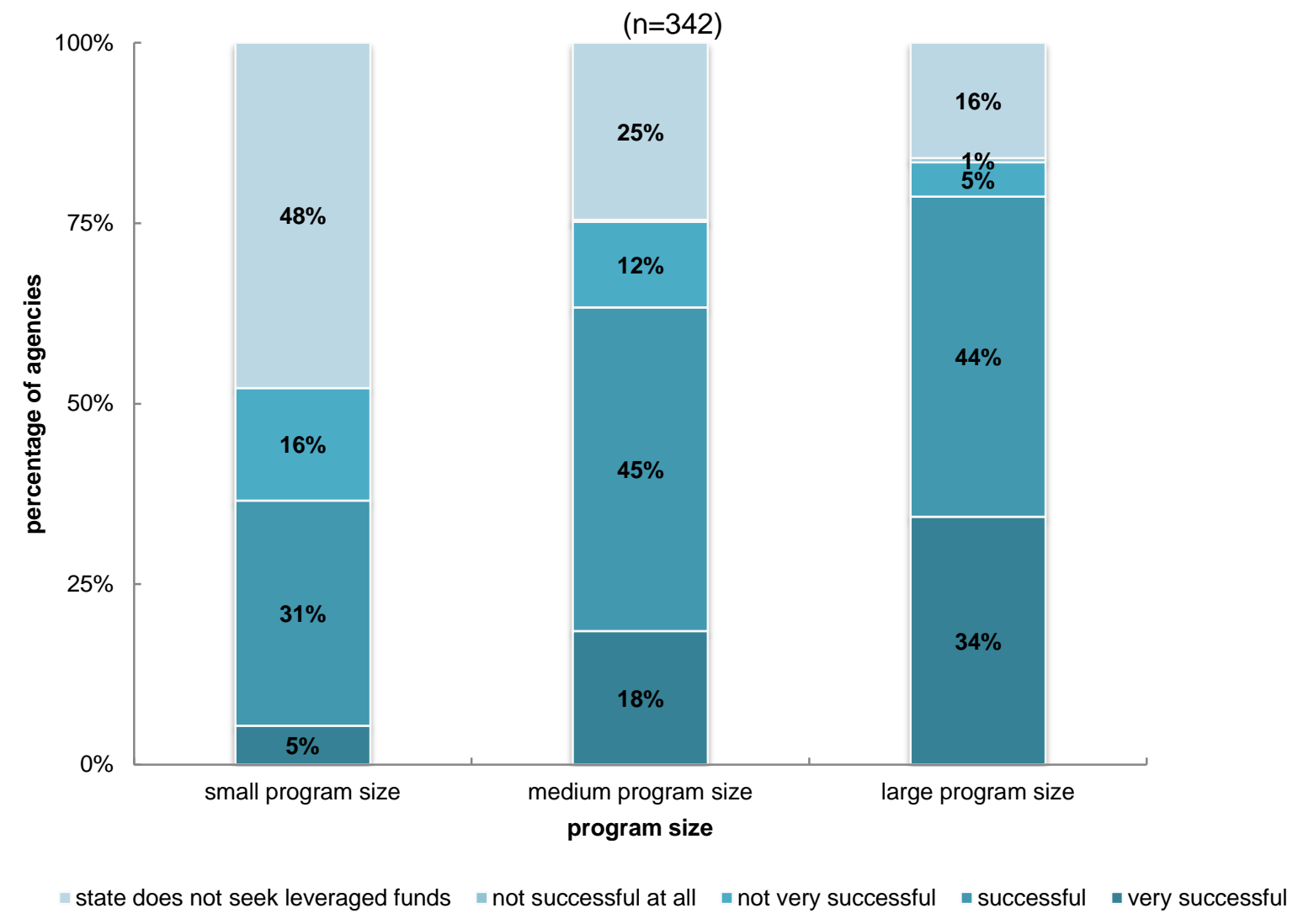

Fig. 2.14:Success of subgrantee efforts to acquire leveraged funds in Program Year 2008 $^{+}$

Subgrantee weatherization programs coordinate with a variety of other energy, housing, and support programs in serving their clients. Cooperation between programs includes use of funds from non-DOE sources to perform repairs to address deferral issues, and also includes referring clients to other services and programs for which they may be eligible. Energy bill paying assistance programs commonly refer clients to the weatherization program, particularly clients that have high energy usage. Subgrantees that were asked to report which programs cooperated with their weatherization initiatives cited the following programs most frequently: energy bill paying assistance programs, health and safety programs, home emergency repair programs, hardship funding programs, and housing rehabilitation programs. Results are presented in Table 2.6. 
Table 2.6: Other programs that cooperated with subgrantee weatherization programs $(n=396)^{+}$

\begin{tabular}{|l|r|r|r|r|}
\hline \multirow{2}{*}{ Type of Program } & \multicolumn{3}{|c|}{$\%$ of agencies using } \\
\cline { 2 - 5 } & $\begin{array}{c}\text { Federal } \\
\text { Funding }\end{array}$ & $\begin{array}{c}\text { State } \\
\text { Funding }\end{array}$ & $\begin{array}{c}\text { Utility } \\
\text { Funding }\end{array}$ & $\begin{array}{c}\text { Other } \\
\text { Funding }\end{array}$ \\
\hline Energy bill paying assistance & $57 \%$ & $30 \%$ & $32 \%$ & $14 \%$ \\
\hline Health and safety & $35 \%$ & $20 \%$ & $14 \%$ & $8 \%$ \\
\hline Home emergency repairs & $39 \%$ & $22 \%$ & $1 \%$ & $9 \%$ \\
\hline $\begin{array}{l}\text { Hardship funds } \\
\text { (other than for energy bill paying) }\end{array}$ & $23 \%$ & $25 \%$ & $5 \%$ & $11 \%$ \\
\hline Housing re-habilitation & $31 \%$ & $15 \%$ & $6 \%$ & $9 \%$ \\
\hline Emergency food & $24 \%$ & $13 \%$ & $1 \%$ & $17 \%$ \\
\hline $\begin{array}{l}\text { Energy education (other than client } \\
\text { education delivered by wx program) }\end{array}$ & $19 \%$ & $11 \%$ & $18 \%$ & $6 \%$ \\
\hline Other & $22 \%$ & $17 \%$ & $1 \%$ & $9 \%$ \\
\hline Home buying education & $15 \%$ & $11 \%$ & $0 \%$ & $7 \%$ \\
\hline Fuel delivery in crisis & $13 \%$ & $8 \%$ & $1 \%$ & $10 \%$ \\
\hline Rehabilitation loan & $15 \%$ & $10 \%$ & $0 \%$ & $5 \%$ \\
\hline Fair housing & $8 \%$ & $6 \%$ & $0 \%$ & $3 \%$ \\
\hline Mortgage loan & $8 \%$ & $5 \%$ & $0 \%$ & $4 \%$ \\
\hline Emergency safety & $6 \%$ & $5 \%$ & $1 \%$ & $2 \%$ \\
\hline
\end{tabular}

\subsubsection{Production}

Subgrantees' production ranged from a low of three DOE units in PY08 to one that completed 2,255 units. The mean production among the responding subgrantees was 106 DOE units, 78 comprehensive non-DOE units, and 24 non-comprehensive units. The median production was 73 DOE units and, among those completing non-DOE projects, 50 comprehensive non-DOE units and 97 non-comprehensive units. Production per agency was greatest in the very cold regions with a median production of 133 DOE units $($ mean $=146)$ and lowest in the hot-humid region with a median production of $38 \mathrm{DOE}$ units $($ mean $=47)$.

About half of the subgrantees reported that their grantee had an official definition for high energy expenditure or high energy burden. Within areas covered by a respective definition, 57 percent of units met the definition of high energy expenditure and 55 percent met the definition of high energy burden.

Sixty-four percent of subgrantees reported having waitlists in PY08 with only the hot-dry climate region reporting a substantially different incidence of waitlists (47 percent). ${ }^{14}$ Waitlists ranged from 1 to 14,000 percent of agencies' annual production for that year. Subgrantees with waitlists reported a median wait time before homes were weatherized of 205 days. Wait times varied substantially by program size and climate region with larger programs and those in the hot-dry region sporting shorter wait times, as shown in Table 2.8 .

\footnotetext{
${ }^{14}$ The actual share of all subgrantees with waitlists may be higher. Thirty-two percent of subgrantees left the question on waitlists blank, so we could not distinguish between respondents who did not answer the question and those who left it blank because they had no waitlist
} 
Table 2.7: Duration on waitlists in PY08 by program size (for subgrantees with waitlists)

\begin{tabular}{|l|c|c|c|c|}
\hline & $\begin{array}{c}\text { Overall } \\
(\mathbf{n = 4 5 2})\end{array}$ & $\begin{array}{c}\text { Small } \\
(\mathbf{n = 1 1 2})\end{array}$ & $\begin{array}{c}\text { Medium } \\
(\mathbf{n = 2 2 6})\end{array}$ & $\begin{array}{c}\text { Large } \\
(\mathbf{n = 1 1 3})\end{array}$ \\
\hline mean (days) & 370 & 389 & 396 & 299 \\
\hline median (days) & 205 & 251 & 238 & 180 \\
\hline
\end{tabular}

Table 2.8: Duration on waitlists in PY08 by climate region (for subgrantees with waitlists)

\begin{tabular}{|l|c|c|c|c|c|}
\hline & $\begin{array}{c}\text { very cold } \\
(\mathbf{n = 8 2})\end{array}$ & $\begin{array}{c}\text { cold } \\
(\mathbf{n = 1 7 0})\end{array}$ & $\begin{array}{c}\text { moderate } \\
(\mathbf{n = 1 1 6})\end{array}$ & $\begin{array}{c}\text { hot-humid } \\
(\mathbf{n = 6 1})\end{array}$ & $\begin{array}{c}\text { hot-dry } \\
(\mathbf{n = 2 3})\end{array}$ \\
\hline mean (days) & 292 & 416 & 334 & 489 & 166 \\
\hline median (days) & 204 & 210 & 195 & 290 & 60 \\
\hline
\end{tabular}

\subsection{SUPPORT FROM THE DEPARTMENT OF ENERGY AND OTHERS}

Although the scope of this evaluation focuses on the weatherization program administered collectively by the grantees and subgrantees, it is important to acknowledge DOE's role in providing not just funding, but also program guidance, rules, training, and other support. Several questions on the program information survey for grantees inquired about DOE's role and support for weatherization program activities. This section summarizes the responses to those questions.

A key issue addressed by the surveys was the flexibility of DOE program rules governing weatherization. Grantee program directors characterized DOE rules as generally flexible. As shown in Fig. 2.15, 87 percent of respondents to our questions on the topic characterized DOE program rules as flexible or very flexible.

At the same time, however, 61 percent of respondents thought that program rules should become more flexible $(46 \%)$ or much more flexible (15\%). (No one who responded thought the programs should become less flexible!) Areas in which program directors sought more flexibility tended to focus on the types of measures that can be implemented in homes, spending limits, and timing of when reweatherization may occur. Other comments focused on client education and the ability to loan funds to multifamily building owners. One respondent suggested that the main issue is not flexibility, but lack of clarity and consistency in the existing program rules. 


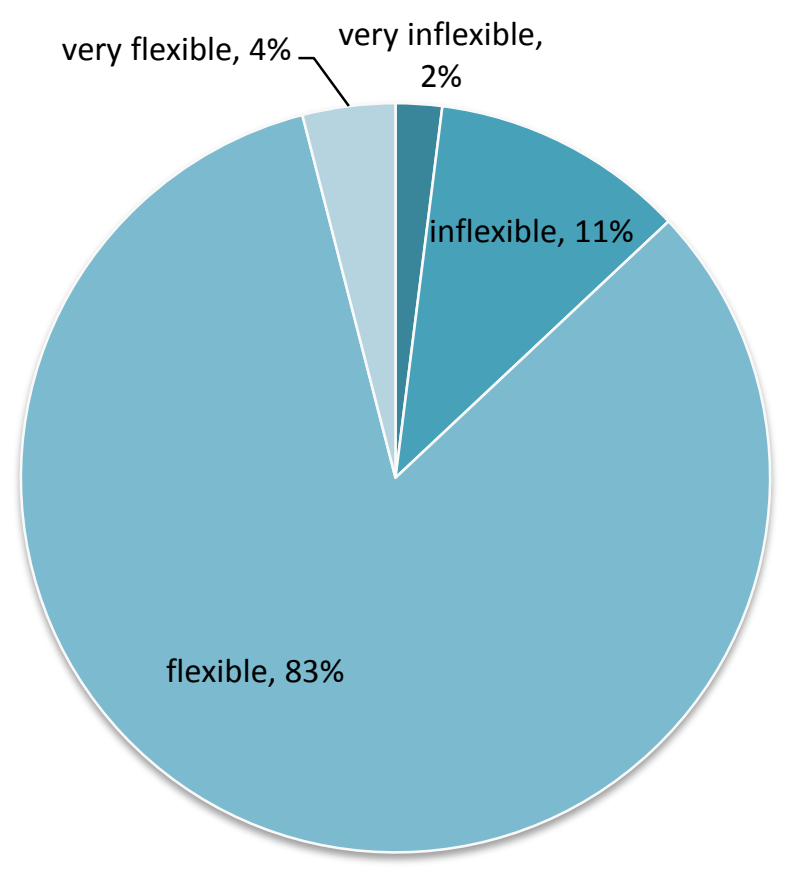

Fig. 2.15: Flexibility in federal program rules as perceived by grantee program directors

Grantees rated the quality of DOE support in management, training, and technical support in PY08 as moderate to high, as shown in Fig. 2.16. Grantees were more negative on DOE support for client education and funding assistance, however. Slightly fewer than half of the respondents provided ratings of moderate or higher, and more than a third marked "not applicable." Open-ended comments suggested that some grantees do not think they receive support in these areas, which may be what is driving the overtly negative responses as well.

When asked about the importance of improving DOE support in various areas, the majority of grantees indicated that increased weatherization funding and improved data and information systems were very important. (See Fig. 2.17.) About a third of grantees also rated improving technical support and training as very important. Readers should note that the questions in the survey instrument asked about levels of support in PY08 and the importance of improving from those levels of support. It is possible that grantees would rate DOE support differently today. ${ }^{15}$ Repeating these questions in the upcoming ARRA-era evaluation will provide fresher insights about grantee perceptions of DOE support.

\footnotetext{
${ }^{15}$ It is also plausible that some respondents rated current levels of support despite the question wording referencing PY08, but there is no way for us to assess this.
} 


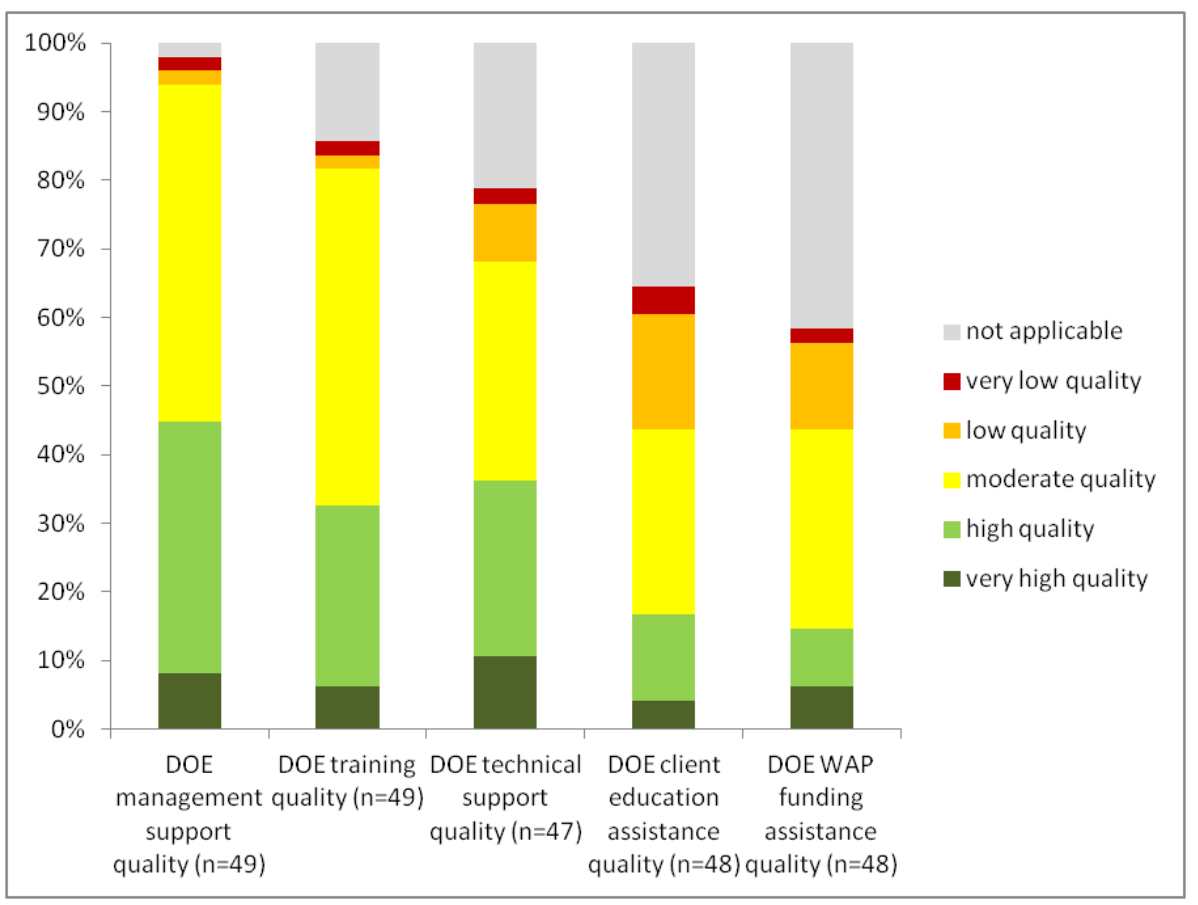

Fig. 2.16:Grantee perceptions of DOE support in selected areas

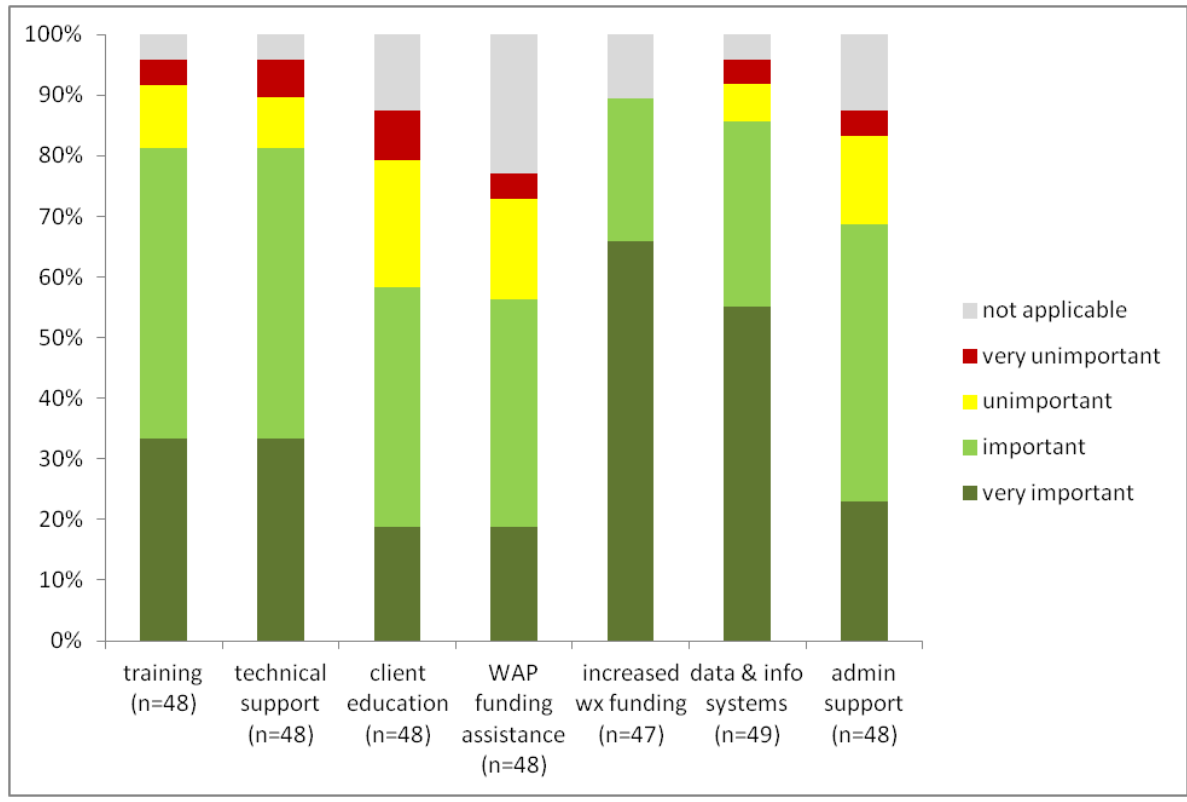

Fig. 2.17:Importance of improvement in DOE support in selected areas

Beyond DOE, other entities that provide support to the weatherization network include ORNL, NASCSP, the National Community Action Foundation (NCAF), and various state-level associations to which subgrantees belong. Furthermore, the Network Planning Committee provides insight and serves as a sounding board on weatherization issues and, as previously noted, provided input to ORNL in developing the evaluation plan we are following on this project. 


\section{TRAINING AND STAFF DEVELOPMENT}

The Weatherization Assistance Program focuses substantial attention on training and staff development at both the federal and state levels. Nine percent of DOE funds spent in PY08 plus another three percent in non-DOE funds were allocated to training and technical assistance. ${ }^{16}$ Grantees retained slightly more than half of these funds to provide training and technical assistance to subgrantees and obtain training for their staff, while the remainder of the funding was passed through to subgrantees for training and development of their staff and related activities.

\subsection{TRAINING PROVIDED}

The evaluation team received self-reports from grantees and subgrantees on the state of their training as well as training activities during PY08. This section reports on both.

\subsubsection{Grantee training and staff preparedness}

The evaluation explored how well-trained agency staff were in PY08 and in what areas they had received training during that program year. We included questions on these topics on survey instruments completed by grantee weatherization program staff. Respondents were asked to rate staff knowledge in key areas using a five-point scale that ranged from not at all well trained to very well trained. In the analysis, we collapsed these ratings into three main categories as shown in the table below.

Table 3.1: Rating categories

\begin{tabular}{|l|l|}
\hline \multirow{2}{*}{ Analysis category } & Response category \\
\hline \multirow{2}{*}{ Well trained } & Very well trained \\
\cline { 2 - 2 } & Well trained \\
\hline \multirow{2}{*}{ Moderately well trained } & Moderately well trained \\
\hline \multirow{2}{*}{ Not well trained } & Not well trained \\
\cline { 2 - 2 } & Not at all well trained \\
\hline
\end{tabular}

We present the results of these self-assessments below.

\footnotetext{
${ }^{16}$ According to financial data provided by grantees, DOE funds spent on training and technical assistance in PY08 totaled $\$ 22$ million (distributed across virtually all grantees), while 18 grantees indicated they spent a total of $\$ 17$ million in non-DOE funds for training and technical assistance.
} 
Staff preparedness - Grantee weatherization managers perceive their staff to be generally well-trained in the areas in which they require particular knowledge to perform their jobs. We asked grantee respondents to rate staff knowledge across seven technical categories pertaining to weatherization in PY08: diagnostic procedures, insulation, HVAC, infiltration (air leakage), doors and windows, water heating, and electric baseloads. The results of these self-assessments are presented in Fig. 3.1.

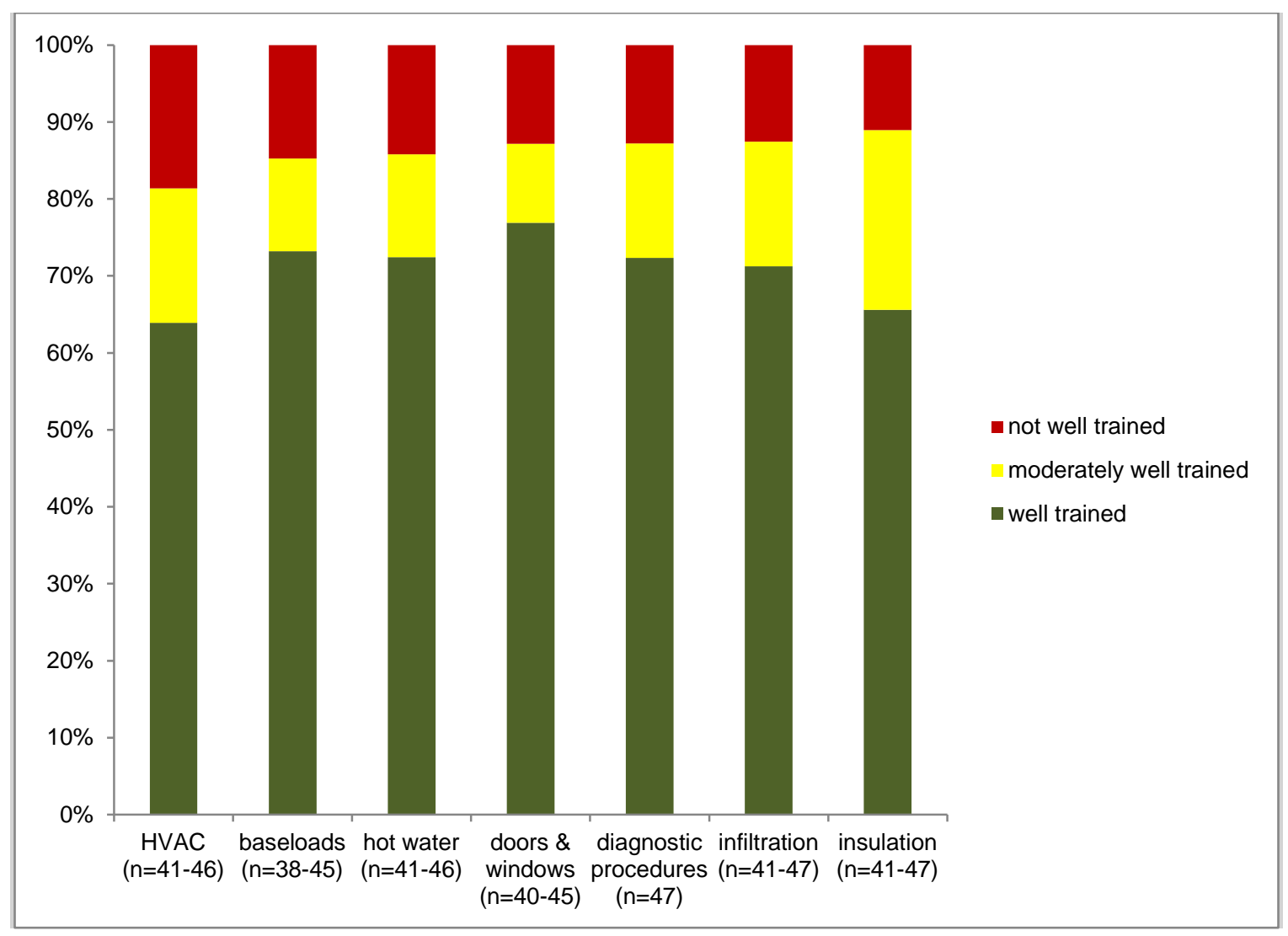

Fig. 3.1: Grantee assessment of staff knowledge on technical weatherization topics in PY08

On average across the seven technical categories, 71 percent of grantees reported that their office staff were well trained, 15 percent reported that staff were moderately well trained, and 14 percent reported that staff were not well trained. The largest number of "not well trained" responses (19\%) was reported in the HVAC category. In the other six categories, the "not well trained" category ranged from 11 to 15 percent of respondents.

The measure installation categories (all of the above categories except diagnostic procedures) were also broken out by housing type: single family, mobile home, and multifamily. Fig. 3.2 compares grantee assessments of staff knowledge across all measure installation categories by housing type. 


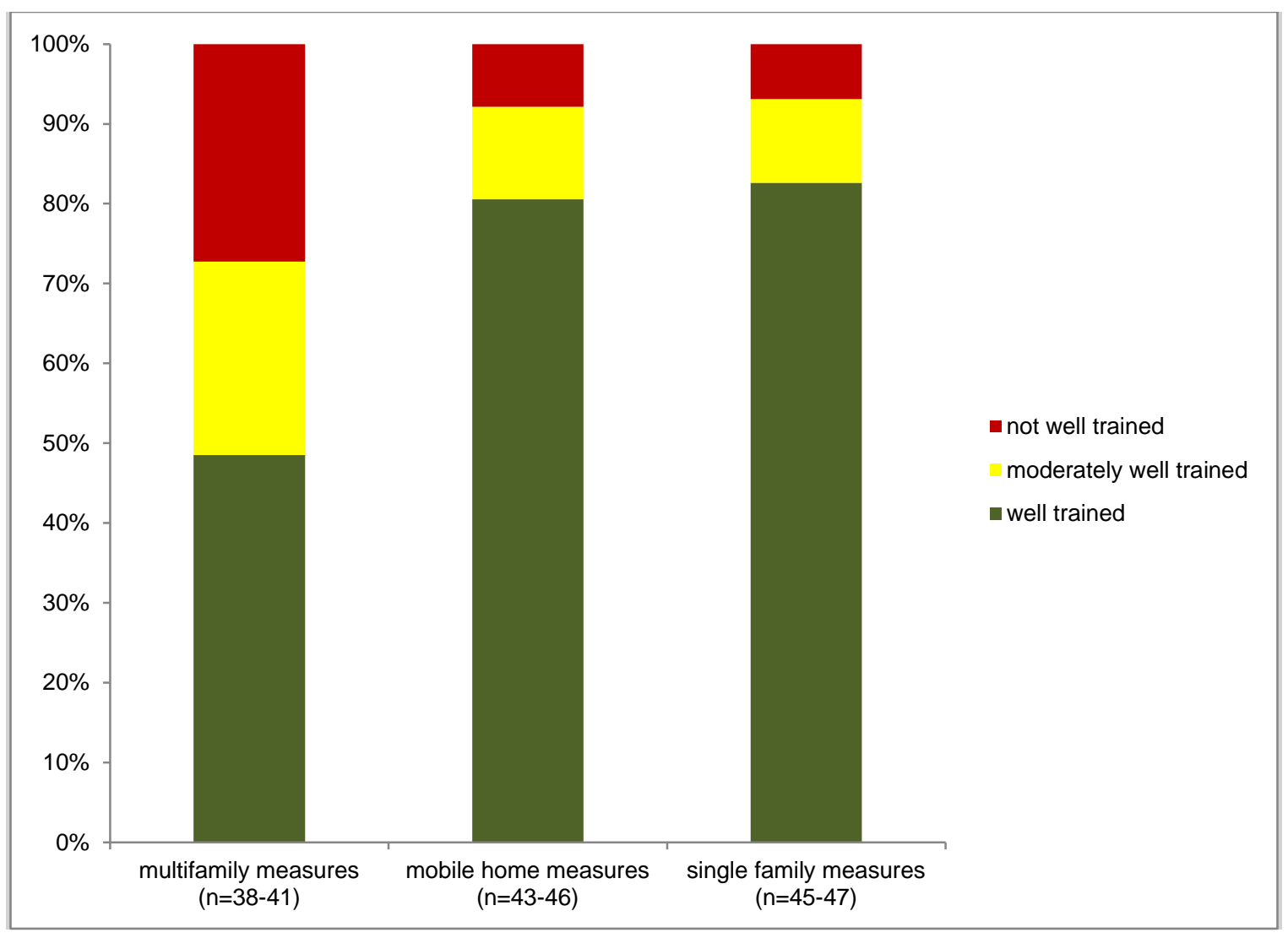

Fig. 3.2:Grantee assessment of staff knowledge on technical weatherization topics, by housing type

Grantees rated their staff knowledge most favorably in the single family ( $83 \%$ well trained) and mobile home ( $81 \%$ well trained) weatherization categories. On average across the multifamily weatherization categories, only 49 percent of grantees rated staff as well trained. This result is not surprising given that in PY08 fewer grantees had incorporated multifamily weatherization into their programs than during the ARRA period.

Grantees were also asked to rate staff knowledge across a number of technical and administrative topics (Fig. 3.3), as well as health and safety topics (Fig. 3.4). On average across all technical and administrative topics, 66 percent of grantees rated their staff as being well trained, compared with 56 percent across all health and safety topics. 


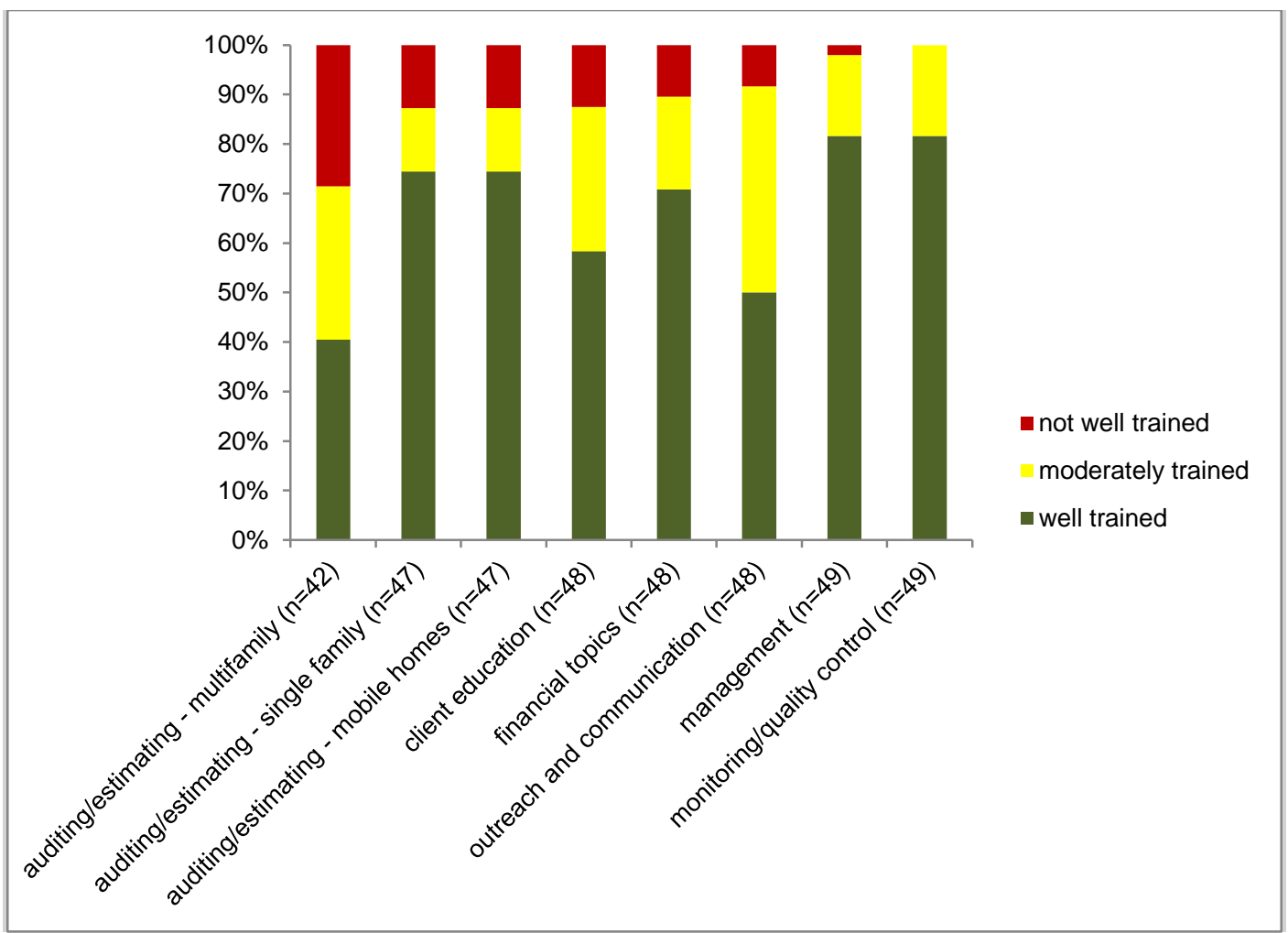

Fig. 3.3: Grantee assessment of staff knowledge on technical/administrative topics in PY08 


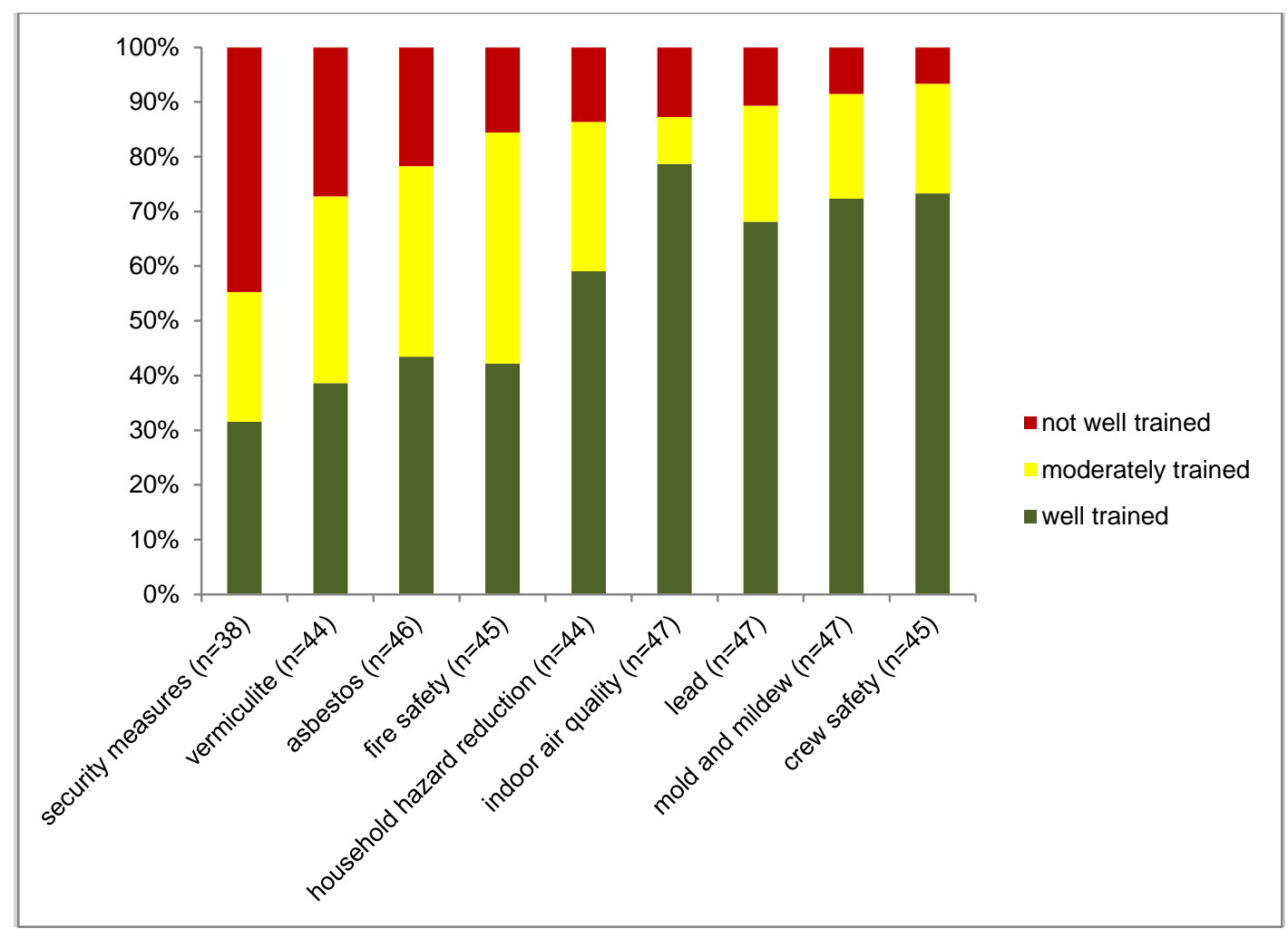

Fig. 3.4: Grantee assessment of staff knowledge on health and safety topics in PY08

In the technical and administrative arena, grantees rated staff as most knowledgeable in the areas of monitoring/quality control and management (both at $82 \%$ well trained), and least knowledgeable in the area of auditing/estimating for multifamily buildings ( $40 \%$ well trained). Again, these results are not surprising given the prevalence of multifamily weatherization in PY08. In the health and safety arena, grantees rated their staff as most knowledgeable about indoor air quality (79\% well trained), and least knowledgeable about household security measures (32\% well trained).

Training - Training by grantee staff ensures that they are well-equipped to support and monitor subgrantee weatherization work. Grantee staff receive training in a variety of venues. Table 3.2 lists the common types of training at which grantees participate and their primary uses. 
Table 3.2: Training supported by grantee weatherization programs

\begin{tabular}{|l|c|l|}
\hline Training Venue & $\begin{array}{c}\text { \% of } \\
\text { grantees } \\
\text { using }\end{array}$ & \multicolumn{1}{|l|}{ Primary use } \\
\hline National Weatherization Program Conference & $63 \%$ & Mgmnt and monitoring classroom training \\
\hline Affordable Comfort Conference & $24 \%$ & T\&TA and monitoring classroom training \\
\hline Other national conference & $16 \%$ & Mgmnt classroom training \\
\hline Regional weatherization conference & $49 \%$ & Mgmnt and monitoring classroom training \\
\hline State's weatherization conference & $29 \%$ & Mgmnt classroom training \\
\hline Other in-state conference & $8 \%$ & Monitoring classroom training \\
\hline State or regional training center class & $24 \%$ & Monitoring and T\&TA classroom training \\
\hline Manufacturer's training school class & $18 \%$ & Monitoring and T\&TA classroom training \\
\hline Utility training class & $12 \%$ & Monitoring classroom and field training \\
\hline Training class provided by responding agency & $35 \%$ & $\begin{array}{l}\text { Mgmnt, monitoring and T\&TA classroom training, } \\
\text { mgmnt field training }\end{array}$ \\
\hline Other class & & T\&TA and monitoring classroom training \\
\hline Expert visit to agency & $20 \%$ & T\&TA and monitoring classroom training \\
\hline
\end{tabular}

Grantee training for its staff tends to span a range of weatherization topics, with health \& safety topics, monitoring, auditing, and management being the most common. Fig. 3.5 shows the six most common topics on which grantee staff received training in PY08. 


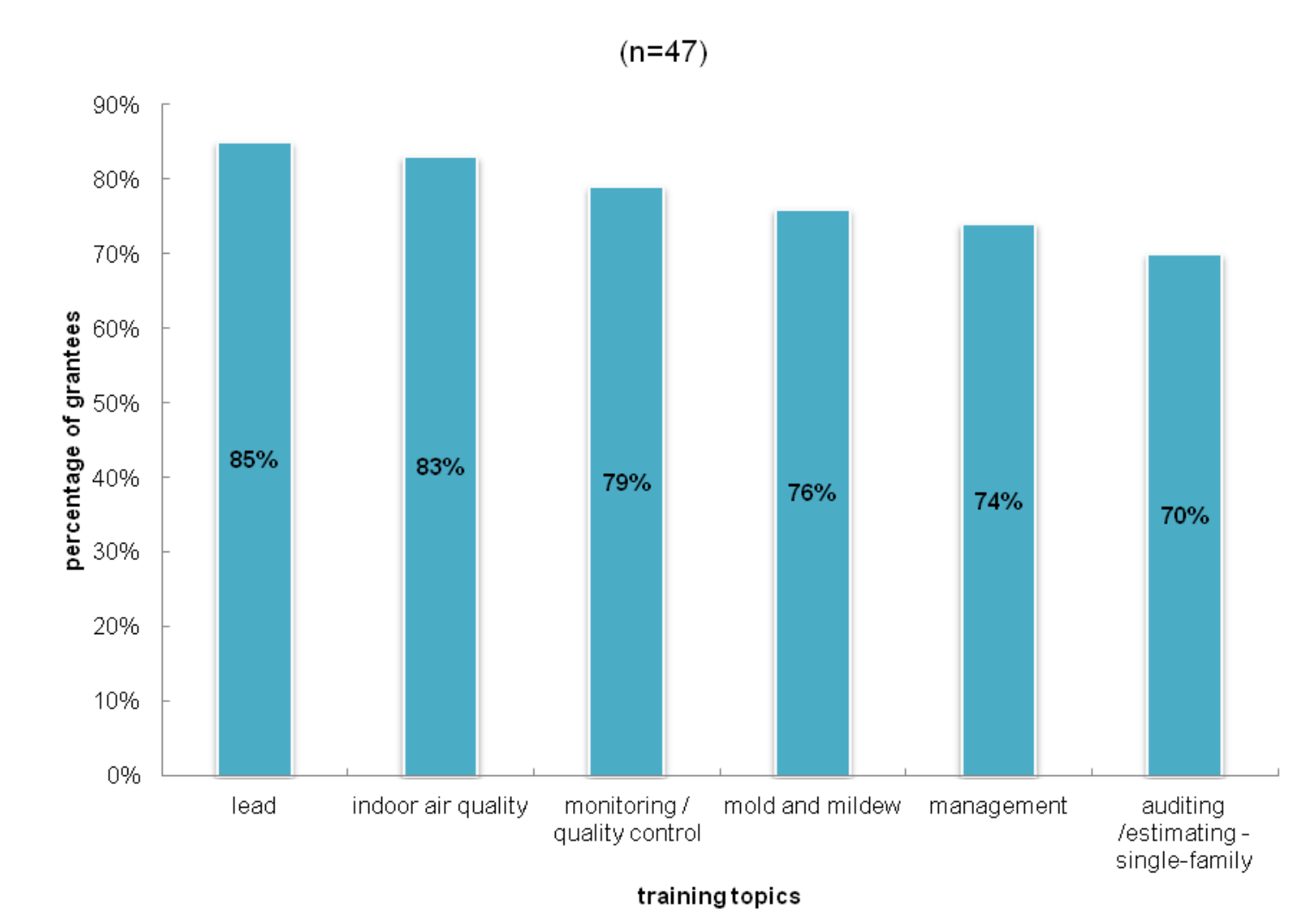

Fig. 3.5: Grantee training for its staff - most common topics

\subsubsection{Staff preparedness and training in subgrantees}

Similar to the approach used for assessing training among grantee program staff, the evaluation explored how well-trained subgrantee staff were in PY08 and in what areas they received training during that year. We included questions on these topics on survey instruments completed by program directors at the grantee level and their counterparts within subgrantee agencies. This allowed us to compare grantee and subgrantee assessments of agency staff knowledge in the following areas: diagnostic procedures, measure installation for single family homes, mobile homes and multifamily buildings, administrative topics, and health and safety topics. We compare grantee and subgrantee perceptions of agency staff knowledge in Table 3.3, which shows that grantees tend to perceive ${ }^{17}$ subgrantee weatherization staff preparedness slightly higher than subgrantees do for the installation of measures. Conversely, subgrantees perceive their staff as better prepared on diagnostic procedures than grantees do.

\footnotetext{
${ }^{17} \mathrm{We}$ did not conduct grantee-level comparisons, however. These comments are based on national level summary data.
} 
Table 3.3: Perceptions of grantees and subgrantees on training/preparedness in key topic areas by subgrantee weatherization staff ${ }^{+}$

\begin{tabular}{|c|c|c|c|c|c|c|c|c|}
\hline & \multicolumn{2}{|c|}{ Well trained } & \multicolumn{2}{|c|}{$\begin{array}{c}\text { Moderately well } \\
\text { trained }\end{array}$} & \multicolumn{2}{|c|}{ Not well trained } & \multicolumn{2}{|c|}{ Not applicable } \\
\hline & grantee & subgr. & grantee & subgr. & grantee & subgr. & grantee & subgr. \\
\hline $\begin{array}{l}\text { Diagnostic procedures } \\
(\mathrm{n}=49-\text { state }) \\
(\mathrm{n}=321-\text { local })\end{array}$ & $62 \%$ & $74 \%$ & $28 \%$ & $18 \%$ & $11 \%$ & $4 \%$ & $0 \%$ & $5 \%$ \\
\hline $\begin{array}{l}\text { single family measures } \\
(\mathrm{n}=49 \text { - state }) \\
(\mathrm{n}=321 \text { - local })\end{array}$ & $77 \%$ & $67 \%$ & $17 \%$ & $18 \%$ & $5 \%$ & $7 \%$ & $0 \%$ & $8 \%$ \\
\hline $\begin{array}{l}\text { mobile home measures } \\
(\mathrm{n}=49 \text { - state }) \\
(\mathrm{n}=321 \text { - local })\end{array}$ & $72 \%$ & $61 \%$ & $20 \%$ & $15 \%$ & $6 \%$ & $8 \%$ & $2 \%$ & $15 \%$ \\
\hline $\begin{array}{l}\text { multifamily measures } \\
(\mathrm{n}=49 \text { - state }) \\
(\mathrm{n}=321-\text { local })\end{array}$ & $40 \%$ & $31 \%$ & $20 \%$ & $11 \%$ & $23 \%$ & $13 \%$ & $17 \%$ & $44 \%$ \\
\hline $\begin{array}{l}\text { administrative topics } \\
(\mathrm{n}=49 \text { - state }) \\
(\mathrm{n}=320-\text { local })\end{array}$ & $55 \%$ & $60 \%$ & $27 \%$ & $19 \%$ & $16 \%$ & $6 \%$ & $2 \%$ & $15 \%$ \\
\hline $\begin{array}{l}\text { health \& safety topics } \\
(\mathrm{n}=49 \text { - state) } \\
(\mathrm{n}=319-\text { local })\end{array}$ & $40 \%$ & $44 \%$ & $31 \%$ & $21 \%$ & $25 \%$ & $16 \%$ & $4 \%$ & $19 \%$ \\
\hline
\end{tabular}

Staff preparedness - Grantees and subgrantees rated agency staff knowledge most favorably in the areas of diagnostic procedures and measure installation for single family and mobile homes. A larger share of subgrantees (74\%) perceived their staff to be well trained on diagnostic procedures, compared with $62 \%$ of grantee staff who rated subgrantees as well trained in this area. This differential was reversed in the area of single family and mobile home weatherization measures, where grantees rated subgrantees knowledge somewhat more favorably than the subgrantees did themselves. Grantees and subgrantees also agreed that the areas where subgrantees were less well trained were on multifamily weatherization measures and health and safety topics. For multifamily, more subgrantees listed multifamily weatherization measures as "not applicable" (44\%) compared with just 17\% of grantee respondents.

As shown in Fig. 3.6, more than 70 percent of subgrantees rated staff as moderately well or well trained on diagnostic procedures, insulation and infiltration. The areas where at least ten percent of subgrantees rated their staff as not well trained were water heating measures (11\%), HVAC measures (10\%), and baseload measures $(10 \%)$. These results are reasonable given that many subgrantees outsource work on HVAC and water heaters. 


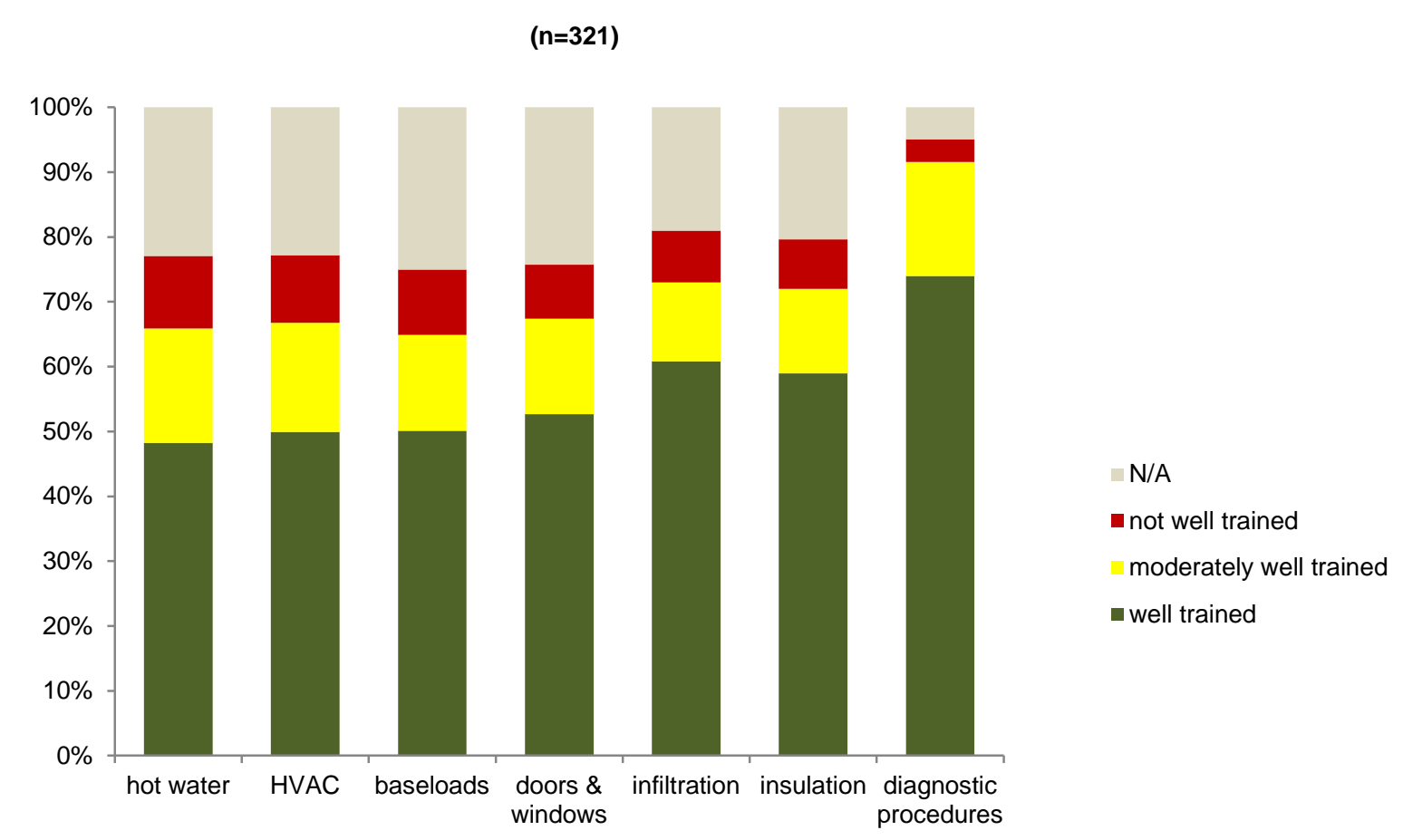

Fig. 3.6: Subgrantee assessment of staff knowledge on technical weatherization topics in $\mathrm{PYOB}^{+}$

The large proportion of "not applicable" ratings across all categories except diagnostic procedures in the above chart is due to a high proportion of "not applicable" ratings in the multifamily category. Fig. 3.7 compares subgrantee perceptions of staff knowledge of measure installation topics by housing type. In PY08 there was clearly a need for subgrantee staff training on multifamily weatherization topics. Given the emphasis on multifamily weatherization under ARRA, it will be interesting to compare these results with the forthcoming ARRA evaluation results. 


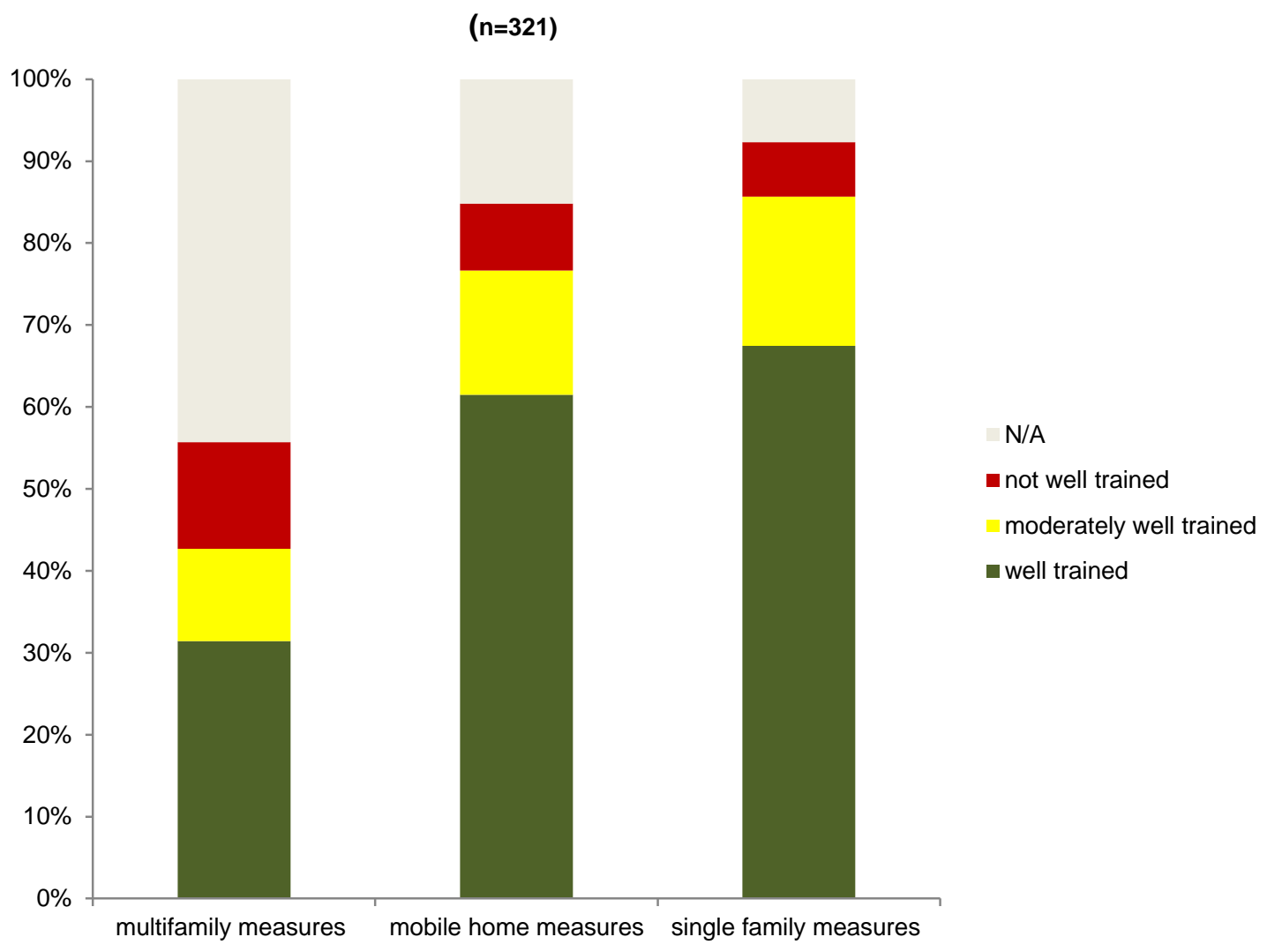

Fig. 3.7: Subgrantee assessment of staff knowledge on technical weatherization topics, by housing type ${ }^{+}$

Subgrantees were also asked to rate staff knowledge across a number of technical/administrative and health and safety topics. Results are presented in Fig. 3.8 and

. These charts again highlight the need for additional training on multifamily topics - in this case, auditing and estimating for multifamily buildings, as well as outreach, communication and client education. Over 20 percent of subgrantees rated staff as not well trained on the following health and safety topics: security measures, vermiculite, and asbestos. 


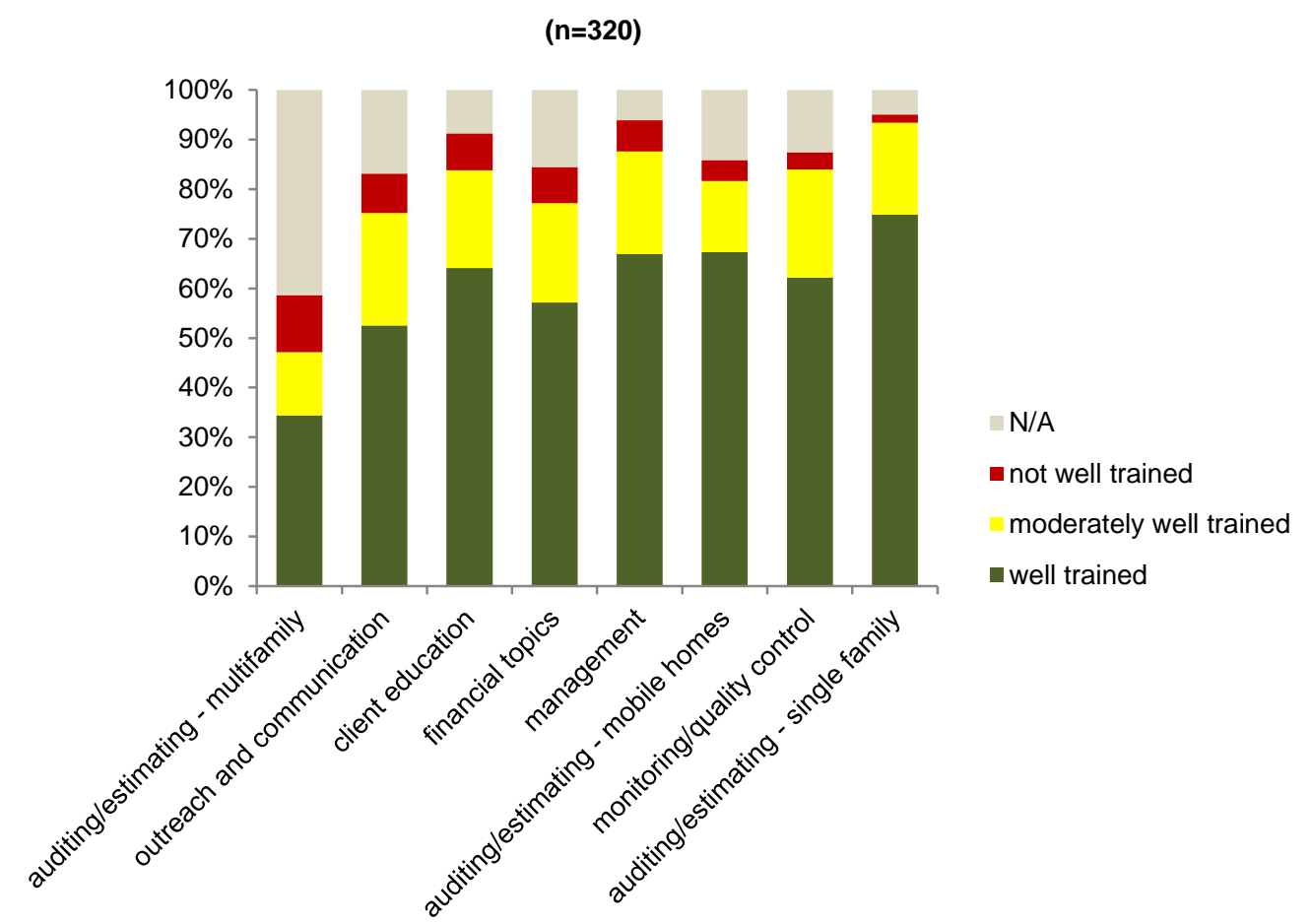

Fig. 3.8: Subgrantee assessment of staff knowledge on technical/administrative topics in $\mathrm{PYOB}^{+}$

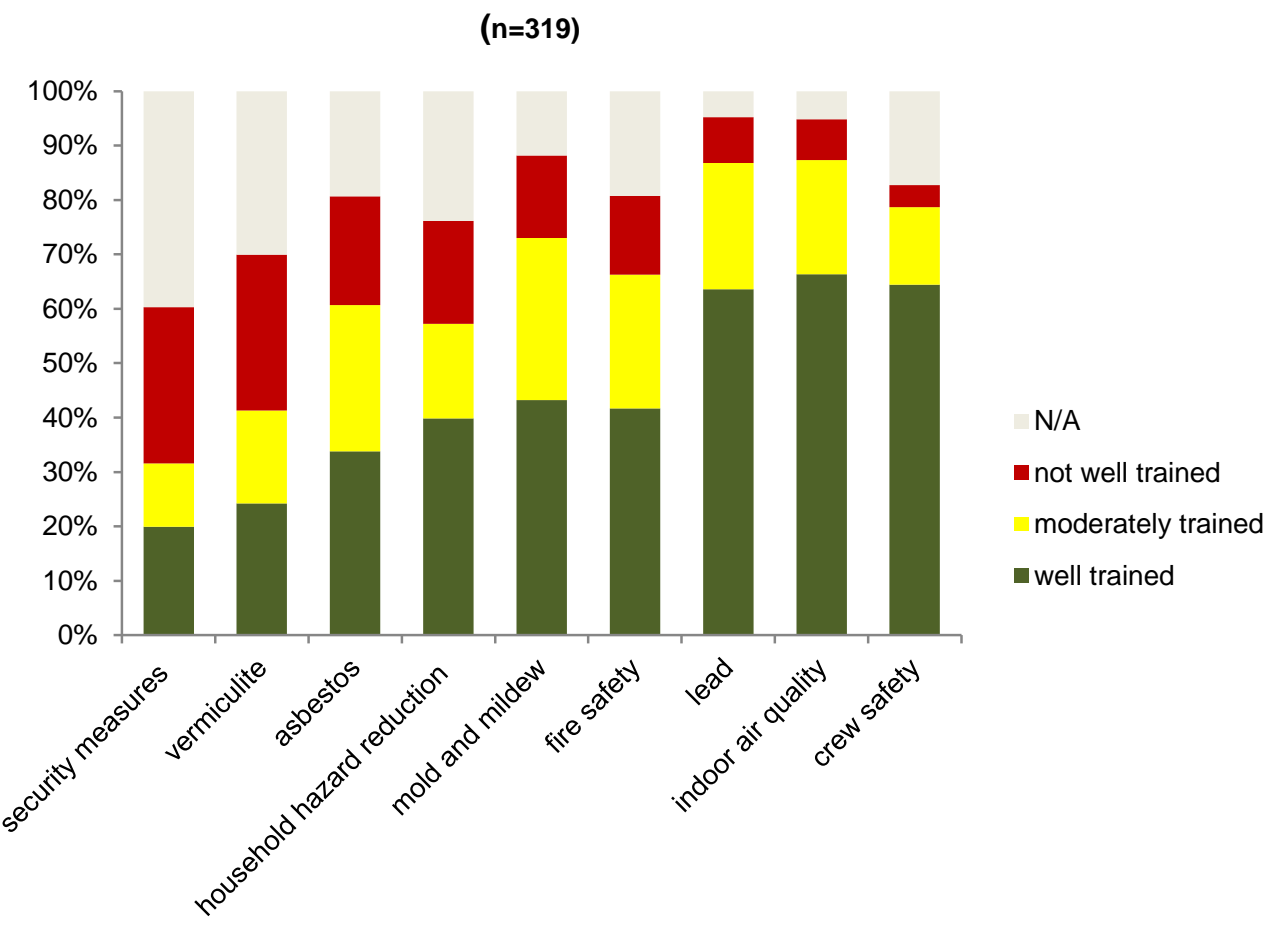

Fig. 3.9: Subgrantee assessment of staff knowledge on health and safety topics in $\mathrm{PYOB}^{+}$ 
Training - Subgrantees reported that they used a wide assortment of training opportunities. The most commonly used venues for training were state and national weatherization conferences (used by 51 and 45 percent of subgrantees, respectively) and state or regional training centers ( 40 percent). In total, subgrantees sent staff to at least 10,600 training opportunities ${ }^{18}$, which computes to at least 1.4 events per person for our estimated weatherization workforce of 7,600 individuals in PY08. Table 3.4 lists usage and average numbers of staff sent by training venue.

Table 3.4: Training venues used by subgrantees $(n=353)^{+}$

\begin{tabular}{|l|c|c|c|}
\hline Training Venue & $\begin{array}{c}\text { \% of agencies } \\
\text { using }\end{array}$ & $\begin{array}{c}\text { Mean \# of staff } \\
\text { sent (of agencies } \\
\text { that used) }\end{array}$ & $\begin{array}{c}\text { Mean \% of staff } \\
\text { sent (of agencies } \\
\text { that used) }\end{array}$ \\
\hline National Weatherization Program Conference & $45 \%$ & 3 & $42 \%$ \\
\hline Affordable Comfort Conference & $35 \%$ & 3 & $41 \%$ \\
\hline Other national conference & $13 \%$ & 3 & $28 \%$ \\
\hline Regional weatherization conference & $32 \%$ & 4 & $47 \%$ \\
\hline State's weatherization conference & $51 \%$ & 4 & $35 \%$ \\
\hline Other in-state conference & $26 \%$ & 3 & $39 \%$ \\
\hline Weatherization conference in another state & $5 \%$ & 2 & $40 \%$ \\
\hline Other conference given in another state & $4 \%$ & 4 & $51 \%$ \\
\hline State or regional training center class & $40 \%$ & 5 & $36 \%$ \\
\hline Manufacturer's training school class & $11 \%$ & 4 & $36 \%$ \\
\hline Utility training class & $14 \%$ & 3 & $57 \%$ \\
\hline Training class provided by responding agency & $29 \%$ & 6 & $46 \%$ \\
\hline One-time state-sponsored class & $19 \%$ & 5 & $38 \%$ \\
\hline Other external class & $8 \%$ & 4 & $39 \%$ \\
\hline Visit to another agency & $20 \%$ & 3 & $47 \%$ \\
\hline State instruction to responding agency & $28 \%$ & 5 & $42 \%$ \\
\hline In-person expert visit & $26 \%$ & 4 & $44 \%$ \\
\hline Webcast & $8 \%$ & 5 & \\
\hline
\end{tabular}

Training for subgrantee staff in PY08 covered the major functional categories comprising weatherization work. Collectively, this training included some aspect of diagnostic procedures, weatherization measures, technical and administrative functions, and health and safety for staff in most agencies. As shown in Fig. 3.10:

- 94 percent of subgrantees reported that staff received training on diagnostic procedures, with staff at 20 percent of agencies covering a solid majority ${ }^{19}$ of the diagnostic procedures listed in our

\footnotetext{
${ }^{18}$ This number does not include any repeated attendances at a single training venue by the same staff, such as a staff person taking multiple classes at regional training centers. The number of training opportunities shown is the sum of the total number of staff sent to each of the 18 training venues included in our questionnaire (plus a $19^{\text {th }}$ venue labeled as other) across all the sampled agencies responding to $\mathrm{S} 3$ and then weighted and scaled up to the full population of local agencies.

${ }^{19}$ The shades in the figure indicate the share of diagnostic procedures from our questionnaire included in the training that subgrantee staff collectively received. The darkest shade represent agencies whose staff, collectively, received training on $2 / 3$ or more of the 28 diagnostic procedures included in our questionnaire. The medium shade represents agencies covering between $1 / 3$ and $2 / 3$ of the procedures in the training received by staff. The lightest shade represents agencies covering less than $1 / 3$ of procedures in the training.
} 
questionnaire. (See Sampled Agencies Detailed Program Information Survey (section 5, question 2 ) in the appendices for a list of procedures included in the questionnaire.)

- 92 percent of subgrantees reported that staff received training on weatherization measures, with half of those covering a solid majority of measures included in the questionnaire (see Sampled Agencies Detailed Program Information Survey, section 5, question 1).

- 90 percent of subgrantees reported that staff received training on technical and administrative activities, including auditing, client education, and some management topics (section 5, question 1a).

- 96 percent of subgrantees made training on health and safety topics available to some staff (section 5, question $1 \mathrm{~b}$ ).

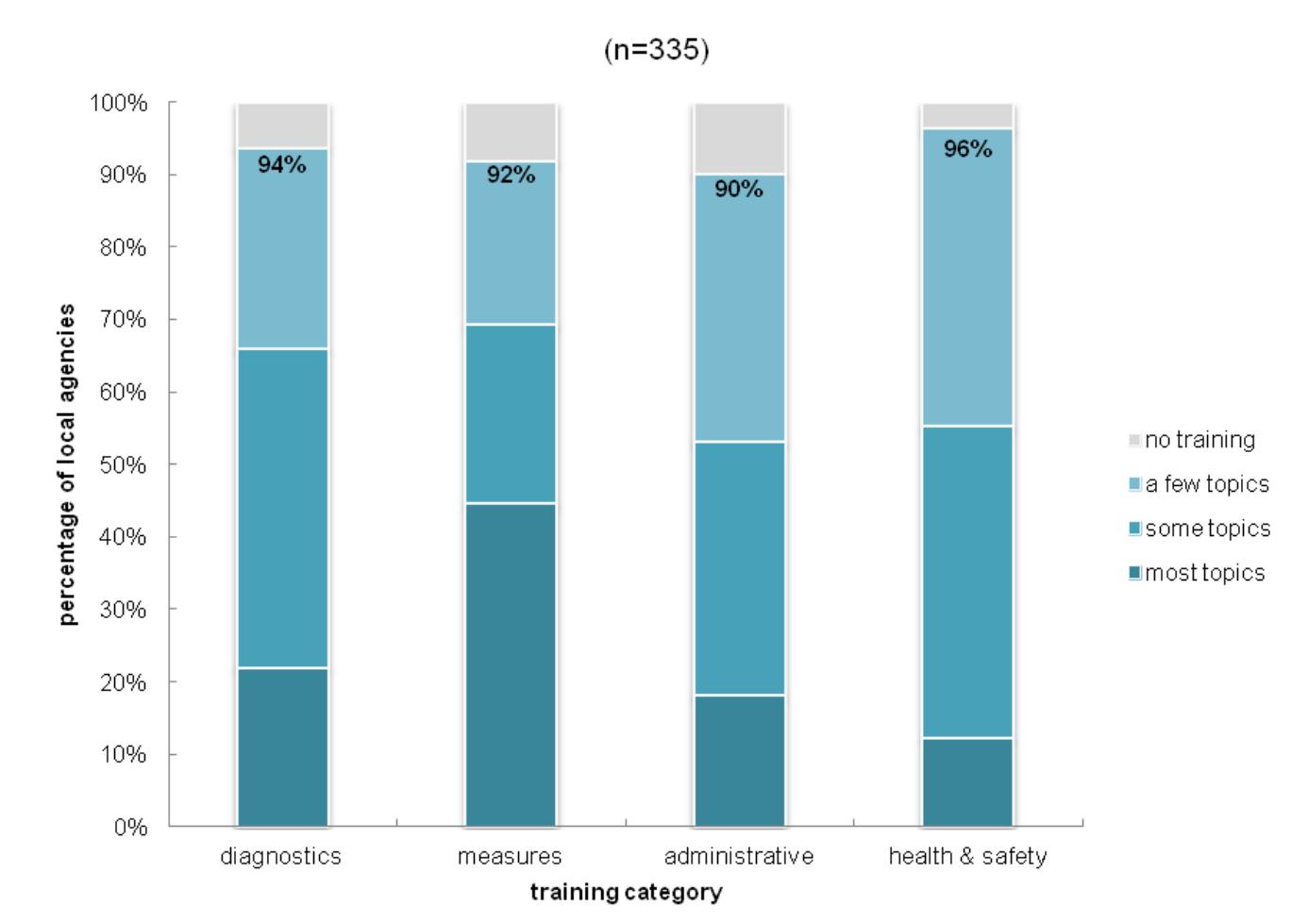

Fig. 3.10: Training received by subgrantee staff by functional category ${ }^{+}$

The diagnostic procedures on which training for subgrantee staff was most widespread are:

- Blower door tests to measure house leakage rates. Eighty-four percent of subgrantees reported that their staff received training on this procedure in PY08. This topic was the most widespread diagnostic training topic in all program size groupings and in all climate regions.

○ By subgrantee size

- Large $89 \%$

- Medium $83 \%$

- Small $83 \%$

○ By climate region

- Hot-dry $94 \%$

- Hot-humid $87 \%$

- Moderate $85 \%$

- Cold 83\%

- Very cold $83 \%$ 
- Carbon monoxide measurements. Four different kinds of carbon monoxide measurements were common training content for subgrantee staff. Seventy-five percent of agencies reported that staff received training on carbon monoxide measurement in space heating flues, 71 percent for water heating system flues, 63 percent for living areas, and 61 percent for equipment rooms. These procedures received fairly widespread coverage across the program size groups and climate regions, although more common in the warmer climate regions. Averages of training rates for all $\mathrm{CO}$ monitoring are shown below.

○ By subgrantee size
- Small
$70 \%$
- Medium
$67 \%$
- Large
$67 \%$
- Hot-dry
$78 \%$
- Moderate
$73 \%$
- Hot-humid
$72 \%$
- Cold
$69 \%$
- Very cold
$53 \%$

- By climate region

- Cooking stove measurements. Sixty-nine percent of subgrantees reported that their staff received training on cooking stove measurements. This training topic was common across all program sizes and in all regions except the very cold climate region.

$\circ$ By subgrantee size

$\begin{array}{ll}\text { - Small } & 72 \% \\ \text { - } \text { Medium } & 70 \% \\ \text { climate region } & 65 \% \\ \text { - Hot-dry } & \\ \text { - Hot-humid } & 93 \% \\ \text { - Moderate } & 87 \% \\ \text { - } \text { Cold } & 76 \% \\ \text { - } \text { Very cold } & 66 \% \\ & \end{array}$

- Flue gas analysis. Sixty-six and fifty-seven percent of subgrantees, respectively, reported that their staff received training on flue gas analysis for space and water heating systems. Training on these topics was more prevalent in the moderate and cold climate regions. Averages of training rates for flue gas analysis for space and water heating shown below.

- By subgrantee size

- Medium 67\%

- Large $63 \%$

- Small $47 \%$

$\circ$ By climate region

- Cold $73 \%$

- Very cold $\quad 57 \%$

- Moderate $56 \%$

- Hot-humid $\quad 50 \%$

- Hot-dry 38\%

- Draft/spillage test. Sixty-four percent of subgrantees reported that their staff were trained on measuring drafting/spillage on space and water heating systems. This training topic followed an 
unusual climate pattern, being most prevalent in the hot-dry region and least prevalent in the hothumid areas. Averages for training for measuring spillage on space and water heating shown below. $\circ \quad$ By subgrantee size

$\begin{array}{ll}\text { - Large } & 72 \% \\ \text { - Medium } & 69 \% \\ \text { - Small } & 45 \% \\ \text { climate region } & \\ \text { - Hot-dry } & 78 \% \\ \text { - Cold } & 73 \% \\ \text { - Very cold } & 65 \% \\ \text { - Moderate } & 56 \% \\ \text { - Hot-humid } & 40 \%\end{array}$

Ninety-two percent of subgrantees said staff received training on at least one measure (Fig. 3.11), but training generally covered multiple measures. Training on measures was widespread across all program size groupings and in all climate regions, although staff in the hot-dry region received training on a greater number of measures than those in the other climate regions. 


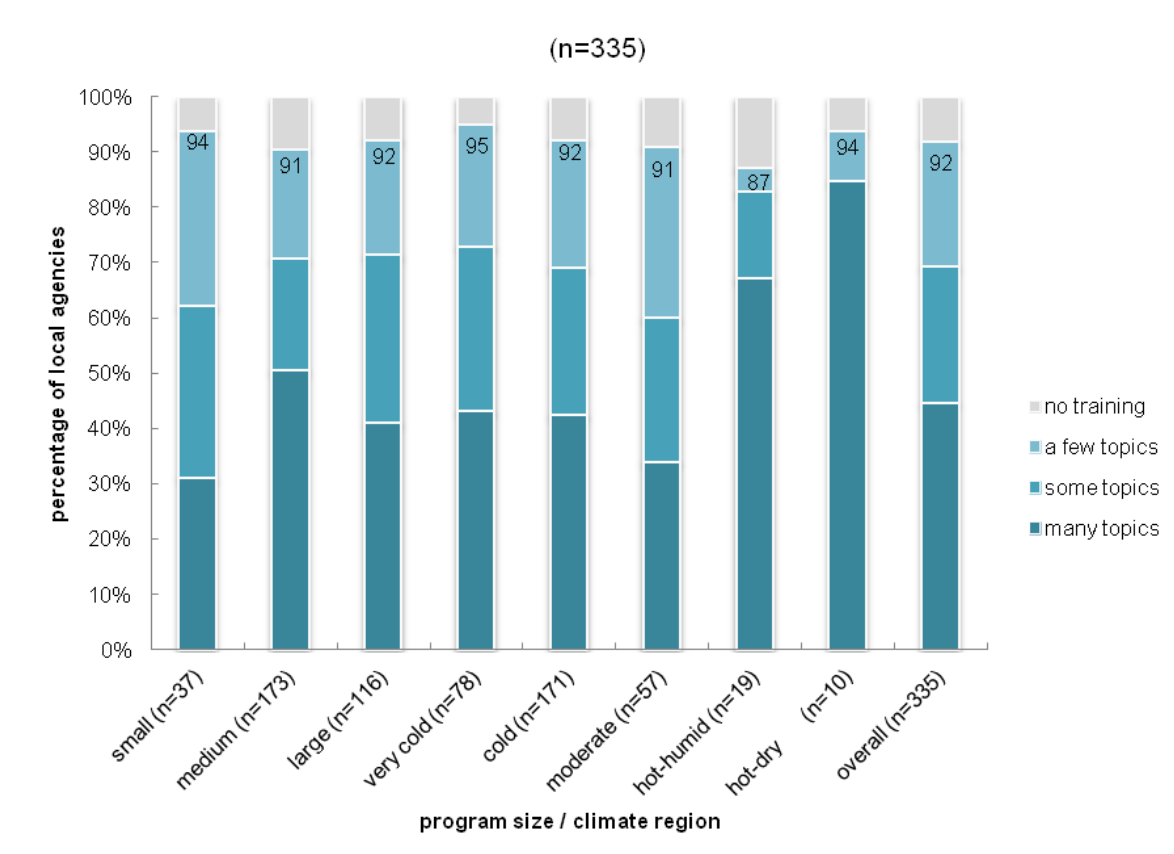

Fig. 3.11: Subgrantee staff training - weatherization measures ${ }^{+}$

The training on measures that subgrantees reported receiving is distributed across six categories roughly equally:

- Doors and windows (83\% of subgrantees reported that staff received training)

- Insulation $(82 \%)$

- Heating systems (78\%)

- Infiltration (78\%)

- Baseload energy usage (72\%)

- Water heating $(71 \%)$

Interestingly, staff from small agencies were most likely to receive training on infiltration and doors and windows (84\% and $81 \%$ of small agencies, respectively, reported these training topics) while those in medium and large agencies were most likely to provide training on insulation (85\% and $86 \%)$ and doors and windows $(83 \%$ and $84 \%)$. Staff in the coldest climate regions were most likely to receive training on insulation ( $88 \%$ very cold, $84 \%$ cold), infiltration ( $87 \%$ very cold, $73 \%$ cold), and doors and windows ( $80 \%$ very cold, $84 \%$ cold). Those in more moderate climates focused more on doors and windows, infiltration, and insulation (79\%, 77\%, and 76\%, respectively). Subgrantee staff in hot-humid climates most commonly received training in insulation, doors and windows, and water-heating measures (86\% each), and those in hot-dry climates received training on HVAC systems and doors and windows most often (94\% each).

The process of installing weatherization measures varies by housing type, so our questionnaire inquired about measure installation training separately for single family homes, multifamily buildings, and mobile homes. We found that subgrantee staff were trained on all three of these housing categories: 89 percent of subgrantees reported that their staff received training on measures for single family homes, 83 percent for multifamily buildings, and 78 percent for mobile homes. 
Ninety percent of subgrantees said staff received training in at least one technical or administrative topic (Fig. 3.12). Technical and administrative training was widespread across all program size groupings and in all climate regions, although staff in the hot-dry region received technical or administrative training in somewhat greater numbers, and those in the hot-humid region a little less.

The most common technical and administrative topics on which subgrantee staff received training were auditing of single-family dwellings (75\% of agencies), auditing of mobile homes (60\%), and monitoring/quality control. Client education and management were part of the training agendas for staff from about half of the subgrantees, while training on financial topics, outreach and communications, and auditing multifamily buildings was less common. ${ }^{20}$

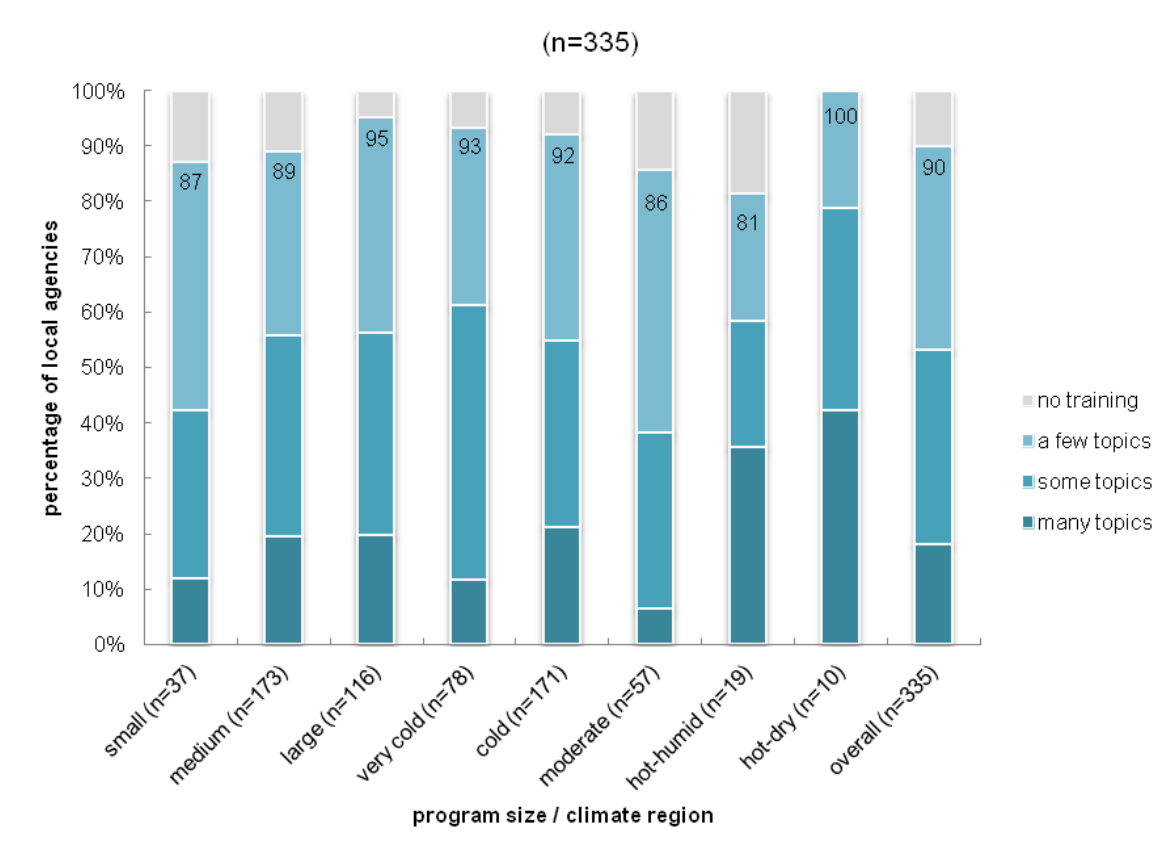

Fig. 3.12: Subgrantee staff training - technical and administrative topics ${ }^{+}$

Nearly all subgrantees (96 percent) reported that staff received training in some health and safety topics (Fig. 3.13). The most common health and safety topics on which staff received training were lead (cited by 82 percent of subgrantees), indoor air quality (69\%), mold and mildew (61\%), and general crew safety $(56 \%)$. All other topics - fire safety, housing unit security, household hazards, asbestos, vermiculitewere identified as training topics for their staff by fewer than half of the subgrantees.

\footnotetext{
${ }^{20}$ The training topic on auditing was labeled as "auditing/estimating" on the survey instrument to distinguish it from the survey questions on various diagnostic procedures that are part of the audit task.
} 


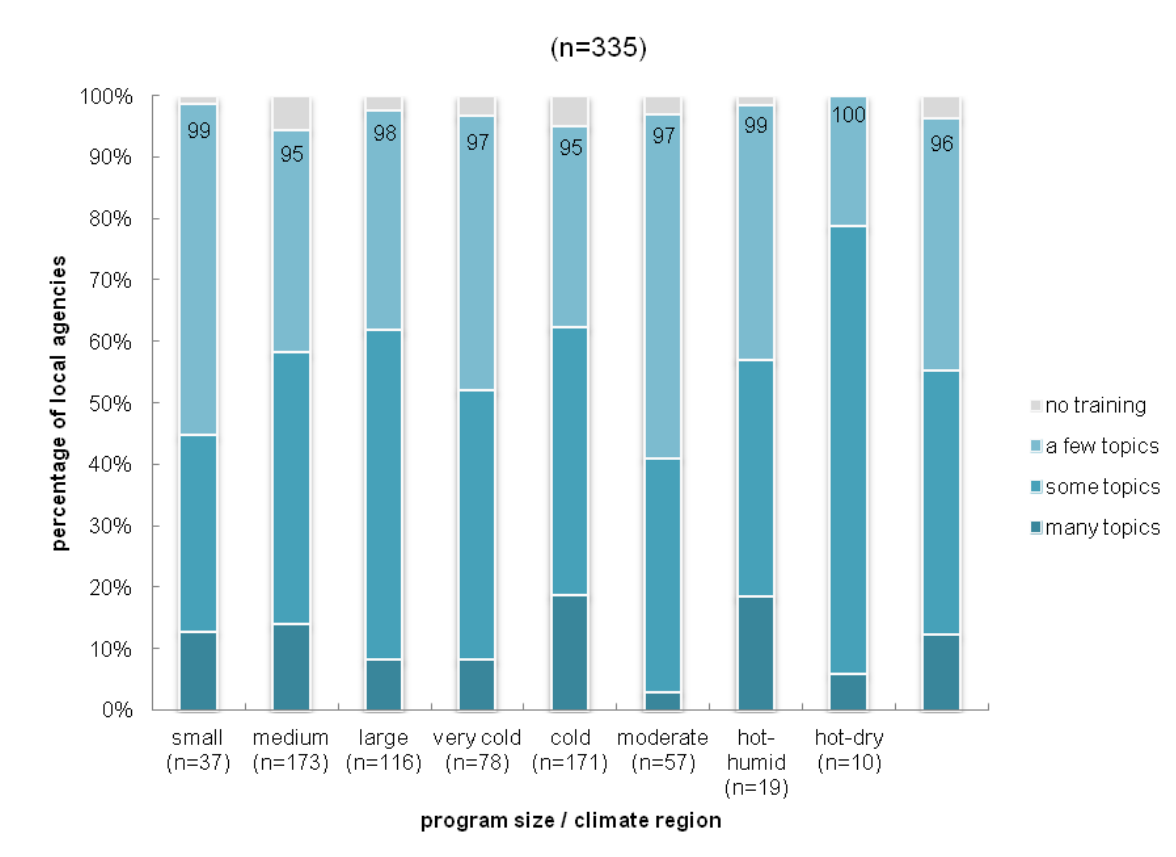

Fig. 3.13: Subgrantee staff training - health $\&$ safety ${ }^{+}$

Barriers to training. When asked what barriers prevented crews from receiving all the training they need, respondents representing two-thirds of subgrantees identified at least one barrier. The most commonly identified barriers ${ }^{21}$ were:

- Poor timing of the existing training options (47\%)

- Inability to take crews out of the field long enough for training (45\%)

- Lack of sufficient training funds (24\%)

- Inconvenient locations for the existing training options (24\%)

Subgrantees with large programs and those in the cold climate region particularly stressed their inability to take crews out of the field. Fifty-four percent of large agencies and 58 percent of those in the very cold climate region noted this barrier, compared to 45 percent of all subgrantees. Agencies with small programs and those in the very cold climate regions reported lack of training funds in greater proportions than their counterparts (30\% and $39 \%$ vs. $24 \%$ ).

\subsubsection{Perceptions of training effectiveness}

Of all the training venues included in our questionnaires, both grantees and subgrantees gave high marks to the quality of training at state/regional training centers and internally provided training. Grantees also gave high marks to the Affordable Comfort Conference. As shown in Table 3.5, grantees singled out training on management, monitoring, and selecting weatherization measures at the training centers, although they provided high ratings to the centers across multiple other topics as well. Grantees thought that internal training is particularly effective for diagnostic procedures and auditing, and they also rated the Affordable Comfort Conference particularly high for training on diagnostic procedures, as well as health and safety and selecting weatherization measures. The National Weatherization Program conference was rated stronger in weatherization installation and health and safety than on other training topics.

\footnotetext{
${ }^{21}$ Respondents chose from a pre-defined list of potential barriers.
} 
Subgrantees rated state/regional training centers particularly highly for health and safety, weatherization skills methods, and outreach and communications, as shown in Table 3.6. Internal training scored well on client education, weatherization skills and methods, and diagnostic procedures. Regional weatherization conferences, collectively, were seen as providing better training in weatherization methods, outreach and communications, and health and safety than they do on other topics, while the National Weatherization Program Conference's relative strengths were auditing and health and safety.

Table 3.5: Training quality by venue - grantee assessment

\begin{tabular}{|l|c|c|c|}
\hline Training Venue & $\mathbf{n}$ & Overall training quality & Top-rated topics \\
\hline State/Regional Training Center & 10 & high & $\begin{array}{c}\text { management (4.60) } \\
\text { monitoring/quality control (4.60) } \\
\text { selecting measures (4.60) }\end{array}$ \\
\hline Internal Training & 28 & high & $\begin{array}{c}\text { diagnostic procedures (4.19) } \\
\text { auditing (4.15) }\end{array}$ \\
\hline Affordable Comfort Conference & 14 & high & $\begin{array}{c}\text { diagnostic procedures (4.60) } \\
\text { health \& safety (4.45) } \\
\text { selecting measures (4.30) }\end{array}$ \\
\hline $\begin{array}{l}\text { National Weatherization Program } \\
\text { Conference }\end{array}$ & 29 & medium-high & $\begin{array}{c}\text { selecting measures (3.86) } \\
\text { management (3.81) }\end{array}$ \\
\hline Regional Weatherization Conference & 24 & medium-high & $\begin{array}{c}\text { weatherization installation (3.95) } \\
\text { health \& safety (3.94) }\end{array}$ \\
\hline State Weatherization Conference & 17 & medium-high & $\begin{array}{c}\text { weatherization installation (4.21) } \\
\text { health \& safety (3.91) } \\
\text { diagnostic procedures (3.91) }\end{array}$ \\
\hline
\end{tabular}

Table 3.6: Training quality by venue - subgrantee assessment

\begin{tabular}{|l|c|c|c|}
\hline Training Venue & $\mathbf{n}$ & Overall training quality & Top-rated topics \\
\hline State/Regional Training Center & 139 & high & $\begin{array}{c}\text { health \& safety (4.27) } \\
\text { wx methods (4.26) } \\
\text { outreach (4.17) }\end{array}$ \\
\hline Internal Training & 156 & high & $\begin{array}{c}\text { client education (4.40) } \\
\text { wx methods (4.11) } \\
\text { diagnostic procedures (4.09) }\end{array}$ \\
\hline Affordable Comfort Conference & 88 & medium-high & $\begin{array}{c}\text { wx methods (3.72) } \\
\text { health \& safety (3.68) }\end{array}$ \\
\hline $\begin{array}{l}\text { National Weatherization Program } \\
\text { Conference }\end{array}$ & 115 & medium-high & $\begin{array}{c}\text { auditing (3.72) } \\
\text { health \& safety (3.69) }\end{array}$ \\
\hline Regional Weatherization Conference & 84 & medium-high & $\begin{array}{c}\text { wx methods (3.92) } \\
\text { outreach (3.89) }\end{array}$ \\
\hline State Weatherization Conference & 129 & medium-high & diagnostic procedures (3.8)
\end{tabular}

While both grantees and subgrantees rated the overall training quality at these venues similarly, the topics they rated highest differed. When asked which of various types of training represented by these training venues were most effective in imparting key skills and information, grantees clearly favored direct instructions given to individual subgrantees. This means of providing training rated first for all subject 
areas included in our questionnaire. ${ }^{22}$ In-person expert visits and grantee-sponsored classes also rated highly, while conferences, the state/regional training centers, and webcasts were rated lower.

On a similar question, subgrantees distinguished clearly between technical skills required in the field and those used in the office or in client interactions. Clear majorities of subgrantees believe field training is the most effective for weatherization skills and methods ( $93 \%$ of agencies classified field work as effective), auditing (77\%), monitoring (69\%), health and safety (71\%), diagnostic procedures (87\%), and selecting weatherization measures $(64 \%)$. In contrast, conferences and classroom training are viewed as more effective for management skills, financial topics, outreach and communications, and client education.

\subsubsection{Certifications}

Technical certification and licensing requirements seek to ensure quality and consistency in the performance of weatherization work. The program information surveys for grantees (S1) and sampled subgrantees (S3) provided high-level information on the extent to which certifications or licensing requirements were in place for weatherization staff at the grantee and subgrantee level during PY2008. Survey questions focused on certification requirements across broad job categories. For grantee weatherization offices, the survey addressed credentialing for trainers, post-weatherization quality control inspectors, and administrative monitors. For subgrantees, the survey addressed requirements for management/administrative staff, auditing/inspection staff, and weatherization installers (in-house and contractors).

\subsubsection{Grantee level}

Sixty-four percent of grantees reported that personnel involved in training subgrantee weatherization agencies or their contractors were required to have technical certifications in PY08. As shown in Fig. 3.14 , such requirements were more prevalent in large grantee programs $(75 \%)$ than small programs (56\%). Seventy-three percent of grantee respondents $(n=41)$ indicated that certification requirements for trainers were important or very important.

\footnotetext{
${ }^{22}$ Those subject areas were: management, weatherization installation, auditing/estimating/measure selection, monitoring and quality control, financial topics, outreach and communications, health and safety, diagnostics procedures, and client education.
} 


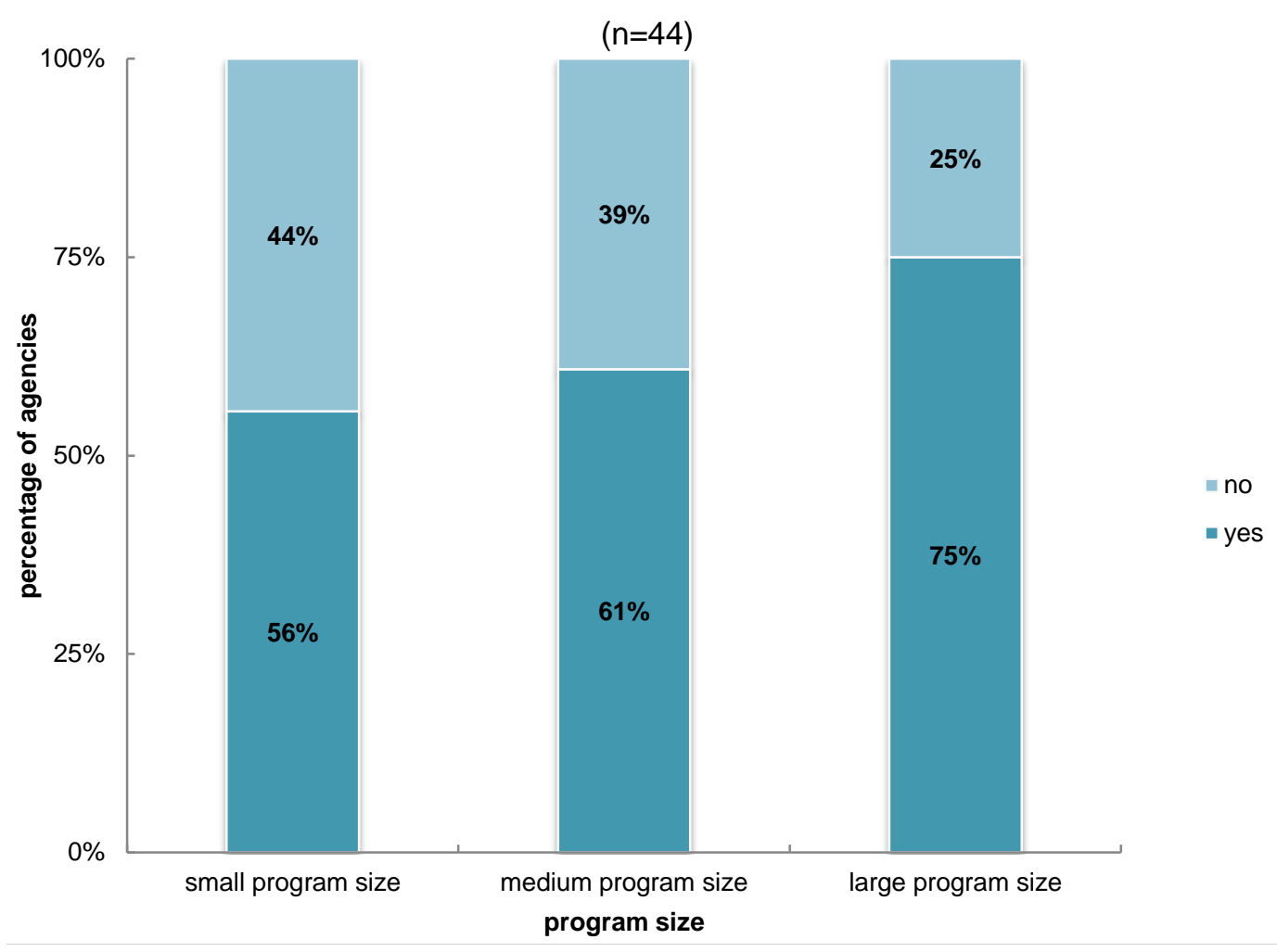

Fig. 3.14: Technical certification requirements for grantee trainers in PY2008

As shown in Fig. 3.15, technical certification requirements at the grantee level were most prevalent in the training arena (64\%). Roughly half of grantee respondents reported that technical certifications were required for staff conducting post-inspections. Certification requirements for post-inspectors were more common among large and medium-sized grantee programs (58\%) than among small programs (22\%). Only 30 percent of grantee respondents reported that technical certifications were required for staff involved in administrative monitoring. These requirements were slightly more prevalent among large and medium-sized grant programs (33 percent) than among small programs (20 percent). 


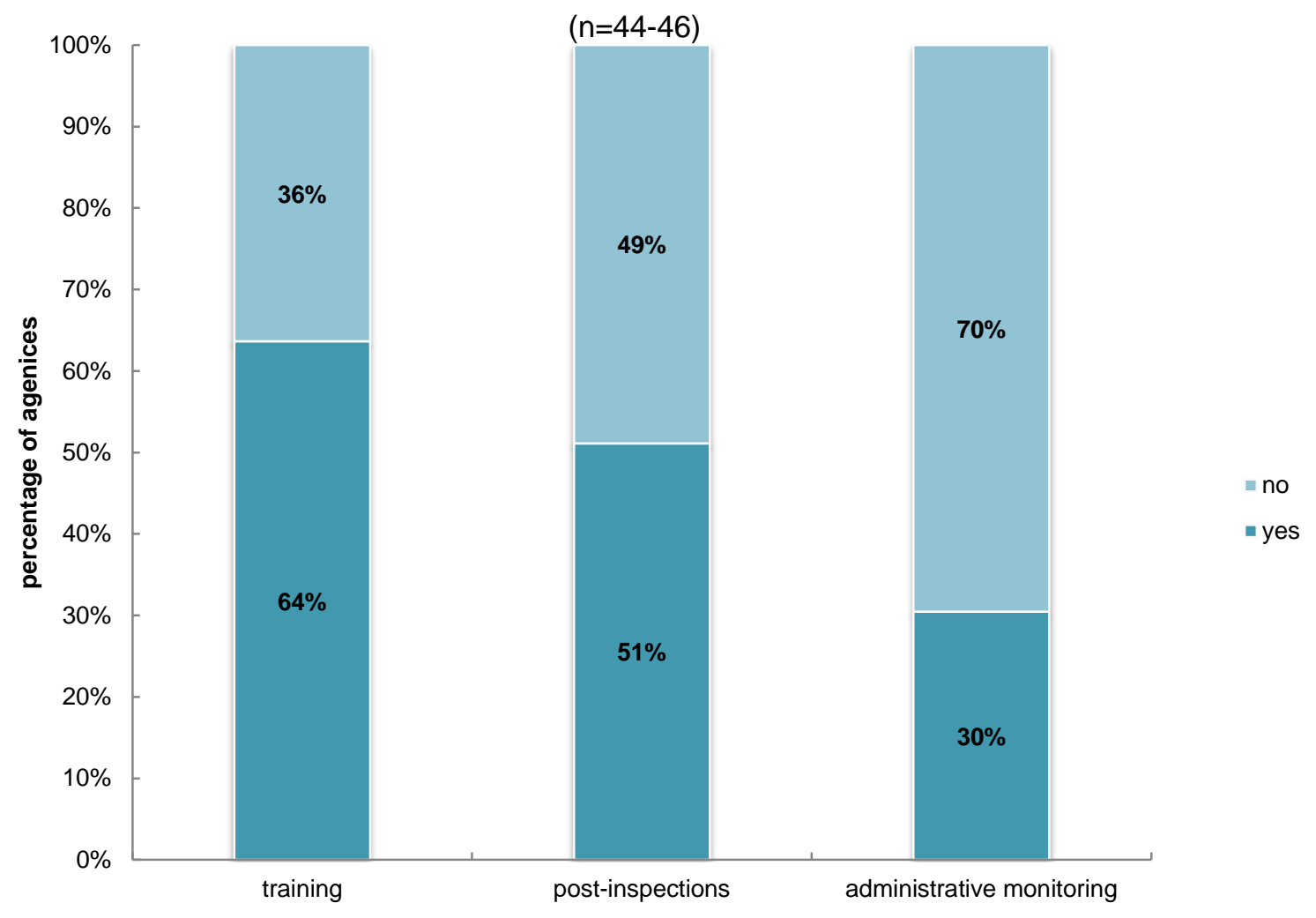

Fig. 3.15: Comparison of technical certification requirements for key grantee roles in PY2008 


\subsubsection{Subgrantee level}

Subgrantees were asked whether their grantee program required certification or licensing in key functional areas: program management/administration, auditing/inspection, and home weatherization. As shown in Table 3.7, certification requirements were most prevalent in the home weatherization category. Subgrantees from 45 states reported that technical certifications or licenses were required for staff or contractors involved in weatherization installation. Subgrantees from 43 states reported that technical certifications or licenses were required for staff or contractors involved in conducting audits or postinspections. Subgrantees from 28 states reported that technical certifications were required for staff or contractors involved in providing weatherization training.

Table 3.7: Grantee certification and licensing requirements

\begin{tabular}{|l|c|}
\hline & $\begin{array}{c}\text { Number of grantees with certification or } \\
\text { licensing requirements for staff/contractors } \\
\text { (as reported by sampled subgrantees) }\end{array}$ \\
\hline Program management/administration & 27 \\
\hline Auditing/inspection & 43 \\
\hline Home weatherization & 45 \\
\hline
\end{tabular}

The program information survey for subgrantees also addressed agency-level certification requirements for staff involved in four key areas: diagnostic procedures, measure selection, post-inspection and client education.

compares the prevalence of technical certification requirements across these categories. Certification requirements were similarly prevalent across diagnostics (78\%), measure selection (77\%) and post inspection (75\%). They were less common for subgrantee personnel involved in client education (51\%). At the subgrantee level, program size had less of an effect on the prevalence of these requirements than at the grantee level. 


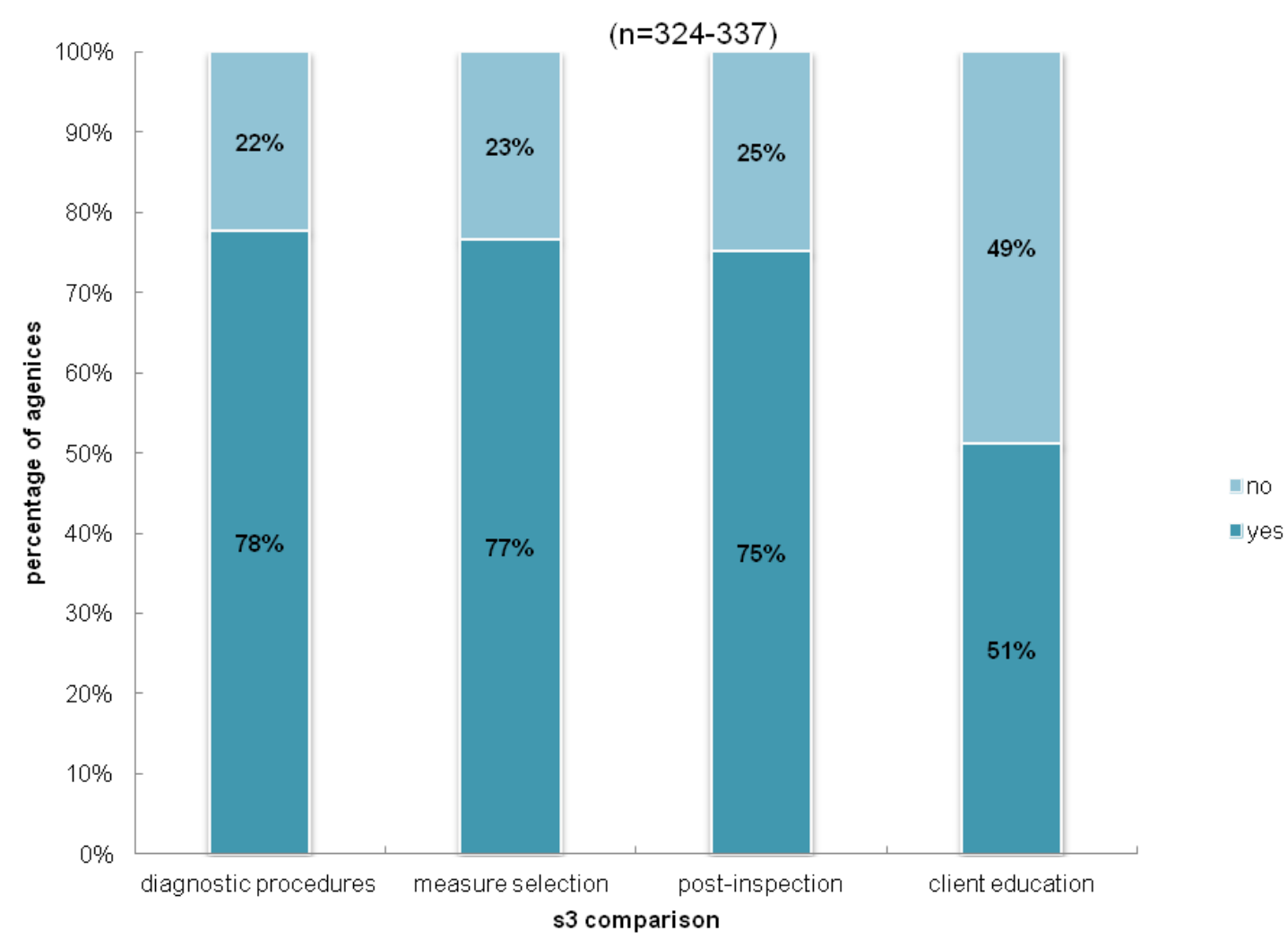

Fig. 3.16: Comparison of technical certification requirements for key subgrantee roles in PY2008 ${ }^{+}$ 


\section{HOW WEATHERIZATION SERVICES ARE DELIVERED}

The full weatherization process involves a multi-step sequence, which comprises an important aspect of the weatherization process. The main steps involving the participant are:

Client intake - Office staff, typically at the subgrantee or sometimes other low-income energy service organizations, explains the program to interested applicants. Once client eligibility is determined, priority for delivery of services is determined among the pool of eligible applicants.

Audit - Once a household is deemed to be eligible, a trained auditor visits the home to conduct various diagnostic tests and determine what measures would save energy and be cost-effective for that home. This audit process is more comprehensive than assessments conducted by some other residential programs and more complete than diagnostics performed by many private sector remodelers who provide energy efficiency improvements as part of their services.

Weatherization - After the audit, a weatherization crew employed by the subgrantee, private contractors, or a combination visits the home over the course of several weeks and performs the work specified by the audit.

Client education - In addition to physical work on the premises, the vast majority of weatherization projects include some form of client education about energy efficient practices and/or the measures being installed. Client education can be conducted at various stages of the weatherization process.

Post-weatherization inspection - After weatherization is complete, an agency inspector visits the home to ensure that all measures were installed and that the work was done well. The inspection component of weatherization results in rework that increases energy efficiency, improves durability of the installed measures, and/or assures health and safety.

\subsection{DISTRIBUTION OF EFFORT AMONG THE COMPONENTS OF WEATHERIZATION}

One measure of the relative effort expended on these various activities is spending by category. As shown in Table 4.1, subgrantees spent approximately two-thirds of their weatherization funds on the installation of measures and another tenth on health and safety measures in PY08. However, it is noteworthy that about seven percent of local weatherization funds were spent on auditing and inspecting homes - two important functions that help support program effectiveness. Program management costs were about 12 percent of the total amount spent at the local level. (The amounts shown in the table are based on all funding sources used by subgrantees, not just DOE funds.)

Table 4.1: Distribution of subgrantees' expenditures by category

\begin{tabular}{|l|c|c|}
\hline Expenditure Category & $\begin{array}{c}\text { Amount Spent by Reporting Subgrantees } \\
(\text { PY 2008) }\end{array}$ & Share of Total \\
\hline Weatherization measure installation & $\$ 470$ million & $70 \%$ \\
\hline Health and safety measures & $\$ 70$ million & $10 \%$ \\
\hline Audits and inspections & $\$ 50$ million & $7 \%$ \\
\hline Training and technical assistance & $\$ 10$ million & $1 \%$ \\
\hline Program management & $\$ 80$ million & $12 \%$ \\
\hline
\end{tabular}

Note: Amounts shown here are approximate and based on responses received from subgrantees. They have not been adjusted for non-respondents. 


\subsection{RECRUITMENT AND SCREENING}

Most subgrantees take some proactive steps to market their weatherization programs. As shown in Fig. 4.1, more than half market their programs through other social service agencies and post information about weatherization on their websites. Many also advertise in printed media and send direct mail to potential clients.

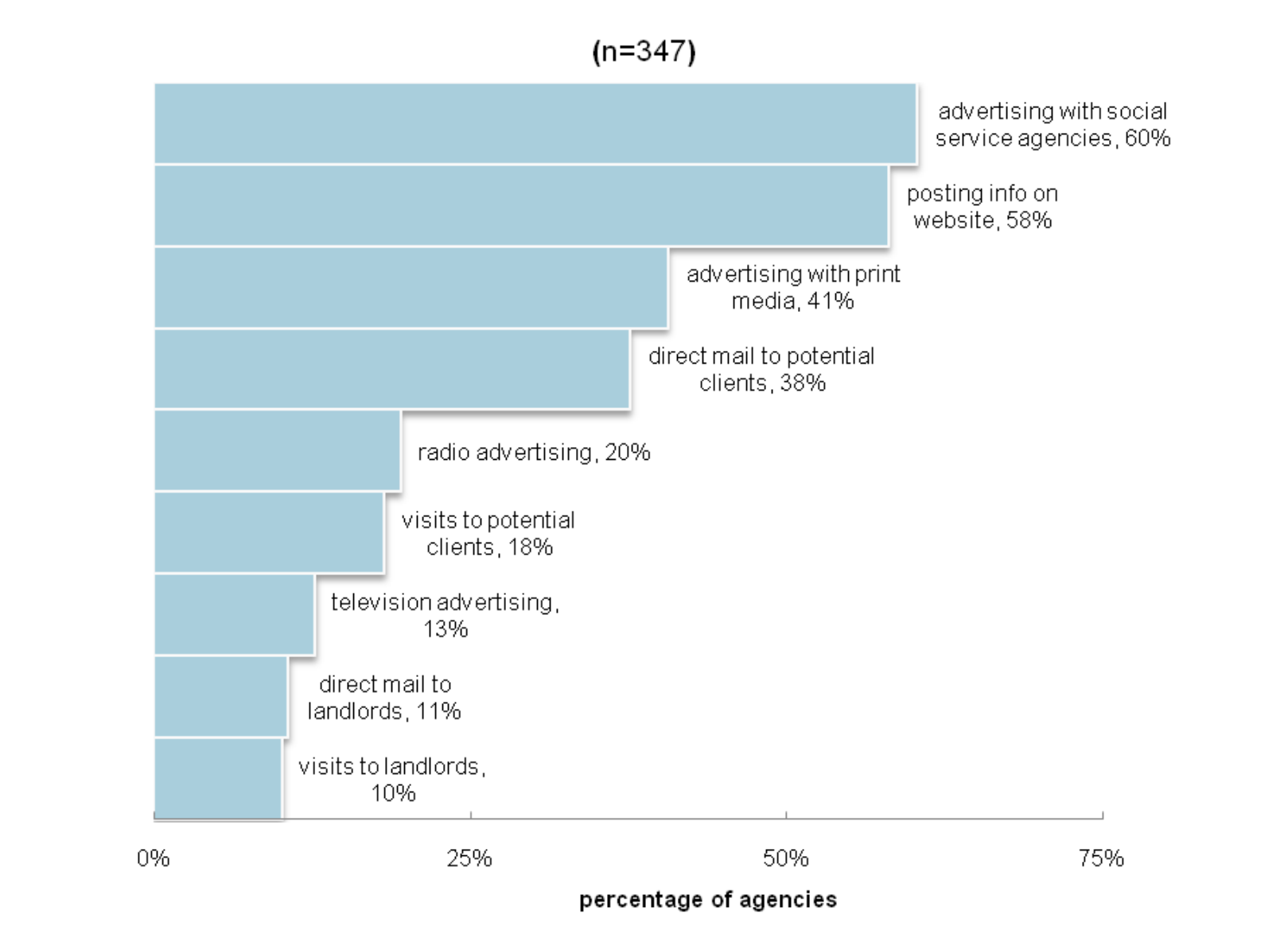

Fig. 4.1: Marketing for weatherization services by subgrantees ${ }^{+23}$

Subgrantees with large programs are more likely to use direct mail or visit potential clients and landlords than those from smaller programs. Fifty-three percent of large subgrantees use direct mail and 34 percent visit either potential clients or their landlords, while only 29 and 13 percent of small agencies do so. However, subgrantees of all sizes advertise with other social service agencies, on their own websites, and in the mass media in similar proportions (large, 46\%; medium, 47\%; small 55\%). In the majority of subgrantees $(73 \%)$, agency management takes responsibility for leading marketing and outreach efforts, while 29 percent of subgrantees indicate that an in-house outreach coordinator leads this function.

The first step in the actual weatherization process comprises the application and qualification process and the scheduling of an audit. Most subgrantees (86\%) indicated that they received needed information to qualify and process applicants from an application, although half received applicable data from LIHEAP

\footnotetext{
${ }^{23}$ The term advertising in this figure may encompass broader marketing activities, such as outreach to-and coordination with —other organizations. The figure shows the response options offered to subgrantees in the surveys they completed.
} 
and a quarter collected some of the household information they need at the time the home is audited. Approximately half of the subgrantees collected household energy consumption information from the household, and half did so from the local energy utility. (Some did both.) Meanwhile, about a fifth of subgrantees did not collect energy consumption data in PY08.

Among eligible applications, subgrantees prioritized weatherization projects based on numerous factors. Nearly all agencies indicated that they give priority to households that include elderly occupants (92\% of agencies), disabled occupants (90\%), or children (85\%). Roughly half of the subgrantees assigned priorities based on the amount of time an applicant had been on the waiting list, a client's energy expenditures or energy burden. Energy consumption, fuel type, and dwelling unit characteristics resulted in priority status in a minority of agencies. Fig. 4.2 displays these priorities graphically.

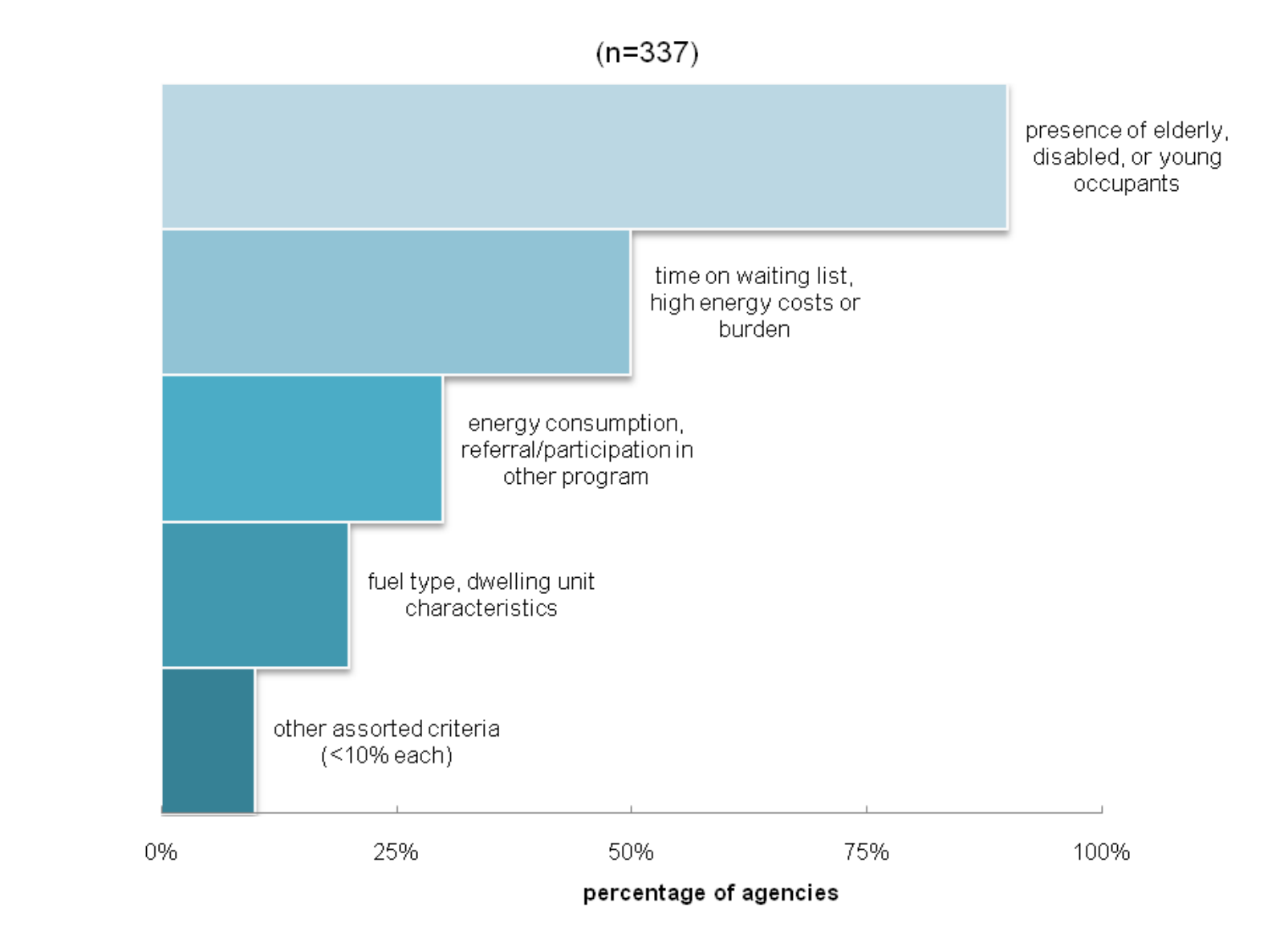

Fig. 4.2: Priority applicants ${ }^{+}$

Although subgrantees from all size groupings and climate regions are consistent in giving priority to households with elderly, disabled, and young occupants, there was some regional variation in the secondary priority factors. For example, subgrantees in the very cold and hot-dry regions more likely to prioritize based on a client's energy consumption, costs, or burden. In contrast, subgrantees in the moderate region were more likely to prioritize based on time spent on a waiting list and on fuel type.

When asked explicitly about setting targets or actively seeking participation in their weatherization programs by households in particular housing types, a minority of subgrantees indicated that they do. About a third of subgrantees set target numbers of single-family units to be weatherized in PY08 (with lower shares setting targets for other housing types), and about the same proportion actively encouraged 
the participation of single-family housing units in their weatherization program. Multifamily buildings were not frequently targeted in PY08. Twelve percent of subgrantees set targets for small multifamily buildings, and six percent did so for large multifamily buildings. Some more-21 percent and 13 percent, respectively - actively sought participation by small and large multifamily buildings.

Most subgrantees also referred clients to non-energy programs for additional social services. Between 57 and 70 percent of subgrantees indicated that they referred weatherization clients to other programs in PY08. ${ }^{24}$

\subsection{IDENTIFYING MEASURES NEEDED}

The first step in weatherizing a home is an audit in which a trained professional visits the home to identify the energy-saving measures that would provide cost-effective efficiency improvements and health and safety concerns that need to be addressed. The audit comprises diagnostic measurements and a decisionmaking process to select the measures that the weatherization crew or subcontractors will be directed to perform.

\subsubsection{Diagnostics}

Weatherization programs have some fairly sophisticated tools to identify heat loss, system efficiency, and other sources of energy waste. Most subgrantees - regardless of size or region-perform a range of procedures to test air infiltration and the safety and performance of space and water heating systems. Table 4.2 presents a list of diagnostic procedures reported by at least half of our respondents, the share of subgrantees that use them, agency perceptions of their comparative effectiveness, and agency perceptions about the level of effort (in cost, time, and training) needed to use them.

\footnotetext{
${ }^{24}$ We presented this share of subgrantees as a range because there was moderately high non-response to the relevant question (19 percent of sampled subgrantees who completed S3). Seventy percent of respondents indicated that they refer at least some of their cases to other programs. However, if the blanks left by the non-respondents actually were intended to mean " 0 ," that would imply that 57 percent of subgrantees refer some clients. We also sought to identify the share of clients referred, but could not reliably do so because the responses subgrantees entered suggest that respondents were inconsistent in whether they entered counts or percentages of units.
} 
Table 4.2: Diagnostic procedures performed by subgrantees in Program Year $\mathbf{2 0 0 8}^{+}$

\begin{tabular}{|l|c|c|c|}
\hline \multirow{2}{*}{ Diagnostic procedure } & \multicolumn{3}{|c|}{ Percentage of subgrantees that } \\
\cline { 2 - 4 } & $\begin{array}{c}\text { perform the } \\
\text { procedure }\end{array}$ & $\begin{array}{c}\text { perceive the } \\
\text { effectiveness as } \\
\text { high }\end{array}$ & $\begin{array}{c}\text { perceive the } \\
\text { investment needed } \\
\text { as high }\end{array}$ \\
\hline Blower door (house air leakage rate) & $99 \%$ & $94 \%$ & $32 \%$ \\
\hline $\begin{array}{l}\text { CO measurements in flues (space } \\
\text { heating system) }\end{array}$ & $91 \%$ & $84 \%$ & $25 \%$ \\
\hline $\begin{array}{l}\text { CO measurements in flues (water } \\
\text { heating system) }\end{array}$ & $88 \%$ & $81 \%$ & $22 \%$ \\
\hline $\begin{array}{l}\text { Flue gas analysis (space heating system } \\
\text { steady-state eff. test) }\end{array}$ & $83 \%$ & $88 \%$ & $31 \%$ \\
\hline CO measurement from cooking stove & $82 \%$ & $77 \%$ & $19 \%$ \\
\hline $\begin{array}{l}\text { Draft/spillage (space heating system, } \\
\text { normal operation) }\end{array}$ & $78 \%$ & $87 \%$ & $24 \%$ \\
\hline CO measurements in living areas & $78 \%$ & $82 \%$ & $21 \%$ \\
\hline $\begin{array}{l}\text { Draft/spillage (water heating system, } \\
\text { normal operation) }\end{array}$ & $77 \%$ & $85 \%$ & $23 \%$ \\
\hline $\begin{array}{l}\text { Flue gas analysis (water heating steady- } \\
\text { state eff. test) }\end{array}$ & $72 \%$ & $80 \%$ & $27 \%$ \\
\hline CO measurements in equipment rooms & $69 \%$ & $80 \%$ & $17 \%$ \\
\hline Worst case draft/spillage (CAZ) & $63 \%$ & $85 \%$ & $35 \%$ \\
\hline Refrigerator energy use & $63 \%$ & $67 \%$ & $14 \%$ \\
\hline Duct pressure pan measurements & $59 \%$ & $63 \%$ & $23 \%$ \\
\hline Zonal pressure measurements & $58 \%$ & $71 \%$ & $33 \%$ \\
\hline $\begin{array}{l}\text { Heat rise measurements (space heating } \\
\text { system) }\end{array}$ & $55 \%$ & $68 \%$ & $17 \%$ \\
\hline Infrared scanning (camera) & $51 \%$ & & \\
\hline & & & \\
\hline
\end{tabular}

* Investment comprises both training and time needed.

Diagnostic procedures performed and perceived as effective vary slightly by program size and a bit more by climate region. As shown in Table 4.3, the most commonly performed diagnostic procedures in smaller and larger subgrantee agencies are generally the same, although smaller agencies more commonly test cooking stoves while larger agencies do more draft/spillage tests. 
Table 4.3: Common diagnostic procedures by program size

\begin{tabular}{|c|c|}
\hline Program Size & Most used diagnostic procedures \\
\hline $\begin{array}{l}\text { small } \\
(n=45)\end{array}$ & $\begin{array}{l}\text { blower door test }(98 \%) \\
\text { cooking stove }(86 \%) \\
\text { CO in living areas }(85 \%) \\
\text { CO in flues (water heat) }(81 \%) \\
\text { CO in flues (space heat) }(81 \%)\end{array}$ \\
\hline $\begin{array}{l}\text { medium } \\
(n=182)\end{array}$ & $\begin{array}{l}\text { blower door test (99\%) } \\
\text { CO in flues (space heat) }(93 \%) \\
\text { flue gas analysis (space heat) }(90 \%) \\
\text { CO in flues (water heat) }(88 \%) \\
\text { draft/spillage }(84 \%)\end{array}$ \\
\hline $\begin{array}{l}\text { large } \\
(n=118)\end{array}$ & $\begin{array}{l}\text { blower door test (98\%) } \\
\mathrm{CO} \text { in flues (space heat) }(96 \%) \\
\mathrm{CO} \text { in flues (water heat) }(94 \%) \\
\mathrm{draft} / \text { spillage (water heat) }(92 \%) \\
\mathrm{draft} / \text { spillage (space heat) }(89 \%)\end{array}$ \\
\hline
\end{tabular}

As shown in Table 4.4, the most commonly performed diagnostic procedures in cooler and warmer climate regions varied somewhat as well, although the results for the hot-dry region are affected by the small respondent pool from that region. While blower door tests were widespread across the regions, colder climates emphasized space and water heating system tests. These tests were less common in warmer climates, so the carbon monoxide tests by those agencies appeared higher in the ranking of diagnostic procedures performed. 
Table 4.4: Common diagnostic procedures by climate region ${ }^{+}$

\begin{tabular}{|c|c|}
\hline Program Size & Most used diagnostic procedures \\
\hline $\begin{array}{l}\text { very cold } \\
(n=80)\end{array}$ & $\begin{array}{l}\text { blower door test }(100 \%) \\
\text { flue gas analysis (space heat) }(88 \%) \\
\text { draft/spillage (space heat) }(88 \%) \\
\text { draft/spillage (water heat) }(87 \%) \\
\text { CO in flues (space heat) }(87 \%)\end{array}$ \\
\hline $\begin{array}{l}\text { cold } \\
(n=172)\end{array}$ & $\begin{array}{l}\text { blower door test (99\%) } \\
\text { CO in flues (space heat) }(98 \%) \\
\text { CO in flues (water heat) }(95 \%) \\
\text { flue gas analysis (space heat) }(94 \%) \\
\text { draft/spillage (space heat) }(93 \%)\end{array}$ \\
\hline $\begin{array}{l}\text { moderate } \\
(n=58)\end{array}$ & $\begin{array}{l}\text { blower door test }(100 \%) \\
\text { CO in living areas }(90 \%) \\
\text { CO from cooking stoves }(89 \%) \\
\text { CO in flues (space heat) }(86 \%) \\
\text { flue gas analysis (space heat) }(85 \%)\end{array}$ \\
\hline $\begin{array}{l}\text { hot-humid } \\
(\mathrm{n}=20)\end{array}$ & $\begin{array}{l}\text { blower door test }(95 \%) \\
\text { CO from cooking stove }(92 \%) \\
\text { CO in flues (space heat) }(82 \%) \\
\text { CO in living areas }(82 \%) \\
\text { CO in flues (water heat) }(77 \%)\end{array}$ \\
\hline $\begin{array}{l}\text { hot-dry } \\
(n=9)\end{array}$ & $\begin{array}{l}\text { CO in flues (space heat) (100\%) } \\
\mathrm{CO} \text { in flues (water heat) }(100 \%) \\
\text { draft/spillage (water heat) }(100 \%) \\
\mathrm{CO} \text { in living areas }(93 \%) \\
\text { blower door test }(93 \%)\end{array}$ \\
\hline
\end{tabular}

\subsubsection{Decision-making tools used}

The diagnostic results from the audit lead to the selection of measures to be installed or implemented on the home being weatherized. Subgrantees can use either a priority list or a calculation procedure, such as Weatherization Assistant (NEAT/MHEA). A slight majority of subgrantees used priority lists as their primary measure section tool in PY08, as shown in 
Table 4.5. About a third used calculation procedures as their primary tool, while 12 percent of subgrantees used priority lists on some units and a calculation procedure on other units. There is relative stability in the use of these decision-making tools; on average, subgrantees have been using their current type of tool for over a decade.

Table 4.5: Primary measure selection tools used in Program Year $\mathbf{2 0 0 8}^{+}$

\begin{tabular}{|l|c|c|}
\hline Tools used & Percentage of subgrantees & $\begin{array}{c}\text { Mean \# of years } \\
\text { used }\end{array}$ \\
\hline priority list & $53 \%$ & 15 \\
\hline calculation procedure & $33 \%$ & 11 \\
\hline both (for different units) & $12 \%$ & 11 \\
\hline ambiguous responses & $2 \%$ & $\mathrm{n} / \mathrm{a}$ \\
\hline
\end{tabular}

Priority lists were more commonly used by subgrantees with small programs (68\% of these used priority lists exclusively) and those in all climate regions except the very coldest - usage ranged from $57 \%$ in the cold region to $71 \%$ in the hot-dry region. Large agencies and those in very cold climate region were more likely to use calculation procedures exclusively ( $42 \%$ and 63\%, respectively).

Subgrantees that used priority lists found them to be easy (67\% of agencies) or very easy (26\%) to use. They also perceived them to be effective $(67 \%)$ or very effective $(21 \%)$.

Weatherization Assistant was the most commonly used calculation procedure in use in PY08 (Table 4.6). Those who used it as their primary measure selection tool rated it, on average, as effective, but not as uniformly easy to use. Sixty-two percent of users rated it as easy or very easy to use, while 38 percent considered it difficult or very difficult to use.

Table 4.6: Most commonly used calculation procedures in Program Year $\mathbf{2 0 0 8}^{+}$

$$
(n=182)
$$

\begin{tabular}{|c|c|c|c|}
\hline Calculation procedure & $\begin{array}{l}\text { Percentage of subgrantees } \\
\text { (of those who use } \\
\text { calculation procedures as a } \\
\text { primary tool) }\end{array}$ & $\begin{array}{c}\text { Ease of use } \\
\text { (\% indicating tool's } \\
\text { use is easy or very } \\
\text { easy) }\end{array}$ & $\begin{array}{l}\text { Effectiveness } \\
\text { (\% indicating tool's } \\
\text { use is effective or } \\
\text { very effective) }\end{array}$ \\
\hline Weatherization Assistant & $53 \%$ & $62 \%$ & $77 \%$ \\
\hline REM/Rate & $8 \%$ & \multirow{4}{*}{\multicolumn{2}{|c|}{$\begin{array}{l}\text { Not presenting means because these } \\
\text { calculation procedures were each used by } \\
\text { fewer than } 20 \text { subgrantees. }\end{array}$}} \\
\hline TREAT & $7 \%$ & & \\
\hline EA-QUIP & $5 \%$ & & \\
\hline EASY & $5 \%$ & & \\
\hline
\end{tabular}

\subsection{CLIENT EDUCATION}

Virtually all subgrantees reported performing some sort of client education in PY08. ${ }^{25}$ Subgrantees reported that, on average, they provide just under 45 minutes of client education at various times during

\footnotetext{
${ }^{25}$ Only five of the sampled subgrantees who responded to the detailed program information survey marked none of the client education measures on the questionnaire. When weighted, these respondents represent three percent of the full population of agencies.
} 
the weatherization process, especially during the intake process, the on-site weatherization work, and the inspection. In most cases, the client education comprised direct interaction with the participantapplicants were the target for client education among $94 \%$ of the subgrantees - and sometimes another household member. ${ }^{26}$ The most common form of client education consisted of the dissemination of some energy literature and in-person interaction with the client.

The plurality of subgrantees reported that they spent between a quarter and a half hour on client education in a typical dwelling (Fig. 4.3). The average amount of time spent was nearly 45 minutes, but that value was buoyed by a few agencies that reported very large amounts of time. The median time spent was about 30 minutes. Fourteen percent of the subgrantees that reported doing client education did not answer our question on the amount of time spent. If these agencies left the question blank because their educational efforts don't involve spending interactive time with the client, then the average time spent may be a bit lower than reported here. The differences between subgrantees with varying program sizes and in different climate regions were small.

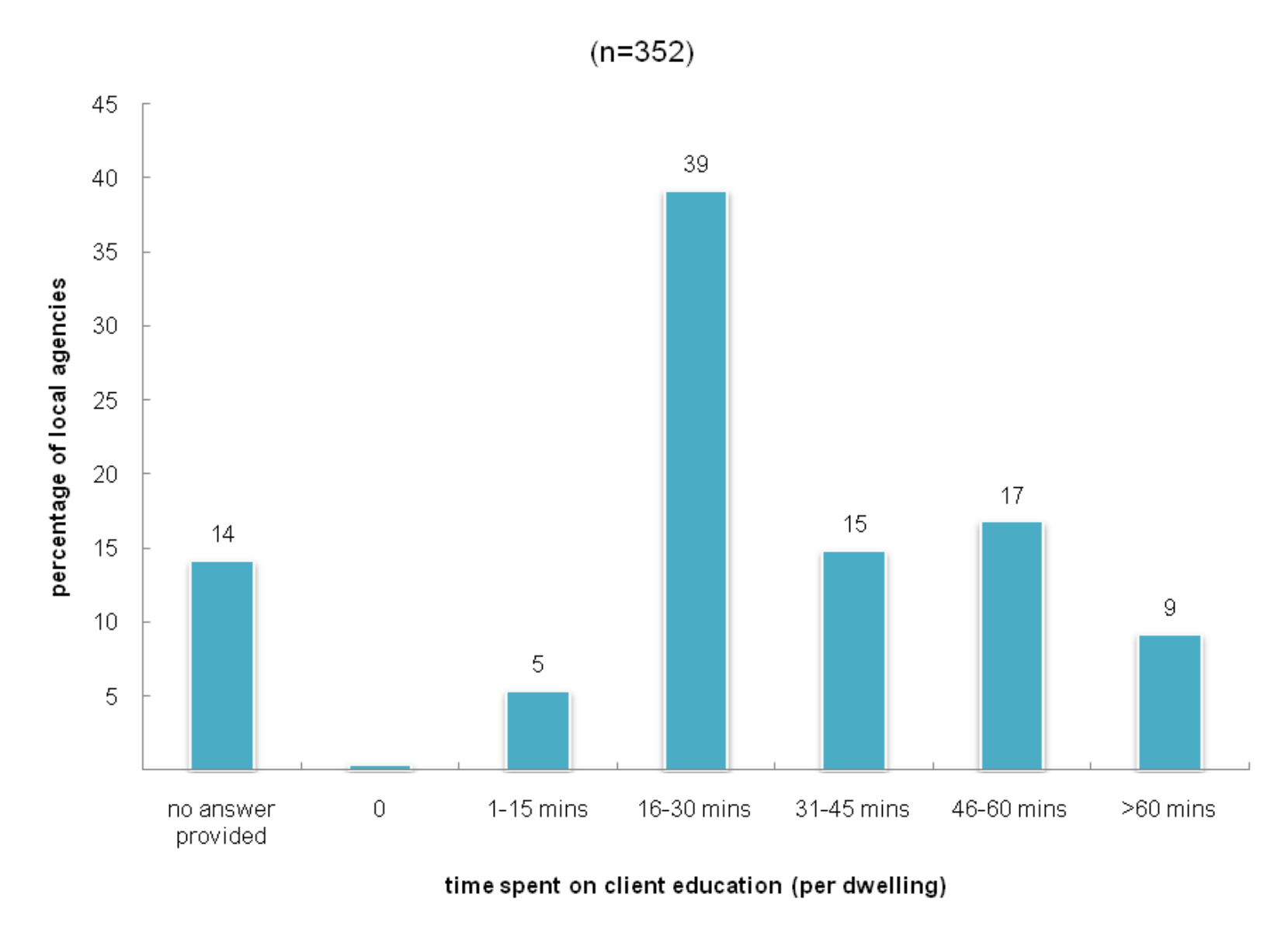

Fig. 4.3: Quantity of client education provided ${ }^{+}$

Table 4.7 below lists some common topics covered as part of client education. The most common topics were management of the client's thermostat, information about insulation, lighting, and the operation and maintenance of the client's heating (or cooling) system. The field process study being done as part of the

\footnotetext{
${ }^{26}$ Twenty-two percent of subgrantees indicated that they typically provide client education to multiple individuals for each weatherization project.
} 
national weatherization evaluation is likely to provide additional insights about the nature and depth of client education.

Table 4.7: Topics covered as part of client education in more than half of weatherization subgrantees+

\begin{tabular}{|l|c|}
\hline Client Education Topics & \% of agencies \\
\hline Thermostat management & 88 \\
\hline Insulation & 86 \\
\hline Lighting & 84 \\
\hline HVAC system operation / maintenance & 80 \\
\hline Windows & 75 \\
\hline Hot water use & 73 \\
\hline Ventilation & 71 \\
\hline Safety monitors (e.g., CO monitors, smoke alarm) & 68 \\
\hline Mold & 67 \\
\hline Refrigerator & 66 \\
\hline Water heating system operation / maintenance & 59 \\
\hline Energy bills & 56 \\
\hline
\end{tabular}

As one might expect, there are regional differences among the topics included in client education. For example, thermostat management was the most commonly cited topic in four of the five climate regions, but was not even among the top five topics for the hot-dry region. Ventilation was commonly discussed in the coldest climate region, but not as frequently in the rest of the country. The warmer climates, on the other hand, more frequently included HVAC system operation and maintenance. Table 4.8 lists the five most common topics by climate region.

Table 4.8: Most common client education topics by climate region

\begin{tabular}{|l|l|l|l|l|l|}
\hline Rank & \multicolumn{1}{|c|}{$\begin{array}{c}\text { very cold } \\
(\mathbf{n = 7 7 )}\end{array}$} & \multicolumn{1}{|c|}{$\begin{array}{c}\text { cold } \\
(\mathbf{n = 1 7 1})\end{array}$} & \multicolumn{1}{|c|}{$\begin{array}{c}\text { moderate } \\
(\mathbf{n = 5 5})\end{array}$} & \multicolumn{1}{c|}{$\begin{array}{c}\text { hot-humid } \\
(\mathbf{n = 2 0})\end{array}$} & \multicolumn{1}{c|}{$\begin{array}{c}\text { hot-dry } \\
(\mathbf{n = 1 0})\end{array}$} \\
\hline 1. & $\begin{array}{l}\text { thermostat } \\
\text { management } \\
(87 \%)\end{array}$ & $\begin{array}{l}\text { thermostat } \\
\text { management } \\
(91 \%)\end{array}$ & $\begin{array}{l}\text { thermostat } \\
\text { management } \\
(85 \%)\end{array}$ & $\begin{array}{l}\text { thermostat } \\
\text { management } \\
(88 \%)\end{array}$ & insulation $(100 \%)$ \\
\hline 2. & ventilation $(86 \%)$ & insulation $(90 \%)$ & insulation $(85 \%)$ & lighting $(79 \%)$ & lighting $(100 \%)$ \\
\hline 3. & lighting $(84 \%)$ & lighting $(88 \%)$ & $\begin{array}{l}\text { HVAC o\&m } \\
(82 \%)\end{array}$ & $\begin{array}{l}\text { safety monitors } \\
(79 \%)\end{array}$ & windows $(100 \%)$ \\
\hline 4. & $\begin{array}{l}\text { hot water use } \\
(77 \%)\end{array}$ & $\begin{array}{l}\text { HVAC o\&m } \\
(83 \%)\end{array}$ & $\begin{array}{l}\text { lighting }(74 \%) \\
(77 \%)\end{array}$ & $\begin{array}{l}\text { HVAC o\&m } \\
(93 \%)\end{array}$ \\
\hline 5. & windows $(77 \%)$ & windows $(79 \%)$ & windows $(69 \%)$ & insulation $(73 \%)$ & energy bills $(93 \%)$ \\
\hline
\end{tabular}

Note: Percentage represent the share of agencies that do client education.

As previously noted, the vast majority of client education consisted of the dissemination of literature or in-person interaction with the client. As shown in Fig. 4.4, 96 and 93 percent of subgrantees, respectively, employed these two methods. Many did both, although small agencies were more likely to rely on literature only. Other forms of client education, such as the dissemination of videos, kits, or separate client education classes were less common and more likely to be done by larger agencies. 


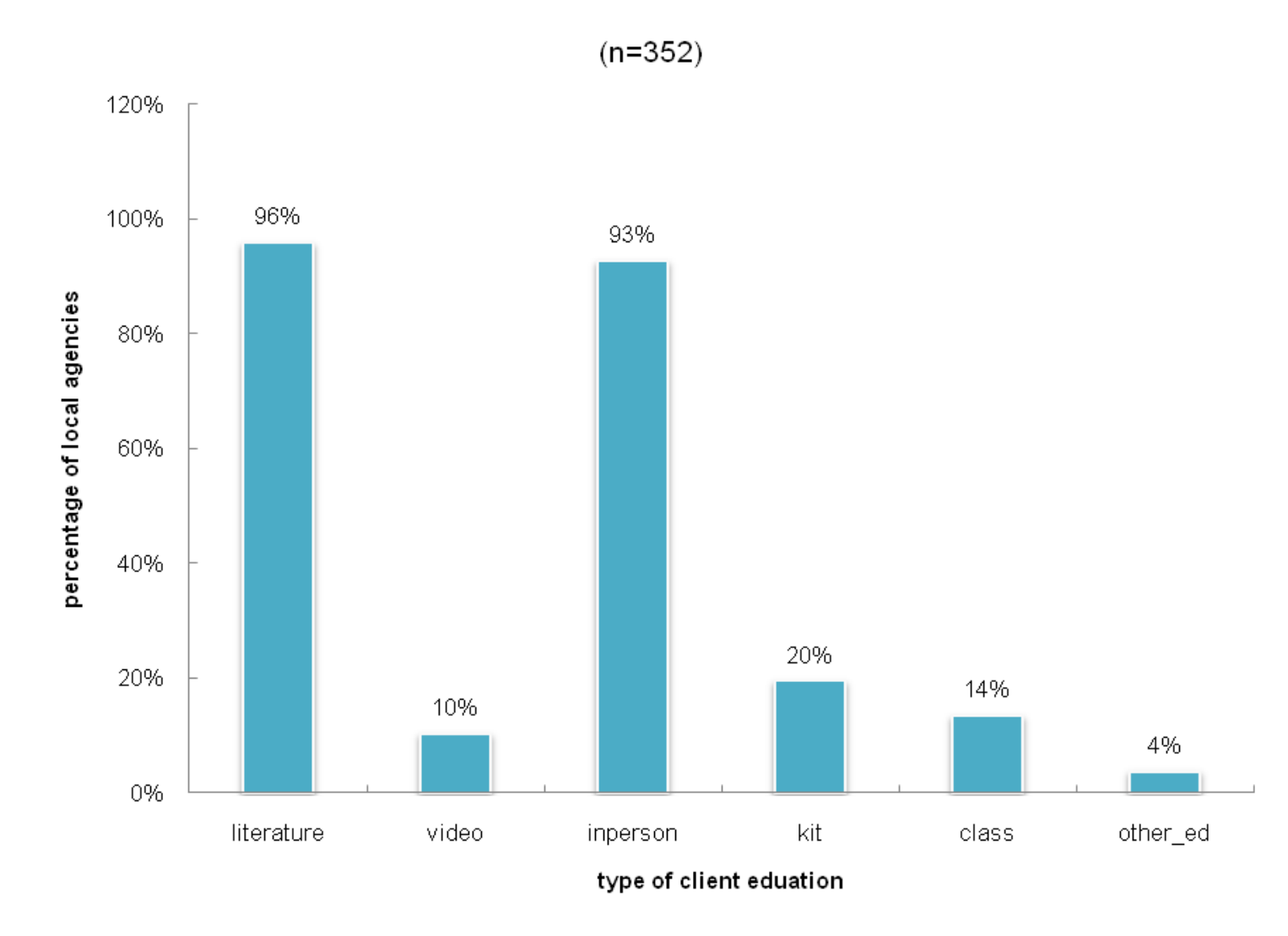

Fig. 4.4: Type of client education ${ }^{+}$

As noted earlier, subgrantees used three separate touch points to conduct their client education. As shown in Fig. 4.5, roughly 90 percent of subgrantees conducted client education at intake, during the weatherization work, and at the inspection. Many used all three of these touch points to conduct client education. Larger agencies were more likely to incorporate client education into the on-site weatherization work, however. 


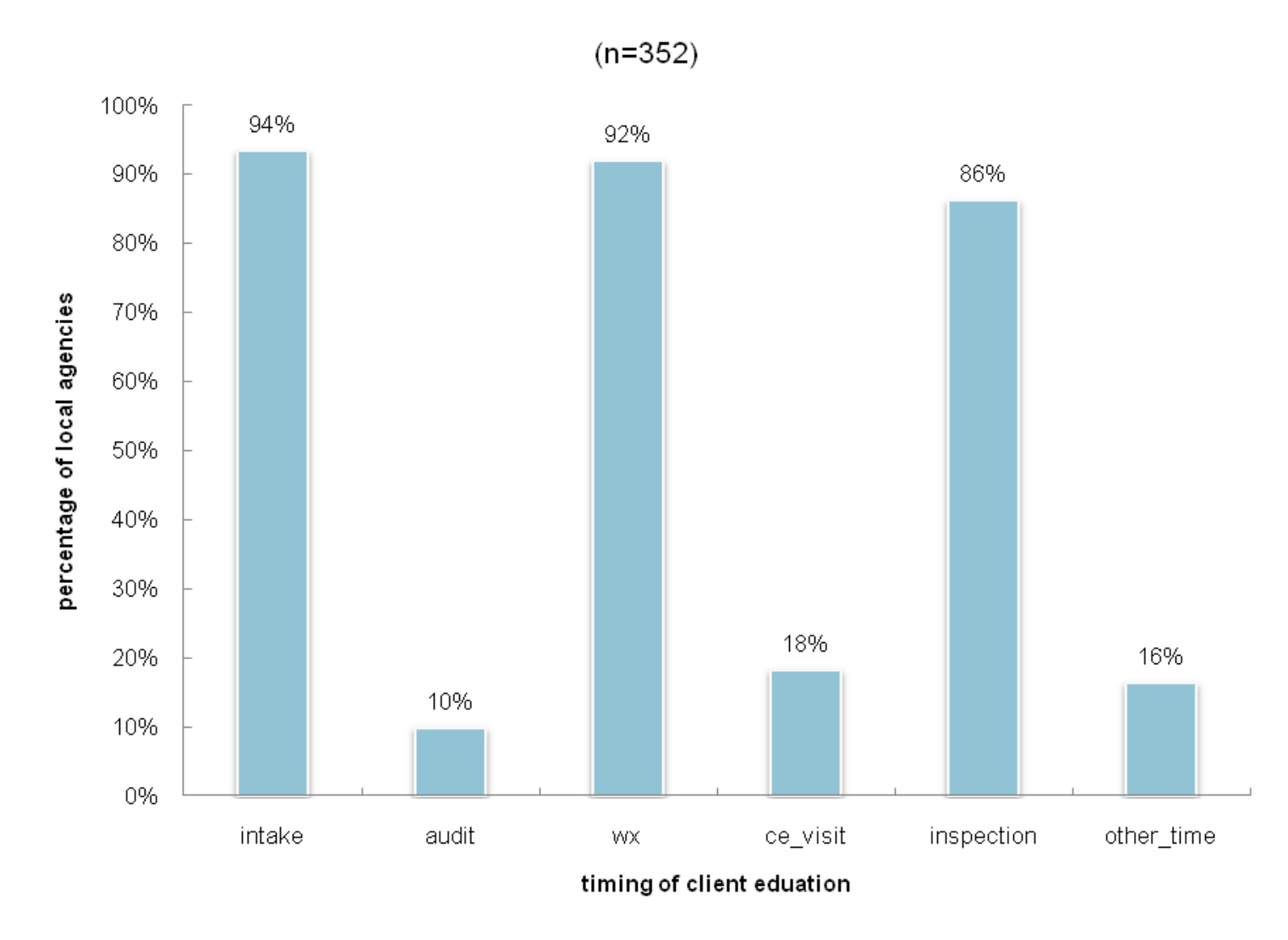

Fig. 4.5: Timing of client education ${ }^{+}$

Not surprisingly, subgrantees reported that staff most involved in client education are auditors (cited by $86 \%$ of subgrantees as providing client education), inspectors (62\%), and intake personnel (54\%).27 Inhouse education specialists or contractor education specialists were used by only 12 and 6 percent of subgrantees, respectively.

When asked about the relative effectiveness and levels of investment needed for each of these client education approaches, subgrantees gave favorable marks to in-person instruction and the dissemination of literature. Respondents rated in-person instruction as being somewhat more effective than the other types of instruction, but also requiring a greater investment of money, time, and staff training. The dissemination of literature was rated as being nearly as effective with a lower overall investment needed. Subgrantees perceive in-home opportunities - either at the time of the audit, the weatherization work, or the inspection - to be relatively more effective and requiring no more investment than other client education opportunities. See Table 4.9 for a more complete summary of subgrantee perceptions.

\footnotetext{
${ }^{27}$ These proportions are lower than one would expect from subgrantee responses on when client education occurs. One possible explanation is that subgrantees did not attribute the dissemination of literature to individual staff. Alternatively, it is possible that there is some underreporting of staff involvement or overreporting of where client education occurs.
} 
Table 4.9: Perceived effectiveness and level of investment needed for various client education approaches ${ }^{+}$

\begin{tabular}{|l|c|c|}
\hline Type of post-client education & $\begin{array}{c}\text { Effectiveness (mean score on } \\
\text { 5-point scale where 5 =very } \\
\text { high") }\end{array}$ & $\begin{array}{c}\text { Investment needed (blended } \\
\text { mean on } \\
\text { 5-point scale where 5 = } \\
\text { "very high") }\end{array}$ \\
\hline Type of Client Education & 3.2 & 2.3 \\
\hline literature & 2.5 & 2.5 \\
\hline video & 3.5 & 2.7 \\
\hline in-person instruction & 2.6 & 2.4 \\
\hline hardware kit & 2.9 & 3.1 \\
\hline group training class & 2.9 & 2.4 \\
\hline Timing of Client Education & 3.1 & 2.4 \\
\hline client intake & 3.1 & 2.4 \\
\hline audit & 2.7 & 2.8 \\
\hline weatherization & 3.1 & 2.4 \\
\hline client education visit & \multicolumn{2}{|c|}{} \\
\hline inspection & \multicolumn{2}{|c|}{} \\
\hline
\end{tabular}

\subsection{INSTALLATION}

Weatherization programs install two general types of measures in client homes: home improvements to save energy and home improvements to address health and safety risks. Measure installation is performed by a weatherization crew employed by the subgrantee, private contractors, or a combination of the two. Diagnostics to determine measures and types of measures installed are discussed more fully in Measures and Services (see Section 5.0 - Whom do Weatherization Agencies Serve with what Services?).

\subsection{QUALITY CONTROL AND ASSURANCE}

Quality monitoring and inspections conducted by grantee staff provide feedback to subgrantees on performance, accountability to funders, and assurance to clients that the weatherization work was done properly. As noted, all grantees conduct quality monitoring of subgrantees, and subgrantees inspect individual projects. These activities encompass both quality control and quality assurance.

\subsubsection{Grantee monitoring}

Grantee quality control and assurance activities consist of both inspections of some weatherized units and agency visits to monitor administrative functions. Together, these activities account for 105 FTEs of activity by grantees nationwide, or about 33 percent of total grantee staff time spent on weatherization program activities (Fig. 4.6). The range of staff time devoted to these activities spans from 5 percent to 60 percent of total staff time at the grantee level. 


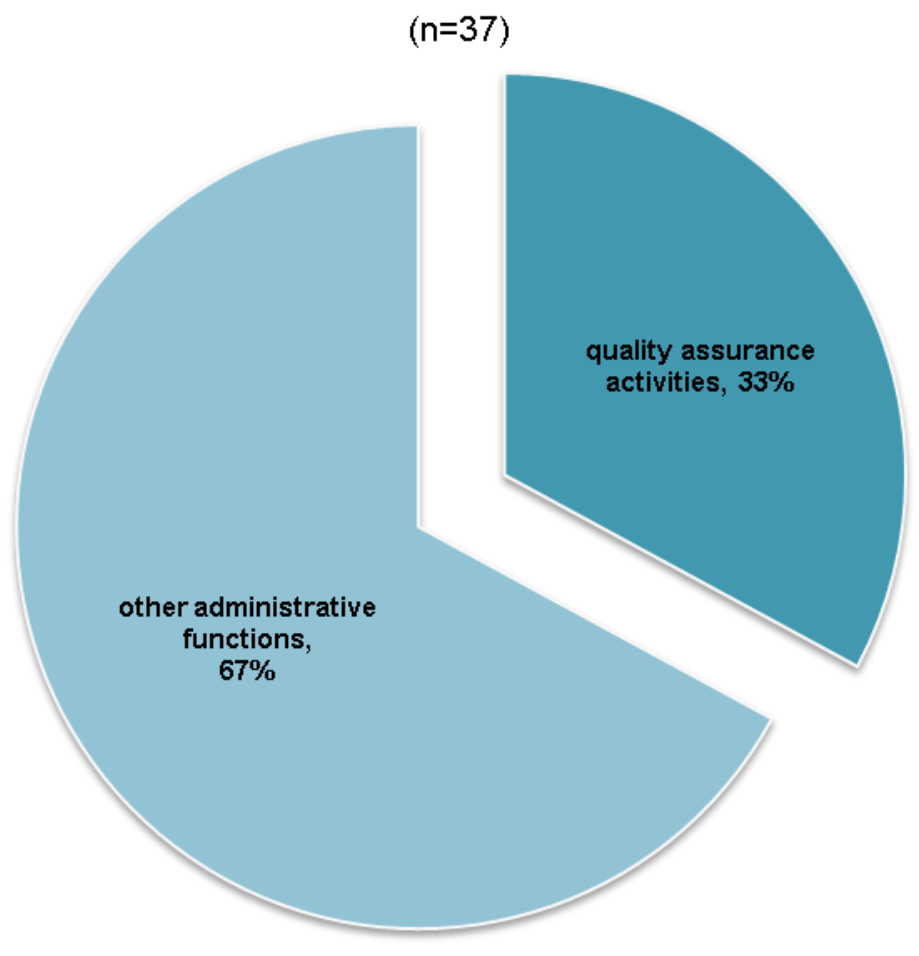

Fig. 4.6: Proportion of staff time (FTEs) spent on quality control and assurance activities

The amount of staff time grantees spend on quality control and assurance varies with program size (Fig. 4.7). Grantees with small weatherization programs tend to spend proportionally less staff time than grantees with medium- or large-sized programs. Roughly one-fifth of total staff time is allocated to these activities among grantees with small programs, whereas grantees with medium-sized programs spend 35 percent of FTEs and those with large programs spend 36 percent. It is important to note that grantee monitoring activities have been expanded under ARRA. The ARRA evaluation may show significant changes in the level of effort devoted to quality control and assurance activities as compared with these PY08 results. 


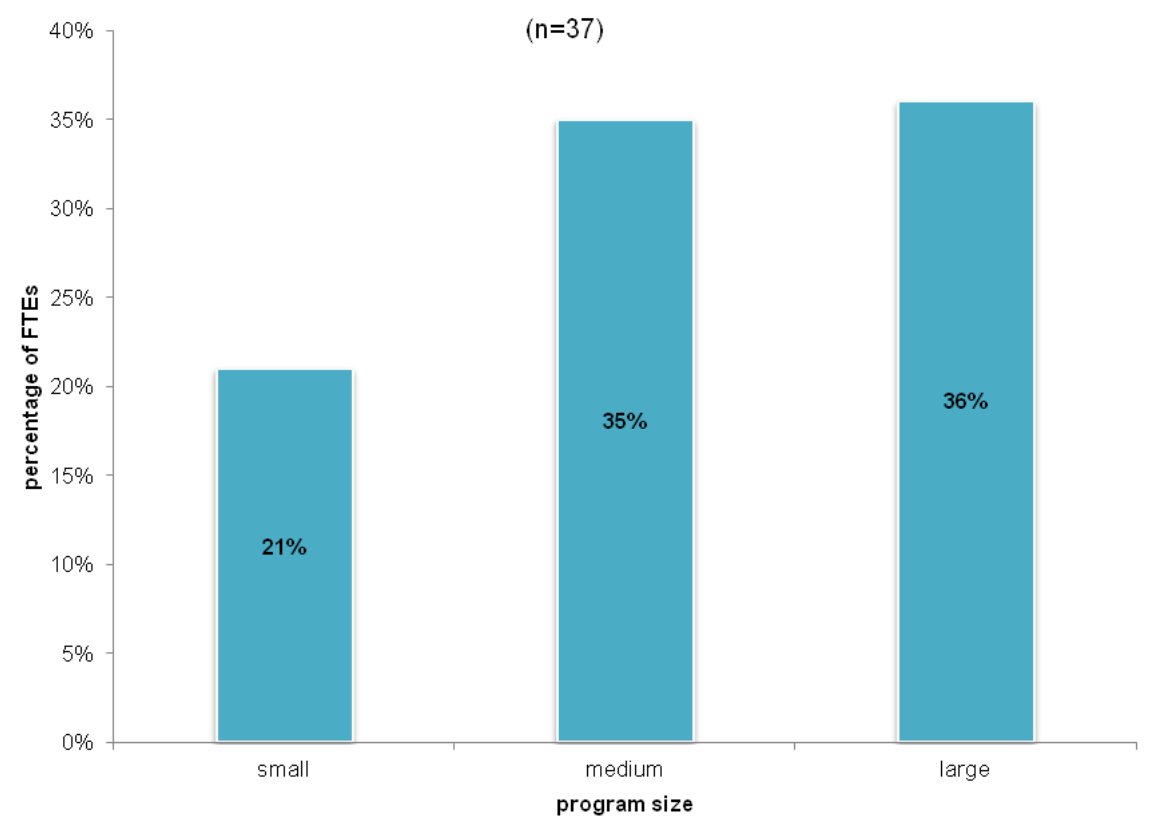

Fig. 4.7: Proportion of staff time spent on quality control and assurance by program size

There is also variation among staff time spent on quality control and assurance among the climate regions (Fig. 4.8). The greatest proportion of FTEs are allocated to inspections and monitoring among grantees located in the cold and hot-humid regions ( $41 \%$ and $37 \%$, respectively) compared to those in very cold, moderate, or hot-dry regions $(28 \%, 29 \%$, and $27 \%$, respectively).

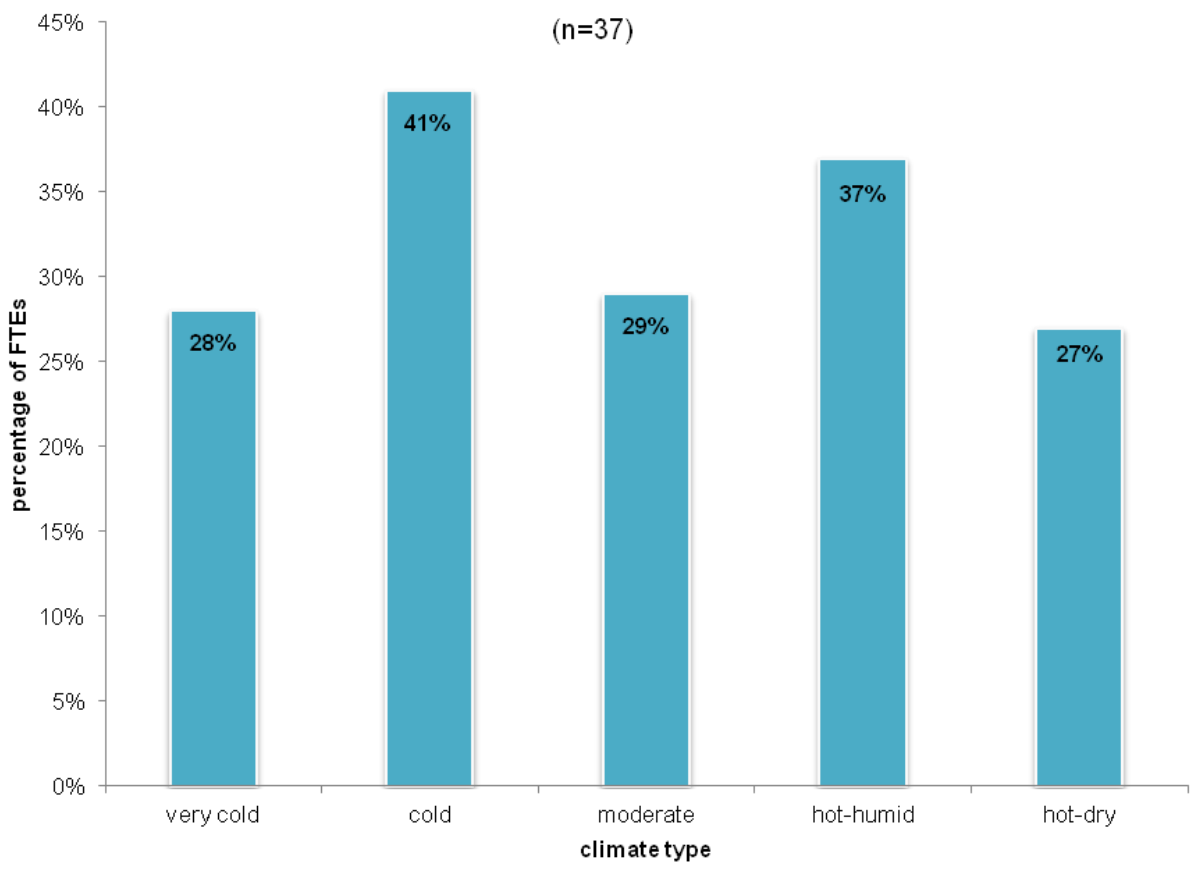

Fig. 4.8: Proportion of staff time spent on quality control and assurance by climate type 
Inspections of units. Most grantees inspected between 23 and 600 weatherized units ${ }^{28}$ in PY08, spending an average of two hours in each home. The most common types of inspections, performed by three-

fourths of states, are:

- Visual inspection of installed measures

- Assessment of quality of measures installed

- Discussion with occupants

- Verification of operation of measures installed

- Identification of unresolved health and safety issues

- Verification of insulation depths/quantities

- Identification of needed measures that were not installed

- Blower door test

- Carbon monoxide monitoring

Grantee weatherization directors consider visual inspection of installed measures, the blower door test and carbon monoxide monitoring to be the most effective monitoring activities, but verification of insulation depths/quantities and discussions with occupants appear to provide good effectiveness for more modest investments in cost, training needs, and time required. Table 4.10 presents all types of inspections included in our surveys and the grantee assessments of their effectiveness and investment required. (Note: The survey instrument inquired about cost, training, and time separately, but we combined the responses, giving equal weight to each factor.)

\footnotetext{
${ }^{28}$ This volume of inspections falls between 4 and 40 percent of these grantees' caseload (with a mean of 14 percent). However, we note that inspections reported appear to include non-DOE cases as well, so we provide these comparative statistics only as a frame of reference.
} 
Table 4.10: Post-weatherization inspections performed by grantees

\begin{tabular}{|l|c|c|c|}
\hline $\begin{array}{l}\text { Type of post- } \\
\text { weatherization inspection }\end{array}$ & $\begin{array}{c}\text { Percentage of } \\
\text { grantees that } \\
\text { perform }\end{array}$ & $\begin{array}{c}\text { Percentage of grantees } \\
\text { that rate effectiveness as } \\
\text { high (4 or 5 on a 5-point } \\
\text { scale) }\end{array}$ & $\begin{array}{c}\text { Percentage of grantees } \\
\text { that rate the investment } \\
\text { needed as high (4 or 5 on } \\
\text { a 5-point scale) }\end{array}$ \\
\hline $\begin{array}{l}\text { Visual inspection of } \\
\text { installed measures }\end{array}$ & $100 \%$ & $90 \%$ & $32 \%$ \\
\hline $\begin{array}{l}\text { Assessment of quality of } \\
\text { measures installed }\end{array}$ & $100 \%$ & $86 \%$ & $33 \%$ \\
\hline Discussion with occupants & $98 \%$ & $74 \%$ & $22 \%$ \\
\hline $\begin{array}{l}\text { Verification of operation of } \\
\text { measures installed }\end{array}$ & $94 \%$ & $80 \%$ & $28 \%$ \\
\hline $\begin{array}{l}\text { Identification of unresolved } \\
\text { health and safety issues }\end{array}$ & $92 \%$ & $78 \%$ & $34 \%$ \\
\hline $\begin{array}{l}\text { Verification of insulation } \\
\text { depths/quantities }\end{array}$ & $92 \%$ & $83 \%$ & $36 \%$ \\
\hline $\begin{array}{l}\text { Identification of needed } \\
\text { measures that were not } \\
\text { installed }\end{array}$ & $92 \%$ & $78 \%$ & $42 \%$ \\
\hline Blower door test & $87 \%$ & $93 \%$ & $29 \%$ \\
\hline $\begin{array}{l}\text { Carbon monoxide (CO) } \\
\text { monitoring }\end{array}$ & $51 \%$ & $90 \%$ & $32 \%$ \\
\hline $\begin{array}{l}\text { Draft/spillage tests of } \\
\text { heating systems }\end{array}$ & $51 \%$ & $70 \%$ & $39 \%$ \\
\hline $\begin{array}{l}\text { Heating system efficiency } \\
\text { test (flue gas analysis) }\end{array}$ & $64 \%$ & $71 \%$ & $46 \%$ \\
\hline Infrared scanning & $54 \%$ & \\
\hline
\end{tabular}

The frequency of inspection types decreases with program size. Grantees with large programs tend to undertake all inspection types more frequently than grantees with small programs. However, the types of inspections that occur most commonly are the same across program size. Fig. 4.9 shows the frequency of the three most common inspection types by program size. 


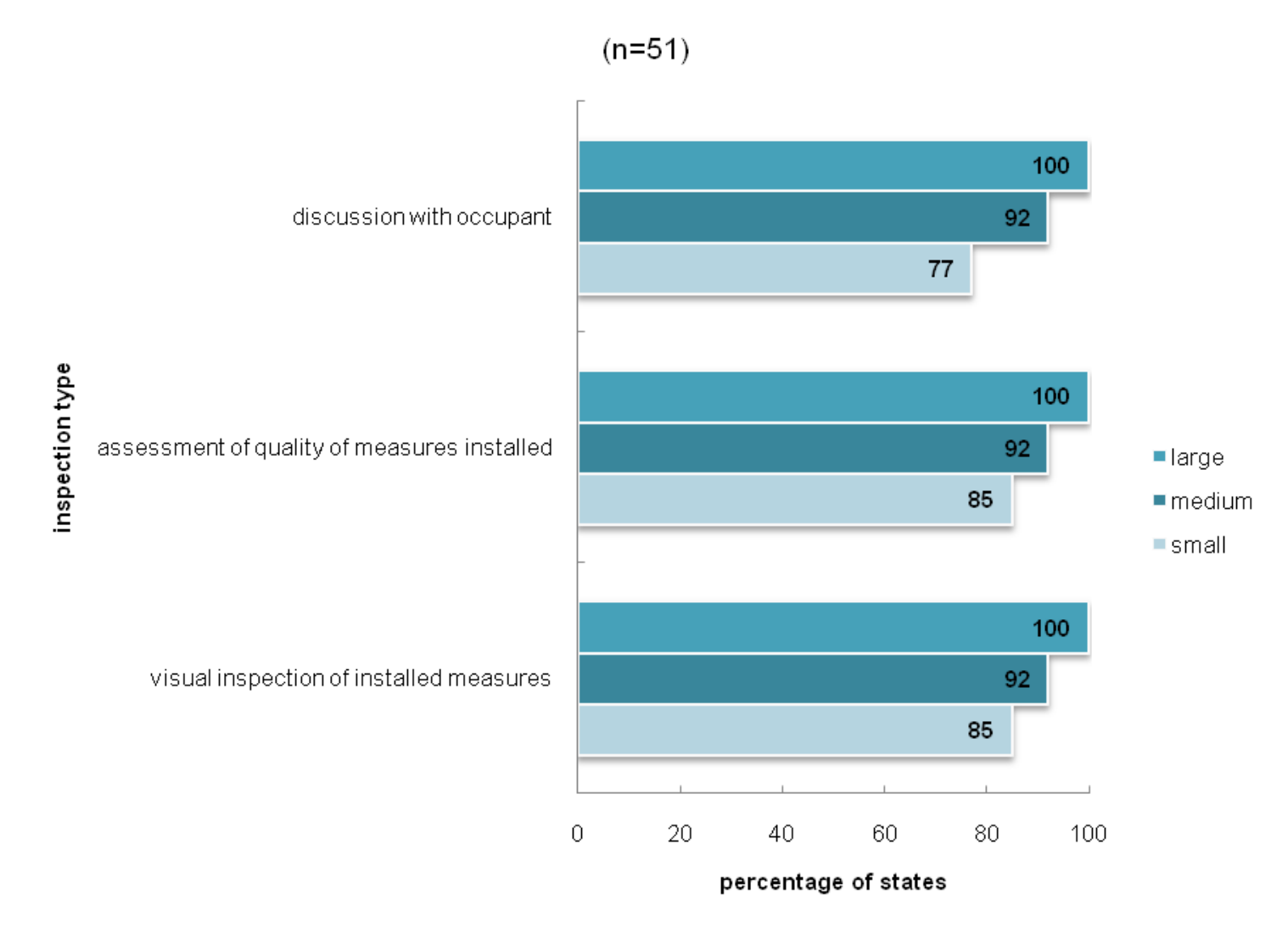

Fig. 4.9: Frequency of most common inspection types by program size

The pattern between frequency of inspection types and climate region is less clear. Among the most common inspection types, grantees in cold or dry climates tend to perform inspections more frequently than those in other climates; however, this does not hold for all inspection types. A few types of inspections - most notably, flue gas analysis and infrared scanning - are performed frequently in colder climates but less often in moderate and hot climates.

Post-weatherization inspections found problems that warranted a return visit by the subgrantee in 21 percent of units inspected with a range from zero in one state to 70 percent in another. The most common problems pertain to insulation (23\%), work quality (14\%), and air sealing (12\%). Other categories of problems (including health and safety measures) occurred fewer than 10 percent of the time. The most common remedy, by far, is to have the subgrantee send a crew back to correct the problem $(90 \%)$.

Grantees in the hot-dry region reported the fewest problems detected during post-weatherization inspections (10\%) while all other regions reported problems with 18 to 26 percent of cases that resulted in a return visit by a subgrantee.

Administrative monitoring. The amount of staff time grantee weatherization programs allocate to monitoring agency administrative activities ranges widely. Most grantees (76\%) conduct subgrantee administrative monitoring visits annually; however, actual staff hours spent at each agency ranges from one hour to 72 hours per subgrantee in most states. The national average for PY08 was 18 staff hours per subgrantee. 


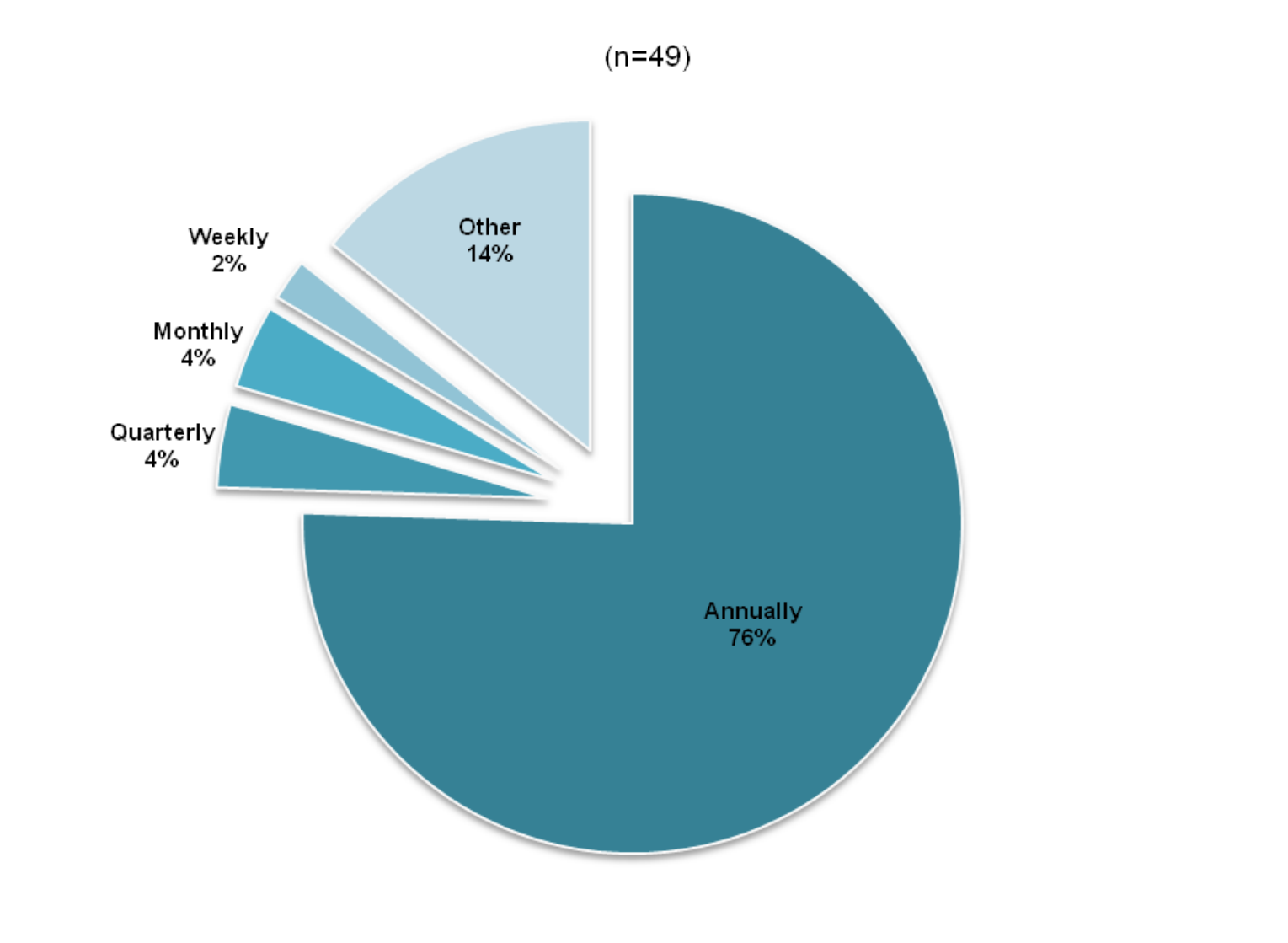

Fig. 4.10: Frequency of grantee administrative monitoring visits to subgrantees

As shown in Fig. 4.11, grantees with large weatherization programs conduct more frequent monitoring visits to subgrantees compared to those with small or medium programs. Sixteen percent of large program grantees make monthly or quarterly visits, while only eight percent of grantees with small or medium programs make visits more regularly than once per year. Furthermore, only 58 percent of grantees with large programs conduct annual monitoring visits compared to 80 to 83 percent of grantees with small or medium programs. In terms of program staff hours, grantees spent a similar amount of time conducting administrative monitoring activities regardless of program size, ranging from an annual average of 13 to 20 hours per subgrantee. 


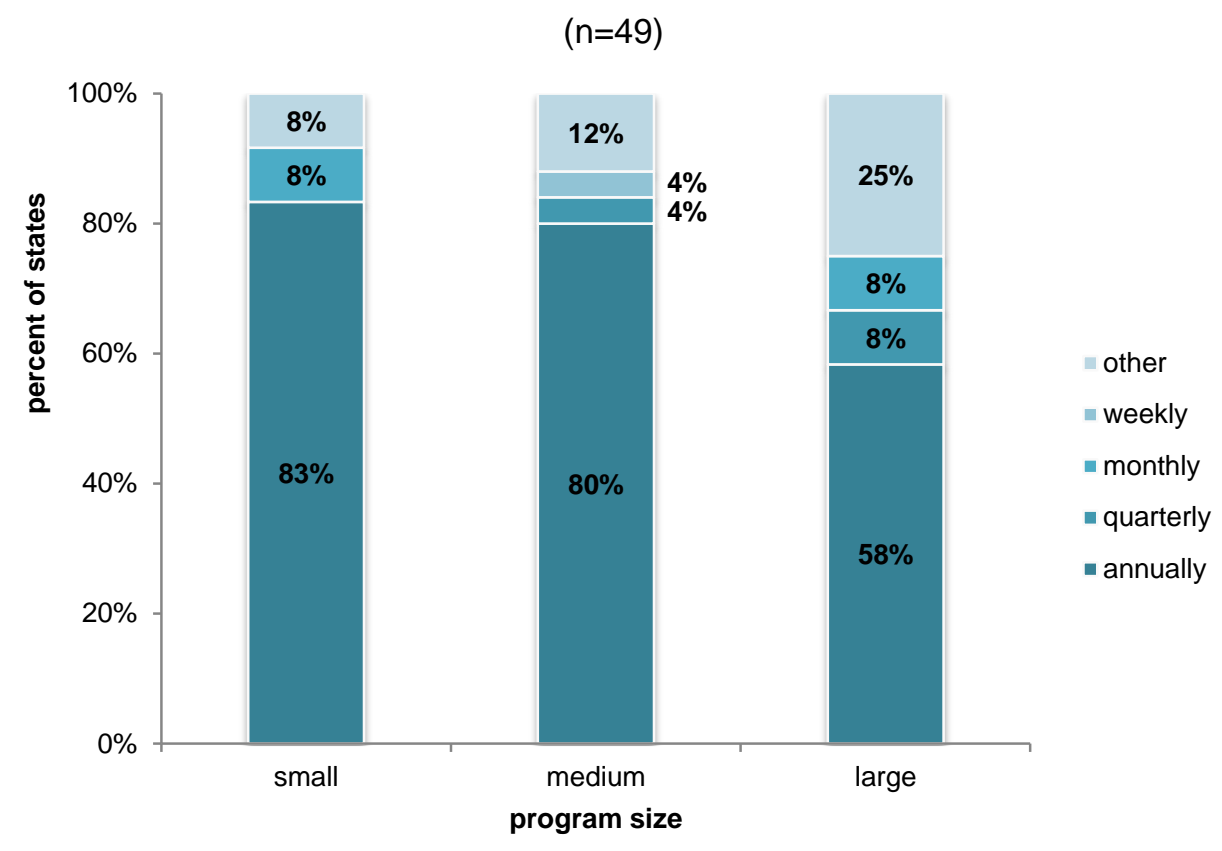

Fig. 4.11: Frequency of grantee administrative monitoring visits to subgrantees (by program size)

Grantees in the cold and moderate climate regions conduct administrative monitoring visits on a variety of schedules - ranging from weekly to annually - while most grantees in the other climate regions make such visits annually. In terms of program staff hours, grantees spent a similar amount of time conducting administrative monitoring activities regardless of climate region, ranging from an annual average of 12 to 22 hours per subgrantee.

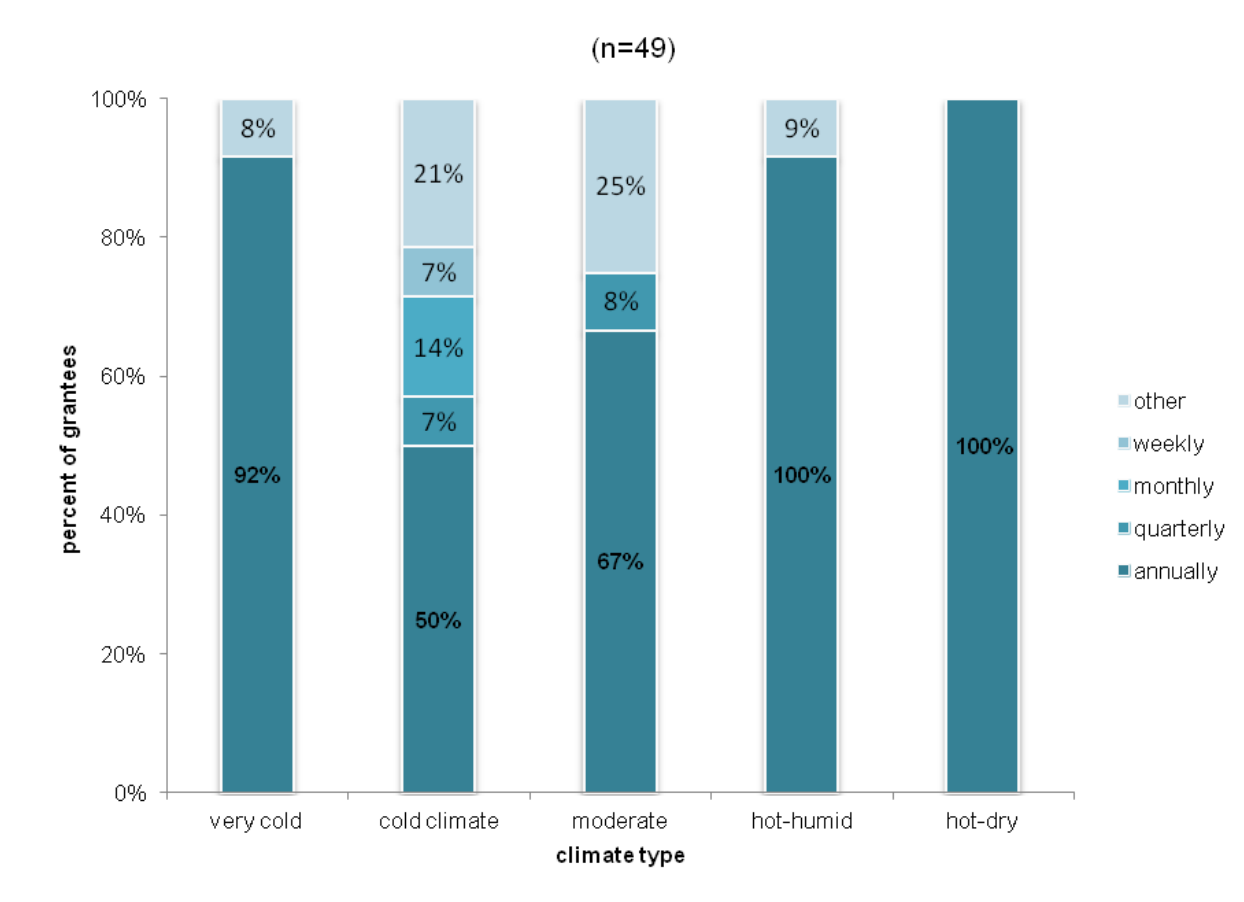

Fig. 4.12: Frequency of grantee administrative monitoring visits to subgrantees (by climate region) 
Grantee monitoring revealed a range of subgrantee administrative problems requiring corrective actions. On average, monitoring efforts found problems in seven subgrantees per state; however frequency varied among grantees with regard to climate region. Grantees in the moderate climate region found the most subgrantees with administrative problems (14), on average, followed by those in the very cold region (6). Those in the hot-dry region found the fewest subgrantees with administrative problems (1). The primary issues were incomplete forms, failure to complete weatherization work, fiscal administration, inaccurate inventory counts and inadequate staff knowledge/management.

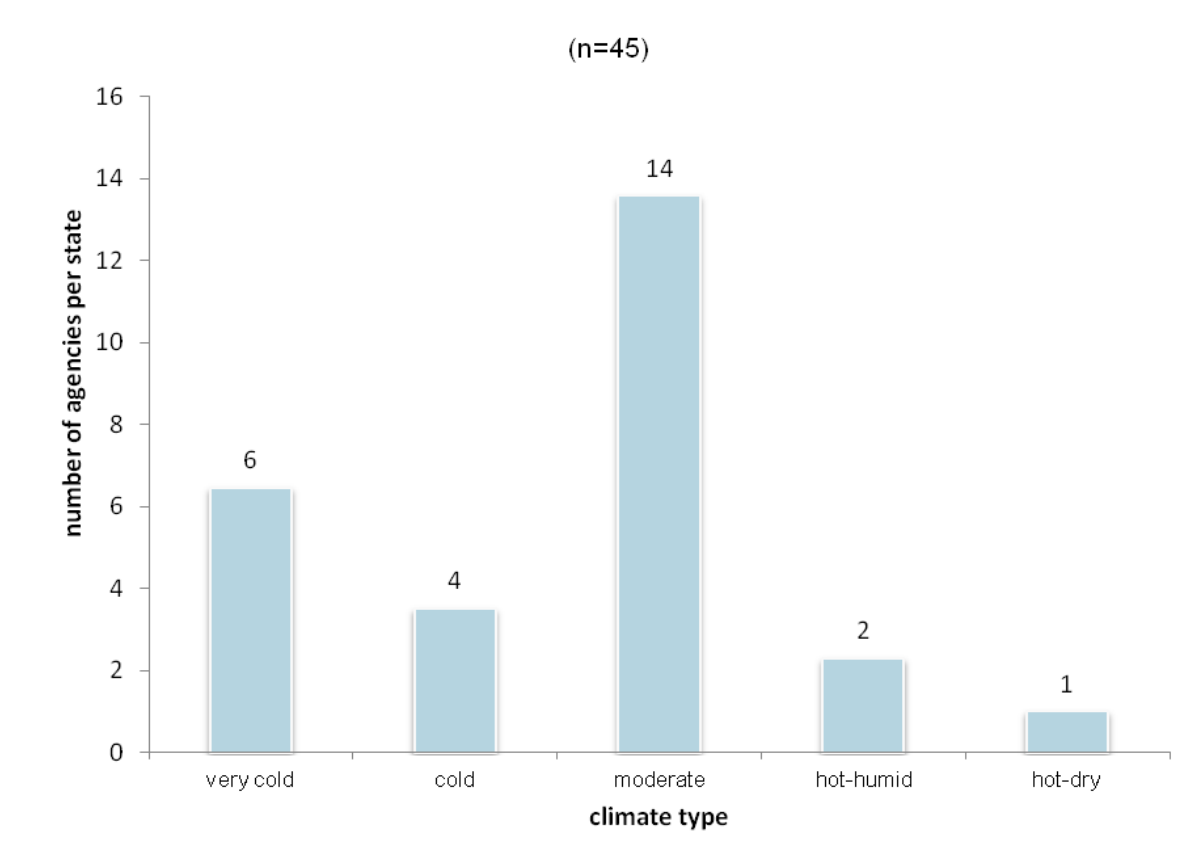

Fig. 4.13: Occurrences of administrative problems per grantee (by climate region)

In response to administrative problems, most grantees undertake multiple corrective actions. The vast majority of grantees (87\%) issue a written report to the subgrantee requiring that corrective action be taken by the agency. Nearly half of grantees (47\%) send someone from the state office to help correct the problem. Two grantees (4\%) reported that they take no action.

\begin{tabular}{|c|c|}
\hline Type of corrective action & $\begin{array}{c}\text { Percentage of grantees } \\
\text { that perform }\end{array}$ \\
\hline Send written report to local agency & $87 \%$ \\
\hline Require local action & $87 \%$ \\
\hline Send state office staff to help correct problem & $47 \%$ \\
\hline Make presentation to local agency & $28 \%$ \\
\hline Send state contractor to help correct problem & $13 \%$ \\
\hline Other & $13 \%$ \\
\hline No action taken & $4 \%$ \\
\hline
\end{tabular}

Fig. 4 14: Corrective actions taken by grantees in response to subgrantee administrative problems 
A number of grantees implemented changes to weatherization training for subgrantee staff after observation of work quality problems. Approximately two-thirds of grantees (65\%) adjusted their training programs. ${ }^{29}$

Implementation of training changes correlates with grantee weatherization program size. Training changes were most frequent among grantees with large programs $(92 \%)$, followed by those with medium programs (67\%) and small programs (33\%). Fig. 4.15 shows the percentage of grantees, by program size.

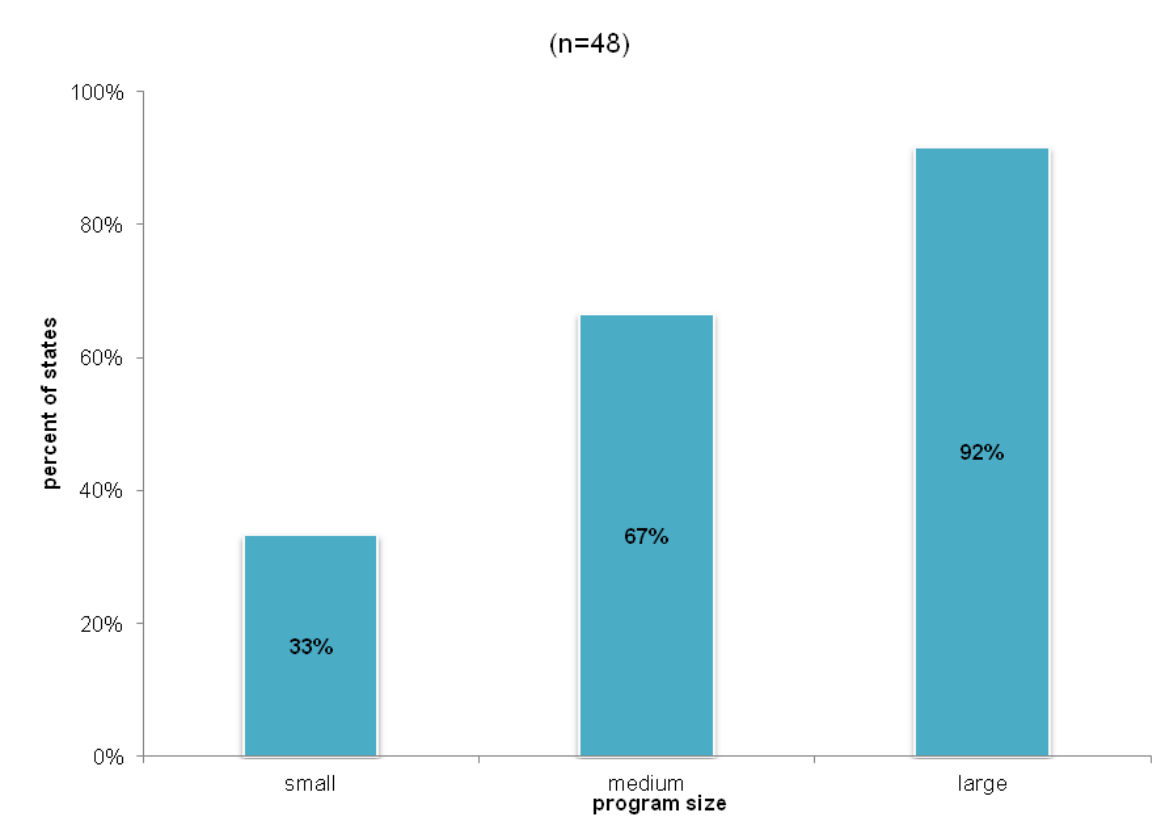

Fig. 4.15: Portion of grantees that made training program changes in response to subgrantee administrative problems (by program size)

There is also a pattern between implementation of training changes and climate region. Comparatively, a larger portion of grantees in cooler climates adjust their training programs in reaction to observed administrative problems among subgrantees. More than two-thirds of grantees in the very cold and cold regions - as well as the hot-dry region - implemented training changes. Among programs in hot-humid and moderate regions, however, only 50 and 58 percent, respectively, made changes (Fig. 4.16).

${ }^{29}$ The primary changes centered on training protocol updates including policy and procedural changes both in the field and in the office. 


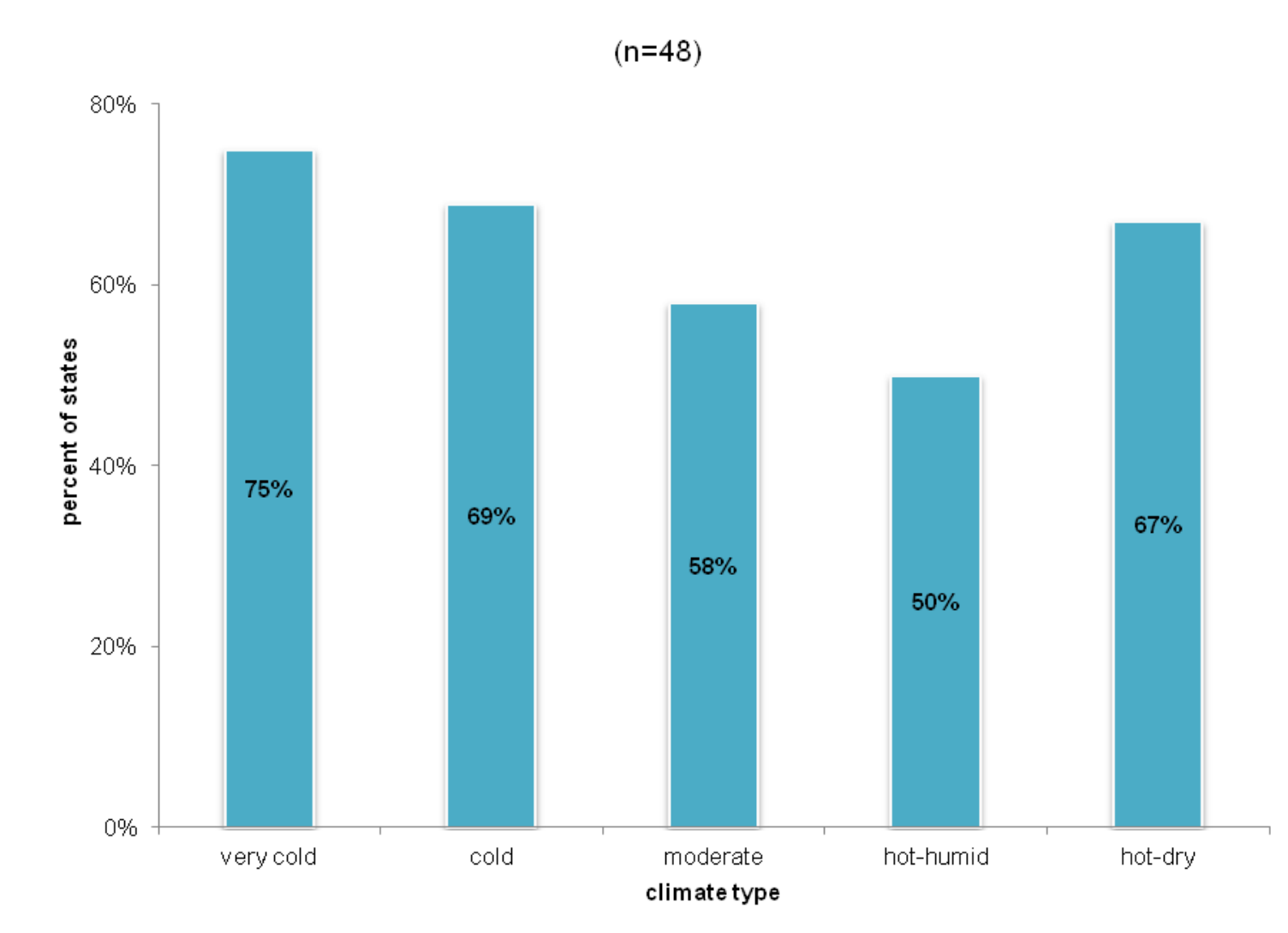

Fig. 4.16: Grantee training program changes in response to subgrantee administrative problems (by climate region)

\subsubsection{Subgrantee inspections}

In PY08, subgrantees performed a range of post-weatherization quality control and assurance inspections on weatherized dwelling units. Typically, subgrantees performed initial inspections one week after weatherization completion; however the time between completion and inspection ranged from same-day inspections to a six month time lapse.

During inspection visits, subgrantees conduct a variety of inspection procedures. The set of procedures conducted during a typical inspection visit varies by subgrantee. The most common types of inspection procedures (performed by more than four-fifths of subgrantees) are listed below:

- Visual inspection of installed measures

- Blower door test

- Verification of insulation depths/quantities

- Verification of operation of measures installed

- Carbon monoxide monitoring

- Discussion with occupants

- Assessment of quality of measures installed 
Other inspection procedures conducted by a sizeable portion of subgrantees included identification of needed measures that were not installed, identification of unresolved health and safety issues, draft/spillage tests of heating systems, heating system efficiency tests (flue gas analysis), and infrared scanning.

Subgrantees regard the blower door test and discussions with occupants as the most effective inspection procedures, along with certain diagnostic tests not specifically asked about in the survey (labeled "other diagnostic tests" in Table 4.11). Verification of insulation depths/quantities, visual inspection of installed measures, and verification of operation of measures installed are also relatively effective inspection procedures, yet require more modest investments in cost, training needs, and time required. Table 4.11 presents all inspection procedures included in our surveys and the subgrantee assessments of their effectiveness and investment required. (Note: The survey instrument inquired about cost, training, and time separately, but we combined the responses, giving equal weight to each factor.)

Table 4.11:Post-weatherization inspections performed by subgrantees

\begin{tabular}{|l|c|c|c|}
\hline $\begin{array}{l}\text { Type of post-weatherization } \\
\text { inspection }\end{array}$ & $\begin{array}{c}\text { Percentage of } \\
\text { subgrantees that } \\
\text { perform }\end{array}$ & $\begin{array}{c}\text { Percentage of subgrantees } \\
\text { that rate effectiveness as high } \\
\text { (4 or 5 on a 5-point scale) }\end{array}$ & $\begin{array}{c}\text { Percentage of subgrantees } \\
\text { that rate the investment } \\
\text { needed as high (4 or 5 on a } \\
\text { 5-point scale) }\end{array}$ \\
\hline $\begin{array}{l}\text { Visual inspection of installed } \\
\text { measures }\end{array}$ & $99 \%$ & $85 \%$ & $24 \%$ \\
\hline Blower door test & $95 \%$ & $89 \%$ & $35 \%$ \\
\hline $\begin{array}{l}\text { Verification of insulation } \\
\text { depths/quantities }\end{array}$ & $92 \%$ & $85 \%$ & $16 \%$ \\
\hline $\begin{array}{l}\text { Verification of operation of } \\
\text { measures installed }\end{array}$ & $89 \%$ & $88 \%$ & $22 \%$ \\
\hline Discussion with occupants & $89 \%$ & $79 \%$ & $25 \%$ \\
\hline $\begin{array}{l}\text { Assessment of quality of } \\
\text { measures installed }\end{array}$ & $88 \%$ & $68 \%$ & $25 \%$ \\
\hline $\begin{array}{l}\text { Identification of needed } \\
\text { measures that were not installed }\end{array}$ & $73 \%$ & $86 \%$ & $25 \%$ \\
\hline $\begin{array}{l}\text { Identification of unresolved } \\
\text { health and safety issues }\end{array}$ & $68 \%$ & $84 \%$ & $29 \%$ \\
\hline Other diagnostic tests & $95 \%$ & & \\
\hline
\end{tabular}

During PY08, most post-weatherization inspections took between one and ten hours, averaging five hours per inspection. Roughly one-third of that time is spent on-site conducting some portion of the inspection types listed above. Another fifth is spent on post-inspection analysis and writing the report. Fig. 4.17 shows the percentage of time spent on the various inspection components. 


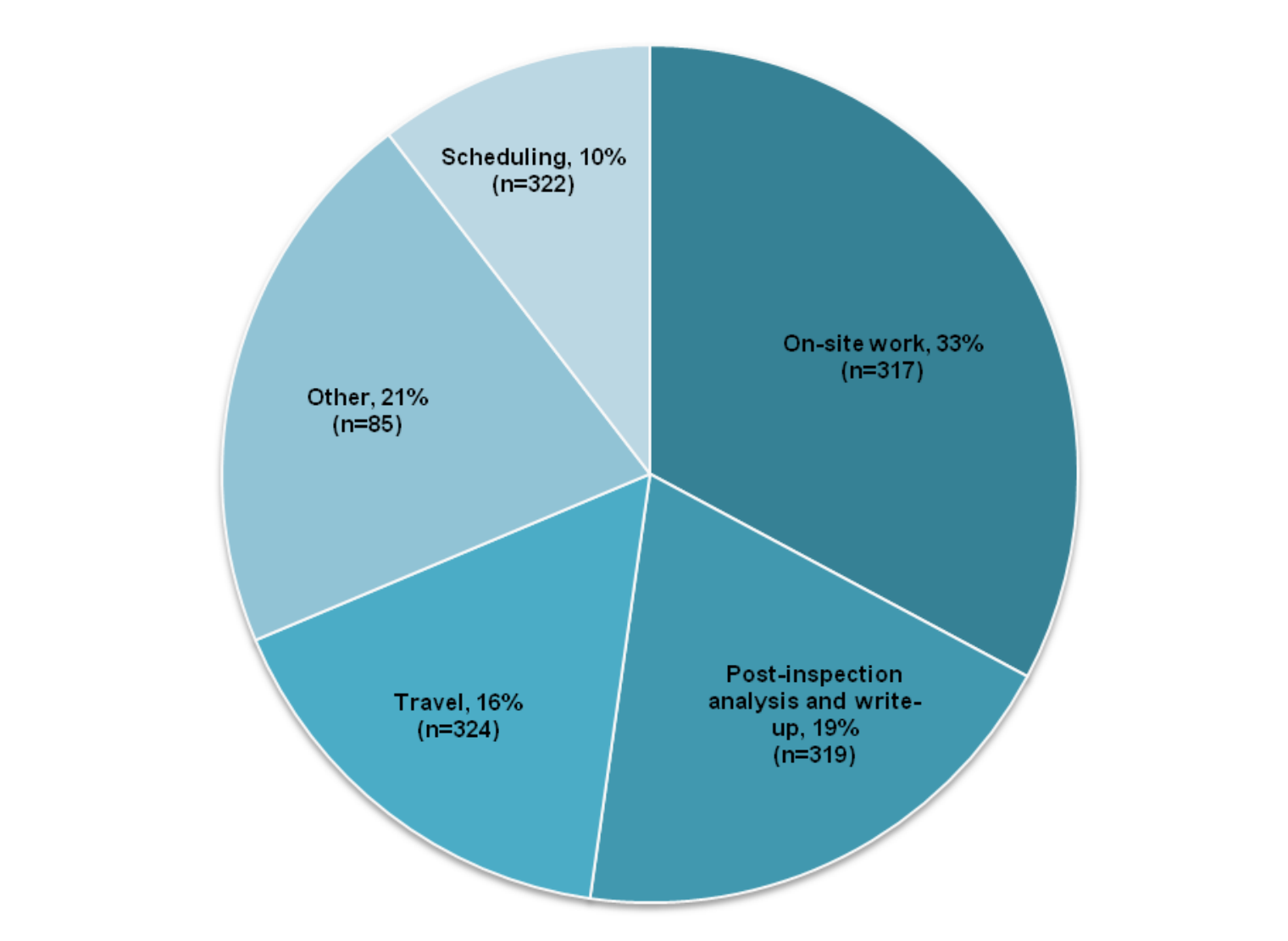

Fig. 4.17: Post-weatherization inspection components

In instances where post-weatherization inspections revealed a problem with the weatherization job performed, nearly all subgrantees (94\%) sent the original crew or contractor back to correct the problem. Large subgrantees also occasionally sent a crew supervisor to correct the problem (6 percent of large subgrantees).

In PY08, eight out of nine subgrantees (89\%) performed additional work on dwelling units as a result of post-weatherization quality control inspections. Among those agencies, half conducted work on fewer than ten units, however, a few agencies performed additional work on a large number of units. This results in an average of 21 dwelling units per subgrantee requiring additional work as the result of postweatherization inspections.

Subgrantees reported that inspections have a significant impact on the quality of future weatherization work. Three-fourths of subgrantees report that the extent of the impact is substantial or very substantial, with 35 percent reporting that inspections have a very substantial impact on the quality of future work (Fig. 4.18). Only seven percent reported little or no impact. 


$$
(n=330)
$$

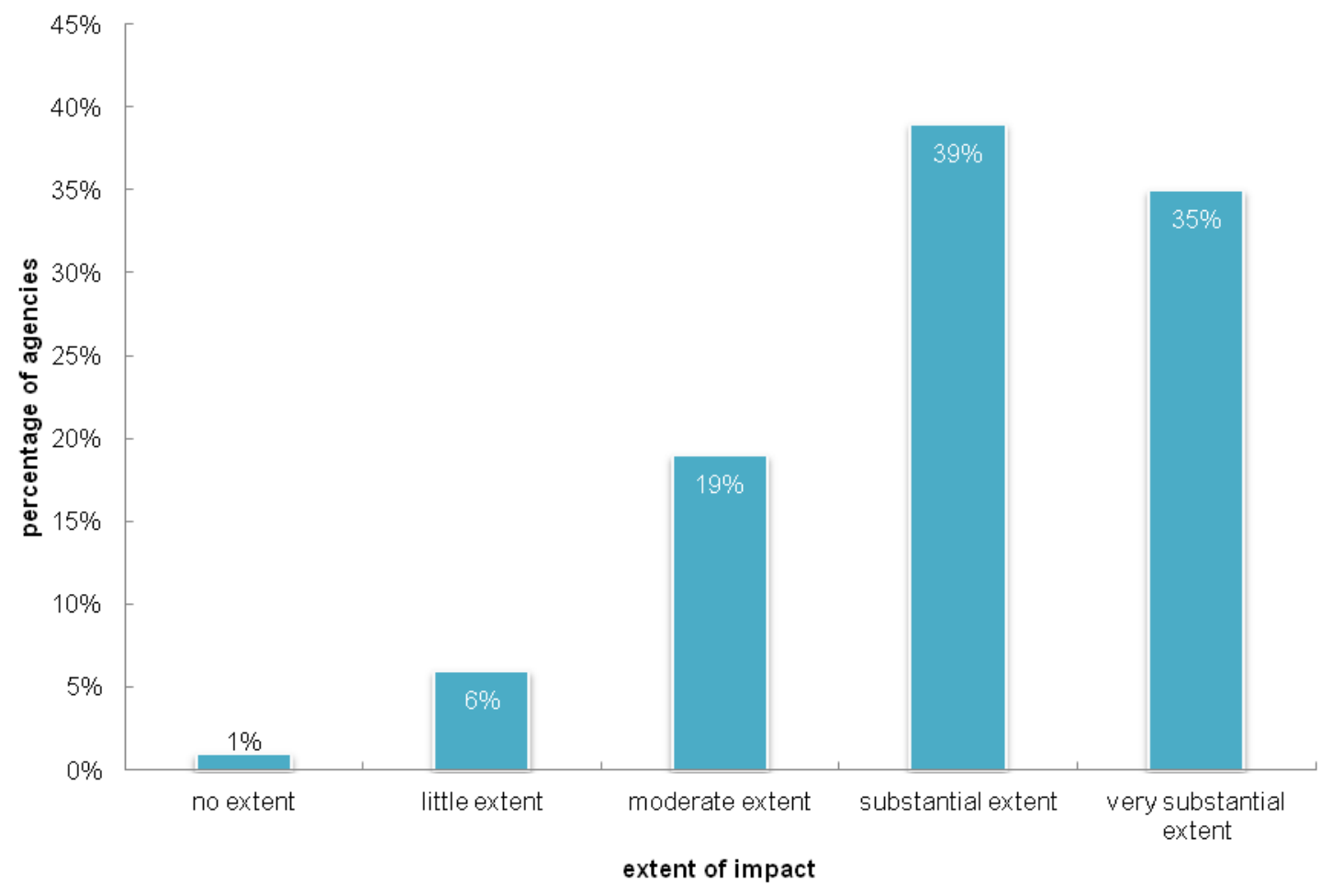

Fig. 4.18: Extent subgrantee inspections affect future weatherization work ${ }^{+}$ 


\section{WHOM DO WEATHERIZATION AGENCIES SERVE WITH WHAT SERVICES?}

The Weatherization Assistance Program serves low-income families. In PY08 DOE rules allowed households at or below 150 percent of the poverty level or 60 percent of the state median income to qualify for weatherization assistance. However, income level is only one characteristic of the eligible population. To fully characterize the clients served by WAP and the services they received, we collected demographic data, housing characteristics and home ownership data, and data on the diagnostic tests performed and measures installed.

\subsection{CLIENTS SERVED}

Weatherization programs across the country serve a wide range of low-income clients, but some particularly vulnerable groups receive priority at the federal or grantee level. Clients that receive special priority — either nationally or regionally_include households with elderly members, disabled residents, or children. Clients with high energy costs or burdens and single parents are also of heightened interest. This section reports on the share of weatherized units served who meet various demographic characteristics and household structures.

Among clients served in PY08, nearly three-fourths own the home in which they live. The remainder rent. Home-ownership, however, is largely correlated with housing type. As noted in Fig. 5.1, most clients living in single-family or mobile homes own their home (87 and 91 percent, respectively), while clients living in multifamily buildings tend to rent their homes ( 89 percent).

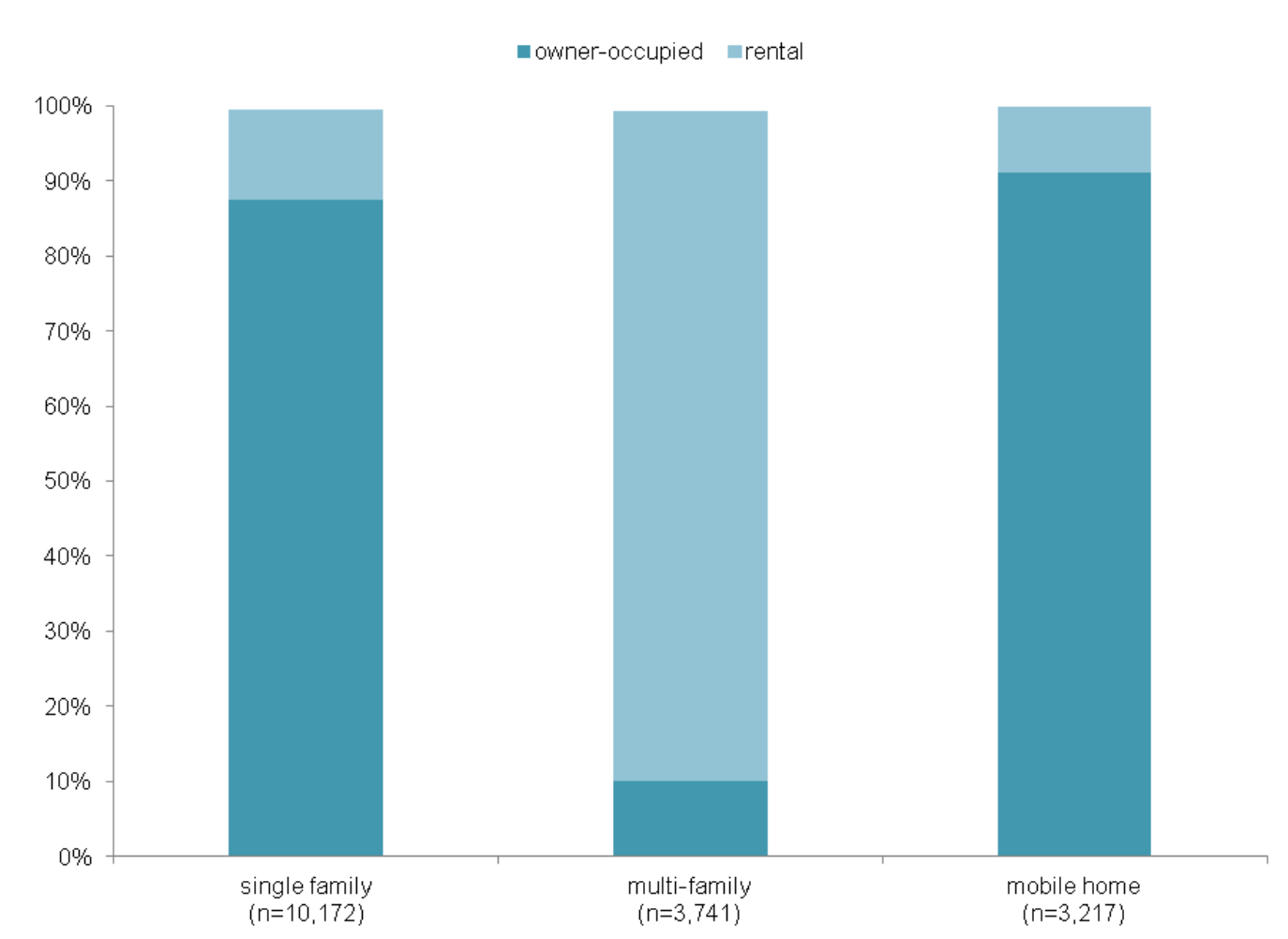

Fig. 5.1: Ownership status by housing type ${ }^{++}$

A number of grantees have defined thresholds used to characterize potential clients as "high energy users." Among the sampled project data we analyzed, 53 percent of households could be classified as either meeting or not meeting a high energy user definition. Of these, a small majority -30 percent of all 
units analyzed - met an applicable definition of high energy user, while the rest-23 percent of units analyzed - did not. The remaining clients either live in states with no defined threshold or their status is unknown. (Fig. 5.2.)

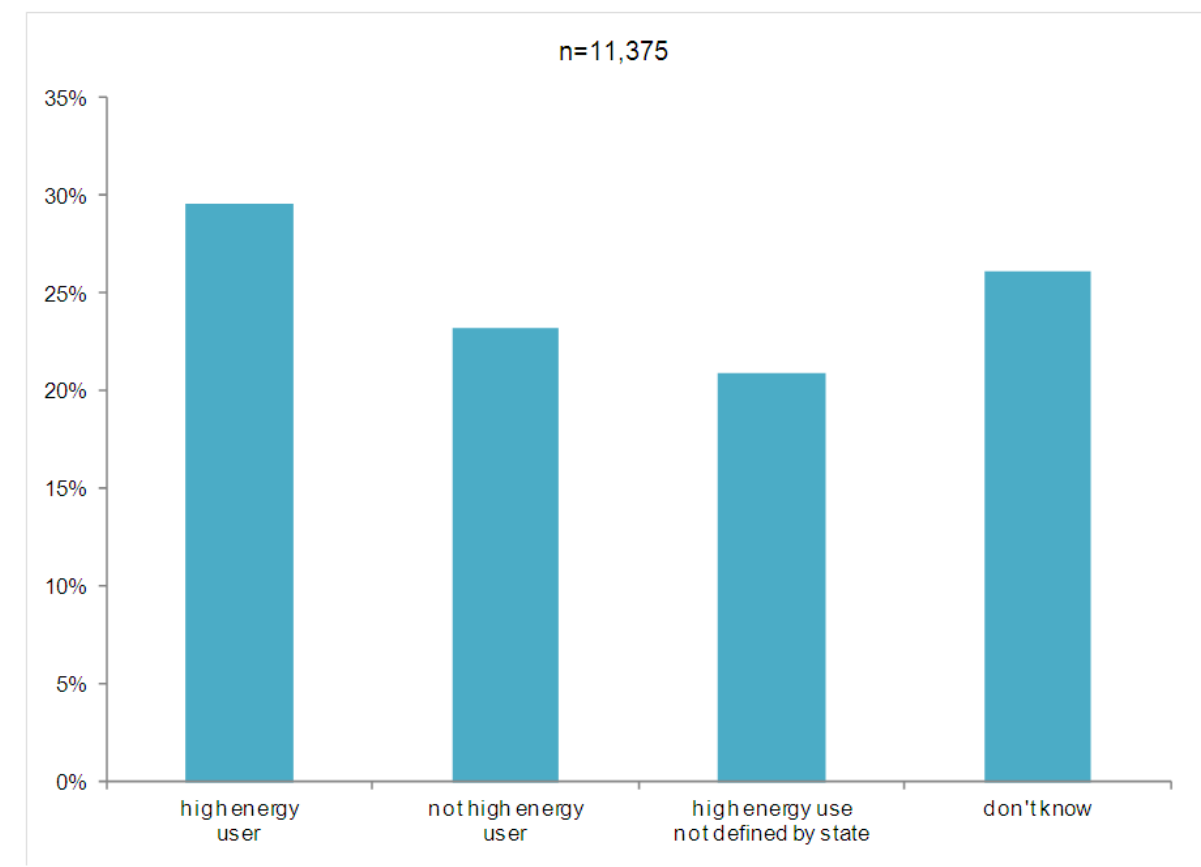

Fig. 5.2: High energy users as share of clients served++

Clients with a high energy burden are defined as those with the lowest income and highest energy use (accounting for household size). The breakdown of PY08 clients having a high energy burden is similar to that for high energy users. As shown in Fig. 5.3, 29 percent of those served are considered to have a high energy burden and 24 percent are not. One-fifth of clients ( 21 percent) live in states that do not have a defined threshold for high energy burden and 26 percent have an unknown energy burden status. 


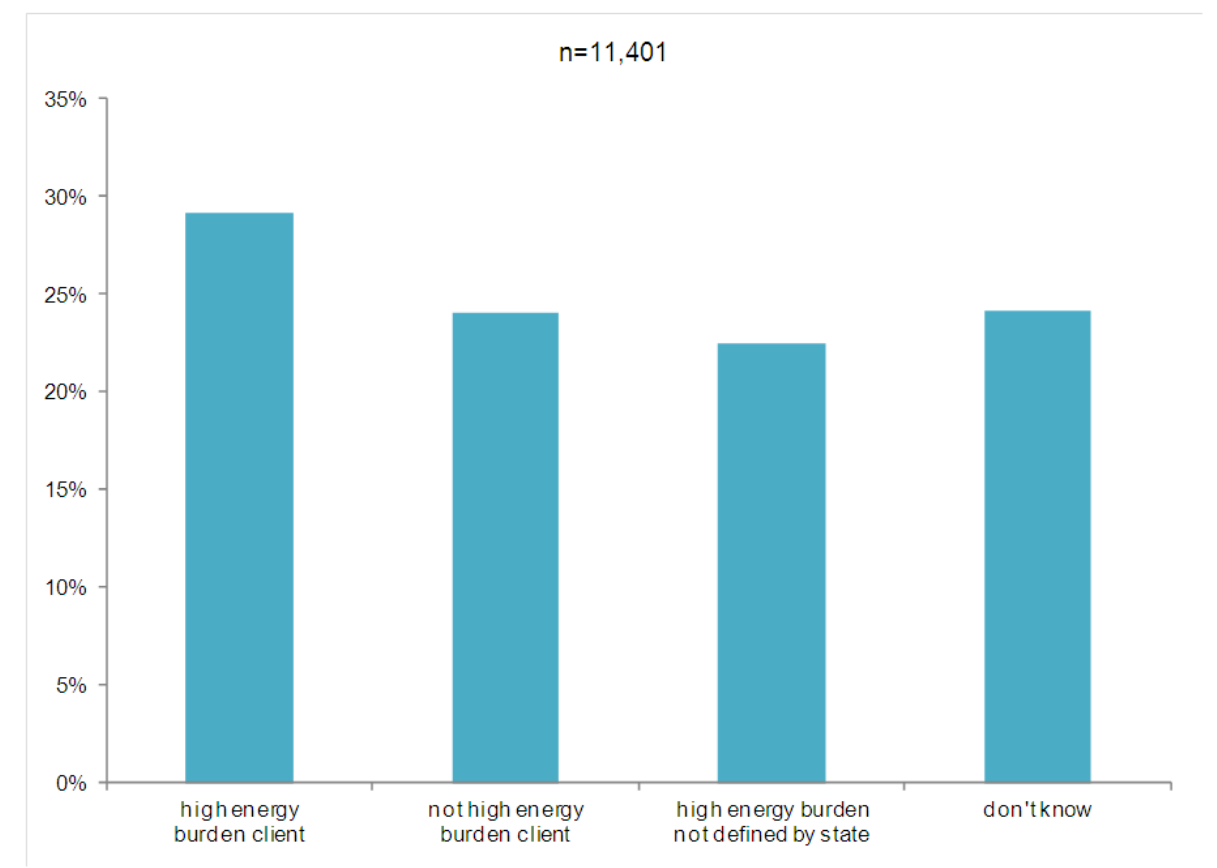

Fig. 5.3: Users with a high energy burden, as share of clients served ${ }^{++}$

As noted above, homes with elderly members, disabled residents, or children receive some priority status. Among homes that received weatherization services in PY08, a sizable portion have residents that are elderly (45 percent), have a qualifying disability (38 percent) or have children living at home (30 percent). (Fig. 5.4.)

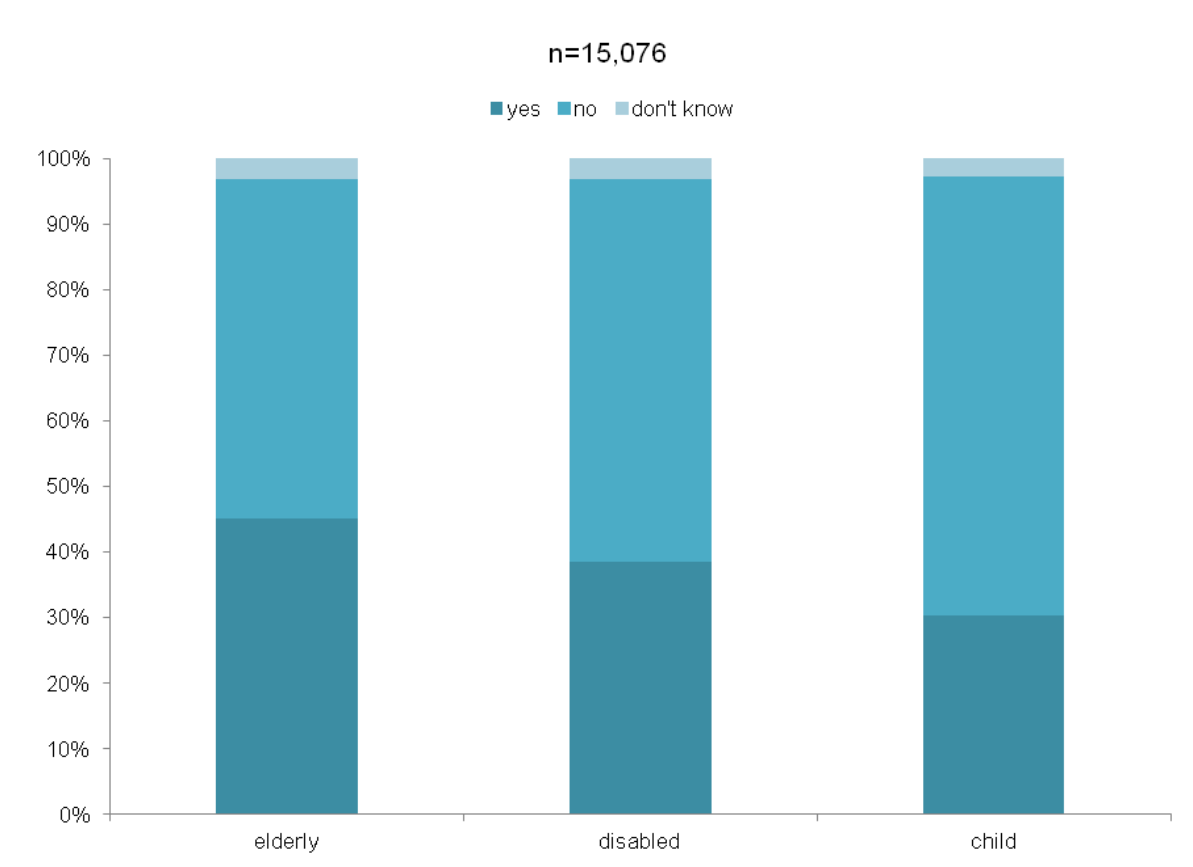

Fig. 5.4: Share of units with presence of priority client types ${ }^{++}$ 
Although race is generally not a priority criterion $^{30}$, racial identity is captured by weatherization programs as part of demographic information gathering. Roughly half of clients who had their homes weatherized in PY08 are white. Sixteen percent are black, five percent are Hispanic and less than four percent are American Indian/Alaskan Native, Asian or Native Hawaiian/Pacific Islander. One percent identifies as more than one race. Data is not available for nearly one-quarter (22 percent) of PY08 clients. (See Fig. 5.5.)

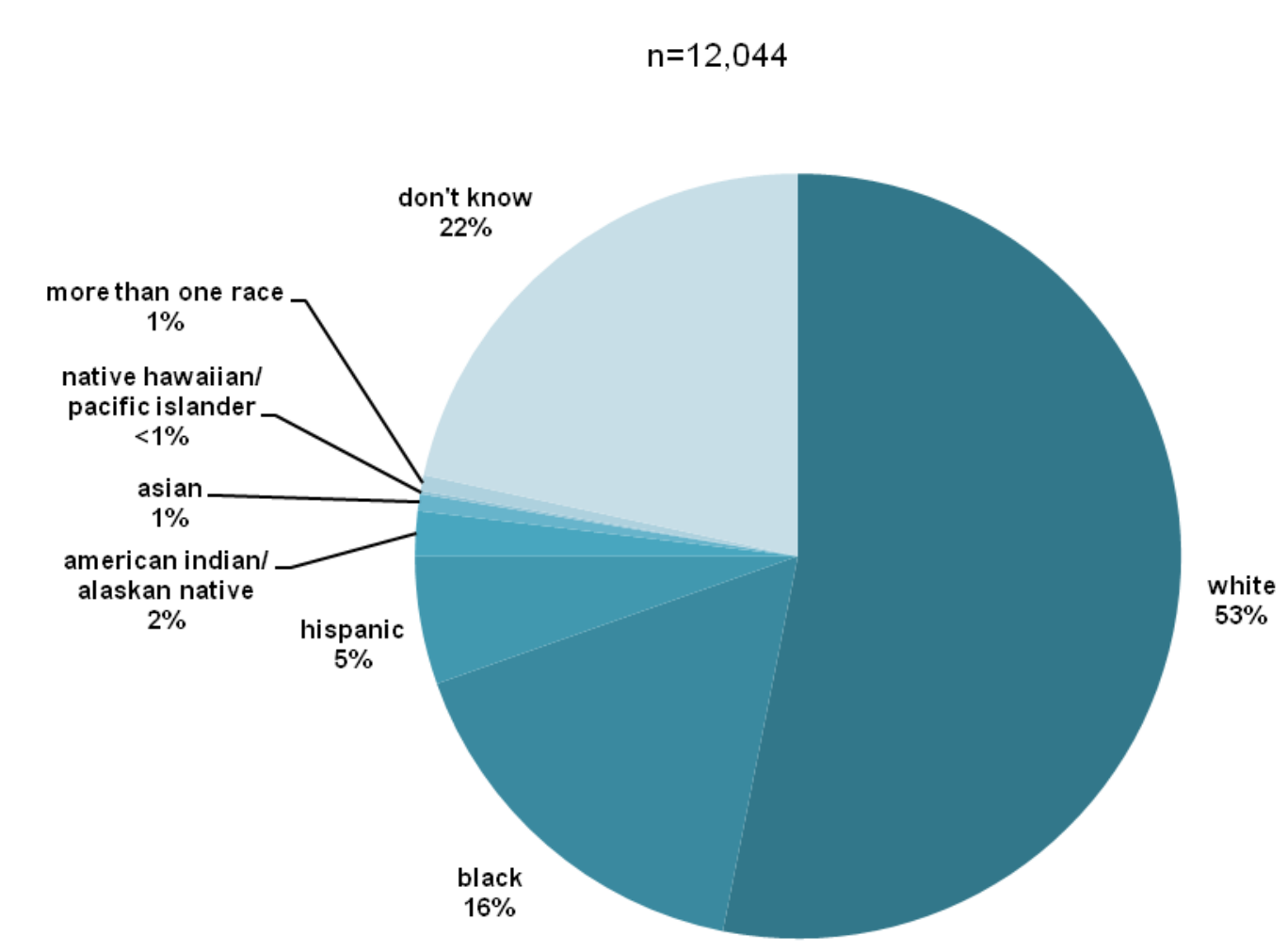

Fig. 5.5: Race of clients ${ }^{++}$

Whether or not a client is a single-parent household is also captured by most weatherization programs. As shown in Fig. 5.6, about one-sixth of PY08 clients are single-parent households, which comprises about half of clients with children. Sixty-nine percent are not headed by a single parent, and data were not available for the remainder of clients.

${ }^{30}$ The allocation of priority status to Native Americans is an exception. 


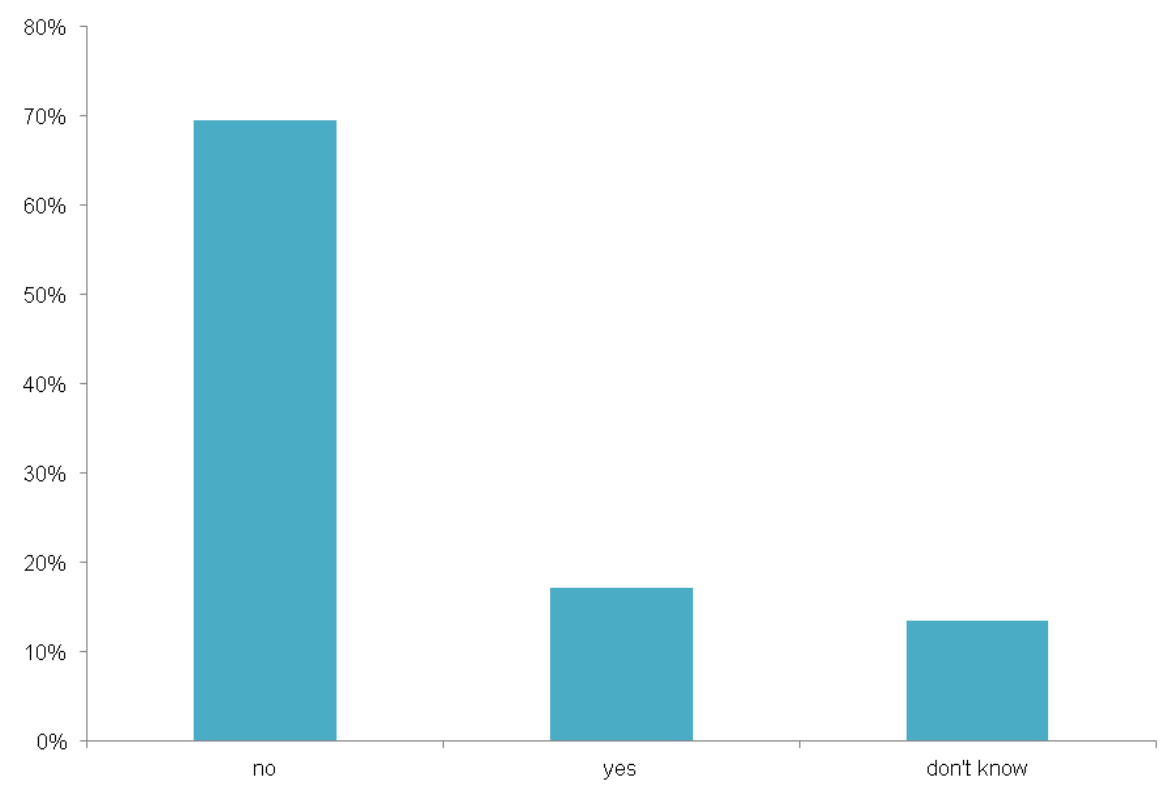

Fig. 5.6: Single-parent status of clients ${ }^{++}$

\subsection{HOUSING STOCK}

The characteristics of pre-weatherized homes are important in understanding the types of homes typically weatherized by grantee and subgrantee assistance programs. Site-built single-family homes constitute over half (58 percent) of the PY08 weatherized housing stock (see Fig. 5.7). Housing units in multifamily buildings account for another quarter ( 24 percent) and mobile homes account for 18 percent. Among multifamily buildings, one-third of weatherized units are located in small buildings (2-4 units) and twothirds are in large buildings (5 or more units). 


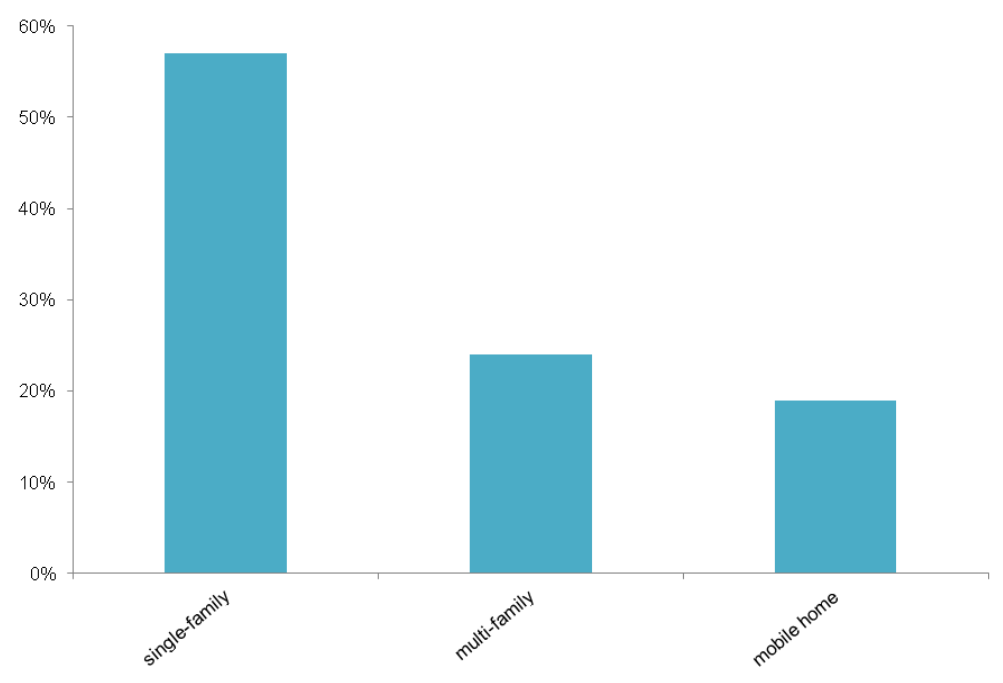

Fig. 5.7: Housing type ${ }^{++}$

Homes weatherized in PY08 span a millennium in terms of the year built. Most commonly, however, weatherized homes were built between 1950 and 1989. Homes built in the 1970s represent 15 percent of all PY08 weatherized homes, followed by the 1980s ( 9 percent), the 1950s ( 8 percent) and the 1960s ( 7 percent).

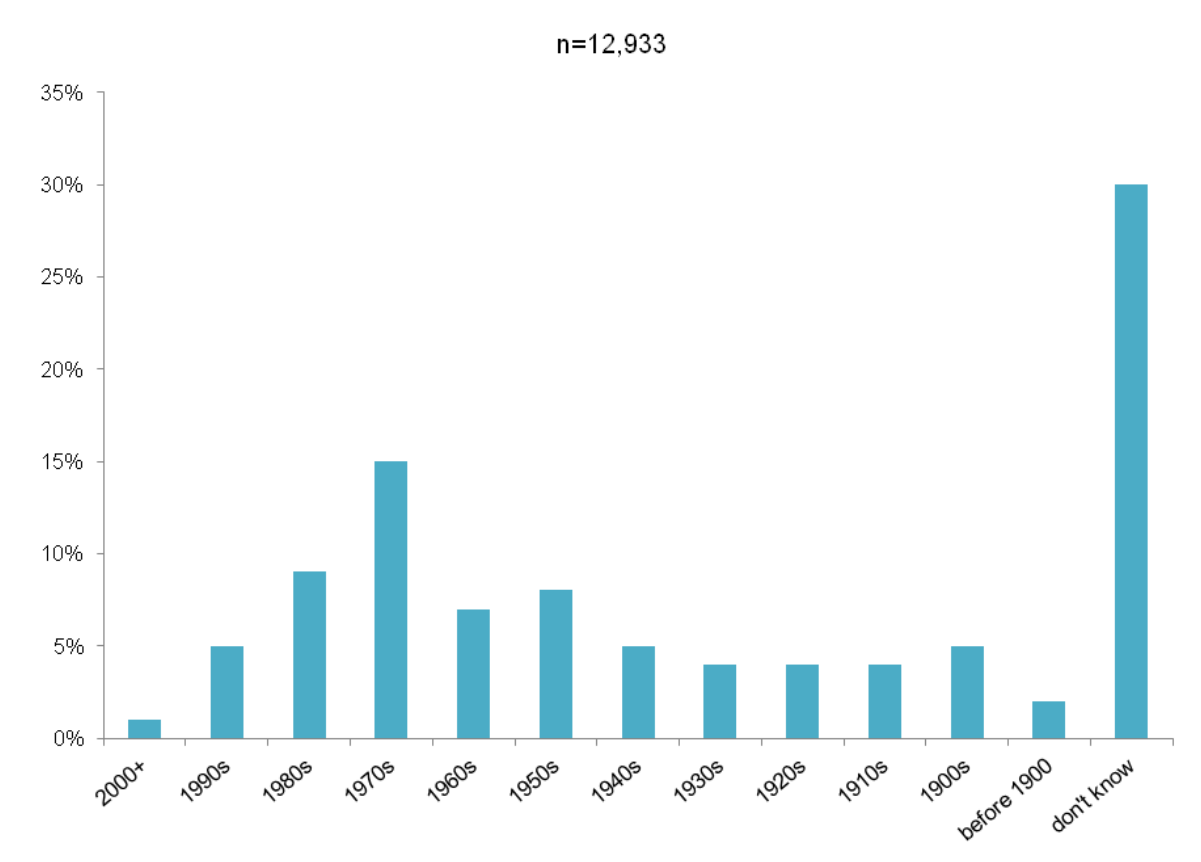

Fig. 5.8: Year built ${ }^{++}$

The majority of PY08 weatherized homes were primarily heated with natural gas ( 55 percent), electricity (20 percent), fuel oil \#2 (10 percent) or propane (9 percent) — prior to any weatherization measures installed. 


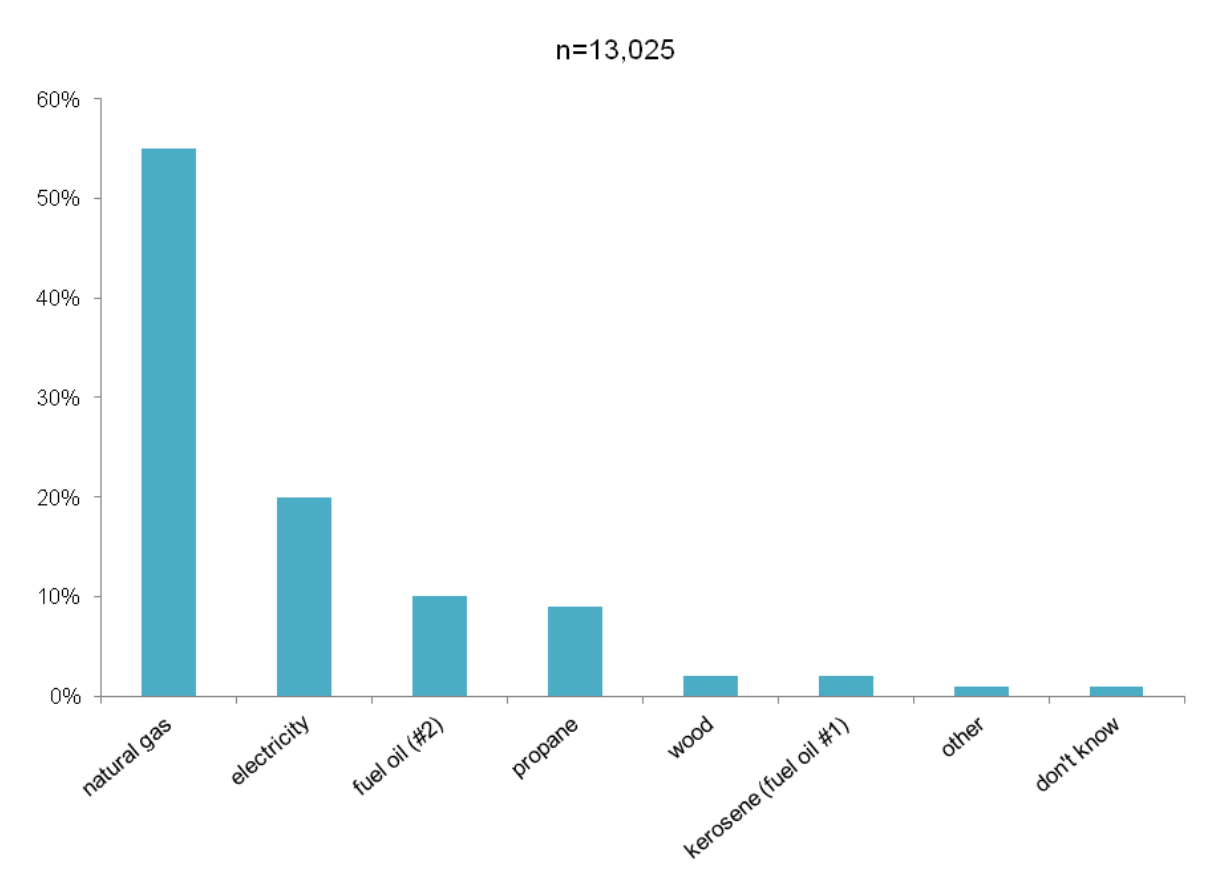

Fig. 5.9: Primary heating fuel type ${ }^{++}$

Mobile homes, while still commonly heated with natural gas (35 percent), were more frequently heated with electricity (35 percent), propane (18 percent) and kerosene ( 7 percent) compared to single-family and multifamily units.

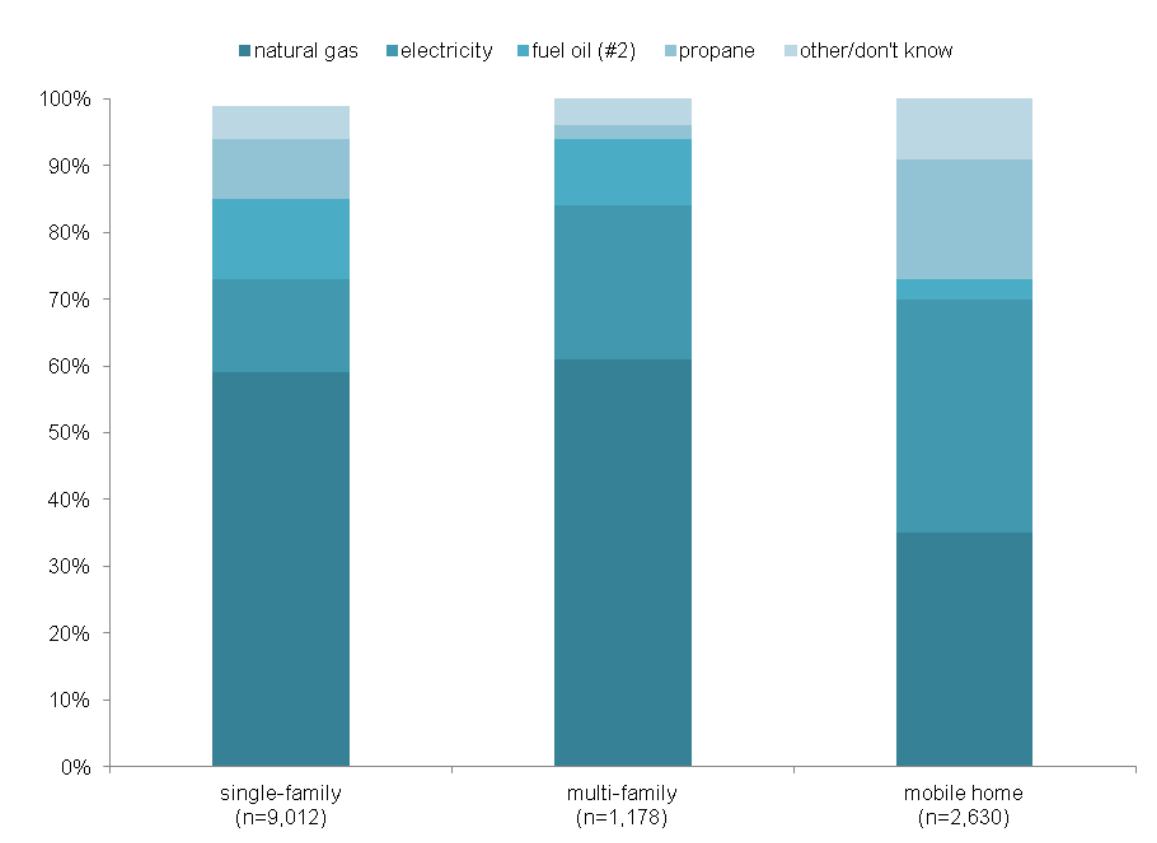

Fig. 5.10: Primary heating fuel type by housing type $e^{++}$ 
Differences among climate regions are notable. The pre-weatherization housing stock in cold and very cold climate regions tends to be more reliant on fuel oil \#2 as a primary heating fuel compared to the housing stock in warmer regions. Conversely, units in warmer regions more frequently use electricity as a primary heating fuel.

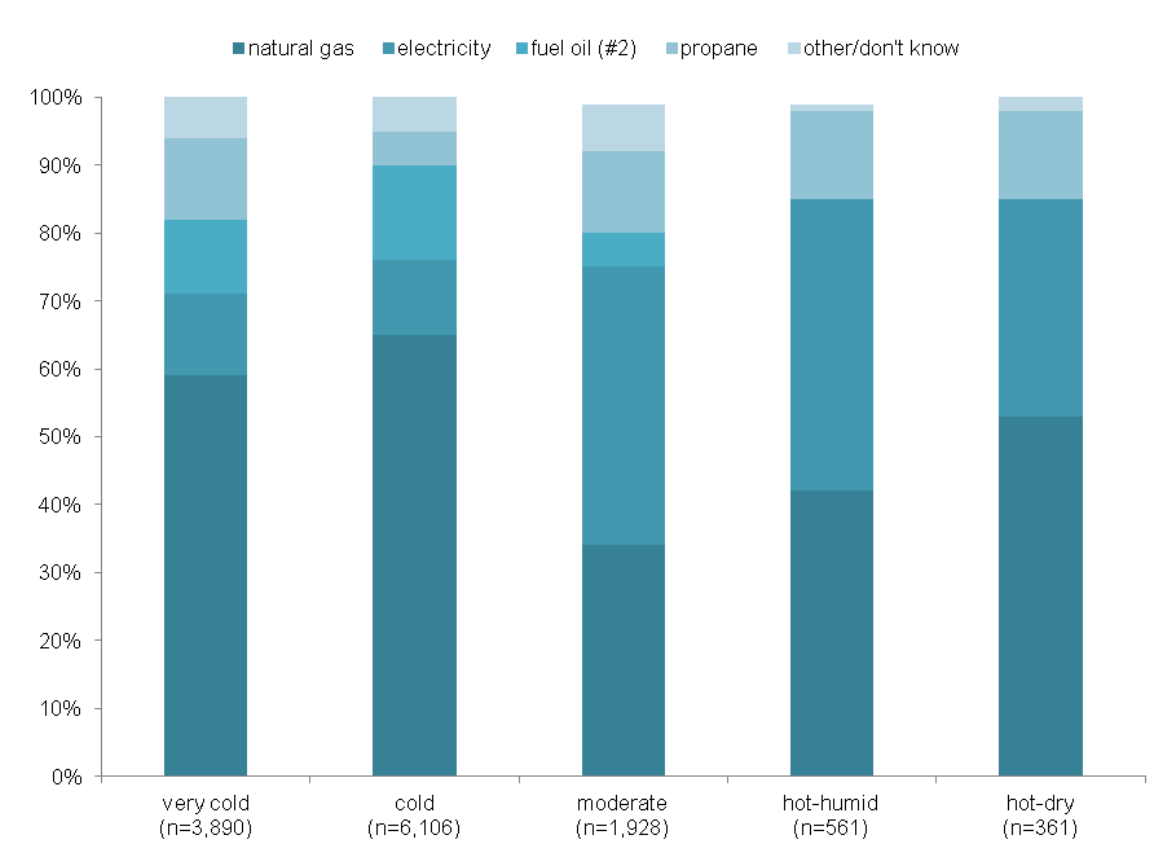

Fig. 5.11: Primary heating fuel type by climate region ${ }^{++}$

In addition to fuel type, heating systems vary among homes weatherized in PY08. The most common primary heating types among pre-weatherized homes is a central furnace system (59 percent), or steam or hydronic system (18 percent). 


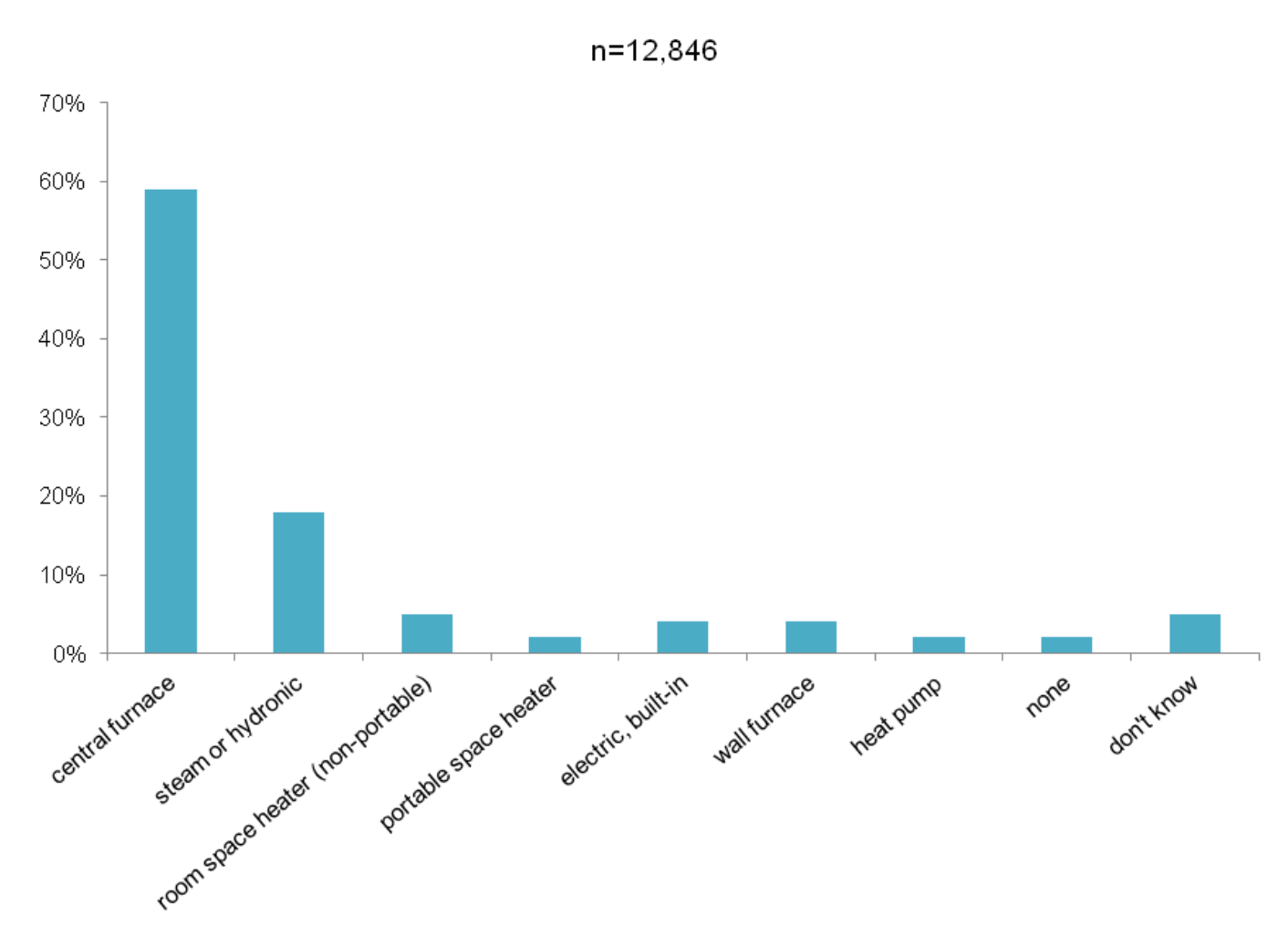

Fig. 5.12: Primary heating system ${ }^{++}$

Central furnace systems are most typical of single-family and mobile homes (63 and 82 percent, respectively), while steam or hydronic systems are more typical of units in multifamily buildings (47 percent). More specifically, steam or hydronic systems are often the primary heat source among multifamily units that have a shared heating system (91 percent of shared heating systems).

The type of air conditioning system is another important characteristic of the PY08 weatherized housing stock. Roughly half of homes had at least one type of air conditioning system. Twenty-two percent had window/wall units, 20 percent had a central system and 2 percent had an evaporative cooling system. Nearly one-fifth (17 percent) of homes had no air conditioning system. 


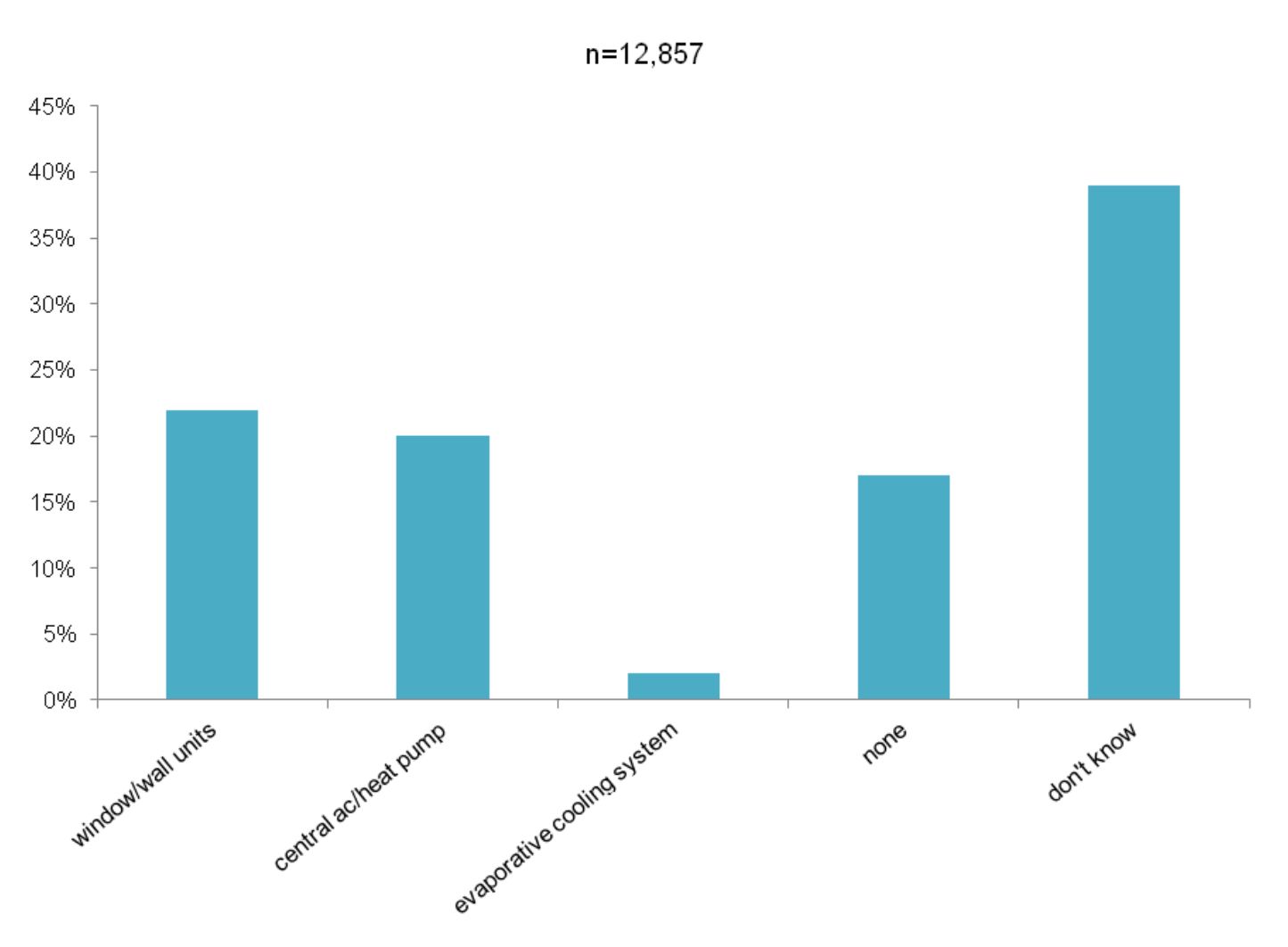

Fig. 5.13: Air conditioning system ${ }^{++}$

\subsection{DIAGNOSTICS PERFORMED}

Diagnostic and inspection procedures are performed on potential weatherization clients' homes to determine the status of the housing unit and the capacity for energy savings. Most homes weatherized during PY08 received at least one pressure diagnostic (83 percent), such as a blower door test, zonal pressure analysis or duct pressure measurements, among others. Similarly, most homes received diagnostic measurements or inspection pertaining to the water-heating system and/or space-heating system ( 82 percent, each). Table 5.1 summarizes the implementation rates, and Fig. 5.14 shows them visually.31

Table 5.1: Percent of weatherized units receiving at least one inspection from diagnostic category ${ }^{++}$

\begin{tabular}{lr}
\hline Diagnostic category & $\begin{array}{r}\text { Percent } \\
\text { of units }\end{array}$ \\
\hline Pressure & $83 \%$ \\
\hline Water-heating system & $82 \%$ \\
\hline Space-heating system & $82 \%$ \\
\hline Other diagnostics & $56 \%$ \\
\hline CO measurements & $47 \%$ \\
\hline HVAC components & $17 \%$ \\
\hline Air conditioning system & $13 \%$ \\
\hline
\end{tabular}

\footnotetext{
${ }^{31}$ These implementation rates are for individual units and may differ from implementation rates reported by subgrantees at the agency-level.
} 


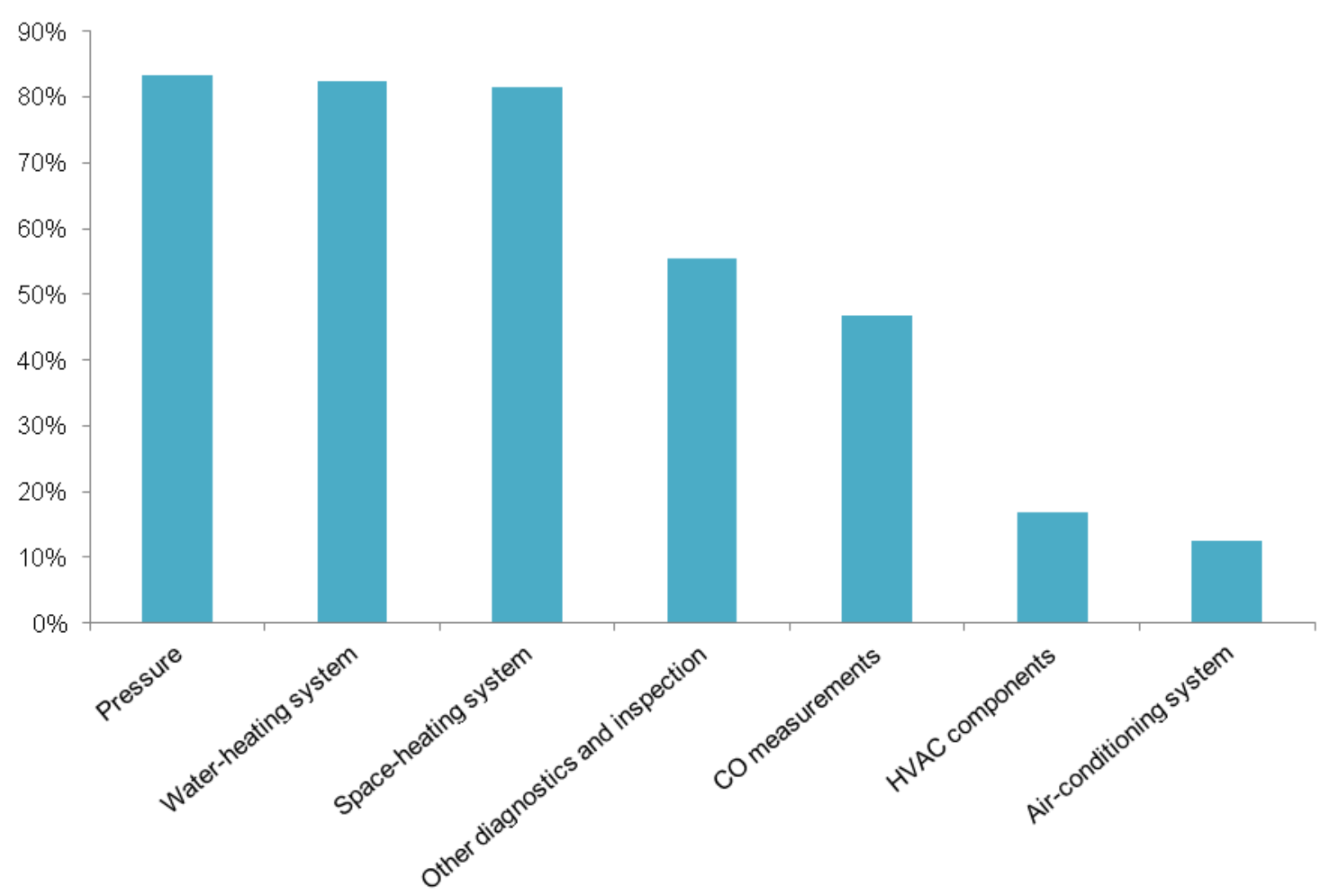

Fig. 5.14: Percent of weatherized units receiving at least one inspection from diagnostic category ${ }^{++}$

\subsubsection{Diagnostics by housing type}

Generally speaking, fewer diagnostic measurements were performed on housing units in multifamily buildings, compared to single-family and mobile homes, although in most cases the differences across building types were slight. Pressure tests were the exception. The likelihood of a multifamily unit receiving a pressure test (51 percent) was roughly half that for the other housing types (over 90 percent).

Table 5.2: Percent of weatherized units receiving at least one inspection from diagnostic category ${ }^{++}$

\begin{tabular}{lccc}
\hline Diagnostic category & $\begin{array}{c}\text { \% single-family units } \\
(\mathbf{n = 9 , 1 3 2})\end{array}$ & $\begin{array}{c}\text { \% multifamily units } \\
(\mathbf{n = 1 , 2 0 0})\end{array}$ & $\begin{array}{c}\text { \% mobile home units } \\
(\mathbf{n = 2 , 6 6 1 )}\end{array}$ \\
\hline Pressure & $92 \%$ & $51 \%$ & $95 \%$ \\
\hline Water-heating system & $84 \%$ & $83 \%$ & $77 \%$ \\
\hline Space-heating system & $84 \%$ & $80 \%$ & $77 \%$ \\
\hline Other diagnostics & $57 \%$ & $51 \%$ & $53 \%$ \\
\hline CO measurements & $49 \%$ & $47 \%$ & $47 \%$ \\
\hline HVAC components & $18 \%$ & $11 \%$ & $22 \%$ \\
\hline Air conditioning system & $13 \%$ & $8 \%$ & $16 \%$ \\
\hline
\end{tabular}




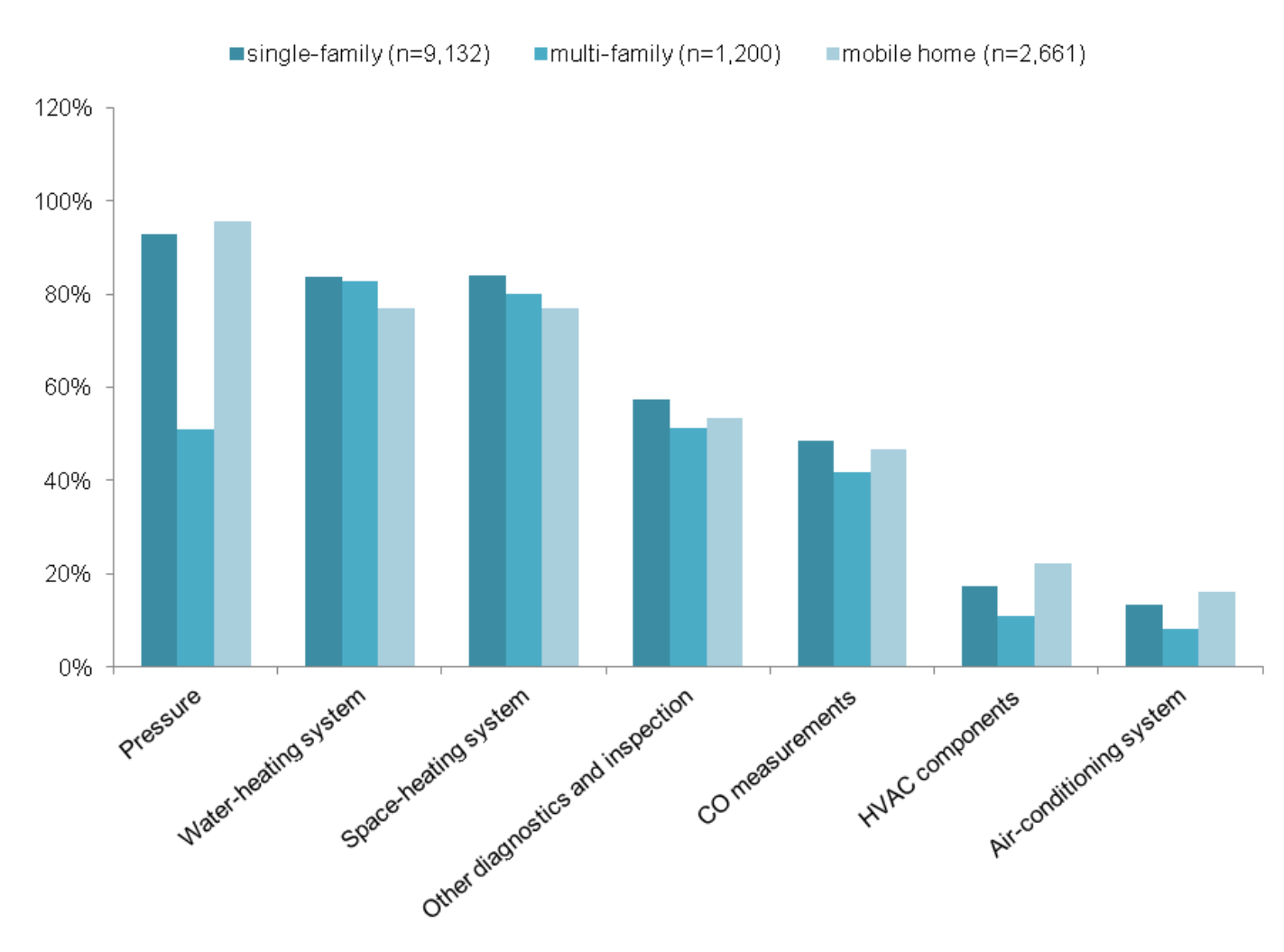

Fig. 5.15:Percent of weatherized units receiving at least one inspection from diagnostic category, by housing type $^{++}$

\subsubsection{Diagnostics by climate zone}

Variation among climate regions is apparent for a handful of diagnostic categories. PY08 weatherized units in hot-dry regions received fewer pressure analyses (58 percent) and fewer 'other' diagnostics (12 percent) than other regions. Measurements included in other diagnostics are refrigerator use, exhaust fan air flow, infrared scanning and radon testing. Interestingly, however, units in hot-dry regions received more space-heating measurements than those in moderate or hot-humid regions. Finally, air conditioning system inspections also proved variable by climate region. Forty-four percent of units in hot-humid regions received diagnostic measures on their air-conditioning systems, 27 percent of units in moderate climates and fewer than 15 percent in each of the other three climate regions. 


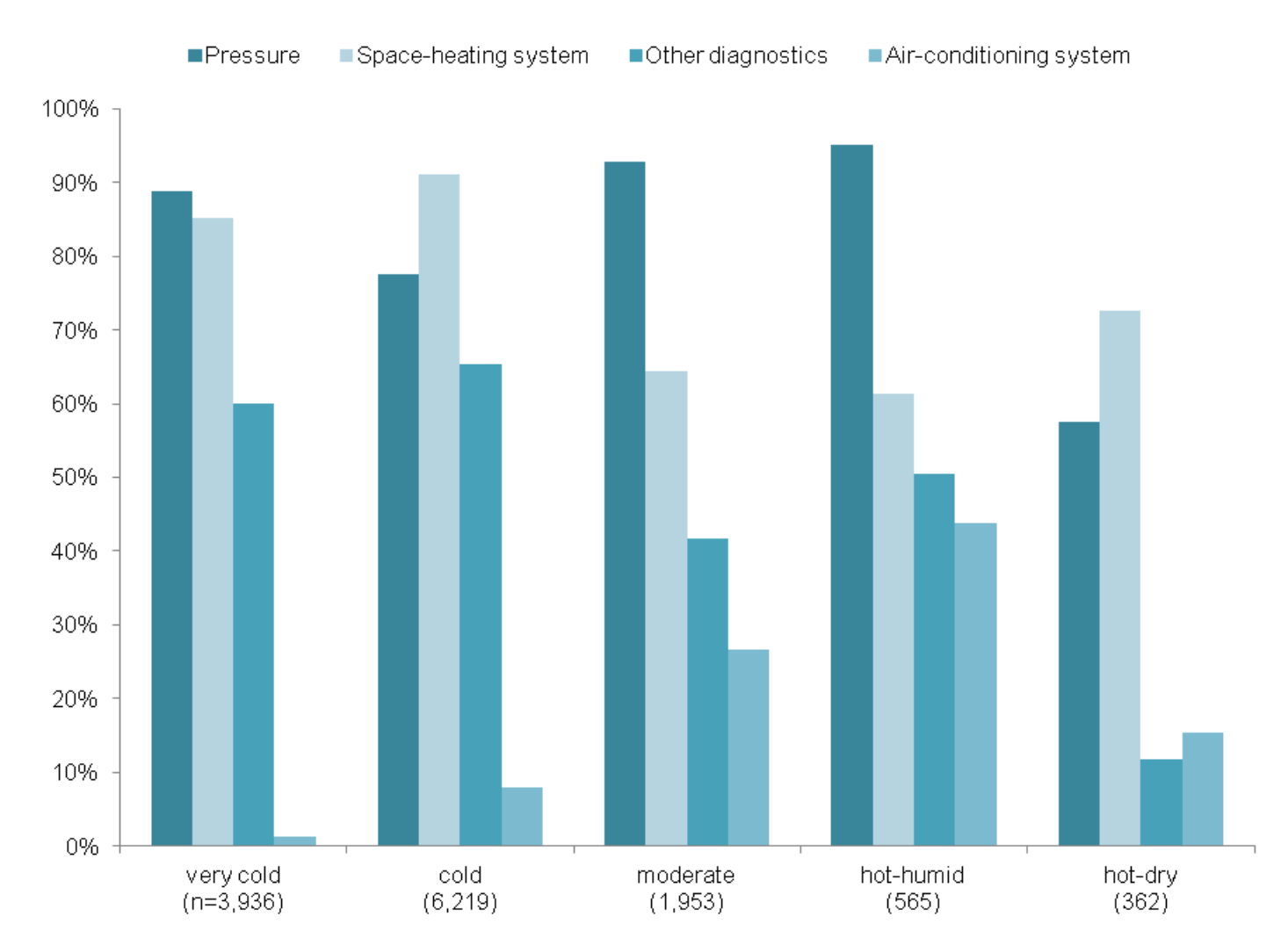

Fig. 5.16:Percent of weatherized units receiving at least one inspection from diagnostic category ${ }^{++}$

\subsection{MEASURES AND SERVICES}

Weatherization programs provide three general types of measures and services to clients: home improvements to save energy, home improvements to address health and safety risks, and client education.

\subsubsection{Energy-saving measures}

The primary service is the installation of measures that save energy, which account for 63 percent of all measures in the 97,965 units weatherized in PY08 for which we collected measure information and 87 percent of funds spent on units and buildings. To qualify as an energy-saving measure under DOE program rules, a measure most be deemed to be cost-effective, which means it needs to have a savings-toinvestment ratio of one or higher.

On average, energy-saving measures make up the largest portion (67 percent) of total measure installations among homes in multifamily buildings. Energy-saving measures constitute 63 percent of all installations among single-family homes and 61 percent among mobile homes.

Weatherization programs across the country tend to install some common measures fairly universally. Nearly all PY08 weatherized homes (91 percent) received some form of air sealing. The next most common types of improvements were insulation (75 percent) and 'other baseload applications' (69 percent). Other baseloads refer to energy-consuming appliances and fixtures that are not part of the heating, ventilation and air conditioning (HVAC) systems. 
Table 5.3: Percent of weatherized units receiving work in various measure categories ${ }^{++}$

\begin{tabular}{lr}
\hline Measure category & $\begin{array}{r}\text { Percent } \\
\text { of units }\end{array}$ \\
\hline Air sealing & $91 \%$ \\
\hline Insulation & $75 \%$ \\
\hline Other baseloads & $69 \%$ \\
\hline Water-heating system & $65 \%$ \\
\hline Space-heating system & $44 \%$ \\
\hline HVAC accessories & $38 \%$ \\
\hline Windows & $37 \%$ \\
\hline Doors & $35 \%$ \\
\hline Ventilation & $26 \%$ \\
\hline Air-conditioning systems & $6 \%$ \\
\hline
\end{tabular}

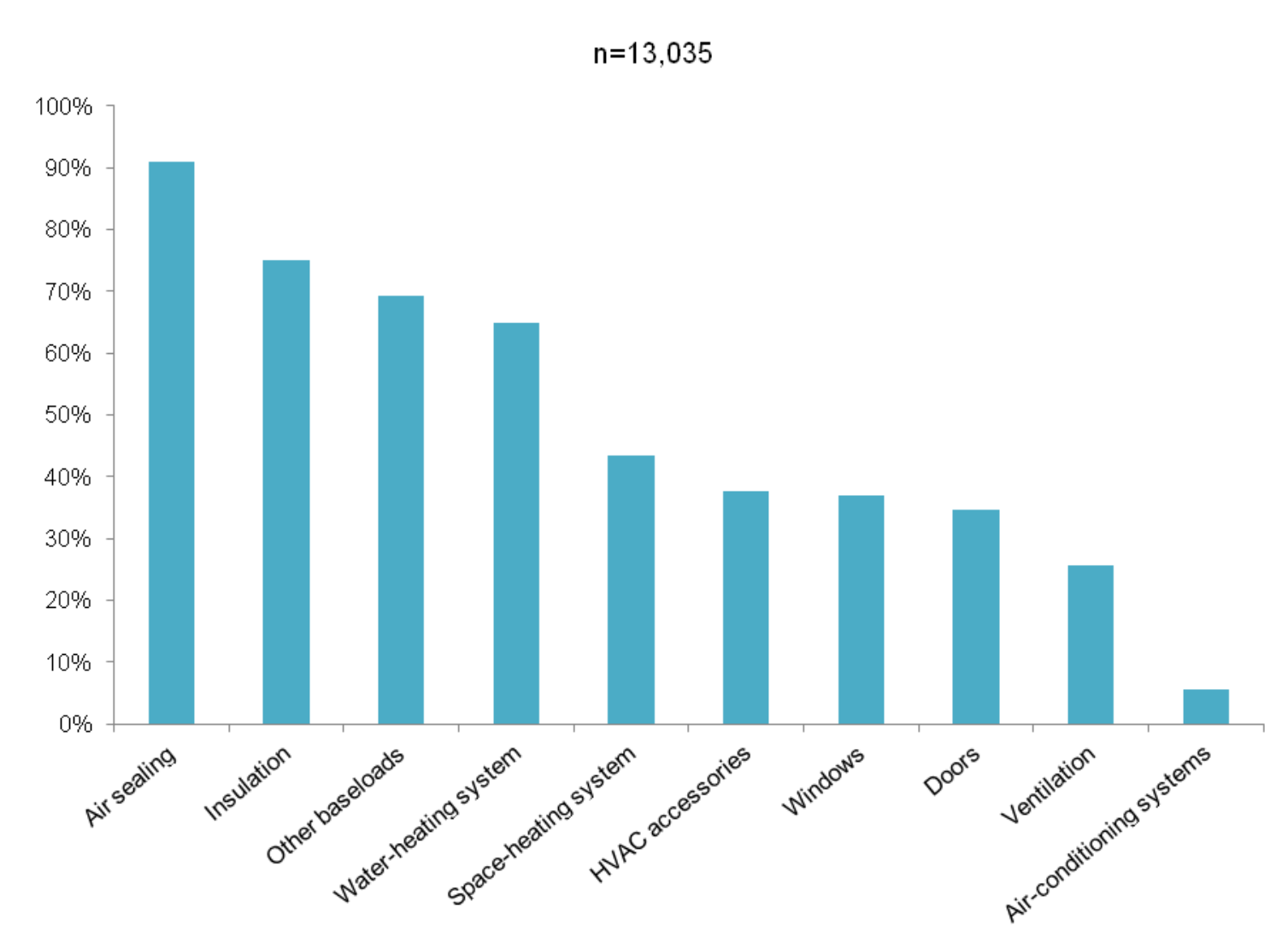

Fig. 5.17:Percent of weatherized units receiving work in various measure categories ${ }^{++}$

In single-family homes, the most commonly installed energy-saving measures are similar to those for all housing types, with air sealing (94 percent), insulation ( 82 percent) and non-HVAC baseload measures (65 percent) leading other measure categories. 
For units in multifamily buildings, air sealing measures are slightly less universal; however, these types of measures and non-HVAC baseloads are the most commonly installed measures (81 percent each). Improvements to water-heating systems (73 percent), insulation (66 percent) and space-heating systems (52 percent) are also commonly installed measure types among weatherized units in multifamily buildings.

Instances of measure types installed in mobile homes differs most notably from single- and multifamily homes in terms of HVAC accessories (52 percent) and window and door improvements (46 percent each).

Table 5.4: Percent of weatherized units receiving at least one measure from broader category, by housing type $^{++}$

\begin{tabular}{lccc}
\hline Measure category & $\begin{array}{c}\text { \% single-family units } \\
(\mathbf{n = 9 , 1 3 2})\end{array}$ & $\begin{array}{c}\text { \% multifamily units } \\
(\mathbf{n = 1 , 2 0 0})\end{array}$ & $\begin{array}{c}\text { \% mobile home units } \\
(\mathbf{n = 2 , 6 6 1 )}\end{array}$ \\
\hline Air sealing & $94 \%$ & $81 \%$ & $94 \%$ \\
\hline Insulation & $82 \%$ & $66 \%$ & $64 \%$ \\
\hline Other baseloads & $65 \%$ & $81 \%$ & $68 \%$ \\
\hline Water-heating system & $63 \%$ & $73 \%$ & $61 \%$ \\
\hline Space-heating system & $41 \%$ & $52 \%$ & $41 \%$ \\
\hline HVAC accessories & $38 \%$ & $26 \%$ & $52 \%$ \\
\hline Windows & $37 \%$ & $19 \%$ & $46 \%$ \\
\hline Doors & $36 \%$ & $33 \%$ & $46 \%$ \\
\hline Ventilation & $24 \%$ & $33 \%$ & $24 \%$ \\
\hline Air-conditioning systems & $6 \%$ & $4 \%$ & $8 \%$ \\
\hline
\end{tabular}

Regional differences are primarily seen in four measure categories: insulation, space-heating systems, airconditioning systems and ventilation. In Fig. 5.18, incidence rates for these measure categories are broken out by climate region. Rates of insulation measures are much lower in hot-dry regions compared to all other regions. ${ }^{32}$ As expected space-heating system measures are more commonly installed in very cold, cold and moderate regions while air-conditioning system measures are more common in hot regions. Ventilation measures have noticeably low incidence rates in hot-dry regions, compared to all other regions.

\footnotetext{
${ }^{32}$ Instances of insulation measures within the hot-dry states tend to be greatest among subgrantees that serve colder portions of the states classified as "hot-dry," suggesting that insulation measures are driven mostly by heating need.
} 


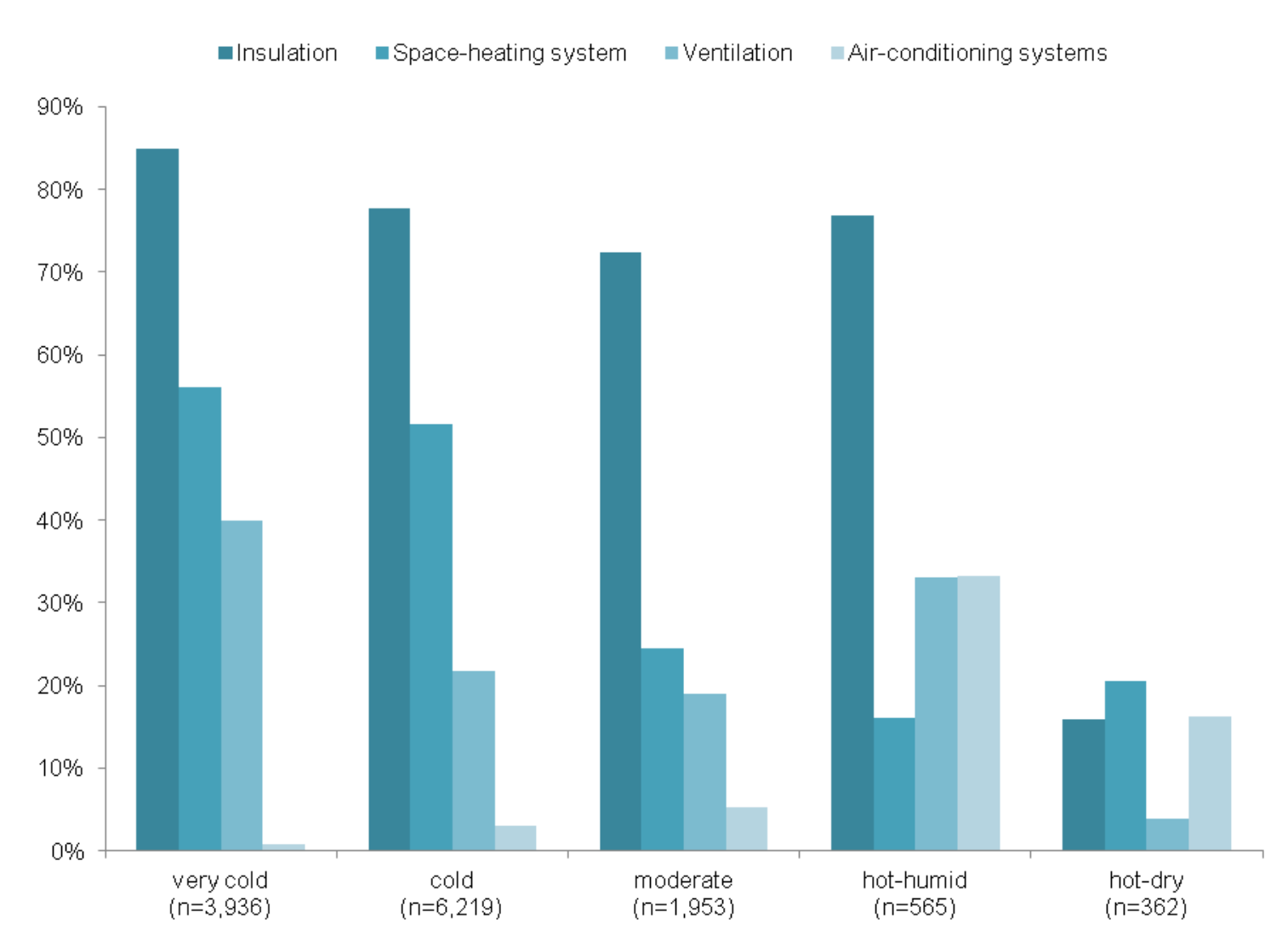

Fig. 5.18: Percent of weatherized units receiving at least one measure from broader category, by climate region $^{++}$

\subsubsection{Heating systems}

Heating system replacements are a significant measure for most weatherization programs where they occur due to the impact and cost involved. During PY08, 16 percent of weatherized homes received a new space-heating system as an energy-saving measure and 11 percent received a new system for health and safety reasons. Eighty-five percent of cost-effective heating system replacements and 75 percent of health and safety-motivated replacements were in the cold and very cold regions.

Changes in heating fuel type and heating system type, before and after weatherization, are shown in the tables below. Table 5.5 shows instances of heating fuel switching among the roughly 2,800 homes that received a new heating system, either as a cost-effective energy conservation measure or for health and safety reasons. Homes switched from the fuel type listed in the left-hand column to the fuel type listed across the first row. As shown, the vast majority of new heating systems use the same fuel as their predecessors (see the shaded cells in the table), but there is a slight net movement toward natural gas systems. 
Table 5.5: Percentage of housing units that switched from old fuel type to new fuel type ${ }^{++}$

\begin{tabular}{lrrrrrr} 
& \multicolumn{7}{c}{ New fuel type } \\
\cline { 2 - 7 } Old fuel type & Natural gas & Propane & $\begin{array}{c}\text { Kerosene } \\
\text { (fuel oil \#1) }\end{array}$ & Fuel oil (\#2) & Electricity & Wood \\
\hline Natural gas & $67 \%$ & $<1 \%$ & - & $0 \%$ & $<1 \%$ & - \\
\hline Propane & $1 \%$ & $8 \%$ & - & $<1 \%$ & $<1 \%$ & - \\
\hline Kerosene (fuel oil \#1) & - & $<1 \%$ & $2 \%$ & $<1 \%$ & $<1 \%$ & - \\
\hline Fuel oil (\#2) & $1 \%$ & $<1 \%$ & $<1 \%$ & $6 \%$ & $<1 \%$ & - \\
\hline Electricity & $3 \%$ & $<1 \%$ & - & - & $9 \%$ & - \\
\hline Wood & - & $<1 \%$ & - & - & - & $1 \%$ \\
\hline Coal & - & - & - & - & $<1 \%$ & - \\
\hline Other & $<1 \%$ & - & - & - & - & - \\
\hline Don't know & $<1 \%$ & - & - & - & - & - \\
\hline $\begin{array}{l}\text { All system } \\
\text { replacements }\end{array}$ & $\mathbf{7 2 \%}$ & $\mathbf{8 \%}$ & $\mathbf{2 \%}$ & $\mathbf{7 \%}$ & $\mathbf{1 0 \%}$ & $\mathbf{1 \%}$ \\
\hline
\end{tabular}

(Note: 'not applicable' and 'don't know' responses for new fuel type not shown in table.)

Table 5.6 shows instances of those same 2,800 homes that switched from one heating system type to another. Again, most new heating systems are of the same type as their predecessors (shaded cells), but there is a slight net movement toward furnaces.

Table 5.6:Percentage of housing units that switched from old heating system to new heating system ${ }^{++}$

\begin{tabular}{|c|c|c|c|c|c|c|c|}
\hline \multirow[b]{2}{*}{ Old heating system } & \multicolumn{7}{|c|}{ New heating system } \\
\hline & $\begin{array}{l}\text { Central } \\
\text { furnace }\end{array}$ & $\begin{array}{l}\text { Heat } \\
\text { pump }\end{array}$ & Electric & $\begin{array}{l}\text { Steam or } \\
\text { hydronic }\end{array}$ & $\begin{array}{c}\text { Wall } \\
\text { furnace }\end{array}$ & $\begin{array}{l}\text { Room } \\
\text { space } \\
\text { heater }\end{array}$ & $\begin{array}{c}\text { Portable } \\
\text { space } \\
\text { heater }\end{array}$ \\
\hline Central furnace & $57 \%$ & $1 \%$ & - & $<1 \%$ & $1 \%$ & $<1 \%$ & $<1 \%$ \\
\hline Heat pump & $<1 \%$ & $1 \%$ & - & - & - & - & - \\
\hline Electric & $<1 \%$ & - & $<1 \%$ & $1 \%$ & - & - & - \\
\hline Steam or hydronic & $<1 \%$ & - & - & $22 \%$ & - & - & - \\
\hline Wall furnace & $1 \%$ & - & - & - & $3 \%$ & $<1 \%$ & - \\
\hline Room space heater & $1 \%$ & - & - & - & $1 \%$ & $4 \%$ & $<1 \%$ \\
\hline Portable space heater & $1 \%$ & - & $<1 \%$ & - & $<1 \%$ & $<1 \%$ & $<1 \%$ \\
\hline Cooking stove & $<1 \%$ & - & - & - & - & - & - \\
\hline None & $<1 \%$ & - & - & - & - & - & $<1 \%$ \\
\hline Don't know & $2 \%$ & - & - & $<1 \%$ & $<1 \%$ & $<1 \%$ & - \\
\hline $\begin{array}{l}\text { All system } \\
\text { replacements }\end{array}$ & $62 \%$ & $2 \%$ & $0 \%$ & $24 \%$ & $5 \%$ & $5 \%$ & $1 \%$ \\
\hline
\end{tabular}

(Note: 'other', 'not applicable' and 'don't know' responses for new heat system not shown in table.) 


\subsubsection{Health and safety measures}

Health and safety measures are installed to help ensure the wellbeing of residents. Some of these measures help residents lower their energy consumptions, but they differ from energy-saving measures because the costs are justified for health and safety reasons, not energy savings. Fig. 5.19 shows the 10 most common health and safety measures installed during PY08. Carbon monoxide (CO) monitors were installed in 53 percent of all weatherized homes and smoke alarms in 42 percent. Four of the 10 top health and safety measures are also common energy-saving measures that are sometimes installed for efficiency reasons: new heating system, new door, new window and new water heater.

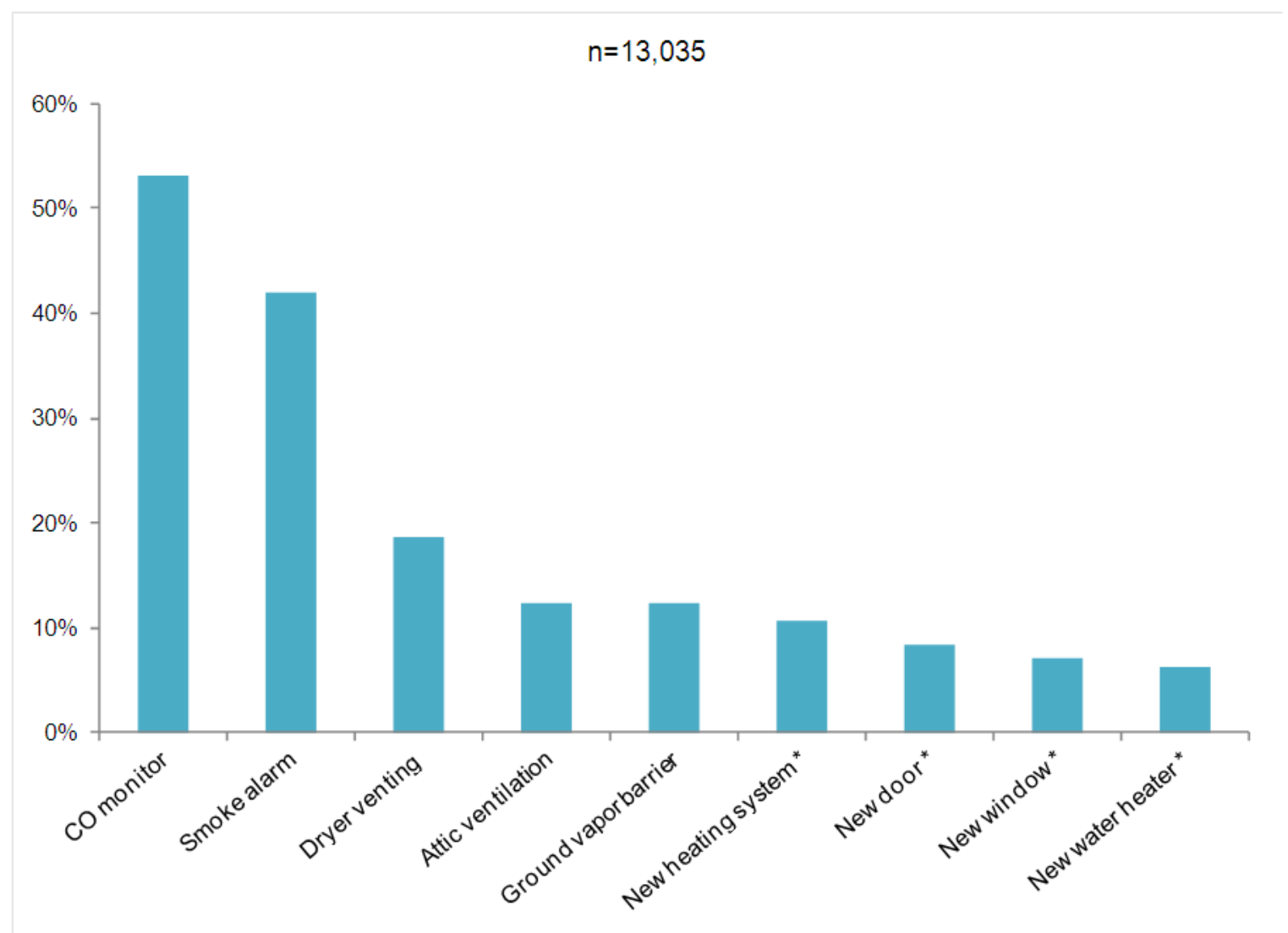

* These measure types could be installed for either energy conservation or for health and safety reasons. Instances reported in this figure were installed for health safety reasons only.

Fig. 5.19: Percent of weatherized units receiving 10 most common health and safety measures ${ }^{++}$

\subsubsection{Client education services}

In addition to physical measures weatherization programs offer clients educational material and training designed to create energy savings or improve home safety through behavioral or usage changes. (The description of client services presented here are taken from a review of project information and may differ from the characterization subgrantees presented - see above.) Overwhelmingly, literature and in-home education were the most common education services offered in PY08. Hardware kits, video/DVD/CDs and classroom education were all less common, ranging from 2 to 7 percent of all homes. 
Data on in-home education instances is broken down by duration: 8 percent of homes received a visit lasting 30 minutes or longer, 17 percent received a 15 to 29 minute visit and 9 percent received a visit lasting no longer than 15 minutes. Additionally, 48 percent of homes received an in-home education service of an undetermined length of time.

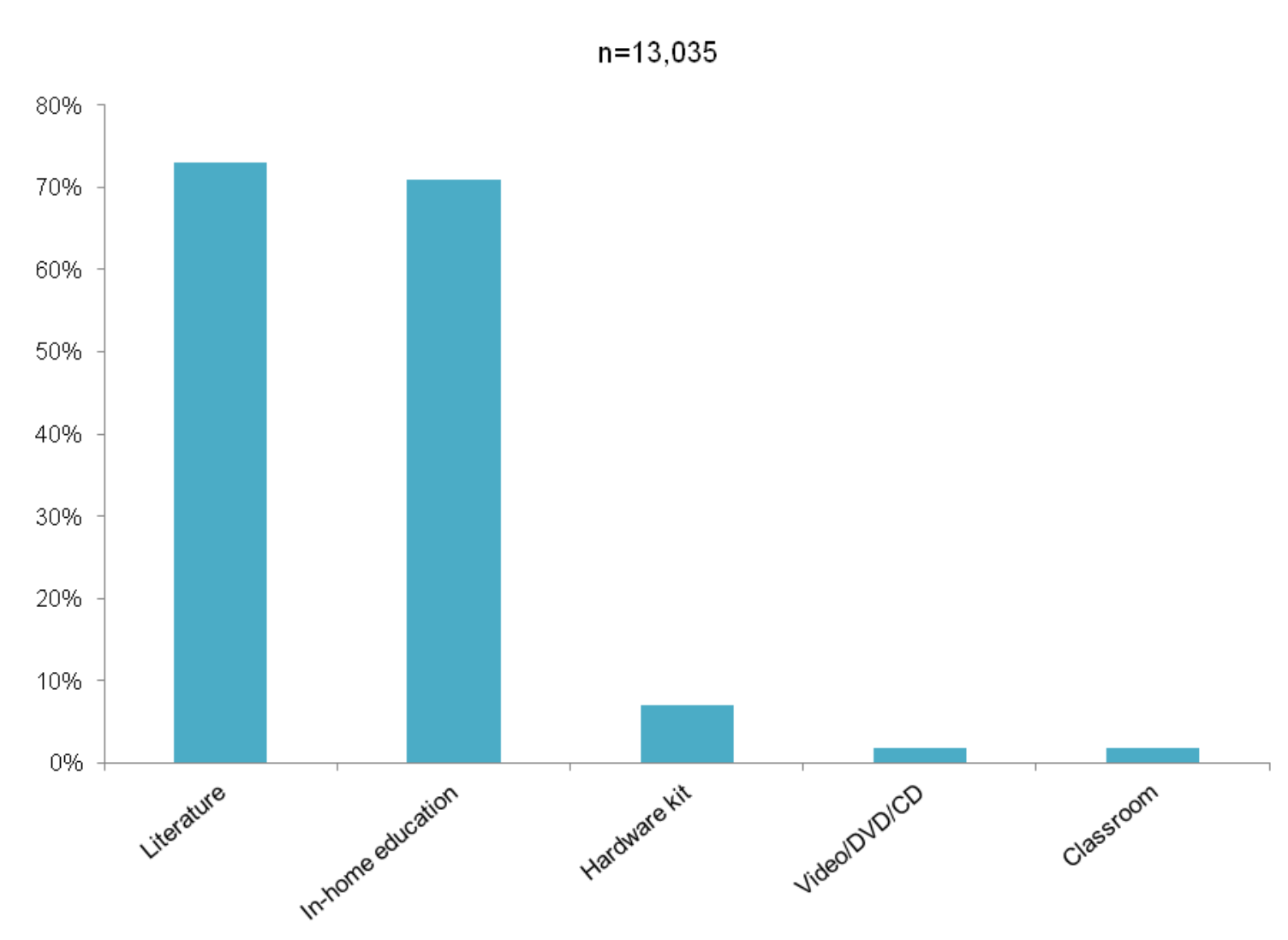

Fig. 5.20: Percent of weatherized units receiving client education measures ${ }^{++}$

\subsection{COST PER UNIT}

Subgrantees reported total project costs to us for 12,113 PY08 weatherization jobs ${ }^{33}$. The project costs varied by housing type and we measured the differences in the costs, broken out by DOE based funding as well as material, labor and overhead costs. The breakdown of in-house crew versus contractors was also determined.

Project costs differed by building type, as shown in Table 5.7, but costs per unit averaged around $\$ 3,500$ regardless of building type. Project costs for individual weatherization jobs also varied widely, as shown by the percentile distribution in the table. (Note: multifamily refers to the entire multifamily building, where multifamily unit refers to the housing unit in which the weatherization project occurred.)

\footnotetext{
${ }^{33}$ Excludes jobs for which cost information was inconsistent across multiple questions and projects for which costs shown were $\$ 0$ or that were extremely high. Two records with overall project costs shown of $\$ 1$ million or more were excluded from the data as either outliers or erroneous data.
} 
Table 5.7: Project costs by building type (all funding sources) ${ }^{++}$

\begin{tabular}{|l|c|c|c|c|c|}
\hline building type & average (mean) & $\begin{array}{c}\text { 25th } \\
\text { percentile }\end{array}$ & $\begin{array}{c}\text { 50th } \\
\text { percentile }\end{array}$ & $\begin{array}{c}75 \text { th } \\
\text { percentile }\end{array}$ & range \\
\hline $\begin{array}{l}\text { overall (building) } \\
(\mathrm{n}=12,066)\end{array}$ & $\$ 4,350$ & $\$ 870$ & $\$ 3,400$ & $\$ 4760$ & $\$ 6-\$ 959,110$ \\
\hline $\begin{array}{l}\text { single family } \\
(\mathrm{n}=8,514)\end{array}$ & $\$ 3,490$ & $\$ 1,500$ & $\$ 2,760$ & $\$ 4,450$ & $\$ 6-\$ 45,030$ \\
\hline $\begin{array}{l}\text { mobile homes } \\
(\mathrm{n}=2,477)\end{array}$ & $\$ 3,350$ & $\$ 1,410$ & $\$ 2,770$ & $\$ 4,490$ & $\$ 16-\$ 17,270$ \\
\hline $\begin{array}{l}\text { multifamily (building) } \\
(\mathrm{n}=1,052)\end{array}$ & $\$ 3,690$ & $\$ 1,670$ & $\$ 3,920$ & $\$ 10,000$ & $\$ 22-\$ 959,110$ \\
\hline $\begin{array}{l}\text { multifamily unit } \\
(\mathrm{n}=1,097)\end{array}$ & $\$ 730$ & $\$ 2,080$ & $\$ 4,890$ & $\$ 5-\$ 44,210$ \\
\hline
\end{tabular}

The majority of projects involving single-family homes were funded using DOE allocations, as shown in Fig. 5.21, whereas costs for multifamily units and mobile homes used greater shares of non-DOE funding.

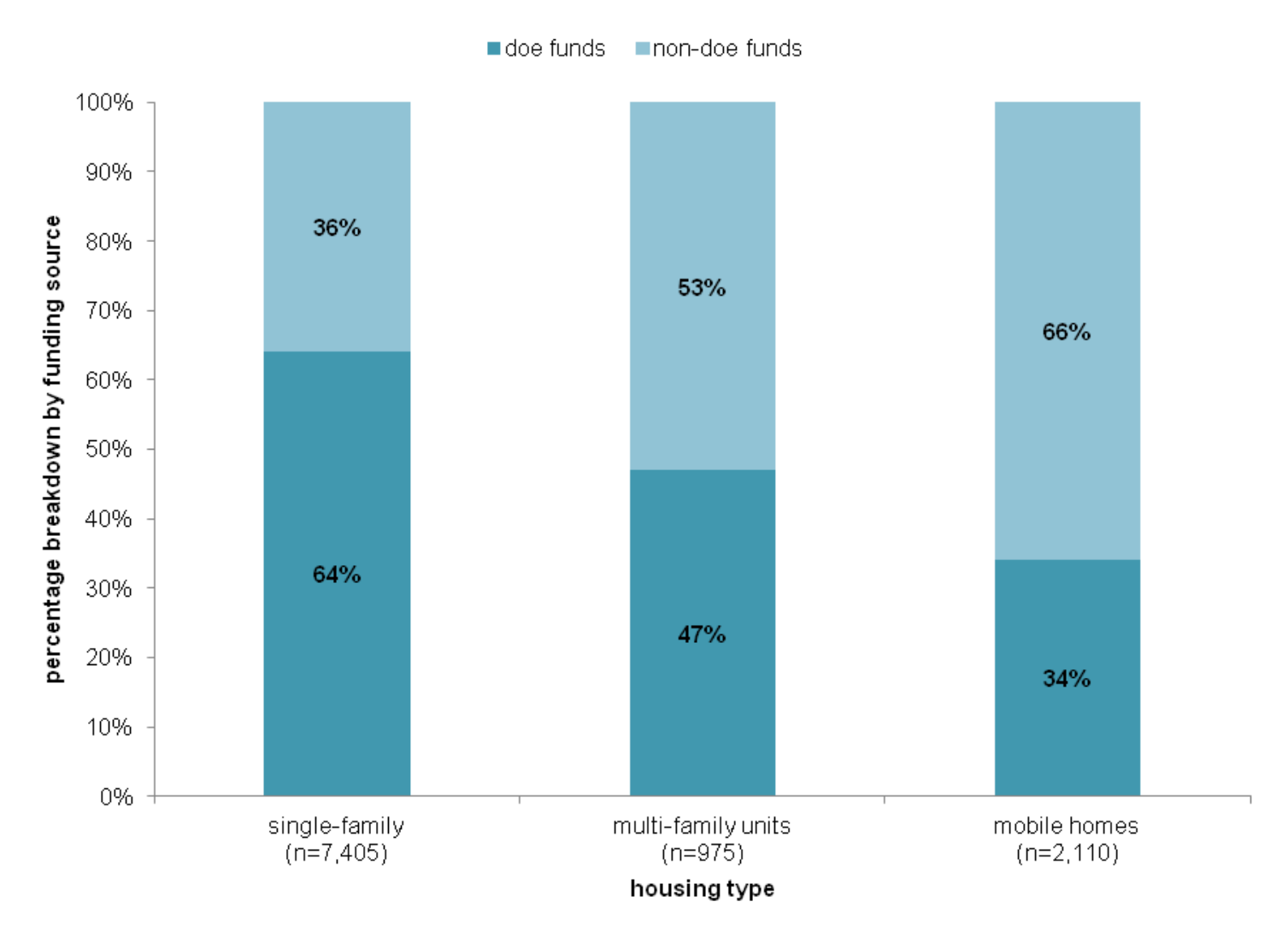

Fig. 5.21: Distribution of costs by funding source and building type ${ }^{++}$

On-site project costs are primarily materials and labor, with labor costs split between subgrantees' inhouse crews and contractors. Based on average costs reported by subgrantees, we developed a "typical" single-family cost structure shown in Table 5.8. For a project costing $\$ 3,500$, materials comprise about $\$ 1,900$ of the project expense while labor accounts for the remaining $\$ 1,600$. On average, labor costs for 
work done by in-house crews is about $\$ 765$ while costs for contracted work is about $\$ 835$, although the distribution can be very different for any individual project.

Table 5.8: Typical project cost breakdown for single family ${ }^{++}$

\begin{tabular}{|r|c|c|}
\hline \multicolumn{3}{|c|}{ Typical total costs for single family project $\sim \mathbf{\$ 3 , 5 0 0}$} \\
\hline Material & \multicolumn{2}{|c|}{$\sim 1,900$} \\
\hline \multirow{2}{*}{ Labor } & $\begin{array}{c}\text { In house crew } \sim \\
\$ 765\end{array}$ & $\begin{array}{c}\text { Contractor } \sim \\
\$ 835\end{array}$ \\
\cline { 2 - 3 } & & \\
\hline
\end{tabular}

As shown in Fig. 5.22, the average distribution of costs between material and labor differs little across building types.

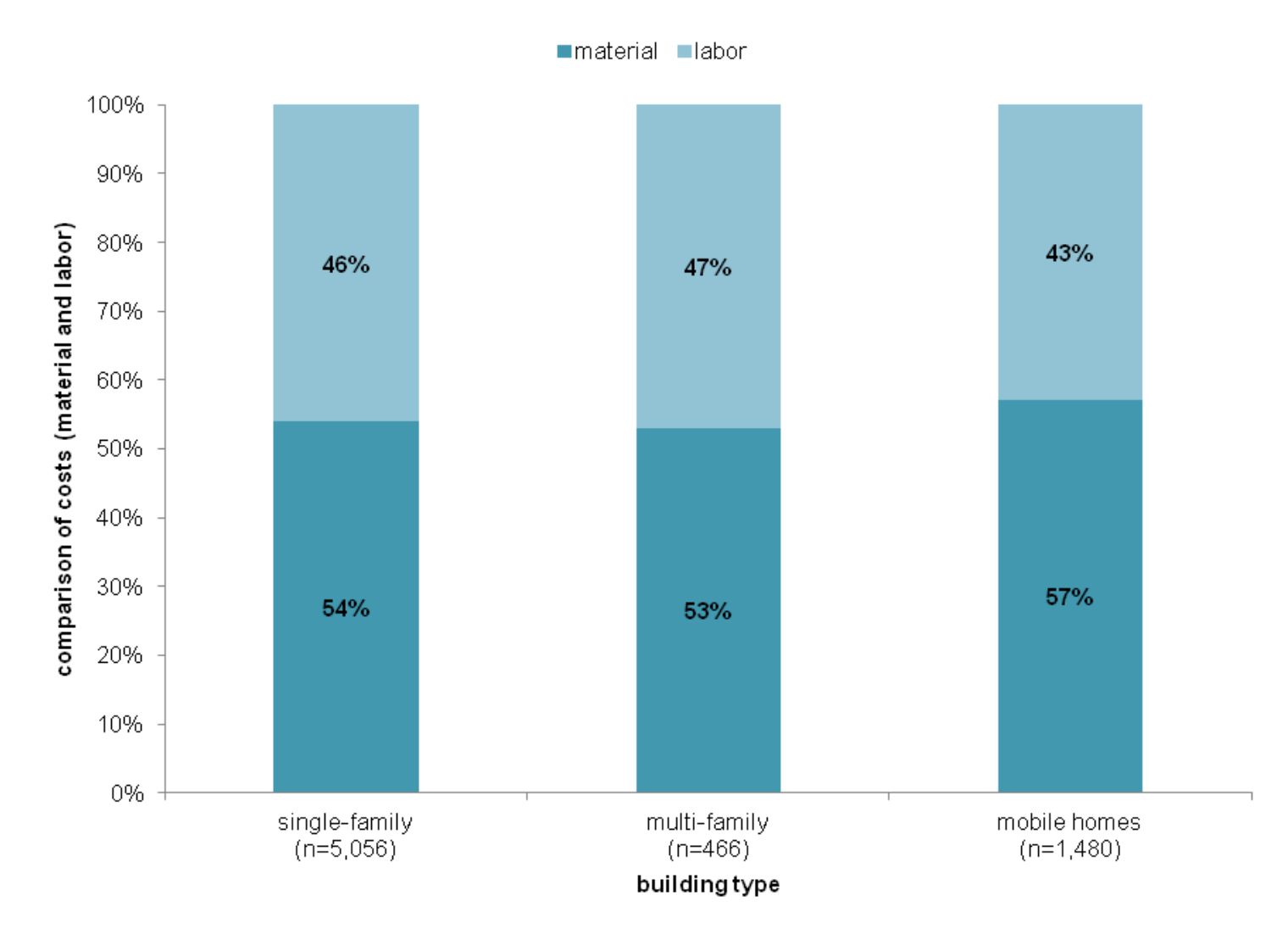

Fig. 5.22:Distribution of material and labor costs by building type ${ }^{++}$ 
shows the project cost breakout of in-house crews against contractor costs by building type. For singlefamily homes and multifamily projects, subgrantees spent a bit more on contractors than for work performed by in-house crews. In contrast, for mobile homes, subgrantees spent more on in-house crews than contractors. 


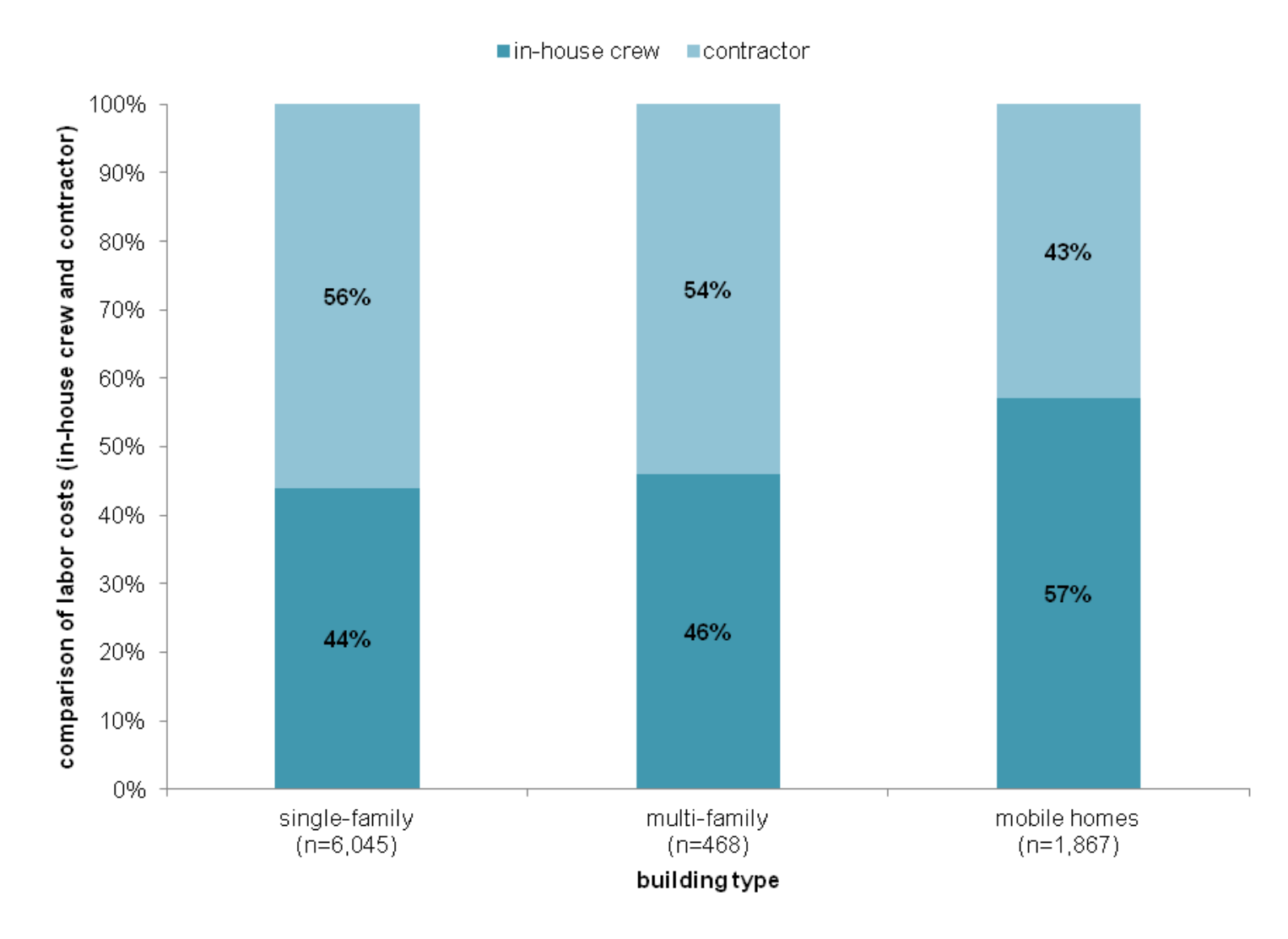

Fig. 5.23: Distribution of labor costs among in-house crews and contractors, by building type ${ }^{++}$

How subgrantees allocate the labor component of weatherization projects is more complex and varied. Possible models include using in-house staff for all (or most) project work, hiring contracted weatherization crews, or doing some work using in-house staff and supplementing their work with specialty contractors, such as HVAC or insulation contractors. We examined labor costs further to assess how commonly subgrantees use these various models.

Project-level analysis of cost data suggests that 60 percent of subgrantees tended to use either in-house crews or contractors on individual projects ${ }^{34}$ for the work on a given single-family project while 40 percent of subgrantees tended to use both in-house crews and contractors on the same single family projects. ${ }^{35}$ Among subgrantees that used either in-house crews or contractors, approximately one third relied on in-house crews and two thirds on contractors. Mobile homes show a similar breakout. For multifamily buildings, we find that it was even more common that subgrantees used either in-house crews or contractors on their projects. Table 5.9 displays the distribution of these labor cost patterns by building type.

\footnotetext{
${ }^{34}$ We used a threshold of $80 \%$ of labor costs spent on either in-house crews or contractors in categorizing projects as being done primarily by one type of labor or the other.

${ }^{35}$ In doing this analysis, we assigned individual projects as being done either primarily by in-house crews (if labor costs for in-house crews exceeded $80 \%$ of total labor costs), primarily by contractors (again using an $80 \%$ threshold), or a mix of both. Then, we examined subgrantees' tendencies across all of their sampled projects for which we had sufficient cost data. We defined subgrantees as using one model or the other as their primary mode if $2 / 3$ or more of their projects fell in that pattern. If both models were in use in at least $1 / 3$ of projects, we classified that subgrantee as using both models.
} 
Table 5.9: Subgrantee cost allocation for labor costs (in-house crew or contractor), by building type ${ }^{++}$

\begin{tabular}{|c|c|c|c|c|}
\hline & $\begin{array}{l}\text { Subgrantees using } \\
\text { mix of labor types }\end{array}$ & $\begin{array}{c}\text { Subgrantees using } \\
\text { either one labor type }\end{array}$ & $\begin{array}{r}\text { Distribution } \\
\text { used by sub }\end{array}$ & $\begin{array}{l}\text { at labor type } \\
\text { "group A" }\end{array}$ \\
\hline \multirow{2}{*}{$\begin{array}{l}\text { Single family } \\
(n=6045)\end{array}$} & \multirow{2}{*}{$40 \%$} & \multirow{2}{*}{$60 \%$} & In-house & $31 \%$ \\
\hline & & & Contractor & $69 \%$ \\
\hline \multirow{2}{*}{$\begin{array}{l}\text { Multifamily } \\
\text { buildings } \\
(\mathrm{n}=\mathbf{4 8 6})\end{array}$} & \multirow[b]{2}{*}{$24 \%$} & \multirow[b]{2}{*}{$76 \%$} & In-house & $46 \%$ \\
\hline & & & Contractor & $54 \%$ \\
\hline \multirow{2}{*}{$\begin{array}{l}\text { Mobile homes } \\
(n=1867)\end{array}$} & \multirow{2}{*}{$32 \%$} & \multirow{2}{*}{$68 \%$} & In-house & $42 \%$ \\
\hline & & & Contractor & $58 \%$ \\
\hline
\end{tabular}

For each building type, expenditures were broken down between energy-related measure, health and safety measures, and incidental repairs. Unsurprisingly, the majority of the effort lies within the category of energy-related measure as shown in Fig. 5.24. For all building types, significantly less was spent on health and safety and incidental repairs. It should be noted that, as with all of the cost analyses, this distribution of expenditure types is taken from those subgrantees that knew the costs and does not include sampled projects for the subgrantees who did not know expenditure allocations. In contrast to material/labor and DOE/non-DOE cost distributions, however, subgrantees tended to have more difficulty providing distributions by measure purpose. 


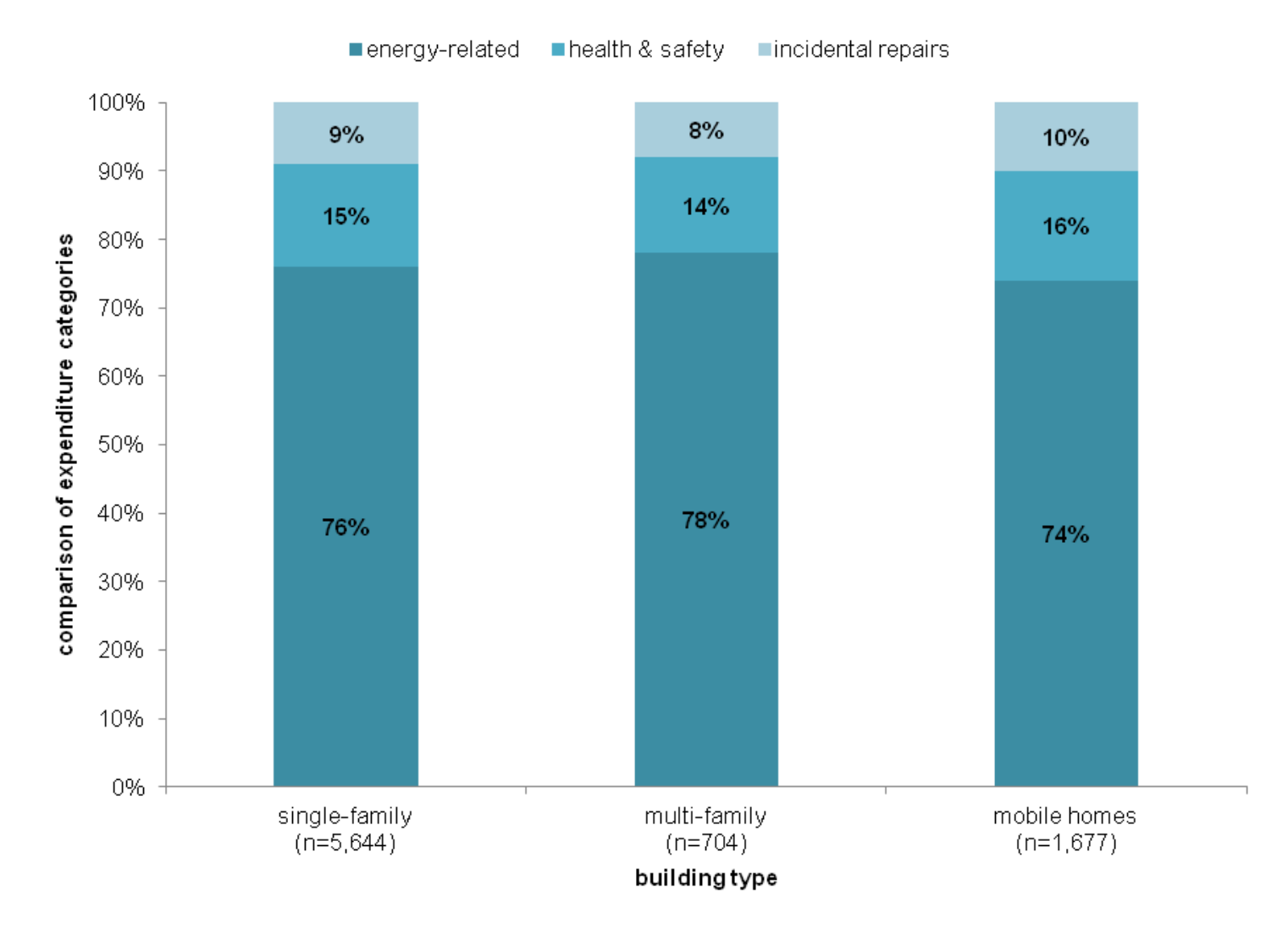

Fig. 5.24: Project cost distribution among energy-saving measures, health \& safety, and incidental reports, by building type $^{++}$ 



\section{CONCLUSIONS}

The three partners in the design and implementation of the WAP program are DOE, the weatherization grantees (i.e., states, tribes, and territories), and the subgrantees. Each plays an important part in the success of the program:

DOE provides:

- the base funding on which the program has been built

- program specifications and boundaries within which grantees need to operate to ensure costeffectiveness and to ensure policy priorities are met

- technical assistance and tools, such as the National Energy Audit Tool

- training funds and staff development opportunities, such as the national weatherization program conference and a network of weatherization training centers

- grant oversight and administration

- participation in national policy development on energy efficiency services for low-income populations.

Grantees contribute:

- program customization and administration to fit the grantee's WAP program to local conditions, needs, and preferences

- development and maintenance of a set of subgrantees to serve eligible households throughout the grantee's jurisdiction

- training opportunities and state-level conferences for subgrantee staff

- quality control and oversight to ensure that homes are weatherized effectively and the program is implemented uniformly

- leveraging of other federal, state, and private funds to supplement DOE grants.

Subgrantees add:

- diagnosis and weatherization of eligible households' homes

- inspection of units served

- recruitment and income screening of eligible households (sometimes performed upstream of WAP subgrantee involvement)

- leveraging of DOE funds by attracting additional funding sources for their work

- (sometimes) complementary services for eligible households and individuals.

Below, we summarize the evaluation project's overall findings about the manner in which these program partners go about performing their functions. Our data collection focused on grantee and subgrantee implementation of the national WAP program, so our findings concentrate on those two levels of the program.

\subsection{DEPARTMENT OF ENERGY}

As the core funder, DOE provides the financial backbone and programmatic structure on which the national WAP program is built. The addition of non-DOE funding - which added about $\$ 541$ million to DOE's \$233 million in PY08 - has built onto this structure. The fact that grantees and subgrantees were able to attract and retain new funding from other sources (including $\$ 210$ million in non-federal funds) over the years speaks well of the program and the confidence that regional and local partners have in it. At the same time, the low adoption of DOE rules by the non-federal funding sources (see suggests that there are unmet needs and priorities at the local level that the DOE structure does not address. 
Grantee input on DOE's administrative and supporting role in their implementation of local WAP programs called for:

- increased financial or leveraging support (than was provided in PY08)

- DOE help with data and information systems

- more flexibility.

\subsection{GRANTEES}

Grantees comprise a diverse set of state agencies (one in each of the 50 states) with disparate other energy or housing-related responsibilities, as well as the District of Columbia, some tribes, and territories. Our data collection from grantees entailed the completion of one data form and one comprehensive survey, as well as the receipt of grantee-level databases of projects completed in PY08 where sufficiently comprehensive datasets existed.

Grantees design and run the actual weatherization programs to meet local conditions, needs, and preferences. They are the ones who assemble a network of local weatherization providers and, in many cases, additional funding sources to support their programs.

We found that grantees:

- received about $\$ 700$ million to administer their weatherization programs in PY08, of which a third was DOE funds

- $\quad$ passed along 94 percent of their weatherization funds (\$677 million) to subgrantees for local weatherization work, retaining 6 percent for program management, oversight, administration, and assistance

- maintained between two and 64 subgrantees, providing an average of $\$ 240,000$ in DOE funds per subgrantee

- maintained an average of eight FTE, typically allocating 2-3 positions for program oversight, 2-3 FTE for agency monitoring and quality assurance, and 1-2 FTE for training and technical assistance.

Certain aspects of the technical work done on homes and oversight are also determined by grantees. As one would expect, we found that some diagnostic tests and measures are more common in certain climate regions or for certain housing types. For example, pressure diagnostics and heating system inspections were almost universal in the colder regions, but performed on only about two-thirds of units in the hot-dry region. Grantee programs also vary by program size, housing stock, and other local factors and preferences.

\subsection{SUBGRANTEES}

Subgrantees are mostly non-profit organizations - some of which provide a full range of services to lowincome households in the local geographies they serve and some of which focus exclusively on weatherization. Our data collection from subgrantees comprised one form and one comprehensive survey requested of each subgrantee in PY08. In addition, we sampled 400 subgrantees for very extensive data collection that included a detailed program survey and requests for project-level details for a share of their weatherization jobs. 
Subgrantees are the face of the weatherization program for clients, providing the actual weatherization services, client education, and in many cases, program intake. Subgrantees vary widely in size, the geography they cover, the housing types they encounter, and the kinds of other services they provide to their clients. For example, some subgrantees employ only a very small number of people to serve a moderate number of houses and mobile homes in sparsely populated rural areas, while others employ or contract with a large number of weatherization professionals in dense urban areas to serve hundreds and even thousands of units in single- and multifamily buildings every year.

We found that subgrantees:

- received about $\$ 753$ million for weatherization work, including $\$ 677$ million from their grantees and $\$ 76$ million from other funding sources

- maintained an average of about ten employees (although staffing varies widely), comprised of about three FTE for management and administration, two FTE for auditing and inspections, three FTE for installation of weatherization measures, and one FTE for other duties

- followed varying funding models-16 percent of subgrantees rely exclusively on DOE funds, but 84 percent receive meaningful shares of their funding from non-DOE sources, and 49 percent include state, utility, or other local funds in their funding mix

- $\quad$ spent an average of $\$ 3,500$ on single-family homes and $\$ 3,200$ per unit on multifamily projects with materials accounting for slightly more than half of the project cost

- often used contractors to install weatherization measures - about 40 percent of subgrantees appear to have used contractors exclusively while nearly 30 percent used contractors for a substantial share of their installation work.

Diagnostic tests and weatherization measures installed vary somewhat by region, but some are fairly uniformly applied in the vast majority of weatherization projects, including:

- blower door tests (99\% of subgrantees apply this procedure)

- CO measurements in flues of space heating systems (91\%) and water heating systems (88\%)

- air sealing measures (performed in 94 percent of single-family homes, but a lesser share of multifamily buildings)

- insulation measures (82\% and 66\%)

- other baseloads (65\% and $81 \%)$.

Virtually all subgrantees also reported providing some level of client education - usually at multiple points in the weatherization process. Client education generally consisted of the distribution of literature and in-person communication when interacting with clients as part of the in-take or weatherization process.

\subsection{TRAINING}

Training receives substantial attention at both the national and state levels. Nine percent of DOE funds and three percent of non-DOE funds were allocated to training and technical assistance. Grantees retained slightly more than half of these funds to train their own staff and provide training and assistance to subgrantees, while passing along the rest to enable subgrantees to build training into their staff development.

From our inquiry into training topics, we found that:

- grantees use primarily the national weatherization program conference and regional weatherization conferences to develop their own staff

- $\quad$ subgrantees use primarily state weatherization conferences, the national weatherization program conference, and state or regional training center classes to develop their staff 
- subgrantee staff are trained most thoroughly in diagnostic procedures and in measures for singlefamily and mobile homes, but less well in health and safety topics and less consistently in multifamily measures

- $\quad$ state and regional training centers and internal training were rated most consistently as providing high quality training by both grantees and subgrantees.

\subsection{QUALITY CONTROL AND ASSURANCE}

Quality control and assurance occurs at both the grantee and subgrantee level. Subgrantees indicated that these inspections have a substantial positive effect on the quality of weatherization installations.

Subgrantees send an inspector to the home after weatherization crews have completed all measure installation. In PY08, post-weatherization inspections averaged five hours per home and most commonly included visual inspections of the installed measures (performed by $99 \%$ of subgrantees), blower door tests (95\%), and verification of insulation quantities (92\%) and operation of measures installed (89\%).

Grantees provide an additional level of quality review. In PY08, grantees inspected between 23 and 600 weatherization units, spending an average of two hours in the inspected homes. These inspections include many of the same activities as the subgrantee inspections.

Post-weatherization inspections by grantees found problems that warranted a return visit by the subgrantee in 21 percent of units inspected. This rate varied greatly among grantees, probably due to differences in either the nature of the inspections or the quality of the initial installations.

\subsection{OVERALL}

Overall, we can see that low-income weatherization is a simple concept built on a sophisticated technical approach that is delivered through a large and varied network of providers. The concept - to reduce the energy burden of low-income households through cost-effective efficiency improvements-led to a DOEfunded program in 1976 that has served several million households. The program has evolved since then, both technically and administratively. It was last evaluated and studied at a national level in 1989. This report — and the data gathering and analysis on which it is based — provide an updated picture of the way weatherization was provided in Program Year 2008. 
APPENDIX A: ALL STATES AGENCIES INFORMATION SURVEY 

OMB Control Number: 1910-5151

\section{DF 1: ALL STATES AGENCIES INFORMATION SURVEY}

Thank you for your prompt response to this data request which is part of the national evaluation of the Weatherization Assistance Program. Evaluation results will provide essential feedback to the weatherization community and inform policymakers about the program's effects on clients' energy consumption, cost savings, and non-energy benefits.

This survey collects data that will be used to compile a complete list of local agencies that provided weatherization services in Program Year 2008.

Thank you in advance for completing this survey.

Public reporting burden for this collection of information is estimated to average four hours per response, including the time for reviewing instructions, searching existing data sources, gathering and maintaining the data needed, and completing and reviewing the collection of information. Send comments regarding this burden estimate or any other aspect of this collection of information, including suggestions for reducing this burden, to Office of the Chief Information Officer, Records Management Division, IM-11, Paperwork Reduction Project (1910-5151), U.S. Department of Energy, 1000 Independence Ave SW, Washington, DC, 20585-1290; and to the Office of Management and Budget (OMB), OIRA, Paperwork Reduction Project (1910-5151), Washington, DC 20503. 
1. Please identify your state.

2. It is important to collect information about the weatherization of homes beyond the standard single family homes that are heated with natural gas or electricity. Please provide the following information about each of the local agencies (subgrantees) that you fund to provide weatherization services in your state:

\begin{tabular}{|c|c|c|c|c|c|c|}
\hline \multirow[b]{2}{*}{$\begin{array}{c}\text { Local } \\
\text { Agency } \\
\text { (Subgrantee) } \\
\text { Name }\end{array}$} & \multirow{2}{*}{$\begin{array}{l}\text { Amount of DOE } \\
\text { Funds Received by } \\
\text { Agency in Program } \\
\text { Year } 2008\end{array}$} & \multicolumn{5}{|c|}{$\begin{array}{l}\text { Indicate, by checking the appropriate boxes below, where a } \\
\text { specific local agency weatherized a substantial number of } \\
\text { dwelling units of a particular type in Program Year } 2008 \text {. }\end{array}$} \\
\hline & & $\begin{array}{l}\text { Large multi- } \\
\text { family }\end{array}$ & $\begin{array}{l}\text { Large multi- } \\
\text { family heated } \\
\text { with fuel oil }\end{array}$ & $\begin{array}{c}\text { Single- } \\
\text { family } \\
\text { heated with } \\
\text { fuel oil }\end{array}$ & $\begin{array}{l}\text { Single- } \\
\text { family } \\
\text { heated } \\
\text { with } \\
\text { propane }\end{array}$ & $\begin{array}{c}\text { Mobile } \\
\text { home } \\
\text { heated with } \\
\text { propane }\end{array}$ \\
\hline & $\begin{array}{l}\text { obs: } 885 \\
\text { missing: } 21 \\
\text { mean: } 237905.81 \\
\text { standard dev: } 276213.51 \\
\text { min: } 5670 \\
\text { 10th percentile: } 64372 \\
\text { 25th percentile: } 102656 \\
\text { median: } 180018 \\
\text { 75th percentile: } 306863 \\
\text { 90th percentile: } 438779 \\
\text { max: } 6009900\end{array}$ & $\begin{array}{l}\text { obs: } 890 \\
\text { yes: } 61 \\
\text { no: } 829\end{array}$ & $\begin{array}{l}\text { obs: } 890 \\
\text { yes: } 25 \\
\text { no: } 865\end{array}$ & $\begin{array}{l}\text { obs: } 890 \\
\text { yes: } 25 \\
\text { no: } 865\end{array}$ & $\begin{array}{l}\text { obs: } 890 \\
\text { yes: } 226 \\
\text { no: } 664\end{array}$ & $\begin{array}{l}\text { obs: } 890 \\
\text { yes: } 249 \\
\text { no: } 641\end{array}$ \\
\hline
\end{tabular}

The definition of substantial will vary from state to state. Please use your best judgment considering the total number of units weatherized and the composition of your housing stock. Include any agencies that tend to handle a large number of the kinds of units listed below, relative to your state's caseload and housing stock. If your state has few units of a particular type, you might not have any agencies with a substantial number of units.

For the purposes of this survey, Program Year 2008 is defined as the program year that includes the heating season spanning late 2008/early 2009.

A large multi-family is a building with five or more housing units (i.e., building that contains living quarters for five or more families or households).

A mobile home is built on a movable chassis, is moved to the site, and may be placed on a permanent or temporary foundation. If rooms are added to the structure, it is considered a mobile home if the added floor area is less than the mobile home's original floor area; otherwise, it is a single-family detached house. A manufactured house assembled on site is a single-family detached house, not a mobile home. 
3. Please provide the following information about each of the local agencies (subgrantees) that you fund to provide weatherization services in your state:

\begin{tabular}{|c|c|c|c|c|c|}
\hline \multirow[b]{2}{*}{$\begin{array}{c}\text { Local } \\
\text { Agency } \\
\text { (Subgrantee) } \\
\text { Name } \\
\end{array}$} & \multicolumn{5}{|c|}{$\begin{array}{c}\text { Indicate, by checking the appropriate boxes below, where you expect a specific local } \\
\text { agency to have the following characteristics. }\end{array}$} \\
\hline & $\begin{array}{c}\text { Average } \\
\text { energy } \\
\text { savings } \\
\text { substantially } \\
\text { higher than } \\
\text { the state } \\
\text { norm } \\
\end{array}$ & $\begin{array}{c}\text { Innovative } \\
\text { and/or } \\
\text { particularly } \\
\text { effective client } \\
\text { education } \\
\text { program } \\
\end{array}$ & $\begin{array}{c}\text { Innovative } \\
\text { and/or } \\
\text { particularly } \\
\text { effective } \\
\text { program for } \\
\text { training } \\
\text { weatherization } \\
\text { staff and/or } \\
\text { contractors } \\
\end{array}$ & $\begin{array}{c}\text { Innovative } \\
\text { and/or } \\
\text { particularly } \\
\text { effective } \\
\text { program for } \\
\text { inspecting } \\
\text { weatherized } \\
\text { units (Quality } \\
\text { Assurance) } \\
\end{array}$ & $\begin{array}{c}\text { Innovative } \\
\text { and/or large } \\
\text { program of } \\
\text { leveraging } \\
\text { DOE funds to } \\
\text { gain non-DOE } \\
\text { funds for } \\
\text { weatherization }\end{array}$ \\
\hline & $\begin{array}{l}\text { obs: } 889 \\
\text { yes: } 130 \\
\text { no: } 759\end{array}$ & $\begin{array}{l}\text { obs: } 889 \\
\text { yes: } 209 \\
\text { no: } 680\end{array}$ & $\begin{array}{l}\text { obs: } 889 \\
\text { yes: } 178 \\
\text { no: } 711\end{array}$ & $\begin{array}{l}\text { obs: } 889 \\
\text { yes: } 168 \\
\text { no: } 721\end{array}$ & $\begin{array}{l}\text { obs: } 889 \\
\text { yes: } 177 \\
\text { no: } 712\end{array}$ \\
\hline
\end{tabular}



APPENDIX B: ALL AGENCIES OVERVIEW DATA FORM 

OMB Control Number: 1910-5151

\section{DF 10: ALL AGENCIES OVERVIEW DATA FORM}

Thank you for your prompt response to this data request which is part of the national evaluation of the Weatherization Assistance Program. Evaluation results will provide essential feedback to the weatherization community and inform policymakers about the program's effects on clients' energy consumption, cost savings, and non-energy benefits.

This survey addresses audit processes, measure selection, client education, and training practices. The data you supply will also be used select a small number of agencies for special process evaluation studies.

All of the information obtained from this data form will be protected and will remain confidential. The data will be analyzed in such a way that the information provided cannot be associated back to your state, your agencies, or the housing units and clients that your state served. Again, please note that the questions refer to Program Year 2008 unless otherwise noted.

Thank you in advance for completing this survey.

Public reporting burden for this collection of information is estimated to average one hour per response, including the time for reviewing instructions, searching existing data sources, gathering and maintaining the data needed, and completing and reviewing the collection of information. Send comments regarding this burden estimate or any other aspect of this collection of information, including suggestions for reducing this burden, to Office of the Chief Information Officer, Records Management Division, IM-11, Paperwork Reduction Project (1910-5151), U.S. Department of Energy, 1000 Independence Ave SW, Washington, DC, 20585-1290; and to the Office of Management and Budget (OMB), OIRA, Paperwork Reduction Project (1910-5151), Washington, DC 20503. 
1. What is the primary method that your agency used in Program Year 2008 to select weatherization measures for clients' dwelling units (excluding health, safety, and repair measures and general heat waste measures)?

\begin{tabular}{|c|c|c|c|}
\hline & Freq. & Percent & Cum. \\
\hline Priority list | & 391 & 45.95 & 45.95 \\
\hline Calculation procedure | & 270 & 31.73 & 77.67 \\
\hline Priority list for some units, calc. pro| & 134 & 15.75 & 93.42 \\
\hline Other | & 56 & 6.58 & 100.00 \\
\hline Total & 851 & 100.00 & \\
\hline
\end{tabular}

2. If your agency used a calculation procedure for at least some dwelling units, what was the name of the procedure or procedures employed. Check all that apply.

AK Warm

$\begin{array}{crcc} & \text { Freq. } & \text { Percent } & \text { Cum. } \\ \text { No } \mid & 707 & 99.30 & 99.30 \\ \text { Yes } \mid & 5 & 0.70 & 100.00 \\ ---------- \\ \text { Total | } & 712 & 100.00 & \end{array}$

EA-3

$\begin{array}{cccc} & \text { Freq. } & \text { Percent } & \text { Cum. } \\ \text { No } \mid & 700 & 98.31 & 98.31 \\ \text { Yes } \mid & 12 & 1.69 & 100.00 \\ ---------- \\ \text { Total| } & 712 & 100.00\end{array}$

EASY

$\begin{array}{rccc} & \text { Freq. } & \text { Percent } & \text { Cum. } \\ \text { No | } & 688 & 96.63 & 96.63 \\ \text { Yes } \mid & 24 & 3.37 & 100.00 \\ ----------- \\ \text { Total | } & 712 & 100.00\end{array}$

_ EA-QUIP

\begin{tabular}{|c|c|c|c|}
\hline 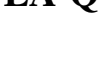 & Freq. & Percent & Cum. \\
\hline No & 673 & 94.52 & 94.52 \\
\hline Yes & 39 & 5.48 & 100.00 \\
\hline Total | & 712 & 100.00 & \\
\hline
\end{tabular}

HomeCheck 
Freq. Percent Cum.

\begin{tabular}{cccc} 
No $\mid$ & 701 & 98.46 & 98.46 \\
Yes $\mid$ & 11 & 1.54 & 100.00 \\
- \hdashline---+--------- & \\
Total & 712 & 100.00
\end{tabular}

\begin{tabular}{|c|c|c|c|}
\hline Mead & $\begin{array}{l}\text { S } \\
\text { Freq. }\end{array}$ & Percent & Cum. \\
\hline No & 705 & 99.02 & 99.02 \\
\hline Yes & 7 & 0.98 & 100.00 \\
\hline Total | & 712 & 100.00 & \\
\hline
\end{tabular}

$\begin{array}{cccc}\text { REES } & & \\ & \text { Freq. } & \text { Percent } & \text { Cum. } \\ \text {--------------------------------- } \\ \text { No | } 712 & 100.00 & 100.00 \\ --------------- & \end{array}$

\begin{tabular}{|c|c|c|c|}
\hline & Freq. & Percent & Cum. \\
\hline No & 679 & 95.37 & 95.37 \\
\hline Yes | & 33 & 4.63 & 100.00 \\
\hline Total & 712 & 100.00 & \\
\hline
\end{tabular}

_MOC-ERS

$\begin{array}{cccc} & \text { Freq. } & \text { Percent } & \text { Cum. } \\ \text { No | } & 701 & 98.46 & 98.46 \\ \text { Yes | } & 11 & 1.54 & 100.00 \\ ---------- & \end{array}$

$\begin{array}{cccc}\text { TIPS } & & & \\ & \text { Freq. } & \text { Percent } & \text { Cum. } \\ \text { No } \mid & 652 & 91.57 & 91.57 \\ \text { Yes } \mid & 60 & 8.43 & 100.00 \\ - \text { Total }\left.\right|^{-} 712 & 100.00 & \\ \text { TREAT } & & \\ & \text { Freq. } & \text { Percent } & \text { Cum. }\end{array}$




$\begin{array}{ccrc}\text { No } \mid & 673 & 94.52 & 94.52 \\ \text { Yes } \mid & 39 & 5.48 & 100.00 \\ ------------------- \\ \text { Total| } & 712 & 100.00\end{array}$

\begin{tabular}{|c|c|c|c|}
\hline & Freq. & Percent & Cum. \\
\hline No & 273 & 38.34 & 38.34 \\
\hline Yes & 439 & 61.66 & 100.00 \\
\hline Total | & 712 & 100.00 & \\
\hline
\end{tabular}

_ WXEOR

$\begin{array}{crrr} & \text { Freq. } & \text { Percent } & \text { Cum. } \\ \text { No | } & 706 & 99.16 & 99.16 \\ \text { Yes } \mid & 6 & 0.84 & 100.00 \\ -------------------------------- \\ \text { Total | } & 712 & 100.00 & \end{array}$

_ Other - Please specify: Freq. Percent Cum.

\begin{tabular}{|c|c|c|c|}
\hline No & 614 & 86.24 & 86.24 \\
\hline Yes & 98 & 13.76 & 100.00 \\
\hline Total | & 712 & 100.00 & \\
\hline
\end{tabular}

3. Which of the following client education approaches did your agency use in Program Year 2008? Check all that apply.

\section{At time of client intake}

_ Provide literature at time of client intake

$\begin{array}{cccc} & \text { Freq. } & \text { Percent } & \text { Cum. } \\ \text { No } \mid & 408 & 47.94 & 47.94 \\ \text { Yes } \mid & 443 & 52.06 & 100.00 \\ ------- & \\ \text { Total } \mid & 851 & 100.00 & \end{array}$


_ Provide video, CD, or DVD at time of client intake Freq. Percent Cum.

\begin{tabular}{|c|c|c|c|}
\hline No & 815 & 95.77 & 95.77 \\
\hline Yes & 36 & 4.23 & 100.00 \\
\hline
\end{tabular}

Provide hardware kit at time of client intake Freq. Percent Cum.

\begin{tabular}{|c|c|c|c|}
\hline No & 831 & 97.65 & 97.65 \\
\hline Yes & 20 & 2.35 & 100.00 \\
\hline Total | & 851 & 100.00 & \\
\hline
\end{tabular}

Provide in-person instruction at time of client intake Freq. Percent Cum.

$\begin{array}{crrr}\text { No } \mid & 558 & 65.57 & 65.57 \\ \text { Yes } \mid & 293 & 34.43 & 100.00 \\ ---------------- \\ \text { Total } & 851 & 100.00\end{array}$

\section{At time of audit}

Provide literature at time of audit Freq. Percent Cum.

$\begin{array}{cccc}\text { No | } & 288 & 33.84 & 33.84 \\ \text { Yes | } & 563 & 66.16 & 100.00 \\ ------------- \\ \text { Total } & 851 & 100.00\end{array}$

_ Provide video, CD, or DVD at time of audit Freq. Percent Cum.

\begin{tabular}{|c|c|c|c|}
\hline No & 828 & 97.30 & 97.30 \\
\hline Yes & 23 & 2.70 & 100.00 \\
\hline Total & 851 & 100.00 & \\
\hline
\end{tabular}


_ Provide hardware kit at time of audit

$\begin{array}{cccc} & \text { Freq. } & \text { Percent } & \text { Cum. } \\ \text { No } \mid & 797 & 93.65 & 93.65 \\ \text { Yes } \mid & 54 & 6.35 & 100.00 \\ ------+--------- \\ \text { Total } & 851 & 100.00\end{array}$

_ Provide in-person instruction at time of audit

$\begin{array}{cccc} & \text { Freq. } & \text { Percent } & \text { Cum. } \\ \text { No } \mid & 214 & 25.15 & 25.15 \\ \text { Yes } \mid & 637 & 74.85 & 100.00 \\ ------------- \\ \text { Total | } & 851 & 100.00 & \end{array}$

\section{At time of weatherization}

_ Provide literature at time of weatherization

$\begin{array}{rrrr} & \text { Freq. } & \text { Percent } & \text { Cum. } \\ \text { No } \mid & 684 & 80.38 & 80.38 \\ \text { Yes } \mid & 167 & 19.62 & 100.00 \\ -------- & \\ \text { Total } & 851 & 100.00 & \end{array}$

_ Provide video, CD, or DVD at time of weatherization

$\begin{array}{cccc} & \text { Freq. } & \text { Percent } & \text { Cum. } \\ \text { No } \mid & 846 & 99.41 & 99.41 \\ \text { Yes } \mid & 5 & 0.59 & 100.00 \\ ------- & \end{array}$

Provide hardware kit at time of weatherization

$\begin{array}{cccc} & \text { Freq. } & \text { Percent } & \text { Cum. } \\ \text { No } \mid & 803 & 94.36 & 94.36 \\ \text { Yes } \mid & 48 & 5.64 & 100.00 \\ ------+----- & \end{array}$


_ Provide in-person instruction at time of weatherization

$\begin{array}{cccc} & \text { Freq. } & \text { Percent } & \text { Cum. } \\ \text { No } \mid & 252 & 29.61 & 29.61 \\ \text { Yes } \mid & 599 & 70.39 & 100.00 \\ ------ & \\ \text { Total } \mid & 851 & 100.00 & \end{array}$

\section{At separate client education visit}

_ Provide literature at separate client education visit

\begin{tabular}{|c|c|c|c|}
\hline & Freq. & Percent & Cum. \\
\hline No & 695 & 81.67 & 81.67 \\
\hline Yes & 156 & 18.33 & 100.00 \\
\hline Total & 851 & 100.00 & \\
\hline
\end{tabular}

_ Provide video, CD, or DVD at separate client education visit

$\begin{array}{rrrr} & \text { Freq. } & \text { Percent } & \text { Cum. } \\ \text { No } \mid & 833 & 97.88 & 97.88 \\ \text { Yes } \mid & 18 & 2.12 & 100.00 \\ ------- & \end{array}$

Provide hardware kit at separate client education visit

$\begin{array}{cccc} & \text { Freq. } & \text { Percent } & \text { Cum. } \\ \text { No } \mid & 810 & 95.18 & 95.18 \\ \text { Yes } \mid & 41 & 4.82 & 100.00 \\ --------------- \\ \text { Total | } & 851 & 100.00 & \end{array}$

Provide in-person instruction at separate client education visit

$\begin{array}{cccc} & \text { Freq. } & \text { Percent } & \text { Cum. } \\ \text { No } \mid & 649 & 76.26 & 76.26 \\ \text { Yes } \mid & 202 & 23.74 & 100.00 \\ ------------- \\ \text { Total| } & 851 & 100.00 & \end{array}$




\section{At time of inspection}

_ Provide literature at time of inspection

$\begin{array}{cccc} & \text { Freq. } & \text { Percent } & \text { Cum. } \\ \text { No } \mid & 607 & 71.33 & 71.33 \\ \text { Yes } \mid & 244 & 28.67 & 100.00 \\ ------- & \end{array}$

Provide video, CD, or DVD at time of inspection Freq. Percent Cum.

\begin{tabular}{|c|c|c|c|}
\hline No & 849 & 99.76 & 99.76 \\
\hline Yes | & 2 & 0.24 & 100.00 \\
\hline Total | & 851 & 100.00 & \\
\hline
\end{tabular}

Provide hardware kit at time of inspection

$\begin{array}{crrr} & \text { Freq. } & \text { Percent } & \text { Cum. } \\ - \text { No } \mid & 827 & 97.18 & 97.18 \\ \text { Yes | } & 24 & 2.82 & 100.00 \\ ------ & \end{array}$

Provide in-person instruction at time of inspection

$\begin{array}{cccc} & \text { Freq. } & \text { Percent } & \text { Cum. } \\ \text { No } \mid & 204 & 23.97 & 23.97 \\ \text { Yes } \mid & 647 & 76.03 & 100.00 \\ ------- & \\ \text { Total } \mid & 851 & 100.00 & \end{array}$

\section{Other approaches}

_ Group training class

\begin{tabular}{|c|c|c|c|}
\hline & Freq. & Percent & Cum. \\
\hline No & 743 & 87.31 & 87.31 \\
\hline Yes & 108 & 12.69 & 100.00 \\
\hline Total | & 851 & 100.00 & \\
\hline
\end{tabular}


Other - Please specify:

Freq. Percent Cum.

$\begin{array}{cccc}\text { No } \mid & 765 & 89.89 & 89.89 \\ \text { Yes } & 86 & 10.11 & 100.00 \\ ------------- & \\ \text { Total } & 851 & 100.00\end{array}$

4. From which of the following sources did your agency obtain needed skills and/or information in Program Year 2008? Check all that apply.

__ National Weatherization Program Conference

$\begin{array}{cccc} & \text { Freq. } & \text { Percent } & \text { Cum. } \\ \text { No | } & 440 & 51.70 & 51.70 \\ \text { Yes | } & 411 & 48.30 & 100.00 \\ ------ & \end{array}$

__ Affordable Comfort Conference

$\begin{array}{cccc} & \text { Freq. } & \text { Percent } & \text { Cum. } \\ \text { No | } & 599 & 70.39 & 70.39 \\ \text { Yes | } & 252 & 29.61 & 100.00 \\ -------- & \end{array}$

_ National Community Action Foundation (NCAF) Conference Freq. Percent Cum.

\begin{tabular}{|c|c|c|c|}
\hline No & 702 & 82.49 & 82.49 \\
\hline Yes & 149 & 17.51 & 100.00 \\
\hline Total | & 851 & 100.00 & \\
\hline
\end{tabular}

National Association for State Community Services Programs (NASCSP) Energy Leveraging Conference Freq. Percent Cum.

\begin{tabular}{|c|c|c|c|}
\hline No & 795 & 93.42 & 93.42 \\
\hline Yes | & 56 & 6.58 & 100.00 \\
\hline Total & 851 & 100.00 & \\
\hline
\end{tabular}


Other national conference

Freq. Percent Cum.

\begin{tabular}{|c|c|c|c|}
\hline No & 796 & 93.54 & 93.54 \\
\hline Yes & 55 & 6.46 & 100.00 \\
\hline Total | & 851 & 100.00 & \\
\hline
\end{tabular}

Regional weatherization conference

\begin{tabular}{|c|c|c|c|}
\hline & Freq. & Percent & Cum. \\
\hline No & 515 & 60.52 & 60.52 \\
\hline Yes & 336 & 39.48 & 100.00 \\
\hline Total | & 851 & 100.00 & \\
\hline
\end{tabular}

_ State weatherization conference

$\begin{array}{cccc} & \text { Freq. } & \text { Percent } & \text { Cum. } \\ \text { No | } & 337 & 39.60 & 39.60 \\ \text { Yes | } & 514 & 60.40 & 100.00 \\ ------- & \end{array}$

Other state conference

Freq. Percent Cum.

\begin{tabular}{|c|c|c|c|}
\hline No & 704 & 82.73 & 82.73 \\
\hline Yes & 147 & 17.27 & 100.00 \\
\hline Total | & 851 & 100.00 & \\
\hline
\end{tabular}

_ State/regional training center class

$\begin{array}{cccc} & \text { Freq. } & \text { Percent } & \text { Cum. } \\ \text { No } \mid & 432 & 50.76 & 50.76 \\ \text { Yes } \mid & 419 & 49.24 & 100.00 \\ ------- & \\ \text { Total | } & 851 & 100.00 & \end{array}$


Manufacturer's training school class

Freq. Percent Cum.

\begin{tabular}{|c|c|c|c|}
\hline No & 738 & 86.72 & 86.72 \\
\hline Yes & 113 & 13.28 & 100.00 \\
\hline Total & 851 & 100.00 & \\
\hline
\end{tabular}

_ Utility training class

$\begin{array}{cccc} & \text { Freq. } & \text { Percent } & \text { Cum. } \\ \text { No } \mid & 720 & 84.61 & 84.61 \\ \text { Yes } \mid & 131 & 15.39 & 100.00 \\ ---------- \\ \text { Total | } & 851 & 100.00 & \end{array}$

State sponsored class taught at central location (e.g., local agency, state office)

$\begin{array}{cccc} & \text { Freq. } & \text { Percent } & \text { Cum. } \\ \text { No } \mid & 310 & 36.43 & 36.43 \\ \text { Yes } \mid & 541 & 63.57 & 100.00 \\ --------- & \\ \text { Total } & 851 & 100.00 & \end{array}$

Class not sponsored by state (e.g., another state, trade organization)

$\begin{array}{cccc} & \text { Freq. } & \text { Percent } & \text { Cum. } \\ \text { No } \mid & 713 & 83.78 & 83.78 \\ \text { Yes } \mid & 138 & 16.22 & 100.00 \\ ------- & \end{array}$

Visiting another agency

$\begin{array}{cccc} & \text { Freq. } & \text { Percent } & \text { Cum. } \\ \text { No } \mid & 635 & 74.62 & 74.62 \\ \text { Yes } \mid & 216 & 25.38 & 100.00 \\ ------------- \\ \text { Total | } & 851 & 100.00 & \end{array}$


Instruction received by just your agency during a visit by state staff

$\begin{array}{cccc} & \text { Freq. } & \text { Percent } & \text { Cum. } \\ \text { No } \mid & 381 & 44.77 & 44.77 \\ \text { Yes } \mid & 470 & 55.23 & 100.00 \\ ---------------- \\ \text { Total| }\end{array}$

__ In-person expert visit to your agency (e.g., peer exchange, consultant)

\begin{tabular}{|c|c|c|c|}
\hline & Freq. & Percent & Cum. \\
\hline No & 643 & 75.56 & 75.56 \\
\hline Yes & 208 & 24.44 & 100.00 \\
\hline Total & 851 & 100.00 & \\
\hline
\end{tabular}

\begin{tabular}{|c|c|c|c|}
\hline web & Freq. & Percent & Cum. \\
\hline No & 707 & 83.08 & 83.08 \\
\hline Yes & 144 & 16.92 & 100.00 \\
\hline Total | & 851 & 100.00 & \\
\hline
\end{tabular}

_ Conference call

\begin{tabular}{|c|c|c|c|}
\hline & Freq. & Percent & Cum. \\
\hline No & 579 & 68.04 & 68.04 \\
\hline Yes & 272 & 31.96 & 100.00 \\
\hline Total | & 851 & 100.00 & \\
\hline
\end{tabular}

Phone call from expert

Freq. Percent Cum.

\begin{tabular}{|c|c|c|c|}
\hline No & 621 & 72.97 & 72.97 \\
\hline Yes & 230 & 27.03 & 100.00 \\
\hline Total | & 851 & 100.00 & \\
\hline
\end{tabular}


E-mail from expert

$\begin{array}{cccc} & \text { Freq. } & \text { Percent } & \text { Cum. } \\ \text { No } \mid & 575 & 67.57 & 67.57 \\ \text { Yes } \mid & 276 & 32.43 & 100.00 \\ ------+--------- \\ \text { Total } & 851 & 100.00 & \end{array}$

__ Other (please specify)

$\begin{array}{cccc} & \text { Freq. } & \text { Percent } & \text { Cum. } \\ \text { No } \mid & 774 & 90.95 & 90.95 \\ \text { Yes } \mid & 77 & 9.05 & 100.00 \\ ----------- & \\ \text { Total | } & 851 & 100.00\end{array}$

5. Which of the following types of post-weatherization quality control inspection did your agency perform in Program Year 2008? Check all that apply.

_ Visual inspection of installed measures

\begin{tabular}{crrr} 
& Freq. & Percent & \multicolumn{1}{c}{ Cum. } \\
No | & 13 & 1.53 & 1.53 \\
Yes $\mid$ & 838 & 98.47 & 100.00 \\
---------- \\
Total| & 851 & 100.00 &
\end{tabular}

_ Verification of insulation depths/quantities

$\begin{array}{crrr} & \text { Freq. } & \text { Percent } & \text { Cum. } \\ \text { No } \mid & 80 & 9.40 & 9.40 \\ \text { Yes } \mid & 771 & 90.60 & 100.00 \\ ------- & \end{array}$

_ Verification of operation of measures installed

$\begin{array}{cccc} & \text { Freq. } & \text { Percent } & \text { Cum. } \\ \text { No } \mid & 120 & 14.10 & 14.10 \\ \text { Yes | } & 731 & 85.90 & 100.00 \\ ------ & \end{array}$


Assessment of quality of measures installed

Freq. Percent Cum.

$\begin{array}{cccc}\text { No } \mid & 103 & 12.10 & 12.10 \\ \text { Yes } \mid & 748 & 87.90 & 100.00 \\ - & & \\ \text { Total } \mid & 851 & 100.00\end{array}$

_ Identification of needed measures that were not installed

$\begin{array}{cccc} & \text { Freq. } & \text { Percent } & \text { Cum. } \\ \text { No } \mid & 286 & 33.61 & 33.61 \\ \text { Yes } \mid & 565 & 66.39 & 100.00 \\ ------ & \end{array}$

\begin{tabular}{|c|c|c|c|}
\hline & Freq. & Percent & Cum. \\
\hline No & 55 & 6.46 & 6.46 \\
\hline Yes | & 796 & 93.54 & 100.00 \\
\hline Total & 851 & 100.00 & \\
\hline
\end{tabular}

_ Heating system efficiency test (flue gas analysis)

$\begin{array}{cccc} & \text { Freq. } & \text { Percent } & \text { Cum. } \\ \text { No } \mid & 211 & 24.79 & 24.79 \\ \text { Yes } \mid & 640 & 75.21 & 100.00 \\ -------------- \\ \text { Total| } & 851 & 100.00 & \end{array}$

_ Draft/spillage tests of heating systems Freq. Percent Cum.

\begin{tabular}{|c|c|c|c|}
\hline No & 246 & 28.91 & 28.91 \\
\hline Yes & 605 & 71.09 & 100.00 \\
\hline Total | & 851 & 100.00 & \\
\hline
\end{tabular}


Carbon monoxide (CO) monitoring Freq. Percent Cum.

$\begin{array}{cccc}\text { No } \mid & 107 & 12.57 & 12.57 \\ \text { Yes } \mid & 744 & 87.43 & 100.00 \\ ------------ & \\ \text { Total } & 851 & 100.00\end{array}$

__ Infrared scanning

$\begin{array}{cccc} & \text { Freq. } & \text { Percent } & \text { Cum. } \\ \text { No } \mid & 490 & 57.58 & 57.58 \\ \text { Yes } \mid & 361 & 42.42 & 100.00 \\ ------- & \\ \text { Total | } & 851 & 100.00 & \end{array}$

_ Identification of unresolved health and safety issues Freq. Percent Cum.

\begin{tabular}{cccc} 
No $\mid$ & 296 & 34.78 & 34.78 \\
Yes $\mid$ & 555 & 65.22 & 100.00 \\
- \hdashline---------- \\
Total & 851 & 100.00
\end{tabular}

_ Discussion with occupants

$\begin{array}{crrr} & \text { Freq. } & \text { Percent } & \text { Cum. } \\ \text { No } \mid & 88 & 10.34 & 10.34 \\ \text { Yes } \mid & 763 & 89.66 & 100.00 \\ ----------- \\ \text { Total| } & 851 & 100.00 & \end{array}$

__ Use of customer sign-off form

$\begin{array}{cccc} & \text { Freq. } & \text { Percent } & \text { Cum. } \\ \text { No } \mid & 95 & 11.16 & 11.16 \\ \text { Yes } \mid & 756 & 88.84 & 100.00 \\ -------- & \\ \text { Total | } & 851 & 100.00 & \end{array}$


Other (specify)

$\begin{array}{cccc} & \text { Freq. } & \text { Percent } & \text { Cum. } \\ \text { No } \mid & 800 & 94.01 & 94.01 \\ \text { Yes } \mid & 51 & 5.99 & 100.00 \\ ------+------------------ & \end{array}$

B-18 
APPENDIX C: ALL STATES PROGRAM INFORMATION SURVEY 

OMB Control Number: 1910-5151

\section{S1: ALL STATES PROGRAM INFORMATION SURVEY}

Thank you for your prompt response to this data request which is part of the national evaluation of the Weatherization Assistance Program. Evaluation results will provide essential feedback to the weatherization community and inform policymakers about the program's effects on clients' energy consumption, cost savings and non-energy benefits.

This survey collects data that will be used to characterize Weatherization Assistance Program activities at the state level during Program Year 2008.

All of the information obtained from this survey will be protected and will remain confidential. The data will be analyzed in such a way that the information provided cannot be associated back to your state, your agencies, or the housing units and clients that your state served. Again, please note that the questions refer to PY 2008 unless otherwise noted.

Thanks you in advance for completing this survey.

Public reporting burden for this collection of information is estimated to average sixteen hours per response, including the time for reviewing instructions, searching existing data sources, gathering and maintaining the data needed, and completing and reviewing the collection of information. Send comments regarding this burden estimate or any other aspect of this collection of information, including suggestions for reducing this burden, to Office of the Chief Information Officer, Records Management Division, IM-11, Paperwork Reduction Project (1910-5151), U.S. Department of Energy, 1000 Independence Ave SW, Washington, DC, 20585-1290; and to the Office of Management and Budget (OMB), OIRA, Paperwork Reduction Project (1910-5151), Washington, DC 20503. 


\section{Section A: PROGRAM CHARACTERIZATION}

1. Please identify your state:

2. How many layers of government (i.e., positions on an organization chart) sat between the governor's office in your state and your weatherization program in Program Year 2008?

For the purposes of this survey, Program Year 2008 is defined as the program year that includes the heating season spanning late 2008/early 2009.

3. During Program Year 2008, was the director of your state's weatherization program (Check best answer):

a civil servant political appointee elected official

$\begin{array}{rrrr} & \text { Freq. } & \text { Percent } & \text { Cum. } \\ \text { political appointee | } & 90 & 81.63 & 81.63 \\ \text { a civil servant } & 9 & 18.37 & 100.00 \\ \text { Total | } & 49 & 100.00 & \end{array}$

4. During Program Year 2008, did the director of your state's weatherization program report to a (Check best answer): civil servant political appointee elected official

\begin{tabular}{rrrr} 
& Freq. & Percent & Cum. \\
\hline a civil servant $\mid$ & 25 & 51.02 & 51.02 \\
political appointee | & 23 & 46.94 & 97.96 \\
elected official $\mid$ & 1 & 2.04 & 100.00 \\
-------------- & \\
Total $\mid$ & 49 & 100.00 &
\end{tabular}


5. For how many years had the current director of your sate's weatherization program served in that capacity prior to Program Year 2008?

\begin{tabular}{|r|r|}
\hline observations: & 50 \\
\hline missing values: & 1 \\
\hline mean: & 8.23 \\
\hline standard deviation: & 8.47 \\
\hline min: & 0 \\
\hline 10th percentile: & 0 \\
\hline 25th percentile: & 1 \\
\hline median: & 6 \\
\hline 75th percentile: & 14 \\
\hline 90th percentile: & 21 \\
\hline max: & 30 \\
\hline
\end{tabular}

6. How many different directors (including the Program Year 2008 director) led your state's weatherization program over the 10 years prior to Program Year 2008?

\begin{tabular}{|r|r|}
\hline observations: & 49 \\
\hline missing values: & 2 \\
\hline mean: & 2.14 \\
\hline standard deviation: & 1.49 \\
\hline min: & 0 \\
\hline 10th percentile: & 1 \\
\hline 25th percentile: & 1 \\
\hline median: & 2 \\
\hline 75th percentile: & 3 \\
\hline 90th percentile: & 4 \\
\hline max: & 7 \\
\hline
\end{tabular}

7. Did your state's weatherization program set annual performance goals of Program Year 2008?

\begin{tabular}{|c|c|c|c|}
\hline \multicolumn{3}{|c|}{$\begin{array}{l}\text { Yes } \\
\text { - No (go to Question 8) }\end{array}$} & \multirow[b]{2}{*}{ Cum. } \\
\hline & Freq. & Percent & \\
\hline no & 7 & 14.00 & 14.00 \\
\hline yes & 43 & 86.00 & 100.00 \\
\hline Total| & 50 & 100.00 & \\
\hline
\end{tabular}


7a. What agency, office, or department was responsible for reviewing the annual performance goals and achievement of goals of your state's weatherization program?

\section{This question refers to a state-level office, agency or department.}

8. Please list other important housing and/or energy-related programs for low-income residents that were administered by the same office that is in charge of your state's weatherization program.

10. For each of the data categories listed, indicate the format in which agencies were supposed to provide the data in Program Year 2008 by checking the appropriate box in the following table:

\begin{tabular}{|c|c|c|c|c|c|}
\hline \multirow[b]{2}{*}{ Data Category } & \multicolumn{5}{|c|}{ Data Format } \\
\hline & $\begin{array}{l}\text { Paper } \\
\text { hardcopy }\end{array}$ & $\begin{array}{l}\text { Excel files or } \\
\text { other } \\
\text { computerized } \\
\text { spreadsheet files }\end{array}$ & $\begin{array}{l}\text { Microsoft Access or } \\
\text { other computerized } \\
\text { database files }\end{array}$ & $\begin{array}{l}\text { Microsoft Word or } \\
\text { other computerized } \\
\text { word processing } \\
\text { files }\end{array}$ & Not Required \\
\hline $\begin{array}{l}\text { Number of homes } \\
\text { weatherized }\end{array}$ & $\begin{array}{l}\text { obs: } 50 \\
\text { no: } 28 \\
\text { yes: } 22 \\
\end{array}$ & $\begin{array}{l}\text { obs: } 50 \\
\text { no: } 31 \\
\text { yes: } 19\end{array}$ & $\begin{array}{l}\text { obs: } 50 \\
\text { no: } 24 \\
\text { yes: } 26\end{array}$ & $\begin{array}{l}\text { obs: } 50 \\
\text { no: } 49 \\
\text { yes: } 1\end{array}$ & $\begin{array}{l}\text { obs: } 50 \\
\text { no: } 50 \\
\text { yes: } 0 \\
\end{array}$ \\
\hline $\begin{array}{l}\text { Number of homes } \\
\text { weatherized in high } \\
\text { priority categories }\end{array}$ & $\begin{array}{l}\text { obs: } 48 \\
\text { no: } 28 \\
\text { yes: } 20\end{array}$ & $\begin{array}{l}\text { obs: } 48 \\
\text { no: } 31 \\
\text { yes: } 17\end{array}$ & $\begin{array}{l}\text { obs: } 48 \\
\text { no: } 26 \\
\text { yes: } 22 \\
\end{array}$ & $\begin{array}{l}\text { obs: } 48 \\
\text { no: } 46 \\
\text { yes: } 2\end{array}$ & $\begin{array}{l}\text { obs: } 48 \\
\text { no: } 43 \\
\text { yes: } 5\end{array}$ \\
\hline $\begin{array}{l}\text { DOE weatherization } \\
\text { funds expended }\end{array}$ & $\begin{array}{l}\text { obs: } 48 \\
\text { no: } 43 \\
\text { yes: } 5\end{array}$ & $\begin{array}{l}\text { obs: } 50 \\
\text { no: } 27 \\
\text { yes: } 23\end{array}$ & $\begin{array}{l}\text { obs: } 50 \\
\text { no: } 28 \\
\text { yes: } 22\end{array}$ & $\begin{array}{l}\text { obs: } 50 \\
\text { no: } 28 \\
\text { yes: } 22\end{array}$ & $\begin{array}{l}\text { obs: } 50 \\
\text { no: } 0 \\
\text { yes: } 50\end{array}$ \\
\hline $\begin{array}{l}\text { Non-DOE } \\
\text { weatherization funds } \\
\text { expended }\end{array}$ & $\begin{array}{l}\text { obs: } 50 \\
\text { no: } 46 \\
\text { yes: } 4\end{array}$ & $\begin{array}{l}\text { obs: } 50 \\
\text { no: } 50 \\
\text { yes: } 0\end{array}$ & $\begin{array}{l}\text { obs: } 47 \\
\text { no: } 29 \\
\text { yes: } 18\end{array}$ & $\begin{array}{l}\text { obs: } 47 \\
\text { no: } 29 \\
\text { yes: } 18\end{array}$ & $\begin{array}{l}\text { obs: } 47 \\
\text { no: } 42 \\
\text { yes: } 5\end{array}$ \\
\hline $\begin{array}{l}\text { Descriptive statistics: } \\
\text { demographics } \\
\text { weatherized homes }\end{array}$ & $\begin{array}{l}\text { obs: } 50 \\
\text { no: } 30 \\
\text { yes: } 20\end{array}$ & $\begin{array}{l}\text { obs: } 50 \\
\text { no: } 34 \\
\text { yes: } 16\end{array}$ & $\begin{array}{l}\text { obs: } 50 \\
\text { no: } 24 \\
\text { yes: } 26\end{array}$ & $\begin{array}{l}\text { obs: } 50 \\
\text { no: } 49 \\
\text { yes: } 1\end{array}$ & $\begin{array}{l}\text { obs: } 50 \\
\text { no: } 47 \\
\text { yes: } 3\end{array}$ \\
\hline $\begin{array}{l}\text { Descriptive statistics: } \\
\text { weatherization } \\
\text { measures installed }\end{array}$ & $\begin{array}{l}\text { obs: } 46 \\
\text { no: } 29 \\
\text { yes: } 17\end{array}$ & $\begin{array}{l}\text { obs: } 46 \\
\text { no: } 34 \\
\text { yes: } 12\end{array}$ & $\begin{array}{l}\text { obs: } 46 \\
\text { no: } 22 \\
\text { yes: } 24\end{array}$ & $\begin{array}{l}\text { obs: } 46 \\
\text { no: } 45 \\
\text { yes: } 1\end{array}$ & $\begin{array}{l}\text { obs: } 46 \\
\text { no: } 40 \\
\text { yes: } 6\end{array}$ \\
\hline $\begin{array}{l}\text { Descriptive statistics: } \\
\text { energy use and } \\
\text { savings in } \\
\text { weatherized homes }\end{array}$ & $\begin{array}{l}\text { obs: } 43 \\
\text { no: } 29 \\
\text { yes: } 14\end{array}$ & $\begin{array}{l}\text { obs: } 43 \\
\text { no: } 36 \\
\text { yes: } 7\end{array}$ & $\begin{array}{l}\text { obs: } 43 \\
\text { no: } 26 \\
\text { yes: } 17\end{array}$ & $\begin{array}{l}\text { obs: } 43 \\
\text { no: } 42 \\
\text { yes: } 1\end{array}$ & $\begin{array}{l}\text { obs: } 43 \\
\text { no: } 28 \\
\text { yes: } 15\end{array}$ \\
\hline $\begin{array}{l}\text { Copies of audits } \\
\text { performed on } \\
\text { weatherized homes }\end{array}$ & $\begin{array}{l}\text { obs: } 42 \\
\text { no: } 16 \\
\text { yes: } 26\end{array}$ & $\begin{array}{l}\text { obs: } 42 \\
\text { no: } 38 \\
\text { yes: } 4\end{array}$ & $\begin{array}{l}\text { obs: } 42 \\
\text { no: } 32 \\
\text { yes: } 10\end{array}$ & $\begin{array}{l}\text { obs: } 42 \\
\text { no: } 39 \\
\text { yes: } 3\end{array}$ & $\begin{array}{l}\text { obs: } 42 \\
\text { no: } 30 \\
\text { yes: } 12\end{array}$ \\
\hline $\begin{array}{l}\text { Results of certain } \\
\text { diagnostic tests }\end{array}$ & $\begin{array}{l}\text { obs: } 42 \\
\text { no: } 15 \\
\text { yes: } 27\end{array}$ & $\begin{array}{l}\text { obs: } 42 \\
\text { no: } 36 \\
\text { yes: } 6\end{array}$ & $\begin{array}{l}\text { obs: } 42 \\
\text { no: } 25 \\
\text { yes: } 17\end{array}$ & $\begin{array}{l}\text { obs: } 42 \\
\text { no: } 41 \\
\text { yes: } 1\end{array}$ & $\begin{array}{l}\text { obs: } 42 \\
\text { no: } 37 \\
\text { yes: } 5\end{array}$ \\
\hline Other & $\begin{array}{l}\text { obs: } 5 \\
\text { no: } 3 \\
\text { yes: } 2\end{array}$ & $\begin{array}{l}\text { obs: } 5 \\
\text { no: } 5 \\
\text { yes: } 0\end{array}$ & $\begin{array}{l}\text { obs: } 5 \\
\text { no: } 4 \\
\text { yes: } 1\end{array}$ & $\begin{array}{l}\text { obs: } 5 \\
\text { no: } 5 \\
\text { yes: } 0\end{array}$ & $\begin{array}{l}\text { obs: } 5 \\
\text { no: } 3 \\
\text { yes: } 2\end{array}$ \\
\hline
\end{tabular}


11. How frequently were the agencies supposed to provide the data in Program Year 2008? Please check the appropriate boxes. Please leave blank for data categories not reported.

\begin{tabular}{|c|c|c|c|c|c|}
\hline \multirow[b]{2}{*}{ Data Category } & \multicolumn{5}{|c|}{ Reporting Frequency } \\
\hline & Weekly & Monthly & Quarterly & Annually & Other \\
\hline $\begin{array}{l}\text { Number of homes } \\
\text { weatherized }\end{array}$ & $\begin{array}{l}\text { obs: } 50 \\
\text { no: } 50 \\
\text { yes: } 0 \\
\end{array}$ & $\begin{array}{l}\text { obs: } 50 \\
\text { no: } 11 \\
\text { yes: } 39 \\
\end{array}$ & $\begin{array}{l}\text { obs: } 50 \\
\text { no: } 39 \\
\text { yes: } 11\end{array}$ & $\begin{array}{l}\text { obs: } 50 \\
\text { no: } 44 \\
\text { yes: } 6\end{array}$ & $\begin{array}{l}\text { obs: } 50 \\
\text { no: } 46 \\
\text { yes: } 4\end{array}$ \\
\hline $\begin{array}{l}\text { Number of homes } \\
\text { weatherized in high priority } \\
\text { categories }\end{array}$ & $\begin{array}{l}\text { obs: } 42 \\
\text { no: } 42 \\
\text { yes: } 0\end{array}$ & $\begin{array}{l}\text { obs: } 42 \\
\text { no: } 11 \\
\text { yes: } 31\end{array}$ & $\begin{array}{l}\text { obs: } 42 \\
\text { no: } 32 \\
\text { yes: } 10\end{array}$ & $\begin{array}{l}\text { obs: } 42 \\
\text { no: } 37 \\
\text { yes: } 5\end{array}$ & $\begin{array}{l}\text { obs: } 42 \\
\text { no: } 38 \\
\text { yes: } 4\end{array}$ \\
\hline $\begin{array}{l}\text { DOE weatherization funds } \\
\text { expended }\end{array}$ & $\begin{array}{l}\text { obs: } 49 \\
\text { no: } 49 \\
\text { yes: } 0\end{array}$ & $\begin{array}{l}\text { obs: } 49 \\
\text { no: } 8 \\
\text { yes: } 41\end{array}$ & $\begin{array}{l}\text { obs: } 49 \\
\text { no: } 39 \\
\text { yes: } 10 \\
\end{array}$ & $\begin{array}{l}\text { obs: } 49 \\
\text { no: } 42 \\
\text { yes: } 7\end{array}$ & $\begin{array}{l}\text { obs: } 49 \\
\text { no: } 46 \\
\text { yes: } 3 \\
\end{array}$ \\
\hline $\begin{array}{l}\text { Non-DOE weatherization } \\
\text { funds expended }\end{array}$ & $\begin{array}{l}\text { obs: } 39 \\
\text { no: } 39 \\
\text { yes: } 0\end{array}$ & $\begin{array}{l}\text { obs: } 39 \\
\text { no: } 6 \\
\text { yes: } 33\end{array}$ & $\begin{array}{l}\text { obs: } 39 \\
\text { no: } 30 \\
\text { yes: } 9\end{array}$ & $\begin{array}{l}\text { obs: } 32 \\
\text { no: } 7 \\
\text { yes: } 39\end{array}$ & $\begin{array}{l}\text { obs: } 39 \\
\text { no: } 36 \\
\text { yes: } 3\end{array}$ \\
\hline $\begin{array}{l}\text { Descriptive statistics: } \\
\text { demographics weatherized } \\
\text { homes }\end{array}$ & $\begin{array}{l}\text { obs: } 46 \\
\text { no: } 46 \\
\text { yes: } 0\end{array}$ & $\begin{array}{l}\text { obs: } 46 \\
\text { no: } 10 \\
\text { yes: } 36\end{array}$ & $\begin{array}{l}\text { obs: } 46 \\
\text { no: } 36 \\
\text { yes: } 10\end{array}$ & $\begin{array}{l}\text { obs: } 46 \\
\text { no: } 40 \\
\text { yes: } 6\end{array}$ & $\begin{array}{l}\text { obs: } 42 \\
\text { no: } 4 \\
\text { yes: } 46\end{array}$ \\
\hline $\begin{array}{l}\text { Descriptive statistics: } \\
\text { weatherization measures } \\
\text { installed }\end{array}$ & $\begin{array}{l}\text { obs: } 39 \\
\text { no: } 39 \\
\text { yes: } 0\end{array}$ & $\begin{array}{l}\text { obs: } 39 \text { : } \\
\text { no: } 11 \\
\text { yes: } 28\end{array}$ & $\begin{array}{l}\text { obs: } 39 \\
\text { no: } 33 \\
\text { yes: } 6\end{array}$ & $\begin{array}{l}\text { obs: } 39 \\
\text { no: } 38 \\
\text { yes: } 1\end{array}$ & $\begin{array}{l}\text { obs: } 39 \\
\text { no: } 32 \\
\text { yes: } 7\end{array}$ \\
\hline $\begin{array}{l}\text { Descriptive statistics: } \\
\text { energy use and savings in } \\
\text { weatherized homes }\end{array}$ & $\begin{array}{l}\text { obs: } 27 \\
\text { no: } 27 \\
\text { yes: } 0\end{array}$ & $\begin{array}{l}\text { obs: } 27 \\
\text { no: } 11 \\
\text { yes: } 16\end{array}$ & $\begin{array}{l}\text { obs: } 27 \\
\text { no: } 24 \\
\text { yes: } 3\end{array}$ & $\begin{array}{l}\text { obs: } 27 \\
\text { no: } 25 \\
\text { yes: } 2\end{array}$ & $\begin{array}{l}\text { obs: } 27 \\
\text { no: } 21 \\
\text { yes: } 6\end{array}$ \\
\hline $\begin{array}{l}\text { Copies of audits performed } \\
\text { on weatherized homes }\end{array}$ & $\begin{array}{l}\text { obs: } 28 \\
\text { no: } 27 \\
\text { yes: } 1\end{array}$ & $\begin{array}{l}\text { obs: } 28 \\
\text { no: } 20 \\
\text { yes: } 8\end{array}$ & $\begin{array}{l}\text { obs: } 28 \\
\text { no: } 26 \\
\text { yes: } 2\end{array}$ & $\begin{array}{l}\text { obs: } 28 \\
\text { no: } 26 \\
\text { yes: } 2\end{array}$ & $\begin{array}{l}\text { obs: } 28 \\
\text { no: } 13 \\
\text { yes: } 15\end{array}$ \\
\hline $\begin{array}{l}\text { Results of certain } \\
\text { diagnostic tests }\end{array}$ & $\begin{array}{l}\text { obs: } 36 \\
\text { no: } 35 \\
\text { yes: } 1\end{array}$ & $\begin{array}{l}\text { obs: } 36 \\
\text { no: } 14 \\
\text { yes: } 22\end{array}$ & $\begin{array}{l}\text { obs: } 36 \\
\text { no: } 33 \\
\text { yes: } 3\end{array}$ & $\begin{array}{l}\text { obs: } 36 \\
\text { no: } 36 \\
\text { yes: } 0\end{array}$ & $\begin{array}{l}\text { obs: } 36 \\
\text { no: } 26 \\
\text { yes: } 10\end{array}$ \\
\hline Other & $\begin{array}{l}\text { obs: } 2 \\
\text { no: } 2 \\
\text { yes: } 0\end{array}$ & $\begin{array}{l}\text { obs: } 2 \\
\text { no: } 2 \\
\text { yes: } 0\end{array}$ & $\begin{array}{l}\text { obs: } 2 \\
\text { no: } 2 \\
\text { yes: } 0\end{array}$ & $\begin{array}{l}\text { obs: } 2 \\
\text { no: } 2 \\
\text { yes: } 0\end{array}$ & $\begin{array}{l}\text { obs: } 2 \\
\text { no: } 0 \\
\text { yes: } 2\end{array}$ \\
\hline
\end{tabular}


12. Overall, how would you rate the quality of the data received from the agencies in Program Year 2008 ? Please check the appropriate boxes. Please leave blank any data categories that are not required to be reported.

Very low data quality means required data was incomplete and inaccurate. Very high data quality means virtually all required data was accurate and complete.

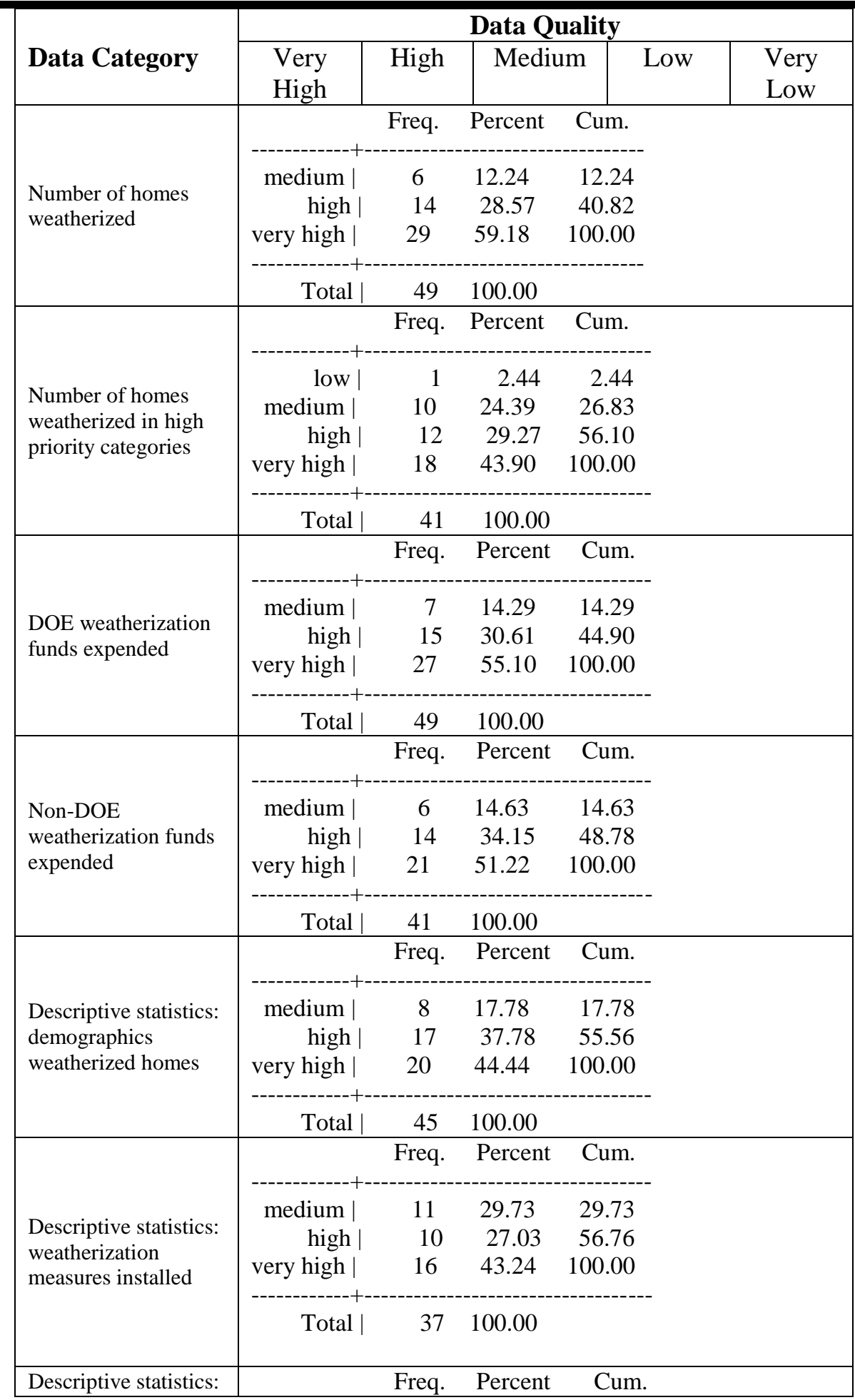




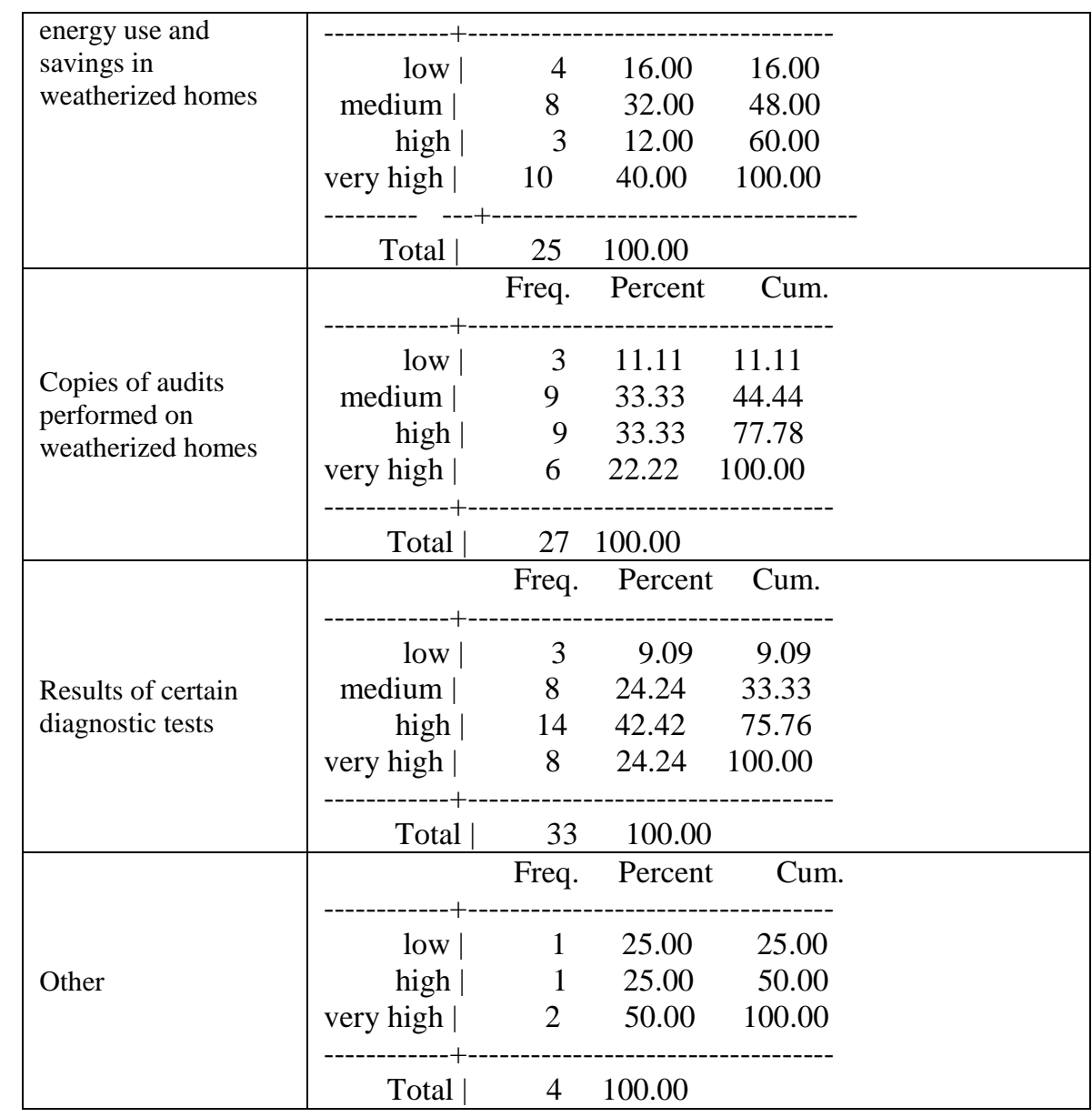

13. Did your state analyze data provided by the weatherization agencies in Program Year 2008 to: (Check all that apply)

\section{Generate descriptive statistics}

Freq. Percent Cum.

\begin{tabular}{|c|c|c|c|}
\hline no | & 19 & 40.43 & 40.43 \\
\hline yes & 28 & 59.57 & 100.00 \\
\hline Total & 47 & 100.00 & \\
\hline
\end{tabular}

Look for trends

Freq. Percent Cum.

$\begin{array}{cccc}\text { no } \mid & 16 & 34.04 & 34.04 \\ \text { yes } \mid & 31 & 65.96 & 100.00 \\ - & \end{array}$

Support state-level strategic planning about its weatherization program 


$\begin{array}{cccc} & \text { Freq. } & \text { Percent } & \text { Cum. } \\ \text { no | } & 16 & 34.04 & 34.04 \\ \text { yes | } & 31 & 65.96 & 100.00 \\ ---------- \\ \text { Total | } & 47 & 100.00 & \end{array}$

Analyze individual agency performance

$\begin{array}{rrrr} & \text { Freq. } & \text { Percent } & \text { Cum. } \\ \text { no } \mid & 5 & 10.64 & 10.64 \\ \text { yes } \mid & 42 & 89.36 & 100.00 \\ ------------ \\ \text { Total } & 47 & 100.00\end{array}$

Other

Freq. Percent Cum.

\begin{tabular}{|c|c|c|c|}
\hline no & 41 & 87.23 & 87.23 \\
\hline yes & 6 & 12.77 & 100.00 \\
\hline Total & 47 & 100.00 & \\
\hline
\end{tabular}

13a. If your state did not analyze data, why did your state not analyze data from the weatherization agencies in Program Year 2008? (Check all that apply)

Not a priority

$\begin{array}{cccc} & \text { Freq. } & \text { Percent } & \text { Cum. } \\ \text { no } \mid & 9 & 90.00 & 90.00 \\ \text { yes } \mid & 1 & 10.00 & 100.00 \\ ---------------- & \end{array}$

Not worth the effort

Freq. Percent Cum.

\begin{tabular}{|c|c|c|c|}
\hline no & 10 & 100.00 & 100.00 \\
\hline Total | & 10 & 100.00 & \\
\hline
\end{tabular}


Insufficient staff resources

\begin{tabular}{cccc} 
& Freq. & Percent & Cum. \\
no $\mid$ & 2 & 20.00 & 20.00 \\
yes $\mid$ & 8 & 80.00 & 100.00 \\
--------- \\
\hline Total $\mid$ & 10 & 100.00
\end{tabular}

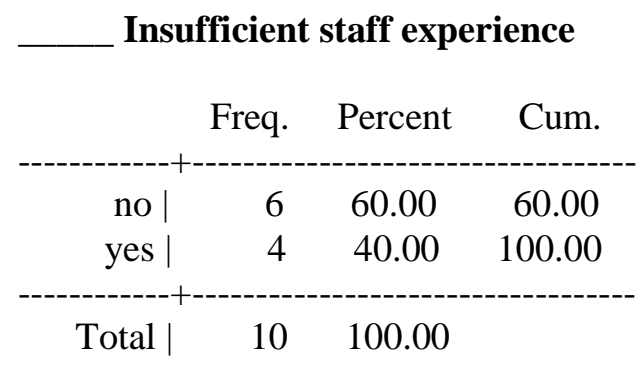

14. What data did your state plan to provide to DOE about its weatherization program in Program Year 2008? (Check all that apply)

Number of homes weatherized

\begin{tabular}{|c|c|c|c|}
\hline & Freq. & Percent & Cum. \\
\hline yes | & 50 & 100.00 & 100.00 \\
\hline Total & 50 & 100.00 & \\
\hline
\end{tabular}

Number of homes weatherized for high priority categories

Freq. Percent Cum.

$\begin{array}{cccc}\text { no } \mid & 16 & 32.00 & 32.00 \\ \text { yes } \mid & 34 & 68.00 & 100.00 \\ -------- & \end{array}$

DOE weatherization funds expended

$\begin{array}{rrrr} & \text { Freq. } & \text { Percent } & \text { Cum. } \\ \text { no } \mid & 2 & 4.00 & 4.00 \\ \text { yes } \mid & 48 & 96.00 & 100.00 \\ ------ & \end{array}$


Descriptive statistics on demographics of households weatherized

\begin{tabular}{|c|c|c|c|}
\hline & Freq. & Percent & Cum. \\
\hline no & 11 & 22.00 & 22.00 \\
\hline yes & 39 & 78.00 & 100.00 \\
\hline Total & 50 & 100.00 & \\
\hline
\end{tabular}

Descriptive statistics on weatherization measures installed in households weatherized

\begin{tabular}{|c|c|c|c|}
\hline & Freq. & Percent & Cum. \\
\hline no & 35 & 70.00 & 70.00 \\
\hline yes & 15 & 30.00 & 100.00 \\
\hline Total & 50 & 100.00 & \\
\hline
\end{tabular}

Descriptive statistics on energy use/savings of households weatherized

$\begin{array}{cccc} & \text { Freq. } & \text { Percent } & \text { Cum. } \\ \text { no } \mid & 29 & 58.00 & 58.00 \\ \text { yes } \mid & 21 & 42.00 & 100.00 \\ ---------- \\ \text { Total | } & 50 & 100.00 & \end{array}$

Copy of audits performed on the households weatherized

Freq. Percent Cum.

\begin{tabular}{cccc} 
no $\mid$ & 45 & 90.00 & 90.00 \\
yes $\mid$ & 5 & 10.00 & 100.00 \\
- \hdashline--- & & \\
- & Total & 50 & 100.00
\end{tabular}

Other

\begin{tabular}{|c|c|c|c|}
\hline & Freq. & Percent & Cum. \\
\hline no & 48 & 96.00 & 96.00 \\
\hline yes $\mid$ & 2 & 4.00 & 100.00 \\
\hline Total & 50 & 100.00 & \\
\hline
\end{tabular}


15. What data did your state actually provide to DOE about its weatherization program during Program Year 2008? (Check all that apply)

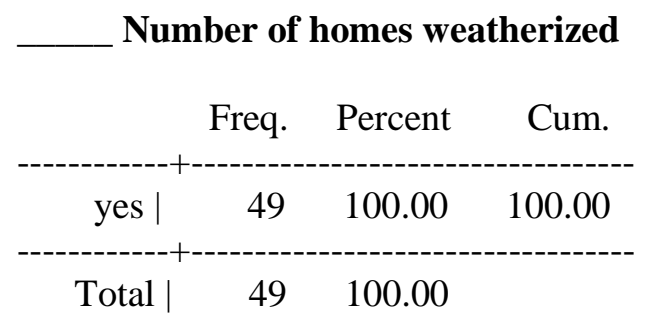

\begin{tabular}{|c|c|c|c|}
\hline & Freq. & Percent & Cum. \\
\hline no & 14 & 28.57 & 28.57 \\
\hline yes | & 35 & 71.43 & 100.00 \\
\hline Total & 49 & 100.00 & \\
\hline
\end{tabular}

\begin{tabular}{cccc}
\multicolumn{4}{c}{ DOE weatherization funds expend } \\
\hline & Freq. & Percent & Cum. \\
no $\mid$ & 1 & 2.04 & 2.04 \\
yes $\mid$ & 48 & 97.96 & 100.00 \\
------+------ \\
Total & 49 & 100.00 &
\end{tabular}

Descriptive statistics on demographics of households weatherized

$\begin{array}{crrr} & & & \\ & \text { Freq. } & \text { Percent } & \text { Cum. } \\ \text { no } \mid & 7 & 14.29 & 14.29 \\ \text { yes | } & 42 & 85.71 & 100.00 \\ ------- & \end{array}$

Descriptive statistics on weatherization measures installed in households weatherized

\begin{tabular}{cccc} 
& Freq. & Percent & Cum. \\
no $\mid$ & 35 & 71.43 & 71.43 \\
yes $\mid$ & 14 & 28.57 & 100.00 \\
- \hdashline--- &
\end{tabular}


Descriptive statistics on energy use/savings of households weatherized

Freq. Percent Cum.

$\begin{array}{cccc}\text { no } \mid & 33 & 67.35 & 67.35 \\ \text { yes } \mid & 16 & 32.65 & 100.00 \\ ------ & \\ \text { Total } & 49 & 100.00\end{array}$

Copy of audits performed on the households weatherized

\begin{tabular}{crrr} 
& Freq. & Percent & Cum. \\
no $\mid$ & 42 & 85.71 & 85.71 \\
yes $\mid$ & 7 & 14.29 & 100.00 \\
- \hdashline--- &
\end{tabular}

Other

$\begin{array}{crrr} & \text { Freq. } & \text { Percent } & \text { Cum. } \\ \text { no } \mid & 44 & 89.80 & 89.80 \\ \text { yes } \mid & 5 & 10.20 & 100.00 \\ -----+----- \\ \text { Total } & 49 & 100.00\end{array}$


16. For each of the non-DOE sources of weatherization funds which your state administered in Program Year 2008, did you require local agencies receiving those funds to follow DOE rules when spending the money? If you did not require DOE rules to be followed for non-DOE jobs, what were the major differences in the rules governing those expenditures in comparison to DOE rules? If you did not administer funding from a particular source in Program Year 2008 for weatherization, please enter N/A.

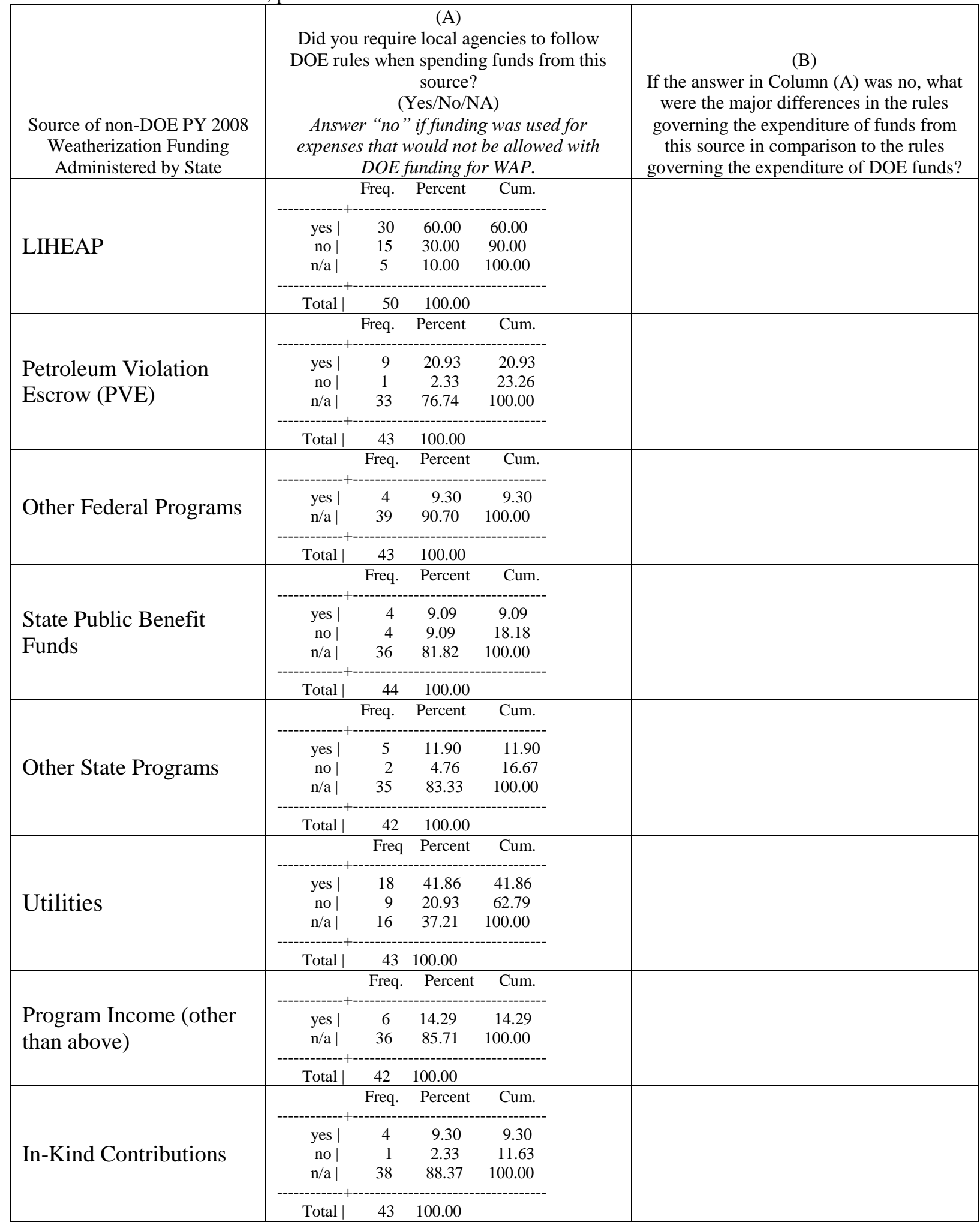




\begin{tabular}{|c|c|c|c|c|}
\hline \multirow{5}{*}{$\begin{array}{l}\text { Non-Profit } \\
\text { Organizations }\end{array}$} & & Freq. & Percent & Cum. \\
\hline & yes $\mid$ & 2 & 4.65 & 4.65 \\
\hline & no & 1 & 2.33 & 6.98 \\
\hline & $\mathrm{n} / \mathrm{a} \mid$ & 40 & 93.02 & 100.00 \\
\hline & Total & 43 & 100.00 & \\
\hline \multirow{4}{*}{ Other } & & Freq. & Percent & Cum. \\
\hline & yes | & 1 & 2.70 & 2.70 \\
\hline & $\mathrm{n} / \mathrm{a} \mid$ & 36 & 97.30 & 100.00 \\
\hline & Total I & 37 & 1 & \\
\hline
\end{tabular}

C-16 
17a. Please indicate the amount of weatherization funding from each source that your state administered in Program Year 2007 that was spent on the different functions or applications shown below. The amount that you list in the right-most cell at the very bottom of the table should equal the total amount of weatherization funding from all sources that your state administered in Program Year 2007.

\begin{tabular}{|c|c|c|c|c|c|c|}
\hline \multirow{2}{*}{$\begin{array}{l}\text { Source of PY } 2007 \\
\text { Weatherization Funding } \\
\text { Administered by State }\end{array}$} & \multicolumn{2}{|c|}{$\begin{array}{c}\text { Training and Technical Assistance } \\
\text { (T\&TA) }\end{array}$} & \multicolumn{2}{|c|}{ Program Management } & \multirow{2}{*}{$\begin{array}{l}\text { Other (funds for } \\
\text { weatherization, } \\
\text { H\&S, and other } \\
\text { measures passed } \\
\text { on to local } \\
\text { agencies) }\end{array}$} & \multirow[b]{2}{*}{ Total } \\
\hline & $\begin{array}{l}\text { Funds retained } \\
\text { and spent by } \\
\text { state }\end{array}$ & $\begin{array}{l}\text { Funds passed on } \\
\text { to local } \\
\text { agencies }\end{array}$ & $\begin{array}{c}\text { Funds retained } \\
\text { and spent by } \\
\text { state }\end{array}$ & $\begin{array}{l}\text { Funds passed on to } \\
\text { local agencies }\end{array}$ & & \\
\hline DOE & $\begin{array}{l}\text { obs: } 50 \\
\text { min: } 0 \\
\text { max: } 1038505 \\
\text { mean: } 176297.83 \\
\text { median: } 83571.52\end{array}$ & $\begin{array}{l}\text { obs: } 50 \\
\text { min: } 0 \\
\text { max: } 3815185 \\
\text { mean: } 200074.99 \\
\text { median: } 77587\end{array}$ & $\begin{array}{l}\text { obs: } 50 \\
\text { min: } 0 \\
\text { max: } 3981946.5 \\
\text { mean: } 256340.14 \\
\text { median: } 109576.5 \\
\end{array}$ & $\begin{array}{l}\text { obs: } 50 \\
\text { min: } 0 \\
\text { max: } 11805719 \\
\text { mean:945961.67 } \\
\text { median: } 365005\end{array}$ & $\begin{array}{l}\text { obs: } 51 \\
\text { min: } 0 \\
\text { max: } 14407620 \\
\text { mean: } 2477823.5 \\
\text { median: } 1437762 \\
\end{array}$ & $\begin{array}{l}\text { obs: } 51 \\
\text { min: } 0 \\
\text { max: } 18009524 \\
\text { mean: } 4043834.9 \\
\text { median: } 2315681\end{array}$ \\
\hline LIHEAP & $\begin{array}{l}\text { obs: } 50 \\
\text { min: } 0 \\
\text { max: } 188000 \\
\text { mean: } 14190.7 \\
\text { median: } 0\end{array}$ & $\begin{array}{l}\text { obs: } 50 \\
\text { min: } 0 \\
\text { max: } 5733524 \\
\text { mean: } 144112.66 \\
\text { median: } 0\end{array}$ & $\begin{array}{l}\text { obs: } 50 \\
\text { min: } 0 \\
\text { max: } 1554524 \\
\text { mean: } 149582.19 \\
\text { median: } 17270.5\end{array}$ & $\begin{array}{l}\text { obs: } 50 \\
\text { min: } 0 \\
\text { max: } 46674732 \\
\text { mean: } 2410325.1 \\
\text { median: } 281160\end{array}$ & $\begin{array}{l}\text { obs: } 50 \\
\text { min: } 0 \\
\text { max: } 34450016 \\
\text { mean: } 2596525.5 \\
\text { median: } 309737\end{array}$ & $\begin{array}{l}\text { obs: } 51 \\
\text { min: } 0 \\
\text { max: } 46840176 \\
\text { mean: } 5322676.8 \\
\text { median: } 2284133\end{array}$ \\
\hline $\begin{array}{l}\text { Petroleum } \\
\text { Violation Escrow } \\
\text { (PVE) }\end{array}$ & $\begin{array}{l}\text { obs: } 51 \\
\text { min: } 0 \\
\text { max: } 0 \\
\text { mean: } 0 \\
\text { median: } 0 \\
\end{array}$ & $\begin{array}{l}\text { obs: } 51 \\
\text { min: } 0 \\
\text { max: } 0 \\
\text { mean: } 0 \\
\text { median: } 0 \\
\end{array}$ & $\begin{array}{l}\text { obs: } 51 \\
\text { min: } 0 \\
\text { max: } 0 \\
\text { mean: } 0 \\
\text { median: } 0 \\
\end{array}$ & $\begin{array}{l}\text { obs: } 51 \\
\text { min: } 0 \\
\text { max: } 680000 \\
\text { mean: } 13333.33 \\
\text { median: } 0\end{array}$ & $\begin{array}{l}\text { obs: } 51 \\
\text { min: } 0 \\
\text { max: } 209792 \\
\text { mean: } 6579.02 \\
\text { median: } 0\end{array}$ & $\begin{array}{l}\text { obs: } 51 \\
\text { min: } 0 \\
\text { max: } 800000 \\
\text { mean: } 19912.35 \\
\text { median: } 0\end{array}$ \\
\hline $\begin{array}{l}\text { Other Federal } \\
\text { Programs }\end{array}$ & $\begin{array}{l}\text { obs: } 51 \\
\text { min: } 0 \\
\text { max: } 2005535 \\
\text { mean: } 41791.94 \\
\text { median: } 0\end{array}$ & $\begin{array}{l}\text { obs: } 51 \\
\text { min: } 0 \\
\text { max: } 69156 \\
\text { mean: } 1653.10 \\
\text { median: } 0\end{array}$ & $\begin{array}{l}\text { obs: } 51 \\
\text { min: } 0 \\
\text { max: } 154740 \\
\text { mean: } 3632.20 \\
\text { median: } 0\end{array}$ & $\begin{array}{l}\text { obs: } 51 \\
\text { min: } 0 \\
\text { max: } 782264 \\
\text { mean: } 23209.92 \\
\text { median: } 0\end{array}$ & $\begin{array}{l}\text { obs: } 51 \\
\text { min: } 0 \\
\text { max: } 206357.59 \\
\text { mean: } 5412.44 \\
\text { median: } 0\end{array}$ & $\begin{array}{l}\text { obs: } 51 \\
\text { min: } 0 \\
\text { max: } 2305212 \\
\text { mean: } 75699.6 \\
\text { median: } 0\end{array}$ \\
\hline $\begin{array}{l}\text { State Public } \\
\text { Benefit Funds }\end{array}$ & $\begin{array}{l}\text { obs: } 51 \\
\text { min: } 0 \\
\text { max: } 168441 \\
\text { mean: } 5099.96 \\
\text { median: } 0\end{array}$ & $\begin{array}{l}\text { obs: } 51 \\
\text { min: } 0 \\
\text { max: } 234959 \\
\text { mean: } 6532.18 \\
\text { median: } 0\end{array}$ & $\begin{array}{l}\text { obs: } 51 \\
\text { min: } 0 \\
\text { max: } 864334 \\
\text { mean: } 31861.94 \\
\text { median: } 0\end{array}$ & $\begin{array}{l}\text { obs: } 51 \\
\text { min: } 0 \\
\text { max: } 6798620 \\
\text { mean: } 363211.08 \\
\text { median: } 0\end{array}$ & $\begin{array}{l}\text { obs: } 51 \\
\text { min: } 0 \\
\text { max: } 42442957 \\
\text { mean: } 1196336.3 \\
\text { median: } 0\end{array}$ & $\begin{array}{l}\text { obs: } 51 \\
\text { min: } 0 \\
\text { max: } 47384020 \\
\text { mean: } 1603041.4 \\
\text { median: } 0\end{array}$ \\
\hline $\begin{array}{l}\text { Other State } \\
\text { Programs }\end{array}$ & $\begin{array}{l}\text { obs: } 50 \\
\text { min: } 0 \\
\text { max: } 0 \\
\text { mean: } 0 \\
\text { median: } 0\end{array}$ & $\begin{array}{l}\text { obs: } 50 \\
\text { min: } 0 \\
\text { max: } 3000000 \\
\text { mean: } 61542.03 \\
\text { median: } 0\end{array}$ & $\begin{array}{l}\text { obs: } 50 \\
\text { min: } 0 \\
\text { max: } 16000 \\
\text { mean: } 320 \\
\text { median: } 0\end{array}$ & $\begin{array}{l}\text { obs: } 50 \\
\text { min: } 0 \\
\text { max: } 1748908 \\
\text { mean: } 57306.20 \\
\text { median: } 0\end{array}$ & $\begin{array}{l}\text { obs: } 50 \\
\text { min: } 0 \\
\text { max: } 1885427.6 \\
\text { mean: } 70479.07 \\
\text { median: } 0\end{array}$ & $\begin{array}{l}\text { obs: } 51 \\
\text { min: } 0 \\
\text { max: } 3000000 \\
\text { mean: } 186542.39 \\
\text { median: } 0\end{array}$ \\
\hline Utilities & $\begin{array}{l}\text { obs: } 51 \\
\text { min: } 0 \\
\text { max: } 20000 \\
\text { mean: } 392.16 \\
\text { median: } 0\end{array}$ & $\begin{array}{l}\text { obs: } 51 \\
\text { min: } 0 \\
\text { max: } 0 \\
\text { mean: } 0 \\
\text { median: } 0\end{array}$ & $\begin{array}{l}\text { obs: } 51 \\
\text { min: } 0 \\
\text { max: } 350000 \\
\text { mean: } 9967.21 \\
\text { median: } 0\end{array}$ & $\begin{array}{l}\text { obs: } 51 \\
\text { min: } 0 \\
\text { max: } 1778170 \\
\text { mean: } 112329.11 \\
\text { median: } 0\end{array}$ & $\begin{array}{l}\text { obs: } 51 \\
\text { min: } 0 \\
\text { max: } 4846840 \\
\text { mean: } 320783.75 \\
\text { median: } 0\end{array}$ & $\begin{array}{l}\text { obs: } 51 \\
\text { min: } 0 \\
\text { max: } 4846840 \\
\text { mean: } 443472.22 \\
\text { median: } 0\end{array}$ \\
\hline $\begin{array}{l}\text { Program Income } \\
\text { (other than } \\
\text { above) }\end{array}$ & $\begin{array}{l}\text { obs: } 51 \\
\text { min: } 0 \\
\text { max: } 0 \\
\text { mean: } 0 \\
\text { median: } 0 \\
\end{array}$ & $\begin{array}{l}\text { obs: } 51 \\
\text { min: } 0 \\
\text { max: } 0 \\
\text { mean: } 0 \\
\text { median: } 0\end{array}$ & $\begin{array}{l}\text { obs: } 51 \\
\text { min: } 0 \\
\text { max: } 0 \\
\text { mean: } 0 \\
\text { median: } 0 \\
\end{array}$ & $\begin{array}{l}\text { obs: } 51 \\
\text { min: } 0 \\
\text { max: } 0 \\
\text { mean: } 0 \\
\text { median: } 0 \\
\end{array}$ & $\begin{array}{l}\text { obs: } 51 \\
\text { min: } 0 \\
\text { max: } 0 \\
\text { mean: } 0 \\
\text { median: } 0 \\
\end{array}$ & $\begin{array}{l}\text { obs: } 51 \\
\text { min: } 0 \\
\text { max: } 0 \\
\text { mean: } 0 \\
\text { median: } 0\end{array}$ \\
\hline $\begin{array}{l}\text { In-Kind } \\
\text { Contributions }\end{array}$ & $\begin{array}{l}\text { obs: } 51 \\
\text { min: } 0 \\
\text { max: } 0 \\
\text { mean: } 0 \\
\text { median: } 0\end{array}$ & $\begin{array}{l}\text { obs: } 51 \\
\text { min: } 0 \\
\text { max: } 0 \\
\text { mean: } 0 \\
\text { median: } 0\end{array}$ & $\begin{array}{l}\text { obs: } 51 \\
\text { min: } 0 \\
\text { max: } 0 \\
\text { mean: } 0 \\
\text { median: } 0\end{array}$ & $\begin{array}{l}\text { obs: } 51 \\
\text { min: } 0 \\
\text { max: } 0 \\
\text { mean: } 0 \\
\text { median: } 0\end{array}$ & $\begin{array}{l}\text { obs: } 51 \\
\text { min: } 0 \\
\text { max: } 0 \\
\text { mean: } 0 \\
\text { median: } 0 \\
\end{array}$ & $\begin{array}{l}\text { obs: } 51 \\
\text { min: } 0 \\
\text { max: } 0 \\
\text { mean: } 0 \\
\text { median: } 0 \\
\end{array}$ \\
\hline $\begin{array}{l}\text { Non-Profit } \\
\text { Organizations }\end{array}$ & $\begin{array}{l}\text { obs: } 51 \\
\text { min: } 0 \\
\text { max: } 0 \\
\text { mean: } 0 \\
\text { median: } 0\end{array}$ & $\begin{array}{l}\text { obs: } 51 \\
\text { min: } 0 \\
\text { max: } 0 \\
\text { mean: } 0 \\
\text { median: } 0 \\
\end{array}$ & $\begin{array}{l}\text { obs: } 51 \\
\text { min: } 0 \\
\text { max: } 0 \\
\text { mean: } 0 \\
\text { median: } 0\end{array}$ & $\begin{array}{l}\text { obs: } 51 \\
\text { min: } 0 \\
\text { max: } 0 \\
\text { mean: } 0 \\
\text { median: } 0\end{array}$ & $\begin{array}{l}\text { obs: } 51 \\
\text { min: } 0 \\
\text { max: } 55000 \\
\text { mean: } 1078.43 \\
\text { median: } 0\end{array}$ & $\begin{array}{l}\text { obs: } 51 \\
\text { min: } 0 \\
\text { max: } 55000 \\
\text { mean: } 1078.43 \\
\text { median: } 0\end{array}$ \\
\hline Other & $\begin{array}{l}\text { obs: } 51 \\
\text { min: } 0 \\
\text { max: } 0 \\
\text { mean: } 0 \\
\text { median: } 0\end{array}$ & $\begin{array}{l}\text { obs: } 51 \\
\text { min: } 0 \\
\text { max: } 0 \\
\text { mean: } 0 \\
\text { median: } 0\end{array}$ & $\begin{array}{l}\text { obs: } 51 \\
\text { min: } 0 \\
\text { max: } 0 \\
\text { mean: } 0 \\
\text { median: } 0\end{array}$ & $\begin{array}{l}\text { obs: } 51 \\
\text { min: } 0 \\
\text { max: } 0 \\
\text { mean: } 0 \\
\text { median: } 0\end{array}$ & $\begin{array}{l}\text { obs: } 51 \\
\text { min: } 0 \\
\text { max: } 21303879 \\
\text { mean: } 428507.43 \\
\text { median: } 0\end{array}$ & $\begin{array}{l}\text { obs: } 51 \\
\text { min: } 0 \\
\text { max: } 21303879 \\
\text { mean: } 428507.43 \\
\text { median: } 0\end{array}$ \\
\hline Total & $\begin{array}{l}\text { obs: } 50 \\
\text { min: } 0 \\
\text { max: } 2324448 \\
\text { mean: } 238718.27 \\
\text { median: } 106920.5\end{array}$ & $\begin{array}{l}\text { obs: } 50 \\
\text { min: } 0 \\
\text { max: } 12617865 \\
\text { mean: } 414078.66 \\
\text { median: } 105269.5 \\
\end{array}$ & $\begin{array}{l}\text { obs: } 50 \\
\text { min: } 0 \\
\text { max: } 4284665 \\
\text { mean: } 452612.9 \\
\text { median:211051.5 }\end{array}$ & $\begin{array}{l}\text { obs: } 50 \\
\text { min: } 0 \\
\text { max: } 47341096 \\
\text { mean: } 3935918.1 \\
\text { median:1016511.5 }\end{array}$ & $\begin{array}{l}\text { obs: } 51 \\
\text { min: } 0 \\
\text { max: } 71046408 \\
\text { mean: } 7101572.6 \\
\text { median: } 2947177 \\
\end{array}$ & $\begin{array}{l}\text { obs: } 51 \\
\text { min: } 0 \\
\text { max: } 78085712 \\
\text { mean: } 12124766 \\
\text { median: } 5838587\end{array}$ \\
\hline
\end{tabular}


17b. Of the Program Year 2007 funds retained by your state's weatherization program for Program Management, how much was used for each function listed below?

\begin{tabular}{|c|c|}
\hline $\begin{array}{c}\text { Type of Management } \\
\text { Function }\end{array}$ & Total \\
\hline Administration* & $\begin{array}{l}\text { obs: } 46 \\
\text { min: } 4129 \\
\text { max: } 2860260 \\
\text { mean: } 301772.96 \\
\text { median: } 144405.12\end{array}$ \\
\hline Agency monitoring & $\begin{array}{l}\text { obs: } 32 \\
\text { min: } 0 \\
\text { max: } 337565 \\
\text { mean: } 122735.74 \\
\text { median: } 101661.5\end{array}$ \\
\hline Other (specify) & $\begin{array}{l}\text { obs: } 12 \\
\text { min: } 0 \\
\text { max: } 737827.63 \\
\text { mean: } 164062.05 \\
\text { median: } 71381.5\end{array}$ \\
\hline TOTAL & $\begin{array}{l}\text { obs: } 51 \\
\text { min: } 0 \\
\text { max: } 2860260 \\
\text { mean: } 387800.87 \\
\text { median: } 208825\end{array}$ \\
\hline
\end{tabular}

* Includes planning, finance and accounting, clerical support, outreach, and evaluation. 
18a. Please indicate the amount of weatherization funding from each source that your state administered in Program Year 2008 that was spent on the different functions or applications shown below. The amount that you list in the right-most cell at the very bottom of the table should equal the total amount of weatherization funding from all sources that your state administered in Program Year 2008.

\begin{tabular}{|c|c|c|c|c|c|c|}
\hline \multirow{2}{*}{$\begin{array}{c}\text { Source of PY } 2008 \\
\text { Weatherization Funding } \\
\text { Administered by State }\end{array}$} & \multicolumn{2}{|c|}{$\begin{array}{l}\text { Training and Technical Assistance } \\
\text { (T\&TA) }\end{array}$} & \multicolumn{2}{|c|}{ Program Management } & \multirow{2}{*}{$\begin{array}{l}\text { Other (funds for } \\
\text { weatherization, } \\
\mathrm{H} \& S \text {, and other } \\
\text { measures passed } \\
\text { on to local } \\
\text { agencies) }\end{array}$} & \multirow[b]{2}{*}{ Total } \\
\hline & $\begin{array}{l}\text { Funds retained and } \\
\text { spent by state }\end{array}$ & $\begin{array}{c}\text { Funds passed on } \\
\text { to local } \\
\text { agencies }\end{array}$ & $\begin{array}{l}\text { Funds retained and } \\
\text { spent by state }\end{array}$ & $\begin{array}{l}\text { Funds passed on } \\
\text { to local agencies }\end{array}$ & & \\
\hline $\mathrm{DOE}$ & $\begin{array}{l}\text { obs: } 51 \\
\text { min: } 0 \\
\text { max: } 898076 \\
\text { mean: } 183343.92 \\
\text { median: } 119396 \\
\end{array}$ & $\begin{array}{l}\text { obs: } 51 \\
\text { min: } 0 \\
\text { max: } 4468840 \\
\text { mean: } 240641.3 \\
\text { median: } 94496\end{array}$ & $\begin{array}{l}\text { obs: } 51 \\
\text { min: } 0 \\
\text { max: } 1003790 \\
\text { mean: } 206716.67 \\
\text { median: } 134617 \\
\end{array}$ & $\begin{array}{l}\text { obs: } 51 \\
\text { min: } 0 \\
\text { max: } 13305394 \\
\text { mean: } 1122956.5 \\
\text { median: } 409447\end{array}$ & $\begin{array}{l}\text { obs: } 51 \\
\text { min: } 0 \\
\text { max: } 15885365 \\
\text { mean: } 2821587.9 \\
\text { median: } 1703174\end{array}$ & $\begin{array}{l}\text { obs: } 51 \\
\text { min: } 0 \\
\text { max: } 20075816 \\
\text { mean: } 4575246.3 \\
\text { median:2890404.5 }\end{array}$ \\
\hline LIHEAP & $\begin{array}{l}\text { obs: } 51 \\
\text { min: } 0 \\
\text { max: } 6042017 \\
\text { mean: } 133522.08 \\
\text { median: } 0\end{array}$ & $\begin{array}{l}\text { obs: } 51 \\
\text { min: } 0 \\
\text { max: } 551466 \\
\text { mean: } 38236.47 \\
\text { median: } 0\end{array}$ & $\begin{array}{l}\text { obs: } 51 \\
\text { min: } 0 \\
\text { max: } 1530148 \\
\text { mean: } 225906.89 \\
\text { median: } 47952\end{array}$ & $\begin{array}{l}\text { obs: } 51 \\
\text { min: } 0 \\
\text { max: } 33345700 \\
\text { mean: } 1705141 \\
\text { median: } 307595\end{array}$ & $\begin{array}{l}\text { obs: } 51 \\
\text { min: } 0 \\
\text { max: } 40724760 \\
\text { mean: } 4148556.3 \\
\text { median: } 854099\end{array}$ & $\begin{array}{l}\text { obs: } 51 \\
\text { min: } 0 \\
\text { max: } 44437348 \\
\text { mean: } 6251362.8 \\
\text { median: } 2709263\end{array}$ \\
\hline $\begin{array}{l}\text { Petroleum } \\
\text { Violation Escrow } \\
\text { (PVE) }\end{array}$ & $\begin{array}{l}\text { obs: } 51 \\
\text { min: } 0 \\
\text { max: } 0 \\
\text { mean: } 0 \\
\text { median: } 0\end{array}$ & $\begin{array}{l}\text { obs: } 51 \\
\text { min: } 0 \\
\text { max: } 0 \\
\text { mean: } 0 \\
\text { median: } 0\end{array}$ & $\begin{array}{l}\text { obs: } 51 \\
\text { min: } 0 \\
\text { max: } 0 \\
\text { mean: } 0 \\
\text { median: } 0\end{array}$ & $\begin{array}{l}\text { obs: } 51 \\
\text { min: } 0 \\
\text { max: } 696870 \\
\text { mean: } 13664.12 \\
\text { median: } 0\end{array}$ & $\begin{array}{l}\text { obs: } 51 \\
\text { min: } 0 \\
\text { max: } 5000000 \\
\text { mean: } 111234.82 \\
\text { median: } 0\end{array}$ & $\begin{array}{l}\text { obs: } 51 \\
\text { min: } 0 \\
\text { max: } 5000000 \\
\text { mean: } 124898.94 \\
\text { median: } 0\end{array}$ \\
\hline $\begin{array}{l}\text { Other Federal } \\
\text { Programs }\end{array}$ & $\begin{array}{l}\text { obs: } 51 \\
\text { min: } 0 \\
\text { max: } 69156 \\
\text { mean: } 3272.88 \\
\text { median: } 0\end{array}$ & $\begin{array}{l}\text { obs: } 51 \\
\text { min: } 0 \\
\text { max: } 2016495 \\
\text { mean: } 41562.61 \\
\text { median: } 0\end{array}$ & $\begin{array}{l}\text { obs: } 51 \\
\text { min: } 0 \\
\text { max: } 170128 \\
\text { mean: } 4165.35 \\
\text { median: } 0\end{array}$ & $\begin{array}{l}\text { obs: } 51 \\
\text { min: } 0 \\
\text { max: } 873532 \\
\text { mean: } 28286.49 \\
\text { median: } 0\end{array}$ & $\begin{array}{l}\text { obs: } 51 \\
\text { min: } 0 \\
\text { max: } 1317932 \\
\text { mean: } 32329.77 \\
\text { median: } 0\end{array}$ & $\begin{array}{l}\text { obs: } 51 \\
\text { min: } 0 \\
\text { max: } 2304828 \\
\text { mean: } 109617.11 \\
\text { median: } 0\end{array}$ \\
\hline $\begin{array}{l}\text { State Public } \\
\text { Benefit Funds }\end{array}$ & $\begin{array}{l}\text { obs: } 51 \\
\text { min: } 0 \\
\text { max: } 128492 \\
\text { mean: } 5233.33 \\
\text { median: } 0\end{array}$ & $\begin{array}{l}\text { obs: } 51 \\
\text { min: } 0 \\
\text { max: } 315054 \\
\text { mean: } 7968.67 \\
\text { median: } 0\end{array}$ & $\begin{array}{l}\text { obs: } 51 \\
\text { min: } 0 \\
\text { max: } 1610995 \\
\text { mean: } 47051.88 \\
\text { median: } 0\end{array}$ & $\begin{array}{l}\text { obs: } 51 \\
\text { min: } 0 \\
\text { max: } 7178416 \\
\text { mean: } 384897.29 \\
\text { median: } 0\end{array}$ & $\begin{array}{l}\text { obs: } 51 \\
\text { min: } 0 \\
\text { max: } 40182405 \\
\text { mean: } 1178866.4 \\
\text { median: } 0\end{array}$ & $\begin{array}{l}\text { obs: } 51 \\
\text { min: } 0 \\
\text { max: } 45735904 \\
\text { mean: } 1624017.6 \\
\text { median: } 0\end{array}$ \\
\hline $\begin{array}{l}\text { Other State } \\
\text { Programs }\end{array}$ & $\begin{array}{l}\text { obs: } 51 \\
\text { min: } 0 \\
\text { max: } 311 \\
\text { mean: } 10.57 \\
\text { median: } 0\end{array}$ & $\begin{array}{l}\text { obs: } 51 \\
\text { min: } 0 \\
\text { max: } 3000000 \\
\text { mean: } 107911.33 \\
\text { median: } 0\end{array}$ & $\begin{array}{l}\text { obs: } 51 \\
\text { min: } 0 \\
\text { max: } 33470 \\
\text { mean: } 1552.35 \\
\text { median: } 0\end{array}$ & $\begin{array}{l}\text { obs: } 51 \\
\text { min: } 0 \\
\text { max: } 14795008 \\
\text { mean: } 344177.89 \\
\text { median: } 0\end{array}$ & $\begin{array}{l}\text { obs: } 51 \\
\text { min: } 0 \\
\text { max: } 3523641 \\
\text { mean: } 163427.86 \\
\text { median: } 0\end{array}$ & $\begin{array}{l}\text { obs: } 51 \\
\text { min: } 0 \\
\text { max: } 14795008 \\
\text { mean: } 617080 \\
\text { median: } 0\end{array}$ \\
\hline Utilities & $\begin{array}{l}\text { obs: } 51 \\
\text { min: } 0 \\
\text { max: } 20000 \\
\text { mean: } 401.29 \\
\text { median: } 0\end{array}$ & $\begin{array}{l}\text { obs: } 51 \\
\text { min: } 0 \\
\text { max: } 0 \\
\text { mean: } 0 \\
\text { median: } 0\end{array}$ & $\begin{array}{l}\text { obs: } 51 \\
\text { min: } 0 \\
\text { max: } 152652 \\
\text { mean: } 4359.11 \\
\text { median: } 0\end{array}$ & $\begin{array}{l}\text { obs: } 51 \\
\text { min: } 0 \\
\text { max: } 2002159 \\
\text { mean: } 115127.97 \\
\text { median: } 0\end{array}$ & $\begin{array}{l}\text { obs: } 51 \\
\text { min: } 0 \\
\text { max: } 4842605 \\
\text { mean: } 289489.6 \\
\text { median: } 0\end{array}$ & $\begin{array}{l}\text { obs: } 51 \\
\text { min: } 0 \\
\text { max: } 4842605 \\
\text { mean: } 409377.97 \\
\text { median: } 0\end{array}$ \\
\hline $\begin{array}{l}\text { Program Income } \\
\text { (other than } \\
\text { above) }\end{array}$ & $\begin{array}{l}\text { obs: } 51 \\
\text { min: } 0 \\
\text { max: } 0 \\
\text { mean: } 0 \\
\text { median: } 0 \\
\end{array}$ & $\begin{array}{l}\text { obs: } 51 \\
\text { min: } 0 \\
\text { max: } 0 \\
\text { mean: } 0 \\
\text { median: } 0\end{array}$ & $\begin{array}{l}\text { obs: } 51 \\
\text { min: } 0 \\
\text { max: } 0 \\
\text { mean: } 0 \\
\text { median: } 0\end{array}$ & $\begin{array}{l}\text { obs: } 51 \\
\text { min: } 0 \\
\text { max: } 0 \\
\text { mean: } 0 \\
\text { median: } 0 \\
\end{array}$ & $\begin{array}{l}\text { obs: } 51 \\
\text { min: } 0 \\
\text { max: } 0 \\
\text { mean: } 0 \\
\text { median: } 0 \\
\end{array}$ & $\begin{array}{l}\text { obs: } 51 \\
\text { min: } 0 \\
\text { max: } 0 \\
\text { mean: } 0 \\
\text { median: } 0\end{array}$ \\
\hline $\begin{array}{l}\text { In-Kind } \\
\text { Contributions }\end{array}$ & $\begin{array}{l}\text { obs: } 51 \\
\text { min: } 0 \\
\text { max: } 0 \\
\text { mean: } 0 \\
\text { median: } 0\end{array}$ & $\begin{array}{l}\text { obs: } 51 \\
\text { min: } 0 \\
\text { max: } 0 \\
\text { mean: } 0 \\
\text { median: } 0\end{array}$ & $\begin{array}{l}\text { obs: } 51 \\
\text { min: } 0 \\
\text { max: } 0 \\
\text { mean: } 0 \\
\text { median: } 0\end{array}$ & $\begin{array}{l}\text { obs: } 51 \\
\text { min: } 0 \\
\text { max: } 0 \\
\text { mean: } 0 \\
\text { median: } 0\end{array}$ & $\begin{array}{l}\text { obs: } 51 \\
\text { min: } 0 \\
\text { max: } 0 \\
\text { mean: } 0 \\
\text { median: } 0 \\
\end{array}$ & $\begin{array}{l}\text { obs: } 51 \\
\text { min: } 0 \\
\text { max: } 0 \\
\text { mean: } 0 \\
\text { median: } 0 \\
\end{array}$ \\
\hline $\begin{array}{l}\text { Non-Profit } \\
\text { Organizations }\end{array}$ & $\begin{array}{l}\text { obs: } 51 \\
\text { min: } 0 \\
\text { max: } 0 \\
\text { mean: } 0 \\
\text { median: } 0 \\
\end{array}$ & $\begin{array}{l}\text { obs: } 51 \\
\text { min: } 0 \\
\text { max: } 0 \\
\text { mean: } 0 \\
\text { median: } 0\end{array}$ & $\begin{array}{l}\text { obs: } 51 \\
\text { min: } 0 \\
\text { max: } 0 \\
\text { mean: } 0 \\
\text { median: } 0\end{array}$ & $\begin{array}{l}\text { obs: } 51 \\
\text { min: } 0 \\
\text { max: } 0 \\
\text { mean: } 0 \\
\text { median: } 0\end{array}$ & $\begin{array}{l}\text { obs: } 51 \\
\text { min: } 0 \\
\text { max: } 33200 \\
\text { mean: } 650.98 \\
\text { median: } 0\end{array}$ & $\begin{array}{l}\text { obs: } 51 \\
\text { min: } 0 \\
\text { max: } 33200 \\
\text { mean: } 650.98 \\
\text { median: } 0\end{array}$ \\
\hline Other & $\begin{array}{l}\text { obs: } 51 \\
\text { min: } 0 \\
\text { max: } 8500 \\
\text { mean: } 166.67 \\
\text { median: } 0\end{array}$ & $\begin{array}{l}\text { obs: } 51 \\
\text { min: } 0 \\
\text { max: } 0 \\
\text { mean: } 0 \\
\text { median: } 0\end{array}$ & $\begin{array}{l}\text { obs: } 51 \\
\text { min: } 0 \\
\text { max: } 0 \\
\text { mean: } 0 \\
\text { median: } 0\end{array}$ & $\begin{array}{l}\text { obs: } 51 \\
\text { min: } 0 \\
\text { max: } 0 \\
\text { mean: } 0 \\
\text { median: } 0\end{array}$ & $\begin{array}{l}\text { obs: } 51 \\
\text { min: } 0 \\
\text { max: } 20006838 \\
\text { mean: } 393663.49 \\
\text { median: } 0\end{array}$ & $\begin{array}{l}\text { obs: } 51 \\
\text { min: } 0 \\
\text { max: } 20006838 \\
\text { mean: } 393663.49 \\
\text { median: } 0\end{array}$ \\
\hline Total & $\begin{array}{l}\text { obs: } 51 \\
\text { min: } 0 \\
\text { max: } 6246423 \\
\text { mean: } 325950.75 \\
\text { median: } 144285\end{array}$ & $\begin{array}{l}\text { obs: } 51 \\
\text { min: } 0 \\
\text { max: } 9941058 \\
\text { mean: } 436320.37 \\
\text { median: } 106936\end{array}$ & $\begin{array}{l}\text { obs: } 51 \\
\text { min: } 0 \\
\text { max: } 2455541 \\
\text { mean: } 489791.48 \\
\text { median: } 270223\end{array}$ & $\begin{array}{l}\text { obs: } 51 \\
\text { min: } 0 \\
\text { max: } 46651096 \\
\text { mean: } 3714251.3 \\
\text { median: } 1470332\end{array}$ & $\begin{array}{l}\text { obs: } 51 \\
\text { min: } 0 \\
\text { max: } 78653192 \\
\text { mean: } 8982620.5 \\
\text { median: } 3141659\end{array}$ & $\begin{array}{l}\text { obs: } 51 \\
\text { min: } 0 \\
\text { max: } 86556232 \\
\text { mean: } 14106082 \\
\text { median: } 7619160\end{array}$ \\
\hline
\end{tabular}


18b. Of the Program Year 2008 funds retained by your state's weatherization program for Program Management, how much was used for each function listed below?

\begin{tabular}{|c|c|}
\hline $\begin{array}{c}\text { Type of Management } \\
\text { Function }\end{array}$ & Total \\
\hline Administration* & $\begin{array}{l}\text { obs: } 51 \\
\text { min: } 4294 \\
\text { max: } 2918600 \\
\text { mean: } 380372.77 \\
\text { median: } 210201\end{array}$ \\
\hline Agency monitoring & $\begin{array}{l}\text { obs: } 51 \\
\text { min: } 0 \\
\text { max: } 363388 \\
\text { mean: } 127001.74 \\
\text { median: } 130201.5\end{array}$ \\
\hline Other (specify) & $\begin{array}{l}\text { obs: } 51 \\
\text { min: } 0 \\
\text { max: } 1032807 \\
\text { mean: } 144389.08 \\
\text { median: } 36704\end{array}$ \\
\hline TOTAL & $\begin{array}{l}\text { obs: } 51 \\
\text { min: } 0 \\
\text { max: } 2918600 \\
\text { mean: } 472012.5 \\
\text { median: } 289151\end{array}$ \\
\hline
\end{tabular}

* Includes planning, finance and accounting, clerical support, outreach, and evaluation.

19. How important were leveraged funds (i.e., funds that support the weatherization program that are not provided by DOE) for your state's weatherization program in PY 2008? (Check best answer). Include leveraged funds not reported by the state but available at the local level.

\begin{tabular}{rrrr} 
& Freq. & Percent & Cum. \\
\hline not important at all | & 5 & 10.20 & 10.20 \\
not very important | & 4 & 8.16 & 18.37 \\
important $\mid$ & 13 & 26.53 & 44.90 \\
very important | & 27 & 55.10 & 100.00 \\
- Total $^{\mid}$ & 49 & 100.00 &
\end{tabular}

20. Did your state set aside funding to advocate for leveraged resources in Program Year 2008 ?

Freq. Percent Cum.

\begin{tabular}{|c|c|c|c|}
\hline no & 35 & 71.43 & 71.43 \\
\hline yes & 14 & 28.57 & 100.00 \\
\hline Total | & 49 & 100.00 & \\
\hline
\end{tabular}


21. What organizations advocated for leveraged resources in Program Year 2008? (Check all that apply)

Your state office

$\begin{array}{crrr} & \text { Freq. } & \text { Percent } & \text { Cum. } \\ \text { no } \mid & 9 & 56.25 & 56.25 \\ \text { yes } \mid & 7 & 43.75 & 100.00 \\ -------- \\ \text { Total | } & 16 & 100.00\end{array}$

Your state's local weatherization agencies or agency associations

\begin{tabular}{|c|c|c|c|}
\hline & Freq. & Percent & Cum. \\
\hline no & 6 & 37.50 & 37.50 \\
\hline yes & 10 & 62.50 & 100.00 \\
\hline Total & 16 & 100.00 & \\
\hline
\end{tabular}

Non-profit organizations funded by your state

$\begin{array}{cccc} & \text { Freq. } & \text { Percent } & \text { Cum. } \\ \text { no } \mid & 13 & 81.25 & 81.25 \\ \text { yes } \mid & 3 & 18.75 & 100.00 \\ ---------- \\ \text { Total | } & 16 & 100.00\end{array}$

Other

Freq. Percent Cum.

$\begin{array}{crrr}\text { no } \mid & 15 & 93.75 & 93.75 \\ \text { yes } \mid & 1 & 6.25 & 100.00 \\ ------- & \\ \text { Total } & 16 & 100.00\end{array}$

22. How successful would you rate your state's efforts to acquire leveraged funds in Program Year 2008 ? (Check best answer). Include leveraged funds not reported by the state but available at the local level.

$\begin{array}{rrrr} & \text { Freq. } & \text { Percent } & \text { Cum. } \\ \text { not successful at all | } & 2 & 4.08 & 4.08 \\ \text { not very successful | } & 7 & 14.29 & 18.37 \\ \text { successful | } & 23 & 46.94 & 65.31 \\ \text { very successful | } & 10 & 20.41 & 85.71 \\ \text { state does not seek leveraged funds | } & 7 & 14.29 & 100.00 \\ - & \text { Total } & 49 & 100.00\end{array}$


23. What factors limited the success of your state's efforts to acquire leveraged funding in Program Year 2008 ?

24. Have you modified your state's weatherization program practices or regulations in the three years prior to Program Year 2008 to facilitate spending and reporting on leveraged resources?

$\begin{array}{cccc} & \text { Freq. } & \text { Percent } & \text { Cum. } \\ \text { no } \mid & 39 & 81.25 & 81.25 \\ \text { yes } \mid & 9 & 18.75 & 100.00 \\ ----------- & \end{array}$

25. Please indicate the number of staff that supported your state's weatherization program and their work effort in Program Year 2008. In considering the number of staff, please include everyone who worked full- or part-time or who worked with the weatherization program as well as other state programs. Also include any non-agency staff supporting the state program who work under contract to the state.

\begin{tabular}{|c|c|c|}
\hline $\begin{array}{c}\text { Type of Administrative } \\
\text { Function }\end{array}$ & $\begin{array}{c}\text { Number of Staff (\# } \\
\text { persons) }\end{array}$ & $\begin{array}{c}\text { Staff Work Effort } \\
\text { (FTE) }\end{array}$ \\
\hline Management/administration & $\begin{array}{l}\text { obs: } 48 \\
\text { min: } .75 \\
\text { max: } 22 \\
\text { mean: } 3.90 \\
\text { median: } 3\end{array}$ & $\begin{array}{l}\text { obs: } 40 \\
\text { min: } .25 \\
\text { max: } 12 \\
\text { mean: } 2.62 \\
\text { median: } 2\end{array}$ \\
\hline Agency monitoring & $\begin{array}{l}\text { obs: } 45 \\
\text { min: } .5 \\
\text { max: } 18 \\
\text { mean: } 3.63 \\
\text { median: } 2\end{array}$ & $\begin{array}{l}\text { obs: } 37 \\
\text { min: } .05 \\
\text { max: } 18 \\
\text { mean: } 2.84 \\
\text { median: } 1.75\end{array}$ \\
\hline $\begin{array}{l}\text { Training and Technical } \\
\text { Assistance }\end{array}$ & $\begin{array}{l}\text { obs: } 39 \\
\text { min: } 0 \\
\text { max: } 32 \\
\text { mean: } 3.12 \\
\text { median: } 1\end{array}$ & $\begin{array}{l}\text { obs: } 34 \\
\text { min: } .3 \\
\text { max: } 13.52 \\
\text { mean: } 2.70 \\
\text { median: } 1\end{array}$ \\
\hline Other (specify) & $\begin{array}{l}\text { obs: } 15 \\
\text { min: } 0 \\
\text { max: } 9 \\
\text { mean: } 2.07 \\
\text { median: } 1\end{array}$ & $\begin{array}{l}\text { obs: } 12 \\
\text { min: } 0 \\
\text { max: } 6.8 \\
\text { mean: } 1.45 \\
\text { median: } .75\end{array}$ \\
\hline TOTAL & $\begin{array}{l}\text { obs: } 51 \\
\text { min: } 0 \\
\text { max: } 57 \\
\text { mean: } 9.86 \\
\text { median: } 7\end{array}$ & $\begin{array}{l}\text { obs: } 51 \\
\text { min: } 0 \\
\text { max: } 34 \\
\text { mean: } 6.26 \\
\text { median: } 4\end{array}$ \\
\hline
\end{tabular}


26. For the in-house staff working on your state's weatherization program in each of the following functional areas in Program Year 2008, please indicate their collective level of experience with the weatherization program.

\begin{tabular}{|c|c|c|c|c|c|c|}
\hline & $\begin{array}{l}\text { Very } \\
\text { High }\end{array}$ & High & & Medium & Low & $\begin{array}{l}\text { Very } \\
\text { Low }\end{array}$ \\
\hline \multirow{6}{*}{ Management/administration } & \multicolumn{4}{|c|}{$\begin{array}{ll} & 1 \\
\text { Percent } & \text { Cum. }\end{array}$} & & \\
\hline & low & 3 & 6.12 & 6.12 & & \\
\hline & medium | & 7 & 14.29 & 20.41 & & \\
\hline & high & 15 & 30.61 & 51.02 & & \\
\hline & very high & 24 & 48.98 & 100.00 & & \\
\hline & Total $\mid$ & 49 & 100.00 & & & \\
\hline \multirow{7}{*}{ Field monitoring/auditing } & & Freq. & Percent & Cum. & & \\
\hline & very low & 1 & 2.04 & 2.04 & & \\
\hline & low & 2 & 4.08 & 6.12 & & \\
\hline & medium | & 5 & 10.20 & 16.33 & & \\
\hline & high & 12 & 24.49 & 40.82 & & \\
\hline & very high | & 29 & 59.18 & 100.00 & & \\
\hline & Total | & 49 & 100.00 & & & \\
\hline \multirow{7}{*}{$\begin{array}{l}\text { Training and Technical } \\
\text { Assistance }\end{array}$} & & Freq. & Percent & Cum. & & \\
\hline & very low & 1 & 2.13 & 2.13 & & \\
\hline & & 2 & 4.26 & 6.38 & & \\
\hline & medium & 6 & 12.77 & 19.15 & & \\
\hline & high & 13 & 27.66 & 46.81 & & \\
\hline & very high | & 25 & 53.19 & 100.00 & & \\
\hline & Total & 47 & 100.00 & & & \\
\hline \multirow{5}{*}{ Other (specify) } & & Freq. & Percent & Cum. & & \\
\hline & very low & 1 & 25.00 & 25.00 & & \\
\hline & low & 1 & 25.00 & 50.00 & & \\
\hline & very high $\mid$ & 2 & 50.00 & 100.00 & & \\
\hline & Total & 4 & 100.00 & & & \\
\hline
\end{tabular}


27. For the in-house staff working on your state's weatherization program in each of the following functional areas in Program Year 2008, please indicate the amount of turnover in staff over a three year period that included Program Year 2008: Turnover is defined as the number of new staff in a functional area in the past three years divided by the total number of staff working in that functional area. If a particular position has had more than one new person during the past three years (e.g., Person 1 leaves, Person 2 is hired to take Person 1's position, then Person 2 leaves and a third person is hired), just count that as one new staff person. (Please check appropriate boxes)

\begin{tabular}{|c|c|c|c|c|c|c|c|}
\hline & $\begin{array}{c}\text { No } \\
\text { Turnover }\end{array}$ & & $10 \%$ & $\begin{array}{l}11 \text { to } \\
25 \%\end{array}$ & 26 to $50 \%$ & $\begin{array}{l}51 \text { to } \\
75 \%\end{array}$ & $\begin{array}{l}76 \text { to } \\
100 \%\end{array}$ \\
\hline \multirow{7}{*}{$\begin{array}{l}\text { Management/ } \\
\text { administration }\end{array}$} & \multicolumn{4}{|c|}{$\begin{array}{ll}\text { Freq. } & \text { Percent }\end{array}$} & & & \\
\hline & 1 to $10 \%$ & 9 & 20.45 & 20.45 & & & \\
\hline & 26 to $50 \%$ & 6 & 13.64 & 34.09 & & & \\
\hline & 51 to $75 \%$ & 2 & 4.55 & 38.64 & & & \\
\hline & 76 to $100 \%$ & 2 & 4.55 & 43.18 & & & \\
\hline & no turnover & 25 & 56.82 & 100.00 & & & \\
\hline & Total | & 44 & 100.00 & & & & \\
\hline \multirow{8}{*}{$\begin{array}{l}\text { Field monitoring/ } \\
\text { Auditing }\end{array}$} & & Freq. & Percent & Cum. & & & \\
\hline & 1 to $10 \%$ & 9 & 20.00 & 20.00 & & & \\
\hline & 11 to $25 \%$ & 1 & 2.22 & 22.22 & & & \\
\hline & 26 to $50 \%$ & 5 & 11.11 & 33.33 & & & \\
\hline & 51 to $75 \%$ & 3 & 6.67 & 40.00 & & & \\
\hline & 76 to $100 \%$ & 5 & 11.11 & 51.11 & & & \\
\hline & no turnover $\mid$ & 22 & 48.89 & 100.00 & & & \\
\hline & Total & 45 & 100.00 & & & & \\
\hline \multirow{7}{*}{$\begin{array}{l}\text { Training and } \\
\text { Technical } \\
\text { Assistance }\end{array}$} & & Freq. & Percent & Cum. & & & \\
\hline & 1 to $10 \%$ & 6 & 15.00 & 15.00 & & & \\
\hline & 26 to $50 \%$ & 4 & 10.00 & 25.00 & & & \\
\hline & 51 to $75 \%$ & 1 & 2.50 & 27.50 & & & \\
\hline & 76 to $100 \%$ & 3 & 7.50 & 35.00 & & & \\
\hline & no turnover $\mid$ & 26 & 65.00 & 100.00 & & & \\
\hline & Total | & 40 & 100.00 & & & & \\
\hline \multirow{5}{*}{ Other (specify) } & & Freq. & Percent & Cum. & & & \\
\hline & 1 to $10 \%$ & 1 & 25.00 & 25.00 & & & \\
\hline & 51 to $75 \%$ & 1 & 25.00 & 50.00 & & & \\
\hline & no turnover $\mid$ & 2 & 50.00 & 100.00 & & & \\
\hline & Total | & 4 & 100.00 & & & & \\
\hline
\end{tabular}


28. The Federal Regulations governing the Weatherization Assistance Program define children as "dependents not exceeding 19 years or a lesser age set forth in the State plan." What age did you state use in your state's definition of children in Program Year 2008?

\begin{tabular}{|l|c|}
\hline observations: & 46 \\
\hline missing values: & 5 \\
\hline mean: & 14.91 \\
\hline standard deviation: & 5.60 \\
\hline min: & 5 \\
\hline 10th percentile: & 6 \\
\hline 25th percentile: & 6 \\
\hline median: & 18 \\
\hline 75th percentile: & 19 \\
\hline 90th percentile: & 19 \\
\hline max: & 19 \\
\hline
\end{tabular}

29. Did your state use a high energy burden category to prioritize the provision of weatherization services in Program Year 2008?

Freq. Percent Cum.

$\begin{array}{cccc}\text { no } \mid & 21 & 42.00 & 42.00 \\ \text { yes } \mid & 29 & 58.00 & 100.00 \\ ------- & \end{array}$

29a. How was 'high energy burden' defined?

30. Did your state use a high energy expenditure category to prioritize the provision of weatherization services in Program Year 2008?

$\begin{array}{rrrr} & \text { Freq. } & \text { Percent } & \text { Cum. } \\ \text { no } \mid & 31 & 62.00 & 62.00 \\ \text { yes } \mid & 19 & 38.00 & 100.00 \\ ---- & \end{array}$

30a. How was 'high energy expenditure' defined?

31. What were the income guidelines for households to be eligible for your state's weatherization program in Program Year 2008? (Check all that apply) 
$100 \%$ of Federal Poverty Guidelines

$\begin{array}{rrrr} & \text { Freq. } & \text { Percent } & \text { Cum. } \\ \text { no | } & 48 & 96.00 & 96.00 \\ \text { yes | } & 2 & 4.00 & 100.00 \\ ----------- & \end{array}$

$125 \%$ of Federal Poverty Guidelines

\begin{tabular}{rrrr} 
& Freq. & Percent & Cum. \\
no | & 44 & 88.00 & 88.00 \\
yes | & 6 & 12.00 & 100.00 \\
\hline Total | & 50 & 100.00 & \\
& & & \\
& $\mathbf{1 5 0 \%}$ of Federal Poverty Guidelines
\end{tabular}

$\begin{array}{rrrr} & \text { Freq. } & \text { Percent } & \text { Cum. } \\ \text { no } \mid & 27 & 54.00 & 54.00 \\ \text { yes } \mid & 23 & 46.00 & 100.00 \\ ----------- \\ \text { Total| } & 50 & 100.00\end{array}$

$185 \%$ of Federal Poverty Guidelines

$\begin{array}{cccc} & \text { Freq. } & \text { Percent } & \text { Cum. } \\ \text { no | } & 48 & 96.00 & 96.00 \\ \text { yes | } & 2 & 4.00 & 100.00 \\ -------- & \end{array}$

More than $185 \%$ of Federal Poverty Guidelines

\begin{tabular}{|c|c|c|c|}
\hline & Freq. & Percent & Cum. \\
\hline no & 50 & 100.00 & 100.00 \\
\hline Total | & 50 & 100.00 & \\
\hline
\end{tabular}


$60 \%$ of state median income

$\begin{array}{rrrr} & \text { Freq. } & \text { Percent } & \text { Cum. } \\ \text { no | } & 37 & 74.00 & 74.00 \\ \text { yes } \mid & 13 & 26.00 & 100.00 \\ ------------ \\ \text { Total| } & 50 & 100.00 & \end{array}$

Other:

$\begin{array}{rrrr} & & & \\ & \text { Freq. } & \text { Percent } & \text { Cum. } \\ \text { no | } & 39 & 78.00 & 78.00 \\ ---- & 11 & 22.00 & 100.00 \\ \text { Total | } & 50 & 100.00 & \end{array}$

\section{Section B: PROGRAM OPERATIONS AND IMPLEMENTATION}

1. Using the following scale, how adequate was the Program Year 2008 funding received by your state from ALL funding sources for weatherizing the stock of eligible low-income dwelling units in your state in a timely fashion? (Check best answer)

\begin{tabular}{|c|c|c|c|}
\hline & Freq. & Percent & Cum. \\
\hline very inadequate | & 8 & 16.33 & 16.33 \\
\hline inadequate & 23 & 46.94 & 63.27 \\
\hline adequate & 16 & 32.65 & 95.92 \\
\hline very adequate & 2 & 4.08 & 100.00 \\
\hline Total & 49 & 100.00 & \\
\hline
\end{tabular}

2. What was the quality of the management support that your state received from DOE and its contractors in Program Year 2008? (Check best answer)

Freq. Percent Cum.

$\begin{array}{rrrr}\text { very low quality | } & 1 & 2.04 & 2.04 \\ \text { low quality | } & 1 & 2.04 & 4.08 \\ \text { moderate quality | } & 24 & 48.98 & 53.06 \\ \text { high quality | } & 18 & 36.73 & 89.80 \\ \text { very high quality | } & 4 & 8.16 & 97.96 \\ \text { not applicable | } & 1 & 2.04 & 100.00 \\ - \text { Total | } & 49 & 100.00 & \end{array}$

2a. If appropriate, why did you rate the quality very low or low?

3. What was the quality of the training that your state received from DOE and its contractors in Program Year 2008? (Check best answer) 


\begin{tabular}{|c|c|c|c|}
\hline & Freq. & Percent & Cum. \\
\hline very low quality & 1 & 2.04 & 2.04 \\
\hline low quality & 1 & 2.04 & 4.08 \\
\hline moderate quality & 24 & 48.98 & 53.06 \\
\hline high quality & 13 & 26.53 & 79.59 \\
\hline very high quality & 3 & 6.12 & 85.71 \\
\hline not applicable & 7 & 14.29 & 100.00 \\
\hline Total & 49 & 100.00 & \\
\hline
\end{tabular}

3a. If appropriate, why did you rate the quality very low or low?

4. What was the quality of the support and assistance on client education that your state received from DOE and its contractors in Program Year 2008? (Check best answer)

Freq. Percent Cum.

\begin{tabular}{rrrr} 
very low quality | & 2 & 4.17 & 4.17 \\
low quality | & 8 & 16.67 & 20.83 \\
moderate quality | & 13 & 27.08 & 47.92 \\
high quality | & 6 & 12.50 & 60.42 \\
very high quality | & 2 & 4.17 & 64.58 \\
not applicable | & 17 & 35.42 & 100.00 \\
\hline------------- \\
Total |
\end{tabular}

4a. If appropriate, why did you rate the quality very low or low?

5. What was the quality of the support and assistance on leveraging the Weatherization Assistance Program funding provided by DOE with other funding sources in Program Year 2008? (Check best answer)

Freq. Percent Cum.

$\begin{array}{rrrr}\text { very low quality | } & 1 & 2.08 & 2.08 \\ \text { low quality } & 6 & 12.50 & 14.58 \\ \text { moderate quality | } & 14 & 29.17 & 43.75 \\ \text { high quality | } & 4 & 8.33 & 52.08 \\ \text { very high quality | } & 3 & 6.25 & 58.33 \\ \text { not applicable | } & 20 & 41.67 & 100.00 \\ - \text { Total }^{-} & 48 & 100.00 & \end{array}$

5a. If appropriate, why did you rate the quality very low or low? 
6. What was the quality of the technical support (e.g., measure selection and installation) that your state received from DOE and its contractors in Program Year 2008? (Check best answer)

\begin{tabular}{|c|c|c|c|}
\hline & Freq. & Percent & Cum. \\
\hline very low quality & 1 & 2.13 & 2.13 \\
\hline low quality & 4 & 8.51 & 10.64 \\
\hline moderate quality & 15 & 31.91 & 42.55 \\
\hline high quality & 12 & 25.53 & 68.09 \\
\hline very high quality | & 5 & 10.64 & 78.72 \\
\hline not applicable & 10 & 21.28 & 100.00 \\
\hline Total & 47 & 100.00 & \\
\hline
\end{tabular}

6a. If appropriate, why did you rate the quality very low or low?

7. How flexible did you find the DOE program rules that governed the weatherization program in Program Year 2008? In other words, did the program rules allow your state to tailor your program to your needs (very flexible) or proscribe your program to only one way of operation (very inflexible)? (Check best answer)

Freq. Percent Cum.

$\begin{array}{rrrr}\text { very inflexible | } & 1 & 2.08 & 2.08 \\ \text { inflexible | } & 5 & 10.42 & 12.50 \\ \text { flexible | } & 40 & 83.33 & 95.83 \\ \text { very flexible | } & 2 & 4.17 & 100.00 \\ ------------- \\ \text { Total | } & 48 & 100.00\end{array}$

7a. Using Program Year 2008 as the reference point, how should the program rules change? (Check best answer)

Become much more flexible

Become more flexible

Stay about the same

Become more inflexible

Become much more inflexible

Freq. Percent Cum.

\begin{tabular}{r|ccc} 
stay about the same | & 19 & 39.58 & 39.58 \\
become more flexible | & 22 & 45.83 & 85.42 \\
become much more flexible | & 7 & 14.58 & 100.00 \\
- Total | & 48 & 100.00 &
\end{tabular}

7b. In what areas should the program rules become more flexible?

7c. In what areas should the program rules become less flexible? 
8. Please describe any important political issues faced by your state's weatherization program in Program Year 2008.

9. Using Program Year 2008 as a reference point, how important was improving administrative support and assistance from DOE and its contractors in improving your state's ability to deliver low-income weatherization services? (Check best answer) Thinking back to Program Year 2008, how great did you perceive the need to be for improvements in administrative support and assistance from DOE and its contractors to help your state deliver low-income weatherization services?

\begin{tabular}{|c|c|c|c|}
\hline & Freq. & Percent & Cum \\
\hline very unimportant & 2 & 4.17 & 4.17 \\
\hline unimportant & 7 & 14.58 & 18.75 \\
\hline important & 22 & 45.83 & 64.5 \\
\hline very important & 11 & 22.92 & 87.50 \\
\hline not applicable & 6 & 12.50 & 100.00 \\
\hline Tota & 48 & 100.00 & \\
\hline
\end{tabular}

10. Using Program Year 2008 as a reference point, how important was improving training from DOE and its contractors in improving your state's ability to deliver low-income weatherization services? (Check best answer) Thinking back to Program Year 2008, how great did you perceive the need to be for improvements in training from DOE and its contractors to help your state deliver low-income weatherization services?

Freq. Percent Cum.

$\begin{array}{rrrr}\text { very unimportant | } & 2 & 4.17 & 4.17 \\ \text { unimportant | } & 5 & 10.42 & 14.58 \\ \text { important | } & 23 & 47.92 & 62.50 \\ \text { very important | } & 16 & 33.33 & 95.83 \\ \text { not applicable | } & 2 & 4.17 & 100.00 \\ -----------------------\end{array}$


11. Using Program Year 2008 as a reference point, how important was improving assistance on client education from DOE and its contractors in improving your state's ability to deliver low-income weatherization services? (Check best answer) Thinking back to Program Year 2008, how great did you perceive the need to be for improvements in assistance on client education from DOE and its contractors to help your state deliver low-income weatherization services?

\begin{tabular}{|c|c|c|c|}
\hline & Freq. & Percent & Cum. \\
\hline very unimportant | & 4 & 8.33 & 8.33 \\
\hline unimportant & 10 & 20.83 & 29.17 \\
\hline important & 19 & 39.58 & 68.75 \\
\hline very important & 9 & 18.75 & 87.50 \\
\hline not applicable & 6 & 12.50 & 100.00 \\
\hline Total & 48 & 100.00 & \\
\hline
\end{tabular}

12. Using Program Year 2008 as a reference point, how important was improving assistance from DOE and its contractors on leveraging the Weatherization Assistance Program provided by DOE with other funding sources? (Check best answer) Thinking back to Program Year 2008, how great did you perceive the need to be for improvements in assistance from DOE and its contractors on leveraging the Weatherization Assistance Program to help your state deliver low-income weatherization services?

\begin{tabular}{|c|c|c|c|}
\hline & Freq. & Percent & Cum \\
\hline very unimportant | & 2 & 4.17 & 4.17 \\
\hline unimportant & 8 & 16.67 & 20.83 \\
\hline important & 18 & 37.50 & 58.33 \\
\hline very important & 9 & 18.75 & 77.08 \\
\hline not applicable & 11 & 22.92 & 100.00 \\
\hline Tota & 48 & 100.00 & \\
\hline
\end{tabular}

13. Using Program Year 2008 as a reference point, how important was improving technical support from DOE and its contractors in improving your state's ability to deliver low-income weatherization services? (Check best answer) Thinking back to Program Year 2008, how great did you perceive the need to be for improvements in technical support from DOE and its contractors to help your state deliver low-income weatherization services?

Freq. Percent Cum.

$\begin{array}{rrrr}\text { very unimportant | } & 3 & 6.25 & 6.25 \\ \text { unimportant | } & 4 & 8.33 & 14.58 \\ \text { important } \mid & 23 & 47.92 & 62.50 \\ \text { very important | } & 16 & 33.33 & 95.83 \\ \text { not applicable | } & 2 & 4.17 & 100.00 \\ -------------- & \end{array}$

14. Using Program Year 2008 as a reference point, how important was increased weatherization funding in improving your state's ability to deliver low-income weatherization services? (Check best 
answer) Thinking back to Program Year 2008, how great did you perceive the need to be for improvements in weatherization funding to help your state deliver low-income weatherization services? "Increased weatherization funding" refers to funding increases going into Program Year 2008. If a state did not experience funding increases going into Program Year 2008, mark not applicable.

Very Important

Somewhat Important

Somewhat Unimportant

Very Unimportant

Not applicable

\begin{tabular}{|c|c|c|c|}
\hline & Freq. & Percent & Cum. \\
\hline important | & 11 & 23.40 & 23.40 \\
\hline very important & 31 & 65.96 & 89.36 \\
\hline not applicable & 5 & 10.64 & 100.00 \\
\hline Tota & 47 & 100.00 & \\
\hline
\end{tabular}

15. Using Program Year 2008 as a reference point, how important was improving data and information systems for managing the delivery of weatherization services? (Check best answer) Thinking back to Program Year 2008, how great did you perceive the need to be for improvements in data and information systems to help your state deliver low-income weatherization services?

Freq. Percent Cum.

\begin{tabular}{r|rrr} 
very unimportant | & 2 & 4.08 & 4.08 \\
unimportant | & 3 & 6.12 & 10.20 \\
important | & 15 & 30.61 & 40.82 \\
very important | & 27 & 55.10 & 95.92 \\
not applicable | & 2 & 4.08 & 100.00
\end{tabular}

Total| $49 \quad 100.00$ 


\section{Section C: TRAINING}

1. For those staff working in your state's weatherization office who need to have knowledge about the following list of weatherization topics, how well trained were they in each area in Program Year 2008? Please use the following scale: 1 -not at all well trained; 2 -not well trained; 3 -moderately well trained; 4 -well trained; 5 - very well trained; 6 - not applicable (Circle best answer).

\section{(1) Diagnostic procedures}

$\begin{array}{rrrr} & \text { Freq. } & \text { Percent } & \text { Cum. } \\ \text { not at all well trained | } & 1 & 2.04 & 2.04 \\ \text { not well trained | } & 5 & 10.20 & 12.24 \\ \text { moderately well trained | } & 7 & 14.29 & 26.53 \\ \text { well trained | } & 15 & 30.61 & 57.14 \\ \text { very well trained | } & 19 & 38.78 & 95.92 \\ \text { not applicable | } & 2 & 4.08 & 100.00 \\ \text { - Total | } & 49 & 100.00 & \end{array}$

\section{(2) Insulation}

\section{-- single family dwellings}

$\begin{array}{rrrr} & \text { Freq. } & \text { Percent } & \text { Cum. } \\ \text { not at all well trained | } & 1 & 2.04 & 2.04 \\ \text { not well trained | } & 1 & 2.04 & 4.08 \\ \text { moderately well trained | } & 6 & 12.24 & 16.33 \\ \text { well trained | } & 15 & 30.61 & 46.94 \\ \text { very well trained | } & 24 & 48.98 & 95.92 \\ \text { not applicable | } & 2 & 4.08 & 100.00 \\ \text { Total | } & 49 & 100.00 & \end{array}$

-- multifamily dwellings

$\begin{array}{rrrr} & \text { Freq. } & \text { Percent } & \text { Cum. } \\ \text { not at all well trained | } & 3 & 6.25 & 6.25 \\ \text { not well trained | } & 7 & 14.58 & 20.83 \\ \text { moderately well trained | } & 16 & 33.33 & 54.17 \\ \text { well trained | } & 4 & 8.33 & 62.50 \\ \text { very well trained | } & 11 & 22.92 & 85.42 \\ \text { not applicable | } & 7 & 14.58 & 100.00 \\ \text { Total | } & 48 & 100.00 & \end{array}$




\section{-- mobile homes}

Freq. Percent Cum.

\begin{tabular}{r|rrr} 
not at all well trained & 1 & 2.13 & 2.13 \\
not well trained | & 1 & 2.13 & 4.26 \\
moderately well trained | & 8 & 17.02 & 21.28 \\
well trained | & 16 & 34.04 & 55.32 \\
very well trained | & 18 & 38.30 & 93.62 \\
not applicable | & 3 & 6.38 & 100.00 \\
- Total | & 47 & 100.00 &
\end{tabular}

(3) Space heating, ventilation, air conditioning -- single family dwellings

Freq. Percent Cum.

$\begin{array}{rrrr}\text { not at all well trained | } & 1 & 2.08 & 2.08 \\ \text { not well trained | } & 4 & 8.33 & 10.42 \\ \text { moderately well trained | } & 7 & 14.58 & 25.00 \\ \text { well trained | } & 14 & 29.17 & 54.17 \\ \text { very well trained | } & 20 & 41.67 & 95.83 \\ \text { not applicable | } & 2 & 4.17 & 100.00 \\ - \text { Total | } & 48 & 100.00 & \end{array}$

-- multifamily dwellings

Freq. Percent Cum.

\begin{tabular}{|c|c|c|c|}
\hline not at all well trained & 2 & 4.17 & 4.17 \\
\hline not well trained & 12 & 25.00 & 29.17 \\
\hline moderately well trained & 9 & 18.75 & 47.92 \\
\hline well trained & 8 & 16.67 & 64.58 \\
\hline very well trained & 10 & 20.83 & 85.42 \\
\hline not applicable & 7 & 14.58 & 100.00 \\
\hline Total & 48 & 100.00 & \\
\hline
\end{tabular}

-- mobile homes

Freq. Percent Cum.

\begin{tabular}{rrrr} 
not at all well trained | & 1 & 2.04 & 2.04 \\
not well trained | & 4 & 8.16 & 10.20 \\
moderately well trained | & 7 & 14.29 & 24.49 \\
well trained | & 14 & 28.57 & 53.06 \\
very well trained | & 20 & 40.82 & 93.88 \\
not applicable | & 3 & 6.12 & 100.00 \\
\hline---+---- &
\end{tabular}




\begin{tabular}{|c|c|c|c|}
\hline \multicolumn{4}{|c|}{$\begin{array}{l}\text { (4) Infiltration measures } \\
\text {-- single family dwellings }\end{array}$} \\
\hline & Freq. & Percent & Cum. \\
\hline not at all well trained & 1 & 2.04 & 2.04 \\
\hline not we & 1 & 2.04 & 4.08 \\
\hline moderately well trained & 5 & 10.20 & 14.29 \\
\hline well trained & 15 & 30.61 & 44.90 \\
\hline very well trained & 25 & 51.02 & 95.92 \\
\hline not applicable & 2 & 4.08 & 100.00 \\
\hline Total & 49 & 100.00 & \\
\hline
\end{tabular}

-- multifamily dwellings

\begin{tabular}{rrrr} 
& Freq. & Percent & Cum. \\
not at all well trained | & 2 & 4.17 & 4.17 \\
not well trained | & 9 & 18.75 & 22.92 \\
moderately well trained | & 12 & 25.00 & 47.92 \\
well trained | & 8 & 16.67 & 64.58 \\
very well trained | & 10 & 20.83 & 85.42 \\
not applicable | & 7 & 14.58 & 100.00 \\
\hline Total | & 48 & 100.00 &
\end{tabular}

-- mobile homes

Freq. Percent Cum.

\begin{tabular}{|c|c|c|c|}
\hline not at all well trained & 1 & 2.04 & 2.04 \\
\hline not well trained & 2 & 4.08 & 6.12 \\
\hline moderately well trained & 4 & 8.16 & 14.29 \\
\hline well trained & 16 & 32.65 & 46.94 \\
\hline very well trained & 23 & 46.94 & 93.88 \\
\hline not applicable & 3 & 6.12 & 100.00 \\
\hline Total & 49 & 100.00 & \\
\hline
\end{tabular}


(5) Doors and windows

-- single family dwellings

Freq. Percent Cum.

\begin{tabular}{|c|c|c|c|}
\hline not at all well trained $\mid$ & 1 & 2.08 & 2.08 \\
\hline not well trained & 2 & 4.17 & 6.25 \\
\hline moderately well trained & 3 & 6.25 & 12.50 \\
\hline well trained & 19 & 39.58 & 52.08 \\
\hline very well trained & 20 & 41.67 & 93.75 \\
\hline not applicable & 3 & 6.25 & 100.00 \\
\hline Total & 48 & 100.00 & \\
\hline
\end{tabular}

-- multifamily dwellings

Freq. Percent Cum.

\begin{tabular}{r|rrr} 
not at all well trained | & 2 & 4.26 & 4.26 \\
not well trained | & 8 & 17.02 & 21.28 \\
moderately well trained | & 6 & 12.77 & 34.04 \\
well trained | & 14 & 29.79 & 63.83 \\
very well trained | & 10 & 21.28 & 85.11 \\
not applicable | & 7 & 14.89 & 100.00 \\
- Total | & 47 & 100.00 &
\end{tabular}

-- mobile homes

Freq. Percent Cum.

\begin{tabular}{|c|c|c|c|}
\hline not at all well trained & 1 & 2.08 & 2.08 \\
\hline not well trained & 2 & 4.17 & 6.25 \\
\hline moderately well trained & 4 & 8.33 & 14.58 \\
\hline well trained & 17 & 35.42 & 50.00 \\
\hline very well trained & 20 & 41.67 & 91.67 \\
\hline not applicable & 4 & 8.33 & 100.00 \\
\hline Total & 48 & 100.00 & \\
\hline
\end{tabular}

(6) Hot water heating

-- single family dwellings

Freq. Percent Cum.

$\begin{array}{rrrr}- & & & \\ \text { not at all well trained | } & 1 & 2.04 & 2.04 \\ \text { not well trained | } & 2 & 4.08 & 6.12 \\ \text { moderately well trained | } & 4 & 8.16 & 14.29 \\ \text { well trained | } & 19 & 38.78 & 53.06 \\ \text { very well trained | } & 20 & 40.82 & 93.88 \\ \text { not applicable | } & 3 & 6.12 & 100.00 \\ - \text { Total } \mid & 49 & 100.00 & \end{array}$

-- multifamily dwellings 
Freq. Percent Cum.

$\begin{array}{rrrr}\text { not at all well trained | } & 2 & 4.26 & 4.26 \\ \text { not well trained | } & 9 & 19.15 & 23.40 \\ \text { moderately well trained | } & 10 & 21.28 & 44.68 \\ \text { well trained | } & 10 & 21.28 & 65.96 \\ \text { very well trained | } & 10 & 21.28 & 87.23 \\ \text { not applicable | } & 6 & 12.77 & 100.00 \\ - \text { Total } & 47 & 100.00 & \end{array}$

-- mobile homes

Freq. Percent Cum.

$\begin{array}{rrrr}\text { not at all well trained | } & 1 & 2.13 & 2.13 \\ \text { not well trained | } & 3 & 6.38 & 8.51 \\ \text { moderately well trained | } & 3 & 6.38 & 14.89 \\ \text { well trained | } & 19 & 40.43 & 55.32 \\ \text { very well trained | } & 17 & 36.17 & 91.49 \\ \text { not applicable | } & 4 & 8.51 & 100.00 \\ \text { Total | } & 47 & 100.00 & \end{array}$

(7) Baseloads (e.g., lighting, refrigerators)

-- single family dwellings

Freq. Percent Cum.

\begin{tabular}{|c|c|c|c|}
\hline not at all well trained & 1 & 2.04 & 2.04 \\
\hline not well trained & 3 & 6.12 & 8.16 \\
\hline moderately well trained & 4 & 8.16 & 16.33 \\
\hline well trained & 17 & 34.69 & 51.02 \\
\hline very well trained & 20 & 40.82 & 91.84 \\
\hline not applicable & 4 & 8.16 & 100.00 \\
\hline Total | & 49 & 100.00 & \\
\hline \multicolumn{4}{|c|}{-- multifamily dwellings } \\
\hline & Freq. & Percent & Cum. \\
\hline not at all well trained & 2 & 4.26 & 4.26 \\
\hline not well trained & 8 & 17.02 & 21.28 \\
\hline moderately well trained & 6 & 12.77 & 34.04 \\
\hline well trained & 11 & 23.40 & 57.45 \\
\hline very well trained & 11 & 23.40 & 80.85 \\
\hline not applicable & 9 & 19.15 & 100.00 \\
\hline Total | & 47 & 100.00 & \\
\hline
\end{tabular}

-- mobile homes

Freq. Percent Cum. 


\begin{tabular}{|c|c|c|c|}
\hline not at all well trained & 1 & 2.04 & 2.04 \\
\hline not well trained & 3 & 6.12 & 8.16 \\
\hline moderately well trained & 5 & 10.20 & 18.37 \\
\hline well trained & 17 & 34.69 & 53.06 \\
\hline very well trained & 18 & 36.73 & 89.80 \\
\hline not applicable & 5 & 10.20 & 100.00 \\
\hline Total & 49 & 100.00 & \\
\hline
\end{tabular}

1a. For those staff working in your state's weatherization office who need to have knowledge about the following list of administrative-related topics, how well trained were they in each area in Program Year 2008? Please use the following scale: 1 - not at all well trained; 2 -not well trained; 3 - moderately well trained; 4 -well trained; 5 - very well trained; 6 - not applicable (Circle best answer).

(1) Management

Freq. Percent Cum.

$\begin{array}{rrrr}\text { not well trained | } & 1 & 2.04 & 2.04 \\ \text { moderately well trained | } & 8 & 16.33 & 18.37 \\ \text { well trained } \mid & 23 & 46.94 & 65.31 \\ \text { very well trained | } & 17 & 34.69 & 100.00 \\ - \text { Total | } & 49 & 100.00 & \end{array}$

\section{(2) Client education}

Freq. Percent Cum.

\begin{tabular}{r|rrr} 
not well trained | & 6 & 12.24 & 12.24 \\
moderately well trained | & 14 & 28.57 & 40.82 \\
well trained | & 12 & 24.49 & 65.31 \\
very well trained | & 16 & 32.65 & 97.96 \\
not applicable | & 1 & 2.04 & 100.00 \\
- Total $\mid$ & 49 & 100.00 &
\end{tabular}

\begin{tabular}{|c|c|c|c|}
\hline \multicolumn{4}{|c|}{$\begin{array}{l}\text { (3) Auditing/estimating } \\
\text {-- single family dwellings }\end{array}$} \\
\hline & Freq. & Percent & Cum. \\
\hline not at all well trained & 1 & 2.04 & 2.04 \\
\hline not well trained & 5 & 10.20 & 12.24 \\
\hline moderately well trained & 6 & 12.24 & 24.49 \\
\hline well trained & 13 & 26.53 & 51.02 \\
\hline very we & 22 & 44.90 & 95.92 \\
\hline not applicable & 2 & 4.08 & 100.00 \\
\hline Total & 49 & 100.00 & \\
\hline
\end{tabular}

-- multifamily dwellings

Freq. Percent Cum. 


$\begin{array}{rrrr}\text { not at all well trained | } & 3 & 6.25 & 6.25 \\ \text { not well trained | } & 9 & 18.75 & 25.00 \\ \text { moderately well trained | } & 13 & 27.08 & 52.08 \\ \text { well trained | } & 6 & 12.50 & 64.58 \\ \text { very well trained | } & 11 & 22.92 & 87.50 \\ \text { not applicable | } & 6 & 12.50 & 100.00 \\ \text { Total | } & 48 & 100.00 & \end{array}$

-- mobile homes

$\begin{array}{rrrr} & \text { Freq. } & \text { Percent } & \text { Cum. } \\ \text { not at all well trained | } & 1 & 2.04 & 2.04 \\ \text { not well trained | } & 5 & 10.20 & 12.24 \\ \text { moderately well trained | } & 6 & 12.24 & 24.49 \\ \text { well trained | } & 12 & 24.49 & 48.98 \\ \text { very well trained | } & 23 & 46.94 & 95.92 \\ \text { not applicable | } & 2 & 4.08 & 100.00 \\ \text { Total | } & 49 & 100.00 & \end{array}$

(4) Monitoring/quality control

$\begin{array}{rrrr} & \text { Freq. } & \text { Percent } & \text { Cum. } \\ \text { moderately well trained | } & 9 & 18.37 & 18.37 \\ \text { well trained | } & 17 & 34.69 & 53.06 \\ \text { very well trained | } & 23 & 46.94 & 100.00 \\ ---------- & \\ \text { Total | } & 49 & 100.00 & \end{array}$

\section{(5) Financial topics}

$\begin{array}{rrrr} & \text { Freq. } & \text { Percent } & \text { Cum. } \\ \text { not well trained | } & 5 & 10.42 & 10.42 \\ \text { moderately well trained | } & 9 & 18.75 & 29.17 \\ \text { well trained | } & 15 & 31.25 & 60.42 \\ \text { very well trained | } & 19 & 39.58 & 100.00 \\ \text {------------ } & \\ \text { Total | } & 48 & 100.00 & \end{array}$


(6) Outreach and communications

$\begin{array}{rrrr} & \text { Freq. } & \text { Percent } & \text { Cum. } \\ \text { not at all well trained | } & 1 & 2.08 & 2.08 \\ \text { not well trained | } & 3 & 6.25 & 8.33 \\ \text { moderately well trained | } & 20 & 41.67 & 50.00 \\ \text { well trained | } & 8 & 16.67 & 66.67 \\ \text { very well trained | } & 16 & 33.33 & 100.00 \\ --------- & \\ \text { Total } & 48 & 100.00 & \end{array}$

\section{(7) Other (please specify)}

1b. For those staff working in your state's weatherization office who need to have knowledge about the following list of health and safety topics, how well trained were they in each area in Program Year 2008? Please use the following scale: 1 - not at all well trained; 2 - not well trained; 3 - moderately well trained; 4 -well trained; 5 - very well trained; 6 - not applicable (Circle best answer).

\section{(1) Fire safety}

$\begin{array}{rrrr} & \text { Freq. } & \text { Percent } & \text { Cum. } \\ \text { not at all well trained | } & 1 & 2.04 & 2.04 \\ \text { not well trained | } & 6 & 12.24 & 14.29 \\ \text { moderately well trained | } & 19 & 38.78 & 53.06 \\ \text { well trained | } & 11 & 22.45 & 75.51 \\ \text { very well trained | } & 8 & 16.33 & 91.84 \\ \text { not applicable | } & 4 & 8.16 & 100.00 \\ \text { Total | } & 49 & 100.00 & \end{array}$

\section{(2) Indoor air quality}

$\begin{array}{rrrr} & \text { Freq. } & \text { Percent } & \text { Cum. } \\ \text { not at all well trained | } & 1 & 2.04 & 2.04 \\ \text { not well trained | } & 5 & 10.20 & 12.24 \\ \text { moderately well trained | } & 4 & 8.16 & 20.41 \\ \text { well trained | } & 14 & 28.57 & 48.98 \\ \text { very well trained | } & 23 & 46.94 & 95.92 \\ \text { not applicable | } & 2 & 4.08 & 100.00 \\ \text { Total | } & 49 & 100.00 & \end{array}$




\section{(3) Measures to increase security of housing unit}

\begin{tabular}{r|rrr} 
& Freq. & Percent & Cum. \\
not at all well trained | & 3 & 6.25 & 6.25 \\
not well trained | & 14 & 29.17 & 35.42 \\
moderately well trained | & 9 & 18.75 & 54.17 \\
well trained | & 6 & 12.50 & 66.67 \\
very well trained | & 6 & 12.50 & 79.17 \\
not applicable | & 10 & 20.83 & 100.00 \\
- Total $^{-}$ & 48 & 100.00 &
\end{tabular}

(4) Measures to reduce common household hazards

Freq. Percent Cum.

$\begin{array}{rrrr}\text { not at all well trained | } & 1 & 2.04 & 2.04 \\ \text { not well trained | } & 5 & 10.20 & 12.24 \\ \text { moderately well trained | } & 12 & 24.49 & 36.73 \\ \text { well trained | } & 13 & 26.53 & 63.27 \\ \text { very well trained | } & 13 & 26.53 & 89.80 \\ \text { not applicable | } & 5 & 10.20 & 100.00 \\ \text { Total | } & 49 & 100.00 & \end{array}$

(5) Mold and mildew

$\begin{array}{rrrr} & \text { Freq. } & \text { Percent } & \text { Cum. } \\ \text { not at all well trained | } & 1 & 2.04 & 2.04 \\ \text { not well trained | } & 3 & 6.12 & 8.16 \\ \text { moderately well trained | } & 9 & 18.37 & 26.53 \\ \text { well trained | } & 16 & 32.65 & 59.18 \\ \text { very well trained | } & 18 & 36.73 & 95.92 \\ \text { not applicable | } & 2 & 4.08 & 100.00 \\ - \text { Total | } & 49 & 100.00 & \end{array}$

(6) Lead

\begin{tabular}{rrrr} 
& Freq. & Percent & Cum. \\
not at all well trained | & 1 & 2.04 & 2.04 \\
not well trained | & 4 & 8.16 & 10.20 \\
moderately well trained | & 10 & 20.41 & 30.61 \\
well trained | & 15 & 30.61 & 61.22 \\
very well trained | & 17 & 34.69 & 95.92 \\
not applicable | & 2 & 4.08 & 100.00 \\
\hline Total | & 49 & 100.00 &
\end{tabular}




\section{(7) Asbestos}

\begin{tabular}{|c|c|c|c|}
\hline & Freq. & Percent & Cum. \\
\hline not at all well trained & 3 & 6.25 & 6.25 \\
\hline not well trained & 7 & 14.58 & 20.83 \\
\hline moderately well trained & 16 & 33.33 & 54.17 \\
\hline well trained & 8 & 16.67 & 70.83 \\
\hline very well trained & 12 & 25.00 & 95.83 \\
\hline not applicable & 2 & 4.17 & 100.00 \\
\hline Tota & 48 & 100.00 & \\
\hline
\end{tabular}

(8) Vermiculite

Freq. Percent Cum.

$\begin{array}{rrrr}\text { not at all well trained | } & 4 & 8.33 & 8.33 \\ \text { not well trained | } & 8 & 16.67 & 25.00 \\ \text { moderately well trained | } & 15 & 31.25 & 56.25 \\ \text { well trained | } & 9 & 18.75 & 75.00 \\ \text { very well trained | } & 8 & 16.67 & 91.67 \\ \text { not applicable | } & 4 & 8.33 & 100.00 \\ \text { Total | } & 48 & 100.00 & \end{array}$

(9) General crew safety

$\begin{array}{rrrr} & \text { Freq. } & \text { Percent } & \text { Cum. } \\ \text { not at all well trained | } & 2 & 4.08 & 4.08 \\ \text { not well trained | } & 1 & 2.04 & 6.12 \\ \text { moderately well trained | } & 9 & 18.37 & 24.49 \\ \text { well trained | } & 18 & 36.73 & 61.22 \\ \text { very well trained | } & 15 & 30.61 & 91.84 \\ \text { not applicable | } & 4 & 8.16 & 100.00 \\ - \text { Total }^{-} & 49 & 100.00 & \end{array}$

(10) Other (please specify)

2. On which of the following weatherization subjects did staff working in your state's weatherization office receive training in Program Year 2008 from DOE, your state, or other entities? (Check all that apply)

(1) Diagnostic procedures

Freq. Percent Cum.

\begin{tabular}{|c|c|c|c|}
\hline no & 3 & 7.14 & 7.14 \\
\hline yes | & 39 & 92.86 & 100.00 \\
\hline Total & 42 & 100.00 & \\
\hline
\end{tabular}

\section{(2) Insulation}


-- single family dwellings

Freq. Percent Cum.

$\begin{array}{crrr}\text { no } \mid & 2 & 4.76 & 4.76 \\ \text { yes } \mid & 40 & 95.24 & 100.00 \\ ------------- \\ \text { Total } & 42 & 100.00\end{array}$

-- multifamily dwellings

Freq. Percent Cum.

$\begin{array}{cccc}\text { no } \mid & 26 & 61.90 & 61.90 \\ \text { yes } \mid & 16 & 38.10 & 100.00 \\ ------------- & \\ \text { Total } & 42 & 100.00\end{array}$

-- mobile homes

Freq. Percent Cum.

\begin{tabular}{|c|c|c|c|}
\hline no & 9 & 21.43 & 21.43 \\
\hline yes & 33 & 78.57 & 100.00 \\
\hline Total & 42 & 100.00 & \\
\hline
\end{tabular}

(3) Space heating, ventilation, air conditioning

-- single family dwellings

Freq. Percent Cum.

\begin{tabular}{|c|c|c|c|}
\hline no & 4 & 9.52 & 9.52 \\
\hline yes | & 38 & 90.48 & 100.00 \\
\hline Total | & 42 & 100.00 & \\
\hline
\end{tabular}

-- multifamily dwellings

Freq. Percent Cum.

\begin{tabular}{|c|c|c|c|}
\hline no & 24 & 57.14 & 57.14 \\
\hline yes | & 18 & 42.86 & 100.00 \\
\hline Total | & 42 & 100.00 & \\
\hline
\end{tabular}

-- mobile homes

Freq. Percent Cum.

\begin{tabular}{|c|c|c|c|}
\hline no & 8 & 19.05 & 19.05 \\
\hline yes | & 34 & 80.95 & 100.00 \\
\hline Total & 42 & 100.00 & \\
\hline
\end{tabular}

(4) Infiltration measures 
-- single family dwellings

Freq. Percent Cum.

$\begin{array}{crrr}\text { no } \mid & 4 & 9.52 & 9.52 \\ \text { yes } \mid & 38 & 90.48 & 100.00 \\ ------------- \\ \text { Total } & 42 & 100.00\end{array}$

-- multifamily dwellings Freq. Percent Cum.

$\begin{array}{cccc}\text { no } \mid & 24 & 57.14 & 57.14 \\ \text { yes } \mid & 18 & 42.86 & 100.00 \\ ------------- & \\ \text { Total } & 42 & 100.00\end{array}$

-- mobile homes Freq. Percent Cum.

\begin{tabular}{|c|c|c|c|}
\hline no & 7 & 16.67 & 16.67 \\
\hline yes $\mid$ & 35 & 83.33 & 100.00 \\
\hline Total & 42 & 100.00 & \\
\hline
\end{tabular}

(5) Doors and windows

-- single family dwellings

Freq. Percent Cum.

\begin{tabular}{|c|c|c|c|}
\hline no & 11 & 26.19 & 26.19 \\
\hline yes & 31 & 73.81 & 100.00 \\
\hline Total & 42 & 100.00 & \\
\hline
\end{tabular}

-- multifamily dwellings

Freq. Percent Cum.

\begin{tabular}{|c|c|c|c|}
\hline no & 27 & 64.29 & 64.29 \\
\hline yes & 15 & 35.71 & 100.00 \\
\hline Total & 42 & 100.00 & \\
\hline
\end{tabular}

-- mobile homes

Freq. Percent Cum.

\begin{tabular}{|c|c|c|c|}
\hline no & 14 & 33.33 & 33.33 \\
\hline yes & 28 & 66.67 & 100.00 \\
\hline Total & 42 & 100.00 & \\
\hline
\end{tabular}

(6) Hot water heating -- single family dwellings 
Freq. Percent Cum.

$\begin{array}{rrrr}\text { no | } & 11 & 26.19 & 26.19 \\ \text { yes | } & 31 & 73.81 & 100.00 \\ ------------------ \\ \text { Total } & 42 & 100.00\end{array}$

\begin{tabular}{|c|c|c|c|}
\hline \multicolumn{4}{|c|}{-- multifamily dwellings } \\
\hline & Freq. & Percent & Cum. \\
\hline no & 27 & 64.29 & 64.29 \\
\hline yes & 15 & 35.71 & 100.00 \\
\hline Total | & 42 & 100.00 & \\
\hline
\end{tabular}

-- mobile homes

Freq. Percent Cum.

$\begin{array}{cccc}\text { no } \mid & 15 & 35.71 & 35.71 \\ \text { yes } \mid & 27 & 64.29 & 100.00 \\ ------------ \\ \text { Total } & 42 & 100.00\end{array}$

(7) Baseloads (e.g., lighting, refrigerators)

-- single family dwellings

Freq. Percent Cum.

\begin{tabular}{|c|c|c|c|}
\hline no & 8 & 19.05 & 19.05 \\
\hline yes & 34 & 80.95 & 100.00 \\
\hline Total | & 42 & 100.00 & \\
\hline
\end{tabular}

-- multifamily dwellings

Freq. Percent Cum.

\begin{tabular}{cccc} 
no $\mid$ & 26 & 61.90 & 61.90 \\
yes $\mid$ & 16 & 38.10 & 100.00 \\
---------- \\
\hline Total & 42 & 100.00
\end{tabular}

-- mobile homes

Freq. Percent Cum.

\begin{tabular}{|c|c|c|c|}
\hline no & 12 & 28.57 & 28.57 \\
\hline yes & 30 & 71.43 & 100.00 \\
\hline Total | & 42 & 100.00 & \\
\hline
\end{tabular}

2a. On which administration-related topics did staff working in your state's weatherization office receive training in Program Year 2008 from DOE, your state, or other entities? (Check all that apply) 
(1) Management

$\begin{array}{cccc} & \text { Freq. } & \text { Percent } & \text { Cum. } \\ \text { no } \mid & 12 & 25.53 & 25.53 \\ \text { yes } \mid & 35 & 74.47 & 100.00 \\ ---------- \\ \text { Total | } & 47 & 100.00 & \end{array}$

\section{(2) Client education}

$\begin{array}{cccc} & \text { Freq. } & \text { Percent } & \text { Cum. } \\ \text { no } \mid & 25 & 53.19 & 53.19 \\ \text { yes } \mid & 22 & 46.81 & 100.00 \\ --------------- \\ \text { Total | } & 47 & 100.00\end{array}$

\section{(3) Auditing/estimating}

\begin{tabular}{cccc}
\multicolumn{4}{c}{-- single family dwellings } \\
Freq. & Percent & Cum. \\
\hline no | & 14 & 29.79 & 29.79 \\
yes | & 33 & 70.21 & 100.00 \\
----+--------- &
\end{tabular}

-- multifamily dwellings Freq. Percent Cum.

\begin{tabular}{|c|c|c|c|}
\hline no & 33 & 70.21 & 70.21 \\
\hline yes & 14 & 29.79 & 100.00 \\
\hline Total | & 47 & 100.00 & \\
\hline
\end{tabular}

-- mobile homes

Freq. Percent Cum.

\begin{tabular}{|c|c|c|c|}
\hline no & 20 & 42.55 & 42.55 \\
\hline yes & 27 & 57.45 & 100.00 \\
\hline Total & 47 & 100.00 & \\
\hline
\end{tabular}


(4) Monitoring/quality control

$\begin{array}{rccc} & \text { Freq. } & \text { Percent } & \text { Cum. } \\ \text { no } \mid & 10 & 21.28 & 21.28 \\ \text { yes } \mid & 37 & 78.72 & 100.00 \\ ----------- & \\ \text { Total } & 47 & 100.00\end{array}$

(5) Financial topics

$\begin{array}{cccc} & \text { Freq. } & \text { Percent } & \text { Cum. } \\ \text { no } \mid & 15 & 31.91 & 31.91 \\ \text { yes } \mid & 32 & 68.09 & 100.00 \\ --------- & \\ \text { Total | } & 47 & 100.00\end{array}$

(6) Outreach and communications

$\begin{array}{rccc} & \text { Freq. } & \text { Percent } & \text { Cum. } \\ \text { no | } & 26 & 55.32 & 55.32 \\ \text { yes | } & 21 & 44.68 & 100.00 \\ --------------- \\ \text { Total | } & 47 & 100.00\end{array}$

\section{(7) Other (please specify)}

2b. On which health and safety topics did staff working in your state's weatherization office receive training in Program Year 2008 from DOE, your state, or other entities? (Check all that apply.)

Fire safety

Freq. Percent Cum.

$\begin{array}{rrrr}\text { no } \mid & 32 & 78.05 & 78.05 \\ \text { yes } \mid & 9 & 21.95 & 100.00 \\ --------------- \\ \text { Total } & 41 & 100.00\end{array}$

Indoor air quality

Freq. Percent Cum.

\begin{tabular}{|c|c|c|c|}
\hline no & 7 & 17.07 & 17.07 \\
\hline yes & 34 & 82.93 & 100.00 \\
\hline Total | & 41 & 100.00 & \\
\hline
\end{tabular}


Measures to increase security of housing unit Freq. Percent Cum.

\begin{tabular}{|c|c|c|c|}
\hline no & 38 & 92.68 & 92.68 \\
\hline yes & 3 & 7.32 & 100.00 \\
\hline Total & 41 & 100.00 & \\
\hline
\end{tabular}

Measures to reduce common household hazards Freq. Percent Cum.

\begin{tabular}{|c|c|c|c|}
\hline no & 25 & 60.98 & 60.98 \\
\hline yes & 16 & 39.02 & 100.00 \\
\hline Total | & 41 & 100.00 & \\
\hline
\end{tabular}

Mold and mildew

\begin{tabular}{cccc}
\multicolumn{4}{c}{ Mold and mildew } \\
& Freq. & Percent & Cum. \\
no $\mid$ & 10 & 24.39 & 24.39 \\
yes $\mid$ & 31 & 75.61 & 100.00 \\
- \hdashline----------- &
\end{tabular}

\begin{tabular}{|c|c|c|c|}
\hline & Freq. & Percent & Cum. \\
\hline no & 6 & 14.63 & 14.63 \\
\hline yes $\mid$ & 35 & 85.37 & 100.00 \\
\hline Total | & 41 & 100.00 & \\
\hline
\end{tabular}

\begin{tabular}{|c|c|c|c|}
\hline & Freq. & Percent & Cum. \\
\hline no & 29 & 70.73 & 70.73 \\
\hline yes | & 12 & 29.27 & 100.00 \\
\hline otal | & 41 & 100.00 & \\
\hline
\end{tabular}

Vermiculite

Freq. Percent Cum.

\begin{tabular}{|c|c|c|c|}
\hline no & 31 & 75.61 & 75.61 \\
\hline yes & 10 & 24.39 & 100.00 \\
\hline Total & 41 & 100.00 & \\
\hline
\end{tabular}


General crew safety

Freq. Percent Cum.

\begin{tabular}{|c|c|c|c|}
\hline no | & 18 & 43.90 & 43.90 \\
\hline yes & 23 & 56.10 & 100.00 \\
\hline Total | & 41 & 100.00 & \\
\hline
\end{tabular}

\section{Other (please specify)}

3. For those staff working in your state's weatherization office who need to have knowledge about the following list of diagnostic topics, how well trained were they in each area in Program Year 2008? Please use the following scale: 1 - not at all well trained; 2 - not well trained; 3 - moderately well trained; 4 well trained; 5 - very well trained; 6 - not applicable (Circle best answer).

\section{Pressure diagnostics:}

- Blower door (house air leakage rate)

$\begin{array}{rrrr} & \text { Freq. } & \text { Percent } & \text { Cum. } \\ \text { not well trained | } & 3 & 6.25 & 6.25 \\ \text { moderatel well trained | } & 5 & 10.42 & 16.67 \\ \text { well trained | } & 12 & 25.00 & 41.67 \\ \text { very well trained | } & 26 & 54.17 & 95.83 \\ \text { not applicable | } & 2 & 4.17 & 100.00 \\ - \text { Total }^{-} & 48 & 100.00 & \end{array}$

- Zonal pressure measurements

$\begin{array}{rrrr} & \text { Freq. } & \text { Percent } & \text { Cum. } \\ \text { not at all well trained | } & 1 & 2.08 & 2.08 \\ \text { not well trained | } & 6 & 12.50 & 14.58 \\ \text { moderately well trained | } & 13 & 27.08 & 41.67 \\ \text { well trained | } & 11 & 22.92 & 64.58 \\ \text { very well trained | } & 13 & 27.08 & 91.67 \\ \text { not applicable | } & 4 & 8.33 & 100.00 \\ - \text { Total }^{-} & 48 & 100.00 & \end{array}$


- Room-to-room pressure measurements

Freq. Percent Cum.

$\begin{array}{rrrr}\text { not at all well trained | } & 1 & 2.08 & 2.08 \\ \text { not well trained | } & 8 & 16.67 & 18.75 \\ \text { moderately well trained | } & 10 & 20.83 & 39.58 \\ \text { well trained | } & 14 & 29.17 & 68.75 \\ \text { very well trained | } & 11 & 22.92 & 91.67 \\ \text { not applicable | } & 4 & 8.33 & 100.00 \\ - \text { Total } \mid & 48 & 100.00 & \end{array}$

- Duct pressure pan measurements

$\begin{array}{rrrr} & \text { Freq. } & \text { Percent } & \text { Cum. } \\ \text { not at all well trained | } & 3 & 6.25 & 6.25 \\ \text { not well trained | } & 3 & 6.25 & 12.50 \\ \text { moderately well trained } \mid & 12 & 25.00 & 37.50 \\ \text { well trained } \mid & 10 & 20.83 & 58.33 \\ \text { very well trained | } & 15 & 31.25 & 89.58 \\ \text { not applicable | } & 5 & 10.42 & 100.00 \\ - \text { Total } \mid & 48 & 100.00 & \end{array}$

- Duct blower measurements (duct air leakage rate) Freq. Percent Cum.

\begin{tabular}{rrrr} 
not at all well trained | & 4 & 8.51 & 8.51 \\
not well trained | & 8 & 17.02 & 25.53 \\
moderately well trained | & 10 & 21.28 & 46.81 \\
well trained | & 7 & 14.89 & 61.70 \\
very well trained | & 11 & 23.40 & 85.11 \\
not applicable | & 7 & 14.89 & 100.00 \\
\hline Total | & 47 & 100.00 &
\end{tabular}

Space-heating system:

- Flue gas analysis (steady-state efficiency measurements)

Freq. Percent Cum.

\begin{tabular}{|c|c|c|c|}
\hline not at all well trained | & 3 & 6.25 & 6.25 \\
\hline not well trained & 7 & 14.58 & 20.83 \\
\hline moderately well trained & 9 & 18.75 & 39.58 \\
\hline well trained & 10 & 20.83 & 60.42 \\
\hline very well trained & 16 & 33.33 & 93.75 \\
\hline not applicable & 3 & 6.25 & 100.00 \\
\hline Total | & 48 & 100.00 & \\
\hline
\end{tabular}


- Heat rise measurements

\begin{tabular}{r|rrr} 
& Freq. & Percent & Cum. \\
not at all well trained | & 2 & 4.17 & 4.17 \\
not well trained | & 6 & 12.50 & 16.67 \\
moderately well trained | & 11 & 22.92 & 39.58 \\
well trained | & 10 & 20.83 & 60.42 \\
very well trained | & 16 & 33.33 & 93.75 \\
not applicable | & 3 & 6.25 & 100.00 \\
- Total $^{-}$ & 48 & 100.00 &
\end{tabular}

- CO measurements in flues

$\begin{array}{rrrr} & \text { Freq. } & \text { Percent } & \text { Cum. } \\ \text { not at all well trained | } & 1 & 2.08 & 2.08 \\ \text { not well trained | } & 3 & 6.25 & 8.33 \\ \text { moderately well trained | } & 10 & 20.83 & 29.17 \\ \text { well trained | } & 10 & 20.83 & 50.00 \\ \text { very well trained | } & 23 & 47.92 & 97.92 \\ \text { not applicable | } & 1 & 2.08 & 100.00 \\ - \text { Total }^{-} & 48 & 100.00 & \end{array}$

- Draft/spillage (normal operation)

Freq. Percent Cum.

$\begin{array}{rrrr}\text { not at all well trained | } & 2 & 4.17 & 4.17 \\ \text { not well trained | } & 4 & 8.33 & 12.50 \\ \text { moderately well trained | } & 9 & 18.75 & 31.25 \\ \text { well trained | } & 11 & 22.92 & 54.17 \\ \text { very well trained | } & 19 & 39.58 & 93.75 \\ \text { not applicable | } & 3 & 6.25 & 100.00 \\ - \text { Total | } & 48 & 100.00 & \end{array}$

\section{Air-conditioning system:}

- Refrigerant charge (e.g., superheat, subcooling)

Freq. Percent Cum.

$\begin{array}{rrrr}\text { not at all well trained | } & 10 & 21.74 & 21.74 \\ \text { not well trained | } & 12 & 26.09 & 47.83 \\ \text { moderately well trained | } & 7 & 15.22 & 63.04 \\ \text { well trained | } & 1 & 2.17 & 65.22 \\ \text { very well trained | } & 3 & 6.52 & 71.74 \\ \text { not applicable | } & 13 & 28.26 & 100.00 \\ \text { Total } & 46 & 100.00 & \end{array}$


HVAC components and cross-cutting diagnostics:

- Air handler flow rate

Freq. Percent Cum.

$\begin{array}{rrrr}\text { not at all well trained | } & 7 & 14.58 & 14.58 \\ \text { not well trained | } & 15 & 31.25 & 45.83 \\ \text { moderately well trained | } & 5 & 10.42 & 56.25 \\ \text { well trained | } & 6 & 12.50 & 68.75 \\ \text { very well trained | } & 9 & 18.75 & 87.50 \\ \text { not applicable | } & 6 & 12.50 & 100.00 \\ - \text { Total | } & 48 & 100.00 & \end{array}$

- Thermostat anticipator current

Freq. Percent Cum.

$\begin{array}{rrrr}\text { not at all well trained | } & 8 & 16.67 & 16.67 \\ \text { not well trained | } & 10 & 20.83 & 37.50 \\ \text { moderately well trained | } & 7 & 14.58 & 52.08 \\ \text { well trained | } & 8 & 16.67 & 68.75 \\ \text { very well trained | } & 9 & 18.75 & 87.50 \\ \text { not applicable | } & 6 & 12.50 & 100.00 \\ \text { Total | } & 48 & 100.00 & \end{array}$

- Worst case draft/spillage (CAZ)

Freq. Percent Cum.

$\begin{array}{rrrr}\text { not at all well trained | } & 2 & 4.26 & 4.26 \\ \text { not well trained | } & 8 & 17.02 & 21.28 \\ \text { moderately well trained | } & 6 & 12.77 & 34.04 \\ \text { well trained | } & 9 & 19.15 & 53.19 \\ \text { very well trained | } & 19 & 40.43 & 93.62 \\ \text { not applicable | } & 3 & 6.38 & 100.00 \\ \text { Total | } & 47 & 100.00 & \end{array}$

Hot-water (water-heating) system:

- Flue gas analysis (steady-state efficiency measurements)

Freq. Percent Cum.

$\begin{array}{rrrr}\text { not at all well trained | } & 5 & 10.42 & 10.42 \\ \text { not well trained | } & 6 & 12.50 & 22.92 \\ \text { moderately well trained | } & 6 & 12.50 & 35.42 \\ \text { well trained | } & 10 & 20.83 & 56.25 \\ \text { very well trained | } & 17 & 35.42 & 91.67 \\ \text { not applicable | } & 4 & 8.33 & 100.00 \\ - \text { Total | } & 48 & 100.00 & \end{array}$


- CO measurements in flues

Freq. Percent Cum.

$\begin{array}{rrrr}\text { not at all well trained | } & 1 & 2.08 & 2.08 \\ \text { not well trained | } & 5 & 10.42 & 12.50 \\ \text { moderately well trained | } & 6 & 12.50 & 25.00 \\ \text { well trained | } & 9 & 18.75 & 43.75 \\ \text { very well trained | } & 25 & 52.08 & 95.83 \\ \text { not applicable | } & 2 & 4.17 & 100.00 \\ - \text { Total } & 48 & 100.00 & \end{array}$

- Draft/spillage (normal operation)

Freq. Percent Cum.

$\begin{array}{rrrr}\text { not at all well trained | } & 2 & 4.17 & 4.17 \\ \text { not well trained | } & 5 & 10.42 & 14.58 \\ \text { moderately well trained | } & 7 & 14.58 & 29.17 \\ \text { well trained | } & 11 & 22.92 & 52.08 \\ \text { very well trained | } & 20 & 41.67 & 93.75 \\ \text { not applicable | } & 3 & 6.25 & 100.00 \\ - \text { Total } & 48 & 100.00 & \end{array}$

- Water flow rates (showerheads and faucets)

Freq. Percent Cum.

$\begin{array}{rrrr}\text { not at all well trained | } & 4 & 8.33 & 8.33 \\ \text { not well trained | } & 10 & 20.83 & 29.17 \\ \text { moderately well trained | } & 9 & 18.75 & 47.92 \\ \text { well trained | } & 11 & 22.92 & 70.83 \\ \text { very well trained | } & 10 & 20.83 & 91.67 \\ \text { not applicable | } & 4 & 8.33 & 100.00 \\ - \text { Total | } & 48 & 100.00 & \end{array}$

Other CO measurements:

- CO measurements in equipment rooms

$\begin{array}{rrrr} & \text { Freq. } & \text { Percent } & \text { Cum. } \\ \text { not at all well trained | } & 3 & 6.25 & 6.25 \\ \text { not well trained | } & 3 & 6.25 & 12.50 \\ \text { moderately well trained | } & 11 & 22.92 & 35.42 \\ \text { well trained | } & 8 & 16.67 & 52.08 \\ \text { very well trained | } & 19 & 39.58 & 91.67 \\ \text { not applicable | } & 4 & 8.33 & 100.00 \\ \text { Total } & 48 & 100.00 & \end{array}$

- Cooking stove 
Freq. Percent Cum.

$\begin{array}{rrrr}\text { not at all well trained | } & 1 & 2.08 & 2.08 \\ \text { not well trained | } & 5 & 10.42 & 12.50 \\ \text { moderately well trained | } & 10 & 20.83 & 33.33 \\ \text { well trained | } & 12 & 25.00 & 58.33 \\ \text { very well trained | } & 17 & 35.42 & 93.75 \\ \text { not applicable | } & 3 & 6.25 & 100.00 \\ \text { Total | } & 48 & 100.00 & \end{array}$

- CO measurements in living areas

Freq. Percent Cum.

$\begin{array}{rrrr}\text { not at all well trained | } & 1 & 2.13 & 2.13 \\ \text { not well trained | } & 4 & 8.51 & 10.64 \\ \text { moderately well trained | } & 9 & 19.15 & 29.79 \\ \text { well trained | } & 11 & 23.40 & 53.19 \\ \text { very well trained | } & 17 & 36.17 & 89.36 \\ \text { not applicable | } & 5 & 10.64 & 100.00 \\ \text { Total | } & 47 & 100.00 & \end{array}$

Other diagnostics and inspections:

- Refrigerator energy use

Freq. Percent Cum.

\begin{tabular}{|c|c|c|c|}
\hline not at all well trained & 1 & 2.08 & 2.08 \\
\hline not well trained & 2 & 4.17 & 6.25 \\
\hline moderately well trained & 7 & 14.58 & 20.83 \\
\hline well trained & 17 & 35.42 & 56.25 \\
\hline very well trained & 16 & 33.33 & 89.58 \\
\hline not applicable | & 5 & 10.42 & 100.00 \\
\hline Total & 48 & 100.00 & \\
\hline
\end{tabular}

- Exhaust fan air flow rate measurement

Freq. Percent Cum.

\begin{tabular}{r|rrr} 
not at all well trained | & 3 & 6.25 & 6.25 \\
not well trained | & 6 & 12.50 & 18.75 \\
moderately well trained | & 11 & 22.92 & 41.67 \\
well trained | & 10 & 20.83 & 62.50 \\
very well trained | & 12 & 25.00 & 87.50 \\
not applicable | & 6 & 12.50 & 100.00 \\
Total & 48 & 100.00 &
\end{tabular}


- Infrared scanning (camera)

Freq. Percent Cum.

$\begin{array}{rrrr}\text { not at all well trained | } & 1 & 2.08 & 2.08 \\ \text { not well trained | } & 6 & 12.50 & 14.58 \\ \text { moderately well trained | } & 10 & 20.83 & 35.42 \\ \text { well trained | } & 15 & 31.25 & 66.67 \\ \text { very well trained | } & 11 & 22.92 & 89.58 \\ \text { not applicable | } & 5 & 10.42 & 100.00 \\ \text { Total | } & 48 & 100.00 & \end{array}$

- Radon testing

Freq. Percent Cum.

$\begin{array}{rrrr}\text { not at all well trained | } & 14 & 29.79 & 29.79 \\ \text { not well trained | } & 7 & 14.89 & 44.68 \\ \text { moderately well trained | } & 7 & 14.89 & 59.57 \\ \text { well trained | } & 1 & 2.13 & 61.70 \\ \text { very well trained | } & 4 & 8.51 & 70.21 \\ \text { not applicable | } & 14 & 29.79 & 100.00 \\ - \text { Total | } & 47 & 100.00 & \end{array}$

- $\quad$ Lead testing

$\begin{array}{rrrr} & \text { Freq. } & \text { Percent } & \text { Cum } \\ \text { not at all well trained | } & 6 & 12.77 & 12.77 \\ \text { not well trained | } & 4 & 8.51 & 21.28 \\ \text { moderately well trained } \mid & 11 & 23.40 & 44.68 \\ \text { well trained } \mid & 12 & 25.53 & 70.21 \\ \text { very well trained | } & 7 & 14.89 & 85.11 \\ \text { not applicable | } & 7 & 14.89 & 100.00 \\ - \text { Total } \mid & 47 & 100.00 & \end{array}$


- Mold and mildew testing

\begin{tabular}{r|rrr} 
& Freq. & Percent & Cum. \\
not at all well trained | & 10 & 21.74 & 21.74 \\
not well trained | & 5 & 10.87 & 32.61 \\
moderately well trained | & 8 & 17.39 & 50.00 \\
well trained | & 7 & 15.22 & 65.22 \\
very well trained | & 7 & 15.22 & 80.43 \\
not applicable | & 9 & 19.57 & 100.00 \\
- Total $\mid$ & 46 & 100.00 &
\end{tabular}

- Moisture content testing

$\begin{array}{rrrr} & \text { Freq. } & \text { Percent } & \text { Cum } \\ \text { not at all well trained | } & 9 & 19.15 & 19.15 \\ \text { not well trained | } & 7 & 14.89 & 34.04 \\ \text { moderately well trained | } & 10 & 21.28 & 55.32 \\ \text { well trained | } & 8 & 17.02 & 72.34 \\ \text { very well trained | } & 7 & 14.89 & 87.23 \\ \text { not applicable | } & 6 & 12.77 & 100.00 \\ \text { Total | } & 47 & 100.00 & \end{array}$

- Other (please specify)

4. On which of the following diagnostic procedures did staff working in your state's weatherization office receive training in Program Year 2008 from DOE, your state, or other entities? (Check all that apply.)

Pressure diagnostics:

- Blower door (house air leakage rate)

Freq. Percent Cum.

\begin{tabular}{|c|c|c|c|}
\hline no & 3 & 7.32 & 7.32 \\
\hline yes | & 38 & 92.68 & 100.00 \\
\hline Total | & 41 & 100.00 & \\
\hline
\end{tabular}

- Zonal pressure measurements

\begin{tabular}{cccc} 
& Freq. & Percent & Cum. \\
no $\mid$ & 14 & 34.15 & 34.15 \\
yes $\mid$ & 27 & 65.85 & 100.00 \\
- \hdashline--- &
\end{tabular}


- Room-to-room pressure measurements (distribution balancing) Freq. Percent Cum.

\begin{tabular}{|c|c|c|c|}
\hline no & 16 & 39.02 & 39.02 \\
\hline yes & 25 & 60.98 & 100.00 \\
\hline Total | & 41 & 100.00 & \\
\hline
\end{tabular}

- Duct pressure pan measurements

$\begin{array}{cccc} & \text { Freq. } & \text { Percent } & \text { Cum. } \\ \text { no } \mid & 16 & 39.02 & 39.02 \\ \text { yes } \mid & 25 & 60.98 & 100.00 \\ ---------- \\ \text { Total | } & 41 & 100.00 & \end{array}$

- Duct blower measurements (duct air leakage rate)

$\begin{array}{cccc} & \text { Freq. } & \text { Percent } & \text { Cum. } \\ \text { no } \mid & 21 & 51.22 & 51.22 \\ \text { yes | } & 20 & 48.78 & 100.00 \\ ------- & \\ \text { Total | } & 41 & 100.00\end{array}$

Space-heating system:

- Flue gas analysis (steady-state efficiency measurements) Freq. Percent Cum.

\begin{tabular}{|c|c|c|c|}
\hline no & 13 & 31.71 & 31.71 \\
\hline yes & 28 & 68.29 & 100.00 \\
\hline Total | & 41 & 100.00 & \\
\hline
\end{tabular}

- Heat rise measurements

Freq. Percent Cum.

\begin{tabular}{|c|c|c|c|}
\hline no & 20 & 48.78 & 48.78 \\
\hline yes | & 21 & 51.22 & 100.00 \\
\hline Total | & 41 & 100.00 & \\
\hline
\end{tabular}

- CO measurements in flues

$\begin{array}{crrr} & \text { Freq. } & \text { Percent } & \text { Cum. } \\ \text { no | } & 4 & 9.76 & 9.76 \\ \text { yes } \mid & 37 & 90.24 & 100.00 \\ ------- & \\ \text { Total | } & 41 & 100.00\end{array}$


- Draft/spillage (normal operation)

$\begin{array}{cccc} & \text { Freq. } & \text { Percent } & \text { Cum. } \\ \text { no | } & 13 & 31.71 & 31.71 \\ \text { yes | } & 28 & 68.29 & 100.00 \\ ------------ \\ \text { Total | } & 41 & 100.00 & \end{array}$

Air-conditioning system:

- Refrigerant charge (e.g., superheat, subcooling)

Freq. Percent Cum.

$\begin{array}{crrr}\text { no } \mid & 34 & 82.93 & 82.93 \\ \text { yes } \mid & 7 & 17.07 & 100.00 \\ -------------- \\ \text { Total } & 41 & 100.00\end{array}$

HVAC components and cross-cutting diagnostics:

- Air handler flow rate

Freq. Percent Cum.

\begin{tabular}{|c|c|c|c|}
\hline no & 27 & 65.85 & 65.85 \\
\hline yes & 14 & 34.15 & 100.00 \\
\hline Total & 41 & 100.00 & \\
\hline
\end{tabular}

- Thermostat anticipator current

$\begin{array}{rrrr} & \text { Freq. } & \text { Percent } & \text { Cum. } \\ \text { no | } & 29 & 70.73 & 70.73 \\ \text { yes | } & 12 & 29.27 & 100.00 \\ --------- & \\ \text { Total | } & 41 & 100.00 & \end{array}$

- Worst case draft/spillage (CAZ)

$\begin{array}{cccc} & \text { Freq. } & \text { Percent } & \text { Cum. } \\ \text { no | } & 19 & 46.34 & 46.34 \\ \text { yes } \mid & 22 & 53.66 & 100.00 \\ ------ & \end{array}$

Hot-water (water-heating) system:

- $\quad$ Flue gas analysis (steady-state efficiency measurements) Freq. Percent Cum.

\begin{tabular}{|c|c|c|c|}
\hline no & 19 & 46.34 & 46.34 \\
\hline yes | & 22 & 53.66 & 100.00 \\
\hline Total & 41 & 100.00 & \\
\hline
\end{tabular}


CO measurements in flues

$\begin{array}{crcc} & \text { Freq. } & \text { Percent } & \text { Cum. } \\ \text { no | } & 9 & 21.95 & 21.95 \\ \text { yes | } & 32 & 78.05 & 100.00 \\ -------- & \end{array}$

- Draft/spillage (normal operation) Freq. Percent Cum.

\begin{tabular}{|c|c|c|c|}
\hline no & 17 & 41.46 & 41.46 \\
\hline yes | & 24 & 58.54 & 100.00 \\
\hline Total | & 41 & 100.00 & \\
\hline
\end{tabular}

- Water flow rates (showerheads and faucets)

$\begin{array}{cccc} & \text { Freq. } & \text { Percent } & \text { Cum. } \\ \text { no | } & 26 & 63.41 & 63.41 \\ \text { yes } \mid & 15 & 36.59 & 100.00 \\ ------- & \\ \text { Total | } & 41 & 100.00\end{array}$

Other CO measurements:

- CO measurements in equipment rooms

$\begin{array}{cccc} & \text { Freq. } & \text { Percent } & \text { Cum. } \\ \text { no | } & 21 & 51.22 & 51.22 \\ \text { yes | } & 20 & 48.78 & 100.00 \\ --------------- \\ \text { Total } & 41 & 100.00 & \end{array}$

- Cooking stove

\begin{tabular}{cccc} 
& Freq. & Percent & Cum. \\
no $\mid$ & 17 & 41.46 & 41.46 \\
yes $\mid$ & 24 & 58.54 & 100.00 \\
- \hdashline--- &
\end{tabular}

- CO measurements in living areas

$\begin{array}{cccc} & \text { Freq. } & \text { Percent } & \text { Cum. } \\ \text { no } \mid & 17 & 41.46 & 41.46 \\ \text { yes } \mid & 24 & 58.54 & 100.00 \\ ------- & \\ \text { Total | } & 41 & 100.00 & \end{array}$


Other diagnostics and inspections:

- Refrigerator energy use

\begin{tabular}{|c|c|c|c|}
\hline & Freq. & Percent & Cum. \\
\hline no & 15 & 36.59 & 36.59 \\
\hline yes | & 26 & 63.41 & 100.00 \\
\hline Total & 41 & 100.00 & \\
\hline
\end{tabular}

- Exhaust fan air flow rate measurement Freq. Percent Cum.

$\begin{array}{cccc}\text { no } \mid & 24 & 58.54 & 58.54 \\ \text { yes } \mid & 17 & 41.46 & 100.00 \\ ---------------- \\ \text { Total } & 41 & 100.00\end{array}$

- Infrared scanning (camera)

Freq. Percent Cum.

\begin{tabular}{|c|c|c|c|}
\hline no & 19 & 46.34 & 46.34 \\
\hline yes & 22 & 53.66 & 100.00 \\
\hline Total | & 41 & 100.00 & \\
\hline
\end{tabular}

- Radon testing

$\begin{array}{rrrr} & \text { Freq. } & \text { Percent } & \text { Cum. } \\ \text { no | } & 36 & 87.80 & 87.80 \\ \text { yes | } & 5 & 12.20 & 100.00 \\ ------- & \\ \text { Total | } & 41 & 100.00 & \end{array}$

- Lead testing

$\begin{array}{cccc} & \text { Freq. } & \text { Percent } & \text { Cum. } \\ \text { no } \mid & 20 & 48.78 & 48.78 \\ \text { yes } \mid & 21 & 51.22 & 100.00 \\ -------- & \\ \text { Total | } & 41 & 100.00 & \end{array}$

- Mold and mildew testing

Freq. Percent Cum.

\begin{tabular}{|c|c|c|c|}
\hline no & 26 & 63.41 & 63.41 \\
\hline yes | & 15 & 36.59 & 100.00 \\
\hline Total & 41 & 100.00 & \\
\hline
\end{tabular}


- Moisture content testing

$\begin{array}{rrrr} & \text { Freq. } & \text { Percent } & \text { Cum. } \\ \text { no | } & 28 & 68.29 & 68.29 \\ \text { yes | } & 13 & 31.71 & 100.00 \\ ---------- & \\ \text { Total | } & 41 & 100.00 & \end{array}$

- Other (please specify)

5. Please indicate the number of your state's weatherization office staff who received training in these three job function areas by attending the following events in Program Year 2008. 


\begin{tabular}{|c|c|c|c|c|c|c|}
\hline \multirow[b]{3}{*}{ Training events } & \multicolumn{6}{|c|}{$\begin{array}{l}\text { Job Function } \\
\end{array}$} \\
\hline & \multicolumn{2}{|c|}{ Management and Administration } & \multicolumn{2}{|c|}{ Field Monitoring and Auditing } & \multicolumn{2}{|c|}{ Training and Technical Assistance } \\
\hline & $\begin{array}{c}\text { Primarily } \\
\text { Classroom } \\
\text { Training }\end{array}$ & $\begin{array}{l}\text { Primarily Field } \\
\text { Training }\end{array}$ & $\begin{array}{c}\text { Primarily } \\
\text { Classroom } \\
\text { Training }\end{array}$ & $\begin{array}{l}\text { Primarily Field } \\
\text { Training }\end{array}$ & $\begin{array}{c}\text { Primarily } \\
\text { Classroom } \\
\text { Training }\end{array}$ & $\begin{array}{c}\text { Primarily Field } \\
\text { Training }\end{array}$ \\
\hline $\begin{array}{l}\text { National Weatherization Program } \\
\text { conference }\end{array}$ & $\begin{array}{l}\text { obs: } 30 \\
\text { min: } 0 \\
\text { max: } 6 \\
\text { mean: } 1.43 \\
\text { median: } 1\end{array}$ & $\begin{array}{l}\text { obs: } 10 \\
\text { min: } 0 \\
\text { max: } 2 \\
\text { mean: } 7 \\
\text { median: } 1\end{array}$ & $\begin{array}{l}\text { obs: } 25 \\
\text { min: } 0 \\
\text { max: } 4 \\
\text { mean: } 1.52 \\
\text { median: } 1\end{array}$ & $\begin{array}{l}\text { obs: } 15 \\
\text { min: } 0 \\
\text { max: } 6 \\
\text { mean: } 1.67 \\
\text { median: } 1\end{array}$ & $\begin{array}{l}\text { obs: } 19 \\
\text { min: } 0 \\
\text { max: } 4 \\
\text { mean: } 1.58 \\
\text { median: } 2\end{array}$ & $\begin{array}{l}\text { obs: } 10 \\
\text { min: } 0 \\
\text { max: } 2 \\
\text { mean: } 1.2 \\
\text { median: } 1.5\end{array}$ \\
\hline Affordable Comfort Conference & $\begin{array}{l}\text { obs: } 13 \\
\text { min: } 0 \\
\text { max: } 2 \\
\text { mean: } .77 \\
\text { median: } 1\end{array}$ & $\begin{array}{l}\text { obs: } 7 \\
\text { min: } 0 \\
\text { max: } 2 \\
\text { mean: } 43 \\
\text { median: } 0\end{array}$ & $\begin{array}{l}\text { obs: } 14 \\
\text { min: } 0 \\
\text { max: } 4 \\
\text { mean: } 1.36 \\
\text { median: } 1\end{array}$ & $\begin{array}{l}\text { obs: } 11 \\
\text { min: } 0 \\
\text { max: } 4 \\
\text { mean: } 1 \\
\text { median: } 1\end{array}$ & $\begin{array}{l}\text { obs: } 15 \\
\text { min: } 0 \\
\text { max: } 4 \\
\text { mean: } 1.33 \\
\text { median: } 1\end{array}$ & $\begin{array}{l}\text { obs: } 7 \\
\text { min: } 0 \\
\text { max: } 3 \\
\text { mean: } .71 \\
\text { median: } 0\end{array}$ \\
\hline Other national conference & $\begin{array}{l}\text { obs: } 8 \\
\text { min: } 0 \\
\text { max: } 2 \\
\text { mean: } 1.13 \\
\text { median: } 1\end{array}$ & $\begin{array}{l}\text { obs: } 4 \\
\text { min: } 0 \\
\text { max: } 1 \\
\text { mean: } .25 \\
\text { median: } 0\end{array}$ & $\begin{array}{l}\text { obs: } 5 \\
\text { min: } 0 \\
\text { max: } 1 \\
\text { mean: } .4 \\
\text { median: } 0\end{array}$ & $\begin{array}{l}\text { obs: } 4 \\
\text { min: } 0 \\
\text { max: } 2 \\
\text { mean: } .5 \\
\text { median: } 0\end{array}$ & $\begin{array}{l}\text { obs: } 3 \\
\text { min: } 0 \\
\text { max: } 0 \\
\text { mean: } 0 \\
\text { median: } 0\end{array}$ & $\begin{array}{l}\text { obs: } 3 \\
\text { min: } 0 \\
\text { max: } 0 \\
\text { mean: } 0 \\
\text { median: } 0\end{array}$ \\
\hline $\begin{array}{l}\text { Regional weatherization } \\
\text { conference }\end{array}$ & $\begin{array}{l}\text { obs: } 25 \\
\text { min: } 0 \\
\text { max: } 4 \\
\text { mean: } 1.6 \\
\text { median: } 1\end{array}$ & $\begin{array}{l}\text { obs: } 6 \\
\text { min: } 0 \\
\text { max: } 1 \\
\text { mean: } .5 \\
\text { median: } .5\end{array}$ & $\begin{array}{l}\text { obs: } 20 \\
\text { min: } 0 \\
\text { max: } 4 \\
\text { mean: } 1.75 \\
\text { median: } 2\end{array}$ & $\begin{array}{l}\text { obs: } 7 \\
\text { min: } 0 \\
\text { max: } 2 \\
\text { mean: } .71 \\
\text { median: } 1\end{array}$ & $\begin{array}{l}\text { obs: } 18 \\
\text { min: } 0 \\
\text { max: } 3 \\
\text { mean: } 1.56 \\
\text { median: } 2\end{array}$ & $\begin{array}{l}\text { obs: } 7 \\
\text { min: } 0 \\
\text { max: } 3 \\
\text { mean: } 1.14 \\
\text { median: } 1\end{array}$ \\
\hline State weatherization conference & $\begin{array}{l}\text { obs: } 17 \\
\text { min: } 0 \\
\text { max: } 12 \\
\text { mean: } 2.71 \\
\text { median: } 1\end{array}$ & $\begin{array}{l}\text { obs: } 7 \\
\text { min: } 0 \\
\text { max: } 4 \\
\text { mean: } 1.43 \\
\text { median: } 1\end{array}$ & $\begin{array}{l}\text { obs: } 13 \\
\text { min: } 0 \\
\text { max: } 12 \\
\text { mean: } 2.38 \\
\text { median: } 1\end{array}$ & $\begin{array}{l}\text { obs: } 9 \\
\text { min: } 0 \\
\text { max: } 4 \\
\text { mean: } 1.33 \\
\text { median: } 1\end{array}$ & $\begin{array}{l}\text { obs: } 9 \\
\text { min: } 0 \\
\text { max: } 12 \\
\text { mean: } 3.11 \\
\text { median: } 2\end{array}$ & $\begin{array}{l}\text { obs: } 7 \\
\text { min: } 0 \\
\text { max: } 4 \\
\text { mean: } 1.14 \\
\text { median: } 1\end{array}$ \\
\hline Other state conference & $\begin{array}{l}\text { obs: } 6 \\
\text { min: } 0 \\
\text { max: } 1 \\
\text { mean: } .33 \\
\text { median: } 0\end{array}$ & $\begin{array}{l}\text { obs: } 4 \\
\text { min: } 0 \\
\text { max: } 1 \\
\text { mean: } .25 \\
\text { median: } 0\end{array}$ & $\begin{array}{l}\text { obs: } 6 \\
\text { min: } 0 \\
\text { max: } 8 \\
\text { mean: } 1.67 \\
\text { median: } .5\end{array}$ & $\begin{array}{l}\text { obs: } 3 \\
\text { min: } 0 \\
\text { max: } 0 \\
\text { mean: } 0 \\
\text { median: } 0\end{array}$ & $\begin{array}{l}\text { obs: } 6 \\
\text { min: } 0 \\
\text { max: } 2 \\
\text { mean: } .67 \\
\text { median: } .5\end{array}$ & $\begin{array}{l}\text { obs: } 4 \\
\text { min: } 0 \\
\text { max: } 2 \\
\text { mean: } .5 \\
\text { median: } 0\end{array}$ \\
\hline $\begin{array}{l}\text { State/regional training center } \\
\text { class }\end{array}$ & $\begin{array}{l}\text { obs: } 9 \\
\text { min: } 0 \\
\text { max: } 1 \\
\text { mean: } .56 \\
\text { median: } 1\end{array}$ & $\begin{array}{l}\text { obs: } 4 \\
\text { min: } 0 \\
\text { max: } 0 \\
\text { mean: } 0 \\
\text { median: } 0\end{array}$ & $\begin{array}{l}\text { obs: } 12 \\
\text { min: } 0 \\
\text { max: } 5 \\
\text { mean: } 1.67 \\
\text { median: } 2\end{array}$ & $\begin{array}{l}\text { obs: } 10 \\
\text { min: } 0 \\
\text { max: } 5 \\
\text { mean: } 1.5 \\
\text { median: } 1.5\end{array}$ & $\begin{array}{l}\text { obs: } 9 \\
\text { min: } 0 \\
\text { max: } 10 \\
\text { mean: } 2.22 \\
\text { median: } 1\end{array}$ & $\begin{array}{l}\text { obs: } 7 \\
\text { min: } 0 \\
\text { max: } 5 \\
\text { mean: } 1.43 \\
\text { median: } 1\end{array}$ \\
\hline $\begin{array}{l}\text { Manufacturer's training school } \\
\text { class }\end{array}$ & $\begin{array}{l}\text { obs: } 5 \\
\text { min: } 0 \\
\text { max: } 1 \\
\text { mean: } .2 \\
\text { median: } 0\end{array}$ & $\begin{array}{l}\text { obs: } 4 \\
\text { min: } 0 \\
\text { max: } 1 \\
\text { mean: } .25 \\
\text { median: } 0\end{array}$ & $\begin{array}{l}\text { obs: } 7 \\
\text { min: } 0 \\
\text { max: } 4 \\
\text { mean: } 1.71 \\
\text { median: } 2\end{array}$ & $\begin{array}{l}\text { obs: } 4 \\
\text { min: } 0 \\
\text { max: } 2 \\
\text { mean: } .5 \\
\text { median: } 0\end{array}$ & $\begin{array}{l}\text { obs: } 6 \\
\text { min: } 0 \\
\text { max: } 5 \\
\text { mean: } 1.33 \\
\text { median: } .5\end{array}$ & $\begin{array}{l}\text { obs: } 5 \\
\text { min: } 0 \\
\text { max: } 1 \\
\text { mean: } .4 \\
\text { median: } 0\end{array}$ \\
\hline Utility sponsored training class & $\begin{array}{l}\text { obs: } 4 \\
\text { min: } 0 \\
\text { max: } 0 \\
\text { mean: } 0 \\
\text { median: } 0\end{array}$ & $\begin{array}{l}\text { obs: } 4 \\
\text { min: } 0 \\
\text { max: } 1 \\
\text { mean: } .25 \\
\text { median: } 0\end{array}$ & $\begin{array}{l}\text { obs: } 6 \\
\text { min: } 0 \\
\text { max: } 2 \\
\text { mean: } 1.17 \\
\text { median: } 1.5\end{array}$ & $\begin{array}{l}\text { obs: } 5 \\
\text { min: } 0 \\
\text { max: } 2 \\
\text { mean: } .8 \\
\text { median: } 0\end{array}$ & $\begin{array}{l}\text { obs: } 4 \\
\text { min: } 0 \\
\text { max: } 2 \\
\text { mean: } .5 \\
\text { median: } 0\end{array}$ & $\begin{array}{l}\text { obs: } 3 \\
\text { min: } 0 \\
\text { max: } 0 \\
\text { mean: } 0 \\
\text { median: } 0\end{array}$ \\
\hline $\begin{array}{l}\text { Class sponsored by your state } \\
\text { weatherization office) }\end{array}$ & $\begin{array}{l}\text { obs: } 12 \\
\text { min: } 0 \\
\text { max: } 12 \\
\text { mean: } 2.67 \\
\text { median: } 2.5\end{array}$ & $\begin{array}{l}\text { obs: } 8 \\
\text { min: } 0 \\
\text { max: } 16 \\
\text { mean: } 3 \\
\text { median: } 1\end{array}$ & $\begin{array}{l}\text { obs: } 13 \\
\text { min: } 0 \\
\text { max: } 8 \\
\text { mean: } 2.23 \\
\text { median: } 2\end{array}$ & $\begin{array}{l}\text { obs: } 9 \\
\text { min: } 0 \\
\text { max: } 7 \\
\text { mean: } 1.89 \\
\text { median: } 1\end{array}$ & $\begin{array}{l}\text { obs: } 12 \\
\text { min: } 0 \\
\text { max: } 8 \\
\text { mean: } 2.42 \\
\text { median: } 1.5\end{array}$ & $\begin{array}{l}\text { obs: } 11 \\
\text { min: } 0 \\
\text { max: } 4 \\
\text { mean: } 1.82 \\
\text { median: } 1\end{array}$ \\
\hline $\begin{array}{l}\text { Class not sponsored by any of the } \\
\text { entities listed above (e.g., another } \\
\text { state, trade organization) }\end{array}$ & $\begin{array}{l}\text { obs: } 5 \\
\text { min: } 0 \\
\text { max: } 2 \\
\text { mean: } 6 \\
\text { median: } 0\end{array}$ & $\begin{array}{l}\text { obs: } 5 \\
\text { min: } 0 \\
\text { max: } 2 \\
\text { mean: } 6 \\
\text { median: } 0\end{array}$ & $\begin{array}{l}\text { obs: } 9 \\
\text { min: } 0 \\
\text { max: } 3 \\
\text { mean: } 1.22 \\
\text { median: } 1\end{array}$ & $\begin{array}{l}\text { obs: } 5 \\
\text { min: } 0 \\
\text { max: } 3 \\
\text { mean: } 1 \\
\text { median: } 0\end{array}$ & $\begin{array}{l}\text { obs: } 8 \\
\text { min: } 0 \\
\text { max: } 5 \\
\text { mean: } 1.63 \\
\text { median: } 1.5\end{array}$ & $\begin{array}{l}\text { obs: } 6 \\
\text { min: } 0 \\
\text { max: } 3 \\
\text { mean: } 1.17 \\
\text { median: } 1\end{array}$ \\
\hline $\begin{array}{l}\text { In-person expert visit to state } \\
\text { (e.g., peer exchange, consultant) }\end{array}$ & $\begin{array}{l}\text { obs: } 6 \\
\text { min: } 0 \\
\text { max: } 2 \\
\text { mean: } .83 \\
\text { median: } .5\end{array}$ & $\begin{array}{l}\text { obs: } 6 \\
\text { min: } 0 \\
\text { max: } 2 \\
\text { mean: } .83 \\
\text { median: } .5\end{array}$ & $\begin{array}{l}\text { obs: } 7 \\
\text { min: } 0 \\
\text { max: } 2 \\
\text { mean: } 86 \\
\text { median: } 1\end{array}$ & $\begin{array}{l}\text { obs: } 8 \\
\text { min: } 0 \\
\text { max: } 2 \\
\text { mean: } 1 \\
\text { median: } 1\end{array}$ & $\begin{array}{l}\text { obs: } 7 \\
\text { min: } 0 \\
\text { max: } 4 \\
\text { mean: } 1.57 \\
\text { median: } 2\end{array}$ & $\begin{array}{l}\text { obs: } 8 \\
\text { min: } 0 \\
\text { max: } 2 \\
\text { mean: } 1 \\
\text { median: } 1\end{array}$ \\
\hline Other (please specify: ___ & & & & & & \\
\hline
\end{tabular}


6. For each broad subject listed in the left-most column of the following table, put a check mark in the appropriate cell(s) to indicate which training method(s) you believe were most effective for imparting key skills and information in that area to your state's in-house staff and any non-agency staff supporting the state program who work under contract to the state in Program Year 2008:

\begin{tabular}{|c|c|c|c|c|c|c|}
\hline Subject & Conferences & $\begin{array}{c}\text { Primarily } \\
\text { Classroom } \\
\text { Training }\end{array}$ & $\begin{array}{c}\text { Primarily } \\
\text { Field } \\
\text { Training }\end{array}$ & $\begin{array}{c}\text { In-person } \\
\text { expert visits }\end{array}$ & Web casts & $\begin{array}{c}\text { Other } \\
\text { (specify) }\end{array}$ \\
\hline Management & $\begin{array}{l}\text { obs: } 43 \\
\text { no: } 11 \\
\text { yes: } 32\end{array}$ & $\begin{array}{l}\text { obs: } 43 \\
\text { no: } 23 \\
\text { yes: } 20\end{array}$ & $\begin{array}{l}\text { obs: } 43 \\
\text { no: } 39 \\
\text { yes: } 4\end{array}$ & $\begin{array}{l}\text { obs: } 43 \\
\text { no: } 28 \\
\text { yes: } 15\end{array}$ & $\begin{array}{l}\text { obs: } 43 \\
\text { no: } 34 \\
\text { yes: } 9\end{array}$ & $\begin{array}{l}\text { obs: } 43 \\
\text { no: } 41 \\
\text { yes: } 2\end{array}$ \\
\hline $\begin{array}{l}\text { Weatherization } \\
\text { installation }\end{array}$ & $\begin{array}{l}\text { obs: } 42 \\
\text { no: } 31 \\
\text { yes: } 11\end{array}$ & $\begin{array}{l}\text { obs: } 42 \\
\text { no: } 28 \\
\text { yes: } 14\end{array}$ & $\begin{array}{l}\text { obs: } 42 \\
\text { no: } 3 \\
\text { yes: } 39\end{array}$ & $\begin{array}{l}\text { obs: } 42 \\
\text { no: } 31 \\
\text { yes: } 11\end{array}$ & $\begin{array}{l}\text { obs: } 42 \\
\text { no: } 42 \\
\text { yes: } 0\end{array}$ & $\begin{array}{l}\text { obs: } 42 \\
\text { no: } 40 \\
\text { yes: } 2\end{array}$ \\
\hline $\begin{array}{l}\text { Auditing/Estimating/ } \\
\text { Measure selection }\end{array}$ & $\begin{array}{l}\text { obs: } 42 \\
\text { no: } 37 \\
\text { yes: } 5\end{array}$ & $\begin{array}{l}\text { obs: } 42 \\
\text { no: } 16 \\
\text { yes: } 26\end{array}$ & $\begin{array}{l}\text { obs: } 42 \\
\text { no: } 12 \\
\text { yes: } 30\end{array}$ & $\begin{array}{l}\text { obs: } 42 \\
\text { no: } 29 \\
\text { yes: } 13\end{array}$ & $\begin{array}{l}\text { obs: } 42 \\
\text { no: } 42 \\
\text { yes: } 0\end{array}$ & $\begin{array}{l}\text { obs: } 42 \\
\text { no: } 41 \\
\text { yes: } 1\end{array}$ \\
\hline $\begin{array}{l}\text { Monitoring and quality } \\
\text { control }\end{array}$ & $\begin{array}{l}\text { obs: } 43 \\
\text { no: } 26 \\
\text { yes: } 17\end{array}$ & $\begin{array}{l}\text { obs: } 43 \\
\text { no: } 21 \\
\text { yes: } 22\end{array}$ & $\begin{array}{l}\text { obs: } 43 \\
\text { no: } 16 \\
\text { yes: } 27\end{array}$ & $\begin{array}{l}\text { obs: } 43 \\
\text { no: } 32 \\
\text { yes: } 11\end{array}$ & $\begin{array}{l}\text { obs: } 43 \\
\text { no: } 40 \\
\text { yes: } 3\end{array}$ & $\begin{array}{l}\text { obs: } 43 \\
\text { no: } 42 \\
\text { yes: } 1\end{array}$ \\
\hline Financial topics & $\begin{array}{l}\text { obs: } 40 \\
\text { no: } 15 \\
\text { yes: } 25\end{array}$ & $\begin{array}{l}\text { obs: } 40 \\
\text { no: } 17 \\
\text { yes: } 23\end{array}$ & $\begin{array}{l}\text { obs: } 40 \\
\text { no: } 35 \\
\text { yes: } 5\end{array}$ & $\begin{array}{l}\text { obs: } 40 \\
\text { no: } 31 \\
\text { yes: } 9\end{array}$ & $\begin{array}{l}\text { obs: } 40 \\
\text { no: } 32 \\
\text { yes: } 8\end{array}$ & $\begin{array}{l}\text { obs: } 40 \\
\text { no: } 39 \\
\text { yes: } 1\end{array}$ \\
\hline $\begin{array}{l}\text { Outreach and } \\
\text { communications }\end{array}$ & $\begin{array}{l}\text { obs: } 39 \\
\text { no: } 16 \\
\text { yes: } 23\end{array}$ & $\begin{array}{l}\text { obs: } 39 \\
\text { no: } 19 \\
\text { yes: } 20\end{array}$ & $\begin{array}{l}\text { obs: } 39 \\
\text { no: } 37 \\
\text { yes: } 2\end{array}$ & $\begin{array}{l}\text { obs: } 39 \\
\text { no: } 36 \\
\text { yes: } 3\end{array}$ & $\begin{array}{l}\text { obs: } 39 \\
\text { no: } 33 \\
\text { yes: } 6\end{array}$ & $\begin{array}{l}\text { obs: } 39 \\
\text { no: } 38 \\
\text { yes: } 1\end{array}$ \\
\hline Health and safety & $\begin{array}{l}\text { obs: } 43 \\
\text { no: } 27 \\
\text { yes: } 16\end{array}$ & $\begin{array}{l}\text { obs: } 43 \\
\text { no: } 17 \\
\text { yes: } 26\end{array}$ & $\begin{array}{l}\text { obs: } 43 \\
\text { no: } 15 \\
\text { yes: } 28\end{array}$ & $\begin{array}{l}\text { obs: } 43 \\
\text { no: } 29 \\
\text { yes: } 14\end{array}$ & $\begin{array}{l}\text { obs: } 43 \\
\text { no: } 41 \\
\text { yes: } 2\end{array}$ & $\begin{array}{l}\text { obs: } 43 \\
\text { no: } 42 \\
\text { yes: } 1\end{array}$ \\
\hline Diagnostic procedures & $\begin{array}{l}\text { obs: } 43 \\
\text { no: } 34 \\
\text { yes: } 9\end{array}$ & $\begin{array}{l}\text { obs: } 43 \\
\text { no: } 20 \\
\text { yes: } 23\end{array}$ & $\begin{array}{l}\text { obs: } 43 \\
\text { no: } 6 \\
\text { yes: } 37\end{array}$ & $\begin{array}{l}\text { obs: } 43 \\
\text { no: } 27 \\
\text { yes: } 16\end{array}$ & $\begin{array}{l}\text { obs: } 43 \\
\text { no: } 43 \\
\text { yes: } 0\end{array}$ & $\begin{array}{l}\text { obs: } 43 \\
\text { no: } 43 \\
\text { yes: } 0\end{array}$ \\
\hline Client education & $\begin{array}{l}\text { obs: } 37 \\
\text { no: } 18 \\
\text { yes: } 19\end{array}$ & $\begin{array}{l}\text { obs: } 37 \\
\text { no: } 16 \\
\text { yes: } 21\end{array}$ & $\begin{array}{l}\text { obs: } 37 \\
\text { no: } 29 \\
\text { yes: } 8\end{array}$ & $\begin{array}{l}\text { obs: } 37 \\
\text { no: } 31 \\
\text { yes: } 6\end{array}$ & $\begin{array}{l}\text { obs: } 37 \\
\text { no: } 34 \\
\text { yes: } 3\end{array}$ & $\begin{array}{l}\text { obs: } 37 \\
\text { no: } 36 \\
\text { yes: } 1\end{array}$ \\
\hline Other (specify) & & & & & & \\
\hline
\end{tabular}


7. For each broad subject listed in the left-most column of the following table, please indicate the quality of training received by your in-house staff in Program Year 2008 at the training venues listed in the column headings. Please leave cells blank were your in-house staff did not receive training during this period of time. Please use the following scale: 1-very low; 2 - low; 3-medium; 4- high; 5-very high

\begin{tabular}{|c|c|c|c|c|c|c|}
\hline Subject & $\begin{array}{c}\text { National } \\
\text { Weatherization } \\
\text { Program Conference }\end{array}$ & $\begin{array}{c}\text { Affordable } \\
\text { Comfort } \\
\text { Conference }\end{array}$ & $\begin{array}{c}\text { Regional } \\
\text { Weatherization } \\
\text { Conference } \\
\end{array}$ & $\begin{array}{c}\text { State } \\
\text { Weatherization } \\
\text { Conference } \\
\end{array}$ & $\begin{array}{l}\text { State/ Regional } \\
\text { Training Center }\end{array}$ & $\begin{array}{c}\text { Training } \\
\text { Provided by Your } \\
\text { Own State }\end{array}$ \\
\hline Management & $\begin{array}{l}\text { obs: } 26 \\
\text { very low: } 0 \\
\text { low: } 1 \\
\text { medium: } 10 \\
\text { high: } 8 \\
\text { very high: } 7\end{array}$ & $\begin{array}{l}\text { obs: } 8 \\
\text { very low: } 0 \\
\text { low: } 0 \\
\text { medium: } 3 \\
\text { high: } 2 \\
\text { very high: } 3\end{array}$ & $\begin{array}{l}\text { obs: } 18 \\
\text { very low: } 0 \\
\text { low: } 0 \\
\text { medium: } 11 \\
\text { high: } 5 \\
\text { very high: } 2\end{array}$ & $\begin{array}{l}\text { obs: } 15 \\
\text { very low: } 1 \\
\text { low: } 0 \\
\text { medium: } 3 \\
\text { high: } 8 \\
\text { very high: } 3\end{array}$ & $\begin{array}{l}\text { obs: } 5 \\
\text { very low: } 0 \\
\text { low: } 0 \\
\text { medium: } 1 \\
\text { high: } 0 \\
\text { very high: } 4\end{array}$ & $\begin{array}{l}\text { obs: } 15 \\
\text { very low: } 1 \\
\text { low: } 1 \\
\text { medium: } 1 \\
\text { high: } 8 \\
\text { very high: } 4\end{array}$ \\
\hline $\begin{array}{l}\text { Weatherization } \\
\text { installation }\end{array}$ & $\begin{array}{l}\text { obs: } 18 \\
\text { very low: } 0 \\
\text { low: } 0 \\
\text { medium: } 10 \\
\text { high: } 6 \\
\text { very high: } 2\end{array}$ & $\begin{array}{l}\text { obs: } 13 \\
\text { very low: } 0 \\
\text { low: } 0 \\
\text { medium: } 4 \\
\text { high: } 4 \\
\text { very high: } 5\end{array}$ & $\begin{array}{l}\text { obs: } 21 \\
\text { very low: } 0 \\
\text { low: } 0 \\
\text { medium: } 7 \\
\text { high: } 8 \\
\text { very high: } 6\end{array}$ & $\begin{array}{l}\text { obs: } 14 \\
\text { very low: } 0 \\
\text { low: } 0 \\
\text { medium: } 2 \\
\text { high: } 7 \\
\text { very high: } 5\end{array}$ & $\begin{array}{l}\text { obs: } 9 \\
\text { very low: } 0 \\
\text { low: } 0 \\
\text { medium: } 1 \\
\text { high: } 2 \\
\text { very high: } 6\end{array}$ & $\begin{array}{l}\text { obs: } 20 \\
\text { very low: } 0 \\
\text { low: } 0 \\
\text { medium: } 5 \\
\text { high: } 9 \\
\text { very high: } 6\end{array}$ \\
\hline $\begin{array}{l}\text { Auditing/ } \\
\text { Estimating }\end{array}$ & $\begin{array}{l}\text { obs: } 18 \\
\text { very low: } 0 \\
\text { low: } 0 \\
\text { medium: } 10 \\
\text { high: } 5 \\
\text { very high: } 3\end{array}$ & $\begin{array}{l}\text { obs: } 12 \\
\text { very low: } 0 \\
\text { low: } 0 \\
\text { medium: } 4 \\
\text { high: } 3 \\
\text { very high: } 5\end{array}$ & $\begin{array}{l}\text { obs: } 17 \\
\text { very low: } 0 \\
\text { low: } 1 \\
\text { medium: } 7 \\
\text { high: } 4 \\
\text { very high: } 5\end{array}$ & $\begin{array}{l}\text { obs: } 10 \\
\text { very low: } 0 \\
\text { low: } 1 \\
\text { medium: } 2 \\
\text { high: } 5 \\
\text { very high: } 2\end{array}$ & $\begin{array}{l}\text { obs: } 8 \\
\text { very low: } 0 \\
\text { low: } 0 \\
\text { medium: } 2 \\
\text { high: } 1 \\
\text { very high: } 5\end{array}$ & $\begin{array}{l}\text { obs: } 20 \\
\text { very low: } 0 \\
\text { low: } 0 \\
\text { medium: } 3 \\
\text { high: } 11 \\
\text { very high: } 6\end{array}$ \\
\hline $\begin{array}{l}\text { Monitoring/ } \\
\text { quality control }\end{array}$ & $\begin{array}{l}\text { obs: } 19 \\
\text { very low: } 0 \\
\text { low: } 4 \\
\text { medium: } 6 \\
\text { high: } 7 \\
\text { very high: } 2\end{array}$ & $\begin{array}{l}\text { obs: } 10 \\
\text { very low: } 1 \\
\text { low: } 0 \\
\text { medium: } 1 \\
\text { high: } 4 \\
\text { very high: } 4\end{array}$ & $\begin{array}{l}\text { obs: } 16 \\
\text { very low: } 0 \\
\text { low: } 2 \\
\text { medium: } 6 \\
\text { high: } 2 \\
\text { very high: } 6\end{array}$ & $\begin{array}{l}\text { obs: } 10 \\
\text { very low: } 1 \\
\text { low: } 0 \\
\text { medium: } 2 \\
\text { high: } 4 \\
\text { very high: } 3\end{array}$ & $\begin{array}{l}\text { obs: } 5 \\
\text { very low: } 0 \\
\text { low: } 0 \\
\text { medium: } 1 \\
\text { high: } 0 \\
\text { very high: } 4\end{array}$ & $\begin{array}{l}\text { obs: } 18 \\
\text { very low: } 0 \\
\text { low: } 0 \\
\text { medium: } 3 \\
\text { high: } 10 \\
\text { very high: } 5\end{array}$ \\
\hline Financial topics & $\begin{array}{l}\text { obs: } 20 \\
\text { very low: } 0 \\
\text { low: } 2 \\
\text { medium: } 8 \\
\text { high: } 6 \\
\text { very high: } 4\end{array}$ & $\begin{array}{l}\text { obs: } 5 \\
\text { very low: } 0 \\
\text { low: } 2 \\
\text { medium: } 1 \\
\text { high: } 0 \\
\text { very high: } 2\end{array}$ & $\begin{array}{l}\text { obs: } 14 \\
\text { very low: } 2 \\
\text { low: } 0 \\
\text { medium: } 6 \\
\text { high: } 2 \\
\text { very high: } 4\end{array}$ & $\begin{array}{l}\text { obs: } 8 \\
\text { very low: } 1 \\
\text { low: } 0 \\
\text { medium: } 4 \\
\text { high: } 2 \\
\text { very high: } 1\end{array}$ & $\begin{array}{l}\text { obs: } 4 \\
\text { very low: } 0 \\
\text { low: } 0 \\
\text { medium: } 1 \\
\text { high: } 1 \\
\text { very high: } 2\end{array}$ & $\begin{array}{l}\text { obs: } 16 \\
\text { very low: } 0 \\
\text { low: } 1 \\
\text { medium: } 4 \\
\text { high: } 6 \\
\text { very high: } 5\end{array}$ \\
\hline $\begin{array}{l}\text { Outreach and } \\
\text { communications }\end{array}$ & $\begin{array}{l}\text { obs: } 13 \\
\text { very low: } 1 \\
\text { low: } 4 \\
\text { medium: } 2 \\
\text { high: } 4 \\
\text { very high: } 2\end{array}$ & $\begin{array}{l}\text { obs: } 6 \\
\text { very low: } 0 \\
\text { low: } 0 \\
\text { medium: } 1 \\
\text { high: } 2 \\
\text { very high: } 3\end{array}$ & $\begin{array}{l}\text { obs: } 8 \\
\text { very low: } 1 \\
\text { low: } 1 \\
\text { medium: } 3 \\
\text { high: } 1 \\
\text { very high: } 2\end{array}$ & $\begin{array}{l}\text { obs: } 10 \\
\text { very low: } 1 \\
\text { low: } 1 \\
\text { medium: } 4 \\
\text { high: } 2 \\
\text { very high: } 2\end{array}$ & $\begin{array}{l}\text { obs: } 3 \\
\text { very low: } 0 \\
\text { low: } 0 \\
\text { medium: } 1 \\
\text { high: } 1 \\
\text { very high: } 1\end{array}$ & $\begin{array}{l}\text { obs: } 14 \\
\text { very low: } 0 \\
\text { low: } 1 \\
\text { medium: } 4 \\
\text { high: } 5 \\
\text { very high: } 4\end{array}$ \\
\hline Health and safety & $\begin{array}{l}\text { obs: } 17 \\
\text { very low: } 1 \\
\text { low: } 2 \\
\text { medium: } 4 \\
\text { high: } 6 \\
\text { very high: } 4 \\
\end{array}$ & $\begin{array}{l}\text { obs: } 11 \\
\text { very low: } 0 \\
\text { low: } 0 \\
\text { medium: } 2 \\
\text { high: } 2 \\
\text { very high: } 7 \\
\end{array}$ & $\begin{array}{l}\text { obs: } 18 \\
\text { very low: } 1 \\
\text { low: } 0 \\
\text { medium: } 3 \\
\text { high: } 9 \\
\text { very high: } 5 \\
\end{array}$ & $\begin{array}{l}\text { obs: } 11 \\
\text { very low: } 0 \\
\text { low: } 0 \\
\text { medium: } 4 \\
\text { high: } 4 \\
\text { very high: } 3 \\
\end{array}$ & $\begin{array}{l}\text { obs: } 6 \\
\text { very low: } 0 \\
\text { low: } 0 \\
\text { medium: } 1 \\
\text { high: } 1 \\
\text { very high: } 4 \\
\end{array}$ & $\begin{array}{l}\text { obs: } 17 \\
\text { very low: } 0 \\
\text { low: } 1 \\
\text { medium: } 4 \\
\text { high: } 6 \\
\text { very high: } 6 \\
\end{array}$ \\
\hline $\begin{array}{l}\text { Diagnostic } \\
\text { procedures }\end{array}$ & $\begin{array}{l}\text { obs: } 18 \\
\text { very low: } 0 \\
\text { low: } 1 \\
\text { medium: } 5 \\
\text { high: } 9 \\
\text { very high: } 3\end{array}$ & $\begin{array}{l}\text { obs: } 10 \\
\text { very low: } 0 \\
\text { low: } 0 \\
\text { medium: } 1 \\
\text { high: } 2 \\
\text { very high: } 7\end{array}$ & $\begin{array}{l}\text { obs: } 15 \\
\text { very low: } 0 \\
\text { low: } 0 \\
\text { medium: } 6 \\
\text { high: } 5 \\
\text { very high: } 4\end{array}$ & $\begin{array}{l}\text { obs: } 11 \\
\text { very low: } 0 \\
\text { low: } 1 \\
\text { medium: } 3 \\
\text { high: } 3 \\
\text { very high: } 4\end{array}$ & $\begin{array}{l}\text { obs: } 7 \\
\text { very low: } 0 \\
\text { low: } 0 \\
\text { medium: } 1 \\
\text { high: } 2 \\
\text { very high: } 4\end{array}$ & $\begin{array}{l}\text { obs: } 21 \\
\text { very low: } 0 \\
\text { low: } 1 \\
\text { medium: } 2 \\
\text { high: } 10 \\
\text { very high: } 8\end{array}$ \\
\hline $\begin{array}{l}\text { Procedures for } \\
\text { selecting } \\
\text { weatherization } \\
\text { measures }\end{array}$ & $\begin{array}{l}\text { obs: } 14 \\
\text { very low: } 0 \\
\text { low: } 2 \\
\text { medium: } 3 \\
\text { high: } 4 \\
\text { very high: } 5\end{array}$ & $\begin{array}{l}\text { obs: } 10 \\
\text { very low: } 0 \\
\text { low: } 0 \\
\text { medium: } 2 \\
\text { high: } 3 \\
\text { very high: } 5 \\
\end{array}$ & $\begin{array}{l}\text { obs: } 15 \\
\text { very low: } 0 \\
\text { low: } 1 \\
\text { medium: } 6 \\
\text { high: } 3 \\
\text { very high: } 5 \\
\end{array}$ & $\begin{array}{l}\text { obs: } 10 \\
\text { very low: } 0 \\
\text { low: } 1 \\
\text { medium: } 4 \\
\text { high: } 3 \\
\text { very high: } 2 \\
\end{array}$ & $\begin{array}{l}\text { obs: } 5 \\
\text { very low: } 0 \\
\text { low: } 0 \\
\text { medium: } 1 \\
\text { high: } 0 \\
\text { very high: } 4 \\
\end{array}$ & $\begin{array}{l}\text { obs: } 20 \\
\text { very low: } 0 \\
\text { low: } 1 \\
\text { medium: } 3 \\
\text { high: } 10 \\
\text { very high: } 6\end{array}$ \\
\hline Client education & $\begin{array}{l}\text { obs: } 16 \\
\text { very low: } 0 \\
\text { low: } 5 \\
\text { medium: } 5 \\
\text { high: } 3 \\
\text { very high: } 3\end{array}$ & $\begin{array}{l}\text { obs: } 7 \\
\text { very low: } 1 \\
\text { low: } 0 \\
\text { medium: } 3 \\
\text { high: } 0 \\
\text { very high: } 3\end{array}$ & $\begin{array}{l}\text { obs: } 15 \\
\text { very low: } 1 \\
\text { low: } 2 \\
\text { medium: } 7 \\
\text { high: } 3 \\
\text { very high: } 2\end{array}$ & $\begin{array}{l}\text { obs: } 8 \\
\text { very low: } 0 \\
\text { low: } 3 \\
\text { medium: } 1 \\
\text { high: } 2 \\
\text { very high: } 2\end{array}$ & $\begin{array}{l}\text { obs: } 5 \\
\text { very low: } 0 \\
\text { low: } 1 \\
\text { medium: } 0 \\
\text { high: } 1 \\
\text { very high: } 3\end{array}$ & $\begin{array}{l}\text { obs: } 15 \\
\text { very low: } 0 \\
\text { low: } 1 \\
\text { medium: } 5 \\
\text { high: } 4 \\
\text { very high: } 5\end{array}$ \\
\hline Other (specify) & & & & & & \\
\hline
\end{tabular}


7. On which of the following weatherization topics did your state provide training to your state's local weatherization agencies or their contractors in Program Year 2008? (Check all that apply). Refer only to training that your office provided directly or had an active role in directing local agencies to attend or take.

8.

(1) Diagnostic procedures

Freq. Percent Cum.

\begin{tabular}{|c|c|c|c|}
\hline no & 2 & 4.55 & 4.55 \\
\hline yes | & 42 & 95.45 & 100.00 \\
\hline Total | & 44 & 100.00 & \\
\hline
\end{tabular}

\section{(2) Insulation}

\begin{tabular}{|c|c|c|c|}
\hline \multicolumn{4}{|c|}{-- single family dwellings } \\
\hline & Freq. & Percent & Cum. \\
\hline no & 7 & 15.91 & 15.91 \\
\hline yes & 37 & 84.09 & 100.00 \\
\hline Total | & 44 & 100.00 & \\
\hline \multicolumn{4}{|c|}{-- multifamily dwellings } \\
\hline & Freq & Percent & Cum. \\
\hline no & 28 & 63.64 & 63.64 \\
\hline yes & 16 & 36.36 & 100.00 \\
\hline Total | & 44 & 100.00 & \\
\hline \multicolumn{4}{|c|}{-- mobile homes } \\
\hline & Freq. & Percent & Cum. \\
\hline no $\mid$ & 13 & 29.55 & 29.55 \\
\hline yes $\mid$ & 31 & 70.45 & 100.00 \\
\hline Total | & 44 & 100.00 & \\
\hline
\end{tabular}

(3) Space heating, ventilation, air conditioning

-- single family dwellings

Freq. Percent Cum.

\begin{tabular}{crrr} 
no $\mid$ & 6 & 13.64 & 13.64 \\
yes $\mid$ & 38 & 86.36 & 100.00 \\
- \hdashline------ & \\
Total| & 44 & 100.00
\end{tabular}


-- multifamily dwellings

Freq. Percent Cum.

$\begin{array}{cccc}\text { no } \mid & 28 & 63.64 & 63.64 \\ \text { yes } \mid & 16 & 36.36 & 100.00 \\ ------------ \\ \text { Total } & 44 & 100.00\end{array}$

-- mobile homes

Freq. Percent Cum.

\begin{tabular}{|c|c|c|c|}
\hline no & 10 & 22.73 & 22.73 \\
\hline yes | & 34 & 77.27 & 100.00 \\
\hline Total | & 44 & 100.00 & \\
\hline
\end{tabular}

(4) Infiltration measures

-- single family dwellings

Freq. Percent Cum.

\begin{tabular}{|c|c|c|c|}
\hline no & 4 & 9.09 & 9.09 \\
\hline yes & 40 & 90.91 & 100.00 \\
\hline Total | & 44 & 100.00 & \\
\hline
\end{tabular}

-- multifamily dwellings

Freq. Percent Cum.

\begin{tabular}{|c|c|c|c|}
\hline no & 27 & 61.36 & 61.36 \\
\hline yes & 17 & 38.64 & 100.00 \\
\hline Total | & 44 & 100.00 & \\
\hline
\end{tabular}

-- mobile homes

Freq. Percent Cum.

\begin{tabular}{|c|c|c|c|}
\hline no| & 10 & 22.73 & 22.73 \\
\hline yes & 34 & 77.27 & 100.00 \\
\hline
\end{tabular}

(5) Doors and windows

-- single family dwellings Freq. Percent Cum.

\begin{tabular}{|c|c|c|c|}
\hline no| & 17 & 38.64 & 38.64 \\
\hline yes & 27 & 61.36 & 100.00 \\
\hline Total | & 44 & 100.00 & \\
\hline
\end{tabular}

-- multifamily dwellings 


\begin{tabular}{|c|c|c|c|}
\hline & Freq. & Percent & Cum. \\
\hline no $\mid$ & 31 & 70.45 & 70.45 \\
\hline yes & 13 & 29.55 & 100.00 \\
\hline Total | & 44 & 100.00 & \\
\hline \multicolumn{4}{|c|}{-- mobile homes } \\
\hline & Freq. & Percent & Cum. \\
\hline no $\mid$ & 18 & 40.91 & 40.91 \\
\hline yes | & 26 & 59.09 & 100.00 \\
\hline Total | & 44 & 100.00 & \\
\hline
\end{tabular}

(6) Hot water heating

-- single family dwellings

Freq. Percent Cum.

\begin{tabular}{|c|c|c|c|}
\hline no & 9 & 20.45 & 20.45 \\
\hline yes | & 35 & 79.55 & 100.00 \\
\hline Total | & 44 & 100.00 & \\
\hline
\end{tabular}

-- multifamily dwellings Freq. Percent Cum.

$\begin{array}{cccc}\text { no } \mid & 28 & 63.64 & 63.64 \\ \text { yes } \mid & 16 & 36.36 & 100.00 \\ --------- & \\ - & \end{array}$

\section{-- mobile homes}

Freq. Percent Cum.

\begin{tabular}{cccc} 
no $\mid$ & 11 & 25.00 & 25.00 \\
yes $\mid$ & 33 & 75.00 & 100.00 \\
- \hdashline-------- & \\
Total| & 44 & 100.00
\end{tabular}

(7) Baseloads (e.g., lighting, refrigerators)

-- single family dwellings

Freq. Percent Cum.

\begin{tabular}{|c|c|c|c|}
\hline no & 9 & 20.45 & 20.45 \\
\hline yes & 35 & 79.55 & 100.00 \\
\hline Total | & 44 & 100.00 & \\
\hline
\end{tabular}




\section{-- multifamily dwellings}

Freq. Percent Cum.

\begin{tabular}{|c|c|c|c|}
\hline no| & 24 & 54.55 & 54.55 \\
\hline yes & 20 & 45.45 & 100.00 \\
\hline Total | & 44 & 100.00 & \\
\hline
\end{tabular}

-- mobile homes

Freq. Percent Cum.

\begin{tabular}{|c|c|c|c|}
\hline no| & 13 & 29.55 & 29.55 \\
\hline yes & 31 & 70.45 & 100.00 \\
\hline Total | & 44 & 100.00 & \\
\hline
\end{tabular}

8a. On which of the following administrative-related topics did your state provide training to your state's local weatherization agencies or their contractors in Program Year 2008? (Check all that apply). Refer only to training that your office provided directly or had an active role in directing local agencies to attend or take.

(1) Management

$\begin{array}{rrrr}\text { Freq. } & \text { Percent } & \text { Cum. } \\ \text { no } \mid & 15 & 33.33 & 33.33 \\ \text { yes } \mid & 30 & 66.67 & 100.00 \\ ------- \\ \text { Total| } & 45 & 100.00\end{array}$

(2) Client education

\begin{tabular}{|c|c|c|c|}
\hline & eq. & Percent & Cum. \\
\hline no & 18 & 40.00 & 40.00 \\
\hline yes & 27 & 60.00 & 100.00 \\
\hline Total | & 45 & 100.00 & \\
\hline
\end{tabular}

\section{(3) Auditing/estimating}

-- single family dwellings

Freq. Percent Cum.

\begin{tabular}{|c|c|c|c|}
\hline no & 4 & $\begin{array}{r}8.89 \\
0111\end{array}$ & $\begin{array}{r}8.89 \\
0000\end{array}$ \\
\hline & & & \\
\hline Total | & 45 & 100.00 & \\
\hline
\end{tabular}




\begin{tabular}{|c|c|c|c|}
\hline \multicolumn{4}{|c|}{-- multifamily dwellings } \\
\hline & Freq. & Percent & Cum. \\
\hline no & 30 & 66.67 & 66.67 \\
\hline yes & 15 & 33.33 & 100.00 \\
\hline Total & 45 & 100.00 & \\
\hline & obile & omes & \\
\hline & Freq. & Percent & Cum. \\
\hline no & 14 & 31.11 & 31.11 \\
\hline yes & 31 & 68.89 & 100.00 \\
\hline Total & 45 & 100.00 & \\
\hline
\end{tabular}

(4) Monitoring/quality control Freq. Percent Cum.

$\begin{array}{cccc}\text { no } \mid & 15 & 33.33 & 33.33 \\ \text { yes } \mid & 30 & 66.67 & 100.00 \\ ------------- \\ \text { Total } & 45 & 100.00\end{array}$

(5) Financial topics

Freq. Percent Cum.

\begin{tabular}{|c|c|c|c|}
\hline no & 17 & 37.78 & 37.78 \\
\hline yes & 28 & 62.22 & 100.00 \\
\hline Total | & 45 & 100.00 & \\
\hline
\end{tabular}

(6) Outreach and communications

Freq. Percent Cum.

$\begin{array}{cccc}\text { no } \mid & 24 & 53.33 & 53.33 \\ \text { yes } \mid & 21 & 46.67 & 100.00 \\ ------------ & \\ \text { Total } & 45 & 100.00\end{array}$

(7) Other (please specify) 
8 b. On which of the following health and safety topics did your state provide training to your state's local weatherization agencies or their contractors in Program Year 2008? (Check all that apply). Refer only to training that your office provided directly or had an active role in directing local agencies to attend or take.

\begin{tabular}{|c|c|c|c|}
\hline Fir & $\begin{array}{l}\text { safety } \\
\text { Freq. }\end{array}$ & Percent & Cum. \\
\hline no & 31 & 70.45 & 70.45 \\
\hline yes | & 13 & 29.55 & 100.00 \\
\hline Total | & 44 & 100.00 & \\
\hline
\end{tabular}

Indoor air quality

Freq. Percent Cum.

$\begin{array}{crrr}\text { no } \mid & 8 & 18.18 & 18.18 \\ \text { yes | } & 36 & 81.82 & 100.00 \\ ----------- \\ \text { Total } & 44 & 100.00\end{array}$

Measures to increase security of housing unit

Freq. Percent Cum.

\begin{tabular}{|c|c|c|c|}
\hline no & 40 & 90.91 & 90.91 \\
\hline yes & 4 & 9.09 & 100.00 \\
\hline Total | & 44 & 100.00 & \\
\hline
\end{tabular}

Measures to reduce common household hazards

Freq. Percent Cum.

\begin{tabular}{|c|c|c|c|}
\hline no & 23 & 52.27 & 52.27 \\
\hline yes | & 21 & 47.73 & 100.00 \\
\hline Total | & 44 & 100.00 & \\
\hline
\end{tabular}

Mold and mildew

Freq. Percent Cum.

\begin{tabular}{|c|c|c|c|}
\hline no & 10 & 22.73 & 22.73 \\
\hline yes $\mid$ & 34 & 77.27 & 100.00 \\
\hline Total | & 44 & 100.00 & \\
\hline
\end{tabular}


Lead

Freq. Percent Cum.

$\begin{array}{crrr}\text { no } \mid & 9 & 20.45 & 20.45 \\ \text { yes } \mid & 35 & 79.55 & 100.00 \\ --------------- \\ \text { Total } & 44 & 100.00\end{array}$

\begin{tabular}{|c|c|c|c|}
\hline As & $\begin{array}{l}\text { tos } \\
\text { req. }\end{array}$ & Percent & Cum. \\
\hline no & 23 & 52.27 & 52.27 \\
\hline yes | & 21 & 47.73 & 100.00 \\
\hline otal | & 44 & 100.00 & \\
\hline
\end{tabular}

Vermiculite

\begin{tabular}{|c|c|c|c|}
\hline & Freq. & Percent & Cum. \\
\hline no & 35 & 79.55 & 79.55 \\
\hline yes & 9 & 20.45 & 100.00 \\
\hline Total & 44 & 100.00 & \\
\hline
\end{tabular}

\begin{tabular}{|c|c|c|c|}
\hline Ge & $\begin{array}{l}\text { al cr } \\
\text { treq. }\end{array}$ & $\begin{array}{l}\text { v safety } \\
\text { Percent }\end{array}$ & Cum. \\
\hline nol & 17 & 38.64 & 38.64 \\
\hline yes | & 27 & 61.36 & 100.00 \\
\hline otal & 44 & 100.00 & \\
\hline
\end{tabular}

Other (please specify)

9. On which of the following diagnostic procedures did your state provide training to your state's local weatherization agencies or their contractors in Program Year 2008? (Check all that apply). Refer only to training that your office provided directly or had an active role in directing local agencies to attend or take.

Pressure diagnostics:

- Blower door (house air leakage rate)

Freq. Percent Cum.

\begin{tabular}{|c|c|c|c|}
\hline no & 2 & 4.65 & 4.65 \\
\hline yes | & 41 & 95.35 & 100.00 \\
\hline Total & 43 & 100.00 & \\
\hline
\end{tabular}


- Zonal pressure measurements

\begin{tabular}{|c|c|c|c|}
\hline & Freq. & Percent & Cum. \\
\hline no & 12 & 27.91 & 27.91 \\
\hline yes $\mid$ & 31 & 72.09 & 100.00 \\
\hline Total | & 43 & 100.00 & \\
\hline
\end{tabular}

- Room-to-room pressure measurements (distribution balancing) Freq. Percent Cum.

$\begin{array}{cccc}\text { no } \mid & 18 & 41.86 & 41.86 \\ \text { yes } \mid & 25 & 58.14 & 100.00 \\ ------------ \\ \text { Total } & 43 & 100.00\end{array}$

- Duct pressure pan measurements

\begin{tabular}{|c|c|c|c|}
\hline & Freq. & Percent & Cum. \\
\hline no $\mid$ & 13 & 30.23 & 30.23 \\
\hline yes $\mid$ & 30 & 69.77 & 100.00 \\
\hline Total | & 43 & 100.00 & \\
\hline
\end{tabular}

- Duct blower measurements (duct air leakage rate)

\begin{tabular}{|c|c|c|c|}
\hline & ireq. & Percent & Cum. \\
\hline no & 17 & 39.53 & 39.53 \\
\hline yes & 26 & 60.47 & 100.00 \\
\hline Total | & 43 & 100.00 & \\
\hline
\end{tabular}

Space-heating system:

- Flue gas analysis (steady-state efficiency measurements) Freq. Percent Cum.

\begin{tabular}{|c|c|c|c|}
\hline no & 14 & 32.56 & 32.56 \\
\hline yes & 29 & 67.44 & 100.00 \\
\hline Total & 43 & 100.00 & \\
\hline
\end{tabular}

- Heat rise measurements

Freq. Percent Cum.

\begin{tabular}{cccc} 
no $\mid$ & 18 & 41.86 & 41.86 \\
yes $\mid$ & 25 & 58.14 & 100.00 \\
--------- & \\
\hline Total & 43 & 100.00
\end{tabular}


- CO measurements in flues

$\begin{array}{crrr} & \text { Freq. } & \text { Percent } & \text { Cum. } \\ \text { no } \mid & 4 & 9.30 & 9.30 \\ \text { yes } \mid & 39 & 90.70 & 100.00 \\ ------- & \end{array}$

- Draft/spillage (normal operation) Freq. Percent Cum.

\begin{tabular}{|c|c|c|c|}
\hline no & 16 & 37.21 & 37.21 \\
\hline yes | & 27 & 62.79 & 100.00 \\
\hline Total | & 43 & 100.00 & \\
\hline
\end{tabular}

Air-conditioning system:

- Refrigerant charge (e.g., superheat, subcooling) Freq. Percent Cum.

\begin{tabular}{|c|c|c|c|}
\hline no & 35 & 81.40 & 81.40 \\
\hline yes & 8 & 18.60 & 100.00 \\
\hline otal | & 43 & 100.00 & \\
\hline
\end{tabular}

HVAC components and cross-cutting diagnostics:

- Air handler flow rate

$\begin{array}{rrrr} & \text { Freq. } & \text { Percent } & \text { Cum. } \\ \text { no } \mid & 27 & 62.79 & 62.79 \\ \text { yes } \mid & 16 & 37.21 & 100.00 \\ --------- & \end{array}$

- Thermostat anticipator current

Freq. Percent Cum.

$\begin{array}{crrr}\text { no } \mid & 27 & 62.79 & 62.79 \\ \text { yes } \mid & 16 & 37.21 & 100.00 \\ ----------------- \\ \text { Total | } & 43 & 100.00\end{array}$

- Worst case draft/spillage (CAZ)

Freq. Percent Cum.

\begin{tabular}{|c|c|c|c|}
\hline no & 17 & 39.53 & 39.53 \\
\hline yes | & 26 & 60.47 & 100.00 \\
\hline Total & 43 & 100.00 & \\
\hline
\end{tabular}


Hot-water (water-heating) system:

- Flue gas analysis (steady-state efficiency measurements) Freq. Percent Cum.

\begin{tabular}{|c|c|c|c|}
\hline no $\mid$ & 17 & 39.53 & 39.53 \\
\hline yes | & 26 & 60.47 & 100.00 \\
\hline
\end{tabular}

- CO measurements in flues Freq. Percent Cum.

\begin{tabular}{|c|c|c|c|}
\hline no & 8 & 18.60 & 18.60 \\
\hline yes | & 35 & 81.40 & 100.00 \\
\hline Total | & 43 & 100.00 & \\
\hline
\end{tabular}

- Draft/spillage (normal operation)

$\begin{array}{cccc} & \text { Freq. } & \text { Percent } & \text { Cum. } \\ \text { no } \mid & 12 & 27.91 & 27.91 \\ \text { yes | } & 31 & 72.09 & 100.00 \\ ---------- \\ \text { Total | } & 43 & 100.00\end{array}$

- Water flow rates (showerheads and faucets)

$\begin{array}{cccc} & \text { Freq. } & \text { Percent } & \text { Cum. } \\ \text { no } \mid & 23 & 53.49 & 53.49 \\ \text { yes } \mid & 20 & 46.51 & 100.00 \\ ------ & \\ \text { Total } \mid & 43 & 100.00\end{array}$

Other CO measurements:

- CO measurements in equipment rooms Freq. Percent Cum.

\begin{tabular}{|c|c|c|c|}
\hline no & 13 & 30.23 & 30.23 \\
\hline yes $\mid$ & 30 & 69.77 & 100.00 \\
\hline Total | & 43 & 100.00 & \\
\hline
\end{tabular}

- Cooking stove

Freq. Percent Cum.

\begin{tabular}{cccc} 
no $\mid$ & 11 & 25.58 & 25.58 \\
yes $\mid$ & 32 & 74.42 & 100.00 \\
-------------- \\
\hline Total | & 43 & 100.00
\end{tabular}

- CO measurements in living areas 


$\begin{array}{cccc} & \text { Freq. } & \text { Percent } & \text { Cum. } \\ \text { no } \mid & 11 & 25.58 & 25.58 \\ \text { yes } \mid & 32 & 74.42 & 100.00 \\ ------ & \\ \text { Total | } & 43 & 100.00 & \end{array}$

Other diagnostics and inspections:

- Refrigerator energy use

Freq. Percent Cum.

\begin{tabular}{|c|c|c|c|}
\hline no & 14 & 32.56 & 32.56 \\
\hline yes & 29 & 67.44 & 100.00 \\
\hline Total & 43 & 100.00 & \\
\hline
\end{tabular}

- Exhaust fan air flow rate measurement Freq. Percent Cum.

\begin{tabular}{|c|c|c|c|}
\hline no & 23 & 53.49 & 53.49 \\
\hline yes | & 20 & 46.51 & 100.00 \\
\hline Total | & 43 & 100.00 & \\
\hline
\end{tabular}

- Infrared scanning (camera)

$\begin{array}{rrrr} & \text { Freq. } & \text { Percent } & \text { Cum. } \\ \text { no } \mid & 18 & 41.86 & 41.86 \\ \text { yes } \mid & 25 & 58.14 & 100.00 \\ --------- & \\ \text { Total| } & 43 & 100.00\end{array}$

- Radon testing

\begin{tabular}{crrr} 
& Freq. & Percent & Cum. \\
no $\mid$ & 38 & 88.37 & 88.37 \\
yes | & 5 & 11.63 & 100.00 \\
- \hdashline---- & \\
Total | & 43 & 100.00
\end{tabular}

- Lead testing

Freq. Percent Cum.

\begin{tabular}{|c|c|c|c|}
\hline no & 18 & 41.86 & 41.86 \\
\hline yes & 25 & 58.14 & 100.00 \\
\hline Total & 43 & 100.00 & \\
\hline
\end{tabular}


- Mold and mildew testing

$\begin{array}{cccc} & \text { Freq. } & \text { Percent } & \text { Cum. } \\ \text { no | } & 26 & 60.47 & 60.47 \\ \text { yes | } & 17 & 39.53 & 100.00 \\ ------------- \\ \text { Total | } & 43 & 100.00\end{array}$

- Moisture content testing

Freq. Percent Cum.

\begin{tabular}{|c|c|c|c|}
\hline no & 27 & 62.79 & 62.79 \\
\hline yes & 16 & 37.21 & 100.00 \\
\hline Total & 43 & 100.00 & \\
\hline
\end{tabular}

- Other (please specify)

9. Which of the following types of personnel did your state use to provide training to your state's local weatherization agencies or their contractors in Program Year 2008? (Check all that apply). Refer only to training that your office provided directly or had an active role in directing local agencies to attend or take.

- DOE staff

Freq. Percent Cum.

$\begin{array}{cccc}\text { no } \mid & 41 & 89.13 & 89.13 \\ \text { yes } \mid & 5 & 10.87 & 100.00 \\ --------------- \\ \text { Total } & 46 & 100.00\end{array}$

- DOE contractor

Freq. Percent Cum.

$\begin{array}{rrrr}\text { no } \mid & 38 & 82.61 & 82.61 \\ \text { yes | } & 8 & 17.39 & 100.00 \\ ----------- & \\ \text { Total | } & 46 & 100.00\end{array}$

- State staff

Freq. Percent Cum.

$\begin{array}{crrr}\text { no } \mid & 5 & 10.87 & 10.87 \\ \text { yes } \mid & 41 & 89.13 & 100.00 \\ ------ & \end{array}$


- State contractor

Freq. Percent Cum.

\begin{tabular}{|c|c|c|c|}
\hline no & 30 & 65.22 & 65.22 \\
\hline yes | & 16 & 34.78 & 100.00 \\
\hline Total | & 46 & 100.00 & \\
\hline
\end{tabular}

- Staff from another state

Freq. Percent Cum.

\begin{tabular}{|c|c|c|c|}
\hline no & 35 & 76.09 & 76.09 \\
\hline yes $\mid$ & 11 & 23.91 & 100.00 \\
\hline Cotal | & 46 & 100.00 & \\
\hline
\end{tabular}

- State training center staff Freq. Percent Cum.

\begin{tabular}{cccc} 
no $\mid$ & 34 & 73.91 & 73.91 \\
yes $\mid$ & 12 & 26.09 & 100.00 \\
-------------- \\
\hline Total |
\end{tabular}

- Local agency staff from your state Freq. Percent Cum.

$\begin{array}{rrrr}\text { no } \mid & 18 & 39.13 & 39.13 \\ \text { yes } \mid & 28 & 60.87 & 100.00 \\ ------- & \end{array}$

- Agency staff from another state Freq. Percent Cum.

\begin{tabular}{|c|c|c|c|}
\hline no & 40 & 86.96 & 86.96 \\
\hline yes & 6 & 13.04 & 100.00 \\
\hline otal & 46 & 100.00 & \\
\hline
\end{tabular}

- Manufacturer representative Freq. Percent Cum.

$\begin{array}{cccc}\text { no } \mid & 34 & 73.91 & 73.91 \\ \text { yes } \mid & 12 & 26.09 & 100.00 \\ ------------ & \\ \text { Total } & 46 & 100.00\end{array}$


- Utility staff

Freq. Percent Cum.

$\begin{array}{crrr}\text { no } \mid & 42 & 91.30 & 91.30 \\ \text { yes | } & 4 & 8.70 & 100.00 \\ ----- & & \\ \text { Total } & 46 & 100.00\end{array}$

- Representative from trade organization

Freq. Percent Cum.

$\begin{array}{crrr}\text { no } \mid & 42 & 91.30 & 91.30 \\ \text { yes | } & 4 & 8.70 & 100.00 \\ ---------- & \\ \text { Total | } & 46 & 100.00\end{array}$

- Consultant

Freq. Percent Cum.

\begin{tabular}{|c|c|c|c|}
\hline no & 26 & 56.52 & 56.52 \\
\hline yes & 20 & 43.48 & 100.00 \\
\hline
\end{tabular}

- Other (please specify)

10. What types of credentials or experience were required of the personnel your state used to provide training to your state's local weatherization agencies or their contractors in Program Year 2008? (Check all that apply). Refer only to training that your office provided directly or had an active role in directing local agencies to attend or take.

- Technical certification

Freq. Percent Cum.

\begin{tabular}{|c|c|c|c|}
\hline no & 16 & 36.36 & 36.36 \\
\hline yes & 28 & 63.64 & 100.00 \\
\hline
\end{tabular}

- Extensive weatherization field experience

Freq. Percent Cum.

yes | $44 \quad 100.00100 .00$
------------
Total | 44100.00


- Construction experience

\begin{tabular}{|c|c|c|c|}
\hline & Freq. & Percent & Cum. \\
\hline no & 25 & 56.82 & 56.82 \\
\hline yes & 19 & 43.18 & 100.00 \\
\hline Total & 44 & 100.00 & \\
\hline
\end{tabular}

- Extensive management experience

Freq. Percent Cum.

\begin{tabular}{|c|c|c|c|}
\hline no & 17 & 38.64 & 38.64 \\
\hline yes & 27 & 61.36 & 100.00 \\
\hline Total | & 44 & 100.00 & \\
\hline
\end{tabular}

- Extensive experience with financial matters

$\begin{array}{cccc} & \text { Freq. } & \text { Percent } & \text { Cum. } \\ \text { no } \mid & 24 & 54.55 & 54.55 \\ \text { yes | } & 20 & 45.45 & 100.00 \\ ----------- \\ \text { Total | } & 44 & 100.00\end{array}$

- Other (please specify)

11a. Using the scale below, please indicate how important each credential was for trainers to have in Program Year 2008?

1= Very Unimportant; 2=Unimportant; 3=Important; 4=Very Important

- Technical certification

Freq. Percent Cum.

\begin{tabular}{|c|c|c|c|}
\hline very unimportant & 4 & 9.76 & 9.76 \\
\hline unimportant & 7 & 17.07 & 26.83 \\
\hline important & 14 & 34.15 & 60.98 \\
\hline very important & 16 & 39.02 & 100.00 \\
\hline Tota & 41 & 100.00 & \\
\hline
\end{tabular}

- Extensive weatherization field experience

Freq. Percent Cum.

\begin{tabular}{|c|c|c|c|}
\hline very unimportant & 1 & 2.22 & 2.22 \\
\hline unimportant & 1 & 2.22 & 4.44 \\
\hline important & 6 & 13.33 & 17.78 \\
\hline very important & 37 & 82.22 & 100.00 \\
\hline Total & 45 & 100.00 & \\
\hline
\end{tabular}


- Construction experience

\begin{tabular}{|c|c|c|c|}
\hline & Freq. & Percent & Cum. \\
\hline very unimportant & 3 & 7.50 & 7.50 \\
\hline unimportant & 9 & 22.50 & 30.00 \\
\hline important & 17 & 42.50 & 72.50 \\
\hline very important & 11 & 27.50 & 100.00 \\
\hline Total & 40 & 100.00 & \\
\hline
\end{tabular}

- Extensive management experience

$\begin{array}{rrrr} & \text { Freq. } & \text { Percent } & \text { Cum. } \\ \text { very unimportant | } & 1 & 2.63 & 2.63 \\ \text { unimportant } \mid & 6 & 15.79 & 18.42 \\ \text { important } \mid & 19 & 50.00 & 68.42 \\ \text { very important } \mid & 12 & 31.58 & 100.00 \\ - \text { Total }^{\mid} & 38 & 100.00 & \end{array}$

- Extensive experience with financial matters

$\begin{array}{rcrr} & \text { Freq. } & \text { Percent } & \text { Cum. } \\ \text { very unimportant | } & 2 & 5.56 & 5.56 \\ \text { unimportant } \mid & 8 & 22.22 & 27.78 \\ \text { important } \mid & 16 & 44.44 & 72.22 \\ \text { very important } \mid & 10 & 27.78 & 100.00 \\ -----------------------\end{array}$

- Other (please specify)

12. How many of your state's weatherization office staff acted as instructors at the following training events that your state provided (e.g., funded, organized) to your state's local weatherization agencies or their contractors in Program Year 2008?

- State weatherization conference

\begin{tabular}{|l|c|}
\hline observations: & 29 \\
\hline missing values: & 22 \\
\hline mean: & 2.17 \\
\hline standard deviation: & 1.67 \\
\hline min: & 0 \\
\hline 10th percentile: & 0 \\
\hline 25th percentile: & 1 \\
\hline median: & 2 \\
\hline 75th percentile: & 3 \\
\hline 90th percentile: & 5 \\
\hline max: & 5 \\
\hline
\end{tabular}


- Other state conference

\begin{tabular}{|l|c|}
\hline observations: & 18 \\
\hline missing values: & 33 \\
\hline mean: & 2.56 \\
\hline standard deviation: & 3.60 \\
\hline min: & 0 \\
\hline 10th percentile: & 0 \\
\hline 25th percentile: & 0 \\
\hline median: & 1.5 \\
\hline 75th percentile: & 4 \\
\hline 90th percentile: & 6 \\
\hline max: & 15 \\
\hline
\end{tabular}

\section{- State/regional training center class}

\begin{tabular}{|l|c|}
\hline observations: & 23 \\
\hline missing values: & 28 \\
\hline mean: & 1.70 \\
\hline standard deviation: & 1.64 \\
\hline min: & 0 \\
\hline 10th percentile: & 0 \\
\hline 25th percentile: & 0 \\
\hline median: & 1 \\
\hline 75th percentile: & 2 \\
\hline 90th percentile: & 5 \\
\hline max: & 5 \\
\hline
\end{tabular}

- State-sponsored class taught at central location

\begin{tabular}{|l|c|}
\hline observations: & 28 \\
\hline missing values: & 23 \\
\hline mean: & 2.04 \\
\hline standard deviation: & 1.57 \\
\hline min: & 0 \\
\hline 10th percentile: & 0 \\
\hline 25th percentile: & 1 \\
\hline median: & 2 \\
\hline 75th percentile: & 2.5 \\
\hline 90th percentile: & 5 \\
\hline max: & 5 \\
\hline
\end{tabular}


- In-person expert visit (e.g., peer exchange, consultant)

\begin{tabular}{|l|c|}
\hline observations: & 30 \\
\hline missing values: & 21 \\
\hline mean: & 2.5 \\
\hline standard deviation: & 3.17 \\
\hline min: & 0 \\
\hline 10th percentile: & 0 \\
\hline 25th percentile: & 1 \\
\hline median: & 1.5 \\
\hline 75th percentile: & 3 \\
\hline 90th percentile: & 5 \\
\hline max: & 15 \\
\hline
\end{tabular}

- Instruction given to individual agency during an agency visit

\begin{tabular}{|l|c|}
\hline observations: & 42 \\
\hline missing values: & 9 \\
\hline mean: & 4.36 \\
\hline standard deviation: & 9.24 \\
\hline min: & 0 \\
\hline 10th percentile: & 1 \\
\hline 25th percentile: & 1 \\
\hline median: & 2 \\
\hline 75th percentile: & 4 \\
\hline 90th percentile: & 8 \\
\hline max: & 60 \\
\hline
\end{tabular}

- Web cast

\begin{tabular}{|l|c|}
\hline observations: & 11 \\
\hline missing values: & 40 \\
\hline mean: & .82 \\
\hline standard deviation: & 1.17 \\
\hline min: & 0 \\
\hline 10th percentile: & 0 \\
\hline 25th percentile: & 0 \\
\hline median: & 0 \\
\hline 75th percentile: & 1 \\
\hline 90th percentile: & 3 \\
\hline max: & 3 \\
\hline
\end{tabular}

- Other (please specify) 
13. For each broad subject listed in the left-most column of the following table, put a check mark in the appropriate cell(s) to indicate which training method(s) you believe were most effective for imparting key skills and information in that area to your local weatherization agencies or their contractors in Program Year 2008:

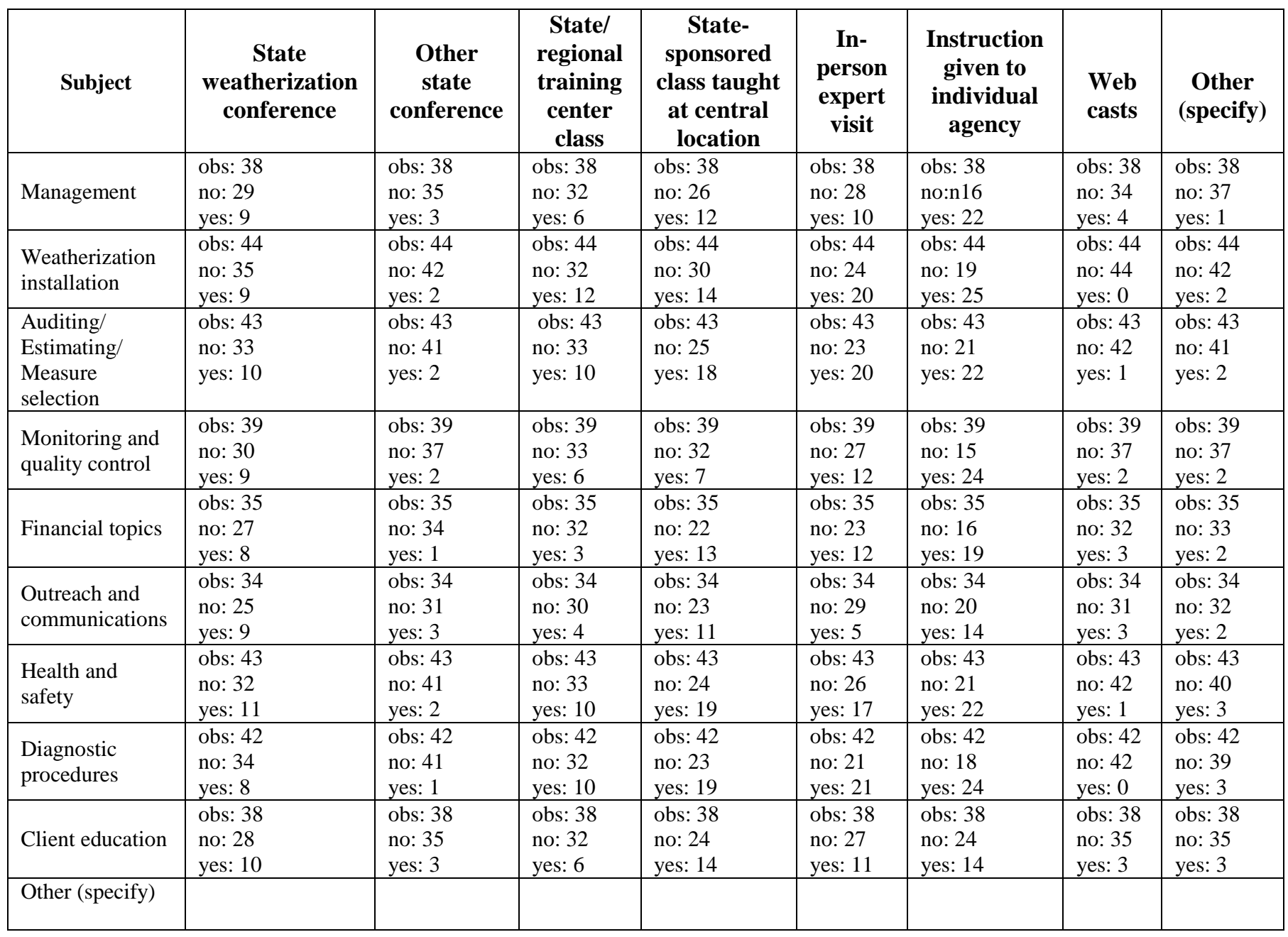


14. In your judgment, how well trained (on average) were local weatherization crews (both agency and contractor) in your state in the following weatherization topics in Program Year 2008? Please use the following scale: 1 -not at all well trained; 2 - not well trained; 3 - moderately well trained; 4 -well trained; 5 - very well trained; 6 - not applicable. (Circle best answer)

(1) Diagnostic procedures

$\begin{array}{rrrr}\text { Freq. } & \text { Percent } & \text { Cum. } \\ \text { not well trained | } & 5 & 10.64 & 10.64 \\ \text { moderately well trained | } & 13 & 27.66 & 38.30 \\ \text { well trained | } & 19 & 40.43 & 78.72 \\ \text { very well trained | } & 10 & 21.28 & 100.00 \\ -{ }_{-1}------ & \end{array}$

(2) Insulation

-- single family dwellings

Freq. Percent Cum.

\begin{tabular}{|c|c|c|c|}
\hline not well trained & 3 & 6.25 & 6.25 \\
\hline moderately well trained & 6 & 12.50 & 18.75 \\
\hline well trained & 26 & 54.17 & 72.92 \\
\hline very well trained & 13 & 27.08 & 100.00 \\
\hline Total & 48 & 100.00 & \\
\hline
\end{tabular}

-- multifamily dwellings

Freq. Percent Cum.

\begin{tabular}{|c|c|c|c|}
\hline not at all well trained & 3 & 6.38 & 6.38 \\
\hline not well trained & 9 & 19.15 & 25.53 \\
\hline moderately well trained & 11 & 23.40 & 48.94 \\
\hline well trained & 12 & 25.53 & 74.47 \\
\hline very well trained & 4 & 8.51 & 82.98 \\
\hline not applicable & 8 & 17.02 & 100.00 \\
\hline Total & 47 & 100.00 & \\
\hline
\end{tabular}

-- mobile homes

Freq. Percent Cum.

\begin{tabular}{|c|c|c|c|}
\hline not well trained & 5 & 10.64 & 10.64 \\
\hline moderately well trained & 8 & 17.02 & 27.66 \\
\hline well trained & 23 & 48.94 & 76.60 \\
\hline very well trained & 10 & 21.28 & 97.87 \\
\hline not applicable & 1 & 2.13 & 100.00 \\
\hline Tota & 47 & 100.00 & \\
\hline
\end{tabular}


(3) Space heating, ventilation, air conditioning

-- single family dwellings

Freq. Percent Cum.

$\begin{array}{rrrr}\text { not well trained | } & 2 & 4.17 & 4.17 \\ \text { moderately well trained | } & 12 & 25.00 & 29.17 \\ \text { well trained | } & 23 & 47.92 & 77.08 \\ \text { very well trained | } & 11 & 22.92 & 100.00 \\ - \text { Total | } & 48 & 100.00 & \end{array}$

-- multifamily dwellings

Freq. Percent Cum.

$\begin{array}{rrrr}\text { not at all well trained | } & 3 & 6.38 & 6.38 \\ \text { not well trained | } & 9 & 19.15 & 25.53 \\ \text { moderately well trained | } & 10 & 21.28 & 46.81 \\ \text { well trained | } & 13 & 27.66 & 74.47 \\ \text { very well trained | } & 4 & 8.51 & 82.98 \\ \text { not applicable | } & 8 & 17.02 & 100.00 \\ \text { Total | } & 47 & 100.00 & \end{array}$

-- mobile homes

$\begin{array}{rrrr} & \text { Freq. } & \text { Percent } & \text { Cum. } \\ \text { not well trained | } & 2 & 4.17 & 4.17 \\ \text { moderately well trained | } & 13 & 27.08 & 31.25 \\ \text { well trained | } & 20 & 41.67 & 72.92 \\ \text { very well trained | } & 12 & 25.00 & 97.92 \\ \text { not applicable | } & 1 & 2.08 & 100.00 \\ \text { Total | } & 48 & 100.00 & \end{array}$

\section{(4) Infiltration measures}

-- single family dwellings

Freq. Percent Cum.

\begin{tabular}{r|crr} 
not well trained & 3 & 6.25 & 6.25 \\
moderately well trained | & 6 & 12.50 & 18.75 \\
well trained & 21 & 43.75 & 62.50 \\
very well trained | & 18 & 37.50 & 100.00 \\
-------------- &
\end{tabular}




\begin{tabular}{r|ccr}
-- multifamily dwellings & & \\
& Freq. & Percent & \multicolumn{1}{c}{ Cum. } \\
not at all well trained | & 4 & 8.51 & 8.51 \\
not well trained | & 8 & 17.02 & 25.53 \\
moderately well trained | & 9 & 19.15 & 44.68 \\
well trained | & 12 & 25.53 & 70.21 \\
very well trained | & 6 & 12.77 & 82.98 \\
not applicable | & 8 & 17.02 & 100.00 \\
Total | & 47 & 100.00 &
\end{tabular}

-- mobile homes

\begin{tabular}{|c|c|c|c|}
\hline & & Percent & Cum. \\
\hline not well trained & 2 & 4.26 & 4.26 \\
\hline moderately well trained & 10 & 21.28 & 25.53 \\
\hline well trained & 20 & 42.55 & 68.09 \\
\hline very well trained & 15 & 31.91 & 100.00 \\
\hline Total & 47 & 100.00 & \\
\hline
\end{tabular}

(5) Doors and windows

-- single family dwellings

Freq. Percent Cum.

$\begin{array}{rrrr}\text { not well trained | } & 2 & 4.17 & 4.17 \\ \text { moderately well trained | } & 8 & 16.67 & 20.83 \\ \text { well trained | } & 28 & 58.33 & 79.17 \\ \text { very well trained | } & 9 & 18.75 & 97.92 \\ \text { not applicable | } & 1 & 2.08 & 100.00 \\ -------------- & \end{array}$

-- multifamily dwellings

Freq. Percent Cum.

$\begin{array}{rrrr}\text { not at all well trained | } & 2 & 4.26 & 4.26 \\ \text { not well trained | } & 7 & 14.89 & 19.15 \\ \text { moderately well trained | } & 8 & 17.02 & 36.17 \\ \text { well trained | } & 17 & 36.17 & 72.34 \\ \text { very well trained | } & 4 & 8.51 & 80.85 \\ \text { not applicable | } & 9 & 19.15 & 100.00 \\ \text { Total | } & 47 & 100.00 & \end{array}$




\begin{tabular}{|c|c|c|c|}
\hline -- mobile homes & Freq. & Percent & Cum. \\
\hline not well trained & 1 & 2.08 & 2.08 \\
\hline moderately well trained & 8 & 16.67 & 18.75 \\
\hline well trained & 31 & 64.58 & 83.33 \\
\hline very well trained & 7 & 14.58 & 97.92 \\
\hline not applicable | & 1 & 2.08 & 100.00 \\
\hline Total | & 48 & 100.00 & \\
\hline
\end{tabular}

(6) Hot water heating

-- single family dwellings

Freq. Percent Cum.

$\begin{array}{rrrr}\text { not well trained | } & 2 & 4.17 & 4.17 \\ \text { moderately well trained | } & 9 & 18.75 & 22.92 \\ \text { well trained | } & 26 & 54.17 & 77.08 \\ \text { very well trained | } & 11 & 22.92 & 100.00 \\ - \text { Total | } & 48 & 100.00 & \end{array}$

-- multifamily dwellings

Freq. Percent Cum.

$\begin{array}{rrrr}\text { not at all well trained | } & 3 & 6.38 & 6.38 \\ \text { not well trained | } & 9 & 19.15 & 25.53 \\ \text { moderately well trained | } & 9 & 19.15 & 44.68 \\ \text { well trained | } & 13 & 27.66 & 72.34 \\ \text { very well trained | } & 5 & 10.64 & 82.98 \\ \text { not applicable | } & 8 & 17.02 & 100.00 \\ \text { Total | } & 47 & 100.00 & \end{array}$

-- mobile homes

Freq. Percent Cum.

\begin{tabular}{|c|c|c|c|}
\hline not well trained & 2 & 4.26 & 4.26 \\
\hline moderately well trained & 9 & 19.15 & 23.40 \\
\hline well trained $\mid$ & 25 & 53.19 & 76.60 \\
\hline very well trained | & 9 & 19.15 & 95.74 \\
\hline not applicable & 2 & 4.26 & 100.00 \\
\hline Total & 47 & 100.00 & \\
\hline
\end{tabular}


(7) Baseloads (e.g., lighting, refrigerators)

-- single family dwellings

Freq. Percent Cum.

$\begin{array}{rrrr}\text { not well trained | } & 3 & 6.25 & 6.25 \\ \text { moderately well trained | } & 9 & 18.75 & 25.00 \\ \text { well trained | } & 21 & 43.75 & 68.75 \\ \text { very well trained | } & 15 & 31.25 & 100.00 \\ - \text { Total | } & 48 & 100.00 & \end{array}$

-- multifamily dwellings

Freq. Percent Cum.

$\begin{array}{rrrr}\text { not at all well trained | } & 2 & 4.26 & 4.26 \\ \text { not well trained | } & 5 & 10.64 & 14.89 \\ \text { moderately well trained | } & 10 & 21.28 & 36.17 \\ \text { well trained | } & 14 & 29.79 & 65.96 \\ \text { very well trained | } & 8 & 17.02 & 82.98 \\ \text { not applicable | } & 8 & 17.02 & 100.00 \\ \text { Total } \mid & 47 & 100.00 & \end{array}$

-- mobile homes

Freq. Percent Cum.

\begin{tabular}{r|rrr} 
not well trained | & 4 & 8.33 & 8.33 \\
moderately well trained | & 9 & 18.75 & 27.08 \\
well trained | & 21 & 43.75 & 70.83 \\
very well trained | & 13 & 27.08 & 97.92 \\
not applicable | & 1 & 2.08 & 100.00 \\
Total | & 48 & 100.00 &
\end{tabular}

14a. In your judgment, how well trained (on average) were local weatherization crews (both agency and contractor) in your state in the following administrative-related topics in Program Year 2008? Please use the following scale: 1 - not at all well trained; 2 - not well trained; 3 - moderately well trained; 4 -well trained; 5 - very well trained; 6 - not applicable. (Circle best answer)

(1) Management

Freq. Percent Cum.

$\begin{array}{rrrr}\text { not well trained | } & 5 & 10.42 & 10.42 \\ \text { moderately well trained | } & 19 & 39.58 & 50.00 \\ \text { well trained | } & 18 & 37.50 & 87.50 \\ \text { very well trained | } & 5 & 10.42 & 97.92 \\ \text { not applicable | } & 1 & 2.08 & 100.00 \\ \text { Total | } & 48 & 100.00 & \end{array}$




\section{(2) Client education}

$\begin{array}{rrrr} & \text { Freq. } & \text { Percent } & \text { Cum. } \\ \text { not well trained | } & 10 & 21.28 & 21.28 \\ \text { moderately well trained | } & 16 & 34.04 & 55.32 \\ \text { well trained | } & 18 & 38.30 & 93.62 \\ \text { very well trained | } & 3 & 6.38 & 100.00 \\ \text { Total | } & 47 & 100.00 & \end{array}$

\begin{tabular}{rrrr}
$\begin{array}{r}\text { (3) Auditing/estimating } \\
\text {-- single family dwellings } \\
\end{array}$ & & \\
& Freq. & Percent & Cum. \\
\hline not at all well trained | & 1 & 2.08 & 2.08 \\
not well trained | & 4 & 8.33 & 10.42 \\
moderately well trained | & 6 & 12.50 & 22.92 \\
well trained | & 27 & 56.25 & 79.17 \\
very well trained | & 10 & 20.83 & 100.00 \\
Total | & 48 & 100.00 &
\end{tabular}

-- multifamily dwellings

Freq. Percent Cum.

$\begin{array}{rrrr}\text { not at all well trained | } & 5 & 10.64 & 10.64 \\ \text { not well trained | } & 11 & 23.40 & 34.04 \\ \text { moderately well trained | } & 10 & 21.28 & 55.32 \\ \text { well trained | } & 12 & 25.53 & 80.85 \\ \text { very well trained | } & 2 & 4.26 & 85.11 \\ \text { not applicable | } & 7 & 14.89 & 100.00 \\ - \text { Total | } & 47 & 100.00 & \end{array}$

-- mobile homes

Freq. Percent Cum.

$\begin{array}{rrrr}\text { not at all well trained | } & 1 & 2.08 & 2.08 \\ \text { not well trained | } & 4 & 8.33 & 10.42 \\ \text { moderately well trained | } & 11 & 22.92 & 33.33 \\ \text { well trained | } & 24 & 50.00 & 83.33 \\ \text { very well trained | } & 7 & 14.58 & 97.92 \\ \text { not applicable | } & 1 & 2.08 & 100.00 \\ \text { Total } & 48 & 100.00 & \end{array}$


(4) Monitoring/quality control

\begin{tabular}{rrrr} 
& Freq. & Percent & \multicolumn{1}{c}{ Cum. } \\
not well trained | & 3 & 6.25 & 6.25 \\
moderately well trained $\mid$ & 10 & 20.83 & 27.08 \\
well trained $\mid$ & 28 & 58.33 & 85.42 \\
very well trained $\mid$ & 7 & 14.58 & 100.00 \\
Total $\mid$ & 48 & 100.00 &
\end{tabular}

\begin{tabular}{rrrr} 
(5) Financial topics & & & \\
& Freq. & Percent & Cum. \\
\hline not at all well trained | & 1 & 2.13 & 2.13 \\
not well trained | & 9 & 19.15 & 21.28 \\
moderately well trained | & 12 & 25.53 & 46.81 \\
well trained | & 21 & 44.68 & 91.49 \\
very well trained | & 4 & 8.51 & 100.00 \\
- Total $\left.\right|^{-} 47$ & 100.00 &
\end{tabular}

(6) Outreach and communications

$\begin{array}{rrrr} & \text { Freq. } & \text { Percent } & \text { Cum. } \\ \text { not at all well trained | } & 2 & 4.17 & 4.17 \\ \text { not well trained | } & 6 & 12.50 & 16.67 \\ \text { moderately well trained | } & 17 & 35.42 & 52.08 \\ \text { well trained | } & 21 & 43.75 & 95.83 \\ \text { very well trained | } & 2 & 4.17 & 100.00 \\ - \text { Total | } & 48 & 100.00 & \end{array}$

\section{(7) Other (please specify)}

14b. In your judgment, how well trained (on average) were local weatherization crews (both agency and contractor) in your state in the following health and safety topics in Program Year 2008? Please use the following scale: 1 - not at all well trained; 2 - not well trained; 3 - moderately well trained; 4 -well trained; 5 - very well trained; 6 - not applicable. (Circle best answer) 


\section{(1) Fire safety}

$\begin{array}{rrrr} & \text { Freq. } & \text { Percent } & \text { Cum. } \\ \text { not at all well trained | } & 5 & 10.42 & 10.42 \\ \text { not well trained | } & 10 & 20.83 & 31.25 \\ \text { moderately well trained | } & 18 & 37.50 & 68.75 \\ \text { well trained | } & 8 & 16.67 & 85.42 \\ \text { very well trained | } & 3 & 6.25 & 91.67 \\ \text { not applicable | } & 4 & 8.33 & 100.00 \\ \text { Total | } & 48 & 100.00 & \end{array}$

(2) Indoor air quality

\begin{tabular}{r|rrr} 
& Freq. & Percent & Cum. \\
not well trained | & 5 & 10.64 & 10.64 \\
moderately well trained | & 16 & 34.04 & 44.68 \\
well trained | & 15 & 31.91 & 76.60 \\
very well trained | & 11 & 23.40 & 100.00 \\
- Total | & 47 & 100.00 &
\end{tabular}

(3) Measures to increase security of housing unit

$\begin{array}{rrrr} & \text { Freq. } & \text { Percent } & \text { Cum. } \\ \text { not at all well trained | } & 4 & 8.51 & 8.51 \\ \text { not well trained | } & 13 & 27.66 & 36.17 \\ \text { moderately well trained | } & 13 & 27.66 & 63.83 \\ \text { well trained | } & 7 & 14.89 & 78.72 \\ \text { not applicable | } & 10 & 21.28 & 100.00 \\ \text { Total } & 47 & 100.00 & \end{array}$

(4) Measures to reduce common household hazards

$\begin{array}{rrrr} & \text { Freq. } & \text { Percent } & \text { Cum. } \\ \text { not at all well trained | } & 1 & 2.08 & 2.08 \\ \text { not well trained } \mid & 8 & 16.67 & 18.75 \\ \text { moderately well trained } \mid & 21 & 43.75 & 62.50 \\ \text { well trained | } & 11 & 22.92 & 85.42 \\ \text { very well trained | } & 4 & 8.33 & 93.75 \\ \text { not applicable | } & 3 & 6.25 & 100.00 \\ \text { Total | } & 48 & 100.00 & \end{array}$


(5) Mold and mildew

Freq. Percent Cum.

$\begin{array}{rrrr}\text { not well trained | } & 8 & 16.67 & 16.67 \\ \text { moderately well trained | } & 16 & 33.33 & 50.00 \\ \text { well trained } \mid & 17 & 35.42 & 85.42 \\ \text { very well trained } \mid & 7 & 14.58 & 100.00 \\ - \text { Total | } & 48 & 100.00 & \end{array}$

(6) Lead

Freq. Percent Cum.

$\begin{array}{rrrr}\text { not at all well trained | } & 1 & 2.08 & 2.08 \\ \text { not well trained | } & 3 & 6.25 & 8.33 \\ \text { moderately well trained | } & 13 & 27.08 & 35.42 \\ \text { well trained | } & 21 & 43.75 & 79.17 \\ \text { very well trained | } & 10 & 20.83 & 100.00 \\ - \text { Total | } & 48 & 100.00 & \end{array}$

(7) Asbestos

$\begin{array}{rrrr} & \text { Freq. } & \text { Percent } & \text { Cum. } \\ \text { not at all well trained | } & 5 & 10.42 & 10.42 \\ \text { not well trained | } & 16 & 33.33 & 43.75 \\ \text { moderately well trained | } & 13 & 27.08 & 70.83 \\ \text { well trained | } & 9 & 18.75 & 89.58 \\ \text { very well trained | } & 4 & 8.33 & 97.92 \\ \text { not applicable | } & 1 & 2.08 & 100.00 \\ \text { Total | } & 48 & 100.00 & \end{array}$

\section{(8) Vermiculite}

Freq. Percent Cum.

$\begin{array}{rrrr}\text { not at all well trained | } & 7 & 15.22 & 15.22 \\ \text { not well trained | } & 15 & 32.61 & 47.83 \\ \text { moderately well trained | } & 14 & 30.43 & 78.26 \\ \text { well trained | } & 5 & 10.87 & 89.13 \\ \text { very well trained | } & 4 & 8.70 & 97.83 \\ \text { not applicable | } & 1 & 2.17 & 100.00 \\ - \text { Total | } & 46 & 100.00 & \end{array}$




\section{(9) General crew safety}

$\begin{array}{rrrr} & \text { Freq. } & \text { Percent } & \text { Cum. } \\ \text { not well trained | } & 4 & 8.33 & 8.33 \\ \text { moderately well trained | } & 10 & 20.83 & 29.17 \\ \text { well trained | } & 21 & 43.75 & 72.92 \\ \text { very well trained | } & 13 & 27.08 & 100.00 \\ ------------ & \\ \text { Total | } & 48 & 100.00 & \end{array}$

\section{(10) Other (please specify)}

16. In your judgment, how well trained (overall) were your state's weatherization crews in Program Year 2008 ? (Check best answer)

Very well trained

Well trained

Moderately well trained

Poorly trained

Very poorly trained

$\begin{array}{rrrr} & \text { Freq. } & \text { Percent } & \text { Cum. } \\ \text { poorly trained | } & 1 & 2.04 & 2.04 \\ \text { moderately well trained | } & 16 & 32.65 & 34.69 \\ \text { well trained | } & 29 & 59.18 & 93.88 \\ \text { very well trained | } & 3 & 6.12 & 100.00 \\ \text { Total } \mid & 49 & 100.00 & \end{array}$

\section{Section D: MONITORING}

1. About how many state weatherization office staff went into the field to monitor local weatherization agencies in your state in Program Year 2008? Note: do not include people who do quality assurance at the local agency level for the local agencies.

\begin{tabular}{|l|c|}
\hline $\begin{array}{l}\text { State staff } \\
\text { observations: }\end{array}$ & 46 \\
\hline missing values: & 5 \\
\hline mean: & 3.68 \\
\hline standard deviation: & 3.41 \\
\hline min: & .5 \\
\hline 10th percentile: & 1 \\
\hline 25th percentile: & 2 \\
\hline median: & 3 \\
\hline 75th percentile: & 4 \\
\hline 90th percentile: & 7 \\
\hline max: & 21 \\
\hline
\end{tabular}




\begin{tabular}{|l|c|}
\hline $\begin{array}{l}\text { State contractor } \\
\text { observations: }\end{array}$ & 21 \\
\hline missing values: & 30 \\
\hline mean: & .5 \\
\hline standard deviation: & .97 \\
\hline min: & 0 \\
\hline 10th percentile: & 0 \\
\hline 25th percentile: & 0 \\
\hline median: & 0 \\
\hline 75th percentile: & .5 \\
\hline 90th percentile: & 2 \\
\hline max: & 3 \\
\hline
\end{tabular}

- Other (please specify)

\begin{tabular}{|l|c|}
\hline observations: & 6 \\
\hline missing values: & 45 \\
\hline mean: & 2.5 \\
\hline standard deviation: & 4.81 \\
\hline min: & 0 \\
\hline 10th percentile: & 0 \\
\hline 25th percentile: & 0 \\
\hline median: & 0 \\
\hline 75th percentile: & 3 \\
\hline 90th percentile: & 12 \\
\hline max: & 12 \\
\hline
\end{tabular}

2. Which of the following types of post-weatherization quality control inspection did your state perform on weatherized dwelling units in Program Year 2008? (Check all that apply)

- Visual inspection of installed measures

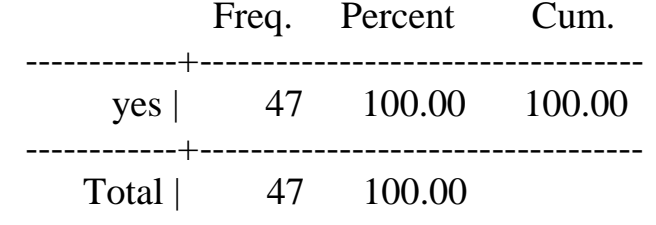

- Verification of insulation depths/quantities

Freq. Percent Cum.

$\begin{array}{crrr}\text { no } \mid & 4 & 8.51 & 8.51 \\ \text { yes } \mid & 43 & 91.49 & 100.00 \\ ------------ \\ \text { Total } & 47 & 100.00\end{array}$


- Verification of operation of measures installed

$\begin{array}{rrrr} & \text { Freq. } & \text { Percent } & \text { Cum. } \\ \text { no | } & 3 & 6.38 & 6.38 \\ \text { yes | } & 44 & 93.62 & 100.00 \\ ---------- & \\ \text { Total } & 47 & 100.00 & \end{array}$

- Assessment of quality of measures installed

Freq. Percent Cum.

yes | $47 \quad 100.00 \quad 100.00$
--------
Total | 47100.00

- Identification of needed measures that were not installed Freq. Percent Cum.

\begin{tabular}{|c|c|c|c|}
\hline no & 4 & 8.51 & 8.51 \\
\hline yes & 43 & 91.49 & 100.00 \\
\hline Total & 47 & 100.00 & \\
\hline
\end{tabular}

- Blower door test

$\begin{array}{crrr} & \text { Freq. } & \text { Percent } & \text { Cum. } \\ \text { no } \mid & 6 & 12.77 & 12.77 \\ \text { yes } \mid & 41 & 87.23 & 100.00 \\ ------- & \end{array}$

- Heating system efficiency test (flue gas analysis)

Freq. Percent Cum.

\begin{tabular}{|c|c|c|c|}
\hline no & 23 & 48.94 & 48.94 \\
\hline yes & 24 & 51.06 & 100.00 \\
\hline Total | & 47 & 100.00 & \\
\hline
\end{tabular}

- Draft/spillage tests of heating systems Freq. Percent Cum.

\begin{tabular}{|c|c|c|c|}
\hline no & 17 & 36.17 & 36.17 \\
\hline yes & 30 & 63.83 & 100.00 \\
\hline Total & 47 & 100.00 & \\
\hline
\end{tabular}


- Carbon monoxide (CO) monitoring

$\begin{array}{crrr} & \text { Freq. } & \text { Percent } & \text { Cum. } \\ \text { no | } & 8 & 17.02 & 17.02 \\ \text { yes } \mid & 39 & 82.98 & 100.00 \\ ------- & \\ \text { Total | } & 47 & 100.00 & \end{array}$

- Infrared scanning

$\begin{array}{cccc} & \text { Freq. } & \text { Percent } & \text { Cum. } \\ \text { no | } & 23 & 48.94 & 48.94 \\ \text { yes } \mid & 24 & 51.06 & 100.00 \\ ------- & \end{array}$

- Identification of unresolved health and safety issues

Freq. Percent Cum.

$\begin{array}{crrr}\text { no } \mid & 4 & 8.51 & 8.51 \\ \text { yes } \mid & 43 & 91.49 & 100.00 \\ ------------------ \\ \text { Total } & 47 & 100.00 & \end{array}$

- Discussion with occupants

$\begin{array}{rccc} & \text { Freq. } & \text { Percent } & \text { Cum. } \\ \text { no } \mid & 1 & 2.13 & 2.13 \\ \text { yes } \mid & 46 & 97.87 & 100.00 \\ ------------ \\ \text { Total } & 47 & 100.00 & \end{array}$

- Other (specify)

3. Please indicate which types of post-weatherization quality control inspection listed below were initiated during three years before Program Year 2008? (Check all that apply)

- Visual inspection of installed measures

$\begin{array}{cccc} & \text { Freq. } & \text { Percent } & \text { Cum. } \\ \text { no | } & 4 & 9.09 & 9.09 \\ \text { yes | } & 40 & 90.91 & 100.00 \\ ------- & \\ \text { Total | } & 44 & 100.00 & \end{array}$


- Verification of insulation depths/quantities

$\begin{array}{rrrr} & \text { Freq. } & \text { Percent } & \text { Cum. } \\ \text { no | } & 10 & 22.73 & 22.73 \\ \text { yes | } & 34 & 77.27 & 100.00 \\ ------- & \\ \text { Total | } & 44 & 100.00 & \end{array}$

- Verification of operation of measures installed Freq. Percent Cum.

$\begin{array}{rrrr}\text { no } \mid & 8 & 18.18 & 18.18 \\ \text { yes } \mid & 36 & 81.82 & 100.00 \\ --------------- \\ \text { Total } & 44 & 100.00 & \end{array}$

- Assessment of quality of measures installed

\begin{tabular}{cccl} 
& Freq. & Percent & \multicolumn{1}{c}{ Cum. } \\
no $\mid$ & 5 & 11.36 & 11.36 \\
yes $\mid$ & 39 & 88.64 & 100.00 \\
--------------- \\
Total & 44 & 100.00 &
\end{tabular}

- Identification of needed measures that were not installed Freq. Percent Cum.

\begin{tabular}{|c|c|c|c|}
\hline no | & 11 & 25.00 & 25.00 \\
\hline yes $\mid$ & 33 & 75.00 & 100.00 \\
\hline Total | & 44 & 100.00 & \\
\hline
\end{tabular}

- Blower door test

\begin{tabular}{|c|c|c|c|}
\hline & Freq. & Percent & Cum. \\
\hline no & 12 & 27.27 & 27.27 \\
\hline yes & 32 & 72.73 & 100.00 \\
\hline Total & 44 & 100.00 & \\
\hline
\end{tabular}

- Heating system efficiency test (flue gas analysis)

$\begin{array}{rrrr} & \text { Freq. } & \text { Percent } & \text { Cum. } \\ \text { no | } & 19 & 43.18 & 43.18 \\ \text { yes | } & 25 & 56.82 & 100.00 \\ ------- & \\ \text { Total | } & 44 & 100.00 & \end{array}$


- Draft/spillage tests of heating systems

$\begin{array}{rrrr} & \text { Freq. } & \text { Percent } & \text { Cum. } \\ \text { no | } & 18 & 40.91 & 40.91 \\ \text { yes | } & 26 & 59.09 & 100.00 \\ ----------- & \end{array}$

- Carbon monoxide (CO) monitoring Freq. Percent Cum.

$\begin{array}{cccc}\text { no } \mid & 10 & 22.73 & 22.73 \\ \text { yes } \mid & 34 & 77.27 & 100.00 \\ ---------------- \\ \text { Total } & 44 & 100.00\end{array}$

- Infrared scanning

Freq. Percent Cum.

\begin{tabular}{|c|c|c|c|}
\hline no & 25 & 56.82 & 56.82 \\
\hline yes & 19 & 43.18 & 100.00 \\
\hline Total & 44 & 100.00 & \\
\hline
\end{tabular}

- Identification of unresolved health and safety issues

\begin{tabular}{|c|c|c|c|}
\hline & Freq. & Percent & Cum. \\
\hline no & 10 & 22.73 & 22.73 \\
\hline yes $\mid$ & 34 & 77.27 & 100.00 \\
\hline Total | & 44 & 100.00 & \\
\hline
\end{tabular}

- Discussion with occupants

$\begin{array}{cccc} & \text { Freq. } & \text { Percent } & \text { Cum. } \\ \text { no | } & 8 & 18.18 & 18.18 \\ \text { yes | } & 36 & 81.82 & 100.00 \\ ----------- \\ \text { Total | } & 44 & 100.00 & \end{array}$

- Other (specify) 
4. For Program Year 2008 please rate key aspects (cost, training needed, time needed and effectiveness) of the quality control inspection procedures listed below using the following scale: 1 - very low; 2 - low; 3 - medium; 4 - high; 5 - very high. For example, if you view visual inspection of installed measures as low-cost, give a rating of 2 in the Cost column. If you view visual inspection of installed measures as highly effective, give a rating of 4 in the Effectiveness column.

\begin{tabular}{|c|c|c|c|c|c|}
\hline $\begin{array}{c}\text { Type of } \\
\text { Post- } \\
\text { Weatheriza } \\
\text { tion } \\
\text { Quality } \\
\text { Control } \\
\text { Inspection }\end{array}$ & Cost & $\begin{array}{c}\text { Traini } \\
\text { ng } \\
\text { Neede } \\
\text { d }\end{array}$ & $\begin{array}{c}\text { Time } \\
\text { Need } \\
\text { ed }\end{array}$ & & a. Effectiveness \\
\hline $\begin{array}{l}\text { Visual } \\
\text { inspection } \\
\text { of installed } \\
\text { measures }\end{array}$ & $\begin{array}{l}\text { obs: } \\
43 \\
\text { very } \\
\text { low: } 7 \\
\text { low: } \\
20 \\
\text { mediu } \\
\text { m: } 11 \\
\text { high: } \\
4 \\
\text { very } \\
\text { high: } \\
1\end{array}$ & $\begin{array}{l}\text { obs: } 42 \\
\text { very } \\
\text { low: } 6 \\
\text { low: } 7 \\
\text { medium: } \\
12 \\
\text { high: } 11 \\
\text { very } \\
\text { high: } 6\end{array}$ & $\begin{array}{l}\text { obs: } 41 \\
\text { very } \\
\text { low: } 2 \\
\text { low: } 8 \\
\text { mediu } \\
\text { m: } 13 \\
\text { high: } \\
14 \\
\text { very } \\
\text { high: } 4\end{array}$ & $\begin{array}{l}\text { obs: } 41 \\
\text { very low: } 0 \\
\text { low: } 1 \\
\text { medium: } 3 \\
\text { high: } 16 \\
\text { very high: } 21\end{array}$ & \\
\hline $\begin{array}{l}\text { Verification } \\
\text { of insulation } \\
\text { depths/quant } \\
\text { ities }\end{array}$ & $\begin{array}{l}\text { obs: } \\
42 \\
\text { very } \\
\text { low: } 8 \\
\text { low: } \\
19 \\
\text { mediu } \\
\text { m: } 13 \\
\text { high: } \\
2 \\
\text { very } \\
\text { high: } \\
0\end{array}$ & $\begin{array}{l}\text { obs: } 41 \\
\text { very } \\
\text { low: } 6 \\
\text { low: } 12 \\
\text { medium: } \\
10 \\
\text { high: } 10 \\
\text { very } \\
\text { high: } 3\end{array}$ & $\begin{array}{l}\text { obs: } 41 \\
\text { very } \\
\text { low: } 4 \\
\text { low: } 13 \\
\text { mediu } \\
\text { m: } 13 \\
\text { high: } 9 \\
\text { very } \\
\text { high: } 2\end{array}$ & $\begin{array}{l}\text { obs: } 40 \\
\text { very low: } 1 \\
\text { low: } 0 \\
\text { medium: } 6 \\
\text { high: } 20 \\
\text { very high: } 13\end{array}$ & \\
\hline $\begin{array}{l}\text { Verification } \\
\text { of operation } \\
\text { of measures } \\
\text { installed }\end{array}$ & $\begin{array}{l}\text { obs: } \\
42 \\
\text { very } \\
\text { low: } 6 \\
\text { low: } \\
19 \\
\text { mediu } \\
\text { m: } 14 \\
\text { high: } \\
3 \\
\text { very } \\
\text { high: } \\
0\end{array}$ & $\begin{array}{l}\text { obs: } 41 \\
\text { very } \\
\text { low: } 2 \\
\text { low: } 6 \\
\text { medium: } \\
17 \\
\text { high: } 10 \\
\text { very } \\
\text { high: } 6\end{array}$ & $\begin{array}{l}\text { obs: } 41 \\
\text { very } \\
\text { low: } 1 \\
\text { low: } 3 \\
\text { mediu } \\
\text { m: } 21 \\
\text { high: } \\
13 \\
\text { very } \\
\text { high: } 3\end{array}$ & $\begin{array}{l}\text { obs: } 41 \\
\text { very low: } 1 \\
\text { low: } 0 \\
\text { medium: } 7 \\
\text { high: } 19 \\
\text { very high: } 14\end{array}$ & \\
\hline $\begin{array}{l}\text { Assessment } \\
\text { of quality of } \\
\text { measures } \\
\text { installed }\end{array}$ & $\begin{array}{l}\text { obs: } \\
43 \\
\text { very } \\
\text { low: } 2 \\
\text { low: } \\
23 \\
\text { mediu } \\
\text { m: } 15 \\
\text { high: } \\
3 \\
\text { very } \\
\text { high: } \\
0 \\
\end{array}$ & $\begin{array}{l}\text { obs: } 42 \\
\text { very } \\
\text { low: } 1 \\
\text { low: } 8 \\
\text { medium: } \\
14 \\
\text { high: } 12 \\
\text { very } \\
\text { high: } 7\end{array}$ & $\begin{array}{l}\text { obs: } 42 \\
\text { very } \\
\text { low: } 1 \\
\text { low: } 6 \\
\text { mediu } \\
\text { m: } 15 \\
\text { high: } \\
17 \\
\text { very } \\
\text { high:3 }\end{array}$ & $\begin{array}{l}\text { obs: } 42 \\
\text { very low: } 1 \\
\text { low: } 0 \\
\text { medium: } 5 \\
\text { high: } 20 \\
\text { very high: } 16\end{array}$ & \\
\hline $\begin{array}{l}\text { Identificatio } \\
n \text { of needed }\end{array}$ & $\begin{array}{l}\text { obs: } \\
41\end{array}$ & $\begin{array}{l}\text { obs: } 40 \\
\text { very }\end{array}$ & $\begin{array}{l}\text { obs: } 40 \\
\text { very }\end{array}$ & $\begin{array}{l}\text { obs: } 40 \\
\text { very low: } 1\end{array}$ & \\
\hline
\end{tabular}




\begin{tabular}{|c|c|c|c|c|}
\hline $\begin{array}{l}\text { measures } \\
\text { that were } \\
\text { not installed }\end{array}$ & $\begin{array}{l}\text { very } \\
\text { low: } 4 \\
\text { low: } \\
18 \\
\text { mediu } \\
\text { m: } 15 \\
\text { high: } \\
4 \\
\text { very } \\
\text { high: } \\
0\end{array}$ & $\begin{array}{l}\text { low: } 1 \\
\text { low: } 5 \\
\text { medium: } \\
12 \\
\text { high: } 14 \\
\text { very } \\
\text { high: } 8\end{array}$ & $\begin{array}{l}\text { low: } 1 \\
\text { low: } 4 \\
\text { mediu } \\
\text { m: } 18 \\
\text { high: } \\
12 \\
\text { very } \\
\text { high: } 5\end{array}$ & $\begin{array}{l}\text { low: } 0 \\
\text { medium: } 8 \\
\text { high: } 17 \\
\text { very high: } 14\end{array}$ \\
\hline $\begin{array}{l}\text { Blower door } \\
\text { test }\end{array}$ & $\begin{array}{l}\text { obs: } \\
43 \\
\text { very } \\
\text { low: } 6 \\
\text { low: } \\
10 \\
\text { mediu } \\
\text { m: } 21 \\
\text { high: } \\
3 \\
\text { very } \\
\text { high: } \\
3\end{array}$ & $\begin{array}{l}\text { obs: } 41 \\
\text { very } \\
\text { low: } 2 \\
\text { low: } 6 \\
\text { medium: } \\
9 \\
\text { high: } 16 \\
\text { very } \\
\text { high: } 8\end{array}$ & $\begin{array}{l}\text { obs: } 41 \\
\text { very } \\
\text { low: } 0 \\
\text { low: } 4 \\
\text { mediu } \\
\text { m: } 15 \\
\text { high: } \\
15 \\
\text { very } \\
\text { high: } 7\end{array}$ & $\begin{array}{l}\text { obs: } 41 \\
\text { very low: } 1 \\
\text { low: } 1 \\
\text { medium: } 1 \\
\text { high: } 18 \\
\text { very high: } 20\end{array}$ \\
\hline $\begin{array}{l}\text { Heating } \\
\text { system } \\
\text { efficiency } \\
\text { test (flue gas } \\
\text { analysis) }\end{array}$ & $\begin{array}{l}\text { obs: } \\
39 \\
\text { very } \\
\text { low: } 3 \\
\text { low: } 9 \\
\text { mediu } \\
\text { m: } 20 \\
\text { high: } \\
5 \\
\text { very } \\
\text { high: } \\
2\end{array}$ & $\begin{array}{l}\text { obs: } 37 \\
\text { very } \\
\text { low: } 2 \\
\text { low: } 3 \\
\text { medium: } \\
10 \\
\text { high: } 13 \\
\text { very } \\
\text { high: } 9\end{array}$ & $\begin{array}{l}\text { obs: } 37 \\
\text { very } \\
\text { low: } 0 \\
\text { low: } 4 \\
\text { mediu } \\
\text { m: } 18 \\
\text { high: } \\
11 \\
\text { very } \\
\text { high: } 4\end{array}$ & $\begin{array}{l}\text { obs: } 37 \\
\text { very low: } 1 \\
\text { low: } 1 \\
\text { medium: } 9 \\
\text { high: } 11 \\
\text { very high: } 15\end{array}$ \\
\hline $\begin{array}{l}\text { Draft/spillag } \\
\text { e tests of } \\
\text { heating } \\
\text { systems }\end{array}$ & $\begin{array}{l}\text { obs: } \\
40 \\
\text { very } \\
\text { low: } 3 \\
\text { low: } \\
17 \\
\text { mediu } \\
\text { m: } 17 \\
\text { high: } \\
2 \\
\text { very } \\
\text { high: } \\
1\end{array}$ & $\begin{array}{l}\text { obs: } 38 \\
\text { very } \\
\text { low: } 3 \\
\text { low: } 5 \\
\text { medium: } \\
9 \\
\text { high: } 13 \\
\text { very } \\
\text { high: } 8\end{array}$ & $\begin{array}{l}\text { obs: } 38 \\
\text { very } \\
\text { low: } 0 \\
\text { low: } 8 \\
\text { mediu } \\
\text { m: } 17 \\
\text { high: } \\
10 \\
\text { very } \\
\text { high: } 3\end{array}$ & $\begin{array}{l}\text { obs: } 38 \\
\text { very low: } 1 \\
\text { low: } 1 \\
\text { medium: } 4 \\
\text { high: } 16 \\
\text { very high: } 16\end{array}$ \\
\hline $\begin{array}{l}\text { Carbon } \\
\text { monoxide } \\
(\mathrm{CO}) \\
\text { monitoring }\end{array}$ & $\begin{array}{l}\text { obs: } \\
42 \\
\text { very } \\
\text { low: } 9 \\
\text { low: } \\
13 \\
\text { mediu } \\
\text { m: } 16 \\
\text { high: } \\
3 \\
\text { very } \\
\text { high: } \\
1\end{array}$ & $\begin{array}{l}\text { obs: } 41 \\
\text { very } \\
\text { low: } 3 \\
\text { low: } 7 \\
\text { medium: } \\
12 \\
\text { high: } 13 \\
\text { very } \\
\text { high: } 6\end{array}$ & $\begin{array}{l}\text { obs: } 41 \\
\text { very } \\
\text { low: } 1 \\
\text { low: } 10 \\
\text { mediu } \\
\text { m: } 17 \\
\text { high: } 9 \\
\text { very } \\
\text { high: } 4\end{array}$ & $\begin{array}{l}\text { obs: } 41 \\
\text { very low: } 1 \\
\text { low: } 1 \\
\text { medium: } 2 \\
\text { high: } 18 \\
\text { very high: } 19\end{array}$ \\
\hline
\end{tabular}




\begin{tabular}{|c|c|c|c|c|}
\hline Infrared scanning & $\begin{array}{l}\text { obs: } 38 \\
\text { very low: } 2 \\
\text { low: } 7 \\
\text { medium: } 15 \\
\text { high: } 5 \\
\text { very high: } 9\end{array}$ & $\begin{array}{l}\text { obs: } 36 \\
\text { very low: } 0 \\
\text { low: } 5 \\
\text { medium: } 11 \\
\text { high: } 12 \\
\text { very high: } 8\end{array}$ & $\begin{array}{l}\text { obs: } 36 \\
\text { very low: } 0 \\
\text { low: } 5 \\
\text { medium: } 15 \\
\text { high: } 10 \\
\text { very high: } 6\end{array}$ & $\begin{array}{l}\text { obs: } 34 \\
\text { very low: } 1 \\
\text { low: } 4 \\
\text { medium: } 5 \\
\text { high: } 12 \\
\text { very high: } 12\end{array}$ \\
\hline $\begin{array}{l}\text { Identification of unresolved } \\
\text { health and safety issues }\end{array}$ & $\begin{array}{l}\text { obs: } 43 \\
\text { very low: } 7 \\
\text { low: } 18 \\
\text { medium: } 14 \\
\text { high: } 4 \\
\text { very high: } 0\end{array}$ & $\begin{array}{l}\text { obs: } 41 \\
\text { very low: } 3 \\
\text { low: } 2 \\
\text { medium: } 13 \\
\text { high: } 16 \\
\text { very high: } 7\end{array}$ & $\begin{array}{l}\text { obs: } 41 \\
\text { very low: } 1 \\
\text { low: } 4 \\
\text { medium: } 21 \\
\text { high: } 10 \\
\text { very high: } 5\end{array}$ & $\begin{array}{l}\text { obs: } 41 \\
\text { very low: } 1 \\
\text { low: } 2 \\
\text { medium: } 6 \\
\text { high: } 13 \\
\text { very high: } 19\end{array}$ \\
\hline Discussion with occupants & $\begin{array}{l}\text { obs: } 44 \\
\text { very low: } 14 \\
\text { low: } 17 \\
\text { medium: } 11 \\
\text { high: } 1 \\
\text { very high: } 1\end{array}$ & $\begin{array}{l}\text { obs: } 42 \\
\text { very low: } 5 \\
\text { low: } 8 \\
\text { medium: } 16 \\
\text { high: } 11 \\
\text { very high: } 2\end{array}$ & $\begin{array}{l}\text { obs: } 42 \\
\text { very low: } 3 \\
\text { low: } 10 \\
\text { medium: } 16 \\
\text { high: } 9 \\
\text { very high: } 4\end{array}$ & $\begin{array}{l}\text { obs: } 42 \\
\text { very low: } 0 \\
\text { low: } 2 \\
\text { medium: } 9 \\
\text { high: } 19 \\
\text { very high: } 12\end{array}$ \\
\hline Other (specify) & & & & \\
\hline
\end{tabular}

5. On average, how many hours were spent by state weatherization office staff on-site conducting postweatherization quality control in a typical weatherized home in Program Year 2008?

6. What types of credentials or experience were required of your post-weatherization quality control inspectors in your state weatherization office in Program Year 2008? (Check all that apply)

\section{- Technical certification}

Freq. Percent Cum.

\begin{tabular}{|c|c|c|c|}
\hline no & 22 & 48.89 & 48.89 \\
\hline yes | & 23 & 51.11 & 100.00 \\
\hline Total | & 45 & 100.00 & \\
\hline
\end{tabular}

- Extensive experience performing pre-weatherization audits

$\begin{array}{cccc} & \text { Freq. } & \text { Percent } & \text { Cum. } \\ \text { no } \mid & 15 & 33.33 & 33.33 \\ \text { yes } \mid & 30 & 66.67 & 100.00 \\ -------- & \\ \text { Total | } & 45 & 100.00 & \end{array}$

- Extensive experience performing weatherization work

Freq. Percent Cum.

\begin{tabular}{|c|c|c|c|}
\hline no & 18 & 40.00 & 40.00 \\
\hline yes & 27 & 60.00 & 100.00 \\
\hline Total & 45 & 100.00 & \\
\hline
\end{tabular}


- Extensive experience supervising weatherization work

\begin{tabular}{|c|c|c|c|}
\hline & req. & Percent & Cum. \\
\hline no & 25 & 55.56 & 55.56 \\
\hline yes & 20 & 44.44 & 100.00 \\
\hline Total | & 45 & 100.00 & \\
\hline
\end{tabular}

- Construction experience

$\begin{array}{cccc} & \text { Freq. } & \text { Percent } & \text { Cum. } \\ \text { no } \mid & 25 & 55.56 & 55.56 \\ \text { yes } \mid & 20 & 44.44 & 100.00 \\ ------- & \\ \text { Total | } & 45 & 100.00 & \end{array}$

- Other (please specify) 
7. Please indicate the level of experience of the post-weatherization quality control inspectors in your state weatherization office for each of the following areas in Program Year 2008.

\begin{tabular}{|c|c|c|c|c|c|c|}
\hline & $\begin{array}{l}\text { Very } \\
\text { High }\end{array}$ & $\mathrm{Hi}$ & & Medium & Low & $\begin{array}{l}\text { Very } \\
\text { Low }\end{array}$ \\
\hline & & req. & Percent & Cum. & & \\
\hline & very low | & 2 & 4.17 & 4.17 & & \\
\hline & low | & 4 & 8.33 & 12.50 & & \\
\hline Performing pre-weatherization audits & medium $\mid$ & 6 & 12.50 & 25.00 & & \\
\hline & & 19 & 39.58 & 64.58 & & \\
\hline & very high | & 17 & 35.42 & 100.00 & & \\
\hline & Total | & 48 & 100.00 & & & \\
\hline & Freq. Perc & & Cum. & & & \\
\hline & very low & 4 & 8.51 & 8.51 & & \\
\hline & low & 5 & 10.64 & 19.15 & & \\
\hline Performing weatherization work & medium & 11 & 23.40 & 42.55 & & \\
\hline & high & 18 & 38.30 & 80.85 & & \\
\hline & very high & 9 & 19.15 & 100.00 & & \\
\hline & Total $\mid$ & 47 & 100.00 & & & \\
\hline & Freq. Perc & & Cum. & & & \\
\hline & very low & 6 & 12.77 & 12.77 & & \\
\hline & low & 7 & 14.89 & 27.66 & & \\
\hline Supervising weatherization work & medium & 10 & 21.28 & 48.94 & & \\
\hline & high & 17 & 36.17 & 85.11 & & \\
\hline & very high 1 & 7 & 14.89 & 100.00 & & \\
\hline & Total $\mid$ & 47 & 100.00 & & & \\
\hline & Freq. $\quad$ Perc & ent & Cum. & & & \\
\hline & very low & 6 & 13.33 & 13.33 & & \\
\hline & low & 8 & 17.78 & 31.11 & & \\
\hline Working in construction & medium & 15 & 33.33 & 64.44 & & \\
\hline & high $\mid$ & 8 & 17.78 & 82.22 & & \\
\hline & very high & 8 & 17.78 & 100.00 & & \\
\hline & Total $\mid$ & 45 & 100.00 & & & \\
\hline & Freq. $\quad$ Perc & ent & Cum. & & & \\
\hline & very low & 1 & 2.13 & 2.13 & & \\
\hline Performing post-weatherization & low & 1 & 2.13 & 4.26 & & \\
\hline inspections post-wedullizallon & medium $\mid$ & 4 & 8.51 & 12.77 & & \\
\hline & high $\mid$ & 24 & 51.06 & 63.83 & & \\
\hline & very high | & 17 & 36.17 & 100.00 & & \\
\hline & Total | & 47 & 100.00 & & & \\
\hline
\end{tabular}




\section{Inspectors with a very high level of experience would be considered experts in weatherization.}

Inspectors with a very low level of experience would be considered new to weatherization.

8. On average, how frequently did state weatherization program office staff visit each local agency to conduct post-weatherization quality control inspections in Program Year 2008? (Check best answer)

Freq. Percent Cum.

$\begin{array}{rrrr}\text { weekly | } & 1 & 2.04 & 2.04 \\ \text { monthly } \mid & 5 & 10.20 & 12.24 \\ \text { quarterly | } & 9 & 18.37 & 30.61 \\ \text { annually | } & 23 & 46.94 & 77.55 \\ \text { other (please specify) } & 11 & 22.45 & 100.00 \\ -{ }_{-}--- & & \end{array}$

9. On how many dwelling units did your state perform post-weatherization quality control inspections in Program Year 2008?

9a. Of those inspected, approximately how many were found to have a problem significant enough to require a return visit by local agency weatherization crews?

9b. Of those requiring a return visit, what were the three most common problems found in the dwelling units inspected by your state?

9c. Of those requiring a return visit, how many had work done that probably resulted in more energy savings?

10. In those cases where a Program Year 2008 post-weatherization quality control inspection revealed a problem with the job performed, what single action was most commonly taken in response to that finding? (Check best answer)

- Made agency send crew back to correct problem

- Made agency send crew supervisor to correct problem

- Sent someone from state office to correct problem

- No action taken

- Other (please specify)

Freq. Percent Cum.

Made agency send crew back to correct problem | $\quad \begin{array}{lll}42 & 87.50 \quad 87.50\end{array}$

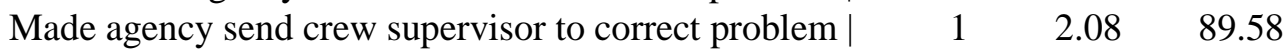

Other (please specify) $\mid \begin{array}{lll}5 & 10.42 \quad 100.00\end{array}$

Total | $\quad 48 \quad 100.00$ 
11. What other actions were taken in Program Year 2008 in response to the discovery of a problem with the weatherization job performed? (Check all that apply)

- Made agency send original crew back to correct problem

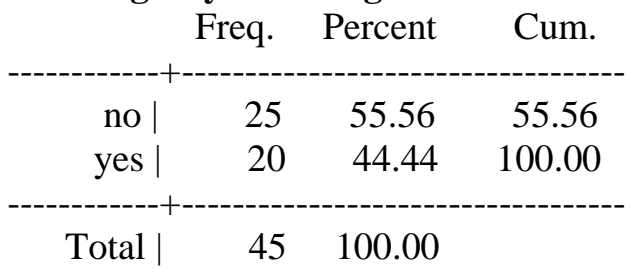

- Made agency send different crew to correct problem

Freq. Percent Cum.

\begin{tabular}{cccc} 
no $\mid$ & 30 & 66.67 & 66.67 \\
yes $\mid$ & 15 & 33.33 & 100.00 \\
-------------- \\
\hline Total & 45 & 100.00
\end{tabular}

- Made agency send crew supervisor to correct problem

$\begin{array}{rrrr} & \text { Freq. } & \text { Percent } & \text { Cum. } \\ \text { no } \mid & 31 & 68.89 & 68.89 \\ \text { yes | } & 14 & 31.11 & 100.00 \\ ------------- \\ \text { Total | } & 45 & 100.00\end{array}$

- Sent someone from state office to correct problem

$\begin{array}{crrr} & \text { Freq. } & \text { Percent } & \text { Cum. } \\ \text { no | } & 41 & 91.11 & 91.11 \\ \text { yes | } & 4 & 8.89 & 100.00 \\ ------------- \\ \text { Total | } & 45 & 100.00 & \end{array}$

- No action taken

$\begin{array}{cccc} & \text { Freq. } & \text { Percent } & \text { Cum. } \\ \text { no | } & 42 & 93.33 & 93.33 \\ \text { yes } \mid & 3 & 6.67 & 100.00 \\ ---------- \\ \text { Total } & 45 & 100.00\end{array}$

- Other (please specify) 
12. Which of the following monitoring tasks did your state perform in Program Year 2008 to check on the administration of local weatherization efforts? (Check all that apply)

- Verification of number of dwelling units weatherized

$\begin{array}{crrr} & \text { Freq. } & \text { Percent } & \text { Cum. } \\ \text { no } \mid & 6 & 12.24 & 12.24 \\ \text { yes | } & 43 & 87.76 & 100.00 \\ -------------- \\ \text { Total | } & 49 & 100.00 & \end{array}$

- Verification of clients' income eligibility Freq. Percent Cum.

\begin{tabular}{|c|c|c|c|}
\hline no & 3 & 6.12 & 6.12 \\
\hline yes & 46 & 93.88 & 100.00 \\
\hline Total | & 49 & 100.00 & \\
\hline
\end{tabular}

- Verification of average expenditure per weatherized unit Freq. Percent Cum.

\begin{tabular}{|c|c|c|c|}
\hline no & 5 & 10.20 & 10.20 \\
\hline yes & 44 & 89.80 & 100.00 \\
\hline Total & 49 & 100.00 & \\
\hline
\end{tabular}

- Verification of material expenditures

$\begin{array}{rrrr} & \text { Freq. } & \text { Percent } & \text { Cum. } \\ \text { no | } & 2 & 4.08 & 4.08 \\ \text { yes | } & 47 & 95.92 & 100.00 \\ ------ & \end{array}$

- Verification that installed measures had an SIR of 1.0 or greater Freq. Percent Cum.

$\begin{array}{crrr}\text { no } \mid & 9 & 18.37 & 18.37 \\ \text { yes } \mid & 40 & 81.63 & 100.00 \\ --------------- \\ \text { Total| }\end{array}$

- Examination of vehicle costs

Freq. Percent Cum.

\begin{tabular}{|c|c|c|c|}
\hline no & 25 & 51.02 & 51.02 \\
\hline yes | & 24 & 48.98 & 100.00 \\
\hline Total | & 49 & 100.00 & \\
\hline
\end{tabular}


- Examination of other equipment costs

\begin{tabular}{|c|c|c|c|}
\hline & Freq. & Percent & Cum. \\
\hline no & 20 & 40.82 & 40.82 \\
\hline yes & 29 & 59.18 & 100.00 \\
\hline Total & 49 & 100.00 & \\
\hline
\end{tabular}

- Examination of training and technical assistance (T\&TA) costs Freq. Percent Cum.

\begin{tabular}{rccc} 
no $\mid$ & 8 & 16.33 & 16.33 \\
yes $\mid$ & 41 & 83.67 & 100.00 \\
---------- \\
\hline Total & 49 & 100.00
\end{tabular}

- Examination of administrative costs Freq. Percent Cum.

$\begin{array}{crrr}\text { no } \mid & 9 & 18.37 & 18.37 \\ \text { yes } \mid & 40 & 81.63 & 100.00 \\ ---------- & \\ \text { Total } & 49 & 100.00\end{array}$

- Examination of material inventory

\begin{tabular}{|c|c|c|c|}
\hline & Freq. & Percent & Cum. \\
\hline no & 19 & 38.78 & 38.78 \\
\hline yes & 30 & 61.22 & 100.00 \\
\hline Total & 49 & 100.00 & \\
\hline
\end{tabular}

- Interviews with agency staff

$\begin{array}{cccc} & \text { Freq. } & \text { Percent } & \text { Cum. } \\ \text { no | } & 3 & 6.12 & 6.12 \\ \text { yes | } & 46 & 93.88 & 100.00 \\ --------- \\ \text { Total } & 49 & 100.00 & \end{array}$

- Interviews with agency contractor staff Freq. Percent Cum.

\begin{tabular}{|c|c|c|c|}
\hline no & 27 & 55.10 & 55.10 \\
\hline yes & 22 & 44.90 & 100.00 \\
\hline Total | & 49 & 100.00 & \\
\hline
\end{tabular}


- Interviews with agency clients

Freq. Percent Cum.

$\begin{array}{crrr}\text { no } & 5 & 10.20 & 10.20 \\ \text { yes } & 44 & 89.80 & 100.00 \\ --------- & \end{array}$

- Other (please specify)

13. Please indicate which types of monitoring tasks listed below were initiated three years prior to Program Year 2008. (Check all that apply)

- Verification of number of dwelling units weatherized

$\begin{array}{crrr} & \text { Freq. } & \text { Percent } & \text { Cum. } \\ \text { no | } & 6 & 13.64 & 13.64 \\ \text { yes | } & 38 & 86.36 & 100.00 \\ ------------ \\ \text { Total | } & 44 & 100.00\end{array}$

- Verification of clients' income eligibility

Freq. Percent Cum.

\begin{tabular}{|c|c|c|c|}
\hline no & 4 & 9.09 & 9.09 \\
\hline yes & 40 & 90.91 & 100.00 \\
\hline Total | & 44 & 100.00 & \\
\hline
\end{tabular}

- Verification of average expenditure per weatherized unit Freq. Percent Cum.

\begin{tabular}{|c|c|c|c|}
\hline no & 8 & 18.18 & 18.18 \\
\hline yes | & 36 & 81.82 & 100.00 \\
\hline Total & 44 & 100.00 & \\
\hline
\end{tabular}

- Verification of material expenditures

$\begin{array}{rrrr} & \text { Freq. } & \text { Percent } & \text { Cum. } \\ \text { no } \mid & 5 & 11.36 & 11.36 \\ \text { yes } \mid & 39 & 88.64 & 100.00 \\ ------- & \\ \text { Total | } & 44 & 100.00\end{array}$


Verification that installed measures had an SIR of 1.0 or greater

$\begin{array}{cccc} & \text { Freq. } & \text { Percent } & \text { Cum. } \\ \text { no } \mid & 11 & 25.00 & 25.00 \\ \text { yes } \mid & 33 & 75.00 & 100.00 \\ ----------- & \end{array}$

- Examination of vehicle costs

Freq. Percent Cum.

\begin{tabular}{|c|c|c|c|}
\hline no & 23 & 52.27 & 52.27 \\
\hline yes & 21 & 47.73 & 100.00 \\
\hline Total & 44 & 100.00 & \\
\hline
\end{tabular}

- Examination of other equipment costs

$\begin{array}{cccc} & \text { Freq. } & \text { Percent } & \text { Cum. } \\ \text { no | } & 19 & 43.18 & 43.18 \\ \text { yes | } & 25 & 56.82 & 100.00 \\ ------ & \\ \text { Total | } & 44 & 100.00\end{array}$

- Examination of training and technical assistance (T\&TA) costs

Freq. Percent Cum.

\begin{tabular}{|c|c|c|c|}
\hline no & 16 & 36.36 & 36.36 \\
\hline yes & 28 & 63.64 & 100.00 \\
\hline Total & 44 & 100.00 & \\
\hline
\end{tabular}

- Examination of administrative costs

$\begin{array}{rrrr} & \text { Freq. } & \text { Percent } & \text { Cum. } \\ \text { no } \mid & 13 & 29.55 & 29.55 \\ \text { yes } \mid & 31 & 70.45 & 100.00 \\ -------- & \end{array}$

- Examination of material inventory

$\begin{array}{cccc} & \text { Freq. } & \text { Percent } & \text { Cum. } \\ \text { no } \mid & 17 & 38.64 & 38.64 \\ \text { yes } \mid & 27 & 61.36 & 100.00 \\ ------- & \\ \text { Total | } & 44 & 100.00 & \end{array}$


Interviews with agency staff

$\begin{array}{rrrr} & \text { Freq. } & \text { Percent } & \text { Cum. } \\ \text { no } \mid & 8 & 18.18 & 18.18 \\ \text { yes | } & 36 & 81.82 & 100.00 \\ ------- \\ \text { Total | } & 44 & 100.00 & \end{array}$

- Interviews with agency contractor staff Freq. Percent Cum.

$\begin{array}{cccc}\text { no } \mid & 24 & 54.55 & 54.55 \\ \text { yes } \mid & 20 & 45.45 & 100.00 \\ -------------- \\ \text { Total | }\end{array}$

- Interviews with agency clients

$\begin{array}{cccc} & \text { Freq. } & \text { Percent } & \text { Cum. } \\ \text { no | } & 8 & 18.18 & 18.18 \\ \text { yes | } & 36 & 81.82 & 100.00 \\ ---------- \\ \text { Total | } & 44 & 100.00 & \end{array}$

- Other (please specify) 
14. For Program Year 2008 please rate key aspects (cost, training needed, time needed and effectiveness) of the monitoring tasks listed below using the following scale: 1 - very low; 2 -low; 3 - medium; 4 high; 5 - very high. For example, if you view verification of number of dwelling units weatherized as requiring a moderate amount of training, give a rating of 3 in the Training Needed column. If you view verification of number of dwelling units weatherized as requiring a low amount of time, give a rating of 2 in the Time Needed column.

\begin{tabular}{|c|c|c|c|c|}
\hline Type of Monitoring Tasks & Cost & $\begin{array}{l}\text { Training } \\
\text { Needed }\end{array}$ & $\begin{array}{l}\text { Time } \\
\text { Needed }\end{array}$ & Effectiveness \\
\hline $\begin{array}{l}\text { Verification of number of dwelling } \\
\text { units weatherized }\end{array}$ & $\begin{array}{l}\text { obs: } 44 \\
\text { very low: } 11 \\
\text { low: } 20 \\
\text { medium: } 8 \\
\text { high: } 4 \\
\text { very high: } 1\end{array}$ & $\begin{array}{l}\text { obs: } 43 \\
\text { very low: } 12 \\
\text { low: } 16 \\
\text { medium: } 10 \\
\text { high: } 4 \\
\text { very high: } 1\end{array}$ & $\begin{array}{l}\text { obs: } 44 \\
\text { very low: } 5 \\
\text { low: } 19 \\
\text { medium: } 12 \\
\text { high: } 5 \\
\text { very high: } 3\end{array}$ & $\begin{array}{l}\text { obs: } 44 \\
\text { very low: } 1 \\
\text { low: } 4 \\
\text { medium: } 7 \\
\text { high: } 16 \\
\text { very high: } 16\end{array}$ \\
\hline $\begin{array}{l}\text { Verification of clients' income } \\
\text { eligibility }\end{array}$ & $\begin{array}{l}\text { obs: } 44 \\
\text { very low: } 11 \\
\text { low: } 21 \\
\text { medium: } 9 \\
\text { high: } 2 \\
\text { very high: } 1\end{array}$ & $\begin{array}{l}\text { obs: } 43 \\
\text { very low: } 8 \\
\text { low: } 8 \\
\text { medium: } 20 \\
\text { high: } 6 \\
\text { very high: } 1\end{array}$ & $\begin{array}{l}\text { obs: } 44 \\
\text { very low: } 3 \\
\text { low: } 14 \\
\text { medium: } 18 \\
\text { high: } 7 \\
\text { very high: } 2 \\
\end{array}$ & $\begin{array}{l}\text { obs: } 44 \\
\text { very low: } 1 \\
\text { low: } 2 \\
\text { medium: } 12 \\
\text { high: } 13 \\
\text { very high: } 16 \\
\end{array}$ \\
\hline $\begin{array}{l}\text { Verification of average expenditure } \\
\text { per weatherized unit }\end{array}$ & $\begin{array}{l}\text { obs: } 43 \\
\text { very low: } 11 \\
\text { low: } 22 \\
\text { medium: } 8 \\
\text { high: } 2 \\
\text { very high: } 0\end{array}$ & $\begin{array}{l}\text { obs: } 42 \\
\text { very low: } 9 \\
\text { low: } 13 \\
\text { medium: } 13 \\
\text { high: } 6 \\
\text { very high: } 1\end{array}$ & $\begin{array}{l}\text { obs: } 43 \\
\text { very low: } 3 \\
\text { low: } 15 \\
\text { medium: } 14 \\
\text { high: } 9 \\
\text { very high: } 2\end{array}$ & $\begin{array}{l}\text { obs: } 43 \\
\text { very low: } 2 \\
\text { low: } 2 \\
\text { medium: } 6 \\
\text { high: } 18 \\
\text { very high: } 15\end{array}$ \\
\hline Verification of material expenditures & $\begin{array}{l}\text { obs: } 43 \\
\text { very low: } 10 \\
\text { low: } 15 \\
\text { medium: } 12 \\
\text { high: } 5 \\
\text { very high: } 1\end{array}$ & $\begin{array}{l}\text { obs: } 42 \\
\text { very low: } 6 \\
\text { low: } 13 \\
\text { medium: } 12 \\
\text { high: } 10 \\
\text { very high: } 1\end{array}$ & $\begin{array}{l}\text { obs: } 43 \\
\text { very low: } 3 \\
\text { low: } 10 \\
\text { medium: } 15 \\
\text { high: } 14 \\
\text { very high: } 1\end{array}$ & $\begin{array}{l}\text { obs: } 43 \\
\text { very low: } 1 \\
\text { low: } 1 \\
\text { medium: } 7 \\
\text { high: } 22 \\
\text { very high: } 12 \\
\end{array}$ \\
\hline $\begin{array}{l}\text { Verification that installed measures } \\
\text { had an SIR of } 1.0 \text { or greater }\end{array}$ & $\begin{array}{l}\text { obs: } 40 \\
\text { very low: } 8 \\
\text { low: } 13 \\
\text { medium: } 13 \\
\text { high: } 6 \\
\text { very high: } 0\end{array}$ & $\begin{array}{l}\text { obs: } 39 \\
\text { very low: } 6 \\
\text { low: } 4 \\
\text { medium: } 11 \\
\text { high: } 14 \\
\text { very high: } 4\end{array}$ & $\begin{array}{l}\text { obs: } 40 \\
\text { very low: } 4 \\
\text { low: } 8 \\
\text { medium: } 13 \\
\text { high: } 10 \\
\text { very high: } 5\end{array}$ & $\begin{array}{l}\text { obs: } 40 \\
\text { very low: } 3 \\
\text { low: } 2 \\
\text { medium: } 7 \\
\text { high: } 15 \\
\text { very high: } 13 \\
\end{array}$ \\
\hline Examination of vehicle costs & $\begin{array}{l}\text { obs: } 34 \\
\text { very low: } 10 \\
\text { low: } 15 \\
\text { medium: } 6 \\
\text { high: } 3 \\
\text { very high: } 0\end{array}$ & $\begin{array}{l}\text { obs: } 33 \\
\text { very low: } 8 \\
\text { low: } 11 \\
\text { medium: } 10 \\
\text { high: } 4 \\
\text { very high: } 0\end{array}$ & $\begin{array}{l}\text { obs: } 33 \\
\text { very low: } 5 \\
\text { low: } 10 \\
\text { medium: } 12 \\
\text { high: } 6 \\
\text { very high: } 0\end{array}$ & $\begin{array}{l}\text { obs: } 33 \\
\text { very low: } 4 \\
\text { low: } 2 \\
\text { medium: } 9 \\
\text { high: } 11 \\
\text { very high: } 7\end{array}$ \\
\hline Examination of other equipment costs & $\begin{array}{l}\text { obs: } 38 \\
\text { very low: } 9 \\
\text { low: } 18 \\
\text { medium: } 7 \\
\text { high: } 4 \\
\text { very high: } 0\end{array}$ & $\begin{array}{l}\text { obs: } 37 \\
\text { very low: } 8 \\
\text { low: } 9 \\
\text { medium: } 16 \\
\text { high: } 4 \\
\text { very high: } 0\end{array}$ & $\begin{array}{l}\text { obs: } 37 \\
\text { very low: } 3 \\
\text { low: } 13 \\
\text { medium: } 14 \\
\text { high: } 7 \\
\text { very high: } 0\end{array}$ & $\begin{array}{l}\text { obs: } 37 \\
\text { very low: } 3 \\
\text { low: } 3 \\
\text { medium: } 13 \\
\text { high: } 11 \\
\text { very high: } 7\end{array}$ \\
\hline $\begin{array}{l}\text { Examination of training and technical } \\
\text { assistance (T\&TA) costs }\end{array}$ & $\begin{array}{l}\text { obs: } 42 \\
\text { very low: } 10 \\
\text { low: } 16 \\
\text { medium: } 10 \\
\text { high: } 6 \\
\text { very high: } 0\end{array}$ & $\begin{array}{l}\text { obs: } 41 \\
\text { very low: } 8 \\
\text { low: } 7 \\
\text { medium: } 19 \\
\text { high: } 5 \\
\text { very high: } 2\end{array}$ & $\begin{array}{l}\text { obs: } 42 \\
\text { very low: } 3 \\
\text { low: } 11 \\
\text { medium: } 17 \\
\text { high: } 10 \\
\text { very high: } 1\end{array}$ & $\begin{array}{l}\text { obs: } 42 \\
\text { very low: } 4 \\
\text { low: } 2 \\
\text { medium: } 14 \\
\text { high: } 17 \\
\text { very high: } 5\end{array}$ \\
\hline Examination of administrative costs & $\begin{array}{l}\text { obs: } 41 \\
\text { very low: } 8 \\
\text { low: } 16 \\
\text { medium: } 10 \\
\text { high: } 7 \\
\text { very high: } 0\end{array}$ & $\begin{array}{l}\text { obs: } 41 \\
\text { very low: } 6 \\
\text { low: } 8 \\
\text { medium: } 14 \\
\text { high: } 10 \\
\text { very high: } 3\end{array}$ & $\begin{array}{l}\text { obs: } 42 \\
\text { very low: } 2 \\
\text { low: } 13 \\
\text { medium: } 13 \\
\text { high: } 11 \\
\text { very high: } 3 \\
\end{array}$ & $\begin{array}{l}\text { obs: } 41 \\
\text { very low: } 2 \\
\text { low: } 3 \\
\text { medium: } 10 \\
\text { high: } 13 \\
\text { very high: } 13 \\
\end{array}$ \\
\hline
\end{tabular}




\begin{tabular}{|c|c|c|c|c|}
\hline Type of Monitoring Tasks & Cost & $\begin{array}{l}\text { Training } \\
\text { Needed }\end{array}$ & $\begin{array}{c}\text { Time } \\
\text { Needed }\end{array}$ & Effectiveness \\
\hline Examination of material inventory & $\begin{array}{l}\text { obs: } 38 \\
\text { very low: } 7 \\
\text { low: } 20 \\
\text { medium: } 6 \\
\text { high: } 5 \\
\text { very high:0 }\end{array}$ & $\begin{array}{l}\text { obs: } 37 \\
\text { very low: } 5 \\
\text { low: } 14 \\
\text { medium: } 11 \\
\text { high: } 7 \\
\text { very high: } 0\end{array}$ & $\begin{array}{l}\text { obs: } 37 \\
\text { very low: } 1 \\
\text { low: } 7 \\
\text { medium: } 13 \\
\text { high: } 14 \\
\text { very high: } 2\end{array}$ & $\begin{array}{l}\text { obs: } 37 \\
\text { very low: } 2 \\
\text { low: } 5 \\
\text { medium: } 10 \\
\text { high: } 17 \\
\text { very high: } 3\end{array}$ \\
\hline Interviews with agency staff & $\begin{array}{l}\text { obs: } 42 \\
\text { very low: } 10 \\
\text { low: } 16 \\
\text { medium: } 11 \\
\text { high: } 5 \\
\text { very high: } 0\end{array}$ & $\begin{array}{l}\text { obs: } 41 \\
\text { very low: } 8 \\
\text { low: } 8 \\
\text { medium: } 18 \\
\text { high: } 7 \\
\text { very high: } 0\end{array}$ & $\begin{array}{l}\text { obs: } 42 \\
\text { very low: } 3 \\
\text { low: } 8 \\
\text { medium: } 21 \\
\text { high: } 10 \\
\text { very high: } 0\end{array}$ & $\begin{array}{l}\text { obs: } 42 \\
\text { very low: } 1 \\
\text { low: } 3 \\
\text { medium: } 14 \\
\text { high: } 16 \\
\text { very high: } 8\end{array}$ \\
\hline $\begin{array}{l}\text { Interviews with agency contractor } \\
\text { staff }\end{array}$ & $\begin{array}{l}\text { obs: } 37 \\
\text { very low: } 7 \\
\text { low: } 13 \\
\text { medium: } 10 \\
\text { high: } 7 \\
\text { very high: } 0\end{array}$ & $\begin{array}{l}\text { obs: } 35 \\
\text { very low: } 6 \\
\text { low: } 7 \\
\text { medium: } 16 \\
\text { high: } 6 \\
\text { very high: } 0\end{array}$ & $\begin{array}{l}\text { obs: } 35 \\
\text { very low: } 3 \\
\text { low: } 7 \\
\text { medium: } 14 \\
\text { high: } 10 \\
\text { very high: } 1\end{array}$ & $\begin{array}{l}\text { obs: } 36 \\
\text { very low: } 2 \\
\text { low: } 2 \\
\text { medium: } 12 \\
\text { high: } 15 \\
\text { very high: } 5\end{array}$ \\
\hline Interviews with agency clients & $\begin{array}{l}\text { obs: } 43 \\
\text { very low: } 7 \\
\text { low: } 18 \\
\text { medium: } 13 \\
\text { high: } 5 \\
\text { very high: } 0\end{array}$ & $\begin{array}{l}\text { obs: } 42 \\
\text { very low: } 7 \\
\text { low: } 9 \\
\text { medium: } 17 \\
\text { high: } 8 \\
\text { very high: } 1\end{array}$ & $\begin{array}{l}\text { obs: } 42 \\
\text { very low: } 2 \\
\text { low: } 10 \\
\text { medium: } 20 \\
\text { high: } 9 \\
\text { very high: } 1\end{array}$ & $\begin{array}{l}\text { obs: } 43 \\
\text { very low: } 1 \\
\text { low: } 1 \\
\text { medium: } 14 \\
\text { high: } 18 \\
\text { very high: } 9\end{array}$ \\
\hline Other (specify) & & & & \\
\hline
\end{tabular}

15. On average, how many hours were spent by state weatherization office staff on-site at each local agency monitoring agency administrative activities in Program Year 2008?

16. What types of credentials or experience were required of those who monitored the administration of local weatherization efforts in your state in Program Year 2008? Check all that apply.

- Technical certification

$\begin{array}{cccc} & \text { Freq. } & \text { Percent } & \text { Cum. } \\ \text { no } \mid & 32 & 69.57 & 69.57 \\ \text { yes | } & 14 & 30.43 & 100.00 \\ ------- & \\ \text { Total | } & 46 & 100.00\end{array}$

- Extensive experience performing pre-weatherization audits

Freq. Percent Cum.

\begin{tabular}{|c|c|c|c|}
\hline no & 26 & 56.52 & 56.52 \\
\hline yes | & 20 & 43.48 & 100.00 \\
\hline Total & 46 & 100.00 & \\
\hline
\end{tabular}

- Extensive experience performing weatherization work

Freq. Percent Cum.

$\begin{array}{rrrr}\text { no } \mid & 31 & 67.39 & 67.39 \\ \text { yes } \mid & 15 & 32.61 & 100.00\end{array}$




\section{Total | $46 \quad 100.00$}

C-113 
- Extensive experience supervising weatherization work

$\begin{array}{rrrr} & \text { Freq. } & \text { Percent } & \text { Cum. } \\ \text { no | } & 27 & 58.70 & 58.70 \\ \text { yes } \mid & 19 & 41.30 & 100.00 \\ ----------- & \\ \text { Total | } & 46 & 100.00\end{array}$

- Construction experience

Freq. Percent Cum.

\begin{tabular}{|c|c|c|c|}
\hline no & 36 & 78.26 & 78.26 \\
\hline yes & 10 & 21.74 & 100.00 \\
\hline Total & 46 & 100.00 & \\
\hline
\end{tabular}

- Extensive management experience

Freq. Percent Cum.

\begin{tabular}{|c|c|c|c|}
\hline no & 20 & 43.48 & 43.48 \\
\hline yes | & 26 & 56.52 & 100.00 \\
\hline Total | & 46 & 100.00 & \\
\hline
\end{tabular}

- Extensive finance experience

$\begin{array}{rrrr} & \text { Freq. } & \text { Percent } & \text { Cum. } \\ \text { no } \mid & 20 & 43.48 & 43.48 \\ \text { yes } \mid & 26 & 56.52 & 100.00 \\ ---------- \\ \text { Total | } & 46 & 100.00 & \end{array}$

- Extensive experience administering local weatherization programs Freq. Percent Cum.

\begin{tabular}{|c|c|c|c|}
\hline no & 26 & 56.52 & 56.52 \\
\hline yes & 20 & 43.48 & 100.00 \\
\hline Total & 46 & 100.00 & \\
\hline
\end{tabular}

- Other (please specify) 
17. Please indicate the level of experience of the state monitors of the local agencies in your state weatherization office for each of the following areas in Program Year 2008.

\begin{tabular}{|c|c|c|c|c|c|c|}
\hline & $\begin{array}{l}\text { Very } \\
\text { High }\end{array}$ & High & Mod & erate & Low & Very Low \\
\hline & & & Freq. & Percent & Cum. & \\
\hline & & low & 2 & 4.17 & 4.17 & \\
\hline & moderate & vell trained | & 18 & 37.50 & 41.67 & \\
\hline Vlanagement & & high $\mid$ & 17 & 35.42 & 77.08 & \\
\hline & & very high | & 11 & 22.92 & 100.00 & \\
\hline & & Total | & 48 & 100.00 & & \\
\hline & & & Freq. & Percent & Cum. & \\
\hline & & low & 7 & 14.58 & 14.58 & \\
\hline & moderatel & vell trained | & 16 & 33.33 & 47.92 & \\
\hline Finance & & high | & 18 & 37.50 & 85.42 & \\
\hline & & very high | & 7 & 14.58 & 100.00 & \\
\hline & & Total | & 48 & 100.00 & & \\
\hline & & & Freq. & Percent & Cum. & \\
\hline & & very low & 3 & 6.25 & 6.25 & \\
\hline Administration of local & & low & 4 & 8.33 & 14.58 & \\
\hline 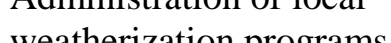 & moderatel & vell trained | & 17 & 35.42 & 50.00 & \\
\hline weatnerization programs & & high | & 12 & 25.00 & 75.00 & \\
\hline & & very high | & 12 & 25.00 & 100.00 & \\
\hline & & Total $\mid$ & 48 & 100.00 & & \\
\hline Other (specify) & & & & & & \\
\hline
\end{tabular}

18. On average, how frequently did state weatherization program office staff visit each local agency to monitor administrative activities in Program Year 2008? (Check best answer)

$\begin{array}{rrrr} & \text { Freq. } & \text { Percent } & \text { Cum. } \\ \text { weekly | } & 1 & 2.04 & 2.04 \\ \text { monthly | } & 2 & 4.08 & 6.12 \\ \text { quarterly | } & 2 & 4.08 & 10.20 \\ \text { annually | } & 37 & 75.51 & 85.71 \\ \text { other (please specify) | } & 7 & 14.29 & 100.00 \\ ----------- & \end{array}$

19. For how many of the local weatherization agencies monitored in your state in Program Year 2008 was an administrative problem found that required corrective actions above and beyond acceptable findings and recommendations? 
20. What were the three most common problems requiring corrective actions above and beyond acceptable findings and recommendations found in the local weatherization agencies monitored in your state in Program Year 2008?

21. In those cases where state monitoring of the administration of local weatherization efforts in Program Year 2008 revealed an administrative problem requiring corrective actions above and beyond acceptable findings and recommendations, what actions were taken in response? (Check all that apply)

- Sent written report to local agency

$\begin{array}{crrr} & \text { Freq. } & \text { Percent } & \text { Cum. } \\ \text { no | } & 6 & 12.77 & 12.77 \\ \text { yes | } & 41 & 87.23 & 100.00 \\ ----- & \\ \text { Total | } & 47 & 100.00 & \end{array}$

- Required corrective action

$\begin{array}{cccc} & \text { Freq. } & \text { Percent } & \text { Cum. } \\ \text { no | } & 6 & 12.77 & 12.77 \\ \text { yes } \mid & 41 & 87.23 & 100.00 \\ --------- \\ \text { Total } & 47 & 100.00 & \end{array}$

- Made presentation to local agency

$\begin{array}{rrrr} & \text { Freq. } & \text { Percent } & \text { Cum. } \\ \text { no } \mid & 34 & 72.34 & 72.34 \\ \text { yes } \mid & 13 & 27.66 & 100.00 \\ ----------- \\ \text { Total | } & 47 & 100.00 & \end{array}$

- Sent someone from state office to help correct problem Freq. Percent Cum.

\begin{tabular}{|c|c|c|c|}
\hline no & 25 & 53.19 & 53.19 \\
\hline yes & 22 & 46.81 & 100.00 \\
\hline Total & 47 & 100.00 & \\
\hline
\end{tabular}


- Sent state contractor to help correct problem

Freq. Percent Cum.

$\begin{array}{crrr}\text { no } \mid & 41 & 87.23 & 87.23 \\ \text { yes } \mid & 6 & 12.77 & 100.00 \\ --------- & \\ \text { Total } & 47 & 100.00\end{array}$

- No action taken

$\begin{array}{cccc} & \text { Freq. } & \text { Percent } & \text { Cum. } \\ \text { no | } & 45 & 95.74 & 95.74 \\ \text { yes } \mid & 2 & 4.26 & 100.00 \\ -------------- \\ \text { Total | } & 47 & 100.00 & \end{array}$

- Other (please specify)

22. Did the observation of problems with the quality of weatherization work lead to changes in weatherization training for local agency staff?

Freq. Percent Cum.

\begin{tabular}{|c|c|c|c|}
\hline no & 17 & 35.42 & 35.42 \\
\hline yes | & 31 & 64.58 & 100.00 \\
\hline Total | & 48 & 100.00 & \\
\hline
\end{tabular}

22a. If Yes, what changes were made?

23. Does your state observe weatherization training sessions to help identify potential problem areas for monitoring in the field (e.g., with respect to installation of measures that trainees seem to have trouble understanding)?

\begin{tabular}{|c|c|c|c|}
\hline & Freq. & Percent & Cum. \\
\hline no & 14 & 28.57 & 28.57 \\
\hline yes | & 35 & 71.43 & 100.00 \\
\hline Total & 49 & 100.00 & \\
\hline
\end{tabular}

24a. If Yes, briefly describe how your in-field monitoring activities were affected by your training session observations. 

APPENDIX D: ALL AGENCIES PROGRAM INFORMATION SURVEY 

MB Control Number: 1910-5151

\section{S2: ALL AGENCIES PROGRAM INFORMATION SURVEY}

Thank you for your prompt response to this data request which is part of the national evaluation of the Weatherization Assistance Program. Evaluation results will provide essential feedback to the weatherization community and inform policymakers about the program's effects on clients' energy consumption, cost savings, and non-energy benefits.

This data is being collected to conduct a process evaluation of the Weatherization Assistance Program at the local level. The data you supply will be used to characterize the program in Program Years 2007 and 2008, with an emphasis on Program Year 2008.

All of the information obtained from this survey will be protected and will remain confidential. The data will be analyzed in such a way that the information provided cannot be associated back to your state, your agencies, or the housing units and clients that your state served. Again, please note that the questions refer to Program Year 2007 and 2008 unless otherwise noted.

Thank you in advance for completing this survey.

Public reporting burden for this collection of information is estimated to average eight hours per response, including the time for reviewing instructions, searching existing data sources, gathering and maintaining the data needed, and completing and reviewing the collection of information. Send comments regarding this burden estimate or any other aspect of this collection of information, including suggestions for reducing this burden, to Office of the Chief Information Officer, Records Management Division, IM-11, Paperwork Reduction Project (1910-5151), U.S. Department of Energy, 1000 Independence Ave SW, Washington, DC, 20585-1290; and to the Office of Management and Budget (OMB), OIRA, Paperwork Reduction Project (1910-5151), Washington, DC 20503. 


\section{Part 1. General Information}

1. Please identify your state.

2. Please identify your local agency.

3. For how many years had the person who was director of your local Weatherization Program in Program Year 2008 served in that capacity prior to Program Year 2008?

\begin{tabular}{|l|c|}
\hline observations: & 777 \\
\hline missing values: & 37 \\
\hline mean: & 10.64 \\
\hline standard deviation: & 9.14 \\
\hline min: & 0 \\
\hline 10th percentile: & 1 \\
\hline 25th percentile: & 2 \\
\hline median: & 8 \\
\hline 75th percentile: & 18 \\
\hline 90th percentile: & 25 \\
\hline max: & 37 \\
\hline
\end{tabular}

4. How many different people have served as director of your local Weatherization Program over the 10 years prior to Program Year 2008 (including the person who was head in Program Year 2008)?

\begin{tabular}{|l|c|}
\hline observations: & 787 \\
\hline missing values: & 27 \\
\hline mean: & 1.78 \\
\hline standard deviation: & 1.30 \\
\hline min: & 0 \\
\hline 10th percentile: & 1 \\
\hline 25th percentile: & 1 \\
\hline median: & 2 \\
\hline 75th percentile: & 2 \\
\hline 90th percentile: & 3 \\
\hline max: & 22 \\
\hline
\end{tabular}

5. What agency, office, or department was responsible for reviewing the performance of your local Weatherization Program in Program Year 2008? 
6. How many layers of management or supervision were there between your weatherization crews and the director of your local Weatherization Program in Program Year 2008?

\begin{tabular}{|l|c|}
\hline observations: & 782 \\
\hline missing values: & 32 \\
\hline mean: & 1.03 \\
\hline standard deviation: & 1.24 \\
\hline min: & 0 \\
\hline 10th percentile: & 0 \\
\hline 25th percentile: & 0 \\
\hline median: & 1 \\
\hline 75th percentile: & 2 \\
\hline 90th percentile: & 2 \\
\hline max: & 20 \\
\hline
\end{tabular}

\section{Part 2. Information about Program Year 2007}

7. Please provide the following information about ALL low-income dwelling units weatherized by your agency in Program Year 2007.

\begin{tabular}{|c|c|c|c|c|}
\hline \multirow[b]{3}{*}{ Type of Unit Weatherized } & \multicolumn{4}{|c|}{ Number of Units Weatherized in PY 2007} \\
\hline & \multirow[b]{2}{*}{ DOE Units ${ }^{1}$} & \multicolumn{2}{|c|}{ Non-DOE Units ${ }^{2}$} & \multirow[b]{2}{*}{ TOTAL Units } \\
\hline & & $\begin{array}{l}\text { Comprehensive } \\
\text { Weatherization }^{3}\end{array}$ & $\begin{array}{c}\text { Non- } \\
\text { comprehensive } \\
\text { Weatherization }^{4}\end{array}$ & \\
\hline $\begin{array}{l}\text { Single Family Attached and } \\
\text { Detached }\end{array}$ & $\begin{array}{l}\text { obs: } 814 \\
\text { min: } 0 \\
\text { max: } 2464 \\
\text { mean: } 64.85 \\
\text { median: } 41 \\
\end{array}$ & $\begin{array}{l}\text { obs: } 814 \\
\text { min: } 0 \\
\text { max: } 1998 \\
\text { mean: } 41.54 \\
\text { median: } 0\end{array}$ & $\begin{array}{l}\text { obs: } 814 \\
\text { min: } 0 \\
\text { max: } 3593 \\
\text { mean: } 16.51 \\
\text { median: } 0\end{array}$ & $\begin{array}{l}\text { obs: } 814 \\
\text { min: } 0 \\
\text { max: } 5435 \\
\text { mean: } 122.90 \\
\text { median: } 65 \\
\end{array}$ \\
\hline Small Multi-family (2-4 units) & $\begin{array}{l}\text { obs: } 814 \\
\text { min: } 0 \\
\text { max: } 785 \\
\text { mean: } 6.90 \\
\text { median: } 0\end{array}$ & $\begin{array}{l}\text { obs: } 814 \\
\text { min: } 0 \\
\text { max: } 423 \\
\text { mean: } 5.36 \\
\text { median: } 0\end{array}$ & $\begin{array}{l}\text { obs: } 814 \\
\text { min: } 0 \\
\text { max: } 423 \\
\text { mean: } 1.41 \\
\text { median: } 0\end{array}$ & $\begin{array}{l}\text { obs: } 814 \\
\text { min: } 0 \\
\text { max: } 790 \\
\text { mean: } 13.68 \\
\text { median: } 0\end{array}$ \\
\hline $\begin{array}{l}\text { Multi-family ( } 5 \text { or More Units per } \\
\text { Building) }\end{array}$ & $\begin{array}{l}\text { obs: } 814 \\
\text { min: } 0 \\
\text { max: } 789 \\
\text { mean: } 13.60 \\
\text { median: } 0\end{array}$ & $\begin{array}{l}\text { obs: } 814 \\
\text { min: } 0 \\
\text { max: } 1956 \\
\text { mean: } 16.07 \\
\text { median: } 0\end{array}$ & $\begin{array}{l}\text { obs: } 814 \\
\text { min: } 0 \\
\text { max: } 1281 \\
\text { mean: } 2.37 \\
\text { median: } 0 \\
\end{array}$ & $\begin{array}{l}\text { obs: } 814 \\
\text { min: } 0 \\
\text { max: } 1956 \\
\text { mean: } 32.03 \\
\text { median: } 0\end{array}$ \\
\hline Mobile Home & $\begin{array}{l}\text { obs: } 814 \\
\text { min: } 0 \\
\text { max: } 191 \\
\text { mean: } 17.18 \\
\text { median: } 9.5 \\
\end{array}$ & $\begin{array}{l}\text { obs: } 814 \\
\text { min: } 0 \\
\text { max: } 715 \\
\text { mean: } 9.15 \\
\text { median: } 0\end{array}$ & $\begin{array}{l}\text { obs: } 814 \\
\text { min: } 0 \\
\text { max: } 381 \\
\text { mean: } 2.65 \\
\text { median: } 0 \\
\end{array}$ & $\begin{array}{l}\text { obs: } 814 \\
\text { min: } 0 \\
\text { max: } 748 \\
\text { mean: } 28.98 \\
\text { median: } 15 \\
\end{array}$ \\
\hline Shelter & $\begin{array}{l}\text { obs: } 814 \\
\text { min: } 0 \\
\text { max: } 22 \\
\text { mean: } 15 \\
\text { median: } 0\end{array}$ & $\begin{array}{l}\text { obs: } 814 \\
\text { min: } 0 \\
\text { max: } 19 \\
\text { mean: } .05 \\
\text { median: } 0\end{array}$ & $\begin{array}{l}\text { obs: } 814 \\
\text { min: } 0 \\
\text { max: } 1 \\
\text { mean: } .001 \\
\text { median: } 0\end{array}$ & $\begin{array}{l}\text { obs: } 814 \\
\text { min: } 0 \\
\text { max: } 34 \\
\text { mean: } .21 \\
\text { median: } 0\end{array}$ \\
\hline TOTAL UNITS & $\begin{array}{l}\text { obs: } 814 \\
\text { min: } 0 \\
\text { max: } 3323 \\
\text { mean: } 102.68 \\
\text { median: } 66\end{array}$ & $\begin{array}{l}\text { obs: } 814 \\
\text { min: } 0 \\
\text { max: } 2866 \\
\text { mean: } 72.17 \\
\text { median: } 0\end{array}$ & $\begin{array}{l}\text { obs: } 814 \\
\text { min: } 0 \\
\text { max: } 4227 \\
\text { mean: } 22.94 \\
\text { median: } 0\end{array}$ & $\begin{array}{l}\text { obs: } 814 \\
\text { min: } 0 \\
\text { max: } 6367 \\
\text { mean: } 197.79 \\
\text { median: } 103 \\
\end{array}$ \\
\hline \multicolumn{5}{|c|}{$\begin{array}{l}{ }^{1} \text { These are dwelling units that your agency weatherized and reported to the State as "DOE Units" } \\
{ }^{2} \text { These are dwelling units that your agency weatherized but did not report as "DOE Units" } \\
{ }^{3} \text { Comprehensive weatherization units are those for which an audit or priority list was used that addressed a large proportion of potential } \\
\text { energy-saving measures. } \\
{ }^{4} \text { Non-comprehensive weatherization units are those for which a limited set of measures was considered (e.g., baseload electric measures only; } \\
\text { low cost/no cost measures only), reflecting the needs and priorities of the funding entity. }\end{array}$} \\
\hline
\end{tabular}


8. For each of the non-DOE sources from which your agency received weatherization funding in Program Year 2007, was any of the money spent in ways not allowed under DOE rules? If so, enter responses in the appropriate rows of Column A. If your agency did spend non-DOE money in ways not allowed under DOE rules, how did those expenditures differ from expenditures made under DOE rules? Enter responses in the appropriate rows of Column B. If your agency received no funding from a particular source in Program Year 2007, please leave that row blank.

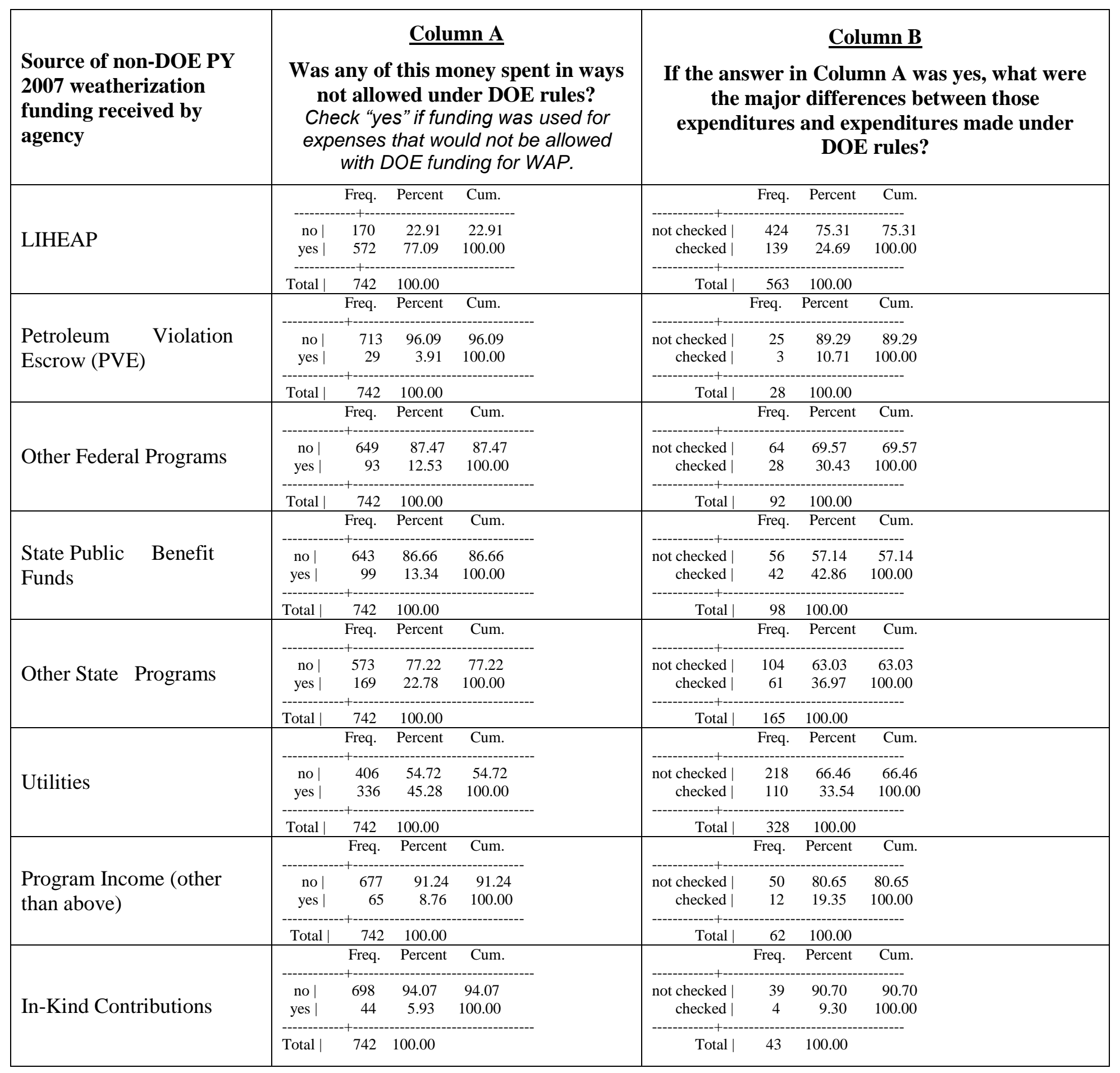




\begin{tabular}{|c|c|c|c|c|c|c|c|c|}
\hline \multirow{4}{*}{ Non-profit Organization } & & Freq & Percent & Cum. & \multicolumn{4}{|c|}{$\begin{array}{lll}\text { Freq. } & \text { Percent } \text { Cum. }\end{array}$} \\
\hline & no & 728 & 98.11 & 98.11 & not checked | & 10 & 100.00 & 100.00 \\
\hline & yes 1 & 14 & & 100.00 & $\pi-1$ & & \multirow{2}{*}{100.00} & \\
\hline & Total | & 742 & 100.00 & & & & \\
\hline \multirow{4}{*}{ Other } & & Freq & Percent & Cum. & \multicolumn{2}{|c|}{ Freq. } & Percent & Cum. \\
\hline & no & 656 & 88.41 & 88.41 & not checked & 34 & 69.39 & 69.39 \\
\hline & yes & 86 & 11.59 & 100.00 & checked & 15 & 30.61 & 100.00 \\
\hline & Total & 742 & 100.00 & & Total | & 49 & 100.00 & \\
\hline
\end{tabular}


9. Please indicate the amount of funding from each source that was spent by your agency in Program Year 2007 on the different functions or applications shown below. The amount that you list in the right-most cell at the very bottom of the table should equal the total amount of weatherization funding from all sources that your agency received in Program Year 2007.

\begin{tabular}{|c|c|c|c|c|c|c|c|}
\hline \multirow[b]{3}{*}{$\begin{array}{l}\text { Source of PY } 2007 \\
\text { Weatherization } \\
\text { Funding Received } \\
\text { by Agency }\end{array}$} & \multirow[b]{3}{*}{$\begin{array}{c}\text { Amount Spent on } \\
\text { Program Manage- } \\
\text { Ment }^{1}\end{array}$} & \multirow[b]{3}{*}{$\begin{array}{c}\text { Amount Spent on } \\
\text { T\&TA }\end{array}$} & \multicolumn{4}{|c|}{ PY 2007 Funds Supporting Weatherization of Units (\$) } & \multirow[b]{3}{*}{$\begin{array}{c}\text { TOTAL Amount } \\
\text { Spent }\end{array}$} \\
\hline & & & \multicolumn{2}{|c|}{ Amount Spent on DOE Units ${ }^{2}$} & \multicolumn{2}{|c|}{ Amount Spent on Non-DOE Units ${ }^{3}$} & \\
\hline & & & $\begin{array}{c}\text { Expenditures for } \\
\text { Health and Safety, } \\
\text { Repairs, and Other } \\
\text { Non Cost-Effective } \\
\text { Measures }^{4}\end{array}$ & $\begin{array}{c}\text { All Expenditures } \\
\text { Defined as } \\
\text { Allowable Costs } \\
\text { for Purpose of } \\
\text { Calculating } \\
\text { Average Cost per } \\
\text { Unit }^{5} \\
\end{array}$ & $\begin{array}{l}\text { Expenditures for } \\
\text { Health and } \\
\text { Safety, Repairs, } \\
\text { and Other Non } \\
\text { Cost-Effective } \\
\text { Measures }^{4}\end{array}$ & $\begin{array}{c}\text { All Expenditures } \\
\text { Defined as } \\
\text { Allowable Costs } \\
\text { for Purpose of } \\
\text { Calculating } \\
\text { Average Cost per } \\
\text { Unit }^{5} \\
\end{array}$ & \\
\hline DOE & $\begin{array}{l}\text { Obs: } 793 \\
\text { Min: } 0 \\
\text { Max: } 5621800 \\
\text { Mean: } 49483.67 \\
\text { Median: } 21837 \\
\end{array}$ & $\begin{array}{l}\text { Obs: } 792 \\
\text { Min: } 0 \\
\text { Max: } 872800 \\
\text { Mean: } 6603.51 \\
\text { Median: } 3363.07\end{array}$ & $\begin{array}{l}\text { Obs: } 792 \\
\text { Min: } 0 \\
\text { Max: } 4319007 \\
\text { Mean: } 31436.15 \\
\text { Median: } 9733.13 \\
\end{array}$ & $\begin{array}{l}\text { Obs: } 792 \\
\text { Min: } 0 \\
\text { Max: } 64577700 \\
\text { Mean: } 270259.55 \\
\text { Median: } 126288.5\end{array}$ & N/A & N/A & $\begin{array}{l}\text { Obs: } 793 \\
\text { Min: } 0 \\
\text { Max: } 74955800 \\
\text { Mean: } 357394.09 \\
\text { Median: } 183321\end{array}$ \\
\hline LIHEAP & $\begin{array}{l}\text { Obs: } 792 \\
\text { Min: } 0 \\
\text { Max: } 548411 \\
\text { Mean: } 23982.10 \\
\text { Median: } 6822.5\end{array}$ & $\begin{array}{l}\text { Obs: } 791 \\
\text { Min: } 0 \\
\text { Max: } 110731 \\
\text { Mean: } 1394.41 \\
\text { Median: } 0\end{array}$ & $\begin{array}{l}\text { Obs: } 791 \\
\text { Min: } 0 \\
\text { Max: } 1253428 \\
\text { Mean: } 13957.89 \\
\text { Median: } 0\end{array}$ & $\begin{array}{l}\text { Obs: } 791 \\
\text { Min: } 0 \\
\text { Max: } 1255000 \\
\text { Mean: } 62681.37 \\
\text { Median: } 0\end{array}$ & $\begin{array}{l}\text { Obs: } 791 \\
\text { Min: } 0 \\
\text { Max: } 2016494 \\
\text { Mean: } 17029.10 \\
\text { Median: } 0\end{array}$ & $\begin{array}{l}\text { Obs: } 791 \\
\text { Min: } 0 \\
\text { Max: } 3858404 \\
\text { Mean: } 83437.33 \\
\text { Median: } 0\end{array}$ & $\begin{array}{l}\text { Obs: } 792 \\
\text { Min: } 0 \\
\text { Max: } 4104275.3 \\
\text { Mean: } 202256.82 \\
\text { Median: } 96134.5\end{array}$ \\
\hline $\begin{array}{l}\text { Petroleum } \\
\text { Violation Escrow } \\
\text { (PVE) }\end{array}$ & $\begin{array}{l}\text { Obs: } 792 \\
\text { Min: } 0 \\
\text { Max: } 52553 \\
\text { Mean: } 167.60 \\
\text { Median: } 0 \\
\end{array}$ & $\begin{array}{l}\text { Obs: } 791 \\
\text { Min: } 0 \\
\text { Max: } 1500 \\
\text { Mean: } 1.90 \\
\text { Median: } 0\end{array}$ & $\begin{array}{l}\text { Obs: } 791 \\
\text { Min: } 0 \\
\text { Max: } 51972 \\
\text { Mean: } 106.32 \\
\text { Median: } 0 \\
\end{array}$ & $\begin{array}{l}\text { Obs: } 791 \\
\text { Min: } 0 \\
\text { Max: } 65759 \\
\text { Mean: } 208.69 \\
\text { Median: } 0 \\
\end{array}$ & $\begin{array}{l}\text { Obs: } 791 \\
\text { Min: } 0 \\
\text { Max: } 171905 \\
\text { Mean: } 335.23 \\
\text { Median: } 0 \\
\end{array}$ & $\begin{array}{l}\text { Obs: } 791 \\
\text { Min: } 0 \\
\text { Max: } 222000 \\
\text { Mean: } 800.63 \\
\text { Median: } 0 \\
\end{array}$ & $\begin{array}{l}\text { Obs: } 792 \\
\text { Min: } 0 \\
\text { Max: } 286000 \\
\text { Mean: } 1618.53 \\
\text { Median: } 0 \\
\end{array}$ \\
\hline $\begin{array}{l}\text { Other Federal } \\
\text { Programs }\end{array}$ & $\begin{array}{l}\text { Obs: } 792 \\
\text { Min: } 0 \\
\text { Max: } 2759000.5 \\
\text { Mean: } 6527.89 \\
\text { Median: } 0 \\
\end{array}$ & $\begin{array}{l}\text { Obs: } 791 \\
\text { Min: } 0 \\
\text { Max: } 16496.16 \\
\text { Mean: } 47.35 \\
\text { Median: } 0\end{array}$ & $\begin{array}{l}\text { Obs: } 791 \\
\text { Min: } 0 \\
\text { Max: } 151555.27 \\
\text { Mean: } 1358.54 \\
\text { Median: } 0 \\
\end{array}$ & $\begin{array}{l}\text { Obs: } 791 \\
\text { Min: } 0 \\
\text { Max: } 831830 \\
\text { Mean: } 6590.93 \\
\text { Median: } 0 \\
\end{array}$ & $\begin{array}{l}\text { Obs: } 791 \\
\text { Min: } 0 \\
\text { Max: } 444016 \\
\text { Mean: } 2467.58 \\
\text { Median: } 0\end{array}$ & $\begin{array}{l}\text { Obs: } 791 \\
\text { Min: } 0 \\
\text { Max: } 8370340.5 \\
\text { Mean: } 18303.53 \\
\text { Median: } 0\end{array}$ & $\begin{array}{l}\text { Obs: } 792 \\
\text { Min: } 0 \\
\text { Max: } 11129341 \\
\text { Mean: } 35259.50 \\
\text { Median: } 0\end{array}$ \\
\hline $\begin{array}{l}\text { State Public } \\
\text { Benefit Funds }\end{array}$ & $\begin{array}{l}\text { Obs: } 792 \\
\text { Min: } 0 \\
\text { Max: } 2327160.5 \\
\text { Mean: } 12361.18 \\
\text { Median: } 0\end{array}$ & $\begin{array}{l}\text { Obs: } 791 \\
\text { Min: } 0 \\
\text { Max: } 42000 \\
\text { Mean: } 220.40 \\
\text { Median: } 0\end{array}$ & $\begin{array}{l}\text { Obs: } 791 \\
\text { Min: } 0 \\
\text { Max: } 671790 \\
\text { Mean: } 3640.32 \\
\text { Median: } 0\end{array}$ & $\begin{array}{l}\text { Obs: } 791 \\
\text { Min: } 0 \\
\text { Max: } 2693708 \\
\text { Mean: } 19357.01 \\
\text { Median: } 0\end{array}$ & $\begin{array}{l}\text { Obs: } 791 \\
\text { Min: } 0 \\
\text { Max: } 549285.19 \\
\text { Mean: } 5595.92 \\
\text { Median: } 0 \\
\end{array}$ & $\begin{array}{l}\text { Obs: } 791 \\
\text { Min: } 0 \\
\text { Max: } 3307116.5 \\
\text { Mean: } 25688.23 \\
\text { Median: } 0 \\
\end{array}$ & $\begin{array}{l}\text { Obs: } 792 \\
\text { Min: } 0 \\
\text { Max: } 6196118.5 \\
\text { Mean: } 66794.24 \\
\text { Median: } 0 \\
\end{array}$ \\
\hline $\begin{array}{l}\text { Other State } \\
\text { Programs }\end{array}$ & $\begin{array}{l}\text { Obs: } 792 \\
\text { Min: } 0 \\
\text { Max: } 876850 \\
\text { Mean: } 7284.20 \\
\text { Median: } 0\end{array}$ & $\begin{array}{l}\text { Obs: } 791 \\
\text { Min: } 0 \\
\text { Max: } 34203 \\
\text { Mean: } 173.87 \\
\text { Median: } 0\end{array}$ & $\begin{array}{l}\text { Obs: } 791 \\
\text { Min: } 0 \\
\text { Max: } 371657.25 \\
\text { Mean: } 2557.82 \\
\text { Median: } 0\end{array}$ & $\begin{array}{l}\text { Obs: } 791 \\
\text { Min: } 0 \\
\text { Max: } 1489874 \\
\text { Mean: } 17851.43 \\
\text { Median: } 0\end{array}$ & $\begin{array}{l}\text { Obs: } 791 \\
\text { Min: } 0 \\
\text { Max: } 126980 \\
\text { Mean: } 1700.40 \\
\text { Median: } 0\end{array}$ & $\begin{array}{l}\text { Obs: } 791 \\
\text { Min: } 0 \\
\text { Max: } 2602744 \\
\text { Mean: } 20814.97 \\
\text { Median: } 0\end{array}$ & $\begin{array}{l}\text { Obs: } 792 \\
\text { Min: } 0 \\
\text { Max: } 3479594 \\
\text { Mean: } 50328.26 \\
\text { Median: } 0\end{array}$ \\
\hline
\end{tabular}




\begin{tabular}{|c|c|c|c|c|c|c|c|}
\hline Utilities & $\begin{array}{l}\text { Obs: } 792 \\
\text { Min: } 0 \\
\text { Max: } 33949300 \\
\text { Mean: } 51147.30 \\
\text { Median: } 0\end{array}$ & $\begin{array}{l}\text { Obs: } 791 \\
\text { Min: } 0 \\
\text { Max: } 43912 \\
\text { Mean: } 231.06 \\
\text { Median: } 0\end{array}$ & $\begin{array}{l}\text { Obs: } 791 \\
\text { Min: } 0 \\
\text { Max: } 372163 \\
\text { Mean: } 3827.23 \\
\text { Median: } 0\end{array}$ & $\begin{array}{l}\text { Obs: } 791 \\
\text { Min: } 0 \\
\text { Max: } 27662500 \\
\text { Mean: } 64548.98 \\
\text { Median: } 0\end{array}$ & $\begin{array}{l}\text { Obs: } 791 \\
\text { Min: } 0 \\
\text { Max: } 296751 \\
\text { Mean: } 1670.62 \\
\text { Median: } 0\end{array}$ & $\begin{array}{l}\text { Obs: } 791 \\
\text { Min: } 0 \\
\text { Max: } 2790000 \\
\text { Mean: } 39156.34 \\
\text { Median: } 0\end{array}$ & $\begin{array}{l}\text { Obs: } 792 \\
\text { Min: } 0 \\
\text { Max: } 61611800 \\
\text { Mean: } 160443.35 \\
\text { Median: } 0\end{array}$ \\
\hline $\begin{array}{l}\text { Program Income } \\
\text { (other than } \\
\text { above) }\end{array}$ & $\begin{array}{l}\text { Obs: } 792 \\
\text { Min: } 0 \\
\text { Max: } 152879.98 \\
\text { Mean: } 499.38 \\
\text { Median: } 0 \\
\end{array}$ & $\begin{array}{l}\text { Obs: } 791 \\
\text { Min: } 0 \\
\text { Max: } 12764 \\
\text { Mean: } 28.34 \\
\text { Median: } 0 \\
\end{array}$ & $\begin{array}{l}\text { Obs: } 791 \\
\text { Min: } 0 \\
\text { Max: } 100000 \\
\text { Mean: } 307.76 \\
\text { Median: } 0\end{array}$ & $\begin{array}{l}\text { Obs: } 791 \\
\text { Min: } 0 \\
\text { Max: } 151982 \\
\text { Mean: } 708.48 \\
\text { Median: } 0 \\
\end{array}$ & $\begin{array}{l}\text { Obs: } 791 \\
\text { Min: } 0 \\
\text { Max: } 126245 \\
\text { Mean: } 322.44 \\
\text { Median: } 0\end{array}$ & $\begin{array}{l}\text { Obs: } 791 \\
\text { Min: } 0 \\
\text { Max: } 383052.19 \\
\text { Mean: } 1365.34 \\
\text { Median: } 0 \\
\end{array}$ & $\begin{array}{l}\text { Obs: } 792 \\
\text { Min: } 0 \\
\text { Max: } 535932.19 \\
\text { Mean: } 3228.28 \\
\text { Median: } 0\end{array}$ \\
\hline $\begin{array}{l}\text { In-Kind } \\
\text { Contributions }\end{array}$ & $\begin{array}{l}\text { Obs: } 792 \\
\text { Min: } 0 \\
\text { Max: } 4156401 \\
\text { Mean: } 5487.37 \\
\text { Median: } 0\end{array}$ & $\begin{array}{l}\text { Obs: } 791 \\
\text { Min: } 0 \\
\text { Max: } 0 \\
\text { Mean:0 } \\
\text { Median: } 0\end{array}$ & $\begin{array}{l}\text { Obs: } 791 \\
\text { Min: } 0 \\
\text { Max: } 629172 \\
\text { Mean: } 938.17 \\
\text { Median: } 0\end{array}$ & $\begin{array}{l}\text { Obs: } 791 \\
\text { Min: } 0 \\
\text { Max: } 300993 \\
\text { Mean: } 1045.64 \\
\text { Median: } 0\end{array}$ & $\begin{array}{l}\text { Obs: } 791 \\
\text { Min: } 0 \\
\text { Max: } 0 \\
\text { Mean: } 0 \\
\text { Median: } 0\end{array}$ & $\begin{array}{l}\text { Obs: } 791 \\
\text { Min: } 0 \\
\text { Max: } 140401 \\
\text { Mean: } 229.49 \\
\text { Median: } 0\end{array}$ & $\begin{array}{l}\text { Obs: } 792 \\
\text { Min: } 0 \\
\text { Max: } 4156401 \\
\text { Mean: } 7697.87 \\
\text { Median: } 0\end{array}$ \\
\hline $\begin{array}{l}\text { Non-Profit } \\
\text { Organizations }\end{array}$ & $\begin{array}{l}\text { Obs: } 792 \\
\text { Min: } 0 \\
\text { Max: } 60152 \\
\text { Mean: } 110.40 \\
\text { Median: } 0\end{array}$ & $\begin{array}{l}\text { Obs: } 791 \\
\text { Min: } 0 \\
\text { Max: } 0 \\
\text { Mean: } 0 \\
\text { Median: } 0\end{array}$ & $\begin{array}{l}\text { Obs: } 791 \\
\text { Min: } 0 \\
\text { Max: } 116476 \\
\text { Mean: } 165.18 \\
\text { Median: } 0\end{array}$ & $\begin{array}{l}\text { Obs: } 791 \\
\text { Min: } 0 \\
\text { Max: } 124398 \\
\text { Mean: } 432.09 \\
\text { Median: } 0\end{array}$ & $\begin{array}{l}\text { Obs: } 791 \\
\text { Min: } 0 \\
\text { Max: } 20000 \\
\text { Mean: } 25.28 \\
\text { Median: } 0\end{array}$ & $\begin{array}{l}\text { Obs: } 791 \\
\text { Min: } 0 \\
\text { Max: } 143786 \\
\text { Mean: } 194.42 \\
\text { Median: } 0\end{array}$ & $\begin{array}{l}\text { Obs:792 } \\
\text { Min: 0 } \\
\text { Max: } 245529 \\
\text { Mean: } 926.34 \\
\text { Median: } 0\end{array}$ \\
\hline TOTAL FUNDS & $\begin{array}{l}\text { Obs: } 793 \\
\text { Min: } 0 \\
\text { Max: } 39571100 \\
\text { Mean: } 156915.45 \\
\text { Median: } 44578\end{array}$ & $\begin{array}{l}\text { Obs: } 792 \\
\text { Min: } 0 \\
\text { Max: } 872800 \\
\text { Mean: } 8698.18 \\
\text { Median: } 4500\end{array}$ & $\begin{array}{l}\text { Obs: } 792 \\
\text { Min: } 0 \\
\text { Max: } 4683982.5 \\
\text { Mean: } 58261.45 \\
\text { Median: } 14960\end{array}$ & $\begin{array}{l}\text { Obs: } 792 \\
\text { Min: } 0 \\
\text { Max: } 92240200 \\
\text { Mean: } 443465.18 \\
\text { Median: } 203627.89\end{array}$ & $\begin{array}{l}\text { Obs: } 792 \\
\text { Min: } 0 \\
\text { Max: } 2016494 \\
\text { Mean: } 29493.79 \\
\text { Median: } 0\end{array}$ & $\begin{array}{l}\text { Obs: } 792 \\
\text { Min: } 0 \\
\text { Max: } 10973084 \\
\text { Mean:192633.02 } \\
\text { Median: } 0\end{array}$ & $\begin{array}{l}\text { Obs: } 793 \\
\text { Min: } 0 \\
\text { Max: } 1.366 \mathrm{e}+08 \\
\text { Mean: } 888263.19 \\
\text { Median: } 437159\end{array}$ \\
\hline \multicolumn{8}{|c|}{$\begin{array}{l}{ }^{1} \text { Program Management costs include: cost of liability insurance; cost of low cost/no cost activities; cost of financial audits; administrative expenses; and funds used for leveraging activities. } \\
2 \text { These are dwelling units that your agency weatherized and reported to the State as "DOE Units" } \\
{ }^{3} \text { These are dwelling units that your agency weatherized but did not report as "DOE Units" } \\
{ }^{4} \text { Non cost-effective measures are those with a Savings to Investment Ratio (SIR) of less than 1.0. } \\
{ }^{5} \text { Under DOE regulations, allowable costs include: the cost of weatherization materials (not for health and safety); labor cost (not for health and safety); transportation of weatherization materials, crews, equipment, } \\
\text { and tools; vehicle maintenance, operations, and insurance; maintenance of tools and equipment; cost of purchasing vehicles; employment of on-site supervisors; storage of weatherization materials, tools, and } \\
\text { equipment; and cost of incidental repairs. }\end{array}$} \\
\hline
\end{tabular}


10. Did your agency classify its expenditures for client intake, audits, and post-weatherization inspections as program management costs or as allowable costs used in the calculation of average cost per unit in Program Year 2007? Please indicate your answer for each type of expenditure by checking the appropriate cell in the table below.

\begin{tabular}{|c|c|c|c|c|}
\hline \multirow[t]{2}{*}{ Type of Expenditure } & $\begin{array}{c}\text { Classified as Program } \\
\text { Management Costs? }\end{array}$ & \multicolumn{3}{|c|}{$\begin{array}{l}\text { Classified as Allowable Costs for } \\
\text { Purpose of Calculating Average } \\
\text { Cost per Unit? }\end{array}$} \\
\hline & & Freq. & Percent & Cum. \\
\hline \multirow{3}{*}{$\begin{array}{l}\text { Expenditures for Client } \\
\text { Intake }\end{array}$} & Classified as Program Management Costs & 312 & 44.51 & 44.51 \\
\hline & Classified as Allowable Costs for Purpose| & 389 & 55.49 & 100.00 \\
\hline & Total | & 701 & 100.00 & \\
\hline \multirow{4}{*}{ Expenditures for Audits } & & Freq. & Percent & Cum. \\
\hline & Classified as Program Management Costs & 288 & 39.56 & 39.56 \\
\hline & Classified as Allowable Costs for Purpose | & 440 & 60.44 & 100.00 \\
\hline & Total & 728 & 100.00 & \\
\hline \multirow{4}{*}{$\begin{array}{l}\text { Expenditures for Post- } \\
\text { Weatherization Quality } \\
\text { Control Inspections }\end{array}$} & & Freq. & Percent & Cum. \\
\hline & Classified as Program Management Costs & 194 & 27.17 & 27.17 \\
\hline & Classified as Allowable Costs for Purpose & 520 & 72.83 & 100.00 \\
\hline & Total | & 714 & 100.00 & \\
\hline
\end{tabular}

11. Of the TOTAL amount spent by your agency in Program Year 2007 using funds from all sources (shown at bottom of right-most column in table for Question 9), please give your best estimate of how much was spent on Audits and Inspections.

\begin{tabular}{|l|c|}
\hline observations: & 719 \\
\hline missing values: & 95 \\
\hline mean: & 61859.06 \\
\hline standard deviation: & 83928.93 \\
\hline min: & 0 \\
\hline 10th percentile: & 2520 \\
\hline 25th percentile: & 10972.5 \\
\hline median: & 32000 \\
\hline 75th percentile: & 74000 \\
\hline 90th percentile: & 172500 \\
\hline max: & 642605 \\
\hline
\end{tabular}


12. Please divide your agency's Program Year 2007 expenditures on DOE units into in-house expenditures and contractor expenditures, as shown in the following table.

\begin{tabular}{|c|c|c|c|}
\hline Type of Expenditure & $\begin{array}{c}\text { PY 2007 } \\
\text { In-house } \\
\text { Expenditures on } \\
\text { DOE Units (in \$) }\end{array}$ & $\begin{array}{l}\text { PY } 2007 \text { Contractor } \\
\text { Expenditures on } \\
\text { DOE Units (in \$) }\end{array}$ & $\begin{array}{l}\text { PY } 2007 \text { Total } \\
\text { Expenditures on } \\
\text { DOE Units (in \$) }\end{array}$ \\
\hline $\begin{array}{l}\text { Expenditures for Health and Safety, Repairs, } \\
\text { and Other Non Cost-Effective Measures }\end{array}$ & $\begin{array}{l}\text { obs: } 814 \\
\text { min: } 0 \\
\text { max: } 1486995 \\
\text { mean: } 17732.84 \\
\text { median: } 925 \\
\end{array}$ & $\begin{array}{l}\text { obs: } 814 \\
\text { min: } 0 \\
\text { max: } 1832528 \\
\text { mean: } 30854.00 \\
\text { median: } 2000 \\
\end{array}$ & $\begin{array}{l}\text { obs: } 814 \\
\text { min: } 0 \\
\text { max: } 3058591 \\
\text { mean: } 48586.85 \\
\text { median: } 13499.5 \\
\end{array}$ \\
\hline $\begin{array}{l}\text { All Expenditures Defined as Allowable Costs } \\
\text { for Purpose of Calculating Average Cost per } \\
\text { Unit }\end{array}$ & $\begin{array}{l}\text { obs: } 814 \\
\text { min: } 0 \\
\text { max: } 2502914 \\
\text { mean: } 155319.08 \\
\text { median: } 62086\end{array}$ & $\begin{array}{l}\text { obs: } 814 \\
\text { min: } 0 \\
\text { max: } 4515651 \\
\text { mean: } 117105.57 \\
\text { median: } 21118\end{array}$ & $\begin{array}{l}\text { obs: } 814 \\
\text { min: } 0 \\
\text { max: } 6048753 \\
\text { mean: } 272424.64 \\
\text { median: } 157601.59\end{array}$ \\
\hline TOTAL FUNDS & $\begin{array}{l}\text { obs: } 814 \\
\text { min: } 0 \\
\text { max: } 2502914 \\
\text { mean: } 173051.92 \\
\text { median: } 72560 \\
\end{array}$ & $\begin{array}{l}\text { obs: } 814 \\
\text { min: } 0 \\
\text { max: } 4515651 \\
\text { mean: } 147959.57 \\
\text { median: } 37614.04\end{array}$ & $\begin{array}{l}\text { obs: } 814 \\
\text { min: } 0 \\
\text { max: } 6048753 \\
\text { mean: } 321011.49 \\
\text { median: } 190532 \\
\end{array}$ \\
\hline \multicolumn{4}{|c|}{$\begin{array}{l}{ }^{1} \text { The amount that you list for Total Expenditures for Health and Safety, Repairs, and Other Non Cost-Effective Measures in the right- } \\
\text { most column of this table should equal the TOTAL FUNDS listed for that same category of expenditures under DOE units at the } \\
\text { bottom of the table for Question 9. The amount that you list for Total Expenditures for All Expenditures Defined as Allowable Costs } \\
\text { for Purpose of Calculating Average Cost per Unit in the right-most column of this table should equal the TOTAL FUNDS listed for that } \\
\text { same category of expenditures under DOE units at the bottom of the table for Question } 9 \text {. }\end{array}$} \\
\hline
\end{tabular}

13. Of your agency's total Program Year 2007 expenditures on DOE units (shown at bottom of right-most column in table for Question 12), please give your best estimate of how much was for labor, how much for materials, and how much for other expenses.

\begin{tabular}{|l|l|}
\hline \multicolumn{1}{|c|}{ Type of Expenditure } & \multicolumn{1}{c|}{ PY 2007 Expenditures on DOE units (in \$) } \\
\hline & $\begin{array}{l}\text { obs: } 814 \\
\text { min: } 0 \\
\text { Lab: } 3313274 \\
\text { mean: } 153708.16 \\
\text { median: } 79353\end{array}$ \\
\hline & $\begin{array}{l}\text { obs: } 814 \\
\text { min: } 0\end{array}$ \\
Materials & $\begin{array}{l}\text { max: } 2636615.3 \\
\text { mean: } 104090.28 \\
\text { median: } 55189.5\end{array}$ \\
\hline Other Expenses (e.g., costs for & obs: 814 \\
vehicles, transportation, maintenance,, & max: 2457000 \\
and storage) & mean: 57829.2 \\
& median: 17837.5 \\
\hline & obs: 814 \\
TOTAL FUNDS & min: 0 \\
& max: 5669722 \\
& mean: 315627.64 \\
median: 181221 \\
\hline
\end{tabular}


14. Of all the DOE units weatherized by your agency in Program Year 2007 (shown at bottom of leftmost column in table for Question 7), how many used each of the following as their main heating fuel (i.e., the fuel providing most of the heat for the dwelling unit) in the winter prior to weatherization?

- Natural gas

\begin{tabular}{|l|c|}
\hline observations: & 727 \\
\hline missing values: & 87 \\
\hline mean: & 58.78 \\
\hline standard deviation: & 114.25 \\
\hline min: & 0 \\
\hline 10th percentile: & 0 \\
\hline 25th percentile: & 8 \\
\hline median: & 29 \\
\hline 75th percentile: & 68 \\
\hline 90th percentile: & 139 \\
\hline max: & 2113 \\
\hline
\end{tabular}

- Fuel oil

\begin{tabular}{|l|c|}
\hline observations: & 727 \\
\hline missing values: & 87 \\
\hline mean: & 15.06 \\
\hline standard deviation: & 38.80 \\
\hline min: & 0 \\
\hline 10th percentile: & 0 \\
\hline 25th percentile: & 0 \\
\hline median: & 1 \\
\hline 75th percentile: & 13 \\
\hline 90th percentile: & 36 \\
\hline max: & 553 \\
\hline
\end{tabular}

\section{- Electricity}

\begin{tabular}{|l|c|}
\hline observations: & 727 \\
\hline missing values: & 87 \\
\hline mean: & 18.51 \\
\hline standard deviation: & 34.47 \\
\hline min: & 0 \\
\hline 10th percentile: & 0 \\
\hline 25th percentile: & 1 \\
\hline median: & 7 \\
\hline 75th percentile: & 21 \\
\hline 90th percentile: & 47 \\
\hline max: & 326 \\
\hline
\end{tabular}


- Propane/LPG

\begin{tabular}{|l|c|}
\hline observations: & 727 \\
\hline missing values: & 87 \\
\hline mean: & 8.70 \\
\hline standard deviation: & 15.25 \\
\hline min: & 0 \\
\hline 10th percentile: & 0 \\
\hline 25th percentile: & 0 \\
\hline median: & 4 \\
\hline 75th percentile: & 11 \\
\hline 90th percentile: & 22 \\
\hline max: & 261 \\
\hline
\end{tabular}

- Kerosene or coal oil

\begin{tabular}{|l|c|}
\hline observations: & 727 \\
\hline missing values: & 87 \\
\hline mean: & 5719.41 \\
\hline standard deviation: & 154152.3 \\
\hline min: & 0 \\
\hline 10th percentile: & 0 \\
\hline 25th percentile: & 0 \\
\hline median: & 0 \\
\hline 75th percentile: & 0 \\
\hline 90th percentile: & 6 \\
\hline max: & 4156401 \\
\hline
\end{tabular}

- Wood

\begin{tabular}{|l|c|}
\hline observations: & 727 \\
\hline missing values: & 87 \\
\hline mean: & 1.91 \\
\hline standard deviation: & 9.77 \\
\hline min: & 0 \\
\hline 10th percentile: & 0 \\
\hline 25th percentile: & 0 \\
\hline median: & 0 \\
\hline 75th percentile: & 1 \\
\hline 90th percentile: & 4 \\
\hline max: & 242 \\
\hline
\end{tabular}


- Other (please specify)

\begin{tabular}{|l|c|}
\hline observations: & 727 \\
\hline missing values: & 87 \\
\hline mean: & 8035.39 \\
\hline standard deviation: & 216586.13 \\
\hline min: & 0 \\
\hline 10th percentile: & 0 \\
\hline 25th percentile: & 0 \\
\hline median: & 0 \\
\hline 75th percentile: & 0 \\
\hline 90th percentile: & 0 \\
\hline max: & 5839801 \\
\hline
\end{tabular}

15. Of all the DOE units weatherized by your agency in Program Year 2007 (shown at bottom of leftmost column in table for Question 7), how many housed members of the following high-priority client populations (leave blank if do not know)?

\section{- Children (according to your state's definition of that term)}

\begin{tabular}{|l|c|}
\hline observations: & 626 \\
\hline missing values: & 188 \\
\hline mean: & 43.72 \\
\hline standard deviation: & 55.02 \\
\hline min: & 0 \\
\hline 10th percentile: & 3 \\
\hline 25th percentile: & 10 \\
\hline median: & 26 \\
\hline 75th percentile: & 56 \\
\hline 90th percentile: & 103 \\
\hline max: & 489 \\
\hline
\end{tabular}

- Elderly (age 60 and older)

\begin{tabular}{|l|c|}
\hline observations: & 663 \\
\hline missing values: & 151 \\
\hline mean: & 44.65 \\
\hline standard deviation: & 63.13 \\
\hline min: & 0 \\
\hline 10th percentile: & 8 \\
\hline 25th percentile: & 16 \\
\hline median: & 29 \\
\hline 75th percentile: & 54 \\
\hline 90th percentile: & 91 \\
\hline max: & 1144 \\
\hline
\end{tabular}


- Disabled

\begin{tabular}{|l|c|}
\hline observations: & 652 \\
\hline missing values: & 162 \\
\hline mean: & 36.41 \\
\hline standard deviation: & 43.71 \\
\hline min: & 0 \\
\hline 10th percentile: & 4 \\
\hline 25th percentile: & 12 \\
\hline median: & 25 \\
\hline 75th percentile: & 48 \\
\hline 90th percentile: & 77 \\
\hline max: & 534 \\
\hline
\end{tabular}

- Native American

\begin{tabular}{|l|c|}
\hline observations: & 493 \\
\hline missing values: & 321 \\
\hline mean: & 4.64 \\
\hline standard deviation: & 19.64 \\
\hline min: & 0 \\
\hline 10th percentile: & 0 \\
\hline 25th percentile: & 0 \\
\hline median: & 0 \\
\hline 75th percentile: & 2 \\
\hline 90th percentile: & 8 \\
\hline max: & 232 \\
\hline
\end{tabular}

16. Did your state have official definitions of "high energy expenditure" or "high energy burden" in Program Year 2007?

$\begin{array}{rrrr} & \text { Freq. } & \text { Percent } & \text { Cum. } \\ \text { no } \mid & 390 & 51.32 & 51.32 \\ \text { yes } \mid & 370 & 48.68 & 100.00 \\ ---------+------------ & \end{array}$

If answer to Question 16 is "No," skip to Question 18. 
17. Of all the DOE units weatherized by your agency in Program Year 2007 (shown at bottom of leftmost column in table for Question 7), how many met your state weatherization program's definition of having "high energy expenditures" and

\begin{tabular}{|l|c|}
\hline observations: & 191 \\
\hline missing values: & 623 \\
\hline mean: & 46.87 \\
\hline standard deviation: & 63.76 \\
\hline min: & 0 \\
\hline 10th percentile: & .5 \\
\hline 25th percentile: & 6 \\
\hline median: & 27 \\
\hline 75th percentile: & 60 \\
\hline 90th percentile: & 103 \\
\hline max: & 410 \\
\hline
\end{tabular}

"high energy burden"

? (Leave blank if state did not have that definition).

\begin{tabular}{|l|c|}
\hline observations: & 201 \\
\hline missing values: & 613 \\
\hline mean: & 40.68 \\
\hline standard deviation: & 68.44 \\
\hline min: & 0 \\
\hline 10th percentile: & 0 \\
\hline 25th percentile: & 4 \\
\hline median: & 22 \\
\hline 75th percentile: & 47 \\
\hline 90th percentile: & 92 \\
\hline max: & 546 \\
\hline
\end{tabular}

18. Were there any differences between households weatherized by your agency in Program Year 2007 as DOE units and those weatherized as non-DOE units?

Freq. Percent Cum.

\begin{tabular}{|c|c|c|c|}
\hline no & 656 & 87.00 & 87.00 \\
\hline yes & 98 & 13.00 & 100.00 \\
\hline Total | & 754 & 100.00 & \\
\hline
\end{tabular}

19. What were the major differences between the rules and conditions governing your weatherization of DOE and non-DOE units in Program Year 2007? 
20. How many homes were on your wait list for weatherization in Program Year 2007 ?

\begin{tabular}{|l|c|}
\hline observations: & 542 \\
\hline missing values: & 272 \\
\hline mean: & 644.76 \\
\hline standard deviation: & 1720.72 \\
\hline min: & 0 \\
\hline 10th percentile: & 18 \\
\hline 25th percentile: & 60 \\
\hline median: & 156 \\
\hline 75th percentile: & 350 \\
\hline 90th percentile: & 1449 \\
\hline max: & 20304 \\
\hline
\end{tabular}

21. On average, how long was a home on the wait list before it was weatherized in Program Year 2007 ?

\begin{tabular}{|l|c|}
\hline observations: & 566 \\
\hline missing values: & 248 \\
\hline mean: & 322.75 \\
\hline standard deviation: & 395.03 \\
\hline min: & 0 \\
\hline 10th percentile: & 30 \\
\hline 25th percentile: & 90 \\
\hline median: & 180 \\
\hline 75th percentile: & 365 \\
\hline 90th percentile: & 730 \\
\hline max: & 3650 \\
\hline
\end{tabular}




\section{Part 3. Information about Program Year 2008}

22. Please provide the following information about ALL low-income dwelling units weatherized by your agency in Program Year 2008.

\begin{tabular}{|c|c|c|c|c|}
\hline \multirow[b]{3}{*}{ Type of Unit Weatherized } & \multicolumn{4}{|c|}{ Number of Units Weatherized in PY 2008} \\
\hline & \multirow[b]{2}{*}{ DOE Units ${ }^{1}$} & \multicolumn{2}{|c|}{ Non-DOE Units ${ }^{2}$} & \multirow[b]{2}{*}{$\begin{array}{l}\text { TOTAL } \\
\text { Units }\end{array}$} \\
\hline & & $\begin{array}{l}\text { Comprehensive } \\
\text { Weatherization }^{3}\end{array}$ & $\begin{array}{c}\text { Non- } \\
\text { comprehensive } \\
\text { Weatherization }\end{array}$ & \\
\hline $\begin{array}{l}\text { Single Family Attached and } \\
\text { Detached }\end{array}$ & $\begin{array}{l}\text { obs: } 814 \\
\text { min: } 0 \\
\text { max: } 1652 \\
\text { mean: } 66.12 \\
\text { median: } 44\end{array}$ & $\begin{array}{l}\text { obs: } 814 \\
\text { min: } 0 \\
\text { max: } 4142 \\
\text { mean: } 45.69 \\
\text { median: } 0\end{array}$ & $\begin{array}{l}\text { obs: } 814 \\
\text { min: } 0 \\
\text { max: } 1033 \\
\text { mean: } 15.63 \\
\text { median: } 0\end{array}$ & $\begin{array}{l}\text { obs: } 814 \\
\text { min: } 0 \\
\text { max: } 4142 \\
\text { mean: } 127.44 \\
\text { median: } 66\end{array}$ \\
\hline Small Multi-family (2-4 units) & $\begin{array}{l}\text { obs: } 814 \\
\text { min: } 0 \\
\text { max: } 560 \\
\text { mean: } 7.25 \\
\text { median: } 0\end{array}$ & $\begin{array}{l}\text { obs: } 814 \\
\text { min: } 0 \\
\text { max: } 579 \\
\text { mean: } 6.08 \\
\text { median: } 0\end{array}$ & $\begin{array}{l}\text { obs: } 814 \\
\text { min: } 0 \\
\text { max: } 180 \\
\text { mean: } 1.58 \\
\text { median: } 0\end{array}$ & $\begin{array}{l}\text { obs: } 814 \\
\text { min: } 0 \\
\text { max: } 694 \\
\text { mean: } 14.91 \\
\text { median: } 0\end{array}$ \\
\hline $\begin{array}{l}\text { Multi-family ( } 5 \text { or More Units per } \\
\text { Building) }\end{array}$ & $\begin{array}{l}\text { obs: } 814 \\
\text { min: } 0 \\
\text { max: } 673 \\
\text { mean: } 13.55 \\
\text { median: } 0\end{array}$ & $\begin{array}{l}\text { obs: } 814 \\
\text { min: } 0 \\
\text { max: } 2275 \\
\text { mean: } 17.58 \\
\text { median: } 0\end{array}$ & $\begin{array}{l}\text { obs: } 814 \\
\text { min: } 0 \\
\text { max: } 575 \\
\text { mean: } 2.59 \\
\text { median: } 0 \\
\end{array}$ & $\begin{array}{l}\text { obs: } 814 \\
\text { min: } 0 \\
\text { max: } 2275 \\
\text { mean: } 33.72 \\
\text { median: } 0\end{array}$ \\
\hline Mobile Home & $\begin{array}{l}\text { obs: } 814 \\
\text { min: } 0 \\
\text { max: } 220 \\
\text { mean: } 18.40 \\
\text { median: } 11\end{array}$ & $\begin{array}{l}\text { obs: } 814 \\
\text { min: } 0 \\
\text { max: } 545 \\
\text { mean: } 8.84 \\
\text { median: } 0\end{array}$ & $\begin{array}{l}\text { obs: } 814 \\
\text { min: } 0 \\
\text { max: } 521 \\
\text { mean: } 3.95 \\
\text { median: } 0\end{array}$ & $\begin{array}{l}\text { obs: } 814 \\
\text { min: } 0 \\
\text { max: } 567 \\
\text { mean: } 31.19 \\
\text { median: } 17\end{array}$ \\
\hline Shelter & $\begin{array}{l}\text { obs: } 814 \\
\text { min: } 0 \\
\text { max: } 136 \\
\text { mean: } .28 \\
\text { median: } 0\end{array}$ & $\begin{array}{l}\text { obs: } 814 \\
\text { min: } 0 \\
\text { max: } 18 \\
\text { mean: } .74 \\
\text { median: } 0\end{array}$ & $\begin{array}{l}\text { obs: } 814 \\
\text { min: } 0 \\
\text { max: } 0 \\
\text { mean: } 0 \\
\text { median: } 0\end{array}$ & $\begin{array}{l}\text { obs: } 814 \\
\text { min: } 0 \\
\text { max: } 136 \\
\text { mean: } 4.91 \\
\text { median: } 0\end{array}$ \\
\hline TOTAL UNITS & $\begin{array}{l}\text { obs: } 814 \\
\text { min: } 0 \\
\text { max: } 2255 \\
\text { mean: } 105.60 \\
\text { median: } 70\end{array}$ & $\begin{array}{l}\text { obs: } 814 \\
\text { min: } 0 \\
\text { max: } 4642 \\
\text { mean: } 78.23 \\
\text { median: } 0\end{array}$ & $\begin{array}{l}\text { obs: } 814 \\
\text { min: } 0 \\
\text { max: } 1723 \\
\text { mean: } 23.75 \\
\text { median: } 0\end{array}$ & $\begin{array}{l}\text { obs: } 814 \\
\text { min: } 0 \\
\text { max: } 5342 \\
\text { mean: } 207.58 \\
\text { median: } 106\end{array}$ \\
\hline \multicolumn{5}{|c|}{$\begin{array}{l}\text { These are dwelling units that your agency weatherized and reported to the State as "DOE Units" } \\
2 \text { These are dwelling units that your agency weatherized but did not report as "DOE Units" } \\
{ }^{3} \text { Comprehensive weatherization units are those for which an audit or priority list was used that addressed a large } \\
\text { proportion of potential energy-saving measures. } \\
{ }^{4} \text { Non-comprehensive weatherization units are those for which a limited set of measures was considered (e.g., baseload } \\
\text { electric measures only; low cost/no cost measures only), reflecting the needs and priorities of the funding entity. }\end{array}$} \\
\hline
\end{tabular}


23. For each of the non-DOE sources from which your agency received weatherization funding in Program Year 2008, was any of the money spent in ways not allowed under DOE rules? If so, enter responses in the appropriate rows of Column A. If your agency did spend non-DOE money in ways not allowed under DOE rules, how did those expenditures differ from expenditures made under DOE rules? Enter responses in the appropriate rows of Column B If your agency received no funding from a particular source in Program Year 2008, please leave that row blank.

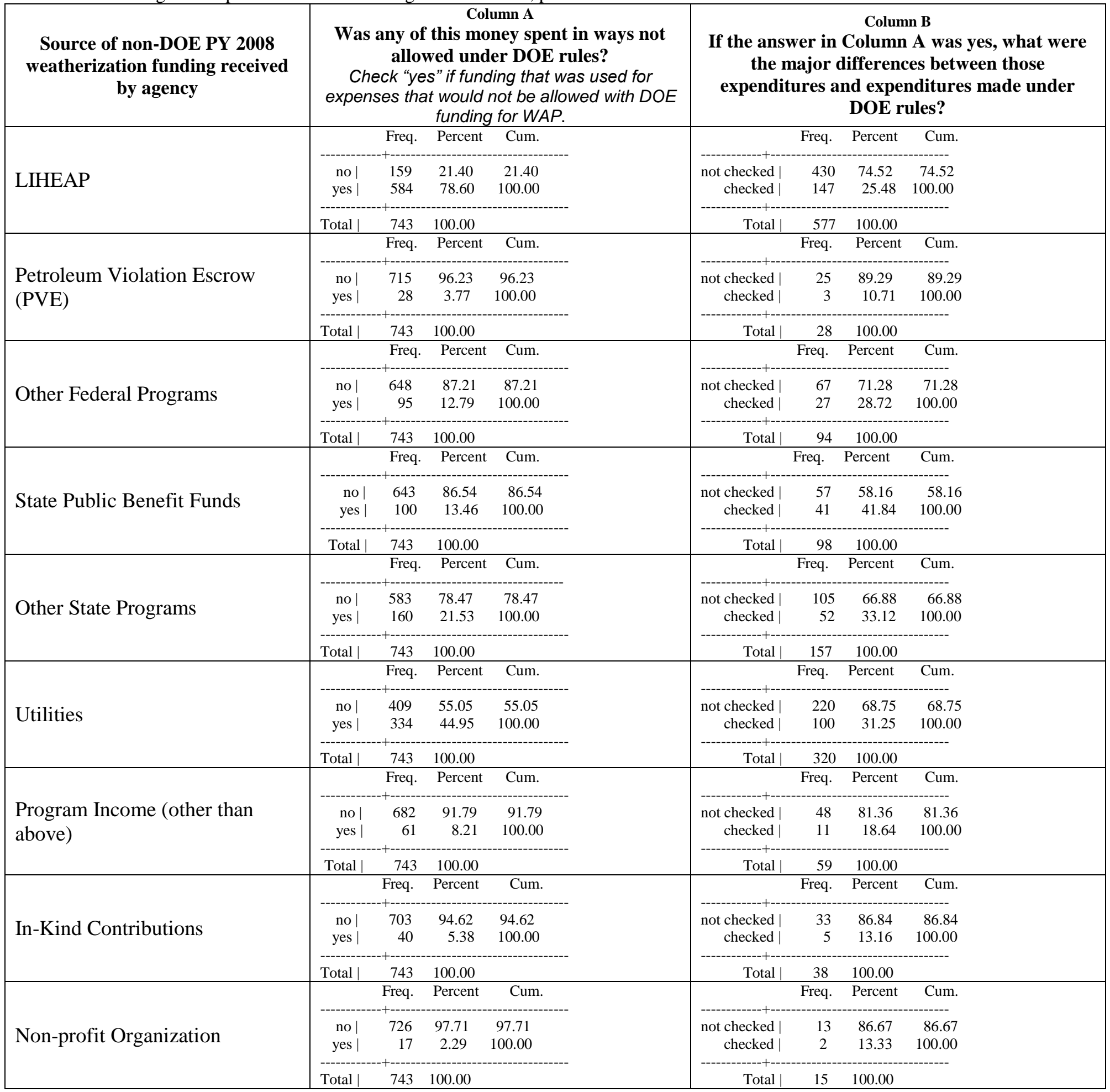




\begin{tabular}{|c|c|c|c|c|c|c|c|c|}
\hline \multirow{3}{*}{ Other } & \multicolumn{4}{|c|}{$\begin{array}{lll}\text { Freq. } & \text { Percent } & \text { Cum. } \\
& & \\
& \end{array}$} & \multicolumn{4}{|c|}{$\begin{array}{lll}\text { Freq. } & \text { Percent } & \text { Cum. }\end{array}$} \\
\hline & $\begin{array}{r}\text { no } \\
\text { yes }\end{array}$ & $\begin{array}{r}657 \\
86\end{array}$ & $\begin{array}{r}88.43 \\
11.57\end{array}$ & $\begin{array}{r}88.43 \\
100.00\end{array}$ & $\begin{array}{r}\text { not checked } \\
\text { checked }\end{array}$ & $\begin{array}{l}35 \\
13\end{array}$ & $\begin{array}{l}72.92 \\
27.08\end{array}$ & $\begin{array}{r}72.92 \\
100.00\end{array}$ \\
\hline & Total & 743 & 100.00 & & Total & 48 & 100.00 & \\
\hline
\end{tabular}


24. Please indicate the amount of funding from each source that was spent by your agency in Program Year 2008 on the different functions or applications shown below. The amount that you list in the right-most cell at the very bottom of the table should equal the total amount of weatherization funding from all sources that your agency received in Program Year 2008.

\begin{tabular}{|c|c|c|c|c|c|c|c|}
\hline \multirow[b]{3}{*}{$\begin{array}{c}\text { Source of PY } 2008 \\
\text { Weatherization } \\
\text { Funding Received by } \\
\text { Agency }\end{array}$} & \multirow[b]{3}{*}{$\begin{array}{l}\text { Amount Spent on } \\
\text { Program Manage- } \\
\text { Ment }^{1}\end{array}$} & \multirow[b]{3}{*}{$\begin{array}{c}\text { Amount Spent } \\
\text { on } \\
\text { T\&TA }\end{array}$} & \multicolumn{4}{|c|}{ PY 2008 Funds Supporting Weatherization of Units (\$) } & \multirow[b]{3}{*}{$\begin{array}{c}\text { TOTAL Amount } \\
\text { Spent }\end{array}$} \\
\hline & & & \multicolumn{2}{|c|}{ Amount Spent on DOE Units ${ }^{2}$} & \multicolumn{2}{|c|}{ Amount Spent on Non-DOE Units ${ }^{3}$} & \\
\hline & & & $\begin{array}{c}\text { Expenditures for } \\
\text { Health and Safety, } \\
\text { Repairs, and Other } \\
\text { Non Cost-Effective } \\
\text { Measures }^{4}\end{array}$ & $\begin{array}{l}\text { All Expenditures } \\
\text { Defined as Allowable } \\
\text { Costs for Purpose of } \\
\text { Calculating Average } \\
\text { Cost per Unit }{ }^{5}\end{array}$ & $\begin{array}{l}\text { Expenditures for } \\
\text { Health and } \\
\text { Safety, Repairs, } \\
\text { and Other Non } \\
\text { Cost-Effective } \\
\text { Measures }\end{array}$ & $\begin{array}{l}\text { All Expenditures } \\
\text { Defined as Allowable } \\
\text { Costs for Purpose of } \\
\text { Calculating Average } \\
\text { Cost per Unit }\end{array}$ & \\
\hline $\mathrm{DOE}$ & $\begin{array}{l}\text { Obs: } 795 \\
\text { Min: } 0 \\
\text { Max:1398125.4 } \\
\text { Mean:48748.89 } \\
\text { Median: } 24997\end{array}$ & $\begin{array}{l}\text { Obs: } 795 \\
\text { Min: } 0 \\
\text { Max: } 175145.05 \\
\text { Mean:6710.92 } \\
\text { Median: } 3761\end{array}$ & $\begin{array}{l}\text { Obs: } 795 \\
\text { Min: } 0 \\
\text { Max: } 1181937 \\
\text { Mean: } 24112.87 \\
\text { Median: } 11549\end{array}$ & $\begin{array}{l}\text { Obs: } 795 \\
\text { Min: } 0 \\
\text { Max: } 4114251 \\
\text { Mean:221764.73 } \\
\text { Median: } 145971\end{array}$ & N/A & N/A & $\begin{array}{l}\text { Obs: } 795 \\
\text { Min: } 0 \\
\text { Max:5325732.5 } \\
\text { Mean:301337.41 } \\
\text { Median:210713 }\end{array}$ \\
\hline LIHEAP & $\begin{array}{l}\text { Obs: } 798 \\
\text { Min: } 0 \\
\text { Max: } 1033957 \\
\text { Mean:30096.08 } \\
\text { Median: } 8218.5\end{array}$ & $\begin{array}{l}\text { Obs: } 798 \\
\text { Min: } 0 \\
\text { Max: } 79065 \\
\text { Mean: } 1798.37 \\
\text { Median: } 0\end{array}$ & $\begin{array}{l}\text { Obs: } 798 \\
\text { Min: } 0 \\
\text { Max: } 2351704 \\
\text { Mean: } 17770.98 \\
\text { Median: } 0\end{array}$ & $\begin{array}{l}\text { Obs: } 798 \\
\text { Min: } 0 \\
\text { Max: } 4887255 \\
\text { Mean: } 82167.45 \\
\text { Median: } 0\end{array}$ & $\begin{array}{l}\text { Obs: } 798 \\
\text { Min: } 0 \\
\text { Max: } 2938369 \\
\text { Mean: } 22660.55 \\
\text { Median: } 0\end{array}$ & $\begin{array}{l}\text { Obs: } 798 \\
\text { Min: } 0 \\
\text { Max: } 4285918 \\
\text { Mean: } 102440.52 \\
\text { Median: } 0\end{array}$ & $\begin{array}{l}\text { Obs: } 798 \\
\text { Min: } 0 \\
\text { Max: } 5320161 \\
\text { Mean: } 257646.92 \\
\text { Median: } 110028.5\end{array}$ \\
\hline $\begin{array}{l}\text { Petroleum } \\
\text { Violation } \\
\text { Escrow (PVE) }\end{array}$ & $\begin{array}{l}\text { Obs: } 798 \\
\text { Min: } 0 \\
\text { Max: } 58760 \\
\text { Mean: } 176.65 \\
\text { Median: } 0 \\
\end{array}$ & $\begin{array}{l}\text { Obs: } 798 \\
\text { Min: } 0 \\
\text { Max: } 2195 \\
\text { Mean: } 4.26 \\
\text { Median: } 0 \\
\end{array}$ & $\begin{array}{l}\text { Obs: } 798 \\
\text { Min: } 0 \\
\text { Max: } 42056 \\
\text { Mean: } 65.66 \\
\text { Median: } 0\end{array}$ & $\begin{array}{l}\text { Obs: } 798 \\
\text { Min: } 0 \\
\text { Max: } 58948 \\
\text { Mean: } 194.51 \\
\text { Median: } 0\end{array}$ & $\begin{array}{l}\text { Obs: } 798 \\
\text { Min: } 0 \\
\text { Max: } 128117.29 \\
\text { Mean: } 361.32 \\
\text { Median: } 0\end{array}$ & $\begin{array}{l}\text { Obs: } 798 \\
\text { Min: } 0 \\
\text { Max: } 226301.2 \\
\text { Mean: } 884.55 \\
\text { Median: } 0\end{array}$ & $\begin{array}{l}\text { Obs: } 798 \\
\text { Min: } 0 \\
\text { Max: } 227264.58 \\
\text { Mean: } 1686.95 \\
\text { Median: } 0\end{array}$ \\
\hline $\begin{array}{l}\text { Other Federal } \\
\text { Programs }\end{array}$ & $\begin{array}{l}\text { Obs: } 798 \\
\text { Min: } 0 \\
\text { Max: } 2088529 \\
\text { Mean: } 6204.53 \\
\text { Median: } 0\end{array}$ & $\begin{array}{l}\text { Obs: } 798 \\
\text { Min: } 0 \\
\text { Max: } 18283 \\
\text { Mean: } 119.25 \\
\text { Median: } 0\end{array}$ & $\begin{array}{l}\text { Obs: } 798 \\
\text { Min: } 0 \\
\text { Max: } 385470 \\
\text { Mean: } 2034.68 \\
\text { Median: } 0\end{array}$ & $\begin{array}{l}\text { Obs: } 798 \\
\text { Min: } 0 \\
\text { Max: } 890114 \\
\text { Mean: } 8595.81 \\
\text { Median: } 0\end{array}$ & $\begin{array}{l}\text { Obs: } 798 \\
\text { Min: } 0 \\
\text { Max: } 760255 \\
\text { Mean: } 3603.22 \\
\text { Median: } 0\end{array}$ & $\begin{array}{l}\text { Obs: } 798 \\
\text { Min: } 0 \\
\text { Max: } 6001593.5 \\
\text { Mean: } 18355.93 \\
\text { Median: } 0\end{array}$ & $\begin{array}{l}\text { Obs: } 798 \\
\text { Min: } 0 \\
\text { Max: } 8090122.5 \\
\text { Mean: } 38913.41 \\
\text { Median: } 0\end{array}$ \\
\hline $\begin{array}{l}\text { State Public } \\
\text { Benefit Funds }\end{array}$ & $\begin{array}{l}\text { Obs: } 798 \\
\text { Min: } 0 \\
\text { Max:2018119.8 } \\
\text { Mean: } 14078.30 \\
\text { Median: } 0\end{array}$ & $\begin{array}{l}\text { Obs: } 798 \\
\text { Min: } 0 \\
\text { Max: } 35762 \\
\text { Mean: } 307.91 \\
\text { Median: } 0\end{array}$ & $\begin{array}{l}\text { Obs: } 798 \\
\text { Min: } 0 \\
\text { Max: } 441853 \\
\text { Mean: } 2796.90 \\
\text { Median: } 0 \\
\end{array}$ & $\begin{array}{l}\text { Obs: } 798 \\
\text { Min: } 0 \\
\text { Max: } 1736106 \\
\text { Mean: } 22064.55 \\
\text { Median: } 0\end{array}$ & $\begin{array}{l}\text { Obs: } 798 \\
\text { Min: } 0 \\
\text { Max: } 698755.5 \\
\text { Mean: } 4980.68 \\
\text { Median: } 0\end{array}$ & $\begin{array}{l}\text { Obs: } 798 \\
\text { Min: } 0 \\
\text { Max: } 3226138.8 \\
\text { Mean: } 31915.09 \\
\text { Median: } 0\end{array}$ & $\begin{array}{l}\text { Obs: } 798 \\
\text { Min: } 0 \\
\text { Max: } 5961014 \\
\text { Mean: } 76143.43 \\
\text { Median: } 0\end{array}$ \\
\hline $\begin{array}{l}\text { Other State } \\
\text { Programs }\end{array}$ & $\begin{array}{l}\text { Obs: } 798 \\
\text { Min: }-16804 \\
\text { Max:1075894.4 } \\
\text { Mean: } 7117.97 \\
\text { Median: } 0\end{array}$ & $\begin{array}{l}\text { Obs: } 798 \\
\text { Min: }-3567 \\
\text { Max: } 102551 \\
\text { Mean: } 368.29 \\
\text { Median: } 0\end{array}$ & $\begin{array}{l}\text { Obs: } 798 \\
\text { Min: } 0 \\
\text { Max: } 468094 \\
\text { Mean: } 3133.10 \\
\text { Median: } 0\end{array}$ & $\begin{array}{l}\text { Obs: } 798 \\
\text { Min: } 0 \\
\text { Max: } 2583139 \\
\text { Mean: } 17923.38 \\
\text { Median: } 0\end{array}$ & $\begin{array}{l}\text { Obs: } 798 \\
\text { Min: } 0 \\
\text { Max: } 109046 \\
\text { Mean: } 1670.62 \\
\text { Median: } 0\end{array}$ & $\begin{array}{l}\text { Obs: } 798 \\
\text { Min: } 0 \\
\text { Max: } 3016580 \\
\text { Mean: } 24633.22 \\
\text { Median: } 0\end{array}$ & $\begin{array}{l}\text { Obs: } 798 \\
\text { Min: } 0 \\
\text { Max: } 4269773 \\
\text { Mean: } 57411.06 \\
\text { Median: } 0\end{array}$ \\
\hline Utilities & $\begin{array}{l}\text { Obs: } 798 \\
\text { Min: } 0 \\
\text { Max: } 1771598 \\
\text { Mean: } 11789.95 \\
\text { Median: } 0\end{array}$ & $\begin{array}{l}\text { Obs: } 798 \\
\text { Min: } 0 \\
\text { Max: } 35000 \\
\text { Mean: } 269.59 \\
\text { Median: } 0\end{array}$ & $\begin{array}{l}\text { Obs: } 798 \\
\text { Min: } 0 \\
\text { Max: } 295202 \\
\text { Mean: } 3568.63 \\
\text { Median: } 0 \\
\end{array}$ & $\begin{array}{l}\text { Obs: } 798 \\
\text { Min: } 0 \\
\text { Max: } 1725312 \\
\text { Mean: } 33452.90 \\
\text { Median: } 0\end{array}$ & $\begin{array}{l}\text { Obs: } 798 \\
\text { Min: } 0 \\
\text { Max: } 336796 \\
\text { Mean: } 2596.29 \\
\text { Median: } 0 \\
\end{array}$ & $\begin{array}{l}\text { Obs: } 798 \\
\text { Min: } 0 \\
\text { Max: } 3635054 \\
\text { Mean: } 44243.38 \\
\text { Median: } 0\end{array}$ & $\begin{array}{l}\text { Obs: } 798 \\
\text { Min: } 0 \\
\text { Max: } 5435354 \\
\text { Mean: } 95920.74 \\
\text { Median: } 0\end{array}$ \\
\hline $\begin{array}{l}\text { Program } \\
\text { Income (other } \\
\text { than above) }\end{array}$ & $\begin{array}{l}\text { Obs: } 798 \\
\text { Min: } 0 \\
\text { Max: } 65000 \\
\text { Mean: } 383.82 \\
\text { Median: } 0\end{array}$ & $\begin{array}{l}\text { Obs: } 798 \\
\text { Min: } 0 \\
\text { Max: } 17920 \\
\text { Mean: } 56.21 \\
\text { Median: } 0\end{array}$ & $\begin{array}{l}\text { Obs: } 798 \\
\text { Min: } 0 \\
\text { Max: } 305255 \\
\text { Mean: } 603.15 \\
\text { Median: } 0\end{array}$ & $\begin{array}{l}\text { Obs: } 798 \\
\text { Min: } 0 \\
\text { Max: } 295978 \\
\text { Mean: } 1382.23 \\
\text { Median: } 0\end{array}$ & $\begin{array}{l}\text { Obs: } 798 \\
\text { Min: } 0 \\
\text { Max: } 28000 \\
\text { Mean: } 74.03 \\
\text { Median: } 0 \\
\end{array}$ & $\begin{array}{l}\text { Obs: } 798 \\
\text { Min: } 0 \\
\text { Max: } 105822 \\
\text { Mean: } 933.41 \\
\text { Median: } 0\end{array}$ & $\begin{array}{l}\text { Obs: } 798 \\
\text { Min: } 0 \\
\text { Max: } 340179 \\
\text { Mean: } 3432.86 \\
\text { Median: } 0\end{array}$ \\
\hline $\begin{array}{l}\text { In-Kind } \\
\text { Contributions }\end{array}$ & $\begin{array}{l}\text { Obs: } 798 \\
\text { Min: } 0 \\
\text { Max: } 181000 \\
\text { Mean: } 363.16 \\
\text { Median: } 0 \\
\end{array}$ & $\begin{array}{l}\text { Obs: } 798 \\
\text { Min: } 0 \\
\text { Max: } 0 \\
\text { Mean: } 0 \\
\text { Median: } 0\end{array}$ & $\begin{array}{l}\text { Obs: } 798 \\
\text { Min: } 0 \\
\text { Max: } 189838.61 \\
\text { Mean: } 490.01 \\
\text { Median: } 0\end{array}$ & $\begin{array}{l}\text { Obs: } 798 \\
\text { Min: } 0 \\
\text { Max: } 567073.38 \\
\text { Mean: } 1772.72 \\
\text { Median: } 0\end{array}$ & $\begin{array}{l}\text { Obs: } 798 \\
\text { Min: } 0 \\
\text { Max: } 10000 \\
\text { Mean: } 12.53 \\
\text { Median: } 0 \\
\end{array}$ & $\begin{array}{l}\text { Obs: } 798 \\
\text { Min: } 0 \\
\text { Max: } 30000 \\
\text { Mean: } 66.28 \\
\text { Median: } 0 \\
\end{array}$ & $\begin{array}{l}\text { Obs: } 798 \\
\text { Min: } 0 \\
\text { Max: } 756912 \\
\text { Mean: } 2704.70 \\
\text { Median: } 0 \\
\end{array}$ \\
\hline $\begin{array}{l}\text { Non-Profit } \\
\text { Organizations }\end{array}$ & $\begin{array}{l}\text { Obs: } 798 \\
\text { Min: } 0 \\
\text { Max: } 69594 \\
\text { Mean: } 200.45 \\
\text { Median: } 0\end{array}$ & $\begin{array}{l}\text { Obs: } 798 \\
\text { Min: } 0 \\
\text { Max: } 879.84 \\
\text { Mean: } 1.10 \\
\text { Median: } 0 \\
\end{array}$ & $\begin{array}{l}\text { Obs: } 798 \\
\text { Min: } 0 \\
\text { Max: } 71298 \\
\text { Mean: } 141.81 \\
\text { Median: } 0\end{array}$ & $\begin{array}{l}\text { Obs: } 798 \\
\text { Min: } 0 \\
\text { Max: } 953480 \\
\text { Mean: } 1608.95 \\
\text { Median: } 0\end{array}$ & $\begin{array}{l}\text { Obs: } 798 \\
\text { Min: } 0 \\
\text { Max: } 76475 \\
\text { Mean: } 113.93 \\
\text { Median: } 0 \\
\end{array}$ & $\begin{array}{l}\text { Obs: } 798 \\
\text { Min: } 0 \\
\text { Max: } 159600 \\
\text { Mean: } 579.12 \\
\text { Median: } 0\end{array}$ & $\begin{array}{l}\text { Obs: } 798 \\
\text { Min: } 0 \\
\text { Max: } 1023074 \\
\text { Mean: } 2645.37 \\
\text { Median: } 0\end{array}$ \\
\hline $\begin{array}{l}\text { TOTAL } \\
\text { FUNDS }\end{array}$ & $\begin{array}{l}\text { Obs: } \\
\text { Min: } 0 \\
\text { Max: } 4562548.5 \\
\text { Mean:119411.53 } \\
\text { Median: } 53919\end{array}$ & $\begin{array}{l}\text { Obs: } \\
\text { Min: } 0 \\
\text { Max:175145.05 } \\
\text { Mean: } 9618.64 \\
\text { Median: } 5298\end{array}$ & $\begin{array}{l}\text { Obs: } \\
\text { Min: 0 } \\
\text { Max: } 3533641 \\
\text { Mean: } 54679.38 \\
\text { Median: } 16851\end{array}$ & $\begin{array}{l}\text { Obs: } \\
\text { Min: } 0 \\
\text { Max: } 5088971 \\
\text { Mean:390527.44 } \\
\text { Median: } 227380\end{array}$ & $\begin{array}{l}\text { Obs: } \\
\text { Min: } 0 \\
\text { Max: } 2938369 \\
\text { Mean: } 36395.05 \\
\text { Median: } 0\end{array}$ & $\begin{array}{l}\text { Obs: } \\
\text { Min: } 0 \\
\text { Max: } 9018173 \\
\text { Mean:229381.22 } \\
\text { Median: 0 }\end{array}$ & $\begin{array}{l}\text { Obs: } \\
\text { Min: } 0 \\
\text { Max: } 17508330 \\
\text { Mean:839679.78 } \\
\text { Median: } 510000\end{array}$ \\
\hline $\begin{array}{l}{ }^{1} \text { Program Manageme } \\
\text { activities. }{ }^{2} \text { These are } \\
{ }^{3} \text { These are dwelling } \\
{ }^{4} \text { Non cost-effective } \\
{ }^{5} \text { Under DOE regulat } \\
\text { materials, crews, equ } \\
\text { supervisors; storage }\end{array}$ & $\begin{array}{l}\text { that your agency } \\
\text {, allowable costs is }\end{array}$ & Savings to Inve & $\begin{array}{l}\text { t Ratio (SIR) of less } \\
\text { t }\end{array}$ & $\begin{array}{l}\text { "DOE Units" } \\
1.0 \text {. } \\
\text { health and safety); la } \\
\text { enance of tools and eq }\end{array}$ & ent; cost of purc & g vehicles; employm & $\begin{array}{l}\text { weatherization } \\
\text { f on-site }\end{array}$ \\
\hline
\end{tabular}


25. Did your agency classify its expenditures for client intake, audits, and post-weatherization inspections as program management costs or as allowable costs used in the calculation of average cost per unit in Program Year 2008? Please indicate your answer for each type of expenditure by checking the appropriate cell in the table below.

\begin{tabular}{|c|c|c|c|c|}
\hline Type of Expenditure & $\begin{array}{c}\text { Classified as Program Management } \\
\text { Costs? }\end{array}$ & $\begin{array}{r}\text { Class } \\
\text { Purpos }\end{array}$ & $\begin{array}{r}\text { fied as Al } \\
\text { of Calcu } \\
\text { per }\end{array}$ & $\begin{array}{l}\text { owable Costs for } \\
\text { ating Average Cost } \\
\text { Unit? }\end{array}$ \\
\hline \multirow{4}{*}{$\begin{array}{l}\text { Expenditures for Client } \\
\text { Intake }\end{array}$} & & Freq. & Percent & Cum. \\
\hline & Classified as Program Management Costs & 303 & 43.98 & 43.98 \\
\hline & Classified as Allowable Costs for Purpose & 386 & 56.02 & 100.00 \\
\hline & Total & 689 & 100.00 & \\
\hline \multirow{4}{*}{ Expenditures for Audits } & & Freq. & Percent & Cum. \\
\hline & Classified as Program Management Costs & 270 & 37.60 & 37.60 \\
\hline & Classified as Allowable Costs for Purpose & 448 & 62.40 & 100.00 \\
\hline & Total & 718 & 100.00 & \\
\hline \multirow{4}{*}{$\begin{array}{l}\text { Expenditures for Post- } \\
\text { Weatherization Quality } \\
\text { Control Inspections }\end{array}$} & & Freq. & Percent & Cum. \\
\hline & Classified as Program Management Costs & 187 & 26.45 & 26.45 \\
\hline & Classified as Allowable Costs for Purpose & 520 & 73.55 & 100.00 \\
\hline & Total & 707 & 100.00 & \\
\hline
\end{tabular}

26. Of the TOTAL amount spent by your agency in Program Year 2008 using funds from all sources (shown at bottom of right-most column in table for Question 24), please give your best estimate of how much was spent on Audits and Inspections. \$

\begin{tabular}{|l|c|}
\hline observations: & 709 \\
\hline missing values: & 105 \\
\hline mean: & 70624.50 \\
\hline standard deviation: & 96081.56 \\
\hline min: & 0 \\
\hline 10th percentile: & 3510 \\
\hline 25th percentile: & 14476.54 \\
\hline median: & 37440 \\
\hline 75th percentile: & 80000 \\
\hline 90th percentile: & 186810 \\
\hline max: & 600000 \\
\hline
\end{tabular}


27. Please divide your agency's Program Year 2008 expenditures on DOE units into in-house expenditures and contractor expenditures, as shown in the following table.

\begin{tabular}{|c|c|c|c|}
\hline Type of Expenditure & $\begin{array}{c}\text { PY } 2008 \\
\text { In-house } \\
\text { Expenditures } \\
\text { on DOE Units } \\
\text { (in \$) }\end{array}$ & $\begin{array}{c}\text { PY } 2008 \\
\text { Contractor } \\
\text { Expenditures } \\
\text { on DOE Units } \\
\text { (in \$) }\end{array}$ & $\begin{array}{c}\text { PY } 2008 \\
\text { Total } \\
\text { Expenditures } \\
\text { on DOE Units } \\
\left(\text { in \$) }{ }^{1}\right.\end{array}$ \\
\hline $\begin{array}{l}\text { Expenditures for Health and Safety, } \\
\text { Repairs, and Other Non Cost- } \\
\text { Effective Measures }\end{array}$ & $\begin{array}{l}\text { obs: } 814 \\
\text { min: } 0 \\
\text { max: } 1899622 \\
\text { mean: } 19163.64 \\
\text { median: } 750.20 \\
\end{array}$ & $\begin{array}{l}\text { obs: } 814 \\
\text { min: } 0 \\
\text { max: } 2473664 \\
\text { mean: } 30547.52 \\
\text { median: } 2948\end{array}$ & $\begin{array}{l}\text { obs: } 814 \\
\text { min: } 0 \\
\text { max: } 4373286 \\
\text { mean: } 49711.16 \\
\text { median: } 15215.5\end{array}$ \\
\hline $\begin{array}{l}\text { All Expenditures Defined as } \\
\text { Allowable Costs for Purpose of } \\
\text { Calculating Average Cost per Unit }\end{array}$ & $\begin{array}{l}\text { obs: } 814 \\
\text { min: } 0 \\
\text { max: } 3111678 \\
\text { mean: } 184821.51 \\
\text { median: } 66660.5 \\
\end{array}$ & $\begin{array}{l}\text { obs: } 814 \\
\text { min: } 0 \\
\text { max: } 3752462 \\
\text { mean: } 136635.26 \\
\text { median: } 20371 \\
\end{array}$ & $\begin{array}{l}\text { obs: } 814 \\
\text { min: } 0 \\
\text { max: } 5325732.5 \\
\text { mean: } 321456.77 \\
\text { median: } 169716 \\
\end{array}$ \\
\hline TOTAL FUNDS & $\begin{array}{l}\text { obs: } 814 \\
\text { min: } 0 \\
\text { max: } 3433798 \\
\text { mean: } 203985.15 \\
\text { median: } 81231.97\end{array}$ & $\begin{array}{l}\text { obs: } 814 \\
\text { min: } 0 \\
\text { max: } 4306182 \\
\text { mean: } 167182.78 \\
\text { median: } 41283.16\end{array}$ & $\begin{array}{l}\text { obs: } 814 \\
\text { min: } 0 \\
\text { max: } 6380606 \\
\text { mean: } 371167.93 \\
\text { median: } 201663.5\end{array}$ \\
\hline \multicolumn{4}{|c|}{ 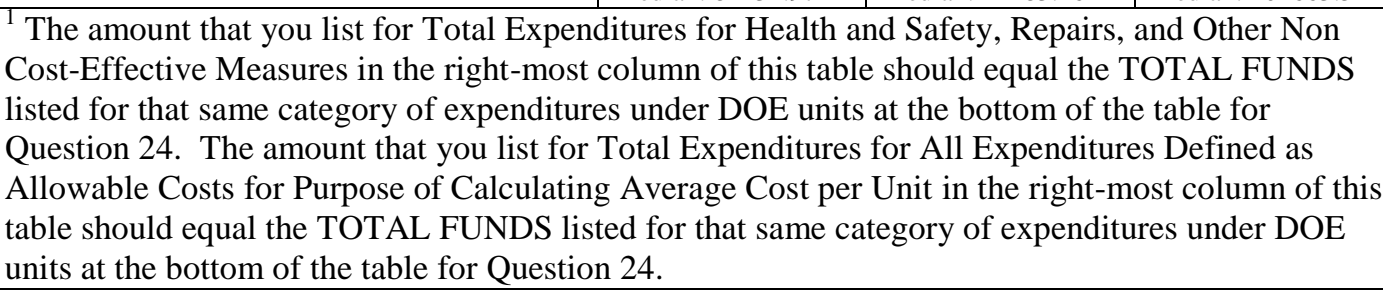 } \\
\hline
\end{tabular}

28. Of your agency's total Program Year 2008 expenditures on DOE units (shown at bottom of right-most column in table for Question 27), please give your best estimate of how much was for labor, how much for materials, and how much for other expenses.

\begin{tabular}{|c|c|}
\hline Type of Expenditure & $\begin{array}{c}\text { PY 2008 } \\
\text { Expenditures on } \\
\text { DOE units (in \$) }\end{array}$ \\
\hline Labor & $\begin{array}{l}\text { obs: } 814 \\
\text { min: } 0 \\
\text { max: } 5544374.5 \\
\text { mean: } 176197.44 \\
\text { median: } 82624.36 \\
\end{array}$ \\
\hline Materials & $\begin{array}{l}\text { obs: } 814 \\
\text { min: } 0 \\
\text { max: } 4367090.5 \\
\text { mean: } 121092.36 \\
\text { median: } 60634.5 \\
\end{array}$ \\
\hline $\begin{array}{l}\text { Other Expenses (e.g., costs for vehicles, } \\
\text { transportation, maintenance, and storage) }\end{array}$ & $\begin{array}{l}\text { obs: } 814 \\
\text { min: } 0 \\
\text { max: } 1072853 \\
\text { mean: } 61359.72 \\
\text { median: } 18798\end{array}$ \\
\hline TOTAL FUNDS & $\begin{array}{l}\text { obs: } 814 \\
\text { min: } 0 \\
\text { max: } 10230263 \\
\text { mean: } 358649.52 \\
\text { median: } 196173.5\end{array}$ \\
\hline
\end{tabular}


29. Of all the DOE units weatherized by your agency in Program Year 2008 (shown at bottom of leftmost column in table for Question 22), how many used each of the following as their main heating fuel (i.e., the fuel providing most of the heat for the dwelling unit) in the winter prior to weatherization?

- Natural gas

\begin{tabular}{|l|c|}
\hline observations: & 729 \\
\hline missing values: & 85 \\
\hline mean: & 6497.87 \\
\hline standard deviation: & 173820.06 \\
\hline min: & 0 \\
\hline 10th percentile: & 1 \\
\hline 25th percentile: & 10 \\
\hline median: & 30 \\
\hline 75th percentile: & 72 \\
\hline 90th percentile: & 140 \\
\hline max: & 4693201 \\
\hline
\end{tabular}

- Fuel oil

\begin{tabular}{|l|c|}
\hline observations: & 729 \\
\hline missing values: & 85 \\
\hline mean: & 15.52 \\
\hline standard deviation: & 37.60 \\
\hline min: & 0 \\
\hline 10th percentile: & 0 \\
\hline 25th percentile: & 0 \\
\hline median: & 1 \\
\hline 75th percentile: & 13 \\
\hline 90th percentile: & 40 \\
\hline max: & 375 \\
\hline
\end{tabular}

\section{- Electricity}

\begin{tabular}{|l|c|}
\hline observations: & 729 \\
\hline missing values: & 85 \\
\hline mean: & 22.05 \\
\hline standard deviation: & 45.33 \\
\hline min: & 0 \\
\hline 10th percentile: & 0 \\
\hline 25th percentile: & 2 \\
\hline median: & 8 \\
\hline 75th percentile: & 24 \\
\hline 90th percentile: & 55 \\
\hline max: & 671 \\
\hline
\end{tabular}


- Propane/LPG

\begin{tabular}{|l|c|}
\hline observations: & 729 \\
\hline missing values: & 85 \\
\hline mean: & 8.84 \\
\hline standard deviation: & 15.31 \\
\hline min: & 0 \\
\hline 10th percentile: & 0 \\
\hline 25th percentile: & 0 \\
\hline median: & 4 \\
\hline 75th percentile: & 11 \\
\hline 90th percentile: & 22 \\
\hline max: & 183 \\
\hline
\end{tabular}

- Kerosene or coal oil

\begin{tabular}{|l|c|}
\hline observations: & 729 \\
\hline missing values: & 85 \\
\hline mean: & 2.01 \\
\hline standard deviation: & 6.80 \\
\hline min: & 0 \\
\hline 10th percentile: & 0 \\
\hline 25th percentile: & 0 \\
\hline median: & 0 \\
\hline 75th percentile: & 0 \\
\hline 90th percentile: & 7 \\
\hline max: & 71 \\
\hline
\end{tabular}

- Wood

\begin{tabular}{|l|c|}
\hline observations: & 729 \\
\hline missing values: & 85 \\
\hline mean: & 1.66 \\
\hline standard deviation: & 4.11 \\
\hline min: & 0 \\
\hline 10th percentile: & 0 \\
\hline 25th percentile: & 0 \\
\hline median: & 0 \\
\hline 75th percentile: & 1 \\
\hline 90th percentile: & 5 \\
\hline max: & 38 \\
\hline
\end{tabular}


- Other (please specify)

\begin{tabular}{|l|c|}
\hline observations: & 729 \\
\hline missing values: & 85 \\
\hline mean: & 3.21 \\
\hline standard deviation: & 46.06 \\
\hline min: & 0 \\
\hline 10th percentile: & 0 \\
\hline 25th percentile: & 0 \\
\hline median: & 0 \\
\hline 75th percentile: & 0 \\
\hline 90th percentile: & 0 \\
\hline max: & 1083 \\
\hline
\end{tabular}

30. Of all the DOE units weatherized by your agency in Program Year 2008 (shown at bottom of left-most column in table for Question 22), how many housed members of the following high-priority client populations (leave blank if do not know)?

\section{- Children (according to your state's definition of that term)}

\begin{tabular}{|l|c|}
\hline observations: & 622 \\
\hline missing values: & 192 \\
\hline mean: & 47.59 \\
\hline standard deviation: & 65.67 \\
\hline min: & 0 \\
\hline 10th percentile: & 4 \\
\hline 25th percentile: & 11 \\
\hline median: & 28 \\
\hline 75th percentile: & 59 \\
\hline 90th percentile: & 107 \\
\hline max: & 932 \\
\hline
\end{tabular}

- Elderly (age 60 and older)

\begin{tabular}{|l|c|}
\hline observations: & 656 \\
\hline missing values: & 158 \\
\hline mean: & 48.67 \\
\hline standard deviation: & 103.77 \\
\hline min: & 0 \\
\hline 10th percentile: & 9 \\
\hline 25th percentile: & 17 \\
\hline median: & 30.5 \\
\hline 75th percentile: & 54 \\
\hline 90th percentile: & 98 \\
\hline max: & 2239 \\
\hline
\end{tabular}


- Disabled

\begin{tabular}{|l|c|}
\hline observations: & 649 \\
\hline missing values: & 165 \\
\hline mean: & 44.43 \\
\hline standard deviation: & 168.49 \\
\hline min: & 0 \\
\hline 10th percentile: & 5 \\
\hline 25th percentile: & 12 \\
\hline median: & 27 \\
\hline 75th percentile: & 50 \\
\hline 90th percentile: & 83 \\
\hline max: & 4192 \\
\hline
\end{tabular}

- Native American

\begin{tabular}{|l|c|}
\hline observations: & 468 \\
\hline missing values: & 346 \\
\hline mean: & 5.82 \\
\hline standard deviation: & 27.42 \\
\hline min: & 0 \\
\hline 10th percentile: & 0 \\
\hline 25th percentile: & 0 \\
\hline median: & 0 \\
\hline 75th percentile: & 3 \\
\hline 90th percentile: & 10 \\
\hline max: & 455 \\
\hline
\end{tabular}

31. Did your state have official definitions of "high energy expenditure" or "high energy burden" in Program Year 2008?

Freq. Percent Cum.

\begin{tabular}{|c|c|c|c|}
\hline no & 352 & 47.00 & 47.00 \\
\hline yes | & 397 & 53.00 & 100.00 \\
\hline otal | & 749 & 100.00 & \\
\hline
\end{tabular}

If answer to Question 31 is "No," skip to Question 33. 
32. Of all the DOE units weatherized by your agency in Program Year 2008 (shown at bottom of leftmost column in table for Question 22), how many met your state's definition of having "high energy expenditures"

\begin{tabular}{|l|c|}
\hline observations: & 198 \\
\hline missing values: & 616 \\
\hline mean: & 49.38 \\
\hline standard deviation: & 62.88 \\
\hline min: & 0 \\
\hline 10th percentile: & 1 \\
\hline 25th percentile: & 9 \\
\hline median: & 29 \\
\hline 75th percentile: & 67 \\
\hline 90th percentile: & 118 \\
\hline max: & 373 \\
\hline
\end{tabular}

and "high energy burden" (leave blank if do not know or if state did not have that definition)?

\begin{tabular}{|l|c|}
\hline observations: & 210 \\
\hline missing values: & 604 \\
\hline mean: & 45.70 \\
\hline standard deviation: & 73.96 \\
\hline min: & 0 \\
\hline 10th percentile: & 0 \\
\hline 25th percentile: & 5 \\
\hline median: & 25 \\
\hline 75th percentile: & 51 \\
\hline 90th percentile: & 110 \\
\hline max: & 606 \\
\hline
\end{tabular}

33. Were there any differences between households weatherized by your agency in Program Year 2008 as DOE units and those weatherized as non-DOE units?

Freq. Percent Cum.

\begin{tabular}{|c|c|c|c|}
\hline no & 646 & 86.25 & 86.25 \\
\hline yes & 103 & 13.75 & 100.00 \\
\hline Total & 749 & 100.00 & \\
\hline
\end{tabular}

34. What were the major differences between the rules and conditions governing your weatherization of DOE and non-DOE units in Program Year 2008? 
35. How many homes were on your wait list for weatherization in Program Year 2008?

\begin{tabular}{|l|c|}
\hline observations: & 554 \\
\hline missing values: & 260 \\
\hline mean: & 689.46 \\
\hline standard deviation: & 1786.06 \\
\hline min: & 0 \\
\hline 10th percentile: & 20 \\
\hline 25th percentile: & 75 \\
\hline median: & 182 \\
\hline 75th percentile: & 402 \\
\hline 90th percentile: & 1500 \\
\hline max: & 19728 \\
\hline
\end{tabular}

36. On average, how long was a home on the wait list before it was weatherized?

\begin{tabular}{|l|c|}
\hline observations: & 563 \\
\hline missing values: & 251 \\
\hline mean: & 325.80 \\
\hline standard deviation: & 410.91 \\
\hline min: & 0 \\
\hline 10th percentile: & 30 \\
\hline 25th percentile: & 90 \\
\hline median: & 180 \\
\hline 75th percentile: & 365 \\
\hline 90th percentile: & 730 \\
\hline max: & 3650 \\
\hline
\end{tabular}

37. Given the rate at which your agency weatherized homes in Program Year 2008, how many years would be needed to weatherize all the homes in your designated area that need to be weatherized?

\begin{tabular}{|l|c|}
\hline observations: & 426 \\
\hline missing values: & 388 \\
\hline mean: & 23.23 \\
\hline standard deviation: & 26.82 \\
\hline min: & 0 \\
\hline 10th percentile: & 2 \\
\hline 25th percentile: & 5 \\
\hline median: & 13.5 \\
\hline 75th percentile: & 30 \\
\hline 90th percentile: & 59 \\
\hline max: & 100 \\
\hline
\end{tabular}



APPENDIX E: SAMPLED AGENCIES DETAILED PROGRAM INFORMATION SURVEY 

OMB Control Number: 1910-5151

\section{Frequency responses are weighted.}

\section{S3: SUBSET OF AGENCIES DETAILED PROGRAM INFORMATION SURVEY}

Thank you for your prompt response to this data request which is part of the national evaluation of the Weatherization Assistance Program. Evaluation results will provide essential feedback to the weatherization community and inform policymakers about the program's effects on clients' energy consumption, cost savings, and non-energy benefits.

This survey collects data that will be used to conduct a detailed process evaluation of the Weatherization Assistance Program at the local level. The data you supply will be used to characterize local agency weatherization activities in Program Year 2008.

All of the information obtained from this survey will be protected and will remain confidential. The data will be analyzed in such a way that the information provided cannot be associated back to your state, your agencies, or the housing units and clients that your state served. Again, please note that the questions refer to Program Year 2008 unless otherwise noted.

Thank you in advance for completing this survey.

Public reporting burden for this collection of information is estimated to average sixteen hours per response, including the time for reviewing instructions, searching existing data sources, gathering and maintaining the data needed, and completing and reviewing the collection of information. Send comments regarding this burden estimate or any other aspect of this collection of information, including suggestions for reducing this burden, to Office of the Chief Information Officer, Records Management Division, IM11, Paperwork Reduction Project (1910-5151), U.S. Department of Energy, 1000 Independence Ave SW, Washington, DC, 20585-1290; and to the Office of Management and Budget (OMB), OIRA, Paperwork Reduction Project (1910-5151), Washington, DC 20503. 


\section{SECTION A: PROGRAM CHARACTERIZATION}

1. Please identify your state.

2. Please identify your local agency.

3. Which of the following best characterizes your agency? Please check the one answer that best applies.

Local Non-Profit Organization

Local Government Agency

County Government Agency

Indian Tribe

Other entity not eligible for CSBG funding

Other (please specify)

Freq. Percent Cum.

\begin{tabular}{|c|c|c|c|}
\hline local non-profit organization | & 305.02 & 87.15 & 87.15 \\
\hline local gov't agency & 14.24 & 4.07 & 91.22 \\
\hline county gov't agency & 16.80 & 4.80 & 96.02 \\
\hline indian tribe & 44 & 0.13 & 96.14 \\
\hline other & 13.50 & 3.86 & 100.00 \\
\hline Total & 350 & 100.00 & \\
\hline
\end{tabular}


4. Please indicate other energy-related, housing, and other programs that cooperated with your agency's weatherization program, by source of funding in Program Year 2008. Please check all that apply.

\begin{tabular}{|c|c|c|c|c|}
\hline Type of Program & Federal Funding & State Funding & Utility Funding & $\begin{array}{c}\text { Other } \\
\text { Funding }\end{array}$ \\
\hline Energy bill paying assistance & $\begin{array}{l}\text { obs: } 247 \\
\text { no: } 70 \\
\text { ves: } 177\end{array}$ & $\begin{array}{l}\text { obs: } 247 \\
\text { no: } 153 \\
\text { ves: } 94\end{array}$ & $\begin{array}{l}\text { obs: } 247 \\
\text { no: } 149 \\
\text { ves: } 98\end{array}$ & $\begin{array}{l}\text { obs: } 247 \\
\text { no: } 205 \\
\text { ves } 42\end{array}$ \\
\hline Fuel delivery in crisis & $\begin{array}{l}\text { obs: } 89 \\
\text { no: } 43 \\
\text { yes: } 46\end{array}$ & $\begin{array}{l}\text { obs: } 89 \\
\text { no: } 61 \\
\text { yes: } 28\end{array}$ & $\begin{array}{l}\text { obs: } 89 \\
\text { no: } 84 \\
\text { yes: } 5\end{array}$ & $\begin{array}{l}\text { obs: } 89 \\
\text { no: } 55 \\
\text { yes: } 34\end{array}$ \\
\hline Housing re-habilitation & $\begin{array}{l}\text { obs: } 145 \\
\text { no: } 42 \\
\text { yes: } 103\end{array}$ & $\begin{array}{l}\text { obs: } 145 \\
\text { no: } 97 \\
\text { yes: } 48\end{array}$ & $\begin{array}{l}\text { obs: } 145 \\
\text { no: } 124 \\
\text { yes: } 21\end{array}$ & $\begin{array}{l}\text { obs: } 145 \\
\text { no: } 115 \\
\text { yes: } 30\end{array}$ \\
\hline Home emergency repairs & $\begin{array}{l}\text { obs: } 180 \\
\text { no: } 51 \\
\text { yes: } 129\end{array}$ & $\begin{array}{l}\text { obs: } 180 \\
\text { no: } 106 \\
\text { yes: } 74\end{array}$ & $\begin{array}{l}\text { obs: } 180 \\
\text { no: } 175 \\
\text { yes: } 5\end{array}$ & $\begin{array}{l}\text { obs: } 180 \\
\text { no: } 150 \\
\text { yes: } 30\end{array}$ \\
\hline $\begin{array}{l}\text { Hardship funds (other than for } \\
\text { energy bill paying) }\end{array}$ & $\begin{array}{l}\text { obs: } 162 \\
\text { no: } 86 \\
\text { yes: } 76\end{array}$ & $\begin{array}{l}\text { obs: } 162 \\
\text { no: } 79 \\
\text { yes: } 83\end{array}$ & $\begin{array}{l}\text { obs: } 162 \\
\text { no: } 146 \\
\text { yes: } 16\end{array}$ & $\begin{array}{l}\text { obs: } 162 \\
\text { no: } 125 \\
\text { yes: } 37\end{array}$ \\
\hline Fair housing & $\begin{array}{l}\text { obs: } 50 \\
\text { no: } 22 \\
\text { yes: } 28\end{array}$ & $\begin{array}{l}\text { obs: } 50 \\
\text { no: } 29 \\
\text { yes: } 21\end{array}$ & $\begin{array}{l}\text { obs: } 50 \\
\text { no: } 50 \\
\text { yes: } 0\end{array}$ & $\begin{array}{l}\text { obs: } 50 \\
\text { no: } 38 \\
\text { yes: } 12\end{array}$ \\
\hline Health and safety & $\begin{array}{l}\text { obs: } 157 \\
\text { no: } 50 \\
\text { yes: } 107\end{array}$ & $\begin{array}{l}\text { obs: } 157 \\
\text { no: } 95 \\
\text { yes: } 62\end{array}$ & $\begin{array}{l}\text { obs: } 157 \\
\text { no: } 115 \\
\text { yes: } 42\end{array}$ & $\begin{array}{l}\text { obs: } 157 \\
\text { no: } 134 \\
\text { yes: } 23\end{array}$ \\
\hline $\begin{array}{l}\text { Energy education (other than client } \\
\text { education delivered by } \\
\text { weatherization program) }\end{array}$ & $\begin{array}{l}\text { obs: } 124 \\
\text { no: } 58 \\
\text { yes: } 66\end{array}$ & $\begin{array}{l}\text { obs: } 124 \\
\text { no: } 88 \\
\text { yes: } 36\end{array}$ & $\begin{array}{l}\text { obs: } 124 \\
\text { no: } 62 \\
\text { yes: } 62\end{array}$ & $\begin{array}{l}\text { obs: } 124 \\
\text { no: } 105 \\
\text { yes: } 19\end{array}$ \\
\hline Home buying education & $\begin{array}{l}\text { obs: } 93 \\
\text { no: } 40 \\
\text { yes: } 53\end{array}$ & $\begin{array}{l}\text { obs: } 93 \\
\text { no: } 52 \\
\text { yes: } 41\end{array}$ & $\begin{array}{l}\text { obs: } 93 \\
\text { no: } 92 \\
\text { yes: } 1\end{array}$ & $\begin{array}{l}\text { obs: } 93 \\
\text { no: } 66 \\
\text { yes: } 27\end{array}$ \\
\hline Rehabilitation loan & $\begin{array}{l}\text { obs: } 86 \\
\text { no: } 35 \\
\text { yes: } 51\end{array}$ & $\begin{array}{l}\text { obs: } 86 \\
\text { no: } 51 \\
\text { yes: } 35\end{array}$ & $\begin{array}{l}\text { obs: } 86 \\
\text { no: } 86 \\
\text { yes: } 0\end{array}$ & $\begin{array}{l}\text { obs: } 86 \\
\text { no: } 70 \\
\text { yes: } 16\end{array}$ \\
\hline Mortgage loan & $\begin{array}{l}\text { obs: } 37 \\
\text { no: } 15 \\
\text { yes: } 22\end{array}$ & $\begin{array}{l}\text { obs: } 37 \\
\text { no: } 22 \\
\text { yes: } 15\end{array}$ & $\begin{array}{l}\text { obs: } 37 \\
\text { no: } 37 \\
\text { yes: } 0\end{array}$ & $\begin{array}{l}\text { obs: } 37 \\
\text { no: } 27 \\
\text { yes: } 10\end{array}$ \\
\hline Emergency food & $\begin{array}{l}\text { obs: } 114 \\
\text { no: } 42 \\
\text { yes: } 72\end{array}$ & $\begin{array}{l}\text { obs: } 114 \\
\text { no: } 76 \\
\text { yes: } 38\end{array}$ & $\begin{array}{l}\text { obs: } 114 \\
\text { no: } 112 \\
\text { yes: } 2\end{array}$ & $\begin{array}{l}\text { obs: } 114 \\
\text { no: } 62 \\
\text { yes: } 52\end{array}$ \\
\hline Emergency safety & $\begin{array}{l}\text { obs: } 40 \\
\text { no: } 16 \\
\text { yes: } 24\end{array}$ & $\begin{array}{l}\text { obs: } 40 \\
\text { no: } 20 \\
\text { yes: } 20\end{array}$ & $\begin{array}{l}\text { obs: } 40 \\
\text { no: } 38 \\
\text { yes: } 2\end{array}$ & $\begin{array}{l}\text { obs: } 40 \\
\text { no: } 31 \\
\text { yes: } 9\end{array}$ \\
\hline Other (please specify) & & & & \\
\hline
\end{tabular}

5. How important were leveraged funds (i.e., funds that support the weatherization program that are not provided by DOE) for your agency's weatherization program in Program Year 2008? Check best answer.

Freq. Percent Cum.

\begin{tabular}{|c|c|c|c|}
\hline not important at all & 38.17 & 11.16 & 11.16 \\
\hline not very important & 32.63 & 9.54 & 20.70 \\
\hline important & 85.87 & 25.11 & 45.81 \\
\hline very important & 185.33 & 54.19 & 100.00 \\
\hline
\end{tabular}

Total | $342 \quad 100.00$ 
6. Did your agency set aside funding to advocate for leveraged resources in Program Year 2008?

$\begin{array}{rrrr} & \text { Freq. } & \text { Percent } & \text { Cum. } \\ \text { no | } & 296.79 & 86.78 & 86.78 \\ \text { yes | } & 45.21 & 13.22 & 100.00 \\ --------- & \end{array}$

7. What organizations worked to acquire leveraged resources in Program Year 2008? Select all that apply.

\begin{tabular}{|c|c|c|c|}
\hline & Freq. & Percent & Cum. \\
\hline not checked | & 199.03 & 58.54 & 58.54 \\
\hline checked & 140.97 & 41.46 & 100.00 \\
\hline Total | & 340 & 100.00 & \\
\hline \multicolumn{4}{|c|}{ Your state's local weatherization } \\
\hline & Freq. & Percent & Cum. \\
\hline not checked | & 195.48 & 57.50 & 57.50 \\
\hline checked | & 144.52 & 42.50 & 100.00 \\
\hline Total | & 340 & 100.00 & \\
\hline \multicolumn{4}{|c|}{ Non-profit organizations funded } \\
\hline & Freq. & Percent & Cum. \\
\hline not checked | & 261.13 & 76.80 & 76.80 \\
\hline checked & 78.87 & 23.20 & 100.00 \\
\hline Total $\mid$ & 340 & 100.00 & \\
\hline \multirow{2}{*}{ _ Other } & & & \\
\hline & Freq. & Percent & Cum. \\
\hline not checked | & 307.42 & 90.42 & 90.42 \\
\hline checked $\mid$ & 32.58 & 9.58 & 100.00 \\
\hline Total | & 340 & 100.00 & \\
\hline \multicolumn{4}{|c|}{ None of the above } \\
\hline & Freq. & Percent & Cum. \\
\hline not checked | & 249.74 & 73.45 & 73.45 \\
\hline checked $\mid$ & 90.26 & 26.55 & 100.00 \\
\hline Total | & 340 & 100.00 & \\
\hline
\end{tabular}


8. How successful would you rate your agency's efforts to acquire leveraged funds in Program Year $2008 ?$

\begin{tabular}{rrrr} 
Freq. & Percent & \multicolumn{1}{c}{ Cum. } \\
\hline not successful at all | & 1.028 & 0.30 & 0.30 \\
not very successful | & 39.00 & 11.40 & 11.70 \\
successful | & 144.55 & 42.27 & 53.97 \\
very successful | & 62.45 & 18.26 & 72.23 \\
state does not seek leveraged funds | & 94.97 & 27.77 & 100.00 \\
---------- & \\
Total | & 342 & 100.00
\end{tabular}

9. What factors limited the success of your agency's efforts to acquire leveraged funding in Program Year 2008 ?

10. Did you modify your agency's weatherization program practices or regulations in the three years prior to Program Year 2008 to facilitate spending and reporting on leveraged resources?

$\begin{array}{rrrr} & \text { Freq. } & \text { Percent } & \text { Cum. } \\ \text { no | } & 223.26 & 64.90 & 64.90 \\ \text { yes | } & 22.17 & 6.44 & 71.35 \\ \text { dk | } & 98.57 & 28.65 & 100.00 \\ -----+------- & \end{array}$


11. Did your agency experience delays or other difficulties in weatherization-related spending for any of these types of non-DOE funds in Program Year 2008?

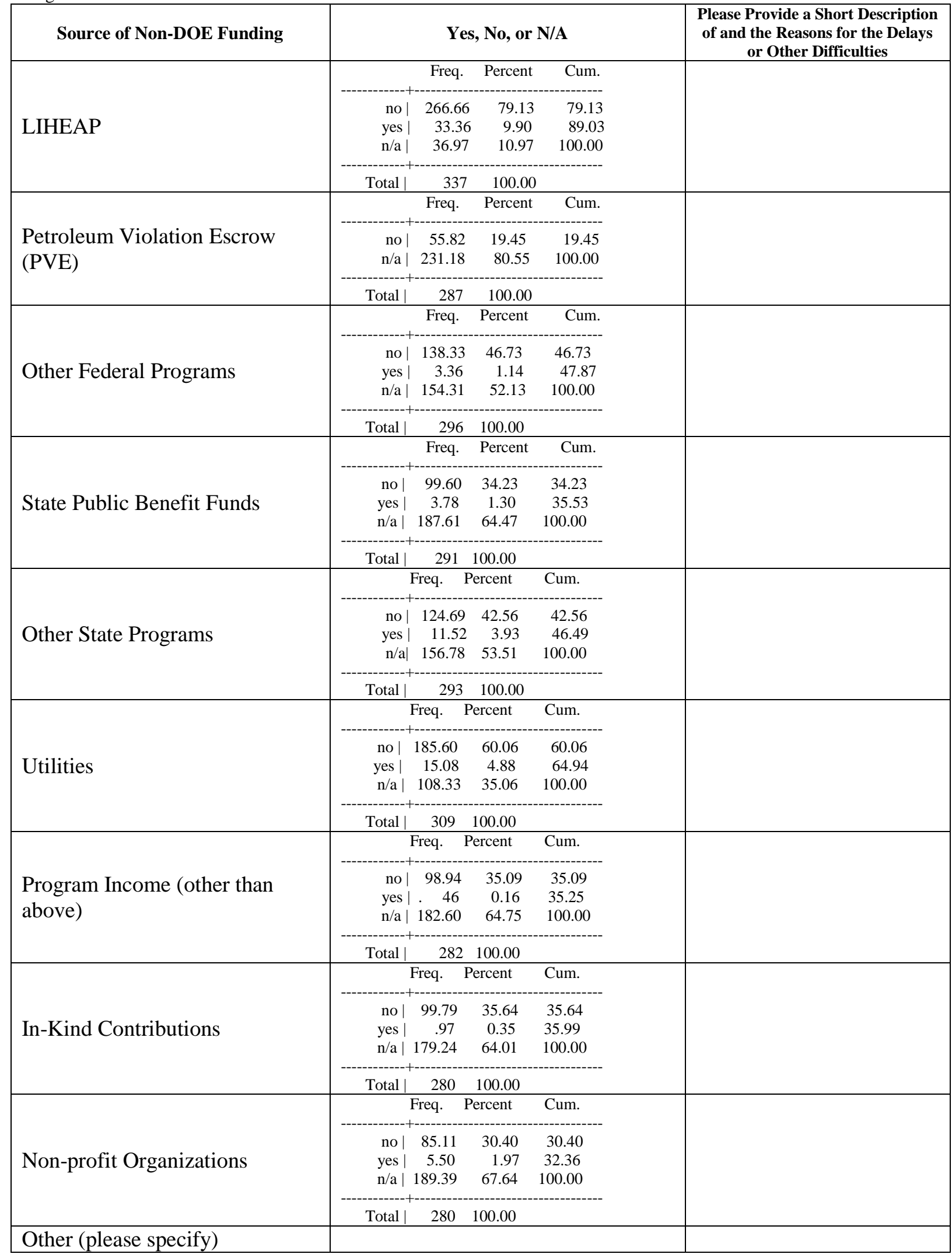


12. Did your agency encounter any of the following problems in spending non-DOE funds in general in Program Year 2008? Check all that apply.

Our agency could not easily increase the number of homes weatherized during the year in order to better spend non-DOE funds

Freq. Percent Cum.

\begin{tabular}{|c|c|c|c|}
\hline $\begin{array}{r}\text { not checked } \\
\text { checked }\end{array}$ & $\begin{array}{r}106.46 \\
36.54\end{array}$ & $\begin{array}{l}74.45 \\
25.55\end{array}$ & $\begin{array}{r}74.45 \\
100.00\end{array}$ \\
\hline Total & 143 & 100.00 & \\
\hline
\end{tabular}

Our agency was required to spend DOE weatherization funds before non-DOE funds were expended

Freq. Percent Cum.

\begin{tabular}{|c|c|c|c|}
\hline $\begin{array}{r}\text { not checked } \\
\text { checked }\end{array}$ & $\begin{array}{r}116.35 \\
26.65\end{array}$ & $\begin{array}{l}81.37 \\
18.63\end{array}$ & $\begin{array}{r}81.37 \\
100.00\end{array}$ \\
\hline Total & 143 & 100.00 & \\
\hline
\end{tabular}

We had insufficient staff to manage the receipt and expenditure of non-DOE funds

\begin{tabular}{rrrr} 
& Freq. & Percent & Cum. \\
not checked | & 112.18 & 78.45 & 78.45 \\
checked | & 30.82 & 21.55 & 100.00 \\
- \hdashline------- & \\
\hline Total | & 143 & 100.00 &
\end{tabular}

We had inadequate accounting systems to manage the receipt and expenditure of non-DOE funds

$\begin{array}{rrrr} & \text { Freq. } & \text { Percent } & \text { Cum. } \\ \text { not checked | } & 131.08 & 91.66 & 91.66 \\ \text { checked | } & 11.92 & 8.34 & 100.00 \\ ----------- & \\ \text { Total | } & 143 & 100.00 & \end{array}$

Guidance received from DOE and/or our state made it difficult to expend non-DOE funds in a timely manner Freq. Percent Cum.

\begin{tabular}{|c|c|c|c|}
\hline not checked & $\begin{array}{r}133.87 \\
9.13\end{array}$ & $\begin{array}{r}93.62 \\
638\end{array}$ & 93.62 \\
\hline & & & \\
\hline Total | & 143 & 100.00 & \\
\hline
\end{tabular}

Other (please specify) 
13. When selecting DOE units to weatherize from the pool of eligible applicants, did your agency give higher priority to specific households based on any of the following characteristics in Program Year 2008? Check all that apply.

- Dwelling unit characteristics

\begin{tabular}{|c|c|c|c|}
\hline & Freq. & Percent & Cum. \\
\hline not checked & 283.23 & 84.04 & 84.04 \\
\hline checked & 53.78 & 15.96 & 100.00 \\
\hline Tot & 337 & 100.00 & \\
\hline
\end{tabular}

- Type of heating system Freq. Percent Cum.

\begin{tabular}{|c|c|c|c|}
\hline not checked & 307.19 & 91.16 & 91.16 \\
\hline checked & 29.81 & 8.84 & 100.00 \\
\hline Total | & 337 & 100.00 & \\
\hline
\end{tabular}

- Fuel type

\begin{tabular}{|c|c|c|c|}
\hline & Freq. & Percent & Cum. \\
\hline not checked & 263.60 & 78.22 & 78.22 \\
\hline checked & 73.40 & 21.78 & 100.00 \\
\hline Total & 337 & 100.00 & \\
\hline
\end{tabular}

- Geographic location

\begin{tabular}{|c|c|c|c|}
\hline & Freq. & Percent & Cum. \\
\hline not checked & 311.40 & 92.40 & 92.40 \\
\hline checked & 25.60 & 7.60 & 100.00 \\
\hline Total | & 337 & 100.00 & \\
\hline
\end{tabular}

- Presence of children

\begin{tabular}{|c|c|c|c|}
\hline & Freq. & Percent & Cum. \\
\hline not checked & 49.75 & 14.76 & 14.76 \\
\hline checked & 287.25 & 85.24 & 100.00 \\
\hline Total & 337 & 100.00 & \\
\hline
\end{tabular}


- Presence of elderly occupants

Freq. Percent Cum.

\begin{tabular}{|c|c|c|c|}
\hline not checked & 23.08 & $\begin{array}{r}6.85 \\
02.15\end{array}$ & 6.85 \\
\hline Total & 337 & 100.00 & \\
\hline
\end{tabular}

- Presence of disabled occupants Freq. Percent Cum.

\begin{tabular}{|c|c|c|c|}
\hline not checked & 34.83 & $\begin{array}{l}10.33 \\
80.67\end{array}$ & 10.33 \\
\hline checked & 302.17 & 89.67 & 100.00 \\
\hline Total | & 337 & 100.00 & \\
\hline
\end{tabular}

- High energy expenditures

\begin{tabular}{|c|c|c|c|}
\hline & Freq. & Percent & Cum. \\
\hline not checked & 171.93 & 51.02 & 51.02 \\
\hline checked & 165.07 & 48.98 & 100.00 \\
\hline
\end{tabular}

- High energy burden

\begin{tabular}{|c|c|c|c|}
\hline & Freq. & Percent & Cum. \\
\hline not checked & 174.22 & 51.70 & 51.70 \\
\hline checked & 162.78 & 48.30 & 100.00 \\
\hline Tota & 337 & 100.00 & \\
\hline
\end{tabular}

- Energy consumption

\begin{tabular}{|c|c|c|c|}
\hline & Freq. & Percent & Cum. \\
\hline not checked & 232.32 & 68.94 & 68.94 \\
\hline checked & 104.68 & 31.06 & 100.00 \\
\hline Total $\mid$ & 337 & 100.00 & \\
\hline
\end{tabular}

- Anticipated cost of weatherization

\begin{tabular}{|c|c|c|c|}
\hline & Freq. & Percent & Cum. \\
\hline not checked & 319.31 & 94.75 & 94.75 \\
\hline checked & 17.69 & 5.25 & 100.00 \\
\hline Total & 337 & 100.00 & \\
\hline
\end{tabular}


- Anticipated savings

\begin{tabular}{|c|c|c|c|}
\hline & Freq. & Percent & Cum. \\
\hline not checked| & 314.84 & 93.42 & 93.42 \\
\hline checked & 22.16 & 6.58 & 100.00 \\
\hline Total & 337 & 100.00 & \\
\hline
\end{tabular}

- Occupant being a renter

\begin{tabular}{|c|c|c|c|}
\hline & Freq. & Percent & Cum. \\
\hline not checked & 329.67 & 97.83 & 97.83 \\
\hline checked & 7.33 & 2.17 & 100.00 \\
\hline Total & 337 & 100.00 & \\
\hline
\end{tabular}

- Landlord or other contributions

Freq. Percent Cum.

\begin{tabular}{|c|c|c|c|}
\hline not checked | & 316.26 & 93.84 & 93.84 \\
\hline checked & 20.74 & 6.16 & 100.00 \\
\hline Total & 337 & 100.00 & \\
\hline
\end{tabular}

- Amount of time on waiting list

\begin{tabular}{|c|c|c|c|}
\hline & Freq. & Percent & Cum. \\
\hline not checked & 165.53 & 49.12 & 49.12 \\
\hline checked & 171.47 & 50.88 & 100.00 \\
\hline Total & 337 & 100.00 & \\
\hline
\end{tabular}

- Referral from, or participation in, another program

\begin{tabular}{|c|c|c|c|}
\hline & Freq. & Percent & Cum. \\
\hline not checked & 232.59 & 69.02 & 69.02 \\
\hline checked & 104.41 & 30.98 & 100.00 \\
\hline Totat & 337 & 100.00 & \\
\hline
\end{tabular}

- Other (please specify) 
14. In Program Year 2008, did your agency set targets and actively solicit participation by dwelling units of the types shown below? Check all that apply.

\begin{tabular}{|c|c|c|c|c|c|c|}
\hline \multirow{4}{*}{$\begin{array}{l}\text { Type of dwelling unit } \\
\text { Single Family Attached and } \\
\text { Detached }\end{array}$} & \multicolumn{2}{|c|}{$\begin{array}{l}\text { Set targets for number of } \\
\text { dwelling units of this type to } \\
\text { weatherize }\end{array}$} & \multicolumn{4}{|c|}{$\begin{array}{c}\text { Actively sought participation } \\
\text { by households residing in this } \\
\text { type of dwelling unit }\end{array}$} \\
\hline & Freq. $\quad$ Percent & Cum. & & Freq. & Percent & Cum. \\
\hline & \begin{tabular}{r|rr} 
not checked & 68.58 & 36.67 \\
checked & 118.42 & 63.33
\end{tabular} & $\begin{array}{r}36.67 \\
100.00\end{array}$ & $\begin{array}{r}\text { not checked } \\
\text { checked }\end{array}$ & $\begin{array}{r}64.40 \\
122.60\end{array}$ & $\begin{array}{l}34.44 \\
65.56\end{array}$ & $\begin{array}{r}34.44 \\
100.00\end{array}$ \\
\hline & $\begin{array}{lll}\text { Total } & 187 \quad 100.00 \\
\end{array}$ & & Total & 187 & 100.00 & \\
\hline \multirow{3}{*}{$\begin{array}{l}\text { Small Multi-family (2-4 } \\
\text { units) }\end{array}$} & Freq. $\quad$ Percent & Cum. & & Freq. & Percent & Cum. \\
\hline & \begin{tabular}{r|rr} 
not checked & 62.60 & 55.40 \\
checked & 50.40 & 44.60
\end{tabular} & $\begin{array}{r}55.40 \\
100.00\end{array}$ & $\begin{array}{r}\text { not checked } \\
\text { checked }\end{array}$ & $\begin{array}{l}26.26 \\
86.74\end{array}$ & $\begin{array}{l}23.23 \\
76.77\end{array}$ & $\begin{array}{r}23.23 \\
100.00\end{array}$ \\
\hline & \begin{tabular}{l|ll} 
Total & $113 \quad 100.00$ \\
\end{tabular} & & Total & 113 & 100.00 & \\
\hline \multirow{3}{*}{$\begin{array}{l}\text { Multifamily ( } 5 \text { or More } \\
\text { Units per Building) }\end{array}$} & Freq. $\quad$ Percent & Cum. & & Freq. & Percent & Cum. \\
\hline & 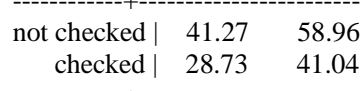 & $\begin{array}{r}58.96 \\
100.00\end{array}$ & $\begin{array}{r}\text { not checked } \\
\text { checked }\end{array}$ & $\begin{array}{l}11.47 \\
58.53\end{array}$ & $\begin{array}{l}16.39 \\
83.61\end{array}$ & $\begin{array}{r}16.39 \\
100.00\end{array}$ \\
\hline & $\begin{array}{lll}\text { Total } & 70 \quad 100.00 \\
\end{array}$ & & Total & 70 & 100.00 & \\
\hline \multirow{3}{*}{ Mobile Home } & Freq. $\quad$ Percent & Cum. & & Freq. & Percent & Cum. \\
\hline & \begin{tabular}{r|r} 
not checked & 58.39 \\
checked & 79.61
\end{tabular} & $\begin{array}{r}42.31 \\
100.00\end{array}$ & $\begin{array}{r}\text { not checked } \\
\text { checked }\end{array}$ & $\begin{array}{l}50.32 \\
87.68\end{array}$ & $\begin{array}{l}36.47 \\
63.53\end{array}$ & $\begin{array}{r}36.47 \\
100.00\end{array}$ \\
\hline & $\begin{array}{lll}\text { Total } & 138 & 100.00 \\
\end{array}$ & & Total | & 138 & 100.00 & \\
\hline \multirow{3}{*}{ Shelter } & Freq. $\quad$ Percent & Cum. & & Freq. & Percent & Cum. \\
\hline & \begin{tabular}{r|r} 
not checked & 12.12 \\
checked & 6.88
\end{tabular} & $\begin{array}{r}63.81 \\
100.00\end{array}$ & $\begin{array}{r}\text { not checked } \\
\text { checked }\end{array}$ & $\begin{array}{r}6.00 \\
13.00\end{array}$ & $\begin{array}{l}31.58 \\
68.42\end{array}$ & $\begin{array}{r}31.58 \\
100.00\end{array}$ \\
\hline & $19 \quad 100.00$ & & Total & 19 & 100.00 & \\
\hline
\end{tabular}

15. Did your state require your agency to provide aggregated data for these four types of data in Program Year 2008? If yes, please indicate how often. Check all that apply.

\begin{tabular}{|l|l|l|l|l|}
\hline & \multicolumn{1}{|c|}{$\begin{array}{c}\text { Household } \\
\text { Data }\end{array}$} & \multicolumn{1}{|c|}{ Audit Data } & \multicolumn{1}{|l|}{$\begin{array}{l}\text { Weatherization } \\
\text { Measures Data }\end{array}$} & Energy Use Data \\
\hline No & $\begin{array}{l}\text { obs: } 333 \\
\text { not checked: } 289 \\
\text { checked: } 44\end{array}$ & $\begin{array}{l}\text { obs: } 298 \\
\text { not checked: } 235 \\
\text { checked: } 63\end{array}$ & $\begin{array}{l}\text { obs: } 312 \\
\text { not checked: } 261 \\
\text { checked: } 51\end{array}$ & $\begin{array}{l}\text { obs: } 265 \\
\text { not checked: } 170 \\
\text { checked: } 95\end{array}$ \\
\hline Yes, annually & $\begin{array}{l}\text { obs: } 333 \\
\text { not checked: } 295 \\
\text { checked: } 28\end{array}$ & $\begin{array}{l}\text { obs: } 298 \\
\text { not checked: } 238 \\
\text { checked: } 60\end{array}$ & $\begin{array}{l}\text { obs: } 312 \\
\text { not checked: } 269 \\
\text { checked: } 43\end{array}$ & $\begin{array}{l}\text { obs: } 265 \\
\text { not checked: } 228 \\
\text { checked: } 37\end{array}$ \\
\hline Yes, quarterly & $\begin{array}{l}\text { obs: } 333 \\
\text { not checked: } 290 \\
\text { checked: } 43\end{array}$ & $\begin{array}{l}\text { obs: } 298 \\
\text { not checked: } 271 \\
\text { checked: } 27\end{array}$ & $\begin{array}{l}\text { obs: } 312 \\
\text { not checked: } 284 \\
\text { checked: } 28\end{array}$ & $\begin{array}{l}\text { obs: } 265 \\
\text { not checked: } 243 \\
\text { checked: } 22\end{array}$ \\
\hline Yes, monthly & $\begin{array}{l}\text { obs: } 333 \\
\text { not checked: } 132 \\
\text { checked: } 201\end{array}$ & $\begin{array}{l}\text { obs: } 298 \\
\text { not checked: } 166 \\
\text { checked: } 132\end{array}$ & $\begin{array}{l}\text { obs: } 312 \\
\text { not checked: } 132 \\
\text { checked: } 180\end{array}$ & $\begin{array}{l}\text { obs: } 265 \\
\text { not checked: } 161 \\
\text { checked: } 104\end{array}$ \\
\hline Yes, other & $\begin{array}{l}\text { obs: } 333 \\
\text { not checked: } 303 \\
\text { checked: } 30\end{array}$ & $\begin{array}{l}\text { obs: } 298 \\
\text { not checked: } 261 \\
\text { checked: } 37\end{array}$ & $\begin{array}{l}\text { obs: } 312 \\
\text { not checked: } 275 \\
\text { checked: } 37\end{array}$ & $\begin{array}{l}\text { obs: } 265 \\
\text { not checked: } 239 \\
\text { checked: } 26\end{array}$ \\
\hline
\end{tabular}


16. Did your state impose any specific requirements on how your agency collected, stored, and used household energy use, weatherization, and/or household data in Program Year 2008?

\begin{tabular}{|c|c|c|c|}
\hline & Freq. & Percent & Cum. \\
\hline nol & 144.49 & 42.00 & 42.00 \\
\hline yes & 199.51 & 58.00 & 100.00 \\
\hline Total & 344 & 100.00 & \\
\hline
\end{tabular}

16a. If Yes, what were the state-imposed requirements?

17. Did your state provide any training to help your agency collect, store and use household energy use, weatherization, and/or household data in Program Year 2008?

Freq. Percent Cum.

\begin{tabular}{|c|c|c|c|}
\hline no & 188.30 & 55.06 & 55.06 \\
\hline yes & 153.70 & 44.94 & 100.00 \\
\hline Total | & 342 & 100.00 & \\
\hline
\end{tabular}

17a. If Yes, what training was provided by your state?

18. How were eligibility and prioritization data about households that applied for weatherization services collected in Program Year 2008? Check all that apply.

Households filled out forms when they apply for weatherization services

Freq. Percent Cum.

\begin{tabular}{|c|c|c|c|}
\hline not checked | & 47.70 & $\begin{array}{l}13.79 \\
8621\end{array}$ & $\begin{array}{r}13.79 \\
10000\end{array}$ \\
\hline checke & 298.30 & 86.21 & 100.00 \\
\hline Total & 346 & 100.00 & \\
\hline
\end{tabular}

Data were provided by LIHEAP

\begin{tabular}{|c|c|c|c|}
\hline & Freq. & Percent & Cum. \\
\hline not checked & 171.37 & 49.53 & 49.53 \\
\hline checked & 174.63 & 50.47 & 100.00 \\
\hline Total | & 346 & 100.00 & \\
\hline
\end{tabular}

The state provided the household data

Freq. Percent Cum.

$\begin{array}{rrr}\text { not checked | } 323.66 & 93.54 & 93.54 \\ \text { checked | } 22.34 & 6.46 & 100.00 \\ ---------- & \\ \text { Total | } 346 & 100.00\end{array}$


Auditors collected the data at the time the home is audited

\begin{tabular}{|c|c|c|c|}
\hline & Freq. & Percent & Cum. \\
\hline not checked & 251.94 & 72.82 & 72.82 \\
\hline checked & 94.06 & 27.18 & 100.00 \\
\hline Total & 346 & 100.00 & \\
\hline
\end{tabular}

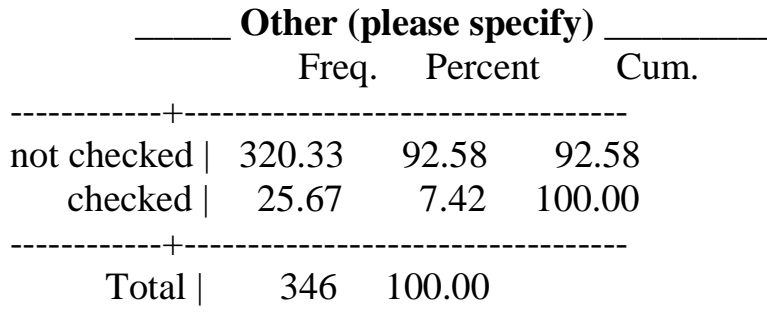

19. How were data needed for audits collected in Program Year 2008? Check all that apply.

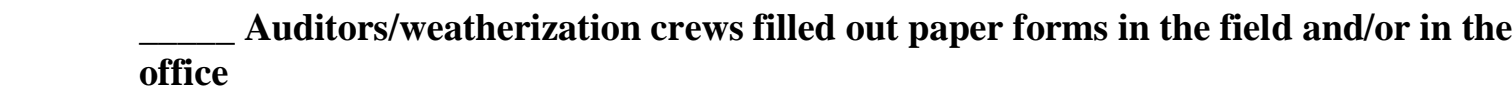

\begin{tabular}{|c|c|c|c|}
\hline & Freq. & Percent & Cum. \\
\hline no & 55.05 & 16.10 & 16.10 \\
\hline yes & 286.95 & 83.90 & 100.00 \\
\hline Total & 342 & 100.00 & \\
\hline
\end{tabular}

Auditors/weatherization crews had laptop computers to enter data in the field

Freq. Percent Cum.

\begin{tabular}{|c|c|c|c|}
\hline no & 315.90 & 92.37 & 92.37 \\
\hline yes | & 26.10 & 7.63 & 100.00 \\
\hline Total & 342 & 100.00 & \\
\hline
\end{tabular}

Auditors/weatherization crews kept notes in the field and then entered the data into computers back at the office

$\begin{array}{ccc}\text { Freq. } & \text { Percent } & \text { Cum. } \\ \text { no | } 110.33 & 32.26 & 32.26 \\ \text { yes | } 231.67 & 67.74 & 100.00 \\ ------------- \\ \text { Total| } 342 & 100.00\end{array}$


Other (please specify)

\begin{tabular}{|c|c|c|c|}
\hline & Freq. & Percent & Cum. \\
\hline no & 339.34 & 99.22 & 99.22 \\
\hline yes & 2.66 & 0.78 & 100.00 \\
\hline otal & 342 & 100.00 & \\
\hline
\end{tabular}

20. How were data about weatherization measures installed in homes collected in Program Year 2008? Check all that apply.

\section{Weatherization crews filed out paper forms and turned them in}

\begin{tabular}{|c|c|c|c|}
\hline & Freq. & cent & Cum. \\
\hline no & 79.58 & 23.27 & 23.27 \\
\hline yes & 262.42 & 76.73 & 100.00 \\
\hline Total & 342 & 100.00 & \\
\hline
\end{tabular}

\section{Weatherization crews had laptop computers to enter data in the field}

\begin{tabular}{|c|c|c|c|}
\hline & Freq. & Percent & Cum. \\
\hline no & 332.48 & 97.22 & 97.22 \\
\hline yes & 9.52 & 2.78 & 100.00 \\
\hline Total & 342 & 100.00 & \\
\hline
\end{tabular}

the office

\section{Weatherization crews kept notes in the field and then entered data into computers at}

\begin{tabular}{ccc} 
Freq. & Percent & Cum. \\
no | 207.89 & 60.79 & 60.79 \\
yes | 134.11 & 39.21 & 100.00 \\
- \hdashline---- \\
Total | 342 & 100.00 &
\end{tabular}

Other (please specify)

\begin{tabular}{|c|c|c|c|}
\hline & Freq. & Percent & Cum. \\
\hline no & 297.59 & 87.01 & 87.01 \\
\hline yes $\mid$ & 44.41 & 12.99 & 100.00 \\
\hline Total | & 342 & 100.00 & \\
\hline
\end{tabular}


21. How were energy use records collected for weatherized houses in Program Year 2008? Check all that apply.

The records were provided by the local utility or utilities

\begin{tabular}{|c|c|c|c|}
\hline & Freq. & Percent & Cum. \\
\hline no & 173.62 & 50.32 & 50.32 \\
\hline yes & 171.38 & 49.68 & 100.00 \\
\hline Total & 345 & 100.00 & \\
\hline
\end{tabular}

The records were provided by the state

Freq. Percent Cum.

\begin{tabular}{|c|c|c|c|}
\hline no & $\begin{array}{r}320.33 \\
24.67\end{array}$ & $\begin{array}{r}92.85 \\
7.15\end{array}$ & $\begin{array}{r}92.85 \\
\end{array}$ \\
\hline & 345 & م & \\
\hline
\end{tabular}

\section{We asked the households for their energy bills}

\begin{tabular}{|c|c|c|c|}
\hline & Freq. & Percent & Cum. \\
\hline no & 145.70 & 42.23 & 42.23 \\
\hline yes & 199.30 & 57.77 & 100.00 \\
\hline Total | & 345 & 100.00 & \\
\hline
\end{tabular}

Did not collect

$\begin{array}{crcc} & \text { Freq. } & \text { Percent } & \text { Cum. } \\ \text { no | } 285.05 & 82.62 & 82.62 \\ \text { yes | } 59.95 & 17.38 & 100.00 \\ --------- \\ \text { Total| } 345 & 100.00 & \end{array}$

Other (please specify)

\begin{tabular}{|c|c|c|c|}
\hline Fr & \multicolumn{2}{|c|}{ Percent } & Cum. \\
\hline no & 323.53 & 93.78 & 93.78 \\
\hline yes & 21.47 & 6.22 & 100.00 \\
\hline Total | & 345 & 100.00 & \\
\hline
\end{tabular}


22. Did your agency computerize the following types of data in Program Year 2008? If no, please indicate why not. Check all that apply.

\begin{tabular}{|c|c|c|c|c|}
\hline & $\begin{array}{l}\text { Household } \\
\text { Data }\end{array}$ & Audit Data & $\begin{array}{l}\text { Weatherization } \\
\text { Measures Data }\end{array}$ & $\begin{array}{c}\text { Energy Use } \\
\text { Data }\end{array}$ \\
\hline Yes & $\begin{array}{l}\text { obs: } 331 \\
\text { not checked: } 57 \\
\text { checked: } 274\end{array}$ & $\begin{array}{l}\text { obs: } 328 \\
\text { not checked: } 79 \\
\text { checked: } 249\end{array}$ & $\begin{array}{l}\text { obs: } 331 \\
\text { not checked: } 71 \\
\text { checked: } 260\end{array}$ & $\begin{array}{l}\text { obs: } 303 \\
\text { not checked: } 126 \\
\text { checked: } 177\end{array}$ \\
\hline No, no need & $\begin{array}{l}\text { obs: } 331 \\
\text { not checked: } 290 \\
\text { checked: } 41\end{array}$ & $\begin{array}{l}\text { obs: } 328 \\
\text { not checked: } 273 \\
\text { checked: } 55\end{array}$ & $\begin{array}{l}\text { obs: } 331 \\
\text { not checked: } 282 \\
\text { checked: } 49\end{array}$ & $\begin{array}{l}\text { obs: } 303 \\
\text { not checked: } 225 \\
\text { checked: } 78\end{array}$ \\
\hline $\begin{array}{l}\text { No, could not } \\
\text { afford }\end{array}$ & $\begin{array}{l}\text { obs: } 331 \\
\text { not checked: } 328 \\
\text { checked: } 3\end{array}$ & $\begin{array}{l}\text { obs: } 328 \\
\text { not checked: } 323 \\
\text { checked: } 5\end{array}$ & $\begin{array}{l}\text { obs: } 331 \\
\text { not checked: } 327 \\
\text { checked: } 4\end{array}$ & $\begin{array}{l}\text { obs: } 303 \\
\text { not checked: } 297 \\
\text { checked: } 6\end{array}$ \\
\hline $\begin{array}{l}\text { No, } \\
\text { insufficient } \\
\text { staff time to } \\
\text { set up } \\
\text { computer }\end{array}$ & $\begin{array}{l}\text { obs: } 331 \\
\text { not checked: } 328 \\
\text { checked: } 3\end{array}$ & $\begin{array}{l}\text { obs: } 328 \\
\text { not checked: } 319 \\
\text { checked: } 9\end{array}$ & $\begin{array}{l}\text { obs: } 311 \\
\text { not checked: } 323 \\
\text { checked: } 8\end{array}$ & $\begin{array}{l}\text { obs: } 303 \\
\text { not checked: } 288 \\
\text { checked: } 15\end{array}$ \\
\hline $\begin{array}{l}\text { No, } \\
\text { insufficient } \\
\text { staff time } \\
\text { enter data }\end{array}$ & $\begin{array}{l}\text { obs: } 331 \\
\text { not checked: } 326 \\
\text { checked: } 5\end{array}$ & $\begin{array}{l}\text { obs: } 328 \\
\text { not checked: } 320 \\
\text { checked: } 8\end{array}$ & $\begin{array}{l}\text { obs: } 331 \\
\text { not checked: } 321 \\
\text { checked: } 10\end{array}$ & $\begin{array}{l}\text { obs: } 303 \\
\text { not checked: } 280 \\
\text { checked: } 23\end{array}$ \\
\hline $\begin{array}{l}\text { Other (please } \\
\text { specify) }\end{array}$ & $\begin{array}{l}\text { obs: } 331 \\
\text { not checked: } 322 \\
\text { checked: } 9\end{array}$ & $\begin{array}{l}\text { obs: } 328 \\
\text { not checked: } 316 \\
\text { checked: } 12\end{array}$ & $\begin{array}{l}\text { obs: } 331 \\
\text { not checked: } 321 \\
\text { checked: } 10\end{array}$ & $\begin{array}{l}\text { obs: } 303 \\
\text { not checked: } 278 \\
\text { checked: } 25\end{array}$ \\
\hline
\end{tabular}

23. Did your agency analyze the following types of data for any of the following reasons in Program Year 2008 ? If yes, please indicate the reason(s) why. Check all that apply.

\begin{tabular}{|c|c|c|c|c|}
\hline Types of Data/Reasons for Use & Household Data & Audit Data & $\begin{array}{l}\text { Weatherization } \\
\text { Measures Data }\end{array}$ & Energy Use Data \\
\hline No & $\begin{array}{l}\text { obs: } 323 \\
\text { not checked: } 184 \\
\text { checked: } 139\end{array}$ & $\begin{array}{l}\text { obs: } 299 \\
\text { not checked: } 134 \\
\text { checked: } 165\end{array}$ & $\begin{array}{l}\text { obs: } 311 \\
\text { not checked: } 161 \\
\text { checked: } 150\end{array}$ & $\begin{array}{l}\text { obs: } 288 \\
\text { not checked: } 113 \\
\text { checked: } 175\end{array}$ \\
\hline $\begin{array}{l}\text { Yes, generated descriptive statistics } \\
\text { (e.g., counts, percentages) }\end{array}$ & $\begin{array}{l}\text { obs: } 323 \\
\text { not checked: } 191 \\
\text { checked: } 132\end{array}$ & $\begin{array}{l}\text { obs: } 299 \\
\text { not checked: } 217 \\
\text { checked: } 82\end{array}$ & $\begin{array}{l}\text { obs: } 311 \\
\text { not checked: } 208 \\
\text { checked: } 103\end{array}$ & $\begin{array}{l}\text { obs: } 288 \\
\text { not checked: } 224 \\
\text { checked: } 64\end{array}$ \\
\hline Yes, looked for trends & $\begin{array}{l}\text { obs: } 323 \\
\text { not checked: } 287 \\
\text { checked: } 36\end{array}$ & $\begin{array}{l}\text { obs: } 299 \\
\text { not checked: } 265 \\
\text { checked: } 34\end{array}$ & $\begin{array}{l}\text { obs: } 311 \\
\text { not checked: } 265 \\
\text { checked: } 46\end{array}$ & $\begin{array}{l}\text { obs: } 288 \\
\text { not checked: } 255 \\
\text { checked: } 33\end{array}$ \\
\hline $\begin{array}{l}\text { Yes, supported agency strategic } \\
\text { planning }\end{array}$ & $\begin{array}{l}\text { obs: } 323 \\
\text { not checked: } 256 \\
\text { checked: } 67\end{array}$ & $\begin{array}{l}\text { obs: } 299 \\
\text { not checked: } 263 \\
\text { checked: } 36\end{array}$ & $\begin{array}{l}\text { obs: } 311 \\
\text { not checked: } 267 \\
\text { checked: } 46\end{array}$ & $\begin{array}{l}\text { obs: } 288 \\
\text { not checked: } 260 \\
\text { checked: } 28\end{array}$ \\
\hline $\begin{array}{l}\text { Yes, supported agency program } \\
\text { performance review }\end{array}$ & $\begin{array}{l}\text { obs: } 323 \\
\text { not checked: } 220 \\
\text { checked: } 103\end{array}$ & $\begin{array}{l}\text { obs: } 299 \\
\text { not checked: } 222 \\
\text { checked: } 77\end{array}$ & $\begin{array}{l}\text { obs: } 311 \\
\text { not checked: } 215 \\
\text { checked: } 96\end{array}$ & $\begin{array}{l}\text { obs: } 288 \\
\text { not checked: } 228 \\
\text { checked: } 60\end{array}$ \\
\hline Other (please specify) & $\begin{array}{l}\text { obs: } 323 \\
\text { not checked: } 313 \\
\text { checked: } 10\end{array}$ & $\begin{array}{l}\text { obs: } 299 \\
\text { not checked: } 291 \\
\text { checked: } 8\end{array}$ & $\begin{array}{l}\text { obs: } 311 \\
\text { not checked: } 302 \\
\text { checked: } 9\end{array}$ & $\begin{array}{l}\text { obs: } 288 \\
\text { not checked: } 274 \\
\text { checked: } 14\end{array}$ \\
\hline
\end{tabular}


24. What percentage of households whose homes were weatherized by your agency in Program Year 2008 registered a complaint regarding the quality or nature of the weatherization job performed on their dwelling unit?

\begin{tabular}{|l|c|}
\hline observations: & 313 \\
\hline missing values: & 44 \\
\hline mean: & 2.05 \\
\hline standard deviation: & 3.83 \\
\hline min: & 0 \\
\hline 10th percentile: & 0 \\
\hline 25th percentile: & 0 \\
\hline median: & 1 \\
\hline 75th percentile: & 2 \\
\hline 90th percentile: & 5 \\
\hline max: & 41 \\
\hline
\end{tabular}

25. Of those households that filed complaints, what percentage of these required some additional work?

\begin{tabular}{|l|c|}
\hline observations: & 288 \\
\hline missing values: & 69 \\
\hline mean: & 11.67 \\
\hline standard deviation: & 24.64 \\
\hline min: & 0 \\
\hline 10th percentile: & 0 \\
\hline 25th percentile: & 0 \\
\hline median: & 1 \\
\hline 75th percentile: & 4 \\
\hline 90th percentile: & 50 \\
\hline max: & 100 \\
\hline
\end{tabular}

26. Of all the homes weatherized by your agency in Program Year 2008, how many did you refer to nonenergy programs for additional services (e.g., nutrition; family counseling)?

\begin{tabular}{|l|c|}
\hline observations: & 290 \\
\hline missing values: & 67 \\
\hline mean: & 38.57 \\
\hline standard deviation: & 221.10 \\
\hline min: & 0 \\
\hline 10th percentile: & 0 \\
\hline 25th percentile: & 0 \\
\hline median: & 7 \\
\hline 75th percentile: & 30 \\
\hline 90th percentile: & 75 \\
\hline max: & 5060 \\
\hline
\end{tabular}


27. How many income-qualified dwelling units were on your agency's waiting list: at the start of Program Year 2008

\begin{tabular}{|l|c|}
\hline observations: & 290 \\
\hline missing values: & 67 \\
\hline mean: & 507.81 \\
\hline standard deviation: & 1179.43 \\
\hline min: & 0 \\
\hline 10th percentile: & 5 \\
\hline 25th percentile: & 46 \\
\hline median: & 127 \\
\hline 75th percentile: & 342 \\
\hline 90th percentile: & 1322 \\
\hline max: & 10000 \\
\hline
\end{tabular}

at the end of Program Year 2008

\begin{tabular}{|l|c|}
\hline observations: & 289 \\
\hline missing values: & 68 \\
\hline mean: & 529.20 \\
\hline standard deviation: & 1251.42 \\
\hline min: & 0 \\
\hline 10th percentile: & 0 \\
\hline 25th percentile: & 42 \\
\hline median: & 125 \\
\hline 75th percentile: & 375 \\
\hline 90th percentile: & 1220 \\
\hline max: & 10000 \\
\hline
\end{tabular}

28. Please list the number of dwelling units that your agency could not weatherize in Program Year 2008 due to housing conditions (i.e., the number you had to "walk away from").

\begin{tabular}{|l|c|}
\hline observations: & 290 \\
\hline missing values: & 67 \\
\hline mean: & 15.44 \\
\hline standard deviation: & 43.44 \\
\hline min: & 0 \\
\hline 10th percentile: & 0 \\
\hline 25th percentile: & 1 \\
\hline median: & 4 \\
\hline 75th percentile: & 10 \\
\hline 90th percentile: & 31 \\
\hline max: & 600 \\
\hline
\end{tabular}


29. Please list the number of dwellings that your agency could not weatherize in Program Year 2008 because they had previously been weatherized?

\begin{tabular}{|l|c|}
\hline observations: & 197 \\
\hline missing values: & 160 \\
\hline mean: & 41.02 \\
\hline standard deviation: & 214.47 \\
\hline min: & 0 \\
\hline 10th percentile: & 0 \\
\hline 25th percentile: & 0 \\
\hline median: & 5 \\
\hline 75th percentile: & 15 \\
\hline 90th percentile: & 40 \\
\hline max: & 2200 \\
\hline
\end{tabular}


30. Please indicate the number of staff that supported your agency's weatherization program and their work effort in Program Year 2008. In considering the number of staff, please include everyone who worked full- or part-time or who worked with the weatherization program as well as other agency programs.

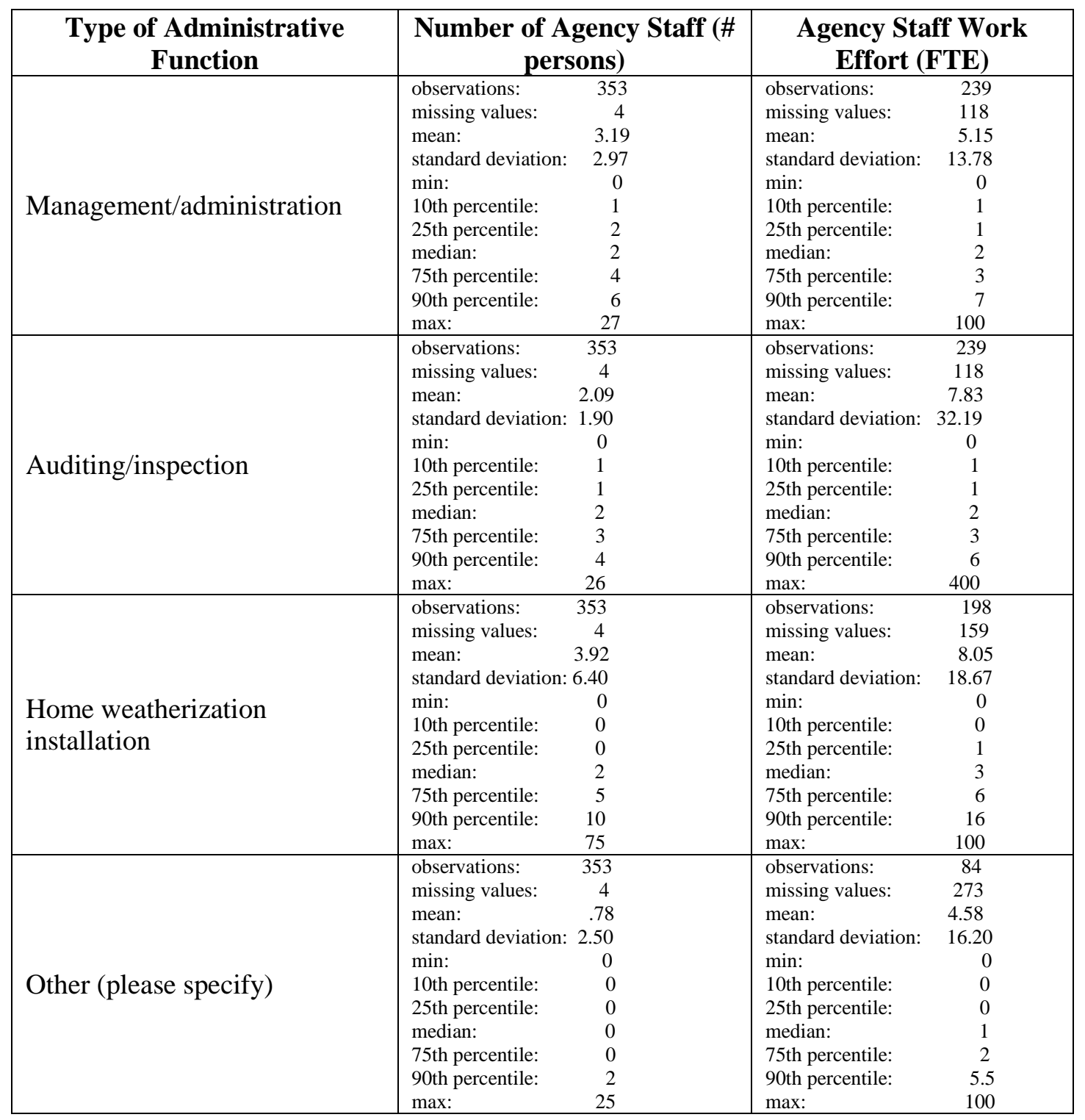


31. For the agency staff working on your agency's weatherization program in each of the following functional areas in Program Year 2008, please indicate their level of experience with the weatherization program:

\begin{tabular}{|c|c|c|c|c|c|c|}
\hline & $\begin{array}{l}\text { Very } \\
\text { High }\end{array}$ & High & \multicolumn{2}{|c|}{ Average } & Low & $\begin{array}{l}\text { Very } \\
\text { Low }\end{array}$ \\
\hline \multirow{7}{*}{ Management/administration } & \multicolumn{4}{|c|}{ Percent $\quad$ Cum. } & & \\
\hline & Very High & 4.53 & 1.28 & 1.28 & & \\
\hline & High & 4.69 & 1.32 & 2.60 & & \\
\hline & Average | & 47.48 & 13.37 & 15.97 & & \\
\hline & & 103.28 & 29.09 & 45.07 & & \\
\hline & Very Low & 195.02 & 54.93 & 100.00 & & \\
\hline & Total & 355 & 100.00 & & & \\
\hline & & $\begin{array}{ll}\text { Freq. } & \mathrm{P}\end{array}$ & Percent & Cum. & & \\
\hline & Very High | & 2.41 & 0.68 & 0.68 & & \\
\hline & High | & 5.141 & 1.46 & 2.14 & & \\
\hline Auditing/inspection & Average & 55.29 & 15.66 & 17.80 & & \\
\hline & Low & 119.26 & 33.78 & 51.59 & & \\
\hline & Very Low & 170.90 & 48.41 & 100.00 & & \\
\hline & Total | & 353 & 100.00 & & & \\
\hline & & Freq. $\quad \mathrm{I}$ & Percent & Cum. & & \\
\hline & Very High & 1.50 & 0.54 & 0.54 & & \\
\hline & High & 7.92 & 2.86 & 3.40 & & \\
\hline Home weatherization & Average | & 64.18 & 23.17 & 26.57 & & \\
\hline & Low & 112.96 & 40.78 & 67.35 & & \\
\hline & Very Low & 90.45 & 32.65 & 100.00 & & \\
\hline & Total | & 277 & 100.00 & & & \\
\hline & & Freq. & Percent & Cum. & & \\
\hline & Very High & 6.32 & 9.44 & 9.44 & & \\
\hline & & 2.75 & 4.10 & 13.53 & & \\
\hline Other (please specify) & Average & 10.27 & 15.34 & 28.87 & & \\
\hline & Low & 29.83 & 44.53 & 73.40 & & \\
\hline & Very Low & 17.82 & 26.60 & 100.00 & & \\
\hline & Total & 67 & 100.00 & & & \\
\hline
\end{tabular}


32. For the agency staff working on your agency's weatherization program in each of the following functional area, please indicate the amount of turnover in staff over a three year period ending with Program Year 2008. Turnover is defined as the number of new staff in a functional area in the past three years divided by the total number of staff working in that functional area. If a particular position has had more than one new person during the past three years (e.g., Person 1 leaves, Person 2 is hired to take Person 1's position, then Person 2 leaves and a third person is hired), just count that as one new staff person. Please check appropriate boxes.

\begin{tabular}{|c|c|c|c|c|c|c|c|}
\hline & $\begin{array}{c}\text { No } \\
\text { Turnover }\end{array}$ & \multicolumn{2}{|c|}{$\begin{array}{l}1 \text { to } \\
10 \%\end{array}$} & $\begin{array}{l}11 \text { to } \\
25 \%\end{array}$ & $\begin{array}{l}26 \text { to } \\
50 \%\end{array}$ & $\begin{array}{l}51 \text { to } \\
75 \%\end{array}$ & $\begin{array}{l}76 \text { to } \\
100 \%\end{array}$ \\
\hline \multirow{8}{*}{$\begin{array}{l}\text { Management/ } \\
\text { administration }\end{array}$} & \multicolumn{7}{|c|}{$\begin{array}{lll}\text { Freq. } & \text { Percent } & \text { Cum. }\end{array}$} \\
\hline & No Turnover & 253.05 & 72.09 & 72.09 & & & \\
\hline & 1 to $10 \%$ & 43.66 & 12.44 & 84.53 & & & \\
\hline & 11 to $25 \%$ & 14.22 & 4.05 & 88.58 & & & \\
\hline & 26 to $50 \%$ & 26.35 & 7.51 & 96.09 & & & \\
\hline & 51 to $75 \%$ & 2.70 & 0.77 & 96.86 & & & \\
\hline & 76 to $100 \%$ & 11.02 & 3.14 & 100.00 & & & \\
\hline & Total | & 351 & 100.00 & & & & \\
\hline & & Freq. $\quad \mathrm{Pe}$ & ercent & Cum. & & & \\
\hline & No Turnover & 248.36 & 71.16 & 71.16 & & & \\
\hline & 1 to $10 \%$ & 46.38 & 13.29 & 84.45 & & & \\
\hline & 11 to $25 \%$ & 11.07 & 3.17 & 87.62 & & & \\
\hline Auditing/1nspection & 26 to $50 \%$ & 31.03 & 8.89 & 96.52 & & & \\
\hline & 51 to $75 \%$ & 1.14 & 0.33 & 96.84 & & & \\
\hline & 76 to $100 \%$ & 11.02 & 3.16 & 100.00 & & & \\
\hline & Total | & 349 & 100.00 & & & & \\
\hline & & Freq. $\quad \mathrm{Pe}$ & rcent & Cum. & & & \\
\hline & No Turnover | & 153.95 & 57.02 & 57.02 & & & \\
\hline & 1 to $10 \%$ & 56.12 & 20.78 & 77.80 & & & \\
\hline & 11 to $25 \%$ & 28.02 & 10.38 & 88.18 & & & \\
\hline Home weatherization & 26 to $50 \%$ & 18.47 & 6.84 & 95.02 & & & \\
\hline & 51 to $75 \%$ & 3.90 & 1.44 & 96.47 & & & \\
\hline & 76 to $100 \%$ & 9.54 & 3.53 & 100.00 & & & \\
\hline & Total | & 270 & 100.00 & & & & \\
\hline & & Freq. $\quad P \epsilon$ & rrcent & Cum. & & & \\
\hline & No Turnover & 27.97 & 50.86 & 50.86 & & & \\
\hline & 1 to $10 \%$ & 14.91 & 27.10 & 77.96 & & & \\
\hline Other (nlease snecifv) & 11 to $25 \%$ & 3.25 & 5.91 & 83.87 & & & \\
\hline Uther (pıease speciıy) & 26 to $50 \%$ & 6.27 & 11.39 & 95.26 & & & \\
\hline & 51 to $75 \%$ & 0.00 & 0.00 & 95.26 & & & \\
\hline & 76 to $100 \%$ & 2.61 & 4.74 & 100.00 & & & \\
\hline & Total & 55 & 100.00 & & & & \\
\hline
\end{tabular}


33. For which of the following functional areas were there certification or licensing requirements in Program Year 2008 for the in-house or contractor staff serving your state's weatherization program? Check all that apply.

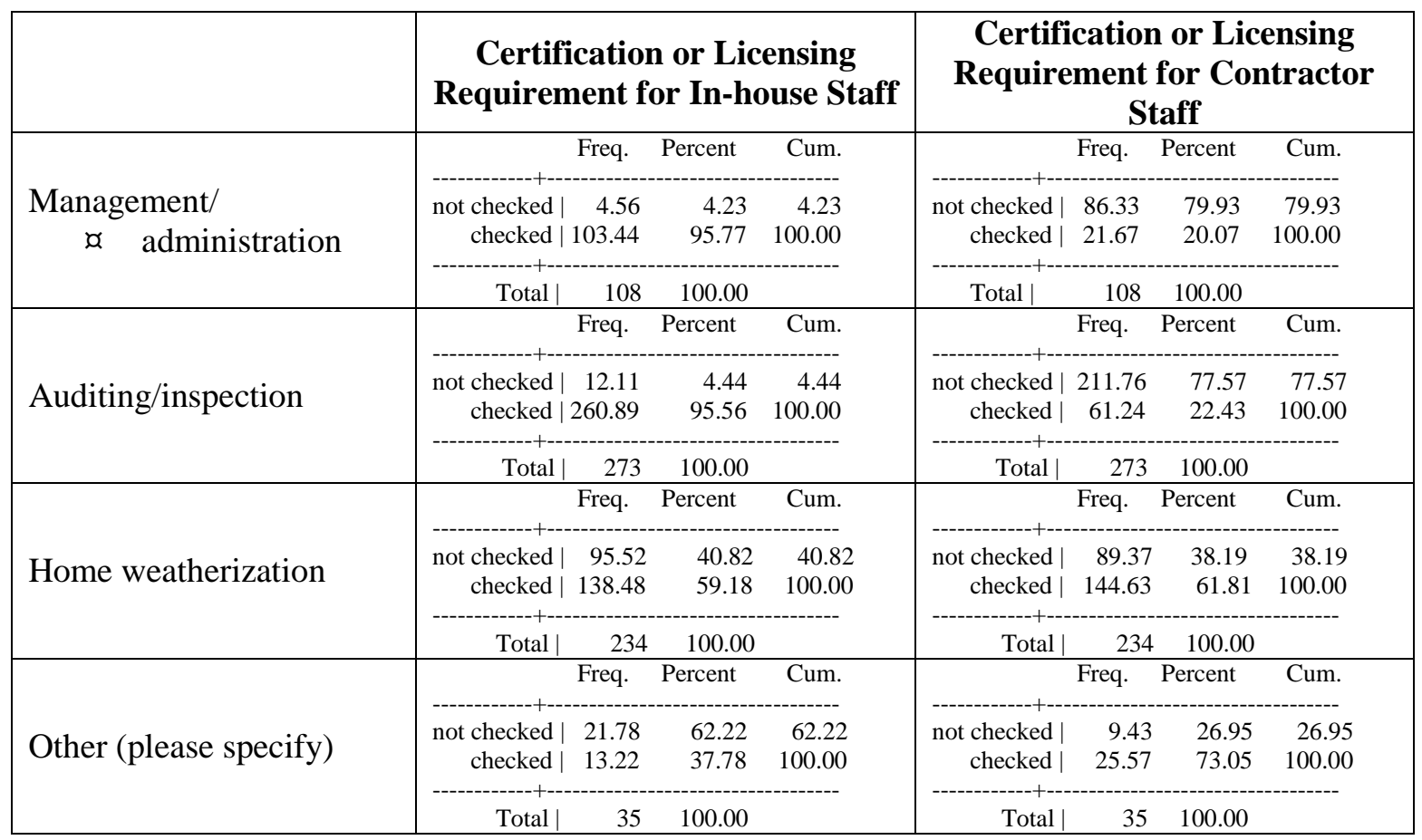

34. Which of the following approaches did your agency use in Program Year 2008 to market your weatherization services to low-income households? Check all that apply.

- Targeted mailings to potential clients

Freq. Percent Cum.

\begin{tabular}{rrr} 
no $\mid 204.47$ & 61.22 & 61.22 \\
Yes $\mid 129.53$ & 38.78 & 100.00 \\
- \hdashline------- & \\
Total| 334 & 100.00
\end{tabular}

- Targeted mailings to landlords of potential clients

Freq. Percent Cum.

\begin{tabular}{|c|c|c|c|}
\hline no & 297.35 & 89.03 & 89.03 \\
\hline Yes | & 36.65 & 10.97 & 100.00 \\
\hline Total | & 334 & 100.00 & \\
\hline
\end{tabular}


- Visits to potential clients

\begin{tabular}{|c|c|c|c|}
\hline & Freq. & Percent & Cum. \\
\hline no & 271.16 & 81.19 & 81.19 \\
\hline Yes & 62.84 & 18.81 & 100.00 \\
\hline Total & 334 & 100.00 & \\
\hline
\end{tabular}

- Visits to landlords of potential clients Freq. Percent Cum.

$\begin{array}{rrr}\text { no | } 299.04 & 89.53 & 89.53 \\ \text { Yes | } 34.96 & 10.47 & 100.00 \\ -------- & \\ \text { Total| } & 334 & 100.00\end{array}$

- Advertising with other social service agencies Freq. Percent Cum.

$\begin{array}{rrr}\text { no | } 126.24 & 37.80 & 37.80 \\ \text { Yes | } 207.76 & 62.20 & 100.00 \\ ---------------- \\ \text { Total| } 334 & 100.00\end{array}$

- Advertising in local newspapers or magazines

\begin{tabular}{|c|c|c|c|}
\hline & Freq. & Percent & Cum. \\
\hline no & 194.18 & 58.14 & 58.14 \\
\hline Yes & 139.82 & 41.86 & 100.00 \\
\hline Total & 334 & 100.00 & \\
\hline
\end{tabular}

- Radio advertising

\begin{tabular}{|c|c|c|c|}
\hline & Freq. & Percent & Cum. \\
\hline no & 266.87 & 79.90 & 79.90 \\
\hline Yes & 67.13 & 20.10 & 100.00 \\
\hline Total | & 334 & 100.00 & \\
\hline
\end{tabular}

- Television advertising

Freq. Percent Cum.

\begin{tabular}{|c|c|c|c|}
\hline no & 289.98 & 86.82 & 86.82 \\
\hline Yes & 44.02 & 13.18 & 100.00 \\
\hline Total & 334 & 100.00 & \\
\hline
\end{tabular}


- Posting information on website

\begin{tabular}{|c|c|c|c|}
\hline & Freq. & Percent & Cum. \\
\hline no & 134.07 & 40.14 & 40.14 \\
\hline Yes & 199.93 & 59.86 & 100.00 \\
\hline Total & 334 & 100.00 & \\
\hline
\end{tabular}

- Other (please specify)

\begin{tabular}{|c|c|c|c|}
\hline & Freq. & Percent & Cum. \\
\hline no & 228.14 & 68.30 & 68.30 \\
\hline Yes & 105.86 & 31.70 & 100.00 \\
\hline Total & 334 & 100.00 & \\
\hline
\end{tabular}

35. Who was responsible for leading the marketing/outreach efforts described above? Check all that apply.

- Agency management

\begin{tabular}{|c|c|c|c|}
\hline & Freq. & Percent & Cum. \\
\hline No & 81.08 & 24.42 & 24.42 \\
\hline Yes & 250.92 & 75.58 & 100.00 \\
\hline Total & 332 & 100.00 & \\
\hline
\end{tabular}

- In-house outreach coordinator

\begin{tabular}{|c|c|c|c|}
\hline & Freq. & Percent & Cum. \\
\hline No & 231.91 & 69.85 & 69.85 \\
\hline Yes & 100.09 & 30.15 & 100.00 \\
\hline Total & 332 & 100.00 & \\
\hline
\end{tabular}

- Contractor outreach coordinator

\begin{tabular}{|c|c|c|c|}
\hline & Freq. & Percent & Cum. \\
\hline No & 323.32 & 97.39 & 97.39 \\
\hline Yes & 8.68 & 2.61 & 100.00 \\
\hline Total | & 332 & 100.00 & \\
\hline
\end{tabular}


- In-house communications staff

\begin{tabular}{|c|c|c|c|}
\hline & Freq. & Percent & Cum. \\
\hline No & 278.23 & 83.80 & 83.80 \\
\hline Yes & 53.77 & 16.20 & 100.00 \\
\hline Total & 332 & 100.00 & \\
\hline
\end{tabular}

- Contractor communications staff Freq. Percent Cum.

\begin{tabular}{|c|c|c|c|}
\hline No & 330.12 & 99.43 & 99.43 \\
\hline Yes & 1.88 & 0.57 & 100.00 \\
\hline Total & 332 & 100.00 & \\
\hline
\end{tabular}

- Other staff (please specify)

\begin{tabular}{|c|c|c|c|}
\hline & Freq. & Percent & Cum. \\
\hline No & 243.47 & 73.33 & 73.33 \\
\hline Yes & 88.53 & 26.67 & 100.00 \\
\hline Total & 332 & 100.00 & \\
\hline
\end{tabular}

\section{SECTION B: PROGRAM OPERATIONS AND IMPLEMENTATION}

1. Please rate the adequacy of the Program Year 2008 funding received by your agency from ALL funding sources in terms of weatherizing the stock of eligible low-income dwelling units in your local jurisdiction in a timely fashion? Check best answer.

\begin{tabular}{r|rrr} 
Freq. & Percent & \multicolumn{1}{c}{ Cum. } \\
\hline-------------+-------- \\
Very inadequate | 26.42 & 7.77 & 7.77 \\
Inadeqate | 110.83 & 32.60 & 40.37 \\
Adequate | 160.53 & 47.22 & 87.58 \\
Very adequate | & 42.22 & 12.42 & 100.00 \\
------------
\end{tabular}


2. What was the quality of the administrative support and assistance that your agency received from the state and its contractors in Program Year 2008? Check best answer.

$\begin{array}{rrrr} & \text { Freq. } & \text { Percent } & \text { Cum. } \\ \text {--------------+------------------------- } \\ \text { very low quality | } & 3.27 & 0.96 & 0.96 \\ \text { low quality | } 11.49 & 3.38 & 4.34 \\ \text { moderate quality | } & 109.38 & 32.17 & 36.51 \\ \text { high quality | } 155.16 & 45.63 & 82.15 \\ \text { very high quality | } & 53.74 & 15.81 & 97.95 \\ \text { not applicable | } & 6.97 & 2.05 & 100.00 \\ -------------+---------------------- \\ \text { Total | } & 340 & 100.00 & \end{array}$

2a. If you gave a rating of "low" or "very low," what were your reasons?

3. What was the quality of the training that your agency received from the state and its contractors in Program Year 2008? Check best answer.

Freq. Percent Cum.

\begin{tabular}{|c|c|c|c|}
\hline very low quality | & 2.48 & 0.73 & 0.73 \\
\hline low quality & 13.75 & 4.04 & 4.77 \\
\hline moderate quality | & 120.04 & 35.31 & 40.08 \\
\hline high quality & 137.08 & 40.32 & 80.40 \\
\hline very high quality & 45.86 & 13.49 & 93.89 \\
\hline not applicable & 20.79 & 6.11 & 100.00 \\
\hline Total | & 340 & 100.00 & \\
\hline
\end{tabular}

3a. If you gave a rating of "low" or "very low," what were your reasons?

4. What was the quality of the support and assistance on client education that your agency received from the state and its contractors in Program Year 2008? Check best answer.

Freq. Percent Cum.

$\begin{array}{rrrr}\text { very low quality } & 8.54 & 2.50 & 2.50 \\ \text { low quality } & 23.23 & 6.81 & 9.32 \\ \text { moderate quality } & 159.41 & 46.75 & 56.06 \\ \text { high quality } & 93.13 & 27.31 & 83.37 \\ \text { very high quality } \mid & 18.88 & 5.54 & 88.91 \\ \text { not applicable | } & 37.82 & 11.09 & 100.00 \\ ---------------\end{array}$

4a. If you gave a rating of "low" or "very low," what were your reasons?

5. What was the quality of the support and assistance on leveraging the Weatherization Assistance Program with other funding sources and related programs that your agency received from the state and its contractors in Program Year 2008? Check best answer. 


\begin{tabular}{|c|c|c|c|}
\hline & Freq. & Percent & Cum. \\
\hline very low quality & 8.65 & 2.54 & 2.54 \\
\hline low quality & 22.19 & 6.53 & 9.07 \\
\hline moderate quality & 128.20 & 37.71 & 46.78 \\
\hline high quality & 86.58 & 25.47 & 72.24 \\
\hline very high quality & 30.75 & 9.04 & 81.29 \\
\hline not applicable & 63.63 & 18.71 & 100.00 \\
\hline Tota & 340 & 100.00 & \\
\hline
\end{tabular}

5a. If you gave a rating of "low" or "very low," what were your reasons?

6. What was the quality of the technical support that your agency received from the state and its contractors in Program Year 2008? Check best answer.

Freq. Percent Cum.

\begin{tabular}{|c|c|c|c|}
\hline very low quality & 3.66 & 1.07 & 1.07 \\
\hline low quality & 11.96 & 3.51 & 4.58 \\
\hline moderate quality & 106.42 & 31.21 & 35.79 \\
\hline high quality & 146.68 & 43.01 & 78.80 \\
\hline very high quality & 56.14 & 16.46 & 95.26 \\
\hline not applicable & 16.15 & 4.74 & 100.00 \\
\hline Total & 341 & 100.00 & \\
\hline
\end{tabular}

6a. If you gave a rating of "low" or "very low," what were your reasons? 
7. How flexible did you find the DOE program rules that governed the weatherization program in Program Year 2008? In other words, did the program rules allow your state to tailor your program to your needs (very flexible) or proscribe your program to only one way of operation (very inflexible)? Check best answer.

\begin{tabular}{r|rrr} 
& Freq. & Percent & Cum. \\
\hline Very Inflexible & 17.77 & 5.21 & 5.21 \\
Inflexible & 128.83 & 37.78 & 42.99 \\
Flexible $\mid$ & 181.52 & 53.23 & 96.22 \\
Very Flexible | & 12.88 & 3.78 & 100.00 \\
-------------+-------------------------- \\
Total & 341 & 100.00
\end{tabular}

7a. Using Program Year 2008 as the reference point, how should the program rules change? Check best answer.

\begin{tabular}{|c|c|c|c|}
\hline & Freq. & Percent & Cum. \\
\hline Become much more inflexible & 1.29 & 0.38 & 0.38 \\
\hline Become more inflexible & 4.68 & 1.37 & 1.75 \\
\hline Stay about the same & 128.02 & 37.54 & 39.29 \\
\hline Become more flexible & 171.40 & 50.26 & 89.56 \\
\hline Become much more flexible & 35.61 & 10.44 & 100.00 \\
\hline Total | & 341 & 100.00 & \\
\hline
\end{tabular}

7b. In what areas should the program rules become more flexible?

7c. In what areas should the program rules become less flexible?

8. Please describe any important political issues faced by your agency's weatherization program in Program Year 2008.

9. Using Program Year 2008 as a reference point, how important was improving administrative support and assistance from the state and its contractors in improving your agency's ability to deliver lowincome weatherization services? Check best answer.

\begin{tabular}{|c|c|c|c|}
\hline & Freq. & Percent & Cum. \\
\hline Very unimportant & 6.30 & 1.85 & 1.85 \\
\hline Unimportant & 51.52 & 15.11 & 16.96 \\
\hline Important & 167.92 & 49.24 & 66.20 \\
\hline Very important & 87.13 & 25.55 & 91.75 \\
\hline Not applicable & 28.13 & 8.25 & 100.00 \\
\hline Total & 341 & 100.00 & \\
\hline
\end{tabular}


10. Using Program Year 2008 as a reference point, how important was improving training from DOE, the state, and their contractors in improving your agency's ability to deliver low-income weatherization services? Check best answer.

\begin{tabular}{rrrr} 
& Freq. & Percent & \multicolumn{1}{c}{ Cum. } \\
\hline Very unimportant | & 4.07 & 1.19 & 1.19 \\
Unimportant | & 38.64 & 11.30 & 12.49 \\
Important | & 144.7 & 42.32 & 54.81 \\
Very important $\mid$ & 133.09 & 38.92 & 93.73 \\
Not applicable | & 21.45 & 6.27 & 100.00 \\
-----------
\end{tabular}

11. Using Program Year 2008 as a reference point, how important was improving assistance on client education from the state and its contractors in improving your agency's ability to deliver low-income weatherization services? Check best answer.

\begin{tabular}{rrrr} 
& Freq. & Percent & \multicolumn{1}{c}{ Cum. } \\
\hline Very unimportant | & 5.47 & 1.61 & 1.61 \\
Unimportant $\mid$ & 79.72 & 23.45 & 25.06 \\
Important | & 143.26 & 42.13 & 67.19 \\
Very important $\mid$ & 75.11 & 22.09 & 89.28 \\
Not applicable | & 36.44 & 10.72 & 100.00 \\
----------- &
\end{tabular}

12. Using Program Year 2008 as a reference point, how important was improving assistance from the state and its contractors on leveraging the Weatherization Assistance Program with other funding sources and related programs in improving your agency's ability to deliver low-income weatherization services? Check best answer.

Freq. Percent Cum.

\begin{tabular}{|c|c|c|c|}
\hline Very unimportant & 6.16 & 1.81 & 1.81 \\
\hline Unimportant & 50.19 & 14.76 & 16.57 \\
\hline Important & 137.34 & 40.39 & 56.97 \\
\hline Very important & 88.98 & 26.17 & 83.14 \\
\hline Not applicable & 57.33 & 16.86 & 100.00 \\
\hline Total | & 340 & 100.00 & \\
\hline
\end{tabular}


13. Using Program Year 2008 as a reference point, how important was improving technical support from the state and its contractors in improving your agency's ability to deliver low-income weatherization services? Check best answer.

\begin{tabular}{rrrr} 
Freq. & Percent & \multicolumn{1}{c}{ Cum. } \\
\hline Very unimportant | & 4.48 & 1.31 & 1.31 \\
Unimportant | & 44.63 & 13.05 & 14.36 \\
Important | 164.95 & 48.23 & 62.59 \\
Very important | & 107.49 & 31.43 & 94.02 \\
Not applicable | & 20.46 & 5.98 & 100.00 \\
--------- &
\end{tabular}

14. Using Program Year 2008 as a reference point, how important was increased weatherization funding in improving your agency's ability to deliver low-income weatherization services? Check best answer.

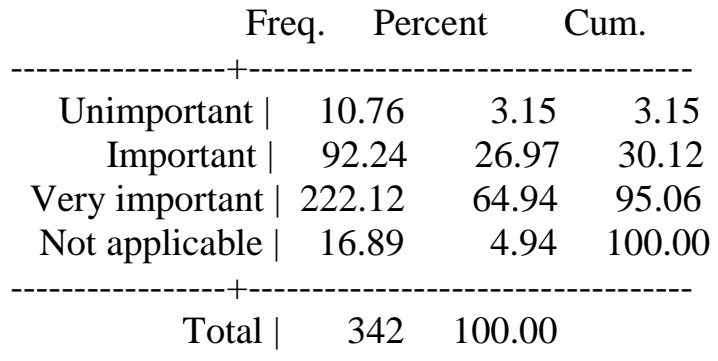

15. Using Program Year 2008 as a reference point, how important was improving data and information systems for managing the delivery of weatherization services? Check best answer.

Freq. Percent Cum.

$\begin{array}{rrrr}\text { Very unimportant | } & 2.20 & 0.65 & 0.65 \\ \text { Unimportant | } & 31.18 & 9.17 & 9.82 \\ \text { Important | } & 147.58 & 43.41 & 53.22 \\ \text { Very important | } & 134.58 & 39.58 & 92.81 \\ \text { Not applicable | } & 24.46 & 7.19 & 100.00 \\ ------------- & \end{array}$

16. In general, how satisfied were you with the length of time between the client's request to have their home weatherized and when it was actually weatherized in Program Year 2008? Check best answer.

Freq. Percent Cum.

$\begin{array}{rrrr}\text { Very dissatisfied | } & 5.49 & 1.62 & 1.62 \\ \text { Dissatisfied | } & 54.94 & 16.16 & 17.77 \\ \text { Not satisfied or dissatisfied | } & 104.79 & 30.82 & 48.59 \\ \text { Satisfied | } & 148.14 & 43.57 & 92.16 \\ \text { Very satisfied | } & 26.65 & 7.84 & 100.00 \\ - \text { Total }^{\mid} & 340 & 100.00 & \end{array}$

17. In general, how easy was it to schedule your visits to client homes to perform audits, weatherization, 
and inspections in Program Year 2008? Check best answer.

$\begin{array}{rrrr} & \text { Freq. } & \text { Percent } & \text { Cum. } \\ \text { Very difficult | } & .92 & 0.27 & 0.27 \\ \text { Difficult | } & 16.25 & 4.76 & 5.03 \\ \text { Not easy or difficult | } & 154.21 & 45.22 & 50.26 \\ \text { Easy | } & 140.73 & 41.27 & 91.53 \\ \text { Very easy | } & 28.90 & 8.47 & 100.00 \\ \text { Total | } & 341 & 100.00 & \end{array}$

\section{SECTION C: AUDIT}

1. What was the primary method that your agency used in Program Year 2008 to select weatherization measures for clients' dwelling units (excluding health, safety, and repair measures and general heat waste measures)? Check best answer.

Freq. Percent Cum.

\begin{tabular}{r|rrr} 
priority list used for all dwelling units | & 183.10 & 51.87 & 51.87 \\
calculation procedure | & 115.02 & 32.58 & 84.45 \\
priority list for units meeting specific | & 41.58 & 11.78 & 96.23 \\
other $\mid$ & 13.30 & 3.77 & 100.00 \\
------------------- & Total | & 353 & 100.00
\end{tabular}

2. Including Program Year 2008, for how many years had your agency used the weatherization measure selection method indicated above?

\begin{tabular}{|l|c|}
\hline observations: & 311 \\
\hline missing values: & 46 \\
\hline mean: & 13.17 \\
\hline standard deviation: & 8.49 \\
\hline min: & 0 \\
\hline 10th percentile: & 3 \\
\hline 25th percentile: & 6 \\
\hline median: & 10 \\
\hline 75th percentile: & 20 \\
\hline 90th percentile: & 25 \\
\hline max: & 40 \\
\hline
\end{tabular}


3. What types of credentials or experience were required of your staff or contractors who were engaged in measure selection in Program Year 2008? Check all that apply.

- Technical certification

\begin{tabular}{|c|c|c|c|}
\hline & Freq. & Percent & Cum. \\
\hline no & 78.87 & 23.40 & 23.40 \\
\hline yes & 258.13 & 76.60 & 100.00 \\
\hline Total & 337 & 100.00 & \\
\hline
\end{tabular}

- Extensive weatherization work experience

\begin{tabular}{|c|c|c|c|}
\hline & Freq. & Percent & Cum. \\
\hline no & 107.96 & 32.04 & 32.04 \\
\hline yes & 229.04 & 67.96 & 100.00 \\
\hline Total | & 337 & 100.00 & \\
\hline
\end{tabular}

- Extensive weatherization supervision experience

\begin{tabular}{|c|c|c|c|}
\hline & Freq. & Percent & Cum. \\
\hline no & 216.26 & 64.17 & 64.17 \\
\hline yes | & 120.74 & 35.83 & 100.00 \\
\hline Total | & 337 & 100.00 & \\
\hline
\end{tabular}

- Construction experience

\begin{tabular}{|c|c|c|c|}
\hline & Freq. & Percent & Cum. \\
\hline no & 150.95 & 44.79 & 44.79 \\
\hline yes | & 186.05 & 55.21 & 100.00 \\
\hline otal & 337 & 100.00 & \\
\hline
\end{tabular}

- Other (please specify)

\begin{tabular}{|c|c|c|c|}
\hline & Freq. & Percent & Cum. \\
\hline no & 314.95 & 93.46 & 93.46 \\
\hline yes & 22.05 & 6.54 & 100.00 \\
\hline Total & 337 & 100.00 & \\
\hline
\end{tabular}


4. Please indicate the level of experience for the agency staff engaged in measure selection in Program Year 2008 in each of the following functional areas.

\begin{tabular}{|c|c|c|c|c|c|c|}
\hline & $\begin{array}{l}\text { Very } \\
\text { High }\end{array}$ & & & Average & \multirow[t]{8}{*}{ Low } & \multirow[t]{2}{*}{ Very Low } \\
\hline \multirow{7}{*}{$\begin{array}{l}\text { Performing weatherization } \\
\text { work }\end{array}$} & \multicolumn{4}{|c|}{ Freq. Percent Cum. } & & \\
\hline & Very low & 10.97 & 3.47 & 3.47 & & \\
\hline & Low & 4.98 & 1.58 & 5.05 & & \\
\hline & Average | & 64.12 & 20.29 & 25.34 & & \\
\hline & High $\mid$ & 114.00 & 36.08 & 61.41 & & \\
\hline & Very High | & 121.94 & 38.59 & 100.00 & & \\
\hline & Total | & 316 & 100.00 & & & \\
\hline & & Freq. & ercent & Cum. & & \\
\hline & Very low & 4.5 & 1.40 & 1.40 & & \\
\hline & Low & 9.61 & 2.94 & 4.34 & & \\
\hline Supervising weatherization & Average & 61.44 & 18.79 & 23.13 & & \\
\hline work & High | & 103.20 & 31.56 & 54.69 & & \\
\hline & Very High & 148.18 & 45.31 & 100.00 & & \\
\hline & Total | & 327 & 100.00 & & & \\
\hline & & $\begin{array}{ll}\text { Freq. } \quad \mathrm{I} \\
\end{array}$ & rcent & Cum. & & \\
\hline & Very low & 9.98 & 3.24 & 3.24 & & \\
\hline & Low & 16.57 & 5.38 & 8.62 & & \\
\hline Working in construction & Average & 86.51 & 28.09 & 36.71 & & \\
\hline & High $\mid$ & 115.35 & 37.45 & 74.16 & & \\
\hline & Very High | & 79.59 & 25.84 & 100.00 & & \\
\hline & Total | & 308 & 100.00 & & & \\
\hline & & $\begin{array}{ll}\text { Freq. } \quad \mathrm{I} \\
\end{array}$ & rcent & Cum. & & \\
\hline & Very low | & 7.63 & 2.26 & 2.26 & & \\
\hline Performing pre- & Low $\mid$ & 7.10 & 2.11 & 4.37 & & \\
\hline (ceatherization audite & Average | & 63.50 & 18.84 & 23.21 & & \\
\hline weatnerization audits & High | & 92.10 & 27.33 & 50.54 & & \\
\hline & Very High | & 166.67 & 49.46 & 100.00 & & \\
\hline & Total | & 337 & 100.00 & & & \\
\hline
\end{tabular}


5. On average, approximately how many hours did it take to select weatherization measures for a typical dwelling unit served by your agency in Program Year 2008, by the major components listed below?

\begin{tabular}{|l|c|}
\hline $\begin{array}{l}\text { Prepartaion/scheduling } \\
\text { observations: }\end{array}$ & 345 \\
\hline missing values: & 12 \\
\hline mean: & 1.09 \\
\hline standard deviation: & 1.83 \\
\hline min: & 0 \\
\hline 10th percentile: & .25 \\
\hline 25th percentile: & .5 \\
\hline median: & 1 \\
\hline 75th percentile: & 1 \\
\hline 90th percentile: & 2 \\
\hline max: & 30 \\
\hline
\end{tabular}

\begin{tabular}{|l|c|}
\hline Travel observations: & 345 \\
\hline missing values: & 12 \\
\hline mean: & 1.24 \\
\hline standard deviation: & 2.33 \\
\hline min: & 0 \\
\hline 10th percentile: & .25 \\
\hline 25th percentile: & .5 \\
\hline median: & 1 \\
\hline 75th percentile: & 1.5 \\
\hline 90th percentile: & 2 \\
\hline max: & 45 \\
\hline
\end{tabular}

\begin{tabular}{|l|c|}
\hline $\begin{array}{l}\text { On-site auditing } \\
\text { observations: }\end{array}$ & 345 \\
\hline missing values: & 12 \\
\hline mean: & 2.91 \\
\hline standard deviation: & 3.84 \\
\hline min: & 0 \\
\hline 10th percentile: & 1 \\
\hline 25th percentile: & 2 \\
\hline median: & 3 \\
\hline 75th percentile: & 3.5 \\
\hline 90th percentile: & 4 \\
\hline max: & 60 \\
\hline
\end{tabular}




\begin{tabular}{|l|c|}
\hline $\begin{array}{l}\text { Post-audit analysis } \\
\text { and write-up } \\
\text { observations: }\end{array}$ & 345 \\
\hline missing values: & 12 \\
\hline mean: & 2.24 \\
\hline standard deviation: & 4.87 \\
\hline min: & 0 \\
\hline 10th percentile: & .5 \\
\hline 25th percentile: & 1 \\
\hline median: & 2 \\
\hline 75th percentile: & 2 \\
\hline 90th percentile: & 4 \\
\hline max: & 120 \\
\hline
\end{tabular}

\begin{tabular}{|l|c|}
\hline $\begin{array}{l}\text { TOTAL of all } \\
\text { components } \\
\text { observations: }\end{array}$ & 357 \\
\hline missing values: & 0 \\
\hline mean: & 7.81 \\
\hline standard deviation: & 10.74 \\
\hline min: & 0 \\
\hline 10th percentile: & 0 \\
\hline 25th percentile: & 5 \\
\hline median: & 7 \\
\hline 75th percentile: & 8.5 \\
\hline 90th percentile: & 11 \\
\hline max: & 145 \\
\hline
\end{tabular}

6. If your agency used a priority list for at least some dwelling units in Program Year 2008, how difficult was it for your staff to use that priority list? Check best answer.

Freq. Percent Cum.

\begin{tabular}{|c|c|c|c|}
\hline Very difficult & 3.36 & 1.14 & 1.14 \\
\hline Difficult & 17.22 & 5.86 & 7.00 \\
\hline Easy & 202.55 & 68.90 & 75.89 \\
\hline Very Easy & 70.87 & 24.11 & 100.00 \\
\hline & 294 & 100.00 & \\
\hline
\end{tabular}


7. If your agency used a priority list in Program Year 2008, how effective was that list? Check best answer.

\begin{tabular}{rrrr} 
& Freq. & Percent & Cum. \\
\hline Very Ineffective | & 19.22 & 6.56 & 6.56 \\
Ineffective | 15.55 & 5.31 & 11.87 \\
Effective | 197.18 & 67.30 & 79.16 \\
Very Effictive | 61.05 & 20.84 & 100.00 \\
------------- &
\end{tabular}

8. If your agency used a calculation procedure for at least some dwelling units in Program Year 2008, what was the name of the procedure or procedures employed? Check all that apply.

a. AK Warm

\begin{tabular}{|c|c|c|c|}
\hline & Freq. & Percent & Cum. \\
\hline No & 296.86 & 99.28 & 99.28 \\
\hline Yes & 2.14 & 0.72 & 100.00 \\
\hline Total & 299 & 100.00 & \\
\hline
\end{tabular}

b. EA-3

$\begin{array}{ccc}\text { Freq. } & \text { Percent } & \text { Cum. } \\ \text { No } \mid 296.42 & 99.14 & 99.14 \\ \text { Yes } \mid 2.58 & 0.86 & 100.00 \\ ------ \\ \text { Total | } 299 & 100.00\end{array}$

c. EASY

\begin{tabular}{|c|c|c|c|}
\hline & Freq. & Percent & Cum. \\
\hline Nol & 292.19 & 97.72 & 97.72 \\
\hline Yes & 6.81 & 2.28 & 100.00 \\
\hline Total | & 299 & 100.00 & \\
\hline
\end{tabular}

d. EA-QUIP

Freq. Percent Cum.

\begin{tabular}{|c|c|c|c|}
\hline No & 281.88 & 94.27 & 94.27 \\
\hline Yes & 17.12 & 5.73 & 100.00 \\
\hline Total | & 299 & 100.00 & \\
\hline
\end{tabular}


e. HomeCheck

\begin{tabular}{|c|c|c|c|}
\hline & Freq. & Percent & Cum. \\
\hline No & 295.43 & 98.81 & 98.81 \\
\hline Yes & 3.57 & 1.19 & 100.00 \\
\hline Total | & 299 & 100.00 & \\
\hline
\end{tabular}

f. Meadows

\begin{tabular}{|c|c|c|c|}
\hline & Freq. & Percent & Cum. \\
\hline No & 293.65 & 98.21 & 98.21 \\
\hline Yes & 5.35 & 1.79 & 100.00 \\
\hline Total | & 299 & 100.00 & \\
\hline
\end{tabular}

g. REES

\begin{tabular}{|c|c|c|c|}
\hline & Freq. & Percent & Cum. \\
\hline No & 299 & 100.00 & 100.00 \\
\hline Total & 299 & 100.00 & \\
\hline
\end{tabular}

h. REM/Rate

\begin{tabular}{crrr} 
& Freq. & Percent & \multicolumn{1}{c}{ Cum. } \\
No $\mid$ & 284.27 & 95.07 & 95.07 \\
Yes $\mid$ & 14.73 & 4.93 & 100.00 \\
--------- \\
Total | 299 & 100.00 &
\end{tabular}

i. SMOC-ERS

Freq. Percent Cum.

\begin{tabular}{|c|c|c|c|}
\hline No & 296.23 & 99.07 & 99.07 \\
\hline Yes & 2.77 & 0.93 & 100.00 \\
\hline Total | & 299 & 100.00 & \\
\hline
\end{tabular}

j. TIPS

\begin{tabular}{|c|c|c|c|}
\hline & Freq. & Percent & Cum. \\
\hline No & 271.91 & 90.94 & 90.94 \\
\hline Yes & 27.09 & 9.06 & 100.00 \\
\hline Total | & 299 & 100.00 & \\
\hline
\end{tabular}


k. TREAT

\begin{tabular}{|c|c|c|c|}
\hline & Freq. & Percent & Cum. \\
\hline No & 281.23 & 94.06 & 94.06 \\
\hline Yes & 17.77 & 5.94 & 100.00 \\
\hline Total | & 299 & 100.00 & \\
\hline
\end{tabular}

1. Weatherization Assistant (NEAT/MHEA)

\begin{tabular}{|c|c|c|c|}
\hline & Freq. & Percent & Cum. \\
\hline No & 109.73 & 36.70 & 36.70 \\
\hline Yes & 189.27 & 63.30 & 100.00 \\
\hline Vtat & 299 & 100.00 & \\
\hline
\end{tabular}

m. WXEOR

\begin{tabular}{|c|c|c|c|}
\hline & Freq. & Percent & Cum. \\
\hline No & 296.28 & 99.09 & 99.09 \\
\hline Yes & 2.72 & 0.91 & 100.00 \\
\hline Total | & 299 & 100.00 & \\
\hline
\end{tabular}

n. Other (pleases specify)

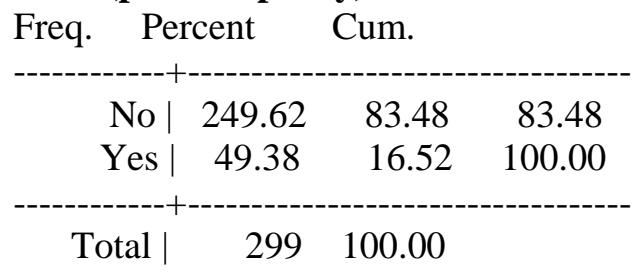

9. If your agency used a calculation procedure in Program Year 2008, use the following scale to describe how difficult it was for your staff to use the applicable procedure(s). Circle best answer. 1= Very Difficult; 2=Difficult; 3= Easy; 4=Very Easy; 5 =N/A

\section{a. AK Warm}

Freq. Percent Cum.

\begin{tabular}{|c|c|c|c|}
\hline Easy & 2.13 & 4.43 & 4.43 \\
\hline Not applicable & 45.87 & 95.57 & 100.00 \\
\hline Total | & 48 & 100.00 & \\
\hline
\end{tabular}


b. EA-3

$\begin{array}{rrrr} & \text { Freq. } & \text { Percent } & \text { Cum. } \\ \text { Easy } \mid & .812 & 1.76 & 1.76 \\ \text { Very Easy } \mid & 1.76 & 3.83 & 5.59 \\ \text { Not applicable } \mid & 43.43 & 94.41 & 100.00 \\ ---------- & \end{array}$

c. EASY

$\begin{array}{rrrr} & \text { Freq. } & \text { Percent } & \text { Cum. } \\ \text { Difficult | } & .42 & 0.87 & 0.87 \\ \text { Easy | } & 5.65 & 11.53 & 12.39 \\ \text { Very Easy | } & .73 & 1.48 & 13.87 \\ \text { Not applicable | } & 42.20 & 86.13 & 100.00 \\ ------------------------------------- \\ \text { Total | } & 49 & 100.00 & \end{array}$

d. EA-QUIP

\begin{tabular}{rrrr} 
& Freq. & Percent & Cum. \\
\hline -----------+-------------------------- \\
Very difficult | & 3.88 & 5.96 & 5.96 \\
Difficult $\mid$ & 3.30 & 5.08 & 11.04 \\
Easy $\mid$ & 3.89 & 5.98 & 17.02 \\
Very Easy $\mid$ & 4.53 & 6.96 & 23.98 \\
Not applicable & 49.41 & 76.02 & 100.00 \\
---------------- \\
Total | & 65 & 100.00 &
\end{tabular}

e. HomeCheck

\begin{tabular}{r|rrr} 
& Freq. & Percent & Cum. \\
Easy & 5.26 & 10.74 & 10.74 \\
Very Easy & 2.44 & 4.97 & 15.71 \\
Not applicable & 41.30 & 84.29 & 100.00 \\
-----+- &
\end{tabular}

f. Meadows

\begin{tabular}{rrrr} 
& Freq. & Percent & Cum. \\
\hline Difficult | & 1.31 & 2.62 & 2.62 \\
Easy $\mid$ & 2.36 & 4.72 & 7.34 \\
Very Easy $\mid$ & 1.58 & 3.17 & 10.51 \\
Not applicable | & 44.75 & 89.49 & 100.00 \\
-----------
\end{tabular}


g. REES

\begin{tabular}{|c|c|c|c|}
\hline & Freq. & Percent & Cum. \\
\hline Not applicable | & 45 & 100.00 & 100.00 \\
\hline Total & 45 & 100.00 & \\
\hline
\end{tabular}

h. REM/Rate

\begin{tabular}{|c|c|c|c|}
\hline & Freq. & Percent & Cum. \\
\hline Very difficult & .45 & 0.83 & 0.83 \\
\hline Difficult & 8.70 & 16.11 & 16.94 \\
\hline Easy & 3.41 & 6.31 & 23.26 \\
\hline Very Easy & .66 & 1.23 & 24.48 \\
\hline Not applicable & 40.78 & 75.52 & 100.00 \\
\hline Total & 54 & 100.00 & \\
\hline
\end{tabular}

i. SMOC-ERS

Freq. Percent Cum.

\begin{tabular}{|c|c|c|c|}
\hline Difficult | & 1.25 & 2.72 & 2.72 \\
\hline Easy & .92 & 1.99 & 4.71 \\
\hline Very Easy & 1.01 & 2.20 & 6.91 \\
\hline Not applicable & 42.82 & 93.09 & 100.00 \\
\hline Total & 46 & 100.00 & \\
\hline
\end{tabular}

j. TIPS

$\begin{array}{rrrr} & \text { Freq. } & \text { Percent } & \text { Cum. } \\ - \text { Difficult } & .63 & 0.65 & 0.65 \\ \text { Easy } \mid & 24.07 & 25.08 & 25.73 \\ \text { Very Easy } \mid & 11.90 & 12.39 & 38.12 \\ \text { Not applicable | } & 59.40 & 61.88 & 100.00 \\ -----------+--------------------------- \\ \text { Total | } & 96 & 100.00 & \end{array}$

k. TREAT

\begin{tabular}{|c|c|c|c|}
\hline & Freq. & Percent & Cum \\
\hline Very difficult & 2.52 & 3.88 & 3.88 \\
\hline Difficult & 4.05 & 6.23 & 10.11 \\
\hline Easy & 11.71 & 18.02 & 28.13 \\
\hline Not applicable & 46.72 & 71.87 & 100.00 \\
\hline Total | & 65 & 100.00 & \\
\hline
\end{tabular}


1. Weatherization Assistant (NEAT/MHEA)

Freq. Percent Cum.

\begin{tabular}{|c|c|c|c|}
\hline Very difficult & 10.00 & 5.98 & 5.98 \\
\hline Difficult & 50.80 & 27.61 & 33.59 \\
\hline Easy & 77.38 & 42.06 & 75.64 \\
\hline Very Easy & 22.05 & 11.99 & 87.63 \\
\hline Not applicable & 22.76 & 12.37 & 100.00 \\
\hline Total & 184 & 100.00 & \\
\hline
\end{tabular}

m. WXEOR

\begin{tabular}{r|rrr} 
& Freq. & Percent & Cum. \\
Difficult | & 3.44 & 6.75 & 6.75 \\
Easy & 3.23 & 6.34 & 13.09 \\
Very Easy $\mid$ & 1.75 & 3.42 & 16.51 \\
Not applicable | & 42.58 & 83.49 & 100.00 \\
---------------- \\
Total | & 51 & 100.00 &
\end{tabular}

n. Other (please specify)

Freq. Percent Cum.

\begin{tabular}{|c|c|c|c|}
\hline Very difficult & 2.21 & 3.88 & 3.88 \\
\hline Difficult & 10.41 & 18.26 & 22.13 \\
\hline Easy & 17.99 & 31.58 & 53.71 \\
\hline Very Easy & 6.31 & 11.08 & 64.79 \\
\hline Not applicable | & 20.07 & 35.21 & 100.00 \\
\hline Total & 57 & 100.00 & \\
\hline
\end{tabular}

10. If your agency used a calculation procedure in Program Year 2008, use the following scale to describe how effective you found the applicable procedure(s). Circle best answer.

1= Very Ineffective; 2=Ineffective; 3= Effective; 4=Very Effective; 5=N/A

\section{a. AK Warm}

Freq. Percent Cum.

\begin{tabular}{r|rrr} 
Effective | & .76 & 1.58 & 1.58 \\
Very Effective | & 1.34 & 2.80 & 4.38 \\
Not applicable | & 45.90 & 95.62 & 100.00 \\
---------------- \\
Total |
\end{tabular}


b. EA-3

\begin{tabular}{r|rrc} 
& Freq. & Percent & Cum. \\
Effective & 1.93 & 4.11 & 4.11 \\
Very Effective | & .62 & 1.33 & 5.44 \\
Not applicable $\mid$ & 44.44 & 94.56 & 100.00 \\
-----------------
\end{tabular}

c. EASY

$\begin{array}{rrrr} & \text { Freq. } & \text { Percent } & \text { Cum. } \\ ------------- \\ \text { Ineffective | } & 2.40 & 4.80 & 4.80 \\ \text { Effective | } & 1.18 & 2.35 & 7.16 \\ \text { Very Effective | } & 2.78 & 5.54 & 12.70 \\ \text { Not applicable | } & 43.65 & 87.30 & 100.00 \\ -------------+-------------------------- \\ \text { Total } & 50 & 100.00 & \end{array}$

d. EA-QUIP

\begin{tabular}{|c|c|c|c|}
\hline & Freq. & Percent & Cum. \\
\hline Very Ineffective | & .96 & 1.46 & 1.46 \\
\hline Ineffective & 2.32 & 3.52 & 4.98 \\
\hline Effective & 6.63 & 10.04 & 15.02 \\
\hline Very Effective & 3.90 & 5.90 & 20.92 \\
\hline Not applicable & 52.19 & 79.08 & 100.00 \\
\hline Total & 66 & 100.00 & \\
\hline
\end{tabular}

\section{e. HomeCheck}

\begin{tabular}{r|rrr} 
& Freq. & Percent & Cum. \\
Effective & 2.08 & 4.24 & 4.24 \\
Very Effective | & 2.54 & 5.19 & 9.44 \\
Not applicable & 44.38 & 90.56 & 100.00 \\
--------------- &
\end{tabular}




\section{f. Meadows}

\begin{tabular}{|c|c|c|c|}
\hline & Freq. & Percent & Cum. \\
\hline Very Ineffective & 1.61 & 3.16 & 3.16 \\
\hline Ineffective & .77 & 1.50 & 4.67 \\
\hline Effective & .54 & 1.05 & 5.72 \\
\hline Very Effective & 2.31 & 4.53 & 10.25 \\
\hline Not applicable & 45.77 & 89.75 & 100.00 \\
\hline Total & 51 & 100.00 & \\
\hline
\end{tabular}

g. REES

\begin{tabular}{cccc} 
& Freq. & Percent & Cum. \\
\hdashline Not applicable | & 46 & 100.00 & 100.00 \\
\hdashline----- & \\
Total | & 46 & 100.00
\end{tabular}

h. REM/Rate

$\begin{array}{rrrr} & \text { Freq. } & \text { Percent } & \text { Cum. } \\ \text {--------------------------------- } \\ \text { Ineffective | } & .66 & 1.20 & 1.20 \\ \text { Effective | } & 12.10 & 22.01 & 23.21 \\ \text { Not applicable | } & 42.23 & 76.79 & 100.00 \\ ------------------------------- \\ \text { Total | } & 55 & 100.00 & \end{array}$

i. SMOC-ERS

Freq. Percent Cum.

\begin{tabular}{|c|c|c|c|}
\hline Very Ineffective & .45 & 0.91 & 0.91 \\
\hline Effective & 1.72 & 3.51 & 4.43 \\
\hline Very Effective & 1.01 & 2.07 & 6.50 \\
\hline Not applicable & 45.82 & 93.50 & 100.00 \\
\hline Total & 49 & 100.00 & \\
\hline
\end{tabular}

j. TIPS

\begin{tabular}{rrrr} 
& Freq. & Percent & Cum. \\
\hline Very Ineffective | & .63 & 0.65 & 0.65 \\
Effective & 20.55 & 21.19 & 21.84 \\
Very Effective | & 15.67 & 16.15 & 37.99 \\
Not applicable | & 60.15 & 62.01 & 100.00 \\
---------- &
\end{tabular}

k. TREAT 


\begin{tabular}{rrrr} 
& Freq. & Percent & Cum. \\
\hline Very Ineffective | & 1.50 & 2.30 & 2.30 \\
Ineffective | & 1.21 & 1.87 & 4.17 \\
Effective | & 13.74 & 21.13 & 25.30 \\
Very Effective | & 1.91 & 2.94 & 28.24 \\
Not applicable | & 46.64 & 71.76 & 100.00 \\
----------- &
\end{tabular}

1. Weatherization Assistant (NEAT/MHEA) Freq. Percent Cum.

\begin{tabular}{|c|c|c|c|}
\hline Very Ineffective | & 7.01 & 3.86 & 3.86 \\
\hline Ineffective & 29.47 & 16.11 & 19.97 \\
\hline Effective & 70.47 & 38.51 & 58.48 \\
\hline Very Effective & 49.24 & 26.91 & 85.38 \\
\hline Not applicable & 26.75 & 14.62 & 100.00 \\
\hline Total | & 183 & 100.00 & \\
\hline
\end{tabular}

m. WXEOR

\begin{tabular}{r|rrr} 
& Freq. & Percent & Cum. \\
Effective & 2.51 & 5.02 & 5.02 \\
Very Effective | & 2.71 & 5.41 & 10.43 \\
Not applicable | & 44.79 & 89.57 & 100.00 \\
----------- \\
Total | & 50 & 100.00 &
\end{tabular}

n. Other (please specify)

Freq. Percent Cum.

\begin{tabular}{|c|c|c|c|}
\hline Very Ineffective & .61 & 1.61 & 1.61 \\
\hline Ineffective & 4.09 & 10.77 & 12.39 \\
\hline Effective & 19.73 & 51.93 & 64.32 \\
\hline Very Effective & 6.98 & 18.37 & 82.69 \\
\hline Not applicable & 6.58 & 17.31 & 100.00 \\
\hline Tota & 38 & 100.00 & \\
\hline
\end{tabular}


11. If your agency used a calculation procedure for at least some dwelling units in Program Year 2008, did your state allow under DOE rules the installation of general heat waste measures (low-cost/nocost weatherization activities) in those units without the need for an energy justification?

\begin{tabular}{crr} 
Freq. & Percent & \multicolumn{1}{c}{ Cum. } \\
no | 115.06 & 38.74 & 38.74 \\
yes | 181.94 & 61.26 & 100.00 \\
-------- \\
Total | 297 & 100.00 &
\end{tabular}

12. Please indicate which of the following general heat waste measures your agency was allowed to install in Program Year 2008. Check all that apply.

\section{- Weatherstripping}

\begin{tabular}{|c|c|c|c|}
\hline & Freq. & Percent & Cum. \\
\hline unchecked & 8.43 & 3.81 & 3.81 \\
\hline checked & 212.57 & 96.19 & 100.00 \\
\hline Total & 221 & 100.00 & \\
\hline
\end{tabular}

\section{- Caulking}

\begin{tabular}{|c|c|c|c|}
\hline & Freq. & Percent & Cum. \\
\hline unchecked & 28.61 & 12.95 & 12.95 \\
\hline checked & 192.39 & 87.05 & 100.00 \\
\hline Total & 221 & 100.00 & \\
\hline
\end{tabular}

- Insulation for plugging air leaks

\begin{tabular}{|c|c|c|c|}
\hline & Freq. & Percent & Cum \\
\hline unchecked & 28.61 & 12.95 & 12.95 \\
\hline checked & 192.39 & 87.05 & 100.00 \\
\hline Total | & 221 & 100.00 & \\
\hline
\end{tabular}

- Low-flow shower heads

\begin{tabular}{|c|c|c|c|}
\hline & Freq. & Percent & Cum. \\
\hline unchecked & 54.18 & 24.52 & 24.52 \\
\hline checked & 166.82 & 75.48 & 100.00 \\
\hline Total & 221 & 100.00 & \\
\hline
\end{tabular}


- Low-flow faucet aerators

\begin{tabular}{|c|c|c|c|}
\hline & Freq. & Percent & Cum. \\
\hline unchecked | & 75.91 & 34.35 & 34.35 \\
\hline checked & 145.09 & 65.65 & 100.00 \\
\hline Total & 221 & 100.00 & \\
\hline
\end{tabular}

- Air filters

\begin{tabular}{|c|c|c|c|}
\hline Freq & Percent & \multicolumn{2}{|c|}{ Cum. } \\
\hline unchecked | & 69.86 & 31.61 & 31.61 \\
\hline checked & 151.14 & 68.39 & 100.00 \\
\hline Total | & 221 & 100.00 & \\
\hline
\end{tabular}

- Glass patching

\begin{tabular}{crr} 
Freq. & Percent & \multicolumn{1}{c}{ Cum. } \\
\hline-------- \\
unchecked | 56.72 & 25.66 & 25.66 \\
checked | 164.28 & 74.34 & 100.00 \\
-------------------------- & \\
Total | 221 & 100.00
\end{tabular}

- Lighting

\begin{tabular}{|c|c|c|c|}
\hline Freq & Percent & \multicolumn{2}{|c|}{ Cum. } \\
\hline unchecked & 40.87 & 18.49 & 18.49 \\
\hline checked & 180.13 & 81.51 & 100.00 \\
\hline Total & 221 & 100.00 & \\
\hline
\end{tabular}

- Hot water tank insulation (water heater wrap)

\begin{tabular}{|c|c|c|c|}
\hline Freq & Percent & \multicolumn{2}{|c|}{ Cum. } \\
\hline unchecked & 34.53 & 15.62 & 15.62 \\
\hline checked & 186.47 & 84.38 & 100.00 \\
\hline Total | & 221 & 100.00 & \\
\hline
\end{tabular}


- Water pipe insulation

\begin{tabular}{|c|c|c|c|}
\hline & Freq. & Percent & Cum. \\
\hline unchecked & 21.35 & 9.66 & 9.66 \\
\hline checked & 199.65 & 90.34 & 100.00 \\
\hline IOtal & 221 & 100.00 & \\
\hline
\end{tabular}

- Other (please specify)

\begin{tabular}{|c|c|c|c|}
\hline & Freq. & Percent & Cum. \\
\hline unchecked & 204.74 & 92.64 & 92.64 \\
\hline checked & 16.26 & 7.36 & 100.00 \\
\hline Total | & 221 & 100.00 & \\
\hline
\end{tabular}

What was the primary justification used by your agency in Program Year 2008 for performing work specifically targeted at reducing air infiltration (i.e., air sealing work)? Check best answer.

Freq. Percent Cum.

\begin{tabular}{|c|c|c|c|}
\hline air leakage rate as measured by a blower & 271.16 & 80.46 & 80.46 \\
\hline work should be performed to address occ & 3.15 & 0.93 & 81.40 \\
\hline all significant air leakage sites shoul & 30.56 & 9.07 & 90.46 \\
\hline air sealing work should be performed on & 27.24 & 8.08 & 98.54 \\
\hline Other & 4.90 & 1.46 & 100.00 \\
\hline Tota & 337 & 100.00 & \\
\hline
\end{tabular}

13. What other justifications were used by your agency in Program Year 2008 for performing work specifically targeted at reducing air infiltration (i.e., air sealing work)? Check all that apply.

- Work should be performed where the air leakage rate as measured by a blower door test is greater than a minimum number (e.g., minimum ventilation guideline) calculated for the dwelling unit in question

\begin{tabular}{|c|c|c|c|}
\hline & Freq. & Percent & Cum. \\
\hline no & 268.17 & 83.28 & 83.28 \\
\hline yes & 53.83 & 16.72 & 100.00 \\
\hline Total & 322 & 100.00 & \\
\hline
\end{tabular}


- Work should be performed to address occupant complaints

\begin{tabular}{|c|c|c|c|}
\hline & Freq. & Percent & Cum. \\
\hline no & 176.13 & 54.70 & 54.70 \\
\hline yes & 145.87 & 45.30 & 100.00 \\
\hline Total & 322 & 100.00 & \\
\hline
\end{tabular}

- All significant air leakage sites should be sealed

\begin{tabular}{|c|c|c|c|}
\hline & Freq. & Percent & Cum. \\
\hline no & 107.45 & 33.37 & 33.37 \\
\hline yes & 214.55 & 66.63 & 100.00 \\
\hline Total | & 322 & 100.00 & \\
\hline
\end{tabular}

- Air sealing work should be performed on all dwelling units

\begin{tabular}{|c|c|c|c|}
\hline & Freq. & Percent & Cum. \\
\hline no & 206.68 & 64.19 & 64.19 \\
\hline yes & 115.32 & 35.81 & 100.00 \\
\hline Total | & 322 & 100.00 & \\
\hline
\end{tabular}

- Other (please specify)

$\begin{array}{rrrr} & \text { Freq. } & \text { Percent } & \text { Cum. } \\ \text { no | } 302.78 & 94.03 & 94.03 \\ \text { yes | } 19.22 & 5.97 & 100.00 \\ --------- \\ \text { Total| } & 322 & 100.00 & \end{array}$

14. What was the primary method used by your agency in Program Year 2008 to identify air leakage sites to seal? Check only one.

Freq. Percent Cum.

\begin{tabular}{|c|c|c|c|}
\hline Auditor identified air leakage sites vi | & 11.44 & 3.37 & 3.37 \\
\hline Auditor identified air leakage sites us & 252.11 & 74.37 & 77.74 \\
\hline Crew identified air leakage sites visua & 2.59 & 0.76 & 78.51 \\
\hline Crew identified air leakage sites using & 65.37 & 19.28 & 97.79 \\
\hline Other $\mid$ & 7.48 & 2.21 & 100.00 \\
\hline Total $\mid$ & 339 & 100.00 & \\
\hline
\end{tabular}


15. What other methods were used by your agency in Program Year 2008 to identify air leakage sites to seal? Check all that apply.

- Auditor identified air leakage sites visually and communicated relevant information to crew

$\begin{array}{rrr}\text { Freq. } & \text { Percent } & \text { Cum. } \\ \text { no | } 139.78 & 41.73 & 41.73 \\ \text { yes | } 195.22 & 58.27 & 100.00 \\ ---------- \\ \text { Total | } 335 & 100.00 & \end{array}$

- Auditor identified air leakage sites using a blower door and/or pressure diagnostics and communicated relevant information to crew

\begin{tabular}{|c|c|c|c|}
\hline & Freq. & Percent & Cum. \\
\hline no & 273.96 & 81.78 & 81.78 \\
\hline yes & 61.04 & 18.22 & 100.00 \\
\hline Total & 335 & 100.00 & \\
\hline
\end{tabular}

- Crew identified air leakage sites visually

\begin{tabular}{ccc} 
Freq. & Percent & \multicolumn{1}{c}{ Cum. } \\
no $\mid 180.10$ & 53.76 & 53.76 \\
yes | 154.90 & 46.24 & 100.00 \\
----------- \\
Total $\quad 335$ & 100.00
\end{tabular}

- Crew identified air leakage sites using a blower door and/or pressure diagnostics

\begin{tabular}{|c|c|c|c|}
\hline & Freq. & Percent & Cum. \\
\hline no & 163.02 & 48.66 & 48.66 \\
\hline yes & 171.98 & 51.34 & 100.00 \\
\hline Total & 335 & 100.00 & \\
\hline
\end{tabular}

- Other (please specify)

\begin{tabular}{|c|c|c|c|}
\hline & Freq. & Percent & Cum. \\
\hline no & 316.00 & 94.33 & 94.33 \\
\hline yes & 19.00 & 5.67 & 100.00 \\
\hline Total & 335 & 100.00 & \\
\hline
\end{tabular}


16. In Program Year 2008, at what point did your agency stop performing air sealing work on a given dwelling unit? Check all that apply.

- When all identified air leakage sites were sealed

\begin{tabular}{|c|c|c|c|}
\hline & Freq. & Percent & Cum. \\
\hline no & 234.24 & 69.51 & 69.51 \\
\hline yes & 102.764 & 30.49 & 100.00 \\
\hline Total & 337 & 100.00 & \\
\hline
\end{tabular}

- When all significant air leakage sites were sealed

\begin{tabular}{|c|c|c|c|}
\hline Freq. & Percent & Cum. & \\
\hline no & 215.73 & 64.02 & 64.02 \\
\hline yes | & 121.27 & 35.98 & 100.00 \\
\hline Total & 337 & 100.00 & \\
\hline
\end{tabular}

- When the air leakage rate as measured by a blower door test dropped below a minimum number calculated for the dwelling unit in question

\begin{tabular}{|c|c|c|c|}
\hline Freq. & Percent & Cum. & \\
\hline no $\mid$ & 94.58 & 28.06 & 28.06 \\
\hline yes & 242.42 & 71.94 & 100.00 \\
\hline Total | & 337 & 100.00 & \\
\hline
\end{tabular}

- When a blower door test indicated that the most recent infiltration reduction measure installed in the dwelling unit was not cost effective

\begin{tabular}{|c|c|c|c|}
\hline & Freq. & Percent & Cum. \\
\hline no & 220.47 & 65.42 & 65.42 \\
\hline yes & 116.53 & 34.58 & 100.00 \\
\hline Total & 337 & 100.00 & \\
\hline
\end{tabular}

- Other (please specify)

Freq. Percent Cum.

\begin{tabular}{|c|c|c|c|}
\hline no & 324.48 & 96.28 & 96.28 \\
\hline yes | & 12.52 & 3.72 & 100.00 \\
\hline Total | & 337 & 100.00 & \\
\hline
\end{tabular}


17. Did your agency do duct sealing work in Program Year 2008?

\begin{tabular}{|c|c|c|c|}
\hline & Freq. & Percent & Cum. \\
\hline no & 40.22 & 11.97 & 11.97 \\
\hline yes & 295.78 & 88.03 & 100.00 \\
\hline Total & 336 & 100.00 & \\
\hline
\end{tabular}

18. How did your agency determine when duct sealing work was needed for a particular dwelling unit in Program Year 2008? Check all that apply.

- All houses with ducts received duct sealing measures

\begin{tabular}{|c|c|c|c|}
\hline & Freq. & Percent & Cum. \\
\hline no & 199.23 & 67.53 & 67.53 \\
\hline yes | & 95.77 & 32.47 & 100.00 \\
\hline Total & 295 & 100.00 & \\
\hline
\end{tabular}

- All houses with return air ducts get sealed

\begin{tabular}{|c|c|c|c|}
\hline & Freq. & Percent & Cum. \\
\hline no & 223.32 & & 75.70 \\
\hline yes & 71.68 & 24.30 & 100.00 \\
\hline
\end{tabular}

- Ducts were sealed in those cases where leakage sites were visible

\begin{tabular}{|c|c|c|c|}
\hline & Freq. & Percent & Cum. \\
\hline no & 182.27 & 61.79 & 61.79 \\
\hline yes & 112.73 & 38.21 & 100.00 \\
\hline Total & 295 & 100.00 & \\
\hline
\end{tabular}

- Ducts were sealed when a blower door test indicated the presence of leaks

$\begin{array}{ccc}\text { Freq. } & \text { Percent } & \text { Cum. } \\ \text { no } \mid 136.44 & 46.25 & 46.25 \\ \text { yes } \mid 158.56 & 53.75 & 100.00 \\ ---------- \\ \text { Total | } 295 & 100.00\end{array}$


- Ducts were sealed when duct diagnostics (blower door subtraction, duct blower, or pressure pan measurements) indicated that the leakage rate was greater than a minimum number calculated for the dwelling unit in question

\begin{tabular}{|c|c|c|c|}
\hline & Freq. & Percent & Cum. \\
\hline no & 118.56 & 40.19 & 40.19 \\
\hline yes & 176.44 & 59.81 & 100.00 \\
\hline Total & 295 & 100.00 & \\
\hline
\end{tabular}

19. What methods were used by your agency in Program Year 2008 to identify duct leakage sites to seal? Check all that apply.

- Auditor identified duct leakage sites visually and communicated relevant information to crew

\begin{tabular}{|c|c|c|c|}
\hline & Freq. & Percent & Cum. \\
\hline no & 132.43 & 44.89 & 44.89 \\
\hline yes | & 162.57 & 55.11 & 100.00 \\
\hline Total | & 295 & 100.00 & \\
\hline
\end{tabular}

- Auditor identified duct leakage sites using a blower door and communicated relevant information to crew

Freq. Percent Cum.

$\begin{array}{ccc}\text { no | } 109.65 & 37.17 & 37.17 \\ \text { yes | } 185.35 & 62.83 & 100.00 \\ ------ & \\ \text { Total| } 295 & 100.00\end{array}$

- Auditor identified duct leakage sites using duct diagnostics and communicated relevant information to crew

Freq. Percent Cum.

\begin{tabular}{|c|c|c|c|}
\hline no & 151.87 & 51.48 & 51.48 \\
\hline yes & 143.13 & 48.52 & 100.00 \\
\hline Total & 295 & 100.00 & \\
\hline
\end{tabular}

- Crew identified duct leakage sites visually

\begin{tabular}{|c|c|c|c|}
\hline & Freq. & Percent & Cum. \\
\hline no & 185.23 & 62.79 & 62.79 \\
\hline yes & 109.77 & 37.21 & 100.00 \\
\hline Total | & 295 & 100.00 & \\
\hline
\end{tabular}


- Crew identified duct leakage sites using a blower door

\begin{tabular}{|c|c|c|c|}
\hline & Freq. & Percent & Cum. \\
\hline no & 181.51 & 61.53 & 61.53 \\
\hline yes & 113.49 & 38.47 & 100.00 \\
\hline Total & 295 & 100.00 & \\
\hline
\end{tabular}

- Crew identified duct leakage sites using duct diagnostics Freq. Percent Cum.

\begin{tabular}{|c|c|c|c|}
\hline no & $\begin{array}{r}216.59 \\
78\end{array}$ & $\begin{array}{r}73.42 \\
26.58\end{array}$ & $\begin{array}{r}73.42 \\
\end{array}$ \\
\hline & 205 & 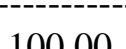 & \\
\hline
\end{tabular}

- Other (please specify)

Freq. Percent Cum.

$\begin{array}{rrr}\text { no | } 283.01 & 95.94 & 95.94 \\ \text { yes | } 11.99 & 4.06 & 100.00 \\ -- & \end{array}$

20. In Program Year 2008, at what point did your agency stop performing duct sealing work on a given dwelling unit? Check all that apply.

- When all identified duct leakage sites were sealed

\begin{tabular}{ccc}
\multicolumn{1}{c}{ Freq. } & Percent & Cum. \\
no | 115.86 & 39.54 & 39.54 \\
yes | 177.14 & 60.46 & 100.00 \\
------------- \\
Total| 293 & 100.00
\end{tabular}

- When a blower door test indicated no more flow from the ducts

Freq. Percent Cum.

$\begin{array}{rrr}\text { no | } 189.91 & 64.81 & 64.81 \\ \text { yes | } 103.09 & 35.19 & 100.00 \\ ------ & \end{array}$


- When the duct leakage rate as measured by duct diagnostics dropped below a minimum number calculated for the dwelling unit in question

\begin{tabular}{|c|c|c|c|}
\hline & Freq. & Percent & Cum. \\
\hline no & 127.78 & 43.61 & 43.61 \\
\hline yes & 165.22 & 56.39 & 100.00 \\
\hline Total & 293 & 100.00 & \\
\hline
\end{tabular}

- Other (please specify)

Freq. Percent Cum.

\begin{tabular}{|c|c|c|c|}
\hline $\begin{array}{c}\text { no } \\
\text { ves }\end{array}$ & 285.96 & 97.60 & $\begin{array}{r}97.60 \\
\end{array}$ \\
\hline Total & 293 & 100.00 & \\
\hline
\end{tabular}

21. How did you determine when a particular refrigerator should be replaced in Program Year 2008? Check all that apply.

- Not allowed to replace refrigerators

\begin{tabular}{|c|c|c|c|}
\hline & Freq. & Percent & Cum. \\
\hline no & 270.03 & 81.34 & 81.34 \\
\hline yes & 61.97 & 18.66 & 100.00 \\
\hline Total & 332 & 100.00 & \\
\hline
\end{tabular}

- Energy use of existing refrigerator was metered

$\begin{array}{ccc}\text { Freq. } & \text { Percent } & \text { Cum. } \\ \text { no } \mid 117.84 & 35.49 & 35.49 \\ \text { yes } \mid 214.16 & 64.51 & 100.00 \\ ---------- \\ \text { Total | } 332 & 100.00\end{array}$

- Energy use of existing refrigerator was assumed base on rated/nameplate value

\begin{tabular}{|c|c|c|c|}
\hline & Freq. & Percent & Cum. \\
\hline no & 260.89 & 78.58 & 78.58 \\
\hline yes & 71.11 & 21.42 & 100.00 \\
\hline Total & 332 & 100.00 & \\
\hline
\end{tabular}


- Non-energy criteria were used (e.g., age, color, physical appearance)

\begin{tabular}{crrr} 
& Freq. & Percent & Cum. \\
no | 302.30 & 91.05 & 91.05 \\
yes | 29.70 & 8.95 & 100.00 \\
- \hdashline--- \\
Total | 332 & 100.00 &
\end{tabular}

- Refrigerator was replaced if it was no longer running or could not maintain desired temperature

\begin{tabular}{|c|c|c|c|}
\hline & Freq. & Percent & Cum. \\
\hline no & 267.95 & 80.71 & 80.71 \\
\hline yes & 64.05 & 19.29 & 100.00 \\
\hline Total | & 332 & 100.00 & \\
\hline
\end{tabular}

- Other (please specify)

\begin{tabular}{crr} 
Freq. & Percent & Cum. \\
no | 275.61 & 83.01 & 83.01 \\
yes | 56.39 & 16.99 & 100.00 \\
- \hdashline--- \\
Total| 332 & 100.00 &
\end{tabular}

22a. How did you determine when a particular air conditioner should be replaced in Program Year 2008 ? Check all that apply.

- Not allowed to replace air conditioner

$\begin{array}{ccc}\text { Freq. } & \text { Percent } & \text { Cum. } \\ \text { no } \mid 143.28 & 42.52 & 42.52 \\ \text { yes | } 193.72 & 57.48 & 100.00 \\ ---------- \\ \text { Total | } 337 & 100.00\end{array}$

- $\quad$ Energy use of existing air conditioner was metered

\begin{tabular}{|c|c|c|c|}
\hline & Freq. & Percent & Cum. \\
\hline no & 324.60 & 96.32 & 96.32 \\
\hline yes & 12.40 & 3.68 & 100.00 \\
\hline Total | & 337 & 100.00 & \\
\hline
\end{tabular}


- Energy use of existing air conditioner was assumed base on rated/nameplate value

\begin{tabular}{|c|c|c|c|}
\hline & Freq. & Percent & Cum. \\
\hline no & 316.68 & 93.97 & 93.97 \\
\hline yes & 20.32 & 6.03 & 100.00 \\
\hline Total & 337 & 100.00 & \\
\hline
\end{tabular}

- Non-energy criteria were used (e.g., age, physical appearance)

\begin{tabular}{|c|c|c|c|}
\hline & Freq. & Percent & Cum. \\
\hline no & 327.85 & 97.29 & 97.29 \\
\hline yes & 9.15 & 2.71 & 100.00 \\
\hline Total & 337 & 100.00 & \\
\hline
\end{tabular}

- Air conditioner was replaced if it was no longer running or could not maintain desired temperature

\begin{tabular}{|c|c|c|c|}
\hline & Freq. & Percent & Cum. \\
\hline no & 305.93 & 90.78 & 90.78 \\
\hline yes & 31.07 & 9.22 & 100.00 \\
\hline Total | & 337 & 100.00 & \\
\hline
\end{tabular}

- Other (please specify)

\begin{tabular}{|c|c|c|c|}
\hline & Freq. & Percent & Cum. \\
\hline no & 299.04 & 88.74 & 88.74 \\
\hline yes $\mid$ & 37.96 & 11.26 & 100.00 \\
\hline Total & 337 & 100.00 & \\
\hline
\end{tabular}

- Not applicable

\begin{tabular}{|c|c|c|c|}
\hline & Freq. & Percent & Cum. \\
\hline no & 299.04 & 88.74 & 88.74 \\
\hline yes & 37.96 & 11.26 & 100.00 \\
\hline Total & 337 & 100.00 & \\
\hline
\end{tabular}


22. Which of the following diagnostic procedures did your agency perform in Program Year 2008? Check all that apply.

Pressure diagnostics:

- Blower door (house air leakage rate)

\begin{tabular}{|c|c|c|c|}
\hline & Freq. & Percent & Cum. \\
\hline no & 4.50 & 1.33 & 1.33 \\
\hline yes & 334.50 & 98.67 & 100.00 \\
\hline Total & 339 & 100.00 & \\
\hline
\end{tabular}

- Zonal pressure measurements

\begin{tabular}{|c|c|c|c|}
\hline & Freq. & Percent & Cum. \\
\hline no & 141.58 & 41.77 & 41.77 \\
\hline yes & 197.42 & 58.23 & 100.00 \\
\hline Total & 339 & 100.00 & \\
\hline
\end{tabular}

- Room-to-room pressure measurements (distribution balancing)

\begin{tabular}{|c|c|c|c|}
\hline & Freq. & Percent & Cum. \\
\hline no & 186.88 & 55.13 & 55.13 \\
\hline yes & 152.12 & 44.87 & 100.00 \\
\hline Total & 339 & 100.00 & \\
\hline
\end{tabular}

- Duct pressure pan measurements

\begin{tabular}{|c|c|c|c|}
\hline & Freq. & Percent & Cum. \\
\hline no & 137.72 & 40.62 & 40.62 \\
\hline yes & 201.28 & 59.38 & 100.00 \\
\hline Total & 339 & 100.00 & \\
\hline
\end{tabular}

- Duct blower measurements (duct air leakage rate)

$\begin{array}{ccc}\text { Freq. } & \text { Percent } & \text { Cum. } \\ \text { no | } 228.37 & 67.36 & 67.36 \\ \text { yes | } 110.63 & 32.64 & 100.00 \\ -------------- \\ \text { Total| } 339 & 100.00 & \end{array}$


Space-heating system:

- Flue gas analysis (steady-state efficiency measurements)

\begin{tabular}{|c|c|c|c|}
\hline & Freq. & Percent & Cum. \\
\hline no & 56.29 & 16.61 & 16.61 \\
\hline yes & 282.71 & 83.39 & 100.00 \\
\hline Total | & 339 & 100.00 & \\
\hline
\end{tabular}

- Heat rise measurements

\begin{tabular}{|c|c|c|c|}
\hline & Freq. & Percent & Cum. \\
\hline no & 153.12 & 45.17 & 45.17 \\
\hline yes & 185.88 & 54.83 & 100.00 \\
\hline Total & 339 & 100.00 & \\
\hline
\end{tabular}

- CO measurements in flues

\begin{tabular}{|c|c|c|c|}
\hline & Freq. & Percent & Cum. \\
\hline no & 30.36 & 8.96 & 8.96 \\
\hline yes & 308.64 & 91.04 & 100.00 \\
\hline Total | & 339 & 100.00 & \\
\hline
\end{tabular}

- Draft/spillage (normal operation)

\begin{tabular}{|c|c|c|c|}
\hline & Freq. & Percent & Cum. \\
\hline no & 75.22 & 22.19 & 22.19 \\
\hline yes & 263.78 & 77.81 & 100.00 \\
\hline Total | & 339 & 100.00 & \\
\hline
\end{tabular}

Air-conditioning system:

- Refrigerant charge (e.g., superheat, subcooling)

$\begin{array}{cccc} & \text { Freq. } & \text { Percent } & \text { Cum. } \\ \text { no | } & 306.23 & 90.33 & 90.33 \\ \text { yes } \mid & 32.77 & 9.67 & 100.00 \\ ------ & \end{array}$


HVAC components and cross-cutting diagnostics:

- Air handler flow rate

\begin{tabular}{|c|c|c|c|}
\hline & Freq. & Percent & Cum. \\
\hline no & 259.97 & 76.69 & 76.69 \\
\hline yes & 79.03 & 23.31 & 100.00 \\
\hline Total & 339 & 100.00 & \\
\hline
\end{tabular}

- Thermostat anticipator current

\begin{tabular}{|c|c|c|c|}
\hline & Freq. & Percent & Cum. \\
\hline no & 232.19 & 68.49 & 68.49 \\
\hline yes & 106.81 & 31.51 & 100.00 \\
\hline Total & 339 & 100.00 & \\
\hline
\end{tabular}

- Worst case draft/spillage (CAZ)

$\begin{array}{ccc}\text { Freq. } & \text { Percent } & \text { Cum. } \\ \text { no | } 124.54 & 36.74 & 36.74 \\ \text { yes | } 214.46 & 63.26 & 100.00 \\ ------------- \\ \text { Total | } 339 & 100.00 & \end{array}$

Hot-water (water-heating) system:

- Flue gas analysis (steady-state efficiency measurements)

\begin{tabular}{|c|c|c|c|}
\hline & Freq. & Percent & Cum. \\
\hline no & 95.20 & 28.08 & 28.08 \\
\hline yes & 243.80 & 71.92 & 100.00 \\
\hline Total & 339 & 100.00 & \\
\hline
\end{tabular}

- CO measurements in flues

\begin{tabular}{|c|c|c|c|}
\hline & Freq. & Percent & Cum. \\
\hline no & 40.20 & 11.86 & 11.86 \\
\hline yes & 298.80 & 88.14 & 100.00 \\
\hline Total | & 339 & 100.00 & \\
\hline
\end{tabular}


- Draft/spillage (normal operation)

\begin{tabular}{rrrr} 
Freq. & Percent & \multicolumn{1}{c}{ Cum. } & \\
no $\mid$ & 77.08 & 22.74 & 22.74 \\
yes | 261.92 & 77.26 & 100.00 \\
------ &
\end{tabular}

- Water flow rates (showerheads and faucets)

Freq. Percent Cum.

$\begin{array}{crr}\text { no | } 257.16 & 75.86 & 75.86 \\ \text { yes | } 81.84 & 24.14 & 100.00 \\ ---------------- \\ \text { Total| } 339 & 100.00\end{array}$

Other CO measurements:

- CO measurements in equipment rooms

\begin{tabular}{ccc} 
Freq. & Percent & Cum. \\
\hline no | 106.73 & 31.48 & 31.48 \\
yes | 232.27 & 68.52 & 100.00 \\
--------------------------------- \\
Total| 339 & 100.00
\end{tabular}

- Cooking stove

\begin{tabular}{|c|c|c|c|}
\hline & Freq. & Percent & Cum. \\
\hline no & 59.77 & 17.63 & 17.63 \\
\hline yes & 279.23 & 82.37 & 100.00 \\
\hline Total & 339 & 100.00 & \\
\hline
\end{tabular}

- $\quad$ CO measurements in living areas

\begin{tabular}{|c|c|c|c|}
\hline & Freq. & Percent & Cum. \\
\hline no & 75.76 & 22.35 & 22.35 \\
\hline yes & 263.24 & 77.65 & 100.00 \\
\hline Total | & 339 & 100.00 & \\
\hline
\end{tabular}


Other diagnostics and inspections:

- Refrigerator energy use

\begin{tabular}{|c|c|c|c|}
\hline & Freq. & Percent & Cum. \\
\hline no & 127.01 & 37.47 & 37.47 \\
\hline yes & 211.99 & 62.53 & 100.00 \\
\hline Total & 339 & 100.00 & \\
\hline
\end{tabular}

- Exhaust fan air flow rate measurement

\begin{tabular}{|c|c|c|c|}
\hline & Freq. & Percent & Cum. \\
\hline no & 231.42 & 68.26 & 68.26 \\
\hline yes | & 107.58 & 31.74 & 100.00 \\
\hline Total | & 339 & 100.00 & \\
\hline
\end{tabular}

- Infrared scanning (camera)

$\begin{array}{ccc}\text { Freq. } & \text { Percent } & \text { Cum. } \\ \text { no | } 167.73 & 49.48 & 49.48 \\ \text { yes | } 171.27 & 50.52 & 100.00 \\ -------- \\ \text { Total | } 339 & 100.00\end{array}$

- Radon testing

\begin{tabular}{|c|c|c|c|}
\hline & Freq. & Percent & Cum. \\
\hline no & 329.18 & 97.10 & 97.10 \\
\hline yes & 9.82 & 2.90 & 100.00 \\
\hline Total & 339 & 100.00 & \\
\hline
\end{tabular}

- Lead

\begin{tabular}{|c|c|c|c|}
\hline & Freq. & Percent & Cum. \\
\hline no & 275.41 & 81.24 & 81.24 \\
\hline yes $\mid$ & 63.59 & 18.76 & 100.00 \\
\hline Total | & 339 & 100.00 & \\
\hline
\end{tabular}


- Mold and mildew testing

\begin{tabular}{|c|c|c|c|}
\hline & Freq. & Percent & Cum. \\
\hline no & 303.64 & 89.57 & 89.57 \\
\hline yes & 35.36 & 10.43 & 100.00 \\
\hline Total & 339 & 100.00 & \\
\hline
\end{tabular}

- Moisture content testing

\begin{tabular}{|c|c|c|c|}
\hline & Freq. & Percent & Cum. \\
\hline no & 286.77 & 84.59 & 84.59 \\
\hline yes & 52.23 & 15.41 & 100.00 \\
\hline Total | & 339 & 100.00 & \\
\hline
\end{tabular}

- Other (please specify)

\begin{tabular}{|c|c|c|c|}
\hline & Freq. & Percent & Cum. \\
\hline no & 330.81 & 97.58 & 97.58 \\
\hline yes & 8.19 & 2.42 & 100.00 \\
\hline Total & 339 & 100.00 & \\
\hline
\end{tabular}

23. Which of the diagnostic procedures listed below were performed for the first time by your agency in Program Year 2008 or in either of the two years prior to Program Year 2008? If your agency did not use a particular procedure, leave that item blank.

Check all that apply.

Pressure diagnostics:

- Blower door (house air leakage rate)

\begin{tabular}{|c|c|c|c|}
\hline & Freq. & Percent & Cum. \\
\hline no & 88.33 & 58.11 & 58.11 \\
\hline yes & 63.67 & 41.89 & 100.00 \\
\hline Total & 152 & 100.00 & \\
\hline
\end{tabular}

- Zonal pressure measurements

\begin{tabular}{|c|c|c|c|}
\hline & Freq. & Percent & Cum. \\
\hline no & 99.17 & 65.25 & 65.25 \\
\hline yes & 52.83 & 34.75 & 100.00 \\
\hline Total & 152 & 100.00 & \\
\hline
\end{tabular}


- Room-to-room pressure measurements (distribution balancing)

\begin{tabular}{|c|c|c|c|}
\hline & Freq. & Percent & Cum. \\
\hline no & 114.31 & 75.20 & 75.20 \\
\hline yes & 37.69 & 24.80 & 100.00 \\
\hline Total & 152 & 100.00 & \\
\hline
\end{tabular}

- Duct pressure pan measurements

\begin{tabular}{|c|c|c|c|}
\hline & Freq. & Percent & Cum. \\
\hline no & 101.03 & 66.47 & 66.47 \\
\hline yes & 50.97 & 33.53 & 100.00 \\
\hline Total | & 152 & 100.00 & \\
\hline
\end{tabular}

- Duct blower measurements (duct air leakage rate)

$\begin{array}{ccc}\text { Freq. } & \text { Percent } & \text { Cum. } \\ \text { no | } 114.87 & 75.58 & 75.58 \\ \text { yes | } 37.11 & 24.42 & 100.00 \\ ---------- \\ \text { Total| } 152 & 100.00\end{array}$

Space-heating system:

- Flue gas analysis (steady-state efficiency measurements)

$\begin{array}{ccc}\text { Freq. } & \text { Percent } & \text { Cum. } \\ \text { no | } 106.88 & 70.32 & 70.32 \\ \text { yes | } 45.12 & 29.68 & 100.00 \\ ---------- \\ \text { Total| } 152 & 100.00\end{array}$

- Heat rise measurements

$\begin{array}{rrr}\text { Freq. } & \text { Percent } & \text { Cum. } \\ \text { no | } 122.51 & 80.60 & 80.60 \\ \text { yes | } 29.49 & 19.40 & 100.00 \\ ---------- \\ \text { Total| } 152 & 100.00\end{array}$


- CO measurements in flues

\begin{tabular}{|c|c|c|c|}
\hline & Freq. & Percent & Cum. \\
\hline no & 88.22 & 58.04 & 58.04 \\
\hline yes & 63.78 & 41.96 & 100.00 \\
\hline Ot & 152 & 100.00 & \\
\hline
\end{tabular}

- Draft/spillage (normal operation) Freq. Percent Cum.

\begin{tabular}{|c|c|c|c|}
\hline no & 106.71 & 70.20 & 70.20 \\
\hline yes | & 45.29 & 29.80 & 100.00 \\
\hline
\end{tabular}

Air-conditioning system:

- Refrigerant charge (e.g., superheat, subcooling) Freq. Percent Cum.

\begin{tabular}{|c|c|c|c|}
\hline no & 142.65 & 93.85 & 93.85 \\
\hline yes | & 9.35 & 6.15 & 100.00 \\
\hline otal & 152 & 100.00 & \\
\hline
\end{tabular}

HVAC components and cross-cutting diagnostics:

- Air handler flow rate

\begin{tabular}{|c|c|c|c|}
\hline & Freq. & Percent & Cum. \\
\hline no & 135.24 & 88.98 & 88.98 \\
\hline yes & 16.76 & 11.02 & 100.00 \\
\hline Total & 152 & 100.00 & \\
\hline
\end{tabular}

- Thermostat anticipator current

Freq. Percent Cum.

\begin{tabular}{|c|c|c|c|}
\hline no & 132.67 & 87.28 & 87.28 \\
\hline yes & 19.33 & 12.72 & 100.00 \\
\hline Total & 152 & 100.00 & \\
\hline
\end{tabular}

- Worst case draft/spillage (CAZ)

Freq. Percent Cum.

\begin{tabular}{|c|c|c|c|}
\hline no & 105.00 & 69.08 & 69.08 \\
\hline yes | & 47.00 & 30.92 & 100.00 \\
\hline Total | & 152 & 100.00 & \\
\hline
\end{tabular}


Hot-water (water-heating) system:

- Flue gas analysis (steady-state efficiency measurements)

Freq. Percent Cum.

\begin{tabular}{|c|c|c|c|}
\hline no & 105.80 & 69.60 & 69.60 \\
\hline yes | & 46.20 & 30.40 & 100.00 \\
\hline Total | & 152 & 100.00 & \\
\hline
\end{tabular}

- CO measurements in flues

\begin{tabular}{|c|c|c|c|}
\hline & Freq. & Percent & Cum. \\
\hline no & 97.79 & 64.34 & 64.34 \\
\hline yes & 54.21 & 35.66 & 100.00 \\
\hline Total & 152 & 100.00 & \\
\hline
\end{tabular}

- Draft/spillage (normal operation)

$\begin{array}{rrr}\text { Freq. } & \text { Percent } & \text { Cum. } \\ \text { no | } 105.64 & 69.50 & 69.50 \\ \text { yes | } 46.36 & 30.50 & 100.00 \\ ------- \\ \text { Total | } 152 & 100.00\end{array}$

- Water flow rates (showerheads and faucets)

\begin{tabular}{|c|c|c|c|}
\hline & Freq. & Percent & Cum. \\
\hline no & 139.33 & 91.67 & 91.67 \\
\hline yes & 12.67 & 8.33 & 100.00 \\
\hline Total & 152 & 100.00 & \\
\hline
\end{tabular}

Other CO measurements:

- CO measurements in equipment rooms

\begin{tabular}{|c|c|c|c|}
\hline & Freq. & Percent & Cum. \\
\hline no & 115.63 & 76.07 & 76.07 \\
\hline yes & 36.37 & 23.93 & 100.00 \\
\hline Total & 152 & 100.00 & \\
\hline
\end{tabular}

- Cooking stove

Freq. Percent Cum.

\begin{tabular}{|c|c|c|c|}
\hline no & 92.57 & 60.90 & 60.90 \\
\hline yes & 59.43 & 39.10 & 100.00 \\
\hline Total | & 152 & 100.00 & \\
\hline
\end{tabular}


- CO measurements in living areas

\begin{tabular}{|c|c|c|c|}
\hline & Freq. & Percent & Cum. \\
\hline no & 105.70 & 69.54 & 69.54 \\
\hline yes & 46.30 & 30.46 & 100.00 \\
\hline Total & 152 & 100.00 & \\
\hline
\end{tabular}

Other diagnostics and inspections:

- Refrigerator energy use

Freq. Percent Cum.

\begin{tabular}{|c|c|c|c|}
\hline no & 92.06 & 60.57 & 60.57 \\
\hline yes & 59.94 & 39.43 & 100.00 \\
\hline Total & 152 & 100.00 & \\
\hline
\end{tabular}

- Exhaust fan air flow rate measurement

\begin{tabular}{|c|c|c|c|}
\hline & Freq. & Percent & Cum. \\
\hline no & 134.04 & 88.18 & 88.18 \\
\hline yes & 17.96 & 11.82 & 100.00 \\
\hline Total & 152 & 100.00 & \\
\hline
\end{tabular}

- Infrared scanning (camera)

\begin{tabular}{|c|c|c|c|}
\hline & Freq. & Percent & Cum. \\
\hline no & 96.80 & 63.69 & 63.69 \\
\hline yes & 55.20 & 36.31 & 100.00 \\
\hline Total & 152 & 100.00 & \\
\hline
\end{tabular}

- Radon testing

\begin{tabular}{|c|c|c|c|}
\hline 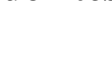 & Freq. & Percent & Cum. \\
\hline no & 151.06 & 99.38 & 99.38 \\
\hline yes | & .94 & 0.62 & 100.00 \\
\hline Total & 152 & 100.00 & \\
\hline
\end{tabular}

- $\quad$ Lead testing

Freq. Percent Cum.

\begin{tabular}{|c|c|c|c|}
\hline $\begin{array}{c}\text { no } \\
\text { yes }\end{array}$ & $\begin{array}{r}128.55 \\
23.45\end{array}$ & $\begin{array}{l}84.57 \\
15.43\end{array}$ & $\begin{array}{r}84.57 \\
100.00\end{array}$ \\
\hline tal & 152 & 100.00 & \\
\hline
\end{tabular}


- Mold and mildew testing

\begin{tabular}{|c|c|c|c|}
\hline & Freq. & Percent & Cum. \\
\hline no & 140.03 & 92.13 & 92.13 \\
\hline yes & 11.97 & 7.87 & 100.00 \\
\hline Total & 152 & 100.00 & \\
\hline
\end{tabular}

- Moisture content testing

Freq. Percent Cum.

\begin{tabular}{|c|c|c|c|}
\hline no & 141.18 & 92.88 & 92.88 \\
\hline yes & 10.82 & 7.12 & 100.00 \\
\hline Total & 152 & 100.00 & \\
\hline
\end{tabular}

- Other (please specify)

\begin{tabular}{|c|c|c|c|}
\hline & Freq. & Percent & Cum. \\
\hline no & 144.99 & 95.39 & 95.39 \\
\hline yes & 7.01 & 4.61 & 100.00 \\
\hline Total & 152 & 100.00 & \\
\hline
\end{tabular}

24. What types of credentials or experience were required of your staff who performed diagnostic procedures in Program Year 2008? Check all that apply.

- Technical certification

\begin{tabular}{|c|c|c|c|}
\hline & Freq. & Percent & Cum. \\
\hline no & 74.69 & 22.36 & 22.36 \\
\hline yes & 259.31 & 77.64 & 100.00 \\
\hline Total & 334 & 100.00 & \\
\hline
\end{tabular}

- Extensive weatherization work experience

Freq. Percent Cum.

$\begin{array}{rrr}\text { no | } 116.01 & 34.73 & 34.73 \\ \text { yes | } 217.99 & 65.27 & 100.00 \\ ---------------- \\ \text { Total| } 334 & 100.00\end{array}$

- Extensive weatherization supervision experience

Freq. Percent Cum.

\begin{tabular}{|c|c|c|c|}
\hline no & 231.66 & 69.36 & 69.36 \\
\hline yes | & 102.34 & 30.64 & 100.00 \\
\hline Total | & 334 & 100.00 & \\
\hline
\end{tabular}


- Construction experience

\begin{tabular}{|c|c|c|c|}
\hline & Freq. & Percent & Cum. \\
\hline no & 201.75 & 60.40 & 60.40 \\
\hline yes & 132.25 & 39.60 & 100.00 \\
\hline Total & 334 & 100.00 & \\
\hline
\end{tabular}

- Other (please specify)

\begin{tabular}{|c|c|c|c|}
\hline & Freq. & Percent & Cum. \\
\hline no & 301.71 & 90.33 & 90.33 \\
\hline yes & 32.29 & 9.67 & 100.00 \\
\hline Total & 334 & 100.00 & \\
\hline
\end{tabular}

25. Approximately how many hours did your agency spend on performing diagnostic procedures for a typical dwelling unit served by your agency in Program Year 2008?

\begin{tabular}{|l|c|}
\hline observations: & 321 \\
\hline missing values: & 36 \\
\hline mean: & 21.52 \\
\hline standard deviation: & 197.83 \\
\hline min: & .25 \\
\hline 10th percentile: & 1 \\
\hline 25th percentile: & 2 \\
\hline median: & 2 \\
\hline 75th percentile: & 3.5 \\
\hline 90th percentile: & 5 \\
\hline max: & 3500 \\
\hline
\end{tabular}


26. For Program Year 2008 please rate key aspects (cost, training needed, time needed and effectiveness) of the diagnostic procedures listed below.

Please use the following scale: 1 - very low; 2 - low; 3 - medium; 4 - high; 5 - very high.

For example, if you view blower door testing as requiring a moderate amount of training, enter a rating of 3 in the Training Needed column. If you view blower door testing as highly effective, enter a rating of 4 in the Effectiveness column.

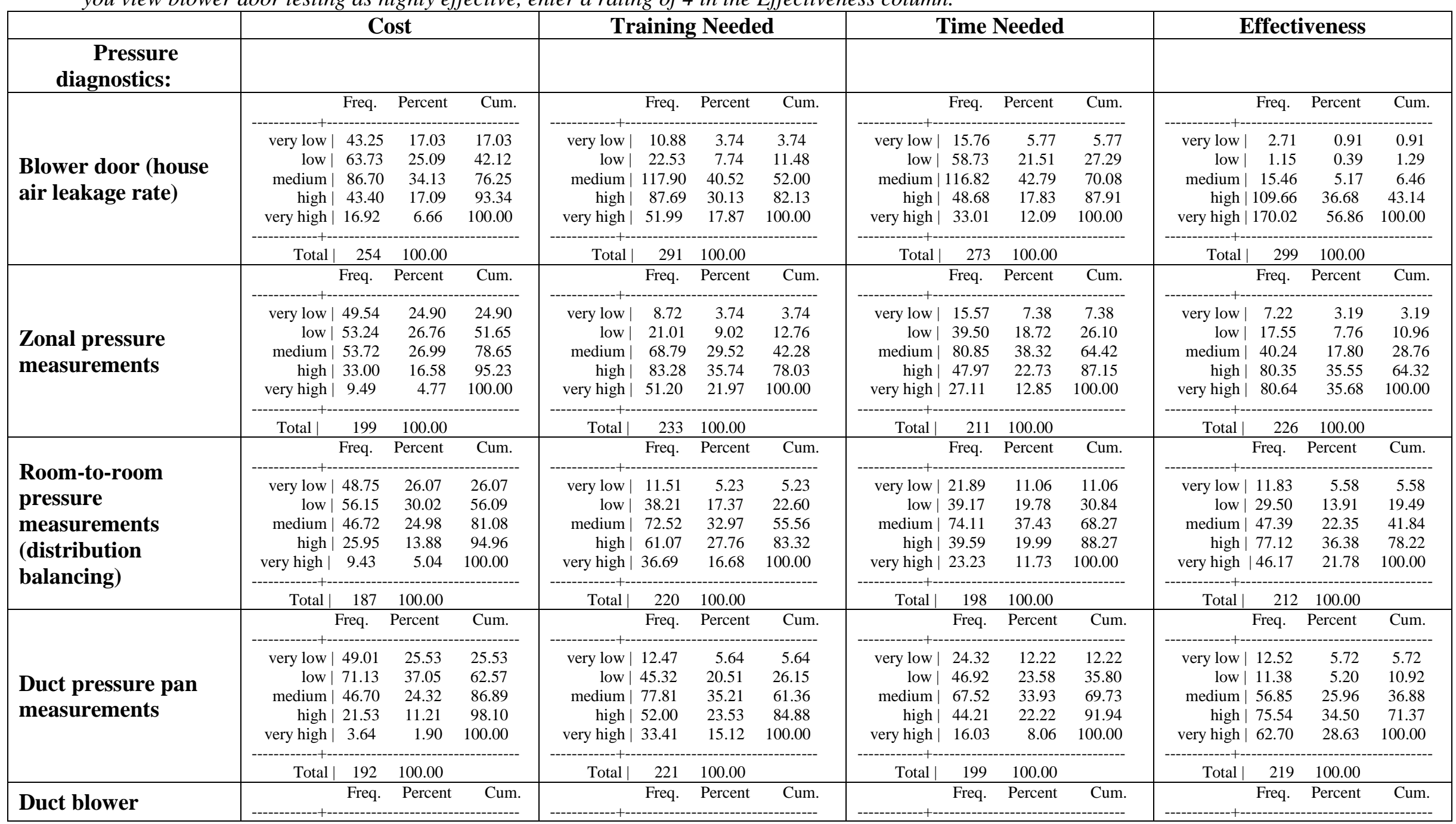




\begin{tabular}{|c|c|c|c|c|c|c|c|c|c|c|c|c|c|c|c|c|}
\hline & \multicolumn{4}{|c|}{ Cost } & \multicolumn{4}{|c|}{ Training Needed } & \multicolumn{4}{|c|}{ Time Needed } & \multicolumn{4}{|c|}{ Effectiveness } \\
\hline \multirow{6}{*}{$\begin{array}{l}\text { measurements (duct } \\
\text { air leakage rate) }\end{array}$} & very low & 34.95 & 21.71 & 21.71 & very low & 17.38 & 9.39 & 9.39 & very low & 17.11 & 10.01 & 10.01 & very low & 19.31 & 10.44 & 10.44 \\
\hline & & 32.08 & 19.93 & 41.63 & low & 23.69 & 12.81 & 22.20 & low & 24.86 & 14.54 & 24.55 & low & 14.45 & 7.81 & 18.25 \\
\hline & medium & 49.98 & 31.04 & 72.68 & medium & 48.33 & 26.13 & 48.33 & medium & 60.98 & 35.66 & 60.21 & medium & 36.61 & 19.79 & 38.04 \\
\hline & high & 29.21 & 18.14 & 90.82 & high & 55.55 & 30.03 & 78.35 & high & 40.27 & 23.55 & 83.76 & high & 64.62 & 34.93 & 72.97 \\
\hline & very high & 14.78 & 9.18 & 100.00 & very high & 40.05 & 21.65 & 100.00 & very high & 27.77 & 16.24 & 100.00 & very high & 50.01 & 27.03 & 100.00 \\
\hline & Total & 161 & 100.00 & & Total & 185 & 100.00 & & Total | & 171 & 100.00 & & Total & 185 & 100.00 & \\
\hline \multicolumn{17}{|l|}{$\begin{array}{c}\text { Space- } \\
\text { heating system: }\end{array}$} \\
\hline \multirow{7}{*}{$\begin{array}{l}\text { Flue gas analysis } \\
\text { (steady-state } \\
\text { efficiency } \\
\text { measurements) }\end{array}$} & & Freq. & Percent & Cum. & & Freq. & Percent & Cum. & & Freq. & Percent & Cum. & & Freq. & Percent & Cum. \\
\hline & very low | & 41.40 & 17.54 & 17.54 & very low | & 15.27 & 5.63 & 5.63 & very low | & 15.28 & 6.11 & 6.11 & very low & 4.33 & 1.60 & 1.60 \\
\hline & & 60.99 & 25.84 & 43.39 & & 22.45 & 8.28 & 13.92 & low & 66.68 & 26.67 & 32.78 & low & 5.82 & 2.15 & 3.75 \\
\hline & medium & 86.62 & 36.70 & 80.09 & medium & 66.05 & 24.37 & 38.29 & medium & 101.59 & 40.64 & 73.42 & medium & 22.02 & 8.12 & 11.87 \\
\hline & high & 34.60 & 14.66 & 94.75 & high & 110.61 & 40.81 & 79.11 & high & 45.58 & 18.23 & 91.65 & high & 109.73 & 40.49 & 52.36 \\
\hline & very high & 12.40 & 5.25 & 100.00 & very high & 56.62 & 20.89 & 100.00 & very high & 20.87 & 8.35 & 100.00 & very high & 129.10 & 47.64 & 100.00 \\
\hline & Total | & 236 & 100.00 & & Total | & 271 & 100.00 & & Total | & 250 & 100.00 & & Total | & 271 & 100.00 & \\
\hline \multirow{7}{*}{$\begin{array}{l}\text { Heat rise } \\
\text { measurements }\end{array}$} & & Freq. & Percent & Cum. & & Freq. & Percent & Cum. & & Freq. & Percent & Cum. & & Freq. & Percent & Cum. \\
\hline & very low | & 62.73 & 32.51 & 32.51 & very low | & 22.13 & 10.01 & 10.01 & very low & 27.62 & 13.54 & 13.54 & very low & 5.83 & 2.65 & 2.65 \\
\hline & & 54.22 & 28.09 & 60.60 & low & 50.16 & 22.70 & 32.71 & low & 70.32 & 34.47 & 48.01 & low & 18.62 & 8.46 & 11.11 \\
\hline & medium & 51.61 & 26.74 & 87.34 & medium & 65.62 & 29.69 & 62.40 & medium & 69.80 & 34.21 & 82.22 & medium & 45.59 & 20.72 & 31.84 \\
\hline & high & 14.57 & 7.55 & 94.89 & high & 48.55 & 21.97 & 84.37 & high & 18.64 & 9.14 & 91.36 & high & 92.59 & 42.08 & 73.92 \\
\hline & very high & 9.87 & 5.11 & 100.00 & very high & 34.54 & 15.63 & 100.00 & very high & 17.62 & 8.64 & 100.00 & very high & 57.37 & 26.08 & 100.00 \\
\hline & Total & 193 & 100.00 & & Total | & 221 & 100.00 & & Total & 204 & 100.00 & & Total & 220 & 100.00 & \\
\hline \multirow{7}{*}{$\begin{array}{l}\text { CO measurements in } \\
\text { flues }\end{array}$} & & Freq. & Percent & Cum. & & Freq. & Percent & Cum. & & Freq. & Percent & Cum. & & Freq. & Percent & Cum. \\
\hline & very low & 58.71 & 24.36 & 24.36 & very low & 16.38 & 5.98 & 5.98 & very low & 37.17 & 14.46 & 14.46 & very low & 1.93 & 0.69 & 0.69 \\
\hline & & 76.01 & 31.54 & 55.90 & low & 49.88 & 18.21 & 24.18 & low & 77.21 & 30.04 & 44.51 & low & 1.50 & 0.53 & 1.22 \\
\hline & medium & 71.97 & 29.86 & 85.76 & medium & 84.26 & 30.75 & 54.94 & medium | & 83.98 & 32.68 & 77.18 & medium & 41.07 & 14.62 & 15.84 \\
\hline & high & 16.94 & 7.03 & 92.79 & high & 70.02 & 25.55 & 80.49 & high & 36.35 & 14.14 & 91.33 & high & 83.83 & 29.83 & 45.67 \\
\hline & very high & 17.38 & 7.21 & 100.00 & very high & 53.46 & 19.51 & 100.00 & very high & 22.29 & 8.67 & 100.00 & very high & 152.67 & 54.33 & 100.00 \\
\hline & Total | & 241 & 100.00 & & Total & 274 & 100.00 & & Total & 257 & 100.00 & & Total & 281 & 100.00 & \\
\hline
\end{tabular}




\begin{tabular}{|c|c|c|c|c|c|c|c|c|c|c|c|c|c|c|c|c|}
\hline \multirow{7}{*}{$\begin{array}{l}\text { Draft/spillage } \\
\text { (normal operation) }\end{array}$} & & Freq. $\quad \mathrm{P}$ & Percent & Cum. & & Freq. & Percent & Cum. & & Freq. & Percent & Cum. & & Freq. & Percent & Cum. \\
\hline & very low & 62.83 & 27.92 & 27.92 & very low & 15.64 & 6.08 & 6.08 & very low & 36.38 & 15.22 & 15.22 & very low & 1.52 & 0.59 & 0.59 \\
\hline & low & 63.90 & 28.40 & 56.32 & low & 33.85 & 13.17 & 19.25 & low & 73.22 & 30.64 & 45.86 & low & 3.54 & 1.37 & 1.96 \\
\hline & medium & 62.83 & 27.93 & 84.25 & medium & 89.64 & 34.88 & 54.13 & medium & 77.20 & 32.30 & 78.16 & medium & 27.10 & 10.49 & 12.45 \\
\hline & high & 21.01 & 9.34 & 93.59 & high & 71.35 & 27.76 & 81.90 & high & 32.94 & 13.78 & 91.94 & high & 88.32 & 34.23 & 46.69 \\
\hline & very high $\mid$ & 14.43 & 6.41 & 100.00 & very high & 46.52 & 18.10 & 100.00 & very high | & 19.25 & 8.06 & 100.00 & very high | & 137.55 & 53.31 & 100.00 \\
\hline & Total & 225 & 100.00 & & Total | & 257 & 100.00 & & Total & 239 & 100.00 & & Total | & 258 & 100.00 & \\
\hline \multicolumn{17}{|l|}{ Air-conditioning system: } \\
\hline \multirow{7}{*}{$\begin{array}{l}\text { Refrigerant charge } \\
\text { (e.g., superheat, } \\
\text { subcooling) }\end{array}$} & & Freq. & Percent & Cum. & & Freq. & Percent & Cum. & & Freq. & Percent & Cum. & & Freq. & Percent & Cum. \\
\hline & very low & 21.04 & 28.83 & 28.83 & very low & 23.26 & 25.28 & 25.28 & very low & 18.73 & 24.32 & 24.32 & very low & 22.86 & 25.98 & 25.98 \\
\hline & low & 10.67 & 14.61 & 43.44 & & 4.33 & 4.71 & 30.00 & low & 12.58 & 16.33 & 40.66 & low & 4.63 & 5.26 & 31.24 \\
\hline & medium & 21.93 & 30.04 & 73.47 & medium & 16.92 & 18.39 & 48.38 & medium & 19.65 & 25.52 & 66.18 & medium & 21.58 & 24.53 & 55.77 \\
\hline & high & 14.83 & 20.31 & 93.78 & high & 23.63 & 25.69 & 74.07 & high & 20.46 & 26.57 & 92.74 & high & 20.51 & 23.31 & 79.07 \\
\hline & very high & 4.54 & 6.22 & 100.00 & very high & 23.85 & 25.93 & 100.00 & very high | & 5.59 & 7.26 & 100.00 & very high & 18.41 & 20.93 & 100.00 \\
\hline & Total & 73 & 100.00 & & Total & 92 & 100.00 & & Total & 77 & 100.00 & & Total & 88 & 100.00 & \\
\hline \multicolumn{17}{|l|}{$\begin{array}{l}\text { HVAC components and } \\
\text { cross-cutting diagnostics: }\end{array}$} \\
\hline \multirow[t]{7}{*}{ Air handler flow rate } & & Freq. & Percent & Cum. & & Freq. & Percent & Cum. & & Freq. & Percent & Cum. & & Freq. & Percent & Cum. \\
\hline & very low & 26.87 & 24.66 & 24.66 & very low & 17.88 & 13.76 & 13.76 & very low & 12.56 & 11.02 & 11.02 & very low & 12.31 & 9.84 & 9.84 \\
\hline & low & 24.07 & 22.08 & 46.74 & low & 12.86 & 9.89 & 23.65 & low & 24.43 & 21.43 & 32.45 & low & 8.84 & 7.08 & 16.92 \\
\hline & medium & 37.31 & 34.23 & 80.97 & medium & 35.13 & 27.03 & 50.67 & medium & 37.83 & 33.19 & 65.63 & medium & 36.43 & 29.14 & 46.06 \\
\hline & high & 13.08 & 12.00 & 92.96 & high & 37.37 & 28.75 & 79.42 & high & 33.65 & 29.52 & 95.15 & high & 42.47 & 33.98 & 80.04 \\
\hline & very high & 7.67 & 7.04 & 100.00 & very high & 26.76 & 20.58 & 100.00 & very high & 5.52 & 4.85 & 100.00 & very high & 24.95 & 19.96 & 100.00 \\
\hline & Total & 109 & 100.00 & & Total & 130 & 100.00 & & Total & 114 & 100.00 & & Total & 125 & 100.00 & \\
\hline \multirow{7}{*}{$\begin{array}{l}\text { Thermostat } \\
\text { anticipator current }\end{array}$} & & Freq. $\quad \mathrm{P}$ & Percent & Cum. & & Freq. & Percent & Cum. & & Freq. & Percent & Cum. & & Freq. & Percent & Cum. \\
\hline & very low & 37.53 & 32.08 & 32.08 & very low & 24.79 & 17.83 & 17.83 & very low & 19.83 & 16.53 & 16.53 & very low & 14.02 & 10.38 & 10.38 \\
\hline & low & 41.82 & 35.74 & 67.82 & low & 17.29 & 12.44 & 30.27 & low & 40.91 & 34.10 & 50.62 & low & 10.89 & 8.07 & 18.45 \\
\hline & medium & 19.39 & 16.57 & 84.39 & medium & 37.21 & 26.77 & 57.04 & medium & 29.74 & 24.78 & 75.40 & medium & 30.56 & 22.64 & 41.09 \\
\hline & high & 13.39 & 11.45 & 95.84 & high & 41.68 & 29.99 & 87.03 & high & 25.91 & 21.59 & 97.00 & high & 53.89 & 39.92 & 81.01 \\
\hline & very high & 4.87 & 4.16 & 100.00 & very high & 18.03 & 12.97 & 100.00 & very high | & 3.60 & 3.00 & 100.00 & very high & 25.64 & 18.99 & 100.00 \\
\hline & Total & 117 & 100.00 & & Total & 139 & 100.00 & & Total & 120 & 100.00 & & Total & 135 & 100.00 & \\
\hline \multirow{7}{*}{$\begin{array}{l}\text { Worst case } \\
\text { draft/spillage }(\mathrm{CAZ})\end{array}$} & & Freq. $\quad \mathrm{P}$ & ercent & Cum. & & Freq. & Percent & Cum. & & Freq. & Percent & Cum. & & Freq. & Percent & Cum. \\
\hline & very low & 36.45 & 21.32 & 21.32 & very low & 13.07 & 6.57 & 6.57 & very low & 18.22 & 10.18 & 10.18 & very low & 6.68 & 3.45 & 3.45 \\
\hline & low & 46.86 & 27.41 & 48.72 & low & 19.62 & 9.86 & 16.43 & low & 29.30 & 16.37 & 26.55 & low & 5.01 & 2.58 & 6.03 \\
\hline & medium & 51.21 & 29.95 & 78.67 & medium | & 50.70 & 25.48 & 41.90 & medium & 66.39 & 37.09 & 63.64 & medium & 18.17 & 9.36 & 15.39 \\
\hline & high & 21.14 & 12.36 & 91.03 & high & 66.52 & 33.43 & 75.33 & high & 38.26 & 21.37 & 85.01 & high & 51.49 & 26.54 & 41.93 \\
\hline & very high & 15.34 & 8.97 & 100.00 & very high & 49.09 & 24.67 & 100.00 & very high | & 26.83 & 14.99 & 100.00 & very high & 112.65 & 58.07 & 100.00 \\
\hline & Total & 171 & 100.00 & & Total & 199 & 100.00 & & Total & 179 & 100.00 & & Total & 194 & 100.00 & \\
\hline
\end{tabular}




\begin{tabular}{|c|c|c|c|c|c|c|c|c|c|c|c|c|c|c|c|c|}
\hline \multirow{7}{*}{$\begin{array}{l}\text { heating) system: } \\
\text { Flue gas } \\
\text { analysis } \\
\text { (steady-state } \\
\text { efficiency } \\
\text { measurements) }\end{array}$} & \multirow{2}{*}{\multicolumn{3}{|c|}{ Freq. Percent }} & \multirow[b]{2}{*}{ Cum. } & \multirow{2}{*}{\multicolumn{4}{|c|}{ Freq. Percent Cum. }} & \multicolumn{4}{|c|}{ 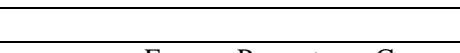 } & \multirow{2}{*}{\multicolumn{2}{|c|}{ Freq. }} & \multirow[b]{2}{*}{ Percent } & \multirow[b]{2}{*}{ Cum. } \\
\hline & & & & & & & & & \multicolumn{4}{|c|}{ Freq. Percent Cum. } & & & & \\
\hline & very low & 47.59 & 23.10 & 23.10 & very low & 10.57 & 4.46 & 4.46 & very low & 20.90 & 9.55 & 9.55 & very low & 3.86 & 1.64 & 1.64 \\
\hline & low & 56.08 & 27.22 & 50.32 & low & 37.65 & 15.89 & 20.34 & low & 60.67 & 27.70 & 37.25 & low & 15.47 & 6.55 & 8.19 \\
\hline & medium & 64.22 & 31.18 & 81.50 & medium & 74.68 & 31.51 & 51.86 & medium & 89.62 & 40.92 & 78.17 & medium & 26.74 & 11.33 & 19.52 \\
\hline & high | & 21.99 & 10.68 & 92.18 & high & 59.42 & 25.07 & 76.93 & high & 28.15 & 12.85 & 91.03 & high & 70.45 & 29.85 & 49.37 \\
\hline & very high | & 16.11 & 7.82 & 100.00 & very high & 54.68 & 23.07 & 100.00 & very high & 19.65 & 8.97 & 100.00 & very high & 119.48 & 50.63 & 100.00 \\
\hline & Total $\mid$ & 206 & 100.00 & & Total | & 237 & 100.00 & & Total | & 219 & 100.00 & & Total & 236 & 100.00 & \\
\hline $\mathrm{CO}$ & & Freq. & Percent & Cum. & & Freq. & Percent & Cum. & & Freq. & Percent & Cum. & & Freq. & Percent & Cum. \\
\hline measurements & very low & 60.12 & 25.05 & 25.05 & very low & 15.78 & 5.85 & 5.85 & very low & 25.49 & 10.08 & 10.08 & very low & 3.35 & 1.22 & 1.22 \\
\hline in flues & low & 73.86 & 30.77 & 55.82 & low & 43.55 & 16.13 & 21.97 & low & 79.73 & 31.51 & 41.59 & low & 4.10 & 1.50 & 2.72 \\
\hline & medium | & 74.65 & 31.10 & 86.93 & medium & 91.15 & 33.76 & 55.73 & medium & 92.06 & 36.39 & 77.98 & medium & 45.47 & 16.60 & 19.31 \\
\hline & high & 14.26 & 5.94 & 92.87 & high & 67.81 & 25.11 & 80.85 & high & 36.95 & 14.60 & 92.58 & high & 79.60 & 29.05 & 48.37 \\
\hline & very high & 17.12 & 7.13 & 100.00 & very high | & 51.72 & 19.15 & 100.00 & very high | & 18.77 & 7.42 & 100.00 & very high & 141.48 & 51.63 & 100.00 \\
\hline & Total | & 240 & 100.00 & & Total | & 270 & 100.00 & & Total & 253 & 100.00 & & Total & 274 & 100.00 & \\
\hline Draft/spillage & & Freq. & Percent & Cum. & & Freq. & Percent & Cum. & & Freq. & Percent & Cum. & & Freq. & Percent & Cum. \\
\hline (normal & very low | & 62.49 & 27.78 & 27.78 & very low & 16.54 & 6.49 & 6.49 & very low | & 34.35 & 14.37 & 14.37 & very low & 4.79 & 1.87 & 1.87 \\
\hline operation) & low & 62.29 & 27.68 & 55.46 & low & 44.26 & 17.35 & 23.84 & low & 72.41 & 30.30 & 44.67 & low & 7.17 & 2.80 & 4.67 \\
\hline & medium & 68.43 & 30.41 & 85.87 & medium & 80.52 & 31.58 & 55.42 & medium & 86.29 & 36.10 & 80.77 & medium & 27.27 & 10.65 & 15.32 \\
\hline & high & 11.32 & 5.03 & 90.90 & high & 62.59 & 24.55 & 79.97 & high & 22.87 & 9.57 & 90.34 & high & 73.95 & 28.89 & 44.21 \\
\hline & very high | & 20.47 & 9.10 & 100.00 & very high | & 51.09 & 20.03 & 100.00 & very high | & 23.09 & 9.66 & 100.00 & very high & 142.82 & 55.79 & 100.00 \\
\hline & Total & 225 & 100.00 & & Total | & 255 & 100.00 & & Total | & 239 & 100.00 & & Total | & 256 & 100.00 & \\
\hline Water flow & & Freq. & Percent & Cum. & & Freq. & Percent & Cum. & & Freq. & Percent & Cum. & & Freq. & Percent & Cum. \\
\hline rates & very low & 60.54 & 44.84 & 44.84 & very low & 48.09 & 30.44 & 30.44 & very low & 42.79 & 29.71 & 29.71 & very low & 20.69 & 13.09 & 13.09 \\
\hline (showerheads & low & 31.48 & 23.32 & 68.16 & low & 50.23 & 31.79 & 62.23 & low & 46.05 & 31.98 & 61.69 & low & 19.49 & 12.34 & 25.43 \\
\hline and faucets) & medium | & 32.34 & 23.96 & 92.12 & medium & 26.40 & 16.71 & 78.94 & medium & 37.43 & 26.00 & 87.69 & medium & 42.00 & 26.58 & 52.01 \\
\hline & high & 1.74 & 1.29 & 93.41 & high & 16.38 & 10.37 & 89.31 & high & 3.64 & 2.52 & 90.22 & high & 46.75 & 29.59 & 81.60 \\
\hline & very high & 8.90 & 6.59 & 100.00 & very high & 16.90 & 10.69 & 100.00 & very high | & 14.09 & 9.78 & 100.00 & very high & 29.08 & 18.40 & 100.00 \\
\hline & Total | & 135 & 100.00 & & Total | & 158 & 100.00 & & Total | & 144 & 100.00 & & Total | & 158 & 100.00 & \\
\hline $\begin{array}{l}\text { Other CO } \\
\text { measurements: }\end{array}$ & & & & & & & & & & & & & & & & \\
\hline $\mathrm{CO}$ & & Freq. & Percent & Cum. & & Freq. & Percent & Cum. & & Freq. & Percent & Cum. & & Freq. & Percent & Cum. \\
\hline measurements & very low & 48.37 & 24.93 & 24.93 & very low & | 18.12 & 8.27 & 8.27 & very low | & 29.86 & 14.78 & 14.78 & very low & 5.02 & 2.31 & 2.31 \\
\hline in equipment & low & 66.84 & 34.45 & 59.39 & low & 54.05 & 24.68 & 32.95 & low & 74.53 & 36.89 & 51.68 & low & 8.81 & 4.06 & 6.37 \\
\hline rooms & medium & 57.94 & 29.87 & 89.25 & medium & 71.62 & 32.70 & 65.65 & medium & 57.83 & 28.63 & 80.30 & medium & 28.90 & 13.32 & 19.69 \\
\hline & high & 11.86 & 6.11 & 95.37 & high & 45.50 & 20.78 & 86.43 & high & 30.00 & 14.85 & 95.16 & high & 70.57 & 32.52 & 52.21 \\
\hline & very high & 8.99 & 4.63 & 100.00 & very high & 29.73 & 13.57 & 100.00 & very high & 9.79 & 4.84 & 100.00 & very high & 103.70 & 47.79 & 100.00 \\
\hline & Total & 194 & 100.00 & & Total | & 219 & 100.00 & & Total & 202 & 100.00 & & Total & 217 & 100.00 & \\
\hline
\end{tabular}




\begin{tabular}{|c|c|c|c|c|c|c|c|c|c|c|c|c|c|c|c|c|}
\hline \multirow[t]{7}{*}{ Cooking stove } & & Freq. & Percent & Cum. & & Freq. & Percent & Cum. & & Freq. & Percent & Cum. & & Freq. & Percent & Cum. \\
\hline & very low & 60.97 & 28.62 & 28.62 & very low | & 16.48 & 6.70 & 6.70 & very low & 37.96 & 16.58 & 16.58 & very low & 8.67 & 3.52 & 3.52 \\
\hline & low & 68.04 & 31.94 & 60.57 & low & 69.86 & 28.40 & 35.10 & low & 77.09 & 33.66 & 50.24 & low & 11.76 & 4.78 & 8.30 \\
\hline & medium & 54.90 & 25.78 & 86.34 & medium & 79.93 & 32.49 & 67.59 & medium & 65.18 & 28.46 & 78.70 & medium & 36.89 & 15.00 & 23.30 \\
\hline & high & 16.89 & 7.93 & 94.27 & high & 46.82 & 19.03 & 86.62 & high & 32.76 & 14.30 & 93.01 & high & 82.47 & 33.53 & 56.83 \\
\hline & very high & 12.21 & 5.73 & 100.00 & very high & 32.91 & 13.38 & 100.00 & very high & 16.02 & 6.99 & 100.00 & very high & 106.21 & 43.17 & 100.00 \\
\hline & Total | & 213 & 100.00 & & Total | & 246 & 100.00 & & Total | & 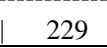 & 100.00 & & Total & 246 & 100.00 & \\
\hline \multirow{6}{*}{$\begin{array}{l}\text { CO } \\
\text { measurements } \\
\text { in living areas }\end{array}$} & & Freq. & Percent & Cum. & & Freq. & Percent & Cum. & & Freq. & Percent & Cum. & & Freq. & Percent & Cum. \\
\hline & low & 66.89 & 34.30 & 64.24 & low & 56.03 & 24.90 & 35.38 & low & 59.38 & 28.97 & 52.47 & low & 5.78 & 2.57 & 4.80 \\
\hline & medium & 40.24 & 20.64 & 84.88 & medium & 72.93 & 32.41 & 67.80 & medium & 55.17 & 26.91 & 79.38 & medium & 29.15 & 12.95 & 17.76 \\
\hline & high & 16.07 & 8.24 & 93.12 & high & 41.47 & 18.43 & 86.23 & high & 28.37 & 13.84 & 93.22 & high & 84.35 & 37.49 & 55.24 \\
\hline & very high & 13.42 & 6.88 & 100.00 & very high & 30.98 & 13.77 & 100.00 & very high & 13.90 & 6.78 & 100.00 & very high & 100.70 & 44.76 & 100.00 \\
\hline & Total & 195 & 100.00 & & Total | & 225 & 100.00 & & Total | & 205 & 100.00 & & Total & 225 & 100.00 & \\
\hline \multicolumn{17}{|l|}{$\begin{array}{l}\text { Other diagnostics } \\
\text { and inspections: }\end{array}$} \\
\hline \multirow{6}{*}{$\begin{array}{l}\text { Refrigerator } \\
\text { energy use }\end{array}$} & & Freq. $\quad \mathrm{P}$ & Percent & Cum. & & Freq. & Percent & Cum. & & Freq. & Percent & Cum. & & Freq. & Percent & Cum. \\
\hline & low & 66.57 & 36.38 & 64.63 & low & 54.69 & 26.17 & 49.72 & low & 41.43 & 21.25 & 46.13 & low & 13.48 & 6.42 & 11.09 \\
\hline & medium & 41.25 & 22.54 & 87.17 & medium & 68.40 & 32.73 & 82.45 & medium & 53.26 & 27.31 & 73.44 & medium & 45.13 & 21.49 & 32.58 \\
\hline & high & 20.58 & 11.25 & 98.42 & high & 24.66 & 11.80 & 94.25 & high & 35.13 & 18.02 & 91.45 & high & 79.94 & 38.07 & 70.65 \\
\hline & very high | & 2.90 & 1.58 & 100.00 & very high | & 12.02 & 5.75 & 100.00 & very high & 16.66 & 8.55 & 100.00 & very high & 61.63 & 29.35 & 100.00 \\
\hline & Total | & 183 & 100.00 & & Total | & 209 & 100.00 & & Total | & 195 & 100.00 & & Total | & 210 & 100.00 & \\
\hline \multirow{7}{*}{$\begin{array}{l}\text { Exhaust fan } \\
\text { air flow rate } \\
\text { measurement }\end{array}$} & & $\begin{array}{ll}\text { Freq. } & \mathrm{P}\end{array}$ & Percent & Cum. & & Freq. & Percent & Cum. & & Freq. & Percent & Cum. & & Freq. & Percent & Cum. \\
\hline & very low & 31.25 & 24.23 & 24.23 & very low & 17.84 & 12.22 & 12.22 & very low & 21.84 & 16.42 & 16.42 & very low | & 10.96 & 7.66 & 7.66 \\
\hline & low & 39.43 & 30.56 & 54.79 & low & 31.59 & 21.64 & 33.85 & low & 36.23 & 27.24 & 43.66 & low & 14.89 & 10.41 & 18.07 \\
\hline & medium & 35.34 & 27.39 & 82.19 & medium & 55.75 & 38.18 & 72.04 & medium & 49.15 & 36.96 & 80.62 & medium & 48.77 & 34.10 & 52.18 \\
\hline & high & 17.96 & 13.92 & 96.11 & high & 27.60 & 18.91 & 90.94 & high & 14.98 & 11.26 & 91.88 & high & 39.74 & 27.79 & 79.97 \\
\hline & very high & 5.03 & 3.89 & 100.00 & very high & 13.22 & 9.06 & 100.00 & very high & 10.80 & 8.12 & 100.00 & very high & 28.64 & 20.03 & 100.00 \\
\hline & Total | & 129 & 100.00 & & Total | & 146 & 100.00 & & Total | & | 133 & 100.00 & & Total & | 143 & 100.00 & \\
\hline \multirow{5}{*}{$\begin{array}{l}\text { Infrared } \\
\text { scanning } \\
\text { (camera) }\end{array}$} & & Freq. $\quad \mathrm{P}$ & Percent & Cum. & & Freq. & Percent & Cum. & & Freq. & Percent & Cum. & & Freq. & Percent & Cum. \\
\hline & very low & 23.21 & 12.97 & 12.97 & very low & 12.83 & 6.11 & 6.11 & very low & 16.39 & 8.49 & 8.49 & very low & 8.31 & 3.94 & 3.94 \\
\hline & low & 15.75 & 8.80 & 21.76 & low & 14.62 & 6.96 & 13.07 & low & 19.90 & 10.31 & 18.81 & low & 4.60 & 2.18 & 6.12 \\
\hline & medium & 38.98 & 21.77 & 43.54 & medium & 62.97 & 29.98 & 43.05 & medium & 75.28 & 39.00 & 57.81 & medium & 29.58 & 14.02 & 20.14 \\
\hline & high & 58.28 & 32.56 & 76.09 & high & 67.33 & 32.06 & 75.12 & high & 50.61 & 26.22 & 84.03 & high & 77.49 & 36.73 & 56.87 \\
\hline
\end{tabular}




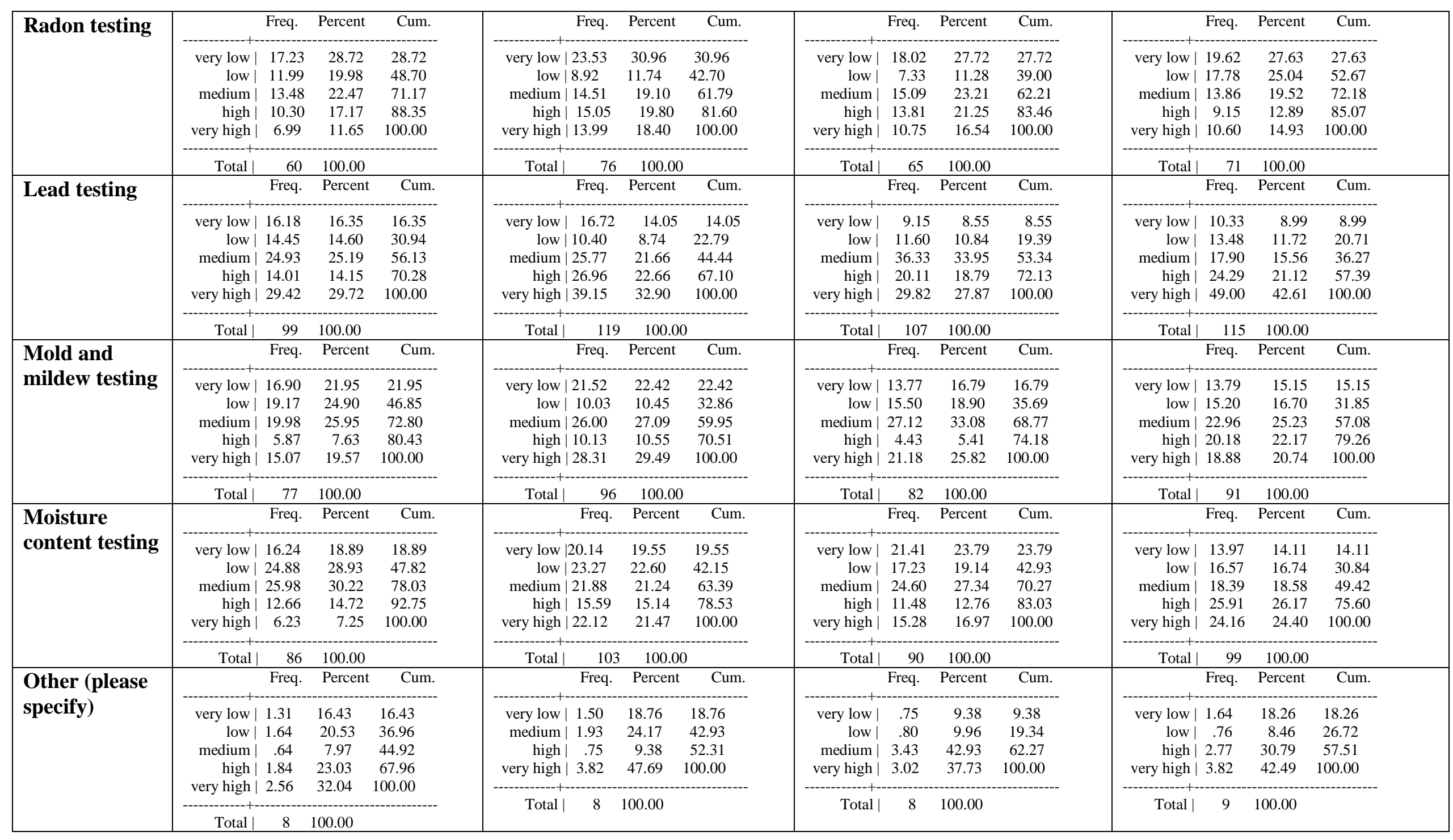




\section{SECTION D: CLIENT EDUCATION}

1. Which of the following client education approaches did your agency use in Program Year 2008 ? Check all that apply.

At time of client intake

a. Provide literature at time of client intake

\begin{tabular}{|c|c|c|c|}
\hline & Freq. & Percent & Cum. \\
\hline no & 139.74 & 39.70 & 39.70 \\
\hline yes | & 212.264 & 60.30 & 100.00 \\
\hline Total & 352 & 100.00 & \\
\hline
\end{tabular}

b. Provide video, CD or DVD at time of client intake

$\begin{array}{crrr} & \text { Freq. } & \text { Percent } & \text { Cum. } \\ \text { no | } 334.264 & 94.96 & 94.96 \\ \text { yes | } & 17.74 & 5.04 & 100.00 \\ ------ & \end{array}$

c. Provide in-person instruction at time of client intake

\begin{tabular}{|c|c|c|c|}
\hline & Freq. & Percent & Cum. \\
\hline no & 205.67 & 58.43 & 58.43 \\
\hline yes | & 146.33 & 41.57 & 100.00 \\
\hline Total | & 352 & 100.00 & \\
\hline
\end{tabular}

d. Provide hardware kit at time of client intake

$\begin{array}{rrrr} & \text { Freq. } & \text { Percent } & \text { Cum. } \\ \text { no } \mid & 329.50 & 93.61 & 93.61 \\ \text { yes } \mid & 22.50 & 6.39 & 100.00 \\ ---------- & \end{array}$


At time of audit

e. Provide literature at time of audit

\begin{tabular}{|c|c|c|c|}
\hline & Freq. & Percent & Cum. \\
\hline no & 88.91 & 25.26 & 25.26 \\
\hline yes | & 263.09 & 74.74 & 100.00 \\
\hline Total | & 352 & 100.00 & \\
\hline
\end{tabular}

f. Provide video, CD or DVD at time of audit

$\begin{array}{rrrr} & \text { Freq. } & \text { Percent } & \text { Cum. } \\ \text { no | } 343.25 & 97.51 & 97.51 \\ \text { yes | } & 8.75 & 2.49 & 100.00 \\ ---------- \\ \text { Total | } & 352 & 100.00 & \end{array}$

g. Provide in-person instruction at time of audit

\begin{tabular}{|c|c|c|c|}
\hline & Freq. & Percent & Cum. \\
\hline no & 61.56 & 17.49 & 17.49 \\
\hline yes & 290.446 & 82.51 & 100.00 \\
\hline Total & 352 & 100.00 & \\
\hline
\end{tabular}

h. Provide hardware kit at time of audit

\begin{tabular}{|c|c|c|c|}
\hline & Freq. & Percent & Cum. \\
\hline no & 331.79 & 94.26 & 94.26 \\
\hline yes | & 20.21 & 5.74 & 100.00 \\
\hline Total | & 352 & 100.00 & \\
\hline
\end{tabular}

At time of weatherization

i. Provide literature at time of weatherization

\begin{tabular}{|c|c|c|c|}
\hline & Freq. & Percent & Cum. \\
\hline no & 280.87 & 79.79 & 79.79 \\
\hline yes & 71.13 & 20.21 & 100.00 \\
\hline Total | & 352 & 100.00 & \\
\hline
\end{tabular}


j. Provide video, CD or DVD at time of weatherization

$\begin{array}{crrr} & \text { Freq. } & \text { Percent } & \text { Cum. } \\ \text { no | } 347.30 & 98.67 & 98.67 \\ \text { yes | } & 4.70 & 1.33 & 100.00 \\ ------- \\ \text { Total | } & 352 & 100.00 & \end{array}$

k. Provide in-person instruction at time of weatherization

\begin{tabular}{|c|c|c|c|}
\hline & Freq. & Percent & Cum. \\
\hline no & 117.28 & 33.32 & 33.32 \\
\hline yes & 234.72 & 66.68 & 100.00 \\
\hline Total & 352 & 100.00 & \\
\hline
\end{tabular}

1. Provide hardware kit at time of weatherization

$\begin{array}{crrr} & \text { Freq. } & \text { Percent } & \text { Cum. } \\ \text { no | } 333.85 & 94.84 & 94.84 \\ \text { yes | } & 18.15 & 5.16 & 100.00 \\ ----------- \\ \text { Total | } 352 & 100.00 & \end{array}$

At separate client education visit

m. Provide literature at separate client education visit

\begin{tabular}{|c|c|c|c|}
\hline & Freq. & Percent & Cum. \\
\hline no & 285.73 & 81.17 & 81.17 \\
\hline yes & 66.27 & 18.83 & 100.00 \\
\hline Total | & 352 & 100.00 & \\
\hline
\end{tabular}

n. Provide video, CD or DVD at separate client education visit

$\begin{array}{rrrr} & \text { Freq. } & \text { Percent } & \text { Cum. } \\ \text { no | } 344.55 & 97.88 & 97.88 \\ \text { yes | } & 7.45 & 2.12 & 100.00 \\ ------------ \\ \text { Total | }\end{array}$


o. Provide in-person instruction at separate client education visit

$\begin{array}{rrrr} & \text { Freq. } & \text { Percent } & \text { Cum. } \\ \text { no | } 274.02 & 77.85 & 77.85 \\ \text { yes | } & 77.98 & 22.15 & 100.00 \\ ------------- \\ \text { Total | } 352 & 100.00 & \end{array}$

p. Provide hardware kit at separate client education visit

$\begin{array}{crrr} & \text { Freq. } & \text { Percent } & \text { Cum. } \\ \text { no | } 337.56 & 95.90 & 95.90 \\ \text { yes | } 14.44 & 4.10 & 100.00 \\ ------ & \end{array}$

At time of inspection

q. Provide literature at time of inspection

\begin{tabular}{|c|c|c|c|}
\hline & Freq. & Percent & Cum. \\
\hline no & 243.71 & 69.24 & 69.24 \\
\hline yes & 108.29 & 30.76 & 100.00 \\
\hline Total & 352 & 100.00 & \\
\hline
\end{tabular}

r. Provide video, CD or DVD at time of inspection

$\begin{array}{cccc} & \text { Freq. } & \text { Percent } & \text { Cum. } \\ \text { no | } 348.55 & 99.02 & 99.02 \\ \text { yes | } & 3.45 & 0.98 & 100.00 \\ ----------- \\ \text { Total | } & 352 & 100.00 & \end{array}$

s. Provide in-person instruction at time of inspection

\begin{tabular}{|c|c|c|c|}
\hline & Freq. & Percent & Cum. \\
\hline no & 89.32 & 25.38 & 25.38 \\
\hline yes & 262.68 & 74.62 & 100.00 \\
\hline Total | & 352 & 100.00 & \\
\hline
\end{tabular}


t. Provide hardware kit at time of inspection

\begin{tabular}{|c|c|c|c|}
\hline & Freq. & Percent & Cum \\
\hline no & 345.24 & 98.08 & 98.08 \\
\hline yes & 6.76 & 1.92 & 100.00 \\
\hline otal | & 352 & 100.00 & \\
\hline
\end{tabular}

\section{Other}

u. Group training class

\begin{tabular}{|c|c|c|c|}
\hline & Freq. & Percent & Cum. \\
\hline no & 304.44 & 86.49 & 86.49 \\
\hline yes & 47.56 & 13.51 & 100.00 \\
\hline Total & 352 & 100.00 & \\
\hline
\end{tabular}

v. Other (please specify)

$\begin{array}{crrr} & \text { Freq. } & \text { Percent } & \text { Cum. } \\ \text { no | } 338.46 & 96.15 & 96.15 \\ \text { yes | } & 13.54 & 3.85 & 100.00 \\ --------- \\ \text { Total | }\end{array}$

2. Which of the following broad topics did your agency cover with clients in Program Year 2008? Check all that apply.

\section{a. Thermostat management}

\begin{tabular}{|c|c|c|c|}
\hline & Freq. & Percent & Cum. \\
\hline no & 40.53 & 12.17 & 12.17 \\
\hline yes & 292.47 & 87.83 & 100.00 \\
\hline Total & 333 & 100.00 & \\
\hline
\end{tabular}

\section{b. HVAC system operation/maintenance}

$\begin{array}{rrrr} & \text { Freq. } & \text { Percent } & \text { Cum. } \\ \text { no | } & 66.38 & 19.93 & 19.93 \\ \text { yes | } 266.62 & 80.07 & 100.00 \\ ---------- \\ \text { Total | } & 333 & 100.00 & \end{array}$




\section{c. Distribution system adjustment and zoning}

\begin{tabular}{|c|c|c|c|}
\hline & Freq. & Percent & Cum. \\
\hline no & 230.55 & 69.24 & 69.24 \\
\hline yes & 102.45 & 30.76 & 100.00 \\
\hline Total | & 333 & 100.00 & \\
\hline
\end{tabular}

d. Cooling load reduction

$\begin{array}{rrrr} & \text { Freq. } & \text { Percent } & \text { Cum. } \\ - \text { no | } 247.38 & 74.29 & 74.29 \\ \text { yes | } 85.62 & 25.71 & 100.00 \\ ------ & \end{array}$

e. Windows

\begin{tabular}{|c|c|c|c|}
\hline & Freq. & Percent & Cum. \\
\hline no & 84.97 & 25.52 & 25.52 \\
\hline yes & 248.03 & 74.48 & 100.00 \\
\hline Total | & 333 & 100.00 & \\
\hline
\end{tabular}

\section{f. Insulation}

Freq. Percent Cum.

\begin{tabular}{|c|c|c|c|}
\hline no & 45.88 & 13.78 & 13.78 \\
\hline yes | & 287.12 & 86.22 & 100.00 \\
\hline Total | & 333 & 100.00 & \\
\hline
\end{tabular}

\section{g. Ventilation}

$\begin{array}{rrrr} & \text { Freq. } & \text { Percent } & \text { Cum. } \\ \text { no } \mid & 95.56 & 28.70 & 28.70 \\ \text { yes | } 237.44 & 71.30 & 100.00 \\ ----- & \end{array}$


h. Mold

\begin{tabular}{|c|c|c|c|}
\hline & Freq. & Percent & Cum. \\
\hline no & 111.59 & 33.51 & 33.51 \\
\hline yes & 221.41 & 66.49 & 100.00 \\
\hline Total | & 333 & 100.00 & \\
\hline
\end{tabular}

i. Refrigerator

\begin{tabular}{|c|c|c|c|}
\hline & Freq. & Percent & Cum. \\
\hline no & 115.00 & 34.54 & 34.54 \\
\hline yes & 218.00 & 65.46 & 100.00 \\
\hline Total | & 333 & 100.00 & \\
\hline
\end{tabular}

j. Hot water use

\begin{tabular}{|c|c|c|c|}
\hline & Freq. & Percent & Cum. \\
\hline no & 88.95 & 26.71 & 26.71 \\
\hline yes & 244.05 & 73.29 & 100.00 \\
\hline Total & 333 & 100.00 & \\
\hline
\end{tabular}

k. Water heating system operation/maintenance

\begin{tabular}{|c|c|c|c|}
\hline & Freq. & Percent & Cum. \\
\hline no & 137.18 & 41.19 & 41.19 \\
\hline yes & 195.82 & 58.81 & 100.00 \\
\hline Total & 333 & 100.00 & \\
\hline
\end{tabular}

\section{Lighting}

\begin{tabular}{|c|c|c|c|}
\hline & Freq. & Percent & Cum. \\
\hline no & 54.60 & 16.40 & 16.40 \\
\hline yes & 278.40 & 83.60 & 100.00 \\
\hline Total & 333 & 100.00 & \\
\hline
\end{tabular}


m. Laundry

\begin{tabular}{|c|c|c|c|}
\hline & Freq. & Percent & Cum. \\
\hline no & 187.69 & 56.36 & 56.36 \\
\hline yes & 145.31 & 43.64 & 100.00 \\
\hline Total & 333 & 100.00 & \\
\hline
\end{tabular}

n. Kitchen appliance operation

\begin{tabular}{|c|c|c|c|}
\hline & Freq. & Percent & Cum. \\
\hline no & 194.41 & 58.38 & 58.38 \\
\hline yes & 138.60 & 41.62 & 100.00 \\
\hline Total & 333 & 100.00 & \\
\hline
\end{tabular}

o. Other baseload electric use

\begin{tabular}{|c|c|c|c|}
\hline & Freq. & Percent & Cum. \\
\hline no & 177.79 & 53.39 & 53.39 \\
\hline yes & 155.21 & 46.61 & 100.00 \\
\hline Total | & 333 & 100.00 & \\
\hline
\end{tabular}

p. Energy Star

\begin{tabular}{|c|c|c|c|}
\hline & Freq. & Percent & Cum. \\
\hline no & 199.86 & 60.02 & 60.02 \\
\hline yes | & 133.14 & 39.98 & 100.00 \\
\hline Total & 333 & 100.00 & \\
\hline
\end{tabular}

q. Safety monitors (e.g., CO monitors, smoke alarm)

\begin{tabular}{|c|c|c|c|}
\hline & Freq. & Percent & Cum. \\
\hline no| & 107.05 & 32.15 & 32.15 \\
\hline yes | & 225.95 & 67.85 & 100.00 \\
\hline Total | & 333 & 100.00 & \\
\hline
\end{tabular}




\section{r. Energy bills}

\begin{tabular}{|c|c|c|c|}
\hline & Freq. & Percent & Cum. \\
\hline no & 148.56 & 44.61 & 44.61 \\
\hline yes | & 184.44 & 55.39 & 100.00 \\
\hline Total | & 333 & 100.00 & \\
\hline
\end{tabular}

s. Other (please specify)

$\begin{array}{rrrr} & \text { Freq. } & \text { Percent } & \text { Cum. } \\ \text { no | } & 321.84 & 96.65 & 96.65 \\ \text { yes | } 11.16 & 3.35 & 100.00 \\ ---------- & \end{array}$

3. Which of the following people provided client education for your agency in Program Year 2008? Check all that apply.

a. In-house manager

\begin{tabular}{|c|c|c|c|}
\hline & Freq. & Percent & Cum. \\
\hline no & 236.42 & 70.78 & 70.78 \\
\hline yes & 97.58 & 29.22 & 100.00 \\
\hline Total & 334 & 100.00 & \\
\hline
\end{tabular}

b. In-house education specialist

\begin{tabular}{|c|c|c|c|}
\hline & Freq. & Percent & Cum. \\
\hline no & 293.88 & 87.99 & 87.99 \\
\hline yes & 40.12 & 12.01 & 100.00 \\
\hline Total & 334 & 100.00 & \\
\hline
\end{tabular}

\section{c. Contractor education specialist}

\begin{tabular}{|c|c|c|c|}
\hline & Freq. & Percent & Cum. \\
\hline no & 313.57 & 93.88 & 93.88 \\
\hline yes & 20.43 & 6.12 & 100.00 \\
\hline Total & 334 & 100.00 & \\
\hline
\end{tabular}


d. Intake staff person

\begin{tabular}{|c|c|c|c|}
\hline & Freq. & Percent & Cum. \\
\hline no & 155.24 & 46.48 & 46.48 \\
\hline yes & 178.76 & 53.52 & 100.00 \\
\hline Total | & 334 & 100.00 & \\
\hline
\end{tabular}

e. Auditor

\begin{tabular}{|c|c|c|c|}
\hline & Freq. & Percent & Cum. \\
\hline no & 47.87 & 14.33 & 14.33 \\
\hline yes & 286.13 & 85.67 & 100.00 \\
\hline Total & 334 & 100.00 & \\
\hline
\end{tabular}

f. In-house weatherization crew chief

\begin{tabular}{|c|c|c|c|}
\hline & Freq. & Percent & Cum. \\
\hline no & 199.37 & 59.69 & 59.69 \\
\hline yes & 134.63 & 40.31 & 100.00 \\
\hline Total & 334 & 100.00 & \\
\hline
\end{tabular}

g. Contractor weatherization crew chief

\begin{tabular}{|c|c|c|c|}
\hline & Freq. & Percent & Cum. \\
\hline no & 267.32 & 80.03 & 80.03 \\
\hline yes & 66.68 & 19.97 & 100.00 \\
\hline Total & 334 & 100.00 & \\
\hline
\end{tabular}

h. In-house weatherization crew member

\begin{tabular}{|c|c|c|c|}
\hline & Freq. & Percent & Cum. \\
\hline no & 247.63 & 74.14 & 74.14 \\
\hline yes & 86.37 & 25.86 & 100.00 \\
\hline Total | & 334 & 100.00 & \\
\hline
\end{tabular}




\section{i. Contractor weatherization crew member}

\begin{tabular}{|c|c|c|c|}
\hline & Freq. & Percent & Cum. \\
\hline no & 289.58 & 86.70 & 86.70 \\
\hline yes & 44.42 & 13.30 & 100.00 \\
\hline Total | & 334 & 100.00 & \\
\hline
\end{tabular}

j. Inspector

\begin{tabular}{|c|c|c|c|}
\hline & Freq. & Percent & Cum. \\
\hline no & 126.54 & 37.89 & 37.89 \\
\hline yes & 207.46 & 62.11 & 100.00 \\
\hline Total & 334 & 100.00 & \\
\hline
\end{tabular}

k. Other (please specify)

\begin{tabular}{|c|c|c|c|}
\hline & Freq. & Percent & Cum. \\
\hline no & 319.68 & 95.71 & 95.71 \\
\hline yes | & 14.32 & 4.29 & 100.00 \\
\hline Total & 334 & 100.00 & \\
\hline
\end{tabular}

4. If in-person instruction was provided by your agency in Program Year 2008, who was your preferred target? Check best answer.

Freq. Percent Cum.

$\begin{array}{rrrr}\text { Applicant | } & 308.45 & 94.33 & 94.33 \\ \text { Other adult member of household | } & 10.51 & 3.21 & 97.54 \\ \text { Child living in household | } & .92 & 0.28 & 97.82 \\ \text { Adult not living in household | } & .46 & 0.14 & 97.96 \\ \text { Other | } & 6.66 & 2.04 & 100.00 \\ \text { Total | } & 327 & 100.00 & \end{array}$

5. If in-person instruction was provided by your agency in Program Year 2008, was it typically provided to a single person or multiple persons? Check best answer.

$\begin{array}{cccc}\text { Freq. } & \text { Percent } & \text { Cum. } & \\ ----- \\ \text { single person | } 256.41 & 78.17 & 78.17 \\ \text { multiple persons | } & 71.59 & 21.83 & 100.00 \\ ------------ & \end{array}$


6. What types of credentials or experience were required of those who provided client education for your agency in Program Year 2008? Check all that apply.

- College degree

\begin{tabular}{|c|c|c|c|}
\hline & Freq. & Percent & Cum. \\
\hline no & 306.20 & 94.51 & 94.51 \\
\hline yes & 17.80 & 5.49 & 100.00 \\
\hline Total & 324 & 100.00 & \\
\hline
\end{tabular}

- Technical certification

\begin{tabular}{|c|c|c|c|}
\hline & Freq. & Percent & Cum. \\
\hline no & 158.17 & 48.82 & 48.82 \\
\hline yes & 165.83 & 51.18 & 100.00 \\
\hline Total & 324 & 100.00 & \\
\hline
\end{tabular}

- Extensive experience in performing weatherization work

\begin{tabular}{|c|c|c|c|}
\hline & Freq. & Percent & Cum. \\
\hline no & 92.88 & 28.67 & 28.67 \\
\hline yes & 231.12 & 71.33 & 100.00 \\
\hline Total | & 324 & 100.00 & \\
\hline
\end{tabular}

- Extensive experience in supervising weatherization work

$\begin{array}{ccc}\text { Freq. } & \text { Percent } & \text { Cum. } \\ \text { no | } 206.92 & 63.86 & 63.86 \\ \text { yes | } 117.08 & 36.14 & 100.00 \\ ------ & \\ \text { Total | } 324 & 100.00\end{array}$

- Educational background

\begin{tabular}{|c|c|c|c|}
\hline & Freq. & Percent & Cum. \\
\hline no & 265.42 & 81.92 & 81.92 \\
\hline yes & 58.58 & 18.08 & 100.00 \\
\hline Total & 324 & 100.00 & \\
\hline
\end{tabular}


- Other (please specify)

\begin{tabular}{|c|c|c|c|}
\hline & Freq. & Percent & Cum. \\
\hline no & 289.04 & 89.21 & 89.21 \\
\hline yes | & 34.96 & 10.79 & 100.00 \\
\hline Total | & 324 & 100.00 & \\
\hline
\end{tabular}

7. Which of the client education approaches listed below were initiated by your agency in Program Year 2008 and the two years prior to Program Year 2008? Check all that apply.

\section{At time of client intake}

a. Provide literature at time of client intake

\begin{tabular}{|c|c|c|c|}
\hline & Freq. & Percent & Cum. \\
\hline unchecked & 120.71 & 46.07 & 46.07 \\
\hline checked & 141.29 & 53.93 & 100.00 \\
\hline Total & 262 & 100.00 & \\
\hline
\end{tabular}

b. Provide video, CD or DVD at time of client intake

Freq. Percent Cum.

\begin{tabular}{|c|c|c|c|}
\hline unchecked & 247.31 & 94.39 & 94.39 \\
\hline checked & 14.69 & 5.61 & 100.00 \\
\hline Total & 262 & 100.00 & \\
\hline
\end{tabular}

c. Provide in-person instruction at time of client intake

\begin{tabular}{|c|c|c|c|}
\hline & Freq. & Percent & Cum. \\
\hline unchecked & 157.27 & 60.03 & 60.03 \\
\hline checked & 104.73 & 39.97 & 100.00 \\
\hline Total & 262 & 100.00 & \\
\hline
\end{tabular}

d. Provide hardware kit at time of client intake

\begin{tabular}{|c|c|c|c|}
\hline & Freq. & rcent & Cum. \\
\hline unchecked & 250.61 & 95.65 & 95.65 \\
\hline checked & 11.39 & 4.35 & 100.00 \\
\hline Total & 262 & 100.00 & \\
\hline
\end{tabular}


At time of audit

e. Provide literature at time of audit

\begin{tabular}{|c|c|c|c|}
\hline & Freq. & Percent & Cum. \\
\hline unchecked & 79.18 & 30.22 & 30.22 \\
\hline checked & 182.82 & 69.78 & 100.00 \\
\hline Total & 262 & 100.00 & \\
\hline
\end{tabular}

f. Provide video, CD or DVD at time of audit

\begin{tabular}{|c|c|c|c|}
\hline & Freq. & Percent & Cum. \\
\hline unchecked & | 252.86 & 96.51 & 96.51 \\
\hline checked & | 9.14 & 3.49 & 100.00 \\
\hline Total | & 262 & 100.00 & \\
\hline
\end{tabular}

g. Provide in-person instruction at time of audit

\begin{tabular}{|c|c|c|c|}
\hline & Freq. & Percent & Cum. \\
\hline unchecked & 62.62 & 23.90 & 23.90 \\
\hline checked & 199.38 & 76.10 & 100.00 \\
\hline Total & 262 & 100.00 & \\
\hline
\end{tabular}

h. Provide hardware kit at time of audit

\begin{tabular}{|c|c|c|c|}
\hline & Freq. & Percent & Cum. \\
\hline unchecked & 240.64 & 91.85 & 91.85 \\
\hline checked & 21.36 & 8.15 & 100.00 \\
\hline Total | & 262 & 100.00 & \\
\hline
\end{tabular}

At time of weatherization

i. Provide literature at time of weatherization

$\begin{array}{rrr}\text { Freq. } & \text { Percent } & \text { Cum. } \\ \text { unchecked | } 195.00 & 74.43 & 74.43 \\ \text { checked | } 67.00 & 25.57 & 100.00 \\ ---------+--------------------------- \\ \text { Total | } 262 & 100.00 & \end{array}$

j. Provide video, CD or DVD at time of weatherization 


\begin{tabular}{|c|c|c|c|}
\hline Freq. & Percent & Cum. & \\
\hline unchecked & 260.98 & 99.61 & 99.61 \\
\hline checked & 1.02 & 0.39 & 100.00 \\
\hline Total | & 262 & 100.00 & \\
\hline
\end{tabular}

k. Provide in-person instruction at time of weatherization

\begin{tabular}{|c|c|c|c|}
\hline & Freq. & rcent & Cum. \\
\hline unchecked | & 94.05 & 35.90 & 35.90 \\
\hline checked & 167.95 & 64.10 & 100.00 \\
\hline 10tai! & 262 & 100.00 & \\
\hline
\end{tabular}

1. Provide hardware kit at time of weatherization

\begin{tabular}{|c|c|c|c|}
\hline & Freq. & Percent & Cum. \\
\hline unchecked & 246.95 & 94.26 & 94.26 \\
\hline checked & 15.05 & 5.74 & 100.00 \\
\hline Total & 262 & 100.00 & \\
\hline
\end{tabular}

At time of separate client education visit

m. Provide literature at separate client education visit

\begin{tabular}{|c|c|c|c|}
\hline & Freq. & Percent & Cum. \\
\hline unchecked & 203.16 & 77.54 & 77.54 \\
\hline checked & 58.84 & 22.46 & 100.00 \\
\hline Total & 262 & 100.00 & \\
\hline
\end{tabular}

n. Provide video, CD or DVD at separate client education visit

Freq. Percent Cum.

\begin{tabular}{|c|c|c|c|}
\hline unchecked & 255.59 & 97.55 & 97.55 \\
\hline checked & 6.41 & 2.45 & 100.00 \\
\hline Total & 262 & 100.00 & \\
\hline
\end{tabular}


o. Provide in-person instruction at separate client education visit

$\begin{array}{rrr}\text { Freq. } & \text { Percent } & \text { Cum. } \\ \text { unchecked | } 196.53 & 75.01 & 75.01 \\ \text { checked | } 65.47 & 24.99 & 100.00 \\ --------+---------------------- \\ \text { Total | } 262 & 100.00\end{array}$

p. Provide hardware kit at separate client education visit

\begin{tabular}{|c|c|c|c|}
\hline & Freq. & Percent & Cum. \\
\hline unchecked & 249.13 & 95.09 & 95.09 \\
\hline checked & 12.87 & 4.91 & 100.00 \\
\hline Total & 262 & 100.00 & \\
\hline
\end{tabular}

\section{At time of inspection}

q. Provide literature at time of inspection

\begin{tabular}{|c|c|c|c|}
\hline & Freq. & Percent & Cum. \\
\hline unchecked & 168.31 & 64.24 & 64.24 \\
\hline checked & 93.69 & 35.76 & 100.00 \\
\hline Total & 262 & 100.00 & \\
\hline
\end{tabular}

r. Provide video, CD or DVD at time of inspection

\begin{tabular}{|c|c|c|c|}
\hline & Freq. & Percent & Cum. \\
\hline unchecked & 259.65 & 99.10 & 99.10 \\
\hline checked & 2.35 & 0.90 & 100.00 \\
\hline Total & 262 & 100.00 & \\
\hline
\end{tabular}

s. Provide in-person instruction at time of inspection

\begin{tabular}{|c|c|c|c|}
\hline & Freq. & Percent & Cum. \\
\hline unchecked & 65.79 & 25.11 & 25.11 \\
\hline checked & 196.21 & 74.89 & 100.00 \\
\hline Total & 262 & 100.00 & \\
\hline
\end{tabular}


t. Provide hardware kit at time of inspection

\begin{tabular}{|c|c|c|c|}
\hline & Freq. & Percent & Cum. \\
\hline unchecked & 254.24 & 97.04 & 97.04 \\
\hline checked & 7.76 & 2.96 & 100.00 \\
\hline Fen & 262 & 100.00 & \\
\hline
\end{tabular}

\section{Other}

u. Group training class

\begin{tabular}{|c|c|c|c|}
\hline & Freq. & Percent & Cum. \\
\hline unchecked & 230.31 & 87.91 & 87.91 \\
\hline checked & 31.69 & 12.09 & 100.00 \\
\hline Total & 262 & 100.00 & \\
\hline
\end{tabular}

v. Other (please specify)

$\begin{array}{crr}\text { Freq. } & \text { Percent } & \text { Cum. } \\ \text {--onchecked | } 257.80 & 98.40 & 98.40 \\ \text { checked | } 4.20 & 1.60 & 100.00 \\ ----------------------------------- \\ \text { Total | } 262 & 100.00\end{array}$


8. For Program Year 2008 please rate key aspects (cost, training needed, time needed and effectiveness) of the diagnostic procedures listed below.

Please use the following scale: 1 - very low; 2 -low; 3 - medium; 4 - high; 5 - very high.

For example, if you view providing literature at the time of client intake as low-cost, enter a rating of 2 in the Cost column. If you view providing literature at the time of client intake as having medium effectiveness, enter a rating of 3 in the Effectiveness column.

\begin{tabular}{|c|c|c|c|c|c|c|c|c|c|c|c|c|c|c|c|c|}
\hline & \multicolumn{4}{|c|}{ Cost } & \multicolumn{4}{|c|}{ Training Needed } & \multicolumn{4}{|c|}{ Time Needed } & \multicolumn{4}{|c|}{ Effectiveness } \\
\hline \multirow{7}{*}{$\begin{array}{l}\text { Provide } \\
\text { literature at } \\
\text { time of client } \\
\text { intake }\end{array}$} & & Freq. & Percent & Cum. & & Freq. & Percent & Cum. & & Freq. & Percent & Cum. & & Freq. & Percent & Cum. \\
\hline & very low & | 71.66 & 34.45 & 34.45 & very low | & 76.74 & 36.54 & 36.54 & very low & 66.04 & 32.22 & 32.22 & very low & | 18.43 & 8.05 & 8.05 \\
\hline & low & 106.31 & 51.11 & 85.56 & low & 61.59 & 29.33 & 65.87 & & 76.71 & 37.42 & 69.64 & low & 42.89 & 18.73 & 26.78 \\
\hline & medium & 15.84 & 7.62 & 93.18 & medium & 41.38 & 19.70 & 85.57 & medium & 40.95 & 19.97 & 89.61 & medium & 68.80 & 30.05 & 56.82 \\
\hline & high & 9.80 & 4.71 & 97.90 & high & 24.24 & 11.54 & 97.12 & high & 16.46 & 8.03 & 97.64 & high & 67.36 & 29.42 & 86.24 \\
\hline & very high & 4.38 & 2.10 & 100.00 & very high & 6.05 & 2.88 & 100.00 & very high & 4.84 & 2.36 & 100.00 & very high & 31.51 & 13.76 & 100.00 \\
\hline & Total | & 208 & 100.00 & & Total | & 210 & 100.00 & & Total & 205 & 100.00 & & Total | & 229 & 100.00 & \\
\hline \multirow{7}{*}{$\begin{array}{l}\text { Provide video, } \\
\text { CD or DVD at } \\
\text { time of client } \\
\text { intake }\end{array}$} & & Freq. & Percent & Cum. & & Freq. & Percent & Cum. & & Freq. & Percent & Cum. & & Freq. & Percent & Cum. \\
\hline & very low & 16.98 & 22.64 & 22.64 & very low & 38.05 & 49.41 & 49.41 & very low & 26.15 & 35.82 & 35.82 & very low & 31.15 & 37.08 & 37.08 \\
\hline & low & 21.01 & 28.01 & 50.66 & & 13.04 & 16.93 & 66.34 & low & 20.12 & 27.57 & 63.38 & low & 13.17 & 15.68 & 52.76 \\
\hline & medium & 14.34 & 19.12 & 69.78 & medium & 14.34 & 18.63 & 84.97 & medium & 13.76 & 18.85 & 82.23 & medium & 15.94 & 18.98 & 71.74 \\
\hline & high & 9.22 & 12.29 & 82.07 & high & 8.48 & 11.02 & 95.99 & high & 9.11 & 12.49 & 94.72 & high & 12.01 & 14.29 & 86.04 \\
\hline & very high & 13.45 & 17.93 & 100.00 & very high & 3.09 & 4.01 & 100.00 & very high & 3.86 & 5.28 & 100.00 & very high & 11.73 & 13.96 & 100.00 \\
\hline & Total & 75 & 100.00 & & Total | & 77 & 100.00 & & Total & 73 & 100.00 & & Total | & 84 & 100.00 & \\
\hline \multirow{7}{*}{$\begin{array}{l}\text { Provide in- } \\
\text { person } \\
\text { instruction at } \\
\text { time of client } \\
\text { intake }\end{array}$} & & Freq. & Percent & Cum. & & Freq. & Percent & Cum. & & Freq. & Percent & Cum. & & Freq. & Percent & Cum. \\
\hline & very low & 49.81 & 28.46 & 28.46 & very low & 32.34 & 17.87 & 17.87 & very low & | 19.53 & 11.10 & 11.10 & very low & 18.09 & 9.28 & 9.28 \\
\hline & low & 69.43 & 39.68 & 68.14 & low & 34.45 & 19.03 & 36.90 & low & 51.07 & 29.02 & 40.11 & low & 25.85 & 13.26 & 22.53 \\
\hline & medium & 31.84 & 18.19 & 86.33 & medium & 57.79 & 31.93 & 68.83 & medium & 70.57 & 40.10 & 80.21 & medium & 57.15 & 29.31 & 51.84 \\
\hline & high & 16.78 & 9.59 & 95.92 & high & 49.31 & 27.24 & 96.07 & high & 30.10 & 17.10 & 97.31 & high & 61.57 & 31.57 & 83.41 \\
\hline & very high & 7.14 & 4.08 & 100.00 & very high & 7.11 & 3.93 & 100.00 & very high & 4.73 & 2.69 & 100.00 & very high & 32.34 & 16.59 & 100.00 \\
\hline & Total | & 175 & 100.00 & & Total | & 181 & 100.00 & & Total & 176 & 100.00 & & Total | & 195 & 100.00 & \\
\hline \multirow{7}{*}{$\begin{array}{l}\text { Provide } \\
\text { hardware kit at } \\
\text { time of client } \\
\text { intake }\end{array}$} & & Freq. & Percent & Cum. & & Freq. & Percent & Cum. & & Freq. & Percent & Cum. & & Freq. & Percent & Cum. \\
\hline & very low & | 24.18 & 31.00 & 31.00 & very low | & 28.59 & 34.86 & 34.86 & very low & 20.37 & 26.81 & 26.81 & very low & 31.58 & 37.59 & 37.59 \\
\hline & low & 16.12 & 20.65 & 51.65 & low & 16.70 & 20.37 & 55.23 & low & 21.79 & 28.68 & 55.48 & low & 14.81 & 17.63 & 55.22 \\
\hline & medium & 14.72 & 18.87 & 70.52 & medium & 13.27 & 16.19 & 71.41 & medium & 17.91 & 23.57 & 79.05 & medium & 13.75 & 16.37 & 71.59 \\
\hline & high & 11.09 & 14.21 & 84.73 & high & 15.46 & 18.85 & 90.26 & high & 8.62 & 11.34 & 90.38 & high & 17.17 & 20.44 & 92.03 \\
\hline & very high & 11.91 & 15.27 & 100.00 & very high & 7.99 & 9.74 & 100.00 & very high & 7.31 & 9.62 & 100.00 & very high & 6.69 & 7.97 & 100.00 \\
\hline & Total & 78 & 100.00 & & Total | & 82 & 100.00 & & Total & 76 & 100.00 & & Total | & 84 & 100.00 & \\
\hline
\end{tabular}




\begin{tabular}{|c|c|c|c|c|c|c|c|c|c|c|c|c|c|c|c|c|}
\hline & \multicolumn{4}{|c|}{ Cost } & \multicolumn{4}{|c|}{ Training Needed } & \multicolumn{4}{|c|}{ Time Needed } & \multicolumn{4}{|c|}{ Effectiveness } \\
\hline \multirow{7}{*}{$\begin{array}{l}\text { Provide } \\
\text { literature at } \\
\text { time of audit }\end{array}$} & & $\begin{array}{ll}\text { Freq. } & \mathrm{F}\end{array}$ & Percent & Cum. & & Freq. & Percent & Cum. & & Freq. & Percent & Cum. & & Freq. & Percent & Cum. \\
\hline & very low & 68.60 & 30.76 & 0.76 & very low | & 57.24 & 25.55 & 25.55 & very low & 57.05 & 25.93 & 25.93 & very low & 14.15 & 5.78 & 5.78 \\
\hline & & 100.11 & 44.89 & 75.65 & low & 62.54 & 27.92 & 53.47 & low & 86.30 & 39.23 & 65.16 & low & 31.48 & 12.85 & 18.62 \\
\hline & medium & 30.97 & 13.89 & 89.54 & medium | & 59.78 & 26.69 & 80.16 & medium & 54.58 & 24.81 & 89.97 & medium | & 78.58 & 32.07 & 50.70 \\
\hline & & 20.31 & 9.11 & 98.65 & high & 33.95 & 15.16 & 95.32 & high & 17.09 & 7.77 & 97.73 & high & 75.33 & 30.75 & 81.44 \\
\hline & very high & 3.02 & 1.35 & 100.00 & very high & 10.49 & 4.68 & 100.00 & very high & 4.99 & 2.27 & 100.00 & very high & 45.46 & 18.56 & 100.00 \\
\hline & Total & 223 & 100.00 & & Total | & 224 & 100.00 & & Total & 220 & 100.00 & & Total & 245 & 100.00 & \\
\hline \multirow{7}{*}{$\begin{array}{l}\text { Provide in- } \\
\text { person } \\
\text { instruction at } \\
\text { time of } \\
\text { weatherization }\end{array}$} & & Freq. $\quad \mathrm{F}$ & Percent & Cum. & & $\begin{array}{ll}\text { Freq. } \quad \mathrm{I} \\
\end{array}$ & Percent & Cum. & & Freq. $\quad \mathrm{P}$ & Percent & Cum. & & Freq. & Percent & Cum. \\
\hline & very low | & 56.03 & 27.33 & 27.33 & very low & 26.10 & 12.43 & 12.43 & very low | & 22.60 & 10.97 & 10.97 & very low | & 8.12 & 3.61 & 3.61 \\
\hline & low & 73.51 & 35.86 & 63.19 & low & 44.29 & 21.09 & 33.52 & low & 64.01 & 31.07 & 42.05 & low & 20.09 & 8.93 & 12.54 \\
\hline & medium | & 45.43 & 22.16 & 85.35 & medium | & 74.42 & 35.44 & 68.96 & medium | & 86.81 & 42.14 & 84.19 & medium | & 60.81 & 27.03 & 39.57 \\
\hline & high & 20.02 & 9.77 & 95.12 & high & 50.99 & 24.28 & 93.24 & high & 23.09 & 11.21 & 95.40 & high & 85.08 & 37.82 & 77.39 \\
\hline & very high & 10.00 & 4.88 & 100.00 & very high & 14.19 & 6.76 & 100.00 & very high & 9.49 & 4.60 & 100.00 & very high & 50.87 & 22.61 & 100.00 \\
\hline & Total | & 205 & 100.00 & & Total | & 210 & 100.00 & & Total & 206 & 100.00 & & Total & 225 & 100.00 & \\
\hline \multirow{7}{*}{$\begin{array}{l}\text { Provide } \\
\text { hardware kit at } \\
\text { time of } \\
\text { weatherization }\end{array}$} & & $\begin{array}{ll}\text { Freq. } & \mathrm{P}\end{array}$ & Percent & Cum. & & Freq. & Percent & Cum. & & Freq. $\quad \mathrm{P}$ & Percent & Cum. & & Freq. & ercent & Cum. \\
\hline & very low | & 20.28 & 29.82 & 29.82 & very low | & 28.10 & 39.03 & 39.03 & very low | & 21.11 & 31.51 & 31.51 & very low | & 22.16 & 28.78 & 28.78 \\
\hline & low & 15.37 & 22.61 & 52.43 & low & 17.69 & 24.57 & 63.60 & low & 22.86 & 34.11 & 65.62 & low & 8.82 & 11.45 & 40.23 \\
\hline & medium & 13.21 & 19.42 & 71.85 & medium & 11.82 & 16.42 & 80.02 & medium & 11.68 & 17.43 & 83.05 & medium & 9.29 & 12.06 & 52.29 \\
\hline & high & 11.41 & 16.79 & 88.64 & high & 8.56 & 11.90 & 91.92 & high & 8.33 & 12.44 & 95.49 & high & 22.40 & 29.09 & 81.38 \\
\hline & very high & 7.73 & 11.36 & 100.00 & very high & 5.82 & 8.08 & 100.00 & very high & 3.02 & 4.51 & 100.00 & very high & 14.33 & 18.62 & 100.00 \\
\hline & Total | & 68 & 100.00 & & Total | & 72 & 100.00 & & Total | & | 67 & 100.00 & & Total | & \begin{tabular}{|l} 
| \\
\end{tabular} & 100.00 & \\
\hline \multirow{7}{*}{$\begin{array}{l}\text { Provide } \\
\text { literature at } \\
\text { separate client } \\
\text { education visit }\end{array}$} & & Freq. $\quad \mathrm{F}$ & Percent & Cum. & & Freq. & Percent & Cum. & & Freq. $\quad \mathrm{P}$ & Percent & Cum. & & Freq. & Percent & Cum. \\
\hline & very low & 17.92 & 19.69 & 19.69 & very low & 37.75 & 39.33 & 39.33 & very low & 20.87 & 23.19 & 23.19 & ry low & 21.31 & 20.89 & 20.89 \\
\hline & low & 23.16 & 25.45 & 45.15 & low & 16.58 & 17.27 & 56.59 & low & 13.72 & 15.24 & 38.43 & low & 15.93 & 15.62 & 36.52 \\
\hline & medium | & 16.98 & 18.66 & 63.80 & medium | & 17.19 & 17.91 & 74.50 & medium & 26.01 & 28.95 & 67.39 & medium | & 29.06 & 28.49 & 65.00 \\
\hline & high & 14.84 & 16.31 & 80.11 & high & 20.49 & 21.34 & 95.84 & high & 17.21 & 19.12 & 86.51 & high & 23.17 & 22.71 & 87.71 \\
\hline & very high & 18.10 & 19.89 & 100.00 & very high & 3.99 & 4.16 & 100.00 & very high & 12.14 & 13.49 & 100.00 & very high & 12.53 & 12.29 & 100.00 \\
\hline & Total | & 91 & 100.00 & & Total | & 96 & 100.00 & & Total | & 90 & 100.00 & & Total & 102 & 100.00 & \\
\hline \multirow{7}{*}{$\begin{array}{l}\text { Provide video, } \\
\text { CD or DVD at } \\
\text { separate client } \\
\text { education visit }\end{array}$} & & Freq. $\quad \mathrm{F}$ & Percent & Cum. & & Freq. & Percent & Cum. & & Freq. $\quad \mathrm{P}$ & Percent & Cum & & Freq. & Percent & Cum. \\
\hline & very low & 13.09 & 21.82 & 21.82 & very low & 28.83 & 45.76 & 45.76 & very low | & 15.74 & 26.67 & 26.67 & very low & 24.21 & 36.68 & 36.68 \\
\hline & low & 6.52 & 10.86 & 32.68 & low & 16.38 & 26.00 & 71.76 & low & 8.16 & 13.82 & 40.50 & low & 13.28 & 20.12 & 56.80 \\
\hline & medium | & 14.82 & 24.69 & 57.37 & medium & 5.82 & 9.24 & 81.00 & medium & 12.66 & 21.46 & 61.95 & medium | & 13.77 & 20.87 & 77.67 \\
\hline & & 7.29 & 12.15 & 69.52 & high & 10.91 & 17.32 & 98.32 & high & 6.68 & 11.32 & 73.28 & high & 11.18 & 16.94 & 94.61 \\
\hline & very high & 18.29 & 30.48 & 100.00 & very high & 1.06 & 1.68 & 100.00 & very high & 15.77 & 26.72 & 100.00 & very high & 3.56 & 5.39 & 100.00 \\
\hline & Total | & 60 & 100.00 & & Total | & 63 & 100.00 & & Total | & 59 & 100.00 & & Total | & 66 & 100.00 & \\
\hline
\end{tabular}




\begin{tabular}{|c|c|c|c|c|c|c|c|c|c|c|c|c|c|c|c|c|}
\hline & \multicolumn{4}{|c|}{ Cost } & \multicolumn{4}{|c|}{ Training Needed } & \multicolumn{4}{|c|}{ Time Needed } & \multicolumn{4}{|c|}{ Effectiveness } \\
\hline \multirow{7}{*}{$\begin{array}{l}\text { Provide in- } \\
\text { person } \\
\text { instruction at } \\
\text { separate client } \\
\text { education visit }\end{array}$} & & $\begin{array}{ll}\text { Freq. } & \mathrm{I}\end{array}$ & Percent & Cum. & & Freq. & Percent & Cum. & & Freq. & Percent & Cum. & & Freq. & Percent & Cum. \\
\hline & very low & 19.64 & 20.46 & 20.46 & very low & 29.42 & 28.84 & 28.84 & very low & 13.20 & 13.89 & 13.89 & very low & 18.69 & 17.47 & 17.47 \\
\hline & low & 20.88 & 21.75 & 42.21 & low & 14.93 & 14.64 & 43.48 & low & 13.15 & 13.84 & 27.73 & low & 12.19 & 11.40 & 28.87 \\
\hline & medium | & 21.94 & 22.85 & 65.06 & medium | & 23.17 & 22.71 & 66.19 & medium | & 31.69 & 33.35 & 61.09 & medium | & 21.58 & 20.17 & 49.04 \\
\hline & high & 11.46 & 11.94 & 77.00 & high & 30.00 & 29.41 & 95.60 & high & 18.67 & 19.65 & 80.74 & high & 34.04 & 31.81 & 80.85 \\
\hline & very high | & 22.08 & 23.00 & 100.00 & very high & 4.48 & 4.40 & 100.00 & very high & 18.30 & 19.26 & 100.00 & very high & 20.49 & 19.15 & 100.00 \\
\hline & Total $\mid$ & 96 & 100.00 & & Total | & 102 & 100.00 & & Total $\mid$ & 95 & 100.00 & & Total | & 107 & 100.00 & \\
\hline \multirow{7}{*}{$\begin{array}{l}\text { Provide } \\
\text { hardware kit at } \\
\text { separate client } \\
\text { education visit }\end{array}$} & & Freq. $\quad \mathrm{P}$ & Percent & Cum. & & $\begin{array}{ll}\text { Freq. } & \mathrm{P}\end{array}$ & Percent & Cum. & & Freq. & Percent & Cum. & & $\begin{array}{ll}\text { Freq. } \quad \mathrm{I} & \\
\end{array}$ & Percent & Cum. \\
\hline & very low | & 15.21 & 23.04 & 23.04 & very low | & 29.26 & 42.41 & 42.41 & very low & 15.96 & 24.94 & 24.94 & very low & 29.04 & 40.33 & 40.33 \\
\hline & low & 5.63 & 8.54 & 31.57 & low & 9.54 & 13.83 & 56.24 & low & 4.88 & 7.62 & 32.56 & low & 13.10 & 18.20 & 58.52 \\
\hline & medium & 18.45 & 27.95 & 59.53 & medium & 16.79 & 24.33 & 80.56 & medium | & 22.70 & 35.47 & 68.0 & medium | & 12.38 & 17.19 & 75.72 \\
\hline & high & 9.98 & 15.13 & 74.66 & high & 10.90 & 15.80 & 96.37 & high & 7.39 & 11.55 & 79.58 & high & 10.11 & 14.04 & 89.76 \\
\hline & very high & 16.73 & 25.34 & 100.00 & very high & 2.51 & 3.63 & 100.00 & very high & 13.07 & 20.42 & 100.00 & very high & 7.38 & 10.24 & 100.00 \\
\hline & Total & 66 & 100.00 & & Total & 69 & 100.00 & & Total & 64 & 100.00 & & Total & 72 & 100.00 & \\
\hline \multirow{7}{*}{$\begin{array}{l}\text { Provide } \\
\text { literature at time } \\
\text { of inspection }\end{array}$} & & Freq. $\quad \mathrm{F}$ & Percent & Cum. & & $\begin{array}{ll}\text { Freq. } & \mathrm{P}\end{array}$ & Percent & Cum. & & Freq. & Percent & Cum. & & $\begin{array}{ll}\text { Freq. } \quad \mathrm{I} \\
\end{array}$ & Percent & Cum. \\
\hline & very low & 46.59 & 32.58 & 32.58 & very low & 40.11 & 27.48 & 27.48 & very low & 37.40 & 26.91 & 26.91 & very low & 12.12 & 7.72 & 7.72 \\
\hline & low & 58.96 & 41.23 & 73.81 & low & 35.45 & 24.28 & 51.76 & low & 50.28 & 36.17 & & low & 19.72 & 12.56 & 20.28 \\
\hline & medium & 22.46 & 15.71 & 89.52 & medium | & 39.31 & 26.92 & 78.68 & medium | & 33.63 & 24.20 & 87.27 & medium | & 43.12 & 27.47 & 47.75 \\
\hline & high & 7.37 & 5.16 & 94.67 & high & 21.80 & 14.93 & 93.62 & high & 12.19 & 8.77 & 96.04 & high & 50.39 & 32.10 & 79.84 \\
\hline & very high & 7.62 & 5.33 & 100.00 & very high & 9.32 & 6.38 & 100.00 & very high & 5.50 & 3.96 & 100.00 & very high & 31.64 & 20.16 & 100.00 \\
\hline & Total | & 143 & 100.00 & & Total | & 146 & 100.00 & & Total | & 139 & 100.00 & & Total | & 157 & 100.00 & \\
\hline \multirow{7}{*}{$\begin{array}{l}\text { Provide video, } \\
\text { CD or DVD at } \\
\text { time of } \\
\text { inspection }\end{array}$} & & $\begin{array}{ll}\text { Freq. } \quad \mathrm{I} \\
\end{array}$ & Percent & Cum. & & Freq. & Percent & Cum. & & Freq. & Percent & Cum. & & $\begin{array}{ll}\text { Freq. } & \mathrm{P}\end{array}$ & Percent & Cum. \\
\hline & very low & 20.36 & 32.31 & 32.31 & very low | & 31.62 & 48.64 & 48.64 & very low | & 23.83 & 40.39 & & very low | & 21.53 & 31.66 & 31.66 \\
\hline & low & 9.75 & 15.47 & 47.78 & low & 10.45 & 16.08 & 64.72 & low & 8.77 & 14.87 & 55.25 & low & 8.37 & 12.32 & 43.97 \\
\hline & medium & 11.72 & 18.61 & 66.39 & medium | & 15.17 & 23.33 & 88.06 & medium | & 12.94 & 21.94 & 77.19 & medium | & 16.67 & 24.52 & 68.49 \\
\hline & high $\mid$ & 4.36 & 6.92 & 73.31 & high $\mid$ & 5.67 & 8.72 & 96.78 & high & 6.94 & 11.76 & 88.95 & high & 13.87 & 20.40 & 88.89 \\
\hline & very high | & 16.82 & 26.69 & 100.00 & very high & 2.09 & 3.22 & 100.00 & very high | & 6.52 & 11.05 & 100.00 & very high & 7.55 & 11.11 & 100.00 \\
\hline & Total | & 63 & 100.00 & & Total | & 65 & 100.00 & & Total | & 59 & 100.00 & & Total | & 68 & 100.00 & \\
\hline \multirow{7}{*}{$\begin{array}{l}\text { Provide in- } \\
\text { person } \\
\text { instruction at } \\
\text { time of } \\
\text { inspection }\end{array}$} & & Freq. & Percent & Cum. & & $\begin{array}{ll}\text { Freq. } & \mathrm{P}\end{array}$ & Percent & Cum. & & Freq. & Percent & Cum. & & $\begin{array}{ll}\text { Freq. } & \mathrm{P}\end{array}$ & Percent & Cum. \\
\hline & very low & 68.02 & 30.23 & 30.23 & very low & 29.86 & 12.98 & 12.98 & very low | & 20.02 & 9.02 & 9.0 & very low & 4.49 & 1.87 & 1.87 \\
\hline & low & 93.98 & 41.77 & 72.00 & low & 58.47 & 25.42 & 38.41 & low & 81.41 & 36.67 & 45.69 & low & 17.84 & 7.43 & 9.31 \\
\hline & medium & 36.70 & 16.31 & 88.31 & medium & 67.26 & 29.24 & 67.65 & medium | & 77.42 & 34.87 & 80.56 & medium | & 64.37 & 26.82 & 36.13 \\
\hline & high $\mid$ & 18.57 & 8.25 & 96.56 & high & 60.96 & 26.51 & 94.15 & high & 29.92 & 13.48 & 94.04 & high & 92.04 & 38.35 & 74.48 \\
\hline & very high & 7.73 & 3.44 & 100.00 & very high & 13.44 & 5.85 & 100.00 & very high | & 13.24 & 5.96 & 100.00 & very high | & 61.26 & 25.52 & 100.00 \\
\hline & Total | & 225 & 100.00 & & Total | & 230 & 100.00 & & Total & 222 & 100.00 & & Total | & 240 & 100.00 & \\
\hline
\end{tabular}




\begin{tabular}{|c|c|c|c|c|c|c|c|c|c|c|c|c|c|c|c|c|}
\hline \multirow{8}{*}{$\begin{array}{l}\text { Provide hardware } \\
\text { kit at time of } \\
\text { inspection }\end{array}$} & \multicolumn{4}{|c|}{ Cost } & \multicolumn{4}{|c|}{ Training Needed } & \multicolumn{4}{|c|}{ Time Needed } & \multicolumn{4}{|c|}{ Effectiveness } \\
\hline & & Freq. & Percent & Cum. & & Freq. & Percent & Cum. & & Freq. & Percent & Cum. & & Freq. & Percent & Cum. \\
\hline & very low & 23.62 & 36.34 & 36.34 & very low & 34.20 & 50.29 & 50.29 & very low & 23.51 & 37.92 & 37.92 & very low & 29.38 & 42.58 & 42.58 \\
\hline & low & 14.78 & 22.73 & 59.07 & & 15.57 & 22.89 & 73.18 & low & 14.40 & 23.23 & 61.14 & low & 10.02 & 14.53 & 57.11 \\
\hline & medium | & 12.04 & 18.53 & 77.60 & medium & 11.02 & 16.21 & 89.39 & medium | & 15.10 & 24.36 & 85.50 & medium & 11.33 & 16.42 & 73.53 \\
\hline & high & 7.07 & 10.87 & 88.47 & high & 6.59 & 9.69 & 99.08 & high & 4.08 & 6.57 & 92.08 & high & 10.30 & 14.93 & 88.46 \\
\hline & very high | & 7.49 & 11.53 & 100.00 & very high & .63 & 0.92 & 100.00 & very high & 4.91 & 7.92 & 100.00 & very high & 7.96 & 11.54 & 100.00 \\
\hline & Total & 65 & 100.00 & & Total & 68 & 100.00 & & Total | & 62 & 100.00 & & Total & 69 & 100.00 & \\
\hline \multirow{7}{*}{$\begin{array}{l}\text { Group training } \\
\text { class }\end{array}$} & & Freq. & Percent & Cum. & & Freq. & Percent & Cum. & & Freq. & Percent & Cum. & & Freq. & Percent & Cum. \\
\hline & very low & 22.39 & 26.66 & 26.66 & very low & 23.28 & 26.76 & 26.76 & very low & 12.92 & 15.21 & 15.21 & very low & 29.88 & 32.13 & 32.13 \\
\hline & low & 11.28 & 13.43 & 40.09 & low & 4.47 & 5.14 & 31.90 & low & 2.91 & 3.42 & 18.63 & low & 4.77 & 5.13 & 37.26 \\
\hline & medium & 20.77 & 24.73 & 64.82 & medium | & 25.20 & 28.97 & 60.86 & medium & 25.75 & 30.29 & 48.92 & medium & 15.56 & 16.76 & 54.02 \\
\hline & high & 11.89 & 14.15 & 78.97 & high & 20.92 & 24.05 & 84.91 & high & 23.64 & 27.82 & 76.73 & high & 29.04 & 31.22 & 85.24 \\
\hline & very high & 17.66 & 21.03 & 100.00 & very high & 13.13 & 15.09 & 100.00 & very high | & 19.78 & 23.27 & 100.00 & very high & 13.73 & 14.76 & 100.00 \\
\hline & Total | & 84 & 100.00 & & Total | & 87 & 100.00 & & Total | & 85 & 100.00 & & Total | & 93 & 100.00 & \\
\hline \multirow{7}{*}{$\begin{array}{l}\text { Other (please } \\
\text { specify) }\end{array}$} & & Freq. $\quad \mathrm{I}$ & Percent & Cum. & & Freq. & Percent & Cum. & & Freq. & Percent & Cum. & & Freq. & Percent & Cum. \\
\hline & very low & 2.94 & 24.50 & 24.50 & very low | & 5.41 & 38.64 & 38.64 & very low | & .61 & 4.70 & 4.70 & very low & 5.55 & 39.65 & 39.65 \\
\hline & low & 3.58 & 29.80 & 54.30 & low & 3.68 & 26.29 & 64.92 & low & 3.62 & 27.85 & 32.54 & low & 3.05 & 21.81 & 61.45 \\
\hline & medium & 2.82 & 23.47 & 77.77 & medium | & 1.28 & 9.17 & 74.10 & medium & 4.08 & 31.41 & 63.95 & medium & 2.32 & 16.59 & 78.04 \\
\hline & high & .92 & 7.67 & 85.44 & high & 2.30 & 16.45 & 90.54 & high & 2.78 & 21.36 & 85.31 & high & 1.66 & 11.86 & 89.90 \\
\hline & very high & 1.75 & 14.56 & 100.00 & very high & 1.32 & 9.46 & 100.00 & very high & 1.91 & 14.69 & 100.00 & very high & 1.41 & 10.10 & 100.00 \\
\hline & Total & 12 & 100.00 & & Total & 14 & 100.00 & & Total & 13 & 100.00 & & Total | & 14 & 100.00 & \\
\hline
\end{tabular}


9. On average, approximately how many minutes were spent in Program Year 2008 on client education in a typical dwelling?

\section{SECTION E: TRAINING}

1. On which of the following weatherization topics did agency staff working on your agency's weatherization efforts receive training in Program Year 2008? Check all that apply.

(1) Diagnostic procedures

\begin{tabular}{|c|c|c|c|}
\hline & Freq. & Percent & Cum. \\
\hline no & 27.84 & 8.78 & 8.78 \\
\hline yes & 289.16 & 91.22 & 100.00 \\
\hline Total & 317 & 100.00 & \\
\hline
\end{tabular}

(2) Insulation

-- single family dwellings

Freq. Percent Cum.

\begin{tabular}{|c|c|c|c|}
\hline no & $\begin{array}{r}51.69 \\
\end{array}$ & $\begin{array}{l}16.30 \\
827\end{array}$ & 16.30 \\
\hline & 317 & 100.00 & \\
\hline
\end{tabular}

-- multifamily dwellings

\begin{tabular}{|c|c|c|c|}
\hline & Freq. & Percent & Cum. \\
\hline no & 208.00 & 65.61 & 65.61 \\
\hline yes & 109.00 & 34.39 & 100.00 \\
\hline Total & 317 & 100.00 & \\
\hline
\end{tabular}

-- mobile homes

Freq. Percent Cum.

$\begin{array}{rrr}\text { no | } 107.68 & 33.97 & 33.97 \\ \text { yes | } 209.32 & 66.03 & 100.00 \\ ------------------ \\ \text { Total| } 317 & 100.00\end{array}$


(3) Space heating, ventilation, air conditioning

-- single family dwellings

\begin{tabular}{|c|c|c|c|}
\hline no & 65.72 & 20.73 & 20.73 \\
\hline yes & 251.28 & 79.27 & 100.00 \\
\hline Total & 317 & 100.00 & \\
\hline
\end{tabular}

-- multifamily dwellings

Freq. Percent Cum.

\begin{tabular}{|c|c|c|c|}
\hline no & 228.05 & 71.94 & 71.94 \\
\hline yes & 88.95 & 28.06 & 100.00 \\
\hline Total & 317 & 100.00 & \\
\hline
\end{tabular}

-- mobile homes

\begin{tabular}{|c|c|c|c|}
\hline & Freq. & Percent & Cum. \\
\hline no & 117.22 & 36.98 & 36.98 \\
\hline yes | & 199.78 & 63.02 & 100.00 \\
\hline Total | & 317 & 100.00 & \\
\hline
\end{tabular}

(4) Infiltration measures

-- single family dwellings

\begin{tabular}{|c|c|c|c|}
\hline & Freq. & Percent & Cum. \\
\hline no & 28.62 & 9.03 & 9.03 \\
\hline yes & 288.38 & 90.97 & 100.00 \\
\hline Total | & 317 & 100.00 & \\
\hline -- multifamily & dwellin & & \\
\hline & Freq. & Percent & Cum. \\
\hline no & 201.31 & 63.51 & 63.51 \\
\hline yes & 115.69 & 36.49 & 100.00 \\
\hline Total | & 317 & 100.00 & \\
\hline
\end{tabular}


-- mobile homes

\begin{tabular}{|c|c|c|c|}
\hline & Freq. & Percent & Cum. \\
\hline no & 76.08 & 24.00 & 24.00 \\
\hline yes | & 240.92 & 76.00 & 100.00 \\
\hline Total & 317 & 100.00 & \\
\hline
\end{tabular}

(5) Doors and windows

-- single family dwellings

\begin{tabular}{|c|c|c|c|}
\hline & Freq. & Percent & Cum. \\
\hline no & 114.63 & 36.16 & 36.16 \\
\hline yes & 202.37 & 63.84 & 100.00 \\
\hline Total & 317 & 100.00 & \\
\hline
\end{tabular}

-- multifamily dwellings

\begin{tabular}{|c|c|c|c|}
\hline & Freq. & Percent & Cum. \\
\hline no & 228.12 & 71.96 & 71.96 \\
\hline yes & 88.88 & 28.04 & 100.00 \\
\hline Total & 317 & 100.00 & \\
\hline
\end{tabular}

-- mobile homes

\begin{tabular}{|c|c|c|c|}
\hline & Freq. & Percent & Cum. \\
\hline no & 138.25 & 43.61 & 43.61 \\
\hline yes & 178.75 & 56.39 & 100.00 \\
\hline Total & 317 & 100.00 & \\
\hline
\end{tabular}

(6) Hot water heating

-- single family dwellings

\begin{tabular}{|c|c|c|c|}
\hline & Freq. & Percent & Cum. \\
\hline no & 101.91 & 32.15 & 32.15 \\
\hline yes & 215.09 & 67.85 & 100.00 \\
\hline Total & 317 & 100.00 & \\
\hline
\end{tabular}

-- multifamily dwellings 


\begin{tabular}{|c|c|c|c|}
\hline & Freq. & Percent & Cum. \\
\hline no & 224.44 & 70.80 & 70.80 \\
\hline yes & 92.56 & 29.20 & 100.00 \\
\hline Total & 317 & 100.00 & \\
\hline
\end{tabular}

-- mobile homes

$\begin{array}{ccc}\text { Freq. } & \text { Percent } & \text { Cum. } \\ \text { no | } 146.27 & 46.14 & 46.14 \\ \text { yes | } 170.73 & 53.86 & 100.00 \\ --------- & \end{array}$

(7) Baseloads (e.g., lighting, refrigerators)

-- single family dwellings

\begin{tabular}{|c|c|c|c|}
\hline & Freq. & Percent & Cum. \\
\hline no & 74.06 & 23.36 & 23.36 \\
\hline yes & 242.94 & 76.64 & 100.00 \\
\hline Total & 317 & 100.00 & \\
\hline
\end{tabular}

-- multifamily dwellings

\begin{tabular}{|c|c|c|c|}
\hline & Freq. & Percent & Cum. \\
\hline no & 221.55 & 69.89 & 69.89 \\
\hline yes & 95.45 & 30.11 & 100.00 \\
\hline Total | & 317 & 100.00 & \\
\hline
\end{tabular}

-- mobile homes

Freq. Percent Cum.

\begin{tabular}{|c|c|c|c|}
\hline no & 107.40 & $\begin{array}{l}33.88 \\
6612\end{array}$ & 33.88 \\
\hline yes | & 209.60 & 66.12 & 100.00 \\
\hline Total | & 317 & 100.00 & \\
\hline
\end{tabular}


1a. On which of the following administrative-related topics did agency staff working on your agency's weatherization efforts receive training in Program Year 2008? Check all that apply.

(1) Management

\begin{tabular}{|c|c|c|c|}
\hline & Freq. & Percent & Cum. \\
\hline no & 148.06 & 48.86 & 48.86 \\
\hline yes & 154.94 & 51.14 & 100.00 \\
\hline Total & 303 & 100.00 & \\
\hline
\end{tabular}

(2) Client education

\begin{tabular}{|c|c|c|c|}
\hline & Freq. & Percent & Cum. \\
\hline no & 127.38 & 42.04 & 42.04 \\
\hline yes & 175.62 & 57.96 & 100.00 \\
\hline Total | & 303 & 100.00 & \\
\hline
\end{tabular}

(3) Auditing/estimating

-- single family dwellings

\begin{tabular}{|c|c|c|c|}
\hline & Freq. & Percent & Cum. \\
\hline no| & 49.78 & 16.43 & 16.43 \\
\hline yes & 253.22 & 83.57 & 100.00 \\
\hline Total | & 303 & 100.00 & \\
\hline
\end{tabular}

-- multifamily dwellings

\begin{tabular}{|c|c|c|c|}
\hline & Freq. & Percent & Cum. \\
\hline nol & 205.94 & 67.97 & 67.97 \\
\hline yes $\mid$ & 97.06 & 32.03 & 100.00 \\
\hline Total | & 303 & 100.00 & \\
\hline -- mobile & omes & & \\
\hline & Freq. & Percent & Cum. \\
\hline no & 99.83 & 32.95 & 32.95 \\
\hline yes & 203.17 & 67.05 & 100.00 \\
\hline Total | & 303 & 100.00 & \\
\hline
\end{tabular}


(4) Monitoring/quality control

\begin{tabular}{|c|c|c|c|}
\hline & Freq. & Percent & Cum. \\
\hline no & 109.77 & 36.23 & 36.23 \\
\hline yes & 193.23 & 63.77 & 100.00 \\
\hline Total & 303 & 100.00 & \\
\hline
\end{tabular}

(5) Financial topics

\begin{tabular}{|c|c|c|c|}
\hline & Freq. & Percent & Cum. \\
\hline no & 168.73 & 55.69 & 55.69 \\
\hline yes & 134.27 & 44.31 & 100.00 \\
\hline Total | & 303 & 100.00 & \\
\hline
\end{tabular}

(6) Outreach and communications

\begin{tabular}{|c|c|c|c|}
\hline & Freq. & Percent & Cum. \\
\hline no & 184.17 & 60.78 & 60.78 \\
\hline yes & 118.83 & 39.22 & 100.00 \\
\hline Total | & 303 & 100.00 & \\
\hline
\end{tabular}

(7) Other (please specify)

$\begin{array}{crrr} & \text { Freq. } & \text { Percent } & \text { Cum. } \\ \text { no | } 291.33 & 96.15 & 96.15 \\ \text { yes | } 11.67 & 3.85 & 100.00 \\ ------- & \end{array}$

1b. On which of the following health and safety topics did agency staff working on your agency's weatherization efforts receive training in Program Year 2008? Check all that apply.

Fire safety

\begin{tabular}{|c|c|c|c|}
\hline & Freq. & Percent & Cum. \\
\hline nol & 240.18 & 74.59 & 74.59 \\
\hline yes | & 81.82 & 25.41 & 100.00 \\
\hline Total & 322 & 100.00 & \\
\hline
\end{tabular}


Indoor air quality

Freq. Percent Cum.

\begin{tabular}{|c|c|c|c|}
\hline no & 90.22 & 28.02 & 28.02 \\
\hline yes | & 231.78 & 71.98 & 100.00 \\
\hline Total | & 322 & 100.00 & \\
\hline
\end{tabular}

Measures to increase security of housing unit Freq. Percent Cum.

\begin{tabular}{|c|c|c|c|}
\hline no & 289.32 & 89.85 & 89.85 \\
\hline yes $\mid$ & 32.68 & 10.15 & 100.00 \\
\hline Total | & 322 & 100.00 & \\
\hline
\end{tabular}

Measures to reduce common household hazards Freq. Percent Cum.

\begin{tabular}{|c|c|c|c|}
\hline no & 216.89 & 67.36 & 67.36 \\
\hline yes & 105.11 & 32.64 & 100.00 \\
\hline Total | & 322 & 100.00 & \\
\hline
\end{tabular}

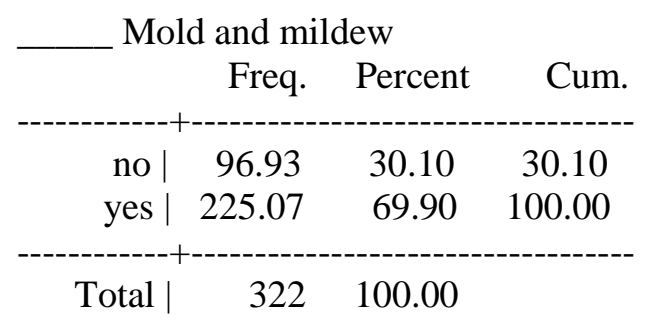

\begin{tabular}{|c|c|c|c|}
\hline & Freq. & Percent & Cum. \\
\hline no & 49.18 & 15.27 & 15.27 \\
\hline yes $\mid$ & 272.82 & 84.73 & 100.00 \\
\hline otal | & 322 & 100.00 & \\
\hline
\end{tabular}

\begin{tabular}{|c|c|c|c|}
\hline & Freq. & Percent & Cum. \\
\hline no & 223.54 & 69.42 & 69.42 \\
\hline yes | & 98.46 & 30.58 & 100.00 \\
\hline Total | & 322 & 100.00 & \\
\hline
\end{tabular}


Vermiculite

$\begin{array}{cccc} & \text { Freq. } & \text { Percent } & \text { Cum. } \\ \text { no } \mid & 251.47 & 78.10 & 78.10 \\ \text { yes } \mid & 70.53 & 21.90 & 100.00 \\ ------------ \\ \text { Total| } 322 & 100.00\end{array}$

\begin{tabular}{|c|c|c|c|}
\hline & Freq. & Percent & Cum. \\
\hline nol & 134.63 & 41.81 & 41.81 \\
\hline yes & 187.37 & 58.19 & 100.00 \\
\hline otal | & 322 & 100.00 & \\
\hline
\end{tabular}

Other (please specify)

$\begin{array}{cccc} & \text { Freq. } & \text { Percent } & \text { Cum. } \\ \text { no | } & 299.98 & 93.16 & 93.16 \\ \text { yes | } & 22.02 & 6.84 & 100.00 \\ ----------- \\ \text { Total| } & 322 & 100.00\end{array}$

2. On which of the following diagnostic procedures did agency staff working on your agency's weatherization efforts receive training in Program Year 2008? Check all that apply.

Pressure diagnostics:

- Blower door (house air leakage rate)

\begin{tabular}{|c|c|c|c|}
\hline & Freq. & Percent & Cum. \\
\hline no & 30.62 & 9.85 & 9.85 \\
\hline yes & 280.38 & 90.15 & 100.00 \\
\hline Total & 311 & 100.00 & \\
\hline
\end{tabular}

- Zonal pressure measurements

\begin{tabular}{|c|c|c|c|}
\hline & Freq. & Percent & Cum. \\
\hline no & 118.82 & 38.21 & 38.21 \\
\hline yes & 192.18 & 61.79 & 100.00 \\
\hline Total & 311 & 100.00 & \\
\hline
\end{tabular}


- Room-to-room pressure measurements (distribution balancing)

\begin{tabular}{|c|c|c|c|}
\hline & Freq. & Percent & Cum. \\
\hline no & 152.69 & 49.10 & 49.10 \\
\hline yes & 158.31 & 50.90 & 100.00 \\
\hline Total & 311 & 100.00 & \\
\hline
\end{tabular}

- Duct pressure pan measurements

$\begin{array}{ccc}\text { Freq. } & \text { Percent } & \text { Cum. } \\ \text { no | } 120.63 & 38.79 & 38.79 \\ \text { yes | } 190.37 & 61.21 & 100.00 \\ -------------- \\ \text { Total| } 311 & 100.00\end{array}$

- Duct blower measurements (duct air leakage rate)

\begin{tabular}{|c|c|c|c|}
\hline & Freq. & Percent & Cum. \\
\hline no & 173.37 & 55.74 & 55.74 \\
\hline yes & 137.63 & 44.26 & 100.00 \\
\hline Total | & 311 & 100.00 & \\
\hline
\end{tabular}

\section{Space-heating system:}

- Flue gas analysis (steady-state efficiency measurements)

\begin{tabular}{|c|c|c|c|}
\hline & Freq. & Percent & Cum. \\
\hline no & 90.12 & 28.97 & 28.97 \\
\hline yes | & 220.89 & 71.03 & 100.00 \\
\hline Total & 311 & 100.00 & \\
\hline
\end{tabular}

- Heat rise measurements

\begin{tabular}{|c|c|c|c|}
\hline & Freq. & Percent & Cum. \\
\hline no & 145.93 & 46.92 & 46.92 \\
\hline yes & 165.07 & 53.08 & 100.00 \\
\hline Total & 311 & 100.00 & \\
\hline
\end{tabular}


- $\mathrm{CO}$ measurements in flues

\begin{tabular}{|c|c|c|c|}
\hline & Freq. & Percent & Cum. \\
\hline no & 60.06 & 19.31 & 19.31 \\
\hline yes & 250.94 & 80.69 & 100.00 \\
\hline Total & 311 & 100.00 & \\
\hline
\end{tabular}

- Draft/spillage (normal operation)

Freq. Percent Cum.

\begin{tabular}{|c|c|c|c|}
\hline no & 97.85 & 31.46 & 31.46 \\
\hline yes & 213.15 & 68.54 & 100.00 \\
\hline Total & 311 & 100.00 & \\
\hline
\end{tabular}

\section{Air-conditioning system:}

- Refrigerant charge (e.g., superheat, subcooling)

\begin{tabular}{|c|c|c|c|}
\hline & Freq. & Percent & Cum. \\
\hline no & 289.66 & 93.14 & 93.14 \\
\hline yes & 21.34 & 6.86 & 100.00 \\
\hline Total | & 311 & 100.00 & \\
\hline
\end{tabular}

HVAC components and cross-cutting diagnostics:

- Air handler flow rate

\begin{tabular}{|c|c|c|c|}
\hline & Freq. & Percent & Cum. \\
\hline no & 242.78 & 78.07 & 78.07 \\
\hline yes & 68.22 & 21.93 & 100.00 \\
\hline Total & 311 & 100.00 & \\
\hline
\end{tabular}

- Thermostat anticipator current

\begin{tabular}{|c|c|c|c|}
\hline & Freq. & Percent & Cum. \\
\hline no & 219.83 & 70.68 & 70.68 \\
\hline yes & 91.17 & 29.32 & 100.00 \\
\hline otal & 311 & 100.00 & \\
\hline
\end{tabular}


- Worst case draft/spillage (CAZ)

\begin{tabular}{|c|c|c|c|}
\hline & Freq. & Percent & Cum. \\
\hline no & 119.52 & 38.43 & 38.43 \\
\hline yes & 191.48 & 61.57 & 100.00 \\
\hline Total & 311 & 100.00 & \\
\hline
\end{tabular}

\section{Hot-water (water-heating) system:}

- Flue gas analysis (steady-state efficiency measurements) Freq. Percent Cum.

$\begin{array}{rrr}\text { no | } 122.16 & 39.28 & 39.28 \\ \text { yes | } 188.84 & 60.72 & 100.00 \\ ------------------ \\ \text { Total| } 311 & 100.00\end{array}$

- $\quad \mathrm{CO}$ measurements in flues

\begin{tabular}{|c|c|c|c|}
\hline & Freq. & Percent & Cum. \\
\hline no & 73.53 & 23.64 & 23.64 \\
\hline yes & 237.47 & 76.36 & 100.00 \\
\hline Total | & 311 & 100.00 & \\
\hline
\end{tabular}

- Draft/spillage (normal operation) Freq. Percent Cum.

\begin{tabular}{|c|c|c|c|}
\hline no & 97.82 & 31.45 & 31.45 \\
\hline yes & 213.18 & 68.55 & 100.00 \\
\hline
\end{tabular}

- Water flow rates (showerheads and faucets) Freq. Percent Cum.

$\begin{array}{crr}\text { no | } 218.50 & 70.26 & 70.26 \\ \text { yes | } 92.50 & 29.74 & 100.00 \\ ------- & \end{array}$




\section{Other CO measurements:}

- $\mathrm{CO}$ measurements in equipment rooms

\begin{tabular}{|c|c|c|c|}
\hline & Freq. & Percent & Cum. \\
\hline no & 107.31 & 34.51 & 34.51 \\
\hline yes & 203.69 & 65.49 & 100.00 \\
\hline Total & 311 & 100.00 & \\
\hline
\end{tabular}

- Cooking stove

\begin{tabular}{|c|c|c|c|}
\hline & Freq. & Percent & Cum. \\
\hline no & 81.92 & 26.34 & 26.34 \\
\hline yes & 229.08 & 73.66 & 100.00 \\
\hline Total | & 311 & 100.00 & \\
\hline
\end{tabular}

- $\mathrm{CO}$ measurements in living areas

\begin{tabular}{|c|c|c|c|}
\hline & Freq. & Percent & Cum. \\
\hline no & 99.81 & 32.09 & 32.09 \\
\hline yes | & 211.19 & 67.91 & 100.00 \\
\hline Total & 311 & 100.00 & \\
\hline
\end{tabular}

\section{Other diagnostics and inspections:}

- Refrigerator energy use

\begin{tabular}{|c|c|c|c|}
\hline & Freq. & rcent & Cum. \\
\hline no & 150.07 & 48.26 & 48.26 \\
\hline yes & 160.93 & 51.74 & 100.00 \\
\hline Total & 311 & 100.00 & \\
\hline
\end{tabular}

- Exhaust fan air flow rate measurement

\begin{tabular}{rrr}
\multicolumn{1}{r}{ Freq. } & Percent & \multicolumn{1}{c}{ Cum. } \\
no | 223.68 & 71.92 & 71.92 \\
yes | 87.32 & 28.08 & 100.00 \\
--------- \\
Total|
\end{tabular}


- Infrared scanning (camera)

$\begin{array}{ccc}\text { Freq. } & \text { Percent } & \text { Cum. } \\ \text { no | } 167.49 & 53.86 & 53.86 \\ \text { yes | } 143.51 & 46.14 & 100.00 \\ ---------- & \end{array}$

- Radon testing

\begin{tabular}{|c|c|c|c|}
\hline & Freq. & Percent & Cum. \\
\hline no & 297.47 & 95.65 & 95.65 \\
\hline yes & 13.53 & 4.35 & 100.00 \\
\hline Total | & 311 & 100.00 & \\
\hline
\end{tabular}

- $\quad$ Lead testing

Freq. Percent Cum.

\begin{tabular}{|c|c|c|c|}
\hline no & 209.30 & 67.30 & 67.30 \\
\hline yes | & 101.70 & 32.70 & 100.00 \\
\hline Total | & 311 & 100.00 & \\
\hline
\end{tabular}

- Mold and mildew testing Freq. Percent Cum.

\begin{tabular}{|c|c|c|c|}
\hline no & 255.99 & 82.31 & 82.31 \\
\hline yes & 55.01 & 17.69 & 100.00 \\
\hline Total | & 311 & 100.00 & \\
\hline
\end{tabular}

- Moisture content testing

Freq. Percent Cum.

\begin{tabular}{|c|c|c|c|}
\hline no & 255.07 & 82.01 & 82.01 \\
\hline yes & 55.93 & 17.99 & 100.00 \\
\hline otal & 311 & 100.00 & \\
\hline
\end{tabular}

- Other (please specify)

\begin{tabular}{|c|c|c|c|}
\hline & Freq. & Percent & Cum. \\
\hline no & 305.95 & 98.37 & 98.37 \\
\hline yes & 5.05 & 1.63 & 100.00 \\
\hline Total & 311 & 100.00 & \\
\hline
\end{tabular}


3. How many of your agency's staff were trained at the following events in Program Year 2008? Enter responses next to any categories that apply.

National Weatherization Program Conference

\begin{tabular}{|l|c|}
\hline observations: & 151 \\
\hline missing values: & 206 \\
\hline mean: & 2.60 \\
\hline standard deviation: & 2.66 \\
\hline min: & 0 \\
\hline 10th percentile: & 1 \\
\hline 25th percentile: & 1 \\
\hline median: & 2 \\
\hline 75th percentile: & 3 \\
\hline 90th percentile: & 5 \\
\hline max: & 30 \\
\hline
\end{tabular}

Affordable Comfort Conference

\begin{tabular}{|l|c|}
\hline observations: & 135 \\
\hline missing values: & 222 \\
\hline mean: & 2.59 \\
\hline standard deviation: & 2.64 \\
\hline min: & 0 \\
\hline 10th percentile: & 0 \\
\hline 25th percentile: & 1 \\
\hline median: & 2 \\
\hline 75th percentile: & 3 \\
\hline 90th percentile: & 6 \\
\hline max: & 30 \\
\hline
\end{tabular}

Other national conference

\begin{tabular}{|l|c|}
\hline observations: & 60 \\
\hline missing values: & 297 \\
\hline mean: & 1.84 \\
\hline standard deviation: & 2.03 \\
\hline min: & 0 \\
\hline 10th percentile: & 0 \\
\hline 25th percentile: & 0 \\
\hline median: & 2 \\
\hline 75th percentile: & 2 \\
\hline 90th percentile: & 4 \\
\hline max: & 10 \\
\hline
\end{tabular}


Regional weatherization conference

\begin{tabular}{|l|c|}
\hline observations: & 115 \\
\hline missing values: & 242 \\
\hline mean: & 3.22 \\
\hline standard deviation: & 2.95 \\
\hline min: & 0 \\
\hline 10th percentile: & 0 \\
\hline 25th percentile: & 1 \\
\hline median: & 2 \\
\hline 75th percentile: & 4 \\
\hline 90th percentile: & 7 \\
\hline max: & 15 \\
\hline
\end{tabular}

Your state's weatherization conference

\begin{tabular}{|l|c|}
\hline observations: & 160 \\
\hline missing values: & 197 \\
\hline mean: & 3.73 \\
\hline standard deviation: & 3.37 \\
\hline min: & 0 \\
\hline 10th percentile: & 1 \\
\hline 25th percentile: & 2 \\
\hline median: & 3 \\
\hline 75th percentile: & 5 \\
\hline 90th percentile: & 7 \\
\hline max: & 30 \\
\hline
\end{tabular}

Some other relevant conference in your state

\begin{tabular}{|l|c|}
\hline observations: & 94 \\
\hline missing values: & 263 \\
\hline mean: & 2.69 \\
\hline standard deviation: & 2.85 \\
\hline min: & 0 \\
\hline 10th percentile: & 0 \\
\hline 25th percentile: & 1 \\
\hline median: & 2 \\
\hline 75th percentile: & 4 \\
\hline 90th percentile: & 5 \\
\hline max: & 20 \\
\hline
\end{tabular}


Weatherization conference given by another state

\begin{tabular}{|l|c|}
\hline observations: & 40 \\
\hline missing values: & 317 \\
\hline mean: & 1.05 \\
\hline standard deviation: & 1.43 \\
\hline min: & 0 \\
\hline 10th percentile: & 0 \\
\hline 25th percentile: & 0 \\
\hline median: & 0 \\
\hline 75th percentile: & 2 \\
\hline 90th percentile: & 3 \\
\hline max: & 7 \\
\hline
\end{tabular}

Some other relevant conference given by another state

\begin{tabular}{|l|c|}
\hline observations: & 35 \\
\hline missing values: & 322 \\
\hline mean: & 1.77 \\
\hline standard deviation: & 2.84 \\
\hline min: & 0 \\
\hline 10th percentile: & 0 \\
\hline 25th percentile: & 0 \\
\hline median: & 0 \\
\hline 75th percentile: & 3 \\
\hline 90th percentile: & 7 \\
\hline max: & 10 \\
\hline
\end{tabular}

Any state or regional training center class

\begin{tabular}{|l|c|}
\hline observations: & 143 \\
\hline missing values: & 214 \\
\hline mean: & 4.77 \\
\hline standard deviation: & 6.83 \\
\hline min: & 0 \\
\hline 10th percentile: & 1 \\
\hline 25th percentile: & 2 \\
\hline median: & 3 \\
\hline 75th percentile: & 6 \\
\hline 90th percentile: & 10 \\
\hline max: & 55 \\
\hline
\end{tabular}


Manufacturer's training school class

\begin{tabular}{|l|c|}
\hline observations: & 63 \\
\hline missing values: & 294 \\
\hline mean: & 2.23 \\
\hline standard deviation: & 3.08 \\
\hline min: & 0 \\
\hline 10th percentile: & 0 \\
\hline 25th percentile: & 0 \\
\hline median: & 2 \\
\hline 75th percentile: & 2 \\
\hline 90th percentile: & 5 \\
\hline max: & 19 \\
\hline
\end{tabular}

Utility training class

\begin{tabular}{|l|c|}
\hline observations: & 68 \\
\hline missing values: & 289 \\
\hline mean: & 2.26 \\
\hline standard deviation: & 2.64 \\
\hline min: & 0 \\
\hline 10th percentile: & 0 \\
\hline 25th percentile: & 0 \\
\hline median: & 2 \\
\hline 75th percentile: & 3 \\
\hline 90th percentile: & 5 \\
\hline max: & 14 \\
\hline
\end{tabular}

Training classes provided by your agency or those agencies you work for

\begin{tabular}{|l|c|}
\hline observations: & 111 \\
\hline missing values: & 246 \\
\hline mean: & 5.68 \\
\hline standard deviation: & 6.85 \\
\hline min: & 0 \\
\hline 10th percentile: & 0 \\
\hline 25th percentile: & 2 \\
\hline median: & 4 \\
\hline 75th percentile: & 6 \\
\hline 90th percentile: & 13 \\
\hline max: & 53 \\
\hline
\end{tabular}


One-time state-sponsored class

\begin{tabular}{|l|c|}
\hline observations: & 77 \\
\hline missing values: & 280 \\
\hline mean: & 3.91 \\
\hline standard deviation: & 5.52 \\
\hline min: & 0 \\
\hline 10th percentile: & 0 \\
\hline 25th percentile: & 1 \\
\hline median: & 2 \\
\hline 75th percentile: & 5 \\
\hline 90th percentile: & 10 \\
\hline max: & 50 \\
\hline
\end{tabular}

Any other class not sponsored by your state (e.g., another state, trade organization)

\begin{tabular}{|l|c|}
\hline observations: & 49 \\
\hline missing values: & 308 \\
\hline mean: & 2.32 \\
\hline standard deviation: & 3.26 \\
\hline min: & 0 \\
\hline 10th percentile: & 0 \\
\hline 25th percentile: & 0 \\
\hline median: & 2 \\
\hline 75th percentile: & 3 \\
\hline 90th percentile: & 6 \\
\hline max: & 20 \\
\hline
\end{tabular}

Visit to an agency you do not work for training

\begin{tabular}{|l|c|}
\hline observations: & 76 \\
\hline missing values: & 281 \\
\hline mean: & 2.05 \\
\hline standard deviation: & 1.92 \\
\hline min: & 0 \\
\hline 10th percentile: & 0 \\
\hline 25th percentile: & 1 \\
\hline median: & 2 \\
\hline 75th percentile: & 3 \\
\hline 90th percentile: & 5 \\
\hline max: & 10 \\
\hline
\end{tabular}


Instruction provided by your state to your individual agency or those agencies you work for

\begin{tabular}{|l|c|}
\hline observations: & 111 \\
\hline missing values: & 246 \\
\hline mean: & 4.02 \\
\hline standard deviation: & 4.8 \\
\hline min: & 0 \\
\hline 10th percentile: & 0 \\
\hline 25th percentile: & 1 \\
\hline median: & 3 \\
\hline 75th percentile: & 6 \\
\hline 90th percentile: & 9 \\
\hline max: & 30 \\
\hline
\end{tabular}

In-person expert visit just to your agency (e.g., peer exchange, consultant)

\begin{tabular}{|l|c|}
\hline observations: & 100 \\
\hline missing values: & 257 \\
\hline mean: & 3.68 \\
\hline standard deviation: & 4.46 \\
\hline min: & 0 \\
\hline 10th percentile: & 0 \\
\hline 25th percentile: & 1 \\
\hline median: & 2 \\
\hline 75th percentile: & 4 \\
\hline 90th percentile: & 7 \\
\hline max: & 30 \\
\hline
\end{tabular}

Web cast

\begin{tabular}{|l|c|}
\hline observations: & 46 \\
\hline missing values: & 311 \\
\hline mean: & 3.14 \\
\hline standard deviation: & 7.10 \\
\hline min: & 0 \\
\hline 10th percentile: & 0 \\
\hline 25th percentile: & 0 \\
\hline median: & 2 \\
\hline 75th percentile: & 2 \\
\hline 90th percentile: & 6 \\
\hline max: & 50 \\
\hline
\end{tabular}


Other (please specify)

\begin{tabular}{|l|c|}
\hline observations: & 20 \\
\hline missing values: & 337 \\
\hline mean: & 3.86 \\
\hline standard deviation: & 5.22 \\
\hline min: & 0 \\
\hline 10th percentile: & 0 \\
\hline 25th percentile: & 0 \\
\hline median: & 2 \\
\hline 75th percentile: & 6 \\
\hline 90th percentile: & 15 \\
\hline max: & 17 \\
\hline
\end{tabular}

4. Which of the following weatherization topics listed below were agency staff first trained on in Program Year 2008 and two years prior to Program Year 2008? Check all that apply.

(1) Diagnostic procedures

Freq. Percent Cum.

\begin{tabular}{|c|c|c|c|}
\hline no & 39.61 & 20.53 & 20.53 \\
\hline yes | & 153.39 & 79.47 & 100.00 \\
\hline Total & 193 & 100.00 & \\
\hline
\end{tabular}

(2) Insulation

-- single family dwellings

Freq. Percent Cum.

\begin{tabular}{|c|c|c|c|}
\hline no & 45.47 & 23.56 & 23.56 \\
\hline yes & 147.53 & 76.44 & 100.00 \\
\hline Total & 193 & 100.00 & \\
\hline
\end{tabular}

-- multifamily dwellings

Freq. Percent Cum.

\begin{tabular}{|c|c|c|c|}
\hline no & 135.69 & 70.31 & 70.31 \\
\hline yes $\mid$ & 57.31 & 29.69 & 100.00 \\
\hline
\end{tabular}

-- mobile homes

\begin{tabular}{|c|c|c|c|}
\hline & Freq. & Percent & Cum. \\
\hline no & 62.92 & 32.60 & 32.60 \\
\hline yes & 130.08 & 67.40 & 100.00 \\
\hline Total | & 193 & 100.00 & \\
\hline
\end{tabular}


(3) Space heating, ventilation, air conditioning

-- single family dwellings

Freq. Percent Cum.

\begin{tabular}{|c|c|c|c|}
\hline no & 64.05 & 33.19 & 33.19 \\
\hline yes & 128.95 & 66.81 & 100.00 \\
\hline Total & 193 & 100.00 & \\
\hline
\end{tabular}

-- multifamily dwellings

Freq. Percent Cum.

\begin{tabular}{|c|c|c|c|}
\hline no & 140.85 & 72.98 & 72.98 \\
\hline yes & 52.15 & 27.02 & 100.00 \\
\hline
\end{tabular}

-- mobile homes

\begin{tabular}{|c|c|c|c|}
\hline no & 81.34 & 42.14 & 42.14 \\
\hline yes & 111.66 & 57.86 & 100.00 \\
\hline Total & 193 & 100.00 & \\
\hline
\end{tabular}

(4) Infiltration measures

-- single family dwellings

Freq. Percent Cum.

\begin{tabular}{|c|c|c|c|}
\hline no & 33.94 & 17.59 & 17.59 \\
\hline yes & 159.06 & 82.41 & 100.00 \\
\hline Total & 193 & 100.00 & \\
\hline
\end{tabular}

-- multifamily dwellings

\begin{tabular}{|c|c|c|c|}
\hline & Freq. & Percent & Cum. \\
\hline no & 138.51 & 71.77 & 71.77 \\
\hline yes & 54.49 & 28.23 & 100.00 \\
\hline Total | & 193 & 100.00 & \\
\hline - mobile hom & & & \\
\hline & Freq. & Percent & Cum. \\
\hline no & 58.73 & 30.43 & 30.43 \\
\hline yes & 134.27 & 69.57 & 100.00 \\
\hline Total | & 193 & 100.00 & \\
\hline
\end{tabular}

(5) Doors and windows 
-- single family dwellings

Freq. Percent Cum.

\begin{tabular}{|c|c|c|c|}
\hline no & 79.21 & 41.04 & 41.04 \\
\hline yes | & 113.79 & 58.96 & 100.00 \\
\hline
\end{tabular}

-- multifamily dwellings

Freq. Percent Cum.

\begin{tabular}{|c|c|c|c|}
\hline no & 144.39 & 74.81 & 74.81 \\
\hline yes | & 48.61 & 25.19 & 100.00 \\
\hline
\end{tabular}

-- mobile homes

\begin{tabular}{|c|c|c|c|}
\hline & Freq. & Percent & Cum. \\
\hline no & 85.21 & 44.15 & 44.15 \\
\hline yes | & 107.79 & 55.85 & 100.00 \\
\hline Total & 193 & 100.00 & \\
\hline
\end{tabular}

(6) Hot water heating

-- single family dwellings

Freq. Percent Cum.

\begin{tabular}{|c|c|c|c|}
\hline no & 66.49 & 34.45 & 34.45 \\
\hline yes & 126.51 & 65.55 & 100.00 \\
\hline Total & 193 & 100.00 & \\
\hline
\end{tabular}

-- multifamily dwellings

Freq. Percent Cum.

$\begin{array}{crr}\text { no | } 145.64 & 75.46 & 75.46 \\ \text { yes | } 47.36 & 24.54 & 100.00 \\ - & & \\ \text { Total | } 193 & 100.00\end{array}$

-- mobile homes

\begin{tabular}{|c|c|c|c|}
\hline & Freq. & Percent & Cum. \\
\hline no & 83.01 & 43.01 & 43.01 \\
\hline yes | & 109.99 & 56.99 & 100.00 \\
\hline Total | & 193 & 100.00 & \\
\hline
\end{tabular}

(7) Baseloads (e.g., lighting, refrigerators)

-- single family dwellings 


\begin{tabular}{|c|c|c|c|}
\hline & Freq. & Percent & Cum. \\
\hline no & 55.10 & 28.55 & 28.55 \\
\hline yes & 137.90 & 71.45 & 100.00 \\
\hline Total & 193 & 100.00 & \\
\hline
\end{tabular}

-- multifamily dwellings

\begin{tabular}{|c|c|c|c|}
\hline & Freq. & Percent & Cum. \\
\hline no & 135.70 & 70.31 & 70.31 \\
\hline yes & 57.30 & 29.69 & 100.00 \\
\hline Total & 193 & 100.00 & \\
\hline
\end{tabular}

-- mobile homes

\begin{tabular}{|c|c|c|c|}
\hline & Freq. & Percent & Cum. \\
\hline no & 78.08 & 40.46 & 40.46 \\
\hline yes & 114.92 & 59.54 & 100.00 \\
\hline Total | & 193 & 100.00 & \\
\hline
\end{tabular}

4a. Which of the following administrative-related topics listed below were agency staff first trained on in Program Year 2008 and in the two years prior to Program Year 2008? If your agency did not receive training on a particular subject, leave that item blank.

Check all that apply.

(1) Management

\begin{tabular}{|c|c|c|c|}
\hline & Freq. & Percent & Cum. \\
\hline uncheck & 109.46 & 58.54 & 58.54 \\
\hline checked & 77.54 & 41.46 & 100.00 \\
\hline
\end{tabular}

(2) Client education

Freq. Percent Cum.

$\begin{array}{rrr}\text { unchecked | } 80.58 & 43.09 & 43.09 \\ \text { checked | } 106.42 & 56.91 & 100.00 \\ --------- & \\ \text { Total | } 187 & 100.00\end{array}$


(3) Auditing/estimating

-- single family dwellings

Freq. Percent Cum.

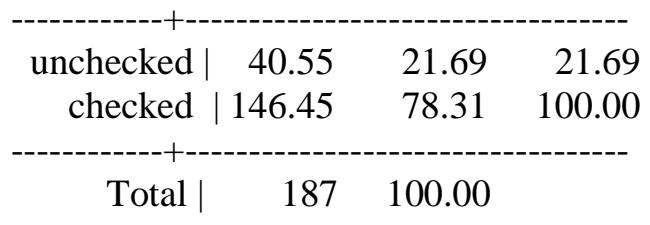

-- multifamily dwellings

Freq. Percent Cum.

\begin{tabular}{|c|c|c|c|}
\hline unchecked & 131.32 & 70.22 & 70.22 \\
\hline checked & 55.68 & 29.78 & 100.00 \\
\hline Total & 187 & 100.00 & \\
\hline
\end{tabular}

-- mobile homes

Freq. Percent Cum.

\begin{tabular}{|c|c|c|c|}
\hline $\begin{array}{l}\text { unchecked } \\
\text { checked }\end{array}$ & $\begin{array}{r}64.57 \\
122.43\end{array}$ & $\begin{array}{l}34.53 \\
65.47\end{array}$ & $\begin{array}{r}34.53 \\
100.00\end{array}$ \\
\hline Total & 187 & 100.00 & \\
\hline
\end{tabular}

(3) Monitoring/quality control

Freq. Percent Cum.

\begin{tabular}{|c|c|c|c|}
\hline $\begin{array}{r}\text { unchecked } \\
\text { checked }\end{array}$ & $\begin{array}{r}83.68 \\
103.32\end{array}$ & $\begin{array}{l}44.75 \\
55.25\end{array}$ & $\begin{array}{r}44.75 \\
100.00\end{array}$ \\
\hline
\end{tabular}

Total | $187 \quad 100.00$

(4) Financial topics

Freq. Percent Cum.

\begin{tabular}{|c|c|c|c|}
\hline unchecked & 104.97 & 56.14 & 56.14 \\
\hline checked & 82.03 & 43.86 & 100.00 \\
\hline Total & 187 & 100.00 & \\
\hline
\end{tabular}

(5) Outreach and communications

Freq. Percent Cum.

\begin{tabular}{|c|c|c|c|}
\hline $\begin{array}{l}\text { unchecked } \\
\text { checked }\end{array}$ & $\begin{array}{r}118.67 \\
68.33\end{array}$ & $\begin{array}{l}63.46 \\
36.54\end{array}$ & $\begin{array}{r}63.46 \\
100.00\end{array}$ \\
\hline Total | & 187 & 100.00 & \\
\hline
\end{tabular}


(7) Other (please specify)

\begin{tabular}{rrrr} 
& Freq. & Percent & \multicolumn{1}{c}{ Cum. } \\
\cline { 3 - 3 } unchecked | & 179.73 & 96.11 & 96.11 \\
checked | & 7.27 & 3.89 & 100.00 \\
---------+-------------------------- \\
Total | & 187 & 100.00 &
\end{tabular}

4b. Which of the following health and safety topics listed below were agency staff first trained on in Program Year 2008 and in the two years prior to Program Year 2008?

Check all that apply.

Fire safety

\begin{tabular}{|c|c|c|c|}
\hline 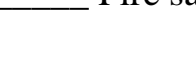 & Freq. & Percent & Cum. \\
\hline unchecked | & 157.10 & 77.01 & 77.01 \\
\hline checked | & 46.90 & 22.99 & 100.00 \\
\hline Total | & 204 & 100.00 & \\
\hline \multicolumn{4}{|c|}{ Indoor air quality } \\
\hline & Freq. & Percent & Cum. \\
\hline unchecked | & 85.44 & 41.88 & 41.88 \\
\hline checked | & 118.56 & 58.12 & 100.00 \\
\hline Total | & 204 & 100.00 & \\
\hline
\end{tabular}

Measures to increase security of housing unit

\begin{tabular}{|c|c|c|c|}
\hline & Freq. & Percent & Cum. \\
\hline unchecked & 176.46 & 86.50 & 86.50 \\
\hline checked & 27.54 & 13.50 & 100.00 \\
\hline Total & 204 & 100.00 & \\
\hline
\end{tabular}

\begin{tabular}{|c|c|c|c|}
\hline & Freq. & Percent & Cum. \\
\hline unchecked & 145.96 & 71.55 & 71.55 \\
\hline checked & 58.04 & 28.45 & 100.00 \\
\hline Total | & 204 & 100.00 & \\
\hline
\end{tabular}


Mold and mildew

Freq. Percent Cum.

\begin{tabular}{rrr} 
unchecked | 90.34 & 44.28 & 44.28 \\
checked | 113.66 & 55.72 & 100.00 \\
- \hdashline---- & \\
\hline Total | 204 & 100.00
\end{tabular}

\begin{tabular}{|c|c|c|c|}
\hline & Freq. & Percent & Cum. \\
\hline unchecked | & 51.99 & 25.48 & 25.48 \\
\hline checked & 152.01 & 74.52 & 100.00 \\
\hline Total | & 204 & 100.00 & \\
\hline
\end{tabular}

Asbestos

\begin{tabular}{|c|c|c|c|}
\hline & Freq. & Percent & Cum. \\
\hline unchecked & 136.93 & 67.12 & 67.12 \\
\hline checked & 67.07 & 32.88 & 100.00 \\
\hline Total & 204 & 100.00 & \\
\hline
\end{tabular}

Vermiculite

\begin{tabular}{|c|c|c|c|}
\hline & Freq. & Percent & Cum. \\
\hline unchecked & 161.49 & 79.16 & 79.16 \\
\hline checked & 42.51 & 20.84 & 100.00 \\
\hline Total & 204 & 100.00 & \\
\hline
\end{tabular}

General crew safety

Freq. Percent Cum.

\begin{tabular}{|c|c|c|c|}
\hline unchecked & 120.23 & 58.93 & 58.93 \\
\hline checked & 83.77 & 41.07 & 100.00 \\
\hline Total & 204 & 100.00 & \\
\hline
\end{tabular}

Other (please specify)

Freq. Percent Cum.

\begin{tabular}{|c|c|c|c|}
\hline unchecked | & 182.49 & 89.45 & 89.45 \\
\hline checked & 21.51 & 10.55 & 100.00 \\
\hline Total | & 204 & 100.00 & \\
\hline
\end{tabular}


5. On which of the following weatherization topics did your agency provide training to your own inhouse staff in Program Year 2008? Check all that apply.

(1) Diagnostic procedures

\begin{tabular}{|c|c|c|c|}
\hline & Freq. & rcent & Cum. \\
\hline unchecked | & 54.27 & 21.88 & 21.88 \\
\hline checked & 193.73 & 78.12 & 100.00 \\
\hline Total | & 248 & 100.00 & \\
\hline
\end{tabular}

(2) Insulation

-- single family dwellings

Freq. Percent Cum.

\begin{tabular}{|c|c|c|c|}
\hline unchecked & 30.75 & 12.40 & 12.40 \\
\hline checked & 217.25 & 87.60 & 100.00 \\
\hline Total | & 248 & 100.00 & \\
\hline
\end{tabular}

-- multifamily dwellings

Freq. Percent Cum.

\begin{tabular}{|c|c|c|c|}
\hline unchecked | & 167.71 & 67.63 & 67.63 \\
\hline checked & 80.29 & 32.37 & 100.00 \\
\hline Total | & 248 & 100.00 & \\
\hline
\end{tabular}

-- mobile homes

Freq. Percent Cum.

\begin{tabular}{|c|c|c|c|}
\hline unchecked & 61.81 & 24.92 & 24.92 \\
\hline checked & 186.19 & 75.08 & 100.00 \\
\hline Total & 248 & 100.00 & \\
\hline
\end{tabular}

(3) Space heating, ventilation, air conditioning

-- single family dwellings

Freq. Percent Cum.

$\begin{array}{rrr}\text { unchecked | } 64.71 & 26.09 & 26.09 \\ \text { checked | } 183.29 & 73.91 & 100.00 \\ ------- & - \\ \text { Total | } 248 & 100.00\end{array}$


-- multifamily dwellings

\begin{tabular}{|c|c|c|c|}
\hline & Freq. & Percent & Cum. \\
\hline unche & 176.62 & 71.22 & 71.22 \\
\hline checked & 71.38 & 28.78 & 100.00 \\
\hline
\end{tabular}

-- mobile homes

\begin{tabular}{|c|c|c|c|}
\hline & Freq. & cent & Cum. \\
\hline unchecked & 95.37 & 38.46 & 38.46 \\
\hline checked & 152.63 & 61.54 & 100.00 \\
\hline Tota & 248 & 100.00 & \\
\hline
\end{tabular}

(4) Infiltration measures

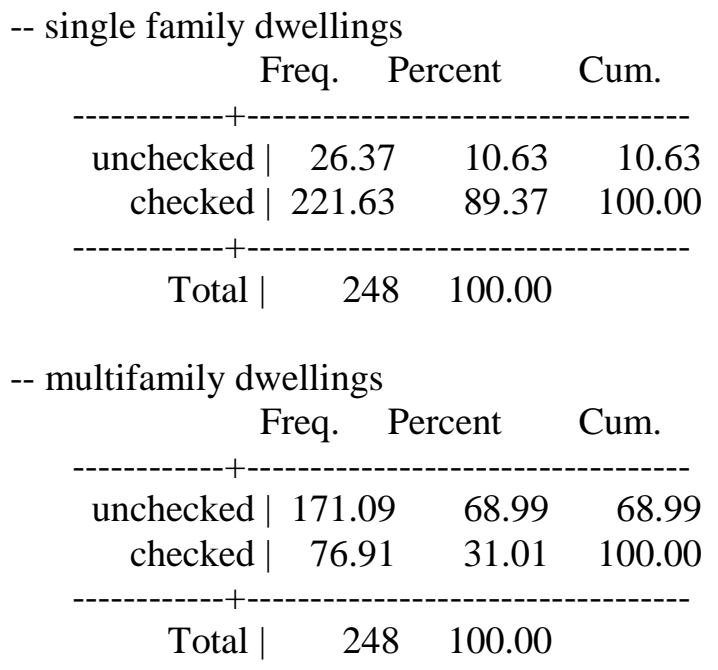

-- mobile homes Freq. Percent Cum.

$\begin{array}{rrr}\text { unchecked | } 57.97 & 23.38 & 23.38 \\ \text { checked | } 190.03 & 76.62 & 100.00 \\ ---------- \\ \text { Total | } 248 & 100.00\end{array}$

(5) Doors and windows

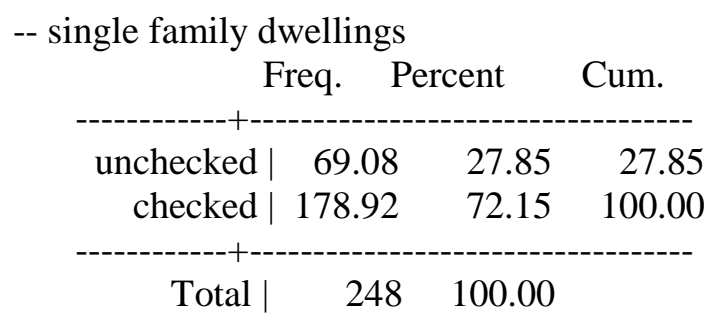

-- multifamily dwellings 


\begin{tabular}{|c|c|c|c|}
\hline & req. & Percent & Cum. \\
\hline unchecked & 180.87 & 72.93 & 72.93 \\
\hline checked & 67.13 & 27.07 & 100.00 \\
\hline Total & 248 & 100.00 & \\
\hline
\end{tabular}

-- mobile homes

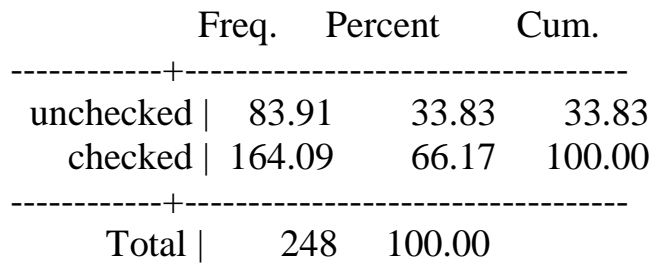

(6) Hot water heating

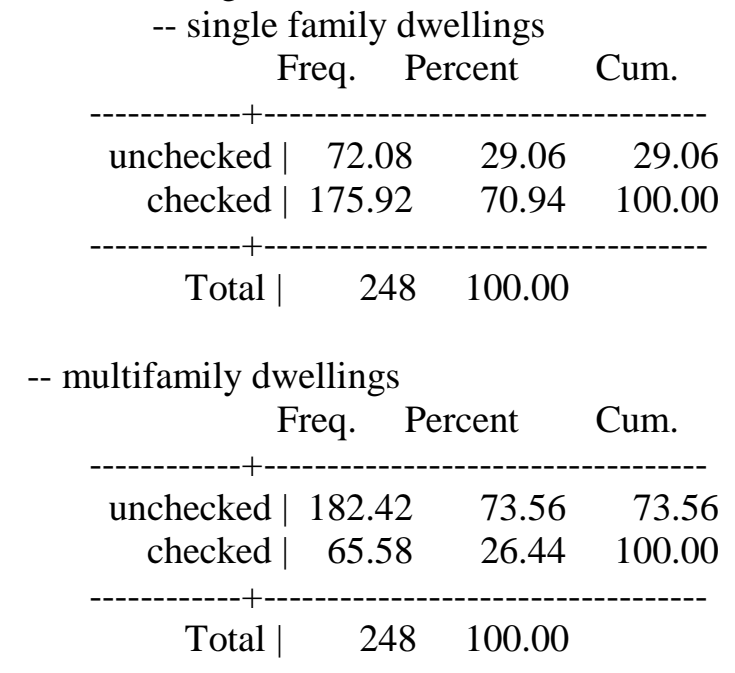

-- mobile homes

\begin{tabular}{|c|c|c|c|}
\hline & Freq. & cent & Cum. \\
\hline unchecked & 93.15 & 37.56 & 37.56 \\
\hline checked & 154.85 & 62.44 & 100.00 \\
\hline Total & 248 & 100.00 & \\
\hline
\end{tabular}

(7) Baseloads (e.g., lighting, refrigerators)

-- single family dwellings

\begin{tabular}{|c|c|c|c|}
\hline & Freq. & cent & Cum. \\
\hline unchecked & 65.43 & 26.38 & 26.38 \\
\hline checked & 182.57 & 73.62 & 100.00 \\
\hline Total & 248 & 100.00 & \\
\hline
\end{tabular}

-- multifamily dwellings

Freq. Percent Cum. 


\begin{tabular}{|c|c|c|c|}
\hline $\begin{array}{r}\text { unchecked } \\
\text { checked }\end{array}$ & $\begin{array}{r}176.68 \\
71.32\end{array}$ & $\begin{array}{l}71.24 \\
28.76\end{array}$ & $\begin{array}{c}71.24 \\
100.00\end{array}$ \\
\hline Total | & 248 & 100.00 & \\
\hline
\end{tabular}

\begin{tabular}{|c|c|c|c|}
\hline & Freq. & Percent & Cum. \\
\hline unchecked & 87.27 & 35.19 & 35.19 \\
\hline checked & 160.73 & 64.81 & 100.00 \\
\hline Total | & 248 & 100.00 & \\
\hline
\end{tabular}

5a. On which of the following administrative-related topics did your agency provide training to your own in-house staff in Program Year 2008? Check all that apply.

(1) Management

\begin{tabular}{|c|c|c|c|}
\hline & Freq. & Percent & Cum. \\
\hline unchecked & 130.82 & 52.12 & 52.12 \\
\hline checked & 120.18 & 47.88 & 100.00 \\
\hline Total & 251 & 100.00 & \\
\hline
\end{tabular}

(2) Client education

\begin{tabular}{|c|c|c|c|}
\hline & Freq. & Percent & Cum. \\
\hline unchecked | & 66.16 & 26.36 & 26.36 \\
\hline checked & 184.84 & 73.64 & 100.00 \\
\hline Total | & 251 & 100.00 & \\
\hline
\end{tabular}

(3) Auditing/estimating

-- single family dwellings

Freq. Percent Cum.

$\begin{array}{rrr}\text { unchecked | } 71.16 & 28.35 & 28.35 \\ \text { checked | } 179.84 & 71.65 & 100.00 \\ ------------- & \\ \text { Total } & 251 & 100.00\end{array}$


-- multifamily dwellings

\begin{tabular}{|c|c|c|c|}
\hline & Freq. & Percent & Cum. \\
\hline unchecked & 176.34 & 70.25 & 70.25 \\
\hline checked & 74.66 & 29.75 & 100.00 \\
\hline Tota & 251 & 100.00 & \\
\hline
\end{tabular}

-- mobile homes

\begin{tabular}{|c|c|c|}
\hline Freq. & Percent & Cum. \\
\hline unchecked |101.31 & 40.36 & 40.36 \\
\hline checked | 149.69 & 59.64 & 100.00 \\
\hline Total | & 100.00 & \\
\hline
\end{tabular}

(3) Monitoring/quality control

\begin{tabular}{|c|c|c|c|}
\hline \multicolumn{3}{|c|}{ Freq. Percent } & \multirow{2}{*}{$\begin{array}{l}\text { Cum. } \\
39.79\end{array}$} \\
\hline unchecked & 99.88 & 39.79 & \\
\hline checked & 151.12 & 60.21 & 100.00 \\
\hline Total & 251 & 100.00 & \\
\hline
\end{tabular}

(4) Financial topics

\begin{tabular}{rrr} 
Freq. & Percent & \multicolumn{1}{c}{ Cum. } \\
unchecked | 133.70 & 53.27 & 53.27 \\
checked | 117.30 & 46.73 & 100.00 \\
-------- \\
Total | 251 & 100.00 &
\end{tabular}

(5) Outreach and communications

\begin{tabular}{|c|c|c|c|}
\hline & Freq. & cent & Cum. \\
\hline unchecked & 137.31 & 54.71 & 54.71 \\
\hline checked & 113.69 & 45.29 & 100.00 \\
\hline Total & 251 & 100.00 & \\
\hline
\end{tabular}

(6) Other (please specify)

\begin{tabular}{|c|c|c|c|}
\hline & req. & ent & Cum. \\
\hline unchecked & 235.16 & 93.70 & 93.70 \\
\hline checked & 15.81 & 6.30 & 100.00 \\
\hline Total & 251 & 100.00 & \\
\hline
\end{tabular}

5b. On which of the following health and safety topics did your agency provide training to your own inhouse staff in Program Year 2008? Check all that apply. 
Fire safety

\begin{tabular}{|c|c|c|c|}
\hline & Freq. & Percent & Cum. \\
\hline unchecked & 154.97 & 64.04 & 64.04 \\
\hline checked & 87.03 & 35.96 & 100.00 \\
\hline Total & 242 & 100.00 & \\
\hline \multicolumn{4}{|c|}{ Indoor air quality } \\
\hline & Freq. & Percent & Cum. \\
\hline unchecked | & 77.08 & 31.85 & 31.85 \\
\hline checked & 164.92 & 68.15 & 100.00 \\
\hline Total & 242 & 100.00 & \\
\hline
\end{tabular}

\begin{tabular}{|c|c|c|c|}
\hline & Freq. & Percent & Cum \\
\hline unchecked & 205.36 & 84.86 & 84.86 \\
\hline checked & 36.64 & 15.14 & 100.00 \\
\hline Total & 242 & 100.00 & \\
\hline
\end{tabular}

\begin{tabular}{|c|c|c|c|}
\hline & Freq. & Percent & Cum. \\
\hline unchecked & 159.27 & 65.81 & 65.81 \\
\hline checked & 82.73 & 34.19 & 100.00 \\
\hline Total & 242 & 100.00 & \\
\hline
\end{tabular}

\begin{tabular}{|c|c|c|c|}
\hline & Freq. & Percent & Cum \\
\hline unchecked & 97.30 & 40.20 & 40.20 \\
\hline checked & 144.70 & 59.80 & 100.00 \\
\hline Total & 242 & 100.00 & \\
\hline
\end{tabular}

\begin{tabular}{rrrr}
\multicolumn{1}{c}{ Lead } & & \\
& Freq. & Percent & Cum. \\
\hline unchecked | & 56.55 & 23.37 & 23.37 \\
checked $\mid$ & 185.45 & 76.63 & 100.00 \\
------------------ & 100.00 &
\end{tabular}

Asbestos
Freq. Percent Cum. 


\begin{tabular}{|c|c|c|c|}
\hline unchecked & 146.20 & 60.42 & 60.42 \\
\hline checked & 95.80 & 39.58 & 100.00 \\
\hline Total & 242 & 100.00 & \\
\hline
\end{tabular}

\begin{tabular}{|c|c|c|c|}
\hline & Freq. & Percent & Cum. \\
\hline unchecked & 180.42 & 74.56 & 74.56 \\
\hline checked & 61.58 & 25.44 & 100.00 \\
\hline Total & 242 & 100.00 & \\
\hline
\end{tabular}

\begin{tabular}{|c|c|c|c|}
\hline & Freq. & Percent & Cum. \\
\hline unchecked | & 98.06 & 40.52 & 40.52 \\
\hline checked | & 143.94 & 59.48 & 100.00 \\
\hline Total | & 242 & 100.00 & \\
\hline \multicolumn{4}{|c|}{ Other (please specify) } \\
\hline & Freq. & Percent & Cum. \\
\hline unchecked | & 213.16 & 88.08 & 88.08 \\
\hline checked | & 28.84 & 11.92 & 100.00 \\
\hline Total & 242 & 100.00 & \\
\hline
\end{tabular}

6. On which of the following diagnostic procedures did your agency provide training to your staff in Program Year 2008? Check all that apply.

\section{Pressure diagnostics:}

- Blower door (house air leakage rate)

\begin{tabular}{|c|c|c|c|}
\hline & Freq. & Percent & Cum. \\
\hline no & 17.99 & 7.37 & 7.37 \\
\hline yes & 226.01 & 92.63 & 100.00 \\
\hline Total | & 244 & 100.00 & \\
\hline
\end{tabular}


- Zonal pressure measurements

$\begin{array}{ccc}\text { Freq. } & \text { Percent } & \text { Cum. } \\ \text { no | } 115.66 & 47.40 & 47.40 \\ \text { yes | } 128.34 & 52.60 & 100.00 \\ -------------- \\ \text { Total| } 244 & 100.00\end{array}$

- Room-to-room pressure measurements (distribution balancing) Freq. Percent Cum.

\begin{tabular}{|c|c|c|c|}
\hline no & 139.62 & 57.22 & 57.22 \\
\hline yes & 104.38 & 42.78 & 100.00 \\
\hline Total & 244 & 100.00 & \\
\hline
\end{tabular}

- Duct pressure pan measurements

\begin{tabular}{|c|c|c|c|}
\hline & Freq. & Percent & Cum. \\
\hline no & 100.18 & 41.06 & 41.06 \\
\hline yes & 143.82 & 58.94 & 100.00 \\
\hline Total | & 244 & 100.00 & \\
\hline
\end{tabular}

- Duct blower measurements (duct air leakage rate)

\begin{tabular}{|c|c|c|c|}
\hline & Freq. & Percent & Cum. \\
\hline no & 146.75 & 60.14 & 60.14 \\
\hline yes & 97.25 & 39.86 & 100.00 \\
\hline Total | & 244 & 100.00 & \\
\hline
\end{tabular}

Space-heating system:

- Flue gas analysis (steady-state efficiency measurements)

\begin{tabular}{|c|c|c|c|}
\hline & Freq. & Percent & Cum. \\
\hline no & 93.45 & 38.30 & 38.30 \\
\hline yes & 150.55 & 61.70 & 100.00 \\
\hline Total & 244 & 100.00 & \\
\hline
\end{tabular}

- Heat rise measurements

Freq. Percent Cum.

\begin{tabular}{|c|c|c|c|}
\hline no & 141.69 & 58.07 & 58.07 \\
\hline yes | & 102.31 & 41.93 & 100.00 \\
\hline Total & 244 & 100.00 & \\
\hline
\end{tabular}


- $\mathrm{CO}$ measurements in flues

\begin{tabular}{|c|c|c|c|}
\hline & Freq. & Percent & Cum. \\
\hline no & 63.65 & 26.09 & 26.09 \\
\hline yes | & 180.35 & 73.91 & 100.00 \\
\hline Total | & 244 & 100.00 & \\
\hline
\end{tabular}

- Draft/spillage (normal operation)

Freq. Percent Cum.

\begin{tabular}{|c|c|c|c|}
\hline no & $\begin{array}{r}96.09 \\
17701\end{array}$ & 39.38 & 39.38 \\
\hline yes | & 147.91 & 60.62 & 100.00 \\
\hline
\end{tabular}

\section{Air-conditioning system:}

- Refrigerant charge (e.g., superheat, subcooling)

Freq. Percent Cum.

\begin{tabular}{|c|c|c|c|}
\hline no | & 230.16 & $\begin{array}{r}94.33 \\
5.67\end{array}$ & $\begin{array}{r}94.33 \\
10000\end{array}$ \\
\hline otal & 244 & 100.00 & \\
\hline
\end{tabular}

HVAC components and cross-cutting diagnostics:

- Air handler flow rate

Freq. Percent Cum.

$\begin{array}{crr}\text { no | } 193.36 & 79.25 & 79.25 \\ \text { yes | } 50.64 & 20.75 & 100.00 \\ -------- & \\ \text { Total| } 244 & 100.00\end{array}$

- Thermostat anticipator current

\begin{tabular}{|c|c|c|c|}
\hline & Freq. & Percent & Cum. \\
\hline no & 181.93 & 74.56 & 74.56 \\
\hline yes & 62.07 & 25.44 & 100.00 \\
\hline Total & 244 & 100.00 & \\
\hline
\end{tabular}

- Worst case draft/spillage (CAZ)

Freq. Percent Cum.

\begin{tabular}{|c|c|c|c|}
\hline no & 108.49 & 44.46 & 44.46 \\
\hline yes $\mid$ & 135.51 & 55.54 & 100.00 \\
\hline
\end{tabular}


Hot-water (water-heating) system:

- Flue gas analysis (steady-state efficiency measurements)

\begin{tabular}{|c|c|c|c|}
\hline & Freq. & Percent & Cum. \\
\hline no & 109.31 & 44.80 & 44.80 \\
\hline yes & 134.69 & 55.20 & 100.00 \\
\hline to & 244 & 100.00 & \\
\hline
\end{tabular}

- $\quad \mathrm{CO}$ measurements in flues

\begin{tabular}{|c|c|c|c|}
\hline & Freq. & Percent & Cum. \\
\hline no & 63.88 & 26.18 & 26.18 \\
\hline yes & 180.12 & 73.82 & 100.00 \\
\hline Total & 244 & 100.00 & \\
\hline
\end{tabular}

- Draft/spillage (normal operation)

\begin{tabular}{|c|c|c|c|}
\hline & Freq. & Percent & Cum. \\
\hline no & 96.55 & 39.57 & 39.57 \\
\hline yes & 147.45 & 60.43 & 100.00 \\
\hline Total & 244 & 100.00 & \\
\hline
\end{tabular}

- Water flow rates (showerheads and faucets)

$\begin{array}{crc}\text { Freq. } & \text { Percent } & \text { Cum. } \\ \text { no | } 174.71 & 71.60 & 71.60 \\ \text { yes | } 69.29 & 28.40 & 100.00 \\ --------- & \end{array}$

\section{Other CO measurements:}

- $\mathrm{CO}$ measurements in equipment rooms

\begin{tabular}{|c|c|c|c|}
\hline & Freq. & Percent & Cum. \\
\hline no & 115.96 & 47.52 & 47.52 \\
\hline yes & 128.04 & 52.48 & 100.00 \\
\hline otal & 244 & 100.00 & \\
\hline
\end{tabular}

- Cooking stove

Freq. Percent Cum.

\begin{tabular}{|c|c|c|c|}
\hline no & 64.81 & 26.56 & 26.56 \\
\hline yes & 179.19 & 73.44 & 100.00 \\
\hline Total & 244 & 100.00 & \\
\hline
\end{tabular}


- $\mathrm{CO}$ measurements in living areas

\begin{tabular}{|c|c|c|c|}
\hline & Freq. & Percent & Cum. \\
\hline no & 92.84 & 38.05 & 38.05 \\
\hline yes & 151.16 & 61.95 & 100.00 \\
\hline Total & 244 & 100.00 & \\
\hline
\end{tabular}

Other diagnostics and inspections:

- Refrigerator energy use

\begin{tabular}{|c|c|c|c|}
\hline & Freq. & Percent & Cum. \\
\hline no & 112.93 & 46.28 & 46.28 \\
\hline yes & 131.07 & 53.72 & 100.00 \\
\hline Total & 244 & 100.00 & \\
\hline
\end{tabular}

- Exhaust fan air flow rate measurement

\begin{tabular}{|c|c|c|c|}
\hline & Freq. & Percent & Cum. \\
\hline no & 171.80 & 70.41 & 70.41 \\
\hline yes & 72.20 & 29.59 & 100.00 \\
\hline Total & 244 & 100.00 & \\
\hline
\end{tabular}

- Infrared scanning (camera)

Freq. Percent Cum.

\begin{tabular}{|c|c|c|c|}
\hline no & 143.30 & 58.73 & 58.73 \\
\hline yes & 100.70 & 41.27 & 100.00 \\
\hline
\end{tabular}

- $\quad$ Radon testing

\begin{tabular}{|c|c|c|c|}
\hline & Freq. & Percent & Cum. \\
\hline no & 227.85 & 93.38 & 93.38 \\
\hline yes & 16.15 & 6.62 & 100.00 \\
\hline Total & 244 & 100.00 & \\
\hline
\end{tabular}

- Lead testing

\begin{tabular}{|c|c|c|c|}
\hline & Freq. & Percent & Cum. \\
\hline no | & 157.91 & 64.72 & 64.72 \\
\hline yes & 86.09 & 35.28 & 100.00 \\
\hline otal | & 244 & 100.00 & \\
\hline
\end{tabular}


- Mold and mildew testing Freq. Percent Cum.

\begin{tabular}{|c|c|c|c|}
\hline no & 207.40 & 85.00 & 85.00 \\
\hline yes & 36.60 & 15.00 & 100.00 \\
\hline Total & 244 & 100.00 & \\
\hline
\end{tabular}

- Moisture content testing

Freq. Percent Cum.

\begin{tabular}{|c|c|c|c|}
\hline no & 207.22 & 84.93 & 84.93 \\
\hline yes | & 36.78 & 15.07 & 100.00 \\
\hline Total | & 244 & 100.00 & \\
\hline
\end{tabular}

- Other (please specify)

Freq. Percent Cum.

\begin{tabular}{|c|c|c|c|}
\hline no & 234.07 & 95.93 & 95.93 \\
\hline yes & 9.93 & 4.07 & 100.00 \\
\hline otal & 244 & 100.00 & \\
\hline
\end{tabular}


7. For each broad subject listed in the left-most column of the following table, put a check mark in the appropriate cell(s) to indicate which training method(s) you believe were most effective for imparting key skills and information in that area to your agency's in-house or contractor weatherization staff in Program Year 2008:

\begin{tabular}{|c|c|c|c|c|c|c|}
\hline Subject & Conferences & $\begin{array}{l}\text { Primarily } \\
\text { Field } \\
\text { training }\end{array}$ & $\begin{array}{l}\text { Primarily } \\
\text { Classroom } \\
\text { training }\end{array}$ & $\begin{array}{c}\text { Agency } \\
\text { visits }\end{array}$ & $\begin{array}{l}\text { Web } \\
\text { casts }\end{array}$ & $\begin{array}{l}\text { Other } \\
\text { (please } \\
\text { specify) }\end{array}$ \\
\hline Management & $\begin{array}{l}\text { obs: } 271 \\
\text { no: } 76 \\
\text { yes: } 195\end{array}$ & $\begin{array}{l}\text { obs: } 271 \\
\text { no: } 220 \\
\text { yes: } 51\end{array}$ & $\begin{array}{l}\text { obs: } 271 \\
\text { no: } 177 \\
\text { yes: } 94\end{array}$ & $\begin{array}{l}\text { obs: } 271 \\
\text { no: } 181 \\
\text { yes: } 90\end{array}$ & $\begin{array}{l}\text { obs: } 271 \\
\text { no: } 239 \\
\text { yes: } 32\end{array}$ & \\
\hline $\begin{array}{l}\text { Weatherization } \\
\text { installation }\end{array}$ & $\begin{array}{l}\text { obs: } 307 \\
\text { no: } 215 \\
\text { yes: } 92\end{array}$ & $\begin{array}{l}\text { obs: } 307 \\
\text { no: } 23 \\
\text { yes: } 284\end{array}$ & $\begin{array}{l}\text { obs: } 307 \\
\text { no: } 199 \\
\text { yes: } 108\end{array}$ & $\begin{array}{l}\text { obs: } 307 \\
\text { no: } 254 \\
\text { yes: } 53\end{array}$ & $\begin{array}{l}\text { obs: } 307 \\
\text { no: } 302 \\
\text { yes: } 5\end{array}$ & \\
\hline $\begin{array}{l}\text { Auditing/ } \\
\text { Estimating }\end{array}$ & $\begin{array}{l}\text { obs: } 309 \\
\text { no: } 206 \\
\text { yes: } 103\end{array}$ & $\begin{array}{l}\text { obs: } 309 \\
\text { no: } 72 \\
\text { yes: } 237\end{array}$ & $\begin{array}{l}\text { obs: } 309 \\
\text { no: } 142 \\
\text { yes: } 167\end{array}$ & $\begin{array}{l}\text { obs: } 309 \\
\text { no: } 253 \\
\text { yes: } 56\end{array}$ & $\begin{array}{l}\text { obs: } 309 \\
\text { no: } 296 \\
\text { yes: } 13\end{array}$ & \\
\hline $\begin{array}{l}\text { Monitoring/ } \\
\text { quality control }\end{array}$ & $\begin{array}{l}\text { obs: } 287 \\
\text { no: } 196 \\
\text { yes: } 91\end{array}$ & $\begin{array}{l}\text { obs: } 287 \\
\text { no: } 90 \\
\text { yes: } 197\end{array}$ & $\begin{array}{l}\text { obs: } 287 \\
\text { no: } 168 \\
\text { yes: } 119\end{array}$ & $\begin{array}{l}\text { obs: } 287 \\
\text { no: } 182 \\
\text { yes: } 105\end{array}$ & $\begin{array}{l}\text { obs: } 287 \\
\text { no: } 275 \\
\text { yes: } 12\end{array}$ & \\
\hline Financial topics & $\begin{array}{l}\text { obs: } 249 \\
\text { no: } 104 \\
\text { yes: } 145\end{array}$ & $\begin{array}{l}\text { obs: } 249 \\
\text { no: } 218 \\
\text { yes: } 31\end{array}$ & $\begin{array}{l}\text { obs: } 249 \\
\text { no: } 140 \\
\text { yes: } 109\end{array}$ & $\begin{array}{l}\text { obs: } 249 \\
\text { no: } 183 \\
\text { yes: } 66\end{array}$ & $\begin{array}{l}\text { obs: } 249 \\
\text { no: } 223 \\
\text { yes: } 26\end{array}$ & \\
\hline $\begin{array}{l}\text { Outreach and } \\
\text { communications }\end{array}$ & $\begin{array}{l}\text { obs: } 230 \\
\text { no: } 93 \\
\text { yes: } 137\end{array}$ & $\begin{array}{l}\text { obs: } 230 \\
\text { no: } 177 \\
\text { yes: } 53\end{array}$ & $\begin{array}{l}\text { obs: } 230 \\
\text { no: } 136 \\
\text { yes: } 94\end{array}$ & $\begin{array}{l}\text { obs: } 230 \\
\text { no: } 186 \\
\text { yes: } 44\end{array}$ & $\begin{array}{l}\text { obs: } 230 \\
\text { no: } 208 \\
\text { yes: } 22\end{array}$ & \\
\hline Health and safety & $\begin{array}{l}\text { obs: } 302 \\
\text { no: } 179 \\
\text { yes: } 123\end{array}$ & $\begin{array}{l}\text { obs: } 302 \\
\text { no: } 89 \\
\text { yes: } 213\end{array}$ & $\begin{array}{l}\text { obs: } 302 \\
\text { no: } 127 \\
\text { yes: } 175\end{array}$ & $\begin{array}{l}\text { obs: } 302 \\
\text { no: } 250 \\
\text { yes: } 52\end{array}$ & $\begin{array}{l}\text { obs: } 302 \\
\text { no: } 292 \\
\text { yes: } 10\end{array}$ & \\
\hline $\begin{array}{l}\text { Diagnostic } \\
\text { procedures }\end{array}$ & $\begin{array}{l}\text { obs: } 298 \\
\text { no: } 202 \\
\text { yes: } 96\end{array}$ & $\begin{array}{l}\text { obs: } 298 \\
\text { no: } 39 \\
\text { yes: } 259\end{array}$ & $\begin{array}{l}\text { obs: } 298 \\
\text { no: } 147 \\
\text { yes: } 151\end{array}$ & $\begin{array}{l}\text { obs: } 298 \\
\text { no: } 250 \\
\text { yes: } 48\end{array}$ & $\begin{array}{l}\text { obs: } 298 \\
\text { no: } 292 \\
\text { yes: } 6\end{array}$ & \\
\hline $\begin{array}{l}\text { Procedures for } \\
\text { selecting } \\
\text { weatherization } \\
\text { measures }\end{array}$ & $\begin{array}{l}\text { obs: } 295 \\
\text { no: } 192 \\
\text { yes: } 103\end{array}$ & $\begin{array}{l}\text { obs: } 295 \\
\text { no: } 107 \\
\text { yes: } 188\end{array}$ & $\begin{array}{l}\text { obs: } 295 \\
\text { no: } 113 \\
\text { yes: } 182\end{array}$ & $\begin{array}{l}\text { obs: } 295 \\
\text { no: } 240 \\
\text { yes: } 55\end{array}$ & $\begin{array}{l}\text { obs: } 295 \\
\text { no: } 287 \\
\text { yes: } 8\end{array}$ & \\
\hline Client education & $\begin{array}{l}\text { obs: } 286 \\
\text { no: } 145 \\
\text { yes: } 141\end{array}$ & $\begin{array}{l}\text { obs: } 286 \\
\text { no: } 198 \\
\text { yes: } 88\end{array}$ & $\begin{array}{l}\text { obs: } 286 \\
\text { no: } 125 \\
\text { yes: } 161\end{array}$ & $\begin{array}{l}\text { obs: } 286 \\
\text { no: } 243 \\
\text { yes: } 43\end{array}$ & $\begin{array}{l}\text { obs: } 286 \\
\text { no: } 271 \\
\text { yes: } 15 \\
\end{array}$ & \\
\hline $\begin{array}{l}\text { Other (please } \\
\text { specify) }\end{array}$ & $\begin{array}{l}\text { obs: } 6 \\
\text { no: } 1 \\
\text { yes: } 5\end{array}$ & $\begin{array}{l}\text { obs: } 6 \\
\text { no: } 5 \\
\text { yes: } 1\end{array}$ & $\begin{array}{l}\text { obs: } 6 \\
\text { no: } 3 \\
\text { yes: } 3\end{array}$ & $\begin{array}{l}\text { obs: } 6 \\
\text { no: } 6 \\
\text { yes: } 0\end{array}$ & $\begin{array}{l}\text { obs: } 6 \\
\text { no: } 5 \\
\text { yes: } 1\end{array}$ & \\
\hline
\end{tabular}


8. For each broad subject listed in the left-most column of the following table, please indicate the quality of training received in Program Year 2008 at the training venues listed in the column headings. Please leave cells blank where your agency did not receive training during this period of time.

Please use the following scale: 1 - very low; 2 - low; 3 - medium; 4 - high; 5 - very high

\begin{tabular}{|c|c|c|c|c|c|c|}
\hline Subject & $\begin{array}{c}\text { National } \\
\text { Weatherization } \\
\text { Program } \\
\text { Conference }\end{array}$ & $\begin{array}{l}\text { Affordable } \\
\text { Comfort } \\
\text { Conference }\end{array}$ & $\begin{array}{c}\text { Regional } \\
\text { Weatherization } \\
\text { Conference }\end{array}$ & $\begin{array}{c}\text { State } \\
\text { Weatherization } \\
\text { Conference }\end{array}$ & $\begin{array}{c}\text { State/ } \\
\text { Regional } \\
\text { Training } \\
\text { Center }\end{array}$ & $\begin{array}{l}\text { Training } \\
\text { Provided by } \\
\text { Your Own } \\
\text { Agency }\end{array}$ \\
\hline Management & $\begin{array}{l}\text { obs: } 115 \\
\text { very low: } 10 \\
\text { low: } 7 \\
\text { medium: } 32 \\
\text { high: } 45 \\
\text { very high: } 21\end{array}$ & $\begin{array}{l}\text { obs: } 80 \\
\text { very low: } 6 \\
\text { low: } 6 \\
\text { medium: } 29 \\
\text { high: } 25 \\
\text { very high: } 14\end{array}$ & $\begin{array}{l}\text { obs: } 84 \\
\text { very low: } 1 \\
\text { low: } 9 \\
\text { medium: } 35 \\
\text { high: } 22 \\
\text { very high: } 17 \\
\end{array}$ & $\begin{array}{l}\text { obs: } 129 \\
\text { very low: } 7 \\
\text { low: } 13 \\
\text { medium: } 32 \\
\text { high: } 51 \\
\text { very high: } 26\end{array}$ & $\begin{array}{l}\text { obs: } 76 \\
\text { very low: } 8 \\
\text { low: } 0 \\
\text { medium: } 20 \\
\text { high: } 30 \\
\text { very high: } 17 \\
\end{array}$ & $\begin{array}{l}\text { obs: } 115 \\
\text { very low: } 8 \\
\text { low: } 3 \\
\text { medium: } 37 \\
\text { high: } 34 \\
\text { very high: } 32\end{array}$ \\
\hline $\begin{array}{l}\text { Weatherization } \\
\text { installation }\end{array}$ & $\begin{array}{l}\text { obs: } 90 \\
\text { very low: } 6 \\
\text { low: } 7 \\
\text { medium: } 13 \\
\text { high: } 50 \\
\text { very high: } 13\end{array}$ & $\begin{array}{l}\text { obs: } 88 \\
\text { very low: } 4 \\
\text { low: } 5 \\
\text { medium: } 26 \\
\text { high: } 30 \\
\text { very high: } 23\end{array}$ & $\begin{array}{l}\text { obs: } 82 \\
\text { very low: } 1 \\
\text { low: } 5 \\
\text { medium: } 17 \\
\text { high: } 36 \\
\text { very high: } 22\end{array}$ & $\begin{array}{l}\text { obs: } 121 \\
\text { very low: } 4 \\
\text { low: } 13 \\
\text { medium: } 32 \\
\text { high: } 51 \\
\text { very high: } 20\end{array}$ & $\begin{array}{l}\text { obs: } 121 \\
\text { very low: } 1 \\
\text { low: } 0 \\
\text { medium: } 15 \\
\text { high: } 57 \\
\text { very high: } 49\end{array}$ & $\begin{array}{l}\text { obs: } 156 \\
\text { very low: } 2 \\
\text { low: } 2 \\
\text { medium: } 32 \\
\text { high: } 61 \\
\text { very high: } 59\end{array}$ \\
\hline $\begin{array}{l}\text { Auditing/ } \\
\text { Estimating }\end{array}$ & $\begin{array}{l}\text { obs: } 90 \\
\text { very low: } 2 \\
\text { low: } 7 \\
\text { medium: } 19 \\
\text { high: } 49 \\
\text { very high: } 13 \\
\end{array}$ & $\begin{array}{l}\text { obs: } 81 \\
\text { very low: } 5 \\
\text { low: } 3 \\
\text { medium: } 24 \\
\text { high: } 31 \\
\text { very high: } 18 \\
\end{array}$ & $\begin{array}{l}\text { obs: } 77 \\
\text { very low: } 1 \\
\text { low: } 6 \\
\text { medium: } 17 \\
\text { high: } 39 \\
\text { very high: } 14 \\
\end{array}$ & $\begin{array}{l}\text { obs: } 118 \\
\text { very low: } 2 \\
\text { low: } 15 \\
\text { medium: } 37 \\
\text { high: } 49 \\
\text { very high: } 15 \\
\end{array}$ & $\begin{array}{l}\text { obs: } 139 \\
\text { very low: } 2 \\
\text { low: } 1 \\
\text { medium: } 26 \\
\text { high: } 59 \\
\text { very high: } 50\end{array}$ & $\begin{array}{l}\text { obs: } 147 \\
\text { very low: } 0 \\
\text { low: } 1 \\
\text { medium: } 34 \\
\text { high: } 70 \\
\text { very high: } 41\end{array}$ \\
\hline $\begin{array}{l}\text { Monitoring/ } \\
\text { quality control }\end{array}$ & $\begin{array}{l}\text { obs: } 82 \\
\text { very low: } 6 \\
\text { low: } 8 \\
\text { medium: } 31 \\
\text { high: } 25 \\
\text { very high: } 12\end{array}$ & $\begin{array}{l}\text { obs: } 61 \\
\text { very low: } 3 \\
\text { low: } 5 \\
\text { medium: } 20 \\
\text { high: } 21 \\
\text { very high: } 12\end{array}$ & $\begin{array}{l}\text { obs: } 70 \\
\text { very low: } 0 \\
\text { low: } 6 \\
\text { medium: } 27 \\
\text { high: } 23 \\
\text { very high: } 15\end{array}$ & $\begin{array}{l}\text { obs: } 103 \\
\text { very low: } 3 \\
\text { low: } 21 \\
\text { medium: } 22 \\
\text { high: } 32 \\
\text { very high: } 25\end{array}$ & $\begin{array}{l}\text { obs: } 91 \\
\text { very low: } 1 \\
\text { low: } 1 \\
\text { medium: } 25 \\
\text { high: } 43 \\
\text { very high: } 20\end{array}$ & $\begin{array}{l}\text { obs: } 126 \\
\text { very low: } 0 \\
\text { low: } 7 \\
\text { medium: } 27 \\
\text { high: } 48 \\
\text { very high: } 43\end{array}$ \\
\hline Financial topics & $\begin{array}{l}\text { obs: } 75 \\
\text { very low: } 8 \\
\text { low: } 14 \\
\text { medium: } 20 \\
\text { high: } 22 \\
\text { very high: } 12\end{array}$ & $\begin{array}{l}\text { obs: } 54 \\
\text { very low: } 3 \\
\text { low: } 13 \\
\text { medium: } 16 \\
\text { high: } 11 \\
\text { very high: } 11\end{array}$ & $\begin{array}{l}\text { obs: } 54 \\
\text { very low: } 1 \\
\text { low: } 11 \\
\text { medium: } 19 \\
\text { high: } 24 \\
\text { very high: } 10\end{array}$ & $\begin{array}{l}\text { obs: } 93 \\
\text { very low: } 6 \\
\text { low: } 15 \\
\text { medium: } 23 \\
\text { high: } 30 \\
\text { very high: } 18\end{array}$ & $\begin{array}{l}\text { obs: } 55 \\
\text { very low: } 6 \\
\text { low: } 5 \\
\text { medium: } 14 \\
\text { high: } 17 \\
\text { very high: } 13\end{array}$ & $\begin{array}{l}\text { obs: } 93 \\
\text { very low: } 2 \\
\text { low: } 15 \\
\text { medium: } 20 \\
\text { high: } 28 \\
\text { very high: } 27\end{array}$ \\
\hline $\begin{array}{l}\text { Outreach and } \\
\text { communications }\end{array}$ & $\begin{array}{l}\text { obs: } 68 \\
\text { very low: } 8 \\
\text { low: } 2 \\
\text { medium: } 28 \\
\text { high: } 15 \\
\text { very high: } 14\end{array}$ & $\begin{array}{l}\text { obs: } 54 \\
\text { very low: } 7 \\
\text { low: } 9 \\
\text { medium: } 19 \\
\text { high: } 13 \\
\text { very high: } 6\end{array}$ & $\begin{array}{l}\text { obs: } 58 \\
\text { very low: } 1 \\
\text { low: } 3 \\
\text { medium: } 29 \\
\text { high: } 12 \\
\text { very high: } 13\end{array}$ & $\begin{array}{l}\text { obs: } 82 \\
\text { very low: } 4 \\
\text { low: } 13 \\
\text { medium: } 28 \\
\text { high: } 23 \\
\text { very high: } 13\end{array}$ & $\begin{array}{l}\text { obs: } 55 \\
\text { very low: } 3 \\
\text { low: } 9 \\
\text { medium: } 17 \\
\text { high: } 10 \\
\text { very high: } 15\end{array}$ & $\begin{array}{l}\text { obs: } 97 \\
\text { very low: } 5 \\
\text { low: } 9 \\
\text { medium: } 21 \\
\text { high: } 33 \\
\text { very high: } 28\end{array}$ \\
\hline $\begin{array}{l}\text { Health and } \\
\text { safety }\end{array}$ & $\begin{array}{l}\text { obs: } 85 \\
\text { very low: } 2 \\
\text { low: } 10 \\
\text { medium: } 21 \\
\text { high: } 36 \\
\text { very high: } 16\end{array}$ & $\begin{array}{l}\text { obs: } 83 \\
\text { very low: } 6 \\
\text { low: } 5 \\
\text { medium: } 24 \\
\text { high: } 28 \\
\text { very high: } 19\end{array}$ & $\begin{array}{l}\text { obs: } 77 \\
\text { very low: } 0 \\
\text { low: } 5 \\
\text { medium: } 20 \\
\text { high: } 31 \\
\text { very high: } 21\end{array}$ & $\begin{array}{l}\text { obs: } 113 \\
\text { very low: } 5 \\
\text { low: } 9 \\
\text { medium: } 35 \\
\text { high: } 42 \\
\text { very high: } 21\end{array}$ & $\begin{array}{l}\text { obs: } 124 \\
\text { very low: } 1 \\
\text { low: } 1 \\
\text { medium: } 19 \\
\text { high: } 60 \\
\text { very high: } 43\end{array}$ & $\begin{array}{l}\text { obs: } 137 \\
\text { very low: } 1 \\
\text { low: } 4 \\
\text { medium: } 28 \\
\text { high: } 61 \\
\text { very high: } 42 \\
\end{array}$ \\
\hline $\begin{array}{l}\text { Diagnostic } \\
\text { procedures }\end{array}$ & $\begin{array}{l}\text { obs: } 83 \\
\text { very low: } 1 \\
\text { low: } 8 \\
\text { medium: } 26 \\
\text { high: } 28 \\
\text { very high: } 20 \\
\end{array}$ & $\begin{array}{l}\text { obs: } 87 \\
\text { very low: } 3 \\
\text { low: } 5 \\
\text { medium: } 31 \\
\text { high: } 25 \\
\text { very high: } 22 \\
\end{array}$ & $\begin{array}{l}\text { obs: } 77 \\
\text { very low: } 0 \\
\text { low: } 3 \\
\text { medium: } 24 \\
\text { high: } 28 \\
\text { very high: } 21 \\
\end{array}$ & $\begin{array}{l}\text { obs: } 106 \\
\text { very low: } 3 \\
\text { low: } 14 \\
\text { medium: } 30 \\
\text { high: } 34 \\
\text { very high: } 24\end{array}$ & $\begin{array}{l}\text { obs: } 135 \\
\text { very low: } 1 \\
\text { low: } 1 \\
\text { medium: } 22 \\
\text { high: } 52 \\
\text { very high: } 60\end{array}$ & $\begin{array}{l}\text { obs: } 139 \\
\text { very low: } 1 \\
\text { low: } 2 \\
\text { medium: } 35 \\
\text { high: } 60 \\
\text { very high: } 41 \\
\end{array}$ \\
\hline $\begin{array}{l}\text { Procedures for } \\
\text { selecting } \\
\text { weatherization } \\
\text { measures }\end{array}$ & $\begin{array}{l}\text { obs: } 79 \\
\text { very low: } 5 \\
\text { low: } 5 \\
\text { medium: } 22 \\
\text { high: } 33 \\
\text { very high: } 13\end{array}$ & $\begin{array}{l}\text { obs: } 69 \\
\text { very low: } 6 \\
\text { low: } 4 \\
\text { medium: } 23 \\
\text { high: } 23 \\
\text { very high: } 13 \\
\end{array}$ & $\begin{array}{l}\text { obs: } 66 \\
\text { very low: } 1 \\
\text { low: } 4 \\
\text { medium: } 20 \\
\text { high: } 23 \\
\text { very high: } 18 \\
\end{array}$ & $\begin{array}{l}\text { obs: } 102 \\
\text { very low: } 5 \\
\text { low: } 12 \\
\text { medium: } 22 \\
\text { high: } 34 \\
\text { very high: } 30\end{array}$ & $\begin{array}{l}\text { obs: } 121 \\
\text { very low: } 1 \\
\text { low: } 0 \\
\text { medium: } 22 \\
\text { high: } 54 \\
\text { very high: } 43\end{array}$ & $\begin{array}{l}\text { obs: } 135 \\
\text { very low: } 0 \\
\text { low: } 1 \\
\text { medium: } 29 \\
\text { high: } 62 \\
\text { very high: } 43\end{array}$ \\
\hline
\end{tabular}




\begin{tabular}{|c|c|c|c|c|c|c|}
\hline Client education & $\begin{array}{l}\text { obs: } 82 \\
\text { very low: } 10 \\
\text { low: } 11 \\
\text { medium: } 23 \\
\text { high: } 26 \\
\text { very high: } 13\end{array}$ & $\begin{array}{l}\text { obs: } 74 \\
\text { very low: } 5 \\
\text { low: } 15 \\
\text { medium: } 23 \\
\text { high: } 20 \\
\text { very high: } 11\end{array}$ & $\begin{array}{l}\text { obs: } 66 \\
\text { very low: } 0 \\
\text { low: } 9 \\
\text { medium: } 17 \\
\text { high: } 25 \\
\text { very high: } 15\end{array}$ & $\begin{array}{l}\text { obs: } 97 \\
\text { very low: } 9 \\
\text { low: } 14 \\
\text { medium: } 27 \\
\text { high: } 29 \\
\text { very high: } 18\end{array}$ & $\begin{array}{l}\text { obs: } 1 \\
\text { very low: } 5 \\
\text { low: } 5 \\
\text { medium: } 32 \\
\text { high: } 32 \\
\text { very high: } 16\end{array}$ & $\begin{array}{l}\text { obs: } 138 \\
\text { very low: } 1 \\
\text { low: } 9 \\
\text { medium: } 39 \\
\text { high: } 48 \\
\text { very high: } 41\end{array}$ \\
\hline $\begin{array}{l}\text { Other (please } \\
\text { specify) }\end{array}$ & $\begin{array}{l}\text { obs: } 3 \\
\text { very low: } 2 \\
\text { low: } 0 \\
\text { medium: } 1 \\
\text { high: } 0 \\
\text { very high: } 0\end{array}$ & $\begin{array}{l}\text { obs: } 2 \\
\text { very low: } 1 \\
\text { low: } 0 \\
\text { medium: } 1 \\
\text { high: } 0 \\
\text { very high: } 0\end{array}$ & $\begin{array}{l}\text { obs: } 2 \\
\text { very low: } 1 \\
\text { low: } 0 \\
\text { medium: } 1 \\
\text { high: } 0 \\
\text { very high: } 0\end{array}$ & $\begin{array}{l}\text { obs: } 2 \\
\text { very low: } 1 \\
\text { low: } 0 \\
\text { medium: } 1 \\
\text { high: } 0 \\
\text { very high: } 0\end{array}$ & $\begin{array}{l}\text { obs: } 3 \\
\text { very low: } 1 \\
\text { low: } 0 \\
\text { medium: } 1 \\
\text { high: } 0 \\
\text { very high: } 1\end{array}$ & $\begin{array}{l}\text { obs: } 5 \\
\text { very low: } 1 \\
\text { low: } 0 \\
\text { medium: } 1 \\
\text { high: } 1 \\
\text { very high: } 2\end{array}$ \\
\hline
\end{tabular}

9. For those staff working in your agency who needed to have knowledge about the following list of weatherization topics in Program Year 2008, how well trained were they in each area in Program Year 2008? Please use the following scale: 1 - not at all well trained; 2 - not well trained; 3 - moderately well trained; 4 -well trained; 5 - very well trained; 6 - not applicable Circle best answer.

(1) Diagnostic procedures

Freq. Percent Cum.

\begin{tabular}{r|rrr} 
not at all well trained & 2.07 & 0.66 & 0.66 \\
not well trained & 9.37 & 2.99 & 3.64 \\
moderately well trained & 56.75 & 18.07 & 21.72 \\
well trained & 125.50 & 39.97 & 61.69 \\
very well trained & 113.67 & 36.20 & 97.89 \\
n/a & 6.64 & 2.11 & 100.00 \\
- Total & 314 & 100.00 &
\end{tabular}

(2) Insulation

-- single family dwellings

Freq. Percent Cum.

\begin{tabular}{|c|c|c|c|}
\hline not well trained & 11.10 & 3.52 & 3.52 \\
\hline moderately well trained & 49.20 & 15.62 & 19.14 \\
\hline well trained & 122.91 & 39.02 & 58.16 \\
\hline very well trained & 122.98 & 39.04 & 97.20 \\
\hline $\mathrm{n} / \mathrm{a}$ & 8.81 & 2.80 & 100.00 \\
\hline Total & 315 & 100.00 & \\
\hline
\end{tabular}

-- multifamily dwellings

Freq. Percent Cum.

\begin{tabular}{r|ccc} 
not at all well trained & 19.21 & 7.22 & 7.22 \\
not well trained & 23.72 & 8.92 & 16.14 \\
moderately well trained & 29.39 & 11.05 & 27.19 \\
well trained & 67.17 & 25.25 & 52.44 \\
very well trained $\mid$ & 46.82 & 17.60 & 70.04 \\
n/a $\mid$ & 79.70 & 29.96 & 100.00 \\
- Total $\mid$ & 266 & 100.00 &
\end{tabular}

E-139 
-- mobile homes

$\begin{array}{rrrr} & \text { Freq. } & \text { Percent } & \text { Cum. } \\ \text { not at all well trained | } & 5.01 & 1.72 & 1.72 \\ \text { not well trained | } & 14.71 & 5.06 & 6.78 \\ \text { moderately well trained } \mid & 46.01 & 15.81 & 22.59 \\ \text { well trained } \mid & 102.38 & 35.18 & 57.77 \\ \text { very well trained } & 101.76 & 34.97 & 92.74 \\ \text { n/a } \mid & 21.12 & 7.26 & 100.00 \\ - \text { Total | } & 291 & 100.00 & \end{array}$

(3) Space heating, ventilation, air conditioning

-- single family dwellings

Freq. Percent Cum.

\begin{tabular}{r|rrr} 
not at all well trained & .92 & 0.30 & 0.30 \\
not well trained & 24.38 & 7.89 & 8.19 \\
moderately well trained & 65.65 & 21.24 & 29.43 \\
well trained $\mid$ & 115.51 & 37.38 & 66.81 \\
very well trained $\mid$ & 89.44 & 28.94 & 95.76 \\
n/a $\mid$ & 13.11 & 4.24 & 100.00 \\
- Total & 309 & 100.00 &
\end{tabular}

-- multifamily dwellings

Freq. Percent Cum.

\begin{tabular}{r|rrr} 
not at all well trained & 18.31 & 7.02 & 7.02 \\
not well trained & 30.48 & 11.68 & 18.69 \\
moderately well trained $\mid$ & 39.21 & 15.02 & 33.72 \\
well trained $\mid$ & 54.83 & 21.01 & 54.73 \\
very well trained $\mid$ & 35.03 & 13.42 & 68.15 \\
n/a $\mid$ & 83.14 & 31.85 & 100.00 \\
- Total & 261 & 100.00 &
\end{tabular}

-- mobile homes

Freq. Percent Cum.

\begin{tabular}{|c|c|c|c|}
\hline not at all well trained & 4.80 & 1.66 & 1.66 \\
\hline not well trained & 21.85 & 7.54 & 9.19 \\
\hline moderately well trained & 57.16 & 19.71 & 28.90 \\
\hline well trained & 101.56 & 35.02 & 63.92 \\
\hline very well trained & 83.63 & 28.84 & 92.76 \\
\hline $\mathrm{n} / \mathrm{a}$ & 20.99 & 7.24 & 100.00 \\
\hline Total & 290 & 100.00 & \\
\hline
\end{tabular}


(4) Infiltration measures

-- single family dwellings

Freq. Percent Cum.

$\begin{array}{rrrr}\text { not at all well trained | } & .82 & 0.26 & 0.26 \\ \text { not well trained | } & 13.62 & 4.30 & 4.56 \\ \text { moderately well trained | } & 43.83 & 13.83 & 18.38 \\ \text { well trained | } & 117.72 & 37.13 & 55.52 \\ \text { very well trained | } & 133.45 & 42.10 & 97.61 \\ \text { n/a | } & 7.57 & 2.39 & 100.00 \\ \text { Total } & 317 & 100.00 & \end{array}$

-- multifamily dwellings

Freq. Percent Cum.

\begin{tabular}{|c|c|c|c|}
\hline not at all well trained & 17.66 & 6.72 & 6.72 \\
\hline not well trained & 23.35 & 8.88 & 15.59 \\
\hline moderately well trained & 34.14 & 12.98 & 28.58 \\
\hline well trained & 55.24 & 21.00 & 49.58 \\
\hline very well trained & 53.74 & 20.43 & 70.01 \\
\hline $\mathrm{n} /\left.\mathrm{a}\right|^{\prime}$ & 78.86 & 29.99 & 100.00 \\
\hline
\end{tabular}

-- mobile homes

Freq. Percent Cum.

\begin{tabular}{r|rrr} 
not at all well trained & 4.48 & 1.51 & 1.51 \\
not well trained | & 16.19 & 5.47 & 6.99 \\
moderately well trained & 38.58 & 13.03 & 20.02 \\
well trained | & 100.28 & 33.88 & 53.90 \\
very well trained & 119.78 & 40.46 & 94.36 \\
n/a & 16.68 & 5.64 & 100.00 \\
- Total $\mid$ & 296 & 100.00 &
\end{tabular}

(5) Doors and windows

-- single family dwellings

Freq. Percent Cum.

\begin{tabular}{|c|c|c|c|}
\hline not at all well trained & 5.63 & 1.85 & 1.85 \\
\hline not well trained & 12.60 & 4.13 & 5.9 \\
\hline moderately well trained & 62.84 & 20.60 & 26.5 \\
\hline well trained & 100.99 & 33.11 & 59.6 \\
\hline very well trained & 101.75 & 33.36 & 93.05 \\
\hline $\mathrm{n} / \mathrm{a}$ & 21.19 & 6.95 & 100.00 \\
\hline Total & 305 & 100.00 & \\
\hline
\end{tabular}


-- multifamily dwellings

\begin{tabular}{r|ccc} 
& Freq. & Percent & Cum. \\
not at all well trained & 17.85 & 6.87 & 6.87 \\
not well trained & 20.60 & 7.92 & 14.79 \\
moderately well trained & 34.34 & 13.21 & 28.00 \\
well trained & 58.00 & 22.31 & 50.30 \\
very well trained $\mid$ & 45.05 & 17.33 & 67.63 \\
n/a & 84.16 & 32.37 & 100.00 \\
- Total $^{\mid}$ & 260 & 100.00 &
\end{tabular}

-- mobile homes

\begin{tabular}{rrrr} 
& Freq. & Percent & \multicolumn{1}{c}{ Cum. } \\
not at all well trained | & 7.12 & 2.46 & 2.46 \\
not well trained | & 15.39 & 5.32 & 7.79 \\
moderately well trained $\mid$ & 43.54 & 15.07 & 22.86 \\
well trained $\mid$ & 98.63 & 34.13 & 56.98 \\
very well trained $\mid$ & 95.96 & 33.21 & 90.19 \\
n/a $\mid$ & 28.35 & 9.81 & 100.00 \\
- Total $\mid$ & 289 & 100.00 &
\end{tabular}

(6) Hot water heating

-- single family dwellings

Freq. Percent Cum.

$\begin{array}{rrrr}\text { not at all well trained | } & 1.57 & 0.51 & 0.51 \\ \text { not well trained | } & 28.06 & 9.17 & 9.68 \\ \text { moderately well trained | } & 68.79 & 22.48 & 32.16 \\ \text { well trained } \mid & 97.81 & 31.96 & 64.13 \\ \text { very well trained | } & 96.49 & 31.53 & 95.66 \\ \text { n/a | } & 13.28 & 4.34 & 100.00 \\ - \text { Total } \mid & 306 & 100.00 & \end{array}$

-- multifamily dwellings

Freq. Percent Cum.

\begin{tabular}{|c|c|c|c|}
\hline not at all well trained | & 19.63 & 7.49 & 7.49 \\
\hline not well trained & 23.80 & 9.09 & 16.58 \\
\hline moderately well trained & 43.60 & 16.64 & 33.22 \\
\hline well trained & 50.23 & 19.17 & 52.39 \\
\hline very well trained & 42.21 & 16.11 & 68.50 \\
\hline $\mathrm{n} / \mathrm{a}$ & 82.53 & 31.50 & 100.00 \\
\hline Total & 262 & 100.00 & \\
\hline
\end{tabular}


-- mobile homes

Freq. Percent Cum.

$\begin{array}{rrrr}\text { not at all well trained | } & 5.22 & 1.82 & 1.82 \\ \text { not well trained } \mid & 28.54 & 9.98 & 11.80 \\ \text { moderately well trained } \mid & 56.04 & 19.60 & 31.40 \\ \text { well trained } \mid & 82.75 & 28.93 & 60.33 \\ \text { very well trained } \mid & 90.18 & 31.53 & 91.87 \\ \text { n/a | } & 23.27 & 8.13 & 100.00 \\ - \text { Total } \mid & 286 & 100.00\end{array}$

(7) Baseloads (e.g., lighting, refrigerators)

-- single family dwellings

Freq. Percent Cum.

\begin{tabular}{r|rrr} 
not at all well trained | & 6.58 & 2.20 & 2.20 \\
not well trained | & 22.00 & 7.36 & 9.56 \\
moderately well trained | & 58.49 & 19.56 & 29.12 \\
well trained | & 96.43 & 32.25 & 61.37 \\
very well trained | & 96.27 & 32.20 & 93.57 \\
n/a | & 19.23 & 6.43 & 100.00 \\
\hline Total | & 299 & 100.00 &
\end{tabular}

-- multifamily dwellings

Freq. Percent Cum.

\begin{tabular}{r|rrr} 
not at all well trained | & 19.48 & 7.64 & 7.64 \\
not well trained | & 17.08 & 6.70 & 14.34 \\
moderately well trained | & 36.84 & 14.45 & 28.79 \\
well trained | & 51.63 & 20.25 & 49.03 \\
very well trained | & 47.54 & 18.64 & 67.68 \\
n/a $\mid$ & 82.43 & 32.32 & 100.00 \\
- Total | & 255 & 100.00 &
\end{tabular}

-- mobile homes

Freq. Percent Cum.

\begin{tabular}{r|rrr} 
not at all well trained & 9.23 & 3.26 & 3.26 \\
not well trained & 21.26 & 7.51 & 10.77 \\
moderately well trained $\mid$ & 44.94 & 15.88 & 26.65 \\
well trained $\mid$ & 89.58 & 31.65 & 58.31 \\
very well trained $\mid$ & 92.89 & 32.82 & 91.13 \\
n/a $\mid$ & 26.00 & 8.87 & 100.00 \\
- Total $\mid$ & 283 & 100.00 &
\end{tabular}


9a. For those staff working in your agency who needed to have knowledge about the following list of administrative-related topics, how well trained were they in each area in Program Year 2008? Please use the following scale: 1 - not at all well trained; 2 - not well trained; 3 - moderately well trained; 4 -well trained; 5 - very well trained; 6 - not applicable

Circle best answer.

(1) Management

$\begin{array}{rrrr} & \text { Freq. } & \text { Percent } & \text { Cum. } \\ \text { not at all well trained | } & .74 & 0.23 & 0.23 \\ \text { not well trained | } & 19.68 & 6.23 & 6.46 \\ \text { moderately well trained | } & 66.86 & 21.16 & 27.62 \\ \text { well trained } \mid & 111.00 & 35.13 & 62.75 \\ \text { very well trained | } & 106.00 & 33.26 & 96.01 \\ \text { n/a | } & 12.62 & 3.99 & 100.00 \\ \text { Total | } & 316 & 100.00 & \end{array}$

(2) Client education

\begin{tabular}{r|rrr} 
& Freq. & Percent & Cum. \\
not at all well trained | & 3.38 & 1.10 & 1.10 \\
not well trained | & 20.56 & 6.70 & 7.80 \\
moderately well trained | & 63.47 & 20.67 & 28.47 \\
well trained | & 119.81 & 39.03 & 67.50 \\
very well trained | & 87.41 & 28.47 & 95.97 \\
n/a | & 12.36 & 4.03 & 100.00 \\
-------------- &
\end{tabular}

(3) Auditing/estimating

-- single family dwellings

\begin{tabular}{|c|c|c|c|}
\hline \multicolumn{3}{|c|}{ Freq. } & Cum. \\
\hline not well trained & 5.42 & 1.72 & 1.72 \\
\hline moderately well trained | & 59.27 & 18.88 & 20.60 \\
\hline well trained & 119.55 & 38.07 & 58.67 \\
\hline very well trained & 120.99 & 38.53 & 97.21 \\
\hline $\mathrm{n} / \mathrm{a}$ & 8.78 & 2.79 & 100.00 \\
\hline Total & 314 & 100.00 & \\
\hline
\end{tabular}


-- multifamily dwellings

Freq. Percent Cum.

\begin{tabular}{|c|c|c|c|}
\hline not at all well trained & 16.09 & 6.02 & 6.02 \\
\hline not well trained & 21.12 & 7.91 & 13.94 \\
\hline moderately well trained & 41.29 & 15.46 & 29.40 \\
\hline well trained & 68.64 & 25.71 & 55.11 \\
\hline very well trained & 42.55 & 15.94 & 71.04 \\
\hline $\mathrm{n} / \mathrm{a}$ & 77.31 & 28.96 & 100.00 \\
\hline Total & 267 & 100.00 & \\
\hline
\end{tabular}

-- mobile homes

$\begin{array}{rrrr} & \text { Freq. } & \text { Percent } & \text { Cum. } \\ \text { not at all well trained | } & 2.87 & 0.97 & 0.97 \\ \text { not well trained | } & 10.77 & 3.63 & 4.59 \\ \text { moderately well trained | } & 46.06 & 15.51 & 20.10 \\ \text { well trained | } & 114.54 & 38.57 & 58.66 \\ \text { very well trained | } & 102.26 & 34.43 & 93.10 \\ \text { n/a | } & 20.50 & 6.90 & 100.00 \\ \text { Total | } & 297 & 100.00 & \end{array}$

(4) Monitoring/quality control

$\begin{array}{rrrr} & \text { Freq. } & \text { Percent } & \text { Cum. } \\ \text { not well trained | } & 11.34 & 3.83 & 3.83 \\ \text { moderately well trained | } & 71.16 & 24.04 & 27.87 \\ \text { well trained | } & 116.94 & 39.51 & 67.38 \\ \text { very well trained | } & 86.60 & 29.26 & 96.63 \\ \text { n/a | } & 9.96 & 3.37 & 100.00 \\ ------------- & \end{array}$

(5) Financial topics

\begin{tabular}{r|rrr} 
& Freq. & Percent & Cum. \\
not at all well trained | & 5.65 & 1.90 & 1.90 \\
not well trained | & 17.80 & 5.99 & 7.90 \\
moderately well trained | & 64.87 & 21.84 & 29.74 \\
well trained | & 107.43 & 36.17 & 65.91 \\
very well trained $\mid$ & 77.52 & 26.10 & 92.01 \\
n/a $\mid$ & 23.76 & 7.99 & 100.00 \\
- Total | & 297 & 100.00 &
\end{tabular}


(7) Outreach and communications

\begin{tabular}{r|rrr} 
& Freq. & Percent & Cum. \\
not at all well trained | & 9.62 & 3.27 & 3.27 \\
not well trained | & 16.33 & 5.55 & 8.82 \\
moderately well trained | & 73.89 & 25.13 & 33.96 \\
well trained | & 108.45 & 36.89 & 70.84 \\
very well trained | & 63.05 & 21.45 & 92.29 \\
n/a $\mid$ & 22.66 & 7.71 & 100.00 \\
---------- &
\end{tabular}

(8) Other (please specify)

Freq. Percent Cum.

\begin{tabular}{|c|c|c|c|}
\hline not at all well trained & 2.77 & 8.66 & 8.66 \\
\hline not well trained & 1.32 & 4.13 & 12.79 \\
\hline moderately well trained & 1.12 & 3.49 & 16.28 \\
\hline well trained & 3.29 & 10.28 & 26.55 \\
\hline very well trained & 3.73 & 11.66 & 38.21 \\
\hline $\mathrm{n} / \mathrm{a}$ & 19.77 & 61.79 & 100.00 \\
\hline Total & 32 & 100.00 & \\
\hline
\end{tabular}

9b. For those staff working in your agency who needed to have knowledge about the following list of health and safety topics, how well trained were they in each area in Program Year 2008? Please use the following scale: 1 - not at all well trained; 2 - not well trained; 3 - moderately well trained; 4 -well trained; 5 - very well trained; 6 - not applicable.

Circle best answer.

(1) Fire safety

Freq. Percent Cum.

\begin{tabular}{r|ccr} 
not at all well trained | & 12.47 & 4.30 & 4.30 \\
not well trained & 34.06 & 11.74 & 16.04 \\
moderately well trained | & 78.91 & 27.21 & 43.26 \\
well trained | & 79.95 & 27.57 & 70.83 \\
very well trained & 53.91 & 18.59 & 89.42 \\
n/a & 30.69 & 10.58 & 100.00 \\
- Total | & 290 & 100.00 &
\end{tabular}


(2) Indoor air quality

\begin{tabular}{rrrr} 
& Freq. & Percent & \multicolumn{1}{c}{ Cum. } \\
not at all well trained | & 3.52 & 1.12 & 1.12 \\
not well trained | & 20.45 & 6.53 & 7.66 \\
moderately well trained | & 67.42 & 21.54 & 29.20 \\
well trained | & 111.71 & 35.69 & 64.89 \\
very well trained | & 101.44 & 32.41 & 97.30 \\
n/a | & 8.46 & 2.70 & 100.00 \\
- Total | & 313 & 100.00 &
\end{tabular}

(3) Measures to increase security of housing unit

Freq. Percent Cum.

\begin{tabular}{r|rrr} 
not at all well trained | & 27.50 & 9.89 & 9.89 \\
not well trained | & 65.85 & 23.69 & 33.58 \\
moderately well trained | & 37.96 & 13.65 & 47.23 \\
well trained | & 38.09 & 13.70 & 60.93 \\
very well trained $\mid$ & 26.62 & 9.57 & 70.50 \\
n/a & 82.00 & 29.50 & 100.00 \\
- Total & 278 & 100.00 &
\end{tabular}

(4) Measures to reduce common household hazards

Freq. Percent Cum.

\begin{tabular}{r|rrr} 
not at all well trained | & 12.98 & 4.52 & 4.52 \\
not well trained | & 48.02 & 16.73 & 21.26 \\
moderately well trained | & 56.24 & 19.59 & 40.85 \\
well trained | & 86.90 & 30.28 & 71.13 \\
very well trained $\mid$ & 41.97 & 14.62 & 85.76 \\
n/a $\mid$ & 40.88 & 14.24 & 100.00 \\
- Total | & 287 & 100.00 &
\end{tabular}

(5) Mold and mildew

Freq. Percent Cum.

\begin{tabular}{|c|c|c|c|}
\hline not at all well trained & 11.28 & 3.75 & 3.75 \\
\hline not well trained & 37.41 & 12.43 & 16.18 \\
\hline moderately well trained & 95.26 & 31.65 & 47.82 \\
\hline well trained & 85.48 & 28.40 & 76.22 \\
\hline very well trained & 52.93 & 17.58 & 93.81 \\
\hline $\mathrm{n} / \mathrm{a} \mid$ & 18.64 & 6.19 & 100.00 \\
\hline Total & 301 & 100.00 & \\
\hline
\end{tabular}


(6) Lead

\begin{tabular}{r|rrr} 
& Freq. & Percent & Cum. \\
not well trained & 26.80 & 8.59 & 8.59 \\
moderately well trained & 73.86 & 23.67 & 32.26 \\
well trained & 113.68 & 36.43 & 68.70 \\
very well trained $\mid$ & 88.71 & 28.43 & 97.13 \\
n/a & 8.96 & 2.87 & 100.00 \\
------------ &
\end{tabular}

(7) Asbestos

\begin{tabular}{|c|c|c|c|}
\hline & eq. & cent & Cum. \\
\hline not at all well trained & 17.06 & 5.88 & 5.88 \\
\hline not well trained & 47.53 & 16.39 & 22.27 \\
\hline moderately well trained & 86.58 & 29.5 & 52.13 \\
\hline well trained & 66.30 & 22.8 & 74.99 \\
\hline very well trained & 42.91 & 14.80 & 89.78 \\
\hline $\mathrm{n} / \mathrm{a}$ & 29.63 & 10.22 & 100.00 \\
\hline Tota & 290 & 100.00 & \\
\hline
\end{tabular}

(9) Vermiculite

\begin{tabular}{r|ccc} 
& Freq. & Percent & Cum. \\
not at all well trained & 33.07 & 12.12 & 12.12 \\
not well trained | & 59.86 & 21.93 & 34.04 \\
moderately well trained & 55.22 & 20.23 & 54.27 \\
well trained & 44.86 & 16.43 & 70.70 \\
very well trained $\mid$ & 33.61 & 12.31 & 83.01 \\
n/a $\mid$ & 46.37 & 16.99 & 100.00 \\
- Total $\mid$ & 273 & 100.00 &
\end{tabular}

(9) General crew safety

Freq. Percent Cum.

\begin{tabular}{r|rrr} 
not at all well trained | & 1.23 & 0.42 & 0.42 \\
not well trained | & 11.85 & 4.00 & 4.42 \\
moderately well trained | & 46.14 & 15.59 & 20.01 \\
well trained | & 105.45 & 35.63 & 55.63 \\
very well trained | & 103.10 & 34.83 & 90.46 \\
n/a $\mid$ & 28.22 & 9.54 & 100.00 \\
- Total & 296 & 100.00 &
\end{tabular}


(10) Other (please specify)

$\begin{array}{rrrr} & \text { Freq. } & \text { Percent } & \text { Cum. } \\ \text { not well trained } \mid & 5.85 & 27.84 & 27.84 \\ \text { moderately well trained } \mid & 3.47 & 16.51 & 44.35 \\ \text { well trained } \mid & 1.09 & 5.17 & 49.52 \\ \text { very well trained } \mid & 3.26 & 15.51 & 65.03 \\ \text { n/a } \mid & 7.34 & 34.97 & 100.00 \\ - \text { Total } \mid & 21 & 100.00 & \end{array}$

10. For those staff working in your agency who needed to have knowledge about the following list of diagnostic topics, how well trained were they in each area in Program Year 2008? Please use the following scale: 1 -not at all well trained; 2 - not well trained; 3 - moderately well trained; 4 -well trained; 5 - very well trained; 6 - not applicable. Circle best answer.

\section{Pressure diagnostics:}

- Blower door (house air leakage rate)

Freq. Percent Cum.

\begin{tabular}{|c|c|c|c|}
\hline not well trained & 8.09 & 2.54 & 2.54 \\
\hline moderately well trained | & 48.49 & 15.25 & 17.79 \\
\hline well trained & 117.06 & 36.81 & 54.60 \\
\hline very well trained & 137.07 & 43.10 & 97.71 \\
\hline $\mathrm{n} / \mathrm{a}$ & 7.29 & 2.29 & 100.00 \\
\hline Total | & 318 & 100.00 & \\
\hline
\end{tabular}

- Zonal pressure measurements

$\begin{array}{rccr} & \text { Freq. } & \text { Percent } & \text { Cum. } \\ \text { not at all well trained | } & 13.29 & 4.43 & 4.43 \\ \text { not well trained | } & 25.13 & 8.38 & 12.81 \\ \text { moderately well trained } \mid & 51.32 & 17.11 & 29.92 \\ \text { well trained | } & 94.71 & 31.57 & 61.48 \\ \text { very well trained | } & 85.51 & 28.50 & 89.99 \\ \text { n/a } \mid & 30.04 & 10.01 & 100.00 \\ ------------\end{array}$


- Room-to-room pressure measurements

Freq. Percent Cum.

\begin{tabular}{|c|c|c|c|}
\hline not at all well trained & 9.30 & 3.20 & 3.20 \\
\hline not well trained & 28.67 & 9.85 & 13.05 \\
\hline moderately well trained & 57.35 & 19.71 & 32. \\
\hline well trained & 79.06 & 27.17 & 59. \\
\hline very well trained & 82.40 & 28.32 & 88.2 \\
\hline $\mathrm{n} / \mathrm{a}$ & 34.21 & 11.76 & 100.0 \\
\hline Fotal & 291 & 100.00 & \\
\hline
\end{tabular}

- Duct pressure pan measurements

Freq. Percent Cum.

$\begin{array}{rrrr}\text { not at all well trained | } & 9.71 & 3.30 & 3.30 \\ \text { not well trained | } & 24.07 & 8.19 & 11.49 \\ \text { moderately well trained | } & 62.17 & 21.15 & 32.64 \\ \text { well trained | } & 87.61 & 29.80 & 62.44 \\ \text { very well trained | } & 82.16 & 27.94 & 90.38 \\ \text { n/a | } & 28.28 & 9.62 & 100.00 \\ - \text { Total } & 294 & 100.00 & \end{array}$

- Duct blower measurements (duct air leakage rate)

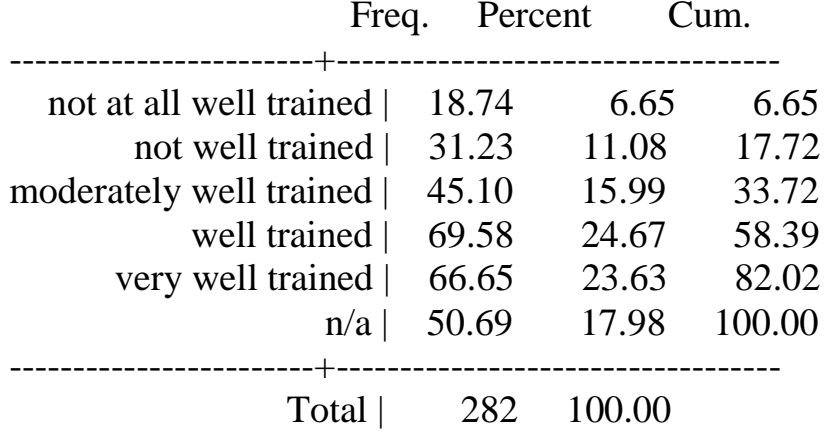




\section{Space-heating system:}

- Flue gas analysis (steady-state efficiency measurements)

Freq. Percent Cum.

\begin{tabular}{|c|c|c|c|}
\hline not at all well trained & 7.38 & 2.43 & 2.4 \\
\hline not well trained & 14.05 & 4.62 & \\
\hline moderately well trained & 47.84 & 15.74 & ש \\
\hline well trained & 100.28 & 32.99 & 55. \\
\hline very well trained & 112.81 & 37.11 & 92. \\
\hline $\mathrm{n} / \mathrm{a}$ & 21.64 & 7.12 & 100.0 \\
\hline Total & 304 & 100.00 & \\
\hline
\end{tabular}

- Heat rise measurements

$\begin{array}{rrrr} & \text { Freq. } & \text { Percent } & \text { Cum. } \\ \text { not at all well trained } \mid & 15.52 & 5.31 & 5.31 \\ \text { not well trained } \mid & 31.16 & 10.67 & 15.98 \\ \text { moderately well trained } \mid & 39.64 & 13.58 & 29.56 \\ \text { well trained } \mid & 86.95 & 29.78 & 59.34 \\ \text { very well trained } \mid & 83.88 & 28.72 & 88.06 \\ \text { n/a } \mid & 34.86 & 11.94 & 100.00 \\ \text { Total } \mid & 292 & 100.00 & \end{array}$

- $\mathrm{CO}$ measurements in flues

Freq. Percent Cum.

\begin{tabular}{|c|c|c|c|}
\hline not at all well trained & 2.70 & 0.86 & 0.86 \\
\hline not well trained & 7.14 & 2.28 & 3.14 \\
\hline moderately well trained & 55.99 & 17.89 & 21.03 \\
\hline well trained & 107.05 & 34.20 & 55.23 \\
\hline very well trained & 127.01 & 40.58 & 95.81 \\
\hline $\mathrm{n} / \mathrm{a} \mid$ & 13.10 & 4.19 & 100.00 \\
\hline Total & 313 & 100.00 & \\
\hline
\end{tabular}

- Draft/spillage (normal operation)

Freq. Percent Cum.

\begin{tabular}{r|rrr} 
not at all well trained & 8.80 & 2.87 & 2.87 \\
not well trained & 12.77 & 4.16 & 7.03 \\
moderately well trained & 47.73 & 15.55 & 22.57 \\
well trained | & 104.39 & 34.00 & 56.58 \\
very well trained $\mid$ & 115.21 & 37.53 & 94.11 \\
n/a & 18.07 & 5.89 & 100.00 \\
- Total & 307 & 100.00 &
\end{tabular}




\section{Air-conditioning system:}

- Refrigerant charge (e.g., superheat, subcooling)

Freq. Percent Cum.

\begin{tabular}{|c|c|c|c|}
\hline not at all well trained & 47.69 & 17.93 & 17.93 \\
\hline not well trained & 38.52 & 14.48 & 32.4 \\
\hline moderately well trained & 21.52 & 8.10 & 40.5 \\
\hline well trained & 18.37 & 6.91 & 47.4 \\
\hline very well trained & 15.49 & 5.82 & 53.2 \\
\hline $\mathrm{n} / \mathrm{a}$ & 124.37 & 46.75 & 100.0 \\
\hline
\end{tabular}

HVAC components and cross-cutting diagnostics:

- Air handler flow rate

\begin{tabular}{r|rrr} 
& Freq. & Percent & Cum. \\
not at all well trained & 25.58 & 9.14 & 9.14 \\
not well trained & 46.62 & 16.65 & 25.79 \\
moderately well trained & 53.77 & 19.20 & 44.99 \\
well trained & 53.39 & 19.07 & 64.06 \\
very well trained $\mid$ & 41.66 & 14.88 & 78.94 \\
n/a & 58.98 & 21.06 & 100.00 \\
- Total $\mid$ & 280 & 100.00 &
\end{tabular}

- Thermostat anticipator current

Freq. Percent Cum.

\begin{tabular}{|c|c|c|c|}
\hline not at all well trained & 20.72 & 7.35 & 7.35 \\
\hline not well trained & 37.87 & 13.43 & 20.78 \\
\hline moderately well trained & 49.05 & 17.39 & 38.17 \\
\hline well trained & 57.68 & 20.45 & 58.62 \\
\hline very well trained & 53.73 & 19.05 & 77.68 \\
\hline $\mathrm{n} / \mathrm{a}$ & 62.95 & 22.32 & 100.00 \\
\hline Total & 282 & 100.00 & \\
\hline
\end{tabular}

- Worst case draft/spillage (CAZ)

Freq. Percent Cum.

\begin{tabular}{r|rrr} 
not at all well trained & 11.8 & 4.02 & 4.02 \\
not well trained & 18.58 & 6.30 & 10.32 \\
moderately well trained $\mid$ & 46.70 & 15.83 & 26.15 \\
well trained & 82.64 & 28.01 & 54.16 \\
very well trained & 104.94 & 35.57 & 89.73 \\
n/a & 30.29 & 10.27 & 100.00 \\
- Total $^{-}$ & 29 & 100.00 &
\end{tabular}




\section{Hot-water (water-heating) system:}

- Flue gas analysis (steady-state efficiency measurements)

\begin{tabular}{rrrr} 
& Freq. & Percent & Cum. \\
\hline not at all well trained | & 8.93 & 2.95 & 2.95 \\
not well trained | & 14.55 & 4.80 & 7.75 \\
moderately well trained $\mid$ & 40.16 & 13.25 & 21.00 \\
well trained | & 99.99 & 33.00 & 54.00 \\
very well trained | & 108.08 & 35.67 & 89.67 \\
n/a | & 31.30 & 10.33 & 100.00 \\
- Total $\left.\right|^{-} 303$ & 100.00 &
\end{tabular}

- $\mathrm{CO}$ measurements in flues

$\begin{array}{cccc} & \text { Freq. } & \text { Percent } & \text { Cum. } \\ \text { not at all well trained | } 4.97 & 1.61 & 1.61 \\ \text { not well trained | } 11.71 & 3.79 & 5.40 \\ \text { moderately well trained | } 37.20 & 12.04 & 17.44 \\ \text { well trained | } 113.23 & 36.64 & 54.08 \\ \text { very well trained | } & 127.37 & 41.22 & 95.30 \\ \text { n/a | } 14.52 & 4.70 & 100.00 & \\ \text { Total | } 309 & 100.00\end{array}$

- Draft/spillage (normal operation)

\begin{tabular}{rrrr} 
& Freq. & Percent & \multicolumn{1}{c}{ Cum. } \\
not at all well trained | & 5.50 & 1.80 & 1.80 \\
not well trained | & 15.14 & 4.95 & 6.74 \\
moderately well trained & 53.16 & 17.37 & 24.12 \\
well trained $\mid$ & 102.19 & 33.39 & 57.51 \\
very well trained $\mid$ & 116.69 & 38.13 & 95.65 \\
n/a $\mid$ & 13.32 & 4.35 & 100.00 \\
- Total | & 306 & 100.00 &
\end{tabular}

- Water flow rates (showerheads and faucets)

Freq. Percent Cum.

\begin{tabular}{r|rrr} 
not at all well trained | & 15.89 & 5.54 & 5.54 \\
not well trained | & 33.63 & 11.72 & 17.26 \\
moderately well trained | & 49.03 & 17.08 & 34.34 \\
well trained | & 79.20 & 27.60 & 61.93 \\
very well trained | & 56.29 & 19.61 & 81.55 \\
n/a & 52.96 & 18.45 & 100.00 \\
- Total | & 287 & 100.00 &
\end{tabular}




\section{Other CO measurements:}

- $\quad \mathrm{CO}$ measurements in equipment rooms

Freq. Percent Cum.

\begin{tabular}{r|rrr} 
not at all well trained | & 5.13 & 1.74 & 1.74 \\
not well trained | & 7.54 & 2.55 & 4.29 \\
moderately well trained | & 52.18 & 17.69 & 21.98 \\
well trained | & 97.98 & 33.21 & 55.20 \\
very well trained | & 102.90 & 34.88 & 90.08 \\
n/a $\mid$ & 29.28 & 9.92 & 100.00 \\
- Total $\mid$ & 295 & 100.00 &
\end{tabular}

- Cooking stove

\begin{tabular}{r|rrr} 
& Freq. & Percent & Cum. \\
not at all well trained & 1.11 & 0.36 & 0.36 \\
not well trained | & 10.29 & 3.35 & 3.71 \\
moderately well trained $\mid$ & 53.08 & 17.29 & 21.01 \\
well trained $\mid$ & 113.12 & 36.85 & 57.85 \\
very well trained $\mid$ & 112.55 & 36.66 & 94.52 \\
n/a $\mid$ & 16.84 & 5.48 & 100.00 \\
- Total $\mid$ & 307 & 100.00 &
\end{tabular}

- $\quad \mathrm{CO}$ measurements in living areas

$\begin{array}{rrrr} & \text { Freq. } & \text { Percent } & \text { Cum. } \\ \text { not at all well trained | } & 1.21 & 0.41 & 0.41 \\ \text { not well trained | } & 8.74 & 2.95 & 3.36 \\ \text { moderately well trained | } & 44.28 & 14.96 & 18.32 \\ \text { well trained | } & 104.99 & 35.47 & 53.79 \\ \text { very well trained | } & 114.34 & 38.63 & 92.42 \\ \text { n/a } \mid & 22.43 & 7.58 & 100.00 \\ - \text { Total | } & 296 & 100.00 & \end{array}$

Other diagnostics and inspections:

- Refrigerator energy use

Freq. Percent Cum.

\begin{tabular}{r|rrr} 
not at all well trained | & 8.26 & 2.80 & 2.80 \\
not well trained | & 17.01 & 5.77 & 8.56 \\
moderately well trained | & 60.69 & 20.57 & 29.14 \\
well trained | & 92.00 & 31.19 & 60.32 \\
very well trained $\mid$ & 83.00 & 28.13 & 88.46 \\
n/a $\mid$ & 34.05 & 11.54 & 100.00 \\
- Total | & 295 & 100.00 &
\end{tabular}


- Exhaust fan air flow rate measurement

Freq. Percent Cum.

\begin{tabular}{|c|c|c|c|}
\hline not at all well trained & 29.07 & 10.65 & 10.65 \\
\hline not well trained & 44.51 & 16.30 & 26.5 \\
\hline moderately well trained & 44.19 & 16.19 & 43.1 \\
\hline well trained & 66.51 & 24.36 & 67.5 \\
\hline very well trained & 45.13 & 16.53 & 84. \\
\hline $\mathrm{n} / \mathrm{a}$ & 43.60 & 15.97 & 100.0 \\
\hline Total & 273 & 100.00 & \\
\hline
\end{tabular}

- Infrared scanning (camera)

Freq. Percent Cum.

\begin{tabular}{r|rrr} 
not at all well trained | & 27.32 & 9.29 & 9.29 \\
not well trained | & 34.99 & 11.90 & 21.19 \\
moderately well trained $\mid$ & 76.19 & 25.91 & 47.11 \\
well trained $\mid$ & 61.98 & 21.08 & 68.19 \\
very well trained $\mid$ & 51.49 & 17.51 & 85.70 \\
n/a $\mid$ & 42.04 & 14.30 & 100.00 \\
- Total $\mid$ & 294 & 100.00 &
\end{tabular}

- Radon testing

$\begin{array}{rrrr} & \text { Freq. } & \text { Percent } & \text { Cum. } \\ \text { not at all well trained } & 57.76 & 22.65 & 22.65 \\ \text { not well trained } & 30.60 & 12.00 & 34.65 \\ \text { moderately well trained } \mid & 13.87 & 5.44 & 40.09 \\ \text { well trained } \mid & 16.10 & 6.31 & 46.40 \\ \text { very well trained } \mid & 12.55 & 4.92 & 51.32 \\ \text { n/a } & 124.13 & 48.68 & 100.00 \\ - \text { Total } \mid & 255 & 100.00 & \end{array}$

- Lead testing

\begin{tabular}{r|rrc} 
& Freq. & Percent & Cum. \\
not at all well trained | & 26.00 & 9.49 & 9.49 \\
not well trained | & 31.00 & 11.31 & 20.80 \\
moderately well trained $\mid$ & 35.85 & 13.08 & 33.89 \\
well trained | & 48.77 & 17.80 & 51.69 \\
very well trained & 60.35 & 22.02 & 73.71 \\
n/a $\mid$ & 72.03 & 26.29 & 100.00 \\
---------------------
\end{tabular}


- Mold and mildew testing

\begin{tabular}{|c|c|c|c|}
\hline & Freq. & Percent & Cum. \\
\hline not at all well trained & 42.90 & 16.13 & 16.13 \\
\hline not well trained & 31.97 & 12.02 & 28.15 \\
\hline moderately well trained & 36.47 & 13.71 & 41.86 \\
\hline well trained & 37.01 & 13.91 & 55.77 \\
\hline very well trained & 24.70 & 9.29 & 65.06 \\
\hline $\mathrm{n} / \mathrm{a}$ & 92.95 & 34.94 & 100.00 \\
\hline Total & 266 & 100.00 & \\
\hline
\end{tabular}

- $\quad$ Moisture content testing

\begin{tabular}{r|rrr} 
& Freq. & Percent & Cum. \\
not at all well trained | & 42.80 & 16.27 & 16.27 \\
not well trained | & 37.52 & 14.27 & 30.54 \\
moderately well trained | & 38.80 & 14.75 & 45.29 \\
well trained | & 40.57 & 15.43 & 60.72 \\
very well trained | & 21.60 & 8.21 & 68.93 \\
n/a $\mid$ & 81.71 & 31.07 & 100.00 \\
- Total $^{-}$ & 263 & 100.00 &
\end{tabular}

- Other (please specify)

$\begin{array}{rrrr}n & \text { Freq. } & \text { Percent } & \text { Cum. } \\ \text { not at all well trained | } & 3.61 & 22.54 & 22.54 \\ \text { well trained } \mid & 1.02 & 6.35 & 28.88 \\ \text { very well trained } \mid & 1.04 & 6.51 & 35.39 \\ \text { n/a | } & 10.34 & 64.61 & 100.00 \\ - \text { Total } \mid & 16 & 100.00 & \end{array}$

11. Overall, how well trained were your agency's weatherization crews in Program Year 2008? Check best answer.

Freq. Percent Cum.

\begin{tabular}{|c|c|c|c|}
\hline very poorly trained & 1.04 & 0.33 & 0.33 \\
\hline poorly trained & 6.21 & 1.97 & 2.30 \\
\hline neither well nor poorly trained & 78.71 & 24.99 & 27.29 \\
\hline well trained & 134.52 & 42.70 & 70.00 \\
\hline very well trained & 94.52 & 30.00 & 100.00 \\
\hline Tota & 315 & 100.00 & \\
\hline
\end{tabular}


11. What were the barriers that prevented your crews from receiving all the training they need? Check all that apply.

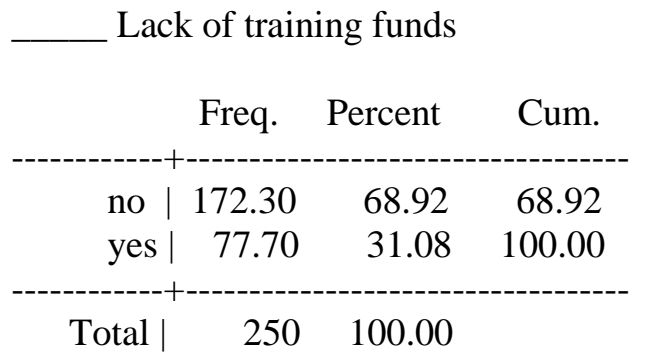

Cannot take crews out of the field long enough for training

\begin{tabular}{|c|c|c|c|}
\hline & Freq. & Percent & Cum. \\
\hline no | & 104.99 & 42.00 & 42.00 \\
\hline yes & 145.01 & 58.00 & 100.00 \\
\hline Total | & 250 & 100.00 & \\
\hline
\end{tabular}

Training not available at the right times

\begin{tabular}{|c|c|c|c|}
\hline & Freq. & Percent & Cum. \\
\hline no | & 100.58 & 40.23 & 40.23 \\
\hline yes | & 149.42 & 59.77 & 100.00 \\
\hline Total | & 250 & 100.00 & \\
\hline
\end{tabular}

Training not available at the right places

\begin{tabular}{|c|c|c|c|}
\hline & Freq. & Percent & Cum. \\
\hline no | & 173.51 & 69.40 & 69.40 \\
\hline yes & 76.49 & 30.60 & 100.00 \\
\hline Total & 250 & 100.00 & \\
\hline
\end{tabular}

Available training is poor in quality

\begin{tabular}{|c|c|c|c|}
\hline Freq. & Percent & \multicolumn{2}{|c|}{ Cum. } \\
\hline no & 230.42 & 92.17 & 92.17 \\
\hline yes & 19.58 & 7.83 & 100.00 \\
\hline Total & 250 & 100.00 & \\
\hline
\end{tabular}




\section{SECTION F: INSPECTION}

1. Which of the following types of post-weatherization quality control inspection did your agency perform on your weatherized dwelling units in Program Year 2008?

Check all that apply.

- Visual inspection of installed measures

\begin{tabular}{|c|c|c|c|}
\hline & Freq. & Percent & Cum. \\
\hline no & 3.14 & 0.90 & 0.90 \\
\hline yes | & 347.86 & 99.10 & 100.00 \\
\hline Total | & 351 & 100.00 & \\
\hline
\end{tabular}

- Verification of insulation depths/quantities

Freq. Percent Cum.

\begin{tabular}{|c|c|c|c|}
\hline no & 28.94 & 8.24 & 8.24 \\
\hline yes & 322.06 & 91.76 & 100.00 \\
\hline Total & 351 & 100.00 & \\
\hline
\end{tabular}

- Verification of operation of measures installed

\begin{tabular}{|c|c|c|c|}
\hline & Freq. & Percent & Cum. \\
\hline no | & 37.11 & 10.57 & 10.57 \\
\hline yes $\mid$ & 313.89 & 89.43 & 100.00 \\
\hline Total | & 351 & 100.00 & \\
\hline
\end{tabular}

- Assessment of quality of measures installed Freq. Percent Cum.

$\begin{array}{crr}\text { no | } 40.97 & 11.67 & 11.67 \\ \text { yes | } 310.03 & 88.33 & 100.00 \\ ---------- \\ \text { Total | } 351 & 100.00\end{array}$

- Identification of needed measures that were not installed

\begin{tabular}{|c|c|c|c|}
\hline & Freq. & Percent & Cum. \\
\hline no & 94.31 & 26.87 & 26.87 \\
\hline yes & 256.69 & 73.13 & 100.00 \\
\hline Total | & 351 & 100.00 & \\
\hline
\end{tabular}


- Blower door test

\begin{tabular}{|c|c|c|c|}
\hline & Freq. & Percent & Cum. \\
\hline no & 19.22 & 5.48 & 5.48 \\
\hline yes & 331.78 & 94.52 & 100.00 \\
\hline Total & 351 & 100.00 & \\
\hline
\end{tabular}

- Heating system efficiency test (flue gas analysis)

\begin{tabular}{|c|c|c|c|}
\hline & Freq. & Percent & Cum. \\
\hline no & 75.01 & 21.37 & 21.37 \\
\hline yes & 275.99 & 78.63 & 100.00 \\
\hline Total & 351 & 100.00 & \\
\hline
\end{tabular}

- Draft/spillage tests of heating systems Freq. Percent Cum.

$\begin{array}{crr}\text { no | } 72.38 & 20.62 & 20.62 \\ \text { yes | } 278.62 & 79.38 & 100.00 \\ ---------------- \\ \text { Total | } 351 & 100.00\end{array}$

- Carbon monoxide (CO) monitoring

\begin{tabular}{|c|c|c|c|}
\hline & Freq. & Percent & Cum. \\
\hline no & 38.16 & 10.87 & 10.87 \\
\hline yes | & 312.84 & 89.13 & 100.00 \\
\hline Total & 351 & 100.00 & \\
\hline
\end{tabular}

- Infrared scanning

\begin{tabular}{|c|c|c|c|}
\hline & Freq. & Percent & Cum. \\
\hline no & 204.00 & 58.12 & 58.12 \\
\hline yes & 147.00 & 41.88 & 100.00 \\
\hline otal & 351 & 100.00 & \\
\hline
\end{tabular}

- Identification of unresolved health and safety issues

\begin{tabular}{|c|c|c|c|}
\hline & Freq. & Percent & Cum. \\
\hline no & 111.69 & 31.82 & 31.82 \\
\hline yes & 239.31 & 68.18 & 100.00 \\
\hline Total & 351 & 100.00 & \\
\hline
\end{tabular}


- Discussion with occupants

\begin{tabular}{|c|c|c|c|}
\hline & Freq. & Percent & Cum. \\
\hline no & 39.27 & 11.19 & 11.19 \\
\hline yes & 311.73 & 88.81 & 100.00 \\
\hline Total & 351 & 100.00 & \\
\hline
\end{tabular}

- Other (please specify)

\begin{tabular}{|c|c|c|c|}
\hline & Freq. & Percent & Cum. \\
\hline no & 331.94 & 94.57 & 94.57 \\
\hline yes & 19.07 & 5.43 & 100.00 \\
\hline Total & 351 & 100.00 & \\
\hline
\end{tabular}

2. Which of the following post-weatherization quality and control inspection topics listed below were agency staff first trained on in Program Year 2008 and in the two years prior to Program Year 2008? If your agency does not use a particular approach, leave that item blank. Check all that apply.

- Visual inspection of installed measures

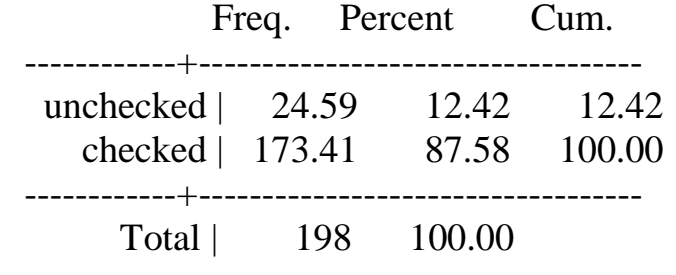

- Verification of insulation depths/quantities

\begin{tabular}{|c|c|c|c|}
\hline & Freq. $\quad \mathrm{P}$ & cent & Cum. \\
\hline unchecked & 29.95 & 15.12 & 15.12 \\
\hline checked & 168.05 & 84.88 & 100.00 \\
\hline Total | & 198 & 100.00 & \\
\hline
\end{tabular}

- Verification of operation of measures installed

\begin{tabular}{rrr} 
Freq. & Percent & \multicolumn{1}{c}{ Cum. } \\
\hline unchecked | 38.89 & 19.64 & 19.64 \\
checked | 159.12 & 80.36 & 100.00 \\
----------- \\
Total | 198 & 100.00 &
\end{tabular}


- Assessment of quality of measures installed

\begin{tabular}{|c|c|c|c|}
\hline & Freq. & Percent & Cum. \\
\hline unchecked & 38.90 & 19.64 & 19.64 \\
\hline checked & 159.10 & 80.36 & 100.00 \\
\hline Toto & 198 & 100.00 & \\
\hline
\end{tabular}

- Identification of needed measures that were not installed

\begin{tabular}{|c|c|c|c|}
\hline & Freq. & Percent & Cum. \\
\hline unchecked | & 60.92 & 30.77 & 30.77 \\
\hline checked & 137.08 & 69.23 & 100.00 \\
\hline Fotat & 198 & 100.00 & \\
\hline
\end{tabular}

- Blower door test

\begin{tabular}{|c|c|c|c|}
\hline & Freq. & Percent & Cum. \\
\hline unchecked & 21.04 & 10.62 & 10.62 \\
\hline checked & 176.96 & 89.38 & 100.00 \\
\hline Total & 198 & 100.00 & \\
\hline
\end{tabular}

- Heating system efficiecy

\begin{tabular}{|c|c|c|c|}
\hline & Freq. & Percent & Cum. \\
\hline unchecked & 54.57 & 27.56 & 27.56 \\
\hline checked & 143.43 & 72.44 & 100.00 \\
\hline 10 tat & 198 & 100.00 & \\
\hline
\end{tabular}

- Draft/spillage tests of heating systems

\begin{tabular}{|c|c|c|c|}
\hline & Freq. & Percent & Cum. \\
\hline unchecked & 58.89 & 29.74 & 29.74 \\
\hline checked & 139.11 & 70.26 & 100.00 \\
\hline Total & 198 & 100.00 & \\
\hline
\end{tabular}

- Other diagnostic tests

- Identification of unresolved health and safety issues

- Discussion with occupants

- Other

\begin{tabular}{|c|c|c|c|}
\hline & req. $\quad \mathrm{P}$ & cent & Cum. \\
\hline unchecked & 191.14 & 96.53 & 96.53 \\
\hline checked & 6.86 & 3.47 & 100.00 \\
\hline Total | & 198 & 100.00 & \\
\hline
\end{tabular}


3. For Program Year 2008 please rate key aspects (cost, training needed, time needed and effectiveness) of the following types of post-weatherization quality control inspection procedures.

Please use the following scale: 1 - very low; 2 -low; 3 - medium; 4 - high; 5 - very high.

For example, if you view visual inspection of installed measures as requiring a low level of training, enter a rating of 2 in the Training Needed column. If you view visual inspection of installed measures as highly effective, enter a rating of 4 in the Effectiveness column.

\begin{tabular}{|c|c|c|c|c|}
\hline & Cost & $\begin{array}{l}\text { Training } \\
\text { Needed }\end{array}$ & $\begin{array}{c}\text { Time } \\
\text { Needed }\end{array}$ & Effectiveness \\
\hline Visual inspection of installed measures & $\begin{array}{l}\text { obs: } 264 \\
\text { very low: } 70 \\
\text { low: } 102 \\
\text { medium: } 73 \\
\text { high: } 14 \\
\text { very high: } 4\end{array}$ & $\begin{array}{l}\text { obs: } 279 \\
\text { very low: } 20 \\
\text { low: } 46 \\
\text { medium: } 97 \\
\text { high: } 73 \\
\text { very high: } 42\end{array}$ & $\begin{array}{l}\text { obs: } 272 \\
\text { very low: } 21 \\
\text { low: } 65 \\
\text { medium: } 117 \\
\text { high: } 54 \\
\text { very high: } 14\end{array}$ & $\begin{array}{l}\text { obs: } 299 \\
\text { very low: } 0 \\
\text { low: } 8 \\
\text { medium: } 37 \\
\text { high: } 127 \\
\text { very high: } 126\end{array}$ \\
\hline Verification of insulation depths/quantities & $\begin{array}{l}\text { obs: } 260 \\
\text { very low: } 79 \\
\text { low: } 120 \\
\text { medium: } 43 \\
\text { high: } 15 \\
\text { very high: } 3\end{array}$ & $\begin{array}{l}\text { obs: } 275 \\
\text { very low: } 36 \\
\text { low: } 88 \\
\text { medium: } 79 \\
\text { high: } 53 \\
\text { very high: } 18\end{array}$ & $\begin{array}{l}\text { obs: } 267 \\
\text { very low: } 39 \\
\text { low: } 78 \\
\text { medium: } 107 \\
\text { high: } 31 \\
\text { very high: } 12\end{array}$ & $\begin{array}{l}\text { obs: } 295 \\
\text { very low: } 1 \\
\text { low: } 12 \\
\text { medium: } 30 \\
\text { high: } 131 \\
\text { very high: } 121\end{array}$ \\
\hline $\begin{array}{l}\text { Verification of operation of measures } \\
\text { installed }\end{array}$ & $\begin{array}{l}\text { obs: } 258 \\
\text { very low: } 66 \\
\text { low: } 103 \\
\text { medium: } 69 \\
\text { high: } 16 \\
\text { very high: } 3\end{array}$ & $\begin{array}{l}\text { obs: } 272 \\
\text { very low: } 24 \\
\text { low: } 59 \\
\text { medium: } 87 \\
\text { high: } 67 \\
\text { very high: } 34\end{array}$ & $\begin{array}{l}\text { obs: } 263 \\
\text { very low: } 17 \\
\text { low: } 73 \\
\text { medium: } 112 \\
\text { high: } 45 \\
\text { very high: } 15\end{array}$ & $\begin{array}{l}\text { obs: } 293 \\
\text { very low: } 1 \\
\text { low: } 7 \\
\text { medium: } 47 \\
\text { high: } 118 \\
\text { very high: } 120\end{array}$ \\
\hline Assessment of quality of measures installed & $\begin{array}{l}\text { obs: } 260 \\
\text { very low: } 66 \\
\text { low: } 108 \\
\text { medium: } 64 \\
\text { high: } 16 \\
\text { very high: } 5\end{array}$ & $\begin{array}{l}\text { obs: } 274 \\
\text { very low: } 21 \\
\text { low: } 47 \\
\text { medium: } 87 \\
\text { high: } 76 \\
\text { very high: } 42\end{array}$ & $\begin{array}{l}\text { obs: } 265 \\
\text { very low: } 13 \\
\text { low: } 61 \\
\text { medium: } 123 \\
\text { high: } 49 \\
\text { very high: } 18\end{array}$ & $\begin{array}{l}\text { obs: } 292 \\
\text { very low: } 1 \\
\text { low: } 10 \\
\text { medium: } 42 \\
\text { high: } 129 \\
\text { very high: } 109\end{array}$ \\
\hline $\begin{array}{l}\text { Identification of needed measures that were } \\
\text { not installed }\end{array}$ & $\begin{array}{l}\text { obs: } 247 \\
\text { very low: } 80 \\
\text { low: } 96 \\
\text { medium: } 44 \\
\text { high: } 20 \\
\text { very high: } 7\end{array}$ & $\begin{array}{l}\text { obs: } 263 \\
\text { very low: } 20 \\
\text { low: } 50 \\
\text { medium: } 76 \\
\text { high: } 64 \\
\text { very high: } 52\end{array}$ & $\begin{array}{l}\text { obs: } 253 \\
\text { very low: } 20 \\
\text { low: } 81 \\
\text { medium: } 93 \\
\text { high: } 35 \\
\text { very high: } 23\end{array}$ & $\begin{array}{l}\text { obs: } 277 \\
\text { very low: } 7 \\
\text { low: } 20 \\
\text { medium: } 65 \\
\text { high: } 100 \\
\text { very high: } 84\end{array}$ \\
\hline Blower door test & $\begin{array}{l}\text { obs: } 257 \\
\text { very low: } 42 \\
\text { low: } 66 \\
\text { medium: } 99 \\
\text { high: } 33 \\
\text { very high: } 16 \\
\end{array}$ & $\begin{array}{l}\text { obs: } 272 \\
\text { very low: } 16 \\
\text { low: } 32 \\
\text { medium: } 85 \\
\text { high: } 92 \\
\text { very high: } 46 \\
\end{array}$ & $\begin{array}{l}\text { obs: } 261 \\
\text { very low: } 8 \\
\text { low: } 60 \\
\text { medium: } 108 \\
\text { high: } 54 \\
\text { very high: } 30 \\
\end{array}$ & $\begin{array}{l}\text { obs: } 291 \\
\text { very low: } 2 \\
\text { low: } 6 \\
\text { medium: } 28 \\
\text { high: } 102 \\
\text { very high: } 153 \\
\end{array}$ \\
\hline $\begin{array}{l}\text { Heating system efficiency test (flue gas } \\
\text { analysis) }\end{array}$ & $\begin{array}{l}\text { obs: } 225 \\
\text { very low: } 42 \\
\text { low: } 60 \\
\text { medium: } 81 \\
\text { high: } 28 \\
\text { very high: } 14 \\
\end{array}$ & $\begin{array}{l}\text { obs: } 244 \\
\text { very low: } 13 \\
\text { low: } 21 \\
\text { medium: } 57 \\
\text { high: } 102 \\
\text { very high: } 51 \\
\end{array}$ & $\begin{array}{l}\text { obs: } 231 \\
\text { very low: } 6 \\
\text { low: } 48 \\
\text { medium: } 105 \\
\text { high: } 49 \\
\text { very high: } 22 \\
\end{array}$ & $\begin{array}{l}\text { obs: } 257 \\
\text { very low: } 0 \\
\text { low: } 3 \\
\text { medium: } 39 \\
\text { high: } 95 \\
\text { very high: } 119\end{array}$ \\
\hline Draft/spillage tests of heating systems & $\begin{array}{l}\text { obs: } 228 \\
\text { very low: } 56 \\
\text { low: } 80 \\
\text { medium: } 62 \\
\text { high: } 19 \\
\text { very high: } 11\end{array}$ & $\begin{array}{l}\text { obs: } 247 \\
\text { very low: } 12 \\
\text { low: } 30 \\
\text { medium: } 78 \\
\text { high: } 78 \\
\text { very high: } 49\end{array}$ & $\begin{array}{l}\text { obs: } 234 \\
\text { very low: } 17 \\
\text { low: } 54 \\
\text { medium: } 97 \\
\text { high: } 45 \\
\text { very high: } 20\end{array}$ & $\begin{array}{l}\text { obs: } 259 \\
\text { very low: } 0 \\
\text { low: } 9 \\
\text { medium: } 29 \\
\text { high: } 101 \\
\text { very high: } 119\end{array}$ \\
\hline Carbon monoxide (CO) monitoring & $\begin{array}{l}\text { obs: } 236 \\
\text { very low: } 59 \\
\text { low: } 80 \\
\text { medium: } 63 \\
\text { high: } 18 \\
\text { very high: } 15\end{array}$ & $\begin{array}{l}\text { obs: } 252 \\
\text { very low: } 14 \\
\text { low: } 51 \\
\text { medium: } 83 \\
\text { high: } 61 \\
\text { very high: } 43\end{array}$ & $\begin{array}{l}\text { obs: } 239 \\
\text { very low: } 19 \\
\text { low: } 80 \\
\text { medium: } 85 \\
\text { high: } 27 \\
\text { very high: } 27\end{array}$ & $\begin{array}{l}\text { obs: } 267 \\
\text { very low: } 0 \\
\text { low: } 6 \\
\text { medium: } 27 \\
\text { high: } 101 \\
\text { very high: } 133\end{array}$ \\
\hline
\end{tabular}




\begin{tabular}{|c|c|c|c|c|}
\hline Infrared scanning & $\begin{array}{l}\text { obs: } 185 \\
\text { very low: } 27 \\
\text { low: } 24 \\
\text { medium: } 46 \\
\text { high: } 40 \\
\text { very high: } 47\end{array}$ & $\begin{array}{l}\text { obs: } 202 \\
\text { very low: } 10 \\
\text { low: } 25 \\
\text { medium: } 66 \\
\text { high: } 57 \\
\text { very high: } 43\end{array}$ & $\begin{array}{l}\text { obs: } 191 \\
\text { very low: } 11 \\
\text { low: } 30 \\
\text { medium: } 82 \\
\text { high: } 39 \\
\text { very high: } 28\end{array}$ & $\begin{array}{l}\text { obs: } 211 \\
\text { very low: } 1 \\
\text { low: } 13 \\
\text { medium: } 37 \\
\text { high: } 72 \\
\text { very high: } 88\end{array}$ \\
\hline $\begin{array}{l}\text { Identification of unresolved health and } \\
\text { safety issues }\end{array}$ & $\begin{array}{l}\text { obs: } 240 \\
\text { very low: } 72 \\
\text { low: } 81 \\
\text { medium: } 60 \\
\text { high: } 17 \\
\text { very high: } 9\end{array}$ & $\begin{array}{l}\text { obs: } 256 \\
\text { very low: } 17 \\
\text { low: } 50 \\
\text { medium: } 65 \\
\text { high: } 72 \\
\text { very high: } 51\end{array}$ & $\begin{array}{l}\text { obs: } 247 \\
\text { very low: } 14 \\
\text { low: } 74 \\
\text { medium: } 95 \\
\text { high: } 39 \\
\text { very high: } 23\end{array}$ & $\begin{array}{l}\text { obs: } 271 \\
\text { very low: } 4 \\
\text { low: } 10 \\
\text { medium: } 61 \\
\text { high: } 104 \\
\text { very high: } 91\end{array}$ \\
\hline Discussion with occupants & $\begin{array}{l}\text { obs: } 252 \\
\text { very low: } 91 \\
\text { low: } 99 \\
\text { medium: } 40 \\
\text { high: } 13 \\
\text { very high: } 8\end{array}$ & $\begin{array}{l}\text { obs: } 265 \\
\text { very low: } 29 \\
\text { low: } 64 \\
\text { medium: } 79 \\
\text { high: } 63 \\
\text { very high: } 30 \\
\end{array}$ & $\begin{array}{l}\text { obs: } 254 \\
\text { very low: } 24 \\
\text { low: } 88 \\
\text { medium: } 87 \\
\text { high: } 36 \\
\text { very high: } 19\end{array}$ & $\begin{array}{l}\text { obs: } 284 \\
\text { very low: } 3 \\
\text { low: } 25 \\
\text { medium: } 77 \\
\text { high: } 93 \\
\text { very high: } 86\end{array}$ \\
\hline Other (please specify) & $\begin{array}{l}\text { obs: } 3 \\
\text { very low: } 1 \\
\text { low: } 1 \\
\text { medium: } 1 \\
\text { high: } 0 \\
\text { very high: } 0\end{array}$ & $\begin{array}{l}\text { obs: } 3 \\
\text { very low: } 1 \\
\text { low: } 1 \\
\text { medium: } 0 \\
\text { high: } 0 \\
\text { very high: } 1\end{array}$ & $\begin{array}{l}\text { obs: } 3 \\
\text { very low: } 1 \\
\text { low: } 1 \\
\text { medium: } 0 \\
\text { high: } 1 \\
\text { very high: } 0\end{array}$ & $\begin{array}{l}\text { obs: } 6 \\
\text { very low: } 0 \\
\text { low: } 0 \\
\text { medium: } 1 \\
\text { high: } 3 \\
\text { very high: } 2\end{array}$ \\
\hline
\end{tabular}

4. Approximately how many hours did it take to perform a typical post-weatherization quality control inspection in Program Year 2008, by the major components listed below?

\begin{tabular}{|l|c|}
\hline $\begin{array}{l}\text { Scheduling } \\
\text { observations: }\end{array}$ & 322 \\
\hline missing values: & 35 \\
\hline mean: & .83 \\
\hline standard deviation: & 1.85 \\
\hline min: & 0 \\
\hline 10th percentile: & .2 \\
\hline 25th percentile: & .5 \\
\hline median: & .5 \\
\hline 75th percentile: & 1 \\
\hline 90th percentile: & 1 \\
\hline max: & 30 \\
\hline
\end{tabular}

\begin{tabular}{|l|c|}
\hline Travel observations: & 324 \\
\hline missing values: & 33 \\
\hline mean: & 1.49 \\
\hline standard deviation: & 5.48 \\
\hline min: & .02 \\
\hline 10th percentile: & .5 \\
\hline 25th percentile: & .5 \\
\hline median: & 1 \\
\hline 75th percentile: & 1.5 \\
\hline 90th percentile: & 2 \\
\hline max: & 90 \\
\hline
\end{tabular}




\begin{tabular}{|l|c|}
\hline $\begin{array}{l}\text { On-site work } \\
\text { observations: }\end{array}$ & 317 \\
\hline missing values: & 40 \\
\hline mean: & 2.72 \\
\hline standard deviation: & 5.52 \\
\hline min: & 0 \\
\hline 10th percentile: & 1 \\
\hline 25th percentile: & 1 \\
\hline median: & 2 \\
\hline 75th percentile: & 2.5 \\
\hline 90th percentile: & 4 \\
\hline max: & 48 \\
\hline
\end{tabular}

\begin{tabular}{|l|c|}
\hline $\begin{array}{l}\text { Post-inspection } \\
\text { analysis and write-up } \\
\text { observations: }\end{array}$ & 319 \\
\hline missing values: & 38 \\
\hline mean: & 1.7 \\
\hline standard deviation: & 5.62 \\
\hline min: & 0 \\
\hline 10th percentile: & .5 \\
\hline 25th percentile: & 1 \\
\hline median: & 1 \\
\hline 75th percentile: & 2 \\
\hline 90th percentile: & 2 \\
\hline max: & 90 \\
\hline
\end{tabular}

\begin{tabular}{|l|c|}
\hline Other observations: & 85 \\
\hline missing values: & 272 \\
\hline mean: & 1.36 \\
\hline standard deviation: & 3.83 \\
\hline min: & 0 \\
\hline 10th percentile: & 0 \\
\hline 25th percentile: & .5 \\
\hline median: & 1 \\
\hline 75th percentile: & 1 \\
\hline 90th percentile: & 1 \\
\hline max: & 30 \\
\hline
\end{tabular}




\begin{tabular}{|l|c|}
\hline $\begin{array}{l}\text { TOTAL of all } \\
\text { components } \\
\text { observations: }\end{array}$ & 357 \\
\hline missing values: & 0 \\
\hline mean: & 6.16 \\
\hline standard deviation: & 14.80 \\
\hline min: & 0 \\
\hline 10th percentile: & 0 \\
\hline 25th percentile: & 3 \\
\hline median: & 4.5 \\
\hline 75th percentile: & 6 \\
\hline 90th percentile: & 9 \\
\hline max: & 240 \\
\hline
\end{tabular}

5. Which of the following parties were involved in performing your agency's post-weatherization quality control inspections in Program Year 2008? Check all that apply.

- In-house manager

\begin{tabular}{|c|c|c|c|}
\hline & Freq. & Percent & Cum. \\
\hline no & 154.51 & 46.68 & 46.68 \\
\hline yes & 176.49 & 53.32 & 100.00 \\
\hline otal & 331 & 100.00 & \\
\hline
\end{tabular}

- In-house inspection specialist

Freq. Percent Cum.

\begin{tabular}{|c|c|c|c|}
\hline no & 89.84 & 27.14 & 27.14 \\
\hline yes & 241.16 & 72.86 & 100.00 \\
\hline Total & 331 & 100.00 & \\
\hline
\end{tabular}

- Contractor inspection specialist

\begin{tabular}{|c|c|c|c|}
\hline & Freq. & Percent & Cum. \\
\hline no & 296.22 & 89.49 & 89.49 \\
\hline yes | & 34.78 & 10.51 & 100.00 \\
\hline at & 331 & 100.00 & \\
\hline
\end{tabular}

- In-house weatherization crew chief

Freq. Percent Cum.

\begin{tabular}{|c|c|c|c|}
\hline no & 249.28 & 75.31 & 75.31 \\
\hline yes & 81.72 & 24.69 & 100.00 \\
\hline Total | & 331 & 100.00 & \\
\hline
\end{tabular}


- Contractor weatherization crew chief

\begin{tabular}{|c|c|c|c|}
\hline & Freq. & Percent & Cum. \\
\hline no & 306.59 & 92.63 & 92.63 \\
\hline yes & 24.41 & 7.37 & 100.00 \\
\hline Total & 331 & 100.00 & \\
\hline
\end{tabular}

- In-house weatherization crew member

Freq. Percent Cum.

\begin{tabular}{|c|c|c|c|}
\hline no & 303.71 & 91.76 & 91.76 \\
\hline yes & 27.29 & 8.24 & 100.00 \\
\hline Total & 331 & 100.00 & \\
\hline
\end{tabular}

- Contractor weatherization crew member

\begin{tabular}{|c|c|c|c|}
\hline & Freq. & Percent & Cum. \\
\hline no & 317.62 & 95.96 & 95.96 \\
\hline yes & 13.38 & 4.04 & 100.00 \\
\hline Total & 331 & 100.00 & \\
\hline
\end{tabular}

- Other (please specify)

\begin{tabular}{|c|c|c|c|}
\hline & Freq. & Percent & Cum. \\
\hline no & 299.31 & 90.42 & 90.42 \\
\hline yes & 31.69 & 9.58 & 100.00 \\
\hline Total | & 331 & 100.00 & \\
\hline
\end{tabular}

5a. Which party was primarily responsible for post-weatherization quality control inspections? Check best answer.

Freq. Percent Cum.

\begin{tabular}{rrrr} 
in-house manager | & 69.62 & 21.10 & 21.10 \\
in-house inspection specialist | & 196.68 & 59.60 & 80.70 \\
contractor inspection specialist | & 10.50 & 3.18 & 83.88 \\
in-house wx crew chief $\mid$ & 21.96 & 6.66 & 90.54 \\
contractor wx crew chief $\mid$ & 2.92 & 0.88 & 91.42 \\
in-house wx crew member | & 4.57 & 1.39 & 92.81 \\
other $\mid$ & 23.74 & 7.19 & 100.00 \\
\hline Total | & 330 & 100.00
\end{tabular}


6. What types of credentials or experience did your agency's post-weatherization quality control inspectors have in Program Year 2008? Check all that apply.

- Technical certification

\begin{tabular}{|c|c|c|c|}
\hline & Freq. & Percent & Cum. \\
\hline no & 82.06 & 24.94 & 24.94 \\
\hline yes & 246.94 & 75.06 & 100.00 \\
\hline Total & 329 & 100.00 & \\
\hline
\end{tabular}

- Extensive experience performing pre-weatherization audits

Freq. Percent Cum.

\begin{tabular}{|c|c|c|c|}
\hline no & 88.03 & 26.76 & 26.76 \\
\hline yes | & 240.97 & 73.24 & 100.00 \\
\hline otal | & 329 & 100.00 & \\
\hline
\end{tabular}

- Extensive experience performing weatherization work

\begin{tabular}{|c|c|c|c|}
\hline & Freq. & Percent & Cum. \\
\hline no & 138.10 & 41.98 & 41.98 \\
\hline yes & 190.90 & 58.02 & 100.00 \\
\hline Total & 329 & 100.00 & \\
\hline
\end{tabular}

- Extensive experience supervising weatherization work

\begin{tabular}{|c|c|c|c|}
\hline & Freq. & Percent & Cum. \\
\hline no & 169.10 & 51.40 & 51.40 \\
\hline yes $\mid$ & 159.90 & 48.60 & 100.00 \\
\hline Total | & 329 & 100.00 & \\
\hline
\end{tabular}

- Construction experience

\begin{tabular}{|c|c|c|c|}
\hline & Freq. & Percent & Cum. \\
\hline no & 149.65 & 45.49 & 45.49 \\
\hline yes | & 179.35 & 54.51 & 100.00 \\
\hline Total & 329 & 100.00 & \\
\hline
\end{tabular}

- Other (please specify)

$\begin{array}{rrr}\text { Freq. } & \text { Percent } & \text { Cum. } \\ \text { no | } 305.48 & 92.85 & 92.85 \\ \text { yes | } 23.52 & 7.15 & 100.00 \\ ------ & \end{array}$


7. Please indicate the level of experience for the agency staff engaged post-weatherization quality control inspections in Program Year 2008 in each of the following functional areas:

\begin{tabular}{|c|c|c|c|c|c|}
\hline & $\begin{array}{l}\text { Very } \\
\text { High }\end{array}$ & High & Averag & Low & Very Low \\
\hline \multirow{7}{*}{$\begin{array}{l}\text { Performing weatherization } \\
\text { work }\end{array}$} & \multicolumn{4}{|c|}{ Percent } & \\
\hline & very low & 6.57 & 2.05 & 2.05 & \\
\hline & low & 19.09 & 5.95 & 7.99 & \\
\hline & average & 61.96 & 19.30 & 27.29 & \\
\hline & high & 114.10 & 35.54 & 62.84 & \\
\hline & very high & 119.29 & 37.16 & 100.00 & \\
\hline & Total & 321 & 100.00 & & \\
\hline & & Freq. & Percent & Cum. & \\
\hline & very low & 7.94 & 2.53 & 2.53 & \\
\hline & low & 18.61 & 5.93 & 8.46 & \\
\hline Supervising weatherization & average & 60.80 & 19.36 & 27.82 & \\
\hline & high & 104.66 & 33.33 & 61.15 & \\
\hline & very high & 122.00 & 38.85 & 100.00 & \\
\hline & Total | & 314 & 100.00 & & \\
\hline & & Freq. & Percent & Cum. & \\
\hline & very low & 14.18 & 4.55 & 4.55 & \\
\hline & low & 20.97 & 6.72 & 11.27 & \\
\hline Working in construction & average & 90.80 & 29.10 & 40.37 & \\
\hline & high & 102.67 & 32.91 & 73.28 & \\
\hline & very high & 83.38 & 26.72 & 100.00 & \\
\hline & Total & 312 & 100.00 & & \\
\hline & 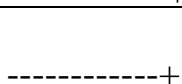 & Freq. & ercent & Cum. & \\
\hline & very low & 3.16 & 0.97 & 0.97 & \\
\hline & low & 20.96 & 6.43 & 7.40 & \\
\hline Performing pre- & average & 49.23 & 15.10 & 22.50 & \\
\hline weatherization audits & high & 105.68 & 32.42 & 54.92 & \\
\hline & very high & 146.97 & 45.08 & 100.00 & \\
\hline & Total | & 326 & 100.00 & & \\
\hline
\end{tabular}


8. For those dwelling units for which post-weatherization quality control inspections were performed by your agency in Program Year 2008, typically how many days after weatherization completion did the initial inspection take place?

\begin{tabular}{|l|c|}
\hline observations: & 322 \\
\hline missing values: & 35 \\
\hline mean: & 9.29 \\
\hline standard deviation: & 13.86 \\
\hline min: & 0 \\
\hline 10th percentile: & 1 \\
\hline 25th percentile: & 3 \\
\hline median: & 5 \\
\hline 75th percentile: & 10 \\
\hline 90th percentile: & 20 \\
\hline max: & 180 \\
\hline
\end{tabular}

9. In those cases where a Program Year 2008 post-weatherization quality control inspection revealed a problem with the job performed, what action was most commonly taken in response to that finding? Check one.

- Sent original crew or contractor back to correct problem

- Sent different crew or contractor to correct problem

- Sent crew supervisor to correct problem

- Sent someone from state office to correct problem

- No action taken

- Other (please specify)

Freq. Percent Cum.

\begin{tabular}{|c|c|c|c|}
\hline Sent original crew or contractor back $\mathrm{t}$ & 310.42 & 94.07 & 94.07 \\
\hline Sent different crew or contractor to co & 3.63 & 1.10 & 95.17 \\
\hline Sent crew supervisor to correct problem | & 10.52 & 3.19 & 98.35 \\
\hline Other & 5.43 & 1.65 & 100.00 \\
\hline
\end{tabular}

10. What other actions were taken in Program Year 2008 in response to the discovery of a problem with the weatherization job performed? Check all that apply.

- Sent original crew or contractor back to correct problem

Freq. Percent Cum.

$\begin{array}{rrr}\text { no | } 282.28 & 94.09 & 94.09 \\ \text { yes | } 17.72 & 5.91 & 100.00 \\ -------- & \end{array}$


- Sent different crew or contractor to correct problem

\begin{tabular}{|c|c|c|c|}
\hline & Freq. & Percent & Cum. \\
\hline no & 167.00 & 55.67 & 55.67 \\
\hline yes & 133.00 & 44.33 & 100.00 \\
\hline ota & 300 & 100.00 & \\
\hline
\end{tabular}

- Sent crew supervisor to correct problem Freq. Percent Cum.

\begin{tabular}{|c|c|c|c|}
\hline no & 161.17 & 53.72 & 53.72 \\
\hline yes & 138.83 & 46.28 & 100.00 \\
\hline Total & 300 & 100.00 & \\
\hline
\end{tabular}

- Sent someone from state office to correct problem Freq. Percent Cum.

\begin{tabular}{|c|c|c|c|}
\hline no & 269.41 & 89.80 & 89.80 \\
\hline yes | & 30.59 & 10.20 & 100.00 \\
\hline Total | & 300 & 100.00 & \\
\hline
\end{tabular}

- No action taken

\begin{tabular}{|c|c|c|c|}
\hline & Freq. & Percent & Cum. \\
\hline no & 276.62 & 92.21 & 92.21 \\
\hline yes & 23.38 & 7.79 & 100.00 \\
\hline Total & 300 & 100.00 & \\
\hline
\end{tabular}

- Other (please specify)

Freq. Percent Cum.

\begin{tabular}{|c|c|c|c|}
\hline no & 236.93 & 78.98 & 78.98 \\
\hline yes & 63.07 & 21.02 & 100.00 \\
\hline
\end{tabular}


11. In Program Year 2008, how many of the dwelling units weatherized by your agency required some additional work as a result of the findings of your post-weatherization quality control inspections?

\begin{tabular}{|l|c|}
\hline observations: & 280 \\
\hline missing values: & 77 \\
\hline mean: & 18.47 \\
\hline standard deviation: & 60.09 \\
\hline min: & 0 \\
\hline 10th percentile: & 0 \\
\hline 25th percentile: & 2 \\
\hline median: & 6 \\
\hline 75th percentile: & 15 \\
\hline 90th percentile: & 30 \\
\hline max: & 1000 \\
\hline
\end{tabular}

11a. Of those requiring some additional work, how many had work done that probably resulted in more energy savings?

\begin{tabular}{|l|c|}
\hline observations: & 267 \\
\hline missing values: & 90 \\
\hline mean: & 14.92 \\
\hline standard deviation: & 46.77 \\
\hline min: & 0 \\
\hline 10th percentile: & 0 \\
\hline 25th percentile: & 1.5 \\
\hline median: & 5 \\
\hline 75th percentile: & 10 \\
\hline 90th percentile: & 25 \\
\hline max: & 560 \\
\hline
\end{tabular}

12. What were the three most common problems found in the dwelling units inspected by your agency in Program Year 2008?

1)

2)

3) 
13. In Program Year 2008, did your agency use findings from your post-weatherization quality control inspections to provide feedback to your in-house or contractor crews on workmanship or related issues?

\begin{tabular}{|c|c|c|c|}
\hline & Freq. & Percent & Cum. \\
\hline no & 13.72 & 4.23 & 4.23 \\
\hline yes & 310.28 & 95.77 & 100.00 \\
\hline Total & 324 & 100.00 & \\
\hline
\end{tabular}

14. To what extent does post-weatherization quality control inspection affect the quality of future weatherization work?

Freq. Percent Cum.

\begin{tabular}{|c|c|c|c|}
\hline No extent & 2.48 & 0.75 & 0.75 \\
\hline Little extent & 17.81 & 5.40 & 6.15 \\
\hline Moderate extent & 63.90 & 19.36 & 25.51 \\
\hline Substantial extent & 128.66 & 38.99 & 64.50 \\
\hline Very substantial extent & 117.15 & 35.50 & 100.00 \\
\hline
\end{tabular}

15. Did the observation of problems with the quality of weatherization work lead to changes in weatherization training for your staff?

\begin{tabular}{|c|c|c|c|}
\hline & Freq. & Percent & Cum. \\
\hline nol & 119.25 & 36.58 & 36.58 \\
\hline yes & 206.75 & 63.42 & 100.00 \\
\hline Total | & 326 & 100.00 & \\
\hline
\end{tabular}

15a. If Yes, what changes were made?

16. Did your agency observe weatherization training sessions to help identify potential problem areas for inspecting in the field (e.g., with respect to installation of measures that trainees seemed to have trouble understanding)?

Freq. Percent Cum.

\begin{tabular}{|c|c|c|c|}
\hline no & 188.81 & 59.37 & 59.37 \\
\hline yes | & 129.19 & 40.63 & 100.00 \\
\hline Total | & 318 & 100.00 & \\
\hline
\end{tabular}

16a. If Yes, briefly describe how your in-field inspection activities were affected by your training session observations. 
APPENDIX F: BUILDING \& HOUSING UNIT INFORMATION SURVEYS 

OMB Control Number: 1910-5151

Frequencies include weighted responses from DF2 and DF3.

\section{DF2: HOUSING UNIT INFORMATION SURVEY}

Thank you for your prompt response to this data request which is part of the national evaluation of the Weatherization Assistance Program. Evaluation results will provide essential feedback to the weatherization community and inform policymakers about the program's effects on clients' energy consumption, cost savings, and non-energy benefits.

This survey collects detailed information about homes weatherized (or waitlisted) by your agency that have been selected for analysis by the national evaluation. The data you supply will be used with billing history data to better understand energy savings attributable to the Weatherization Assistance Program.

Please use this form (DF2) to provide information about any single family detached and attached houses, mobile homes, or individual units within multi-family buildings. The Building Information Survey (DF3) should be used to document information on small or large multifamily buildings in which the whole building and all units in the building were weatherized or are waitlisted. Refer to the definitions of each building type provided at the end of the survey because these definitions are slightly different than those commonly used within the Weatherization Assistance Program.

All of the information obtained from this survey will be protected and will remain confidential. The data will be analyzed in such a way that the information provided cannot be associated back to your state, your agencies, or the housing units and clients that your state served.

Thank you in advance for completing this survey.

Public reporting burden for this collection of information is estimated to average forty hours per weatherization agency, including the time for reviewing instructions, searching existing data sources, gathering and maintaining the data needed, and completing and reviewing the collection of information. Send comments regarding this burden estimate or any other aspect of this collection of information, including suggestions for reducing this burden, to Office of the Chief Information Officer, Records Management Division, IM-11, Paperwork Reduction Project (1910-5151), U.S. Department of Energy, 1000 Independence Ave SW, Washington, DC, 20585-1290; and to the Office of Management and Budget (OMB), OIRA, Paperwork Reduction Project (1910-5151), Washington, DC 20503. 
Form completed by:

Date:

Unit identification number (to be completed by the evaluation team):

\section{IDENTIFICATION}

1. Subgrantee (agency) name:

2. State:

3. Agency job number:

4. Occupant name:

5. Site address:

6. City:

\section{WEATHERIZATION INFORMATION}

[Question 7 intentionally deleted.]

Weatherization dates (not audit or inspection dates):

8a. Started:

8b. Completed:

$$
\text { (month) }
$$
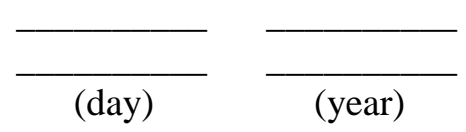

9. Was this a "reweatherized" unit? (check only one)

$$
\text { Freq. Percent Cum. }
$$

\begin{tabular}{|c|c|c|c|}
\hline No & $12,412.69$ & 95.81 & 95.81 \\
\hline Yes & 285.38 & 2.20 & 98.02 \\
\hline DK & 256.93 & 1.98 & 100.00 \\
\hline Cotal & 12,955 & 100.00 & \\
\hline
\end{tabular}

10. Does the housing unit meet your state's definition for being a high residential energy user? (check

\begin{tabular}{|c|c|c|c|}
\hline \multicolumn{2}{|c|}{ Freq. Percent } & \multicolumn{2}{|c|}{ Cum. } \\
\hline No & $3,400.97$ & 28.10 & 28.10 \\
\hline Yes & $3,177.77$ & 26.26 & 54.37 \\
\hline Not Applicable (DF3 only) & 458.54 & 3.79 & 58.15 \\
\hline No state definition & $2,017.51$ & 16.67 & 74.83 \\
\hline DK & $3,046.20$ & 25.17 & 100.00 \\
\hline Tota & 12,101 & 100.00 & \\
\hline
\end{tabular}
only one) 
12. Does the housing unit meet your state's definition for being a household with a high energy burden? (check only one)

\begin{tabular}{|c|c|c|c|}
\hline & Freq. & Percent & Cum. \\
\hline No & | 2,749.27 & 24.11 & 24.11 \\
\hline Yes & $3,330.27$ & 29.21 & 53.32 \\
\hline No state definition & $2,561.66$ & 22.47 & 75.79 \\
\hline DK & $2,759.80$ & 24.21 & 100.00 \\
\hline Total & 11,401 & 100.00 & \\
\hline
\end{tabular}

12. Did this client file a complaint about the weatherization services you provided? (check only one)

\begin{tabular}{|c|c|c|c|}
\hline No & $11,724.50$ & 91.31 & 91.31 \\
\hline Yes & 132.38 & 1.03 & 92.34 \\
\hline DK & 983.11 & 7.66 & 100.00 \\
\hline Total & 12,840 & 100.00 & \\
\hline
\end{tabular}

\section{HOUSING UNIT}

13. Building type - see definitions at the end of the survey: (check only one)

$$
\text { Freq. Percent Cum. }
$$

\begin{tabular}{|c|c|c|c|}
\hline Single-family detached & $6,190.26$ & 47.55 & 47.55 \\
\hline Single-family attached & 374.71 & 2.88 & 50.43 \\
\hline Single-family det/att unknown & 908.16 & 6.98 & 57.41 \\
\hline Mobile home & $2,364.35$ & 18.16 & 75.57 \\
\hline Small multifam (2-4 units) & $1,053.22$ & 8.09 & 83.66 \\
\hline Large multifam (5+ units) & $2,063.61$ & 15.85 & 99.51 \\
\hline Shelter & 0.14 & 0.00 & 99.51 \\
\hline DK & 63.56 & 0.49 & 100.00 \\
\hline Total & 13,018 & 100.00 & \\
\hline
\end{tabular}

14. Number of stories above grade: (check only one)

\section{Freq. Percent Cum.}

\begin{tabular}{|c|c|c|c|}
\hline 1 & |4,515.13 & 45.50 & 45.50 \\
\hline 2 & $3,533.52$ & 35.61 & 81.10 \\
\hline 3 & 346.68 & 3.49 & 84.60 \\
\hline $4+(\mathrm{DF} 2), 4(\mathrm{DF} 3)$ & 217.39 & 2.19 & 86.79 \\
\hline 5-9 (DF3 only) & 489.92 & 4.94 & 91.72 \\
\hline $10-19$ (DF3 only) & 89.75 & 0.90 & 92.63 \\
\hline $20+($ DF3 only) & 86.91 & 0.88 & 93.50 \\
\hline Not applicable & 117.81 & 1.19 & 94.69 \\
\hline DK & 526.90 & 5.31 & 100.00 \\
\hline Totat & 9,924 & 100.00 & \\
\hline
\end{tabular}


15. If single-family attached, number of units attached (adjacent) to this unit: (check only one) Freq. Percent Cum.

$\begin{array}{rrrr}1 \mid & 176.01 & 11.21 & 11.21 \\ 2 \mid & 81.52 & 5.19 & 16.40 \\ 3 \mid & 21.37 & 1.36 & 17.76 \\ 4+\mid & 47.34 & 3.02 & 20.78 \\ \text { Not applicable } \mid & 734.33 & 46.77 & 67.55 \\ \text { DK } \mid & 509.43 & 32.45 & 100.00 \\ ----------- & \end{array}$

16. If mobile home, number of rooms that have been added on: (check only one) Freq. Percent Cum.

\begin{tabular}{|c|c|c|c|}
\hline none | & $1,869.24$ & 70.46 & 70.46 \\
\hline 1 & 188.91 & 7.12 & 77.58 \\
\hline 2 & 38.89 & 1.47 & 79.04 \\
\hline 3 & 13.25 & 0.50 & 79.54 \\
\hline $4+$ & 13.95 & 0.53 & 80.07 \\
\hline 98 & 98.75 & 3.72 & 83.79 \\
\hline $\mathrm{DK}$ & 430.01 & 16.21 & 100.00 \\
\hline Total & 2,653 & 100.00 & \\
\hline
\end{tabular}

17. If small or large multifamily building, number of units in the building: (check only one) Freq. Percent Cum.

\begin{tabular}{|c|c|c|c|}
\hline $1 \mid$ & 4.35 & 1.27 & 1.27 \\
\hline $2 \mid$ & 103.47 & 30.25 & 31.53 \\
\hline 3 & 29.37 & 8.59 & 40.11 \\
\hline $4 \mid$ & 25.15 & 7.35 & 47.47 \\
\hline $5-9$ & 32.15 & 9.40 & 56.87 \\
\hline $10-19$ & 22.33 & 6.53 & 63.39 \\
\hline $20-29$ & 23.36 & 6.83 & 70.23 \\
\hline $30-49$ & 23.36 & 6.83 & 77.06 \\
\hline $50-99$ & 16.54 & 4.84 & 81.89 \\
\hline $100+$ & 35.88 & 10.49 & 92.38 \\
\hline Not applicable & 12.54 & 3.67 & 96.05 \\
\hline DK & 13.51 & 3.95 & 100.00 \\
\hline Total & 342 & 100.00 & \\
\hline
\end{tabular}

18. 
Ownership (check only one)

Freq. Percent Cum.

$\begin{array}{rrrr}\text { Owner occupied | } 10,462.18 & 86.42 & 86.42 \\ \text { Rental | } & 1,584.42 & 13.09 & 99.51 \\ \text { Other | } & 38.39 & 0.32 & 99.83 \\ \text { DK | } & 21.01 & 0.17 & 100.00 \\ ------------ & \end{array}$

19. Year house/building originally built: (check only one)

Freq. Percent Cum.

\begin{tabular}{r|rrr}
$2000+\mid$ & 172.71 & 1.34 & 1.34 \\
$1990 \mathrm{~s} \mid$ & 678.51 & 5.25 & 6.58 \\
$1980 \mathrm{~s} \mid$ & $1,193.36$ & 9.23 & 15.81 \\
$1970 \mathrm{~s} \mid$ & $1,936.15$ & 14.97 & 30.78 \\
$1960 \mathrm{~s} \mid$ & 956.82 & 7.40 & 38.18 \\
$1950 \mathrm{~s} \mid$ & $1,060.87$ & 8.20 & 46.38 \\
$1940 \mathrm{~s} \mid$ & 670.32 & 5.18 & 51.56 \\
$1930 \mathrm{~s} \mid$ & 485.42 & 3.75 & 55.32 \\
$1920 \mathrm{~s} \mid$ & 521.88 & 4.04 & 59.35 \\
$1910 \mathrm{~s} \mid$ & 465.28 & 3.60 & 62.95 \\
$1900 \mathrm{~s} \mid$ & 590.55 & 4.57 & 67.52 \\
Before 1900 | & 311.98 & 2.41 & 69.93 \\
DK $\mid 3,889.15$ & 30.07 & 100.00 \\
---------------------------
\end{tabular}

Conditioned floor area at the time of weatherization: (include the basement only if it is intentionally conditioned)

20a. Heated floor area: $\mathrm{ft}^{2}$

Don't know

20b. Air conditioned floor area: $\mathrm{ft}^{2}$

Don't know 
21a. Primary fuel used to heat the unit during the winter before weatherization: (check only one) Freq. Percent Cum.

\begin{tabular}{|c|c|c|c|}
\hline Natural gas & $7,216.99$ & 55.41 & 55.41 \\
\hline Propane & $1,161.72$ & 8.92 & 64.33 \\
\hline Kerosene (fuel oil \#1) & 281.37 & 2.16 & 66.49 \\
\hline Fuel oil (\#2) & $1,270.73$ & 9.76 & 76.24 \\
\hline Fuel oil (\#6) & 48.40 & 0.37 & 76.62 \\
\hline Electricity & $2,615.57$ & 20.08 & 96.70 \\
\hline Wood & 214.36 & 1.65 & 98.34 \\
\hline Coal & 18.49 & 0.14 & 98.48 \\
\hline District steam & 22.94 & 0.18 & 98.66 \\
\hline Other & 101.27 & 0.78 & 99.44 \\
\hline DK & 73.17 & 0.56 & 100.00 \\
\hline Total & 13,025 & 100.00 & \\
\hline
\end{tabular}

21b. Primary fuel used for water heating before weatherization: (check only one) Freq. Percent Cum.

\begin{tabular}{r|rrr} 
None (post-coded) & 10.41 & 0.08 & 0.08 \\
Natural gas $\mid$ & $6,211.32$ & 48.79 & 48.87 \\
Propane $\mid$ & 673.37 & 5.29 & 54.16 \\
oil/kerosene (post-coded) & 479.27 & 3.76 & 57.92 \\
Electricity $\mid$ & $4,494.14$ & 35.30 & 93.22 \\
Other $\mid$ & 150.89 & 1.19 & 94.40 \\
DK $\mid$ & 712.59 & 5.60 & 100.00 \\
- Total $\mid$ & 12,732 & 100.00 &
\end{tabular}

22. Type of primary space-heating system before weatherization: (check only one)

Freq. Percent Cum.

$\begin{array}{rrrr}\text { Central furnace } \mid & 7,526.02 & 58.59 & 58.59 \\ \text { Heat pump } \mid & 269.70 & 2.10 & 60.69 \\ \text { Electric, built-in | } & 564.45 & 4.39 & 65.08 \\ \text { Steam or hydronic } \mid & 2,260.73 & 17.60 & 82.68 \\ \text { Wall furnace } \mid & 490.68 & 3.82 & 86.50 \\ \text { Room space heater (non-portable) } \mid & 582.04 & 4.53 & 91.03 \\ \text { Portable space heater } \mid & 211.45 & 1.65 & 92.68 \\ \text { Cooking stove } \mid & 7.10 & 0.06 & 92.73 \\ \text { None | } & 233.92 & 1.82 & 94.55 \\ \text { DK } \mid & 699.91 & 5.45 & 100.00\end{array}$


23. If small or large multifamily building, was the primary space-heating system shared with other housing units? (check only one)

Freq. Percent Cum.

\begin{tabular}{|c|c|c|c|}
\hline No & 555.14 & 46.69 & 46.69 \\
\hline Yes $\mid$ & 508.73 & 42.79 & 89.48 \\
\hline Not applicable | & 18.67 & 1.57 & 91.05 \\
\hline DK $\mid$ & 106.45 & 8.95 & 100.00 \\
\hline Total | & 1,189 & 100.00 & \\
\hline
\end{tabular}

24. Supplemental fuel(s) used to heat the unit during the winter before weatherization: (check all that apply)

- Natural gas

Freq. Percent Cum.

\begin{tabular}{|c|c|c|c|}
\hline No & $11,575.19$ & 90.42 & 90.42 \\
\hline Yes & $1,225.81$ & 9.58 & 100.00 \\
\hline Total & 12,801 & 100.00 & \\
\hline
\end{tabular}

- Propane/LPG

Freq. Percent Cum.

\begin{tabular}{|c|c|c|c|}
\hline No & $12,541.16$ & 97.97 & 97.97 \\
\hline Yes & 259.84 & 2.03 & 100.00 \\
\hline Total & 12,801 & 100.00 & \\
\hline
\end{tabular}

- Kerosene (\#1 fuel oil)

\begin{tabular}{crr} 
Freq. & Percent & \multicolumn{1}{c}{ Cum. } \\
No | $12,718.99$ & 99.36 & 99.36 \\
Yes | 82.01 & 0.64 & 100.00 \\
--------- &
\end{tabular}

- Fuel oil (\#2 fuel oil)

Freq. Percent Cum.

\begin{tabular}{|c|c|c|c|}
\hline No & $12,676.47$ & 99.03 & 99.03 \\
\hline Yes & 124.53 & 0.97 & 100.00 \\
\hline Total & 12,801 & 100.00 & \\
\hline
\end{tabular}


- Electricity

\begin{tabular}{rrr} 
Freq. & Percent & \multicolumn{1}{c}{ Cum. } \\
$-\mathrm{-}$ No | $11,615.76$ & 90.74 & 90.74 \\
Yes | $1,185.24$ & 9.26 & 100.00 \\
---------- &
\end{tabular}

- Wood

\begin{tabular}{crr} 
Freq. & Percent & \multicolumn{1}{c}{ Cum. } \\
- No | $12,295.39$ & 96.05 & 96.05 \\
Yes | 505.61 & 3.95 & 100.00 \\
------------ \\
Total| 12,801 & 100.00
\end{tabular}

- Other (specify:

\begin{tabular}{|c|c|c|c|}
\hline & Freq. & Percent & Cum. \\
\hline No & $12,766.58$ & 99.73 & 99.73 \\
\hline Yes & 34.42 & 0.27 & 100.00 \\
\hline Total | & 12,801 & 100.00 & \\
\hline
\end{tabular}

- None

$\begin{array}{lrr}\text { Freq. } & \text { Percent } & \text { Cum. } \\ \text { No } \mid 6,183.68 & 48.31 & 48.31 \\ \text { Yes | 6,617.33 } & 51.69 & 100.00 \\ ---------- \\ \text { Total | } 12,801 & 100.00\end{array}$

- Don't know

$\begin{array}{crr}\text { Freq. } & \text { Percent } & \text { Cum. } \\ \text { No | } 10,099.61 & 78.90 & 78.90 \\ \text { Yes | 2,701.39 } & 21.10 & 100.00 \\ --------------- \\ \text { Total| } 12,801 & 100.00\end{array}$

25. Type of operable air conditioning system present before weatherization: (check all that apply)

- Central air conditioner/heat pump

\begin{tabular}{|c|c|c|c|}
\hline & Freq. & Percent & Cum. \\
\hline No & $10,232.65$ & 79.59 & 79.59 \\
\hline Yes & $2,624.35$ & 20.41 & 100.00 \\
\hline${ }_{0}$ & 12,857 & 100.00 & \\
\hline
\end{tabular}


- Window/wall units

$\begin{array}{rrr}\text { Freq. } & \text { Percent } & \text { Cum. } \\ \text { No| } 9,995.73 & 77.75 & 77.75 \\ \text { Yes | } 2,861.27 & 22.25 & 100.00 \\ ------- & \end{array}$

- Evaporative cooling system ("swamp coolers") Freq. Percent Cum.

$\begin{array}{crr}\text { No | } 12,536.96 & 97.51 & 97.51 \\ \text { Yes | } 320.04 & 2.49 & 100.00 \\ ------ & \\ \text { Total | } 12,857 & 100.00\end{array}$

- None

\begin{tabular}{crr} 
Freq. & Percent & \multicolumn{1}{c}{ Cum. } \\
No | 10,701.76 & 83.24 & 83.24 \\
Yes | 2,155.24 & 16.76 & 100.00 \\
---------------- \\
Total | 12,857 & 100.00
\end{tabular}

- Don't know

$\begin{array}{ccc}\text { Freq. } & \text { Percent } & \text { Cum. } \\ \text { No } \mid 7,873.70 & 61.24 & 61.24 \\ \text { Yes } \mid 4,983.30 & 38.76 & 100.00 \\ ------+---- \\ \text { Total | } 12,857 & 100.00\end{array}$

26. Number of window/wall air conditioning units: (check only one) Freq. Percent Cum.

\begin{tabular}{|c|c|c|c|}
\hline 0 & $4,627.76$ & 38.91 & 38.91 \\
\hline 1 & $1,251.56$ & 10.52 & 49.44 \\
\hline 2 & 532.86 & 4.48 & 53.92 \\
\hline 3 & 161.67 & 1.36 & 55.28 \\
\hline $4+$ & 46.80 & 0.39 & 55.67 \\
\hline DK & $5,271.35$ & 44.33 & 100.00 \\
\hline Total & 11,892 & 100.00 & \\
\hline
\end{tabular}

[Questions 27 and 28 intentionally deleted.] 


\section{HOUSEHOLD}

29. Household annual income (as used to determine program eligibility): \$ per year

\begin{tabular}{|l|c|}
\hline observations: & 11401 \\
\hline missing values: & 2716 \\
\hline mean: & 14819.81 \\
\hline standard deviation: & 13103.42 \\
\hline min: & 0 \\
\hline 10th percentile: & 5489 \\
\hline 25th percentile: & 8424 \\
\hline median: & 12750 \\
\hline 75th percentile: & 19014.94 \\
\hline 90th percentile: & 26833 \\
\hline max: & 1112400 \\
\hline
\end{tabular}

30. Total number of occupants: (check only one)

Freq. Percent Cum.

\begin{tabular}{|c|c|c|c|}
\hline 1 & $4,793.06$ & 39.55 & 39.55 \\
\hline 2 & $2,786.65$ & 22.99 & 62.54 \\
\hline 3 & $1,635.02$ & 13.49 & 76.03 \\
\hline 4 & $1,357.75$ & 11.20 & 87.23 \\
\hline 5 & 805.24 & 6.64 & 93.88 \\
\hline 6 & 419.78 & 3.46 & 97.34 \\
\hline 7 & 155.25 & 1.28 & 98.62 \\
\hline 8 & 50.20 & 0.41 & 99.03 \\
\hline $9+$ & 51.07 & 0.42 & 99.46 \\
\hline DK & 65.98 & 0.54 & 100.00 \\
\hline Tota & 12,120 & 100.00 & \\
\hline
\end{tabular}

31. Check if the housing unit was occupied by at least one person who was: (check all that apply)

- Elderly (60 or older)

Freq. Percent Cum.

$\begin{array}{ccc}\text { No | } 5,052.40 & 50.43 & 50.43 \\ \text { Yes } \mid 4,965.60 & 49.57 & 100.00 \\ --------------- \\ \text { Total| } 10,018 & 100.00\end{array}$

- Disabled

Freq. Percent Cum.

\begin{tabular}{|c|c|c|c|}
\hline No & $5,611.03$ & 56.01 & 56.01 \\
\hline Yes & $4,406.97$ & 43.99 & 100.00 \\
\hline Total & 10,018 & 100.00 & \\
\hline
\end{tabular}

- Native American 


\begin{tabular}{ccc} 
Freq. & Percent & \multicolumn{1}{c}{ Cum. } \\
No $\mid 9,774.63$ & 97.57 & 97.57 \\
Yes | 243.37 & 2.43 & 100.00 \\
-------- \\
Total| 10,018 & 100.00
\end{tabular}

- A child (as defined by your state)

Freq. Percent Cum.

\begin{tabular}{|c|c|c|c|}
\hline No & $6,673.17$ & 66.61 & 66.61 \\
\hline Yes & $3,344.83$ & 33.39 & 100.00 \\
\hline Total | & 10,018 & 100.00 & \\
\hline
\end{tabular}

32. Number of children (as defined by your state): (check only one) Freq. Percent Cum.

\begin{tabular}{|c|c|c|c|}
\hline 0 & $4,102.07$ & 51.30 & 51.30 \\
\hline 1 & $1,510.64$ & 18.89 & 70.19 \\
\hline 2 & $1,301.01$ & 16.27 & 86.45 \\
\hline 3 & 659.12 & 8.24 & 94.70 \\
\hline 4 & 301.87 & 3.77 & 98.47 \\
\hline $5+$ & 116.41 & 1.46 & 99.93 \\
\hline DK & 5.87 & 0.07 & 100.00 \\
\hline Total & 7,997 & 100.00 & \\
\hline
\end{tabular}

33. Number of elderly (60 or older): (check only one)

\begin{tabular}{|c|c|c|c|}
\hline & Freq. & Percent & Cum. \\
\hline 0 & $3,296.35$ & 38.46 & 38.46 \\
\hline 1 & $4,292.27$ & 50.08 & 88.55 \\
\hline 2 & 965.76 & 11.27 & 99.82 \\
\hline 3 & 9.79 & 0.11 & 99.93 \\
\hline 4 & .86 & 0.01 & 99.94 \\
\hline DK & 4.96 & 0.06 & 100.00 \\
\hline Total | & 8,570 & 100.00 & \\
\hline
\end{tabular}


34. Number of disabled : (check only one)

\begin{tabular}{|c|c|c|c|}
\hline & Freq. & Percent & Cum. \\
\hline 0 & | 3,637.86 & 43.07 & 43.07 \\
\hline $1 \mid$ & $4,055.70$ & 48.02 & 91.09 \\
\hline 2 & 664.48 & 7.87 & 98.96 \\
\hline 3 & 57.99 & 0.69 & 99.65 \\
\hline 4 & 12.99 & 0.15 & 99.80 \\
\hline $5+$ & 2.17 & 0.03 & 99.82 \\
\hline DK & 14.80 & 0.18 & 100.00 \\
\hline tal & 8,446 & 100.00 & \\
\hline
\end{tabular}

35. Year moved into housing unit: (check only one) Freq. Percent Cum.

\begin{tabular}{|c|c|c|c|}
\hline 2008 & 177.13 & 1.58 & 1.58 \\
\hline $2007 \mid$ & 295.23 & 2.63 & 4.21 \\
\hline 2006 & 224.02 & 2.00 & 6.21 \\
\hline 2005 & 256.67 & 2.29 & 8.50 \\
\hline 2000-2004 & 715.99 & 6.38 & 14.88 \\
\hline 1995-1999 & 378.28 & 3.37 & 18.26 \\
\hline 1990-1994 & 285.47 & 2.55 & 20.80 \\
\hline 1985-1989 & 226.07 & 2.02 & 22.82 \\
\hline $1980-1984$ & 155.88 & 1.39 & 24.21 \\
\hline $1975-1979$ & 149.67 & 1.33 & 25.54 \\
\hline $1970-1974$ & 132.95 & 1.19 & 26.73 \\
\hline $1965-1969$ & 109.47 & 0.98 & 27.70 \\
\hline 1960-1964 & 76.75 & 0.68 & 28.39 \\
\hline $1955-1959$ & 51.06 & 0.46 & 28.84 \\
\hline 1950-1954 & 53.31 & 0.48 & 29.32 \\
\hline Before 1950 & 69.47 & 0.62 & 29.94 \\
\hline DK & $7,856.58$ & 70.06 & 100.00 \\
\hline
\end{tabular}

36. Is the household headed by a single parent? (check only one) Freq. Percent Cum.

$\begin{array}{ccc}\text { No | 8,356.94 } & 69.48 & 69.48 \\ \text { Yes |2,056.78 } & 17.10 & 86.58 \\ \text { DK | } 1,614.28 & 13.42 & 100.00 \\ -------------- & \\ \text { Total | } 12,028 & 100.00 & \end{array}$


37. Race and ethnicity of the head of household: (check all that apply)

- American Indian or Alaska Native

\begin{tabular}{|c|c|c|c|}
\hline & Freq. & Percent & Cum. \\
\hline No & $11,793.62$ & 97.92 & 97.92 \\
\hline Yes & 250.38 & 2.08 & 100.00 \\
\hline Total & 12,044 & 100.00 & \\
\hline
\end{tabular}

- Asian

\begin{tabular}{|c|c|c|c|}
\hline & Freq. & Percent & Cum. \\
\hline No & $11,950.51$ & 99.22 & 99.22 \\
\hline Yes & 93.49 & 0.78 & 100.00 \\
\hline Total & 12,044 & 100.00 & \\
\hline
\end{tabular}

- Black or African American

$\begin{array}{ccc}\text { Freq. } & \text { Percent } & \text { Cum. } \\ \text { No | 10,040.20 } & 83.36 & 83.36 \\ \text { Yes | 2,003.80 } & 16.64 & 100.00 \\ ------------- \\ \text { Total| } 12,044 & 100.00\end{array}$

- Native Hawaiian or other Pacific Islander

\begin{tabular}{crr} 
Freq. & Percent & \multicolumn{1}{c}{ Cum. } \\
No | $12,021.32$ & 99.81 & 99.81 \\
Yes | 22.68 & 0.19 & 100.00 \\
--------- \\
Total| 12,044 & 100.00
\end{tabular}

- White

$\begin{array}{ccc}\text { Freq. } & \text { Percent } & \text { Cum. } \\ \text { No } \mid 5,559.40 & 46.16 & 46.16 \\ \text { Yes | 6,484.60 } & 53.84 & 100.00 \\ ----------- \\ \text { Total | } 12,044 & 100.00 & \end{array}$

- Hispanic or Latino

Freq. Percent Cum.

\begin{tabular}{|c|c|c|c|}
\hline No & $11,351.06$ & 94.25 & 94.25 \\
\hline Yes & 692.94 & 5.75 & 100.00 \\
\hline Total & 12,044 & 100.00 & \\
\hline
\end{tabular}


- Don't know

\begin{tabular}{|c|c|c|c|}
\hline & Freq. & Percent & Cum. \\
\hline No & $9,442.97$ & 78.40 & 78.40 \\
\hline Yes & $2,601.03$ & 21.60 & 100.00 \\
\hline Total & 12,044 & 100.00 & \\
\hline
\end{tabular}

\section{AUDIT}

38. Primary method used to select weatherization measures for this house (excluding health, safety, and repair measures and general heat waste measures): (check only one)

Freq. Percent Cum.

\begin{tabular}{|c|c|c|c|}
\hline Priority list & 6,607.73 & 51.16 & 51.16 \\
\hline Calculation procedure & | 5,897.08 & 45.65 & 96.81 \\
\hline Other & 412.19 & 3.19 & 100.00 \\
\hline Total & 12,917 & 100.00 & \\
\hline
\end{tabular}

39. If a calculation procedure was used, the name of the procedure(s): (check all that apply)

- AK Warm

Freq. Percent Cum.

\begin{tabular}{|c|c|c|c|}
\hline No & $6,136.51$ & 98.58 & 98.58 \\
\hline Yes & 88.49 & 1.42 & 100.00 \\
\hline Total & 6,225 & 100.00 & \\
\hline
\end{tabular}

- $\mathrm{EA}-3$

\begin{tabular}{|c|c|c|c|}
\hline & Freq. & Percent & Cum. \\
\hline No & $5,967.05$ & 95.86 & 95.86 \\
\hline Yes & 257.95 & 4.14 & 100.00 \\
\hline Total & 6,225 & 100.00 & \\
\hline
\end{tabular}

- $\quad$ EASY

\begin{tabular}{lrr} 
Freq. & Percent & \multicolumn{1}{c}{ Cum. } \\
No | 6,019.25 & 96.69 & 96.69 \\
Yes | 205.75 & 3.31 & 100.00 \\
--------- \\
Total |
\end{tabular}


- EA-QUIP

\begin{tabular}{ccc} 
Freq. & Percent & Cum. \\
No | 5,623.20 & 90.33 & 90.33 \\
Yes | 601.80 & 9.67 & 100.00 \\
- \hdashline---
\end{tabular}

- HomeCheck

\begin{tabular}{|c|c|c|c|}
\hline & Freq. & Percent & Cum. \\
\hline No & | 6,180.06 & 99.28 & 99.28 \\
\hline Yes & 44.94 & 0.72 & 100.00 \\
\hline Total & 6,225 & 100.00 & \\
\hline
\end{tabular}

- Meadows

Freq. Percent Cum.

\begin{tabular}{|c|c|c|c|}
\hline No & $6,140.76$ & 98.65 & 98.65 \\
\hline Yes & 84.24 & 1.35 & 100.00 \\
\hline Total & 6,225 & 100.00 & \\
\hline
\end{tabular}

- REES

\begin{tabular}{|c|c|c|c|}
\hline & Freq. & Percent & Cum. \\
\hline No & 6,225 & 100.00 & 100.00 \\
\hline Total & 6,225 & 100.00 & \\
\hline
\end{tabular}

- REM/Rate

$\begin{array}{ccc}\text { Freq. } & \text { Percent } & \text { Cum. } \\ \text { No |5,981.99 } & 96.10 & 96.10 \\ \text { Yes | 243.01 } & 3.90 & 100.00 \\ ------------ \\ \text { Total| } 6,225 & 100.00\end{array}$

- SMOC-ERS

Freq. Percent Cum.

\begin{tabular}{|c|c|c|c|}
\hline No & 6,225 & 100.00 & 100.00 \\
\hline Total | & 6,225 & 100.00 & \\
\hline
\end{tabular}


- $\quad$ TIPS

$\begin{array}{ccc}\text { Freq. } & \text { Percent } & \text { Cum. } \\ \text {-------------------------------- } \\ \text { No | 5,623.16 } & 90.33 & 90.33 \\ \text { Yes | } 601.84 & 9.67 & 100.00 \\ ------------------------------ \\ \text { Total| } 6,225 & 100.00\end{array}$

- TREAT

$\begin{array}{ccc}\text { Freq. } & \text { Percent } & \text { Cum. } \\ \text { No | 5,912.70 } & 94.98 & 94.98 \\ \text { Yes | } 312.30 & 5.02 & 100.00 \\ ---------- \\ \text { Total | } 6,225 & 100.00\end{array}$

- Weatherization Assistant (NEAT/MHEA)

\begin{tabular}{|c|c|c|c|}
\hline & Freq. & Percent & Cum. \\
\hline No & | 3,567.05 & 57.30 & 57.30 \\
\hline Yes & $2,657.95$ & 42.70 & 100.00 \\
\hline Total | & 6,225 & 100.00 & \\
\hline
\end{tabular}

- WXEOR

\begin{tabular}{|c|c|c|c|}
\hline & Freq. & Percent & Cum. \\
\hline No & $6,106.09$ & 98.09 & 98.09 \\
\hline Yes & 118.91 & 1.91 & 100.00 \\
\hline
\end{tabular}

- Other (specify:

Freq. Percent Cum.

$\begin{array}{ccc}\text { No } \mid 5,331.38 & 85.64 & 85.64 \\ \text { Yes | } 893.62 & 14.36 & 100.00 \\ ------- & \\ \text { Total| } & 6,225 & 100.00\end{array}$

- Not applicable

$\begin{array}{ccc}\text { Freq. } & \text { Percent } & \text { Cum. } \\ \text { No |6,085.90 } & 97.77 & 97.77 \\ \text { Yes | } 139.10 & 2.23 & 100.00 \\ ------- & \end{array}$




\section{DIAGNOSTICS AND INSPECTIONS}

If you know when a diagnostic/inspection procedure was performed, please check the appropriate box(es) in the first three response columns. If a diagnostic/inspection procedure was performed but you do not know when, please check the box in the "Performed?" column.

\begin{tabular}{|c|c|c|c|c|}
\hline \multirow[t]{2}{*}{ Diagnostic measurement or inspection } & \multicolumn{3}{|c|}{ Diagnostic/inspection performed during: } & \multirow[b]{2}{*}{ Performed? } \\
\hline & $\begin{array}{c}\text { Audit/house } \\
\text { assessment }\end{array}$ & $\begin{array}{c}\text { Measure } \\
\text { installation }\end{array}$ & $\begin{array}{c}\text { Post- } \\
\text { inspection }\end{array}$ & \\
\hline \multicolumn{5}{|l|}{ Pressure diagnostics: } \\
\hline $\begin{array}{l}\text { 40a. Blower door measurement (house air } \\
\text { leakage rate) }\end{array}$ & $\begin{array}{l}\text { obs: } 12585 \\
\text { no: } 2782 \\
\text { yes: } 9802\end{array}$ & $\begin{array}{l}\text { obs: } 12585 \\
\text { no: } 8309 \\
\text { yes: } 4276\end{array}$ & $\begin{array}{l}\text { obs: } 12585 \\
\text { no: } 4379 \\
\text { yes: } 8206\end{array}$ & $\begin{array}{l}\text { obs: } 13027 \\
\text { no: } 2321 \\
\text { yes: } 10706\end{array}$ \\
\hline 40b. Zonal pressure & $\begin{array}{l}\text { obs: } 12858 \\
\text { no: } 9882 \\
\text { yes: } 2976\end{array}$ & $\begin{array}{l}\text { obs: } 12858 \\
\text { no: } 11446 \\
\text { yes: } 1412\end{array}$ & $\begin{array}{l}\text { obs: } 12858 \\
\text { no: } 10480 \\
\text { yes: } 2378\end{array}$ & $\begin{array}{l}\text { obs: } 13027 \\
\text { no: } 9687 \\
\text { yes: } 3340\end{array}$ \\
\hline $\begin{array}{l}\text { 40c. Room-to-room pressures (distribution } \\
\text { system balancing) }\end{array}$ & $\begin{array}{l}\text { obs: } 12941 \\
\text { no: } 10877 \\
\text { yes: } 2064\end{array}$ & $\begin{array}{l}\text { obs: } 12941 \\
\text { no: } 11981 \\
\text { yes: } 960\end{array}$ & $\begin{array}{l}\text { obs: } 12941 \\
\text { no: } 11271 \\
\text { yes: } 1670\end{array}$ & $\begin{array}{l}\text { obs: } 13027 \\
\text { no: } 10546 \\
\text { yes: } 2481\end{array}$ \\
\hline 40d. Duct pressure pan measurements & $\begin{array}{l}\text { obs: } 12863 \\
\text { no: } 10567 \\
\text { yes: } 2296\end{array}$ & $\begin{array}{l}\text { obs: } 12863 \\
\text { no: } 12028 \\
\text { yes: } 835\end{array}$ & $\begin{array}{l}\text { obs: } 12863 \\
\text { no: } 11175 \\
\text { yes: } 1688\end{array}$ & $\begin{array}{l}\text { obs: } 13027 \\
\text { no: } 10435 \\
\text { yes: } 2592\end{array}$ \\
\hline $\begin{array}{l}\text { 40e. Duct blower measurement (duct air } \\
\text { leakage rate) }\end{array}$ & $\begin{array}{l}\text { obs: } 12964 \\
\text { no: } 12514 \\
\text { yes: } 450\end{array}$ & $\begin{array}{l}\text { obs: } 12964 \\
\text { no: } 12673 \\
\text { yes: } 291\end{array}$ & $\begin{array}{l}\text { obs: } 12964 \\
\text { no: } 12670 \\
\text { yes: } 274\end{array}$ & $\begin{array}{l}\text { obs: } 13027 \\
\text { no: } 12279 \\
\text { yes: } 748\end{array}$ \\
\hline $\begin{array}{l}\text { 40f. Blower door subtraction meas. (duct } \\
\text { air leakage rate) }\end{array}$ & $\begin{array}{l}\text { obs: } 12945 \\
\text { no: } 12311 \\
\text { yes: } 634\end{array}$ & $\begin{array}{l}\text { obs: } 12945 \\
\text { no: } 12836 \\
\text { yes: } 109\end{array}$ & $\begin{array}{l}\text { obs: } 12945 \\
\text { no: } 12533 \\
\text { yes: } 412\end{array}$ & $\begin{array}{l}\text { obs: } 13027 \\
\text { no: } 12237 \\
\text { yes: } 790\end{array}$ \\
\hline \multicolumn{5}{|l|}{ Space-heating system: } \\
\hline $\begin{array}{l}\text { 41a. Flue gas analysis (steady-state } \\
\text { efficiency measurement) }\end{array}$ & $\begin{array}{l}\text { obs: } 12874 \\
\text { no: } 6778 \\
\text { yes: } 6096\end{array}$ & $\begin{array}{l}\text { obs: } 12874 \\
\text { no: } 10969 \\
\text { yes: } 1905\end{array}$ & $\begin{array}{l}\text { obs: } 12874 \\
\text { no: } 8260 \\
\text { yes: } 4614\end{array}$ & $\begin{array}{l}\text { obs: } 13027 \\
\text { no: } 6096 \\
\text { yes: } 6931\end{array}$ \\
\hline 41b. Heat rise & $\begin{array}{l}\text { obs: } 12827 \\
\text { no: } 9378 \\
\text { yes: } 3449\end{array}$ & $\begin{array}{l}\text { obs: } 12827 \\
\text { no: } 11169 \\
\text { yes: } 1658\end{array}$ & $\begin{array}{l}\text { obs: } 12827 \\
\text { no: } 9866 \\
\text { yes: } 2961\end{array}$ & $\begin{array}{l}\text { obs: } 13027 \\
\text { no: } 8399 \\
\text { yes: } 4628\end{array}$ \\
\hline 41c. CO level in flue & $\begin{array}{l}\text { obs: } 12861 \\
\text { no: } 5683 \\
\text { yes: } 7178\end{array}$ & $\begin{array}{l}\text { obs: } 12861 \\
\text { no: } 10577 \\
\text { yes: } 2284\end{array}$ & $\begin{array}{l}\text { obs: } 12861 \\
\text { no: } 7399 \\
\text { yes: } 5462\end{array}$ & $\begin{array}{l}\text { obs: } 13027 \\
\text { no: } 4871 \\
\text { yes: } 8156\end{array}$ \\
\hline 41d. CO level of equipment room & $\begin{array}{l}\text { obs: } 12852 \\
\text { no: } 8371 \\
\text { yes: } 4481\end{array}$ & $\begin{array}{l}\text { obs: } 12852 \\
\text { no: } 11636 \\
\text { yes: } 1216\end{array}$ & $\begin{array}{l}\text { obs: } 12852 \\
\text { no: } 9116 \\
\text { yes: } 3736\end{array}$ & $\begin{array}{l}\text { obs: } 13027 \\
\text { no: } 7976 \\
\text { yes: } 5051\end{array}$ \\
\hline 41e. Draft/spillage (normal operation) & $\begin{array}{l}\text { obs: } 12837 \\
\text { no: } 7083 \\
\text { yes: } 5754\end{array}$ & $\begin{array}{l}\text { obs: } 12837 \\
\text { no: } 11205 \\
\text { yes: } 1632\end{array}$ & $\begin{array}{l}\text { obs: } 12837 \\
\text { no: } 8165 \\
\text { yes: } 4672\end{array}$ & $\begin{array}{l}\text { obs: } 13027 \\
\text { no: } 6566 \\
\text { yes: } 6461\end{array}$ \\
\hline 41f. Worst case draft/spillage (CAZ) & $\begin{array}{l}\text { obs: } 12893 \\
\text { no: } 8114 \\
\text { yes: } 4779\end{array}$ & $\begin{array}{l}\text { obs: } 12893 \\
\text { no: } 10835 \\
\text { yes: } 2058\end{array}$ & $\begin{array}{l}\text { obs: } 12893 \\
\text { no: } 9119 \\
\text { yes: } 3774\end{array}$ & $\begin{array}{l}\text { obs: } 13027 \\
\text { no: } 7531 \\
\text { yes: } 5496\end{array}$ \\
\hline 41g. Safety inspection & $\begin{array}{l}\text { obs: } 12757 \\
\text { no: } 3781 \\
\text { yes: } 8976 \\
\end{array}$ & $\begin{array}{l}\text { obs: } 12757 \\
\text { no: } 9959 \\
\text { yes: } 2798\end{array}$ & $\begin{array}{l}\text { obs: } 12757 \\
\text { no: } 6367 \\
\text { yes: } 6390\end{array}$ & $\begin{array}{l}\text { obs: } 13027 \\
\text { no: } 3283 \\
\text { yes: } 9744\end{array}$ \\
\hline \multicolumn{5}{|l|}{ Air-conditioning system: } \\
\hline $\begin{array}{l}\text { 42a. Refrigerant charge (e.g., superheat or } \\
\text { subcooling) }\end{array}$ & $\begin{array}{l}\text { obs: } 13007 \\
\text { no: } 12782 \\
\text { yes: } 225\end{array}$ & $\begin{array}{l}\text { obs: } 13007 \\
\text { no: } 12886 \\
\text { yes: } 121\end{array}$ & $\begin{array}{l}\text { obs: } 13007 \\
\text { no: } 12851 \\
\text { yes: } 156\end{array}$ & $\begin{array}{l}\text { obs: } 13027 \\
\text { no: } 12731 \\
\text { yes: } 296\end{array}$ \\
\hline 42b. Safety inspection & $\begin{array}{l}\text { obs: } 12893 \\
\text { no: } 11553 \\
\text { yes: } 1340\end{array}$ & $\begin{array}{l}\text { obs: } 12893 \\
\text { no: } 12576 \\
\text { yes: } 317\end{array}$ & $\begin{array}{l}\text { obs: } 12893 \\
\text { no: } 12085 \\
\text { yes: } 808\end{array}$ & $\begin{array}{l}\text { obs: } 13027 \\
\text { no: } 11452 \\
\text { yes: } 1575\end{array}$ \\
\hline
\end{tabular}




\begin{tabular}{|c|c|c|c|c|}
\hline \multirow[t]{2}{*}{ Diagnostic measurement or inspection } & \multicolumn{3}{|c|}{ Diagnostic/inspection performed during: } & \multirow[b]{2}{*}{ Performed? } \\
\hline & $\begin{array}{c}\text { Audit/house } \\
\text { assessment }\end{array}$ & $\begin{array}{c}\text { Measure } \\
\text { installation }\end{array}$ & $\begin{array}{c}\text { Post- } \\
\text { inspection }\end{array}$ & \\
\hline \multicolumn{5}{|l|}{ HVAC components: } \\
\hline 43a. Air handler flow rate & $\begin{array}{l}\text { obs: } 12911 \\
\text { no: } 12371 \\
\text { yes: } 540\end{array}$ & $\begin{array}{l}\text { obs: } 12911 \\
\text { no: } 12415 \\
\text { yes: } 496\end{array}$ & $\begin{array}{l}\text { obs: } 12911 \\
\text { no: } 12506 \\
\text { yes: } 405\end{array}$ & $\begin{array}{l}\text { obs: } 13027 \\
\text { no: } 11933 \\
\text { yes: } 1094\end{array}$ \\
\hline 43b. Thermostat anticipator current & $\begin{array}{l}\text { obs: } 12883 \\
\text { no: } 11670 \\
\text { yes: } 1213\end{array}$ & $\begin{array}{l}\text { obs: } 12883 \\
\text { no: } 12449 \\
\text { yes: } 434\end{array}$ & $\begin{array}{l}\text { obs: } 12883 \\
\text { no: } 12383 \\
\text { yes: } 500\end{array}$ & $\begin{array}{l}\text { obs: } 13027 \\
\text { no: } 11393 \\
\text { yes: } 1634\end{array}$ \\
\hline \multicolumn{5}{|l|}{ Hot-water (water-heating) system: } \\
\hline $\begin{array}{l}\text { 44a. Flue gas analysis (steady-state } \\
\text { efficiency measurement) }\end{array}$ & $\begin{array}{l}\text { obs: } 12777 \\
\text { no: } 8859 \\
\text { yes: } 3918\end{array}$ & $\begin{array}{l}\text { obs: } 12777 \\
\text { no: } 11836 \\
\text { yes: } 941\end{array}$ & $\begin{array}{l}\text { obs: } 12777 \\
\text { no: } 9861 \\
\text { yes: } 2916\end{array}$ & $\begin{array}{l}\text { obs: } 13027 \\
\text { no: } 8604 \\
\text { yes: } 4423\end{array}$ \\
\hline 44b. CO level in flue & $\begin{array}{l}\text { obs: } 12776 \\
\text { no: } 6648 \\
\text { yes: } 6128\end{array}$ & $\begin{array}{l}\text { obs: } 12776 \\
\text { no: } 10878 \\
\text { yes: } 1898\end{array}$ & $\begin{array}{l}\text { obs: } 12776 \\
\text { no: } 8223 \\
\text { yes: } 4553\end{array}$ & $\begin{array}{l}\text { obs: } 13027 \\
\text { no: } 6087 \\
\text { yes: } 6940\end{array}$ \\
\hline 44c. CO level of equipment room & $\begin{array}{l}\text { obs: } 12884 \\
\text { no: } 9261 \\
\text { yes: } 3623\end{array}$ & $\begin{array}{l}\text { obs: } 12884 \\
\text { no: } 11893 \\
\text { yes: } 991\end{array}$ & $\begin{array}{l}\text { obs: } 12884 \\
\text { no: } 9926 \\
\text { yes: } 2958\end{array}$ & $\begin{array}{l}\text { obs: } 13027 \\
\text { no: } 8943 \\
\text { yes: } 4084\end{array}$ \\
\hline 44d. Draft/spillage (normal operation) & $\begin{array}{l}\text { obs: } 12830 \\
\text { no: } 7742 \\
\text { yes: } 5088\end{array}$ & $\begin{array}{l}\text { obs: } 12830 \\
\text { no: } 11286 \\
\text { yes: } 1544\end{array}$ & $\begin{array}{l}\text { obs: } 12830 \\
\text { no: } 8807 \\
\text { yes: } 4023\end{array}$ & $\begin{array}{l}\text { obs: } 13027 \\
\text { no: } 7369 \\
\text { yes: } 5658\end{array}$ \\
\hline 44e. Worst case draft/spillage (CAZ) & $\begin{array}{l}\text { obs: } 12942 \\
\text { no: } 9317 \\
\text { yes: } 3625\end{array}$ & $\begin{array}{l}\text { obs: } 12942 \\
\text { no: } 11343 \\
\text { yes: } 1599\end{array}$ & $\begin{array}{l}\text { obs: } 12942 \\
\text { no: } 10056 \\
\text { yes: } 2887\end{array}$ & $\begin{array}{l}\text { obs: } 13027 \\
\text { no: } 8942 \\
\text { yes: } 4085\end{array}$ \\
\hline 44f. Hot water temperature & $\begin{array}{l}\text { obs: } 12765 \\
\text { no: } 7625 \\
\text { yes: } 5140\end{array}$ & $\begin{array}{l}\text { obs: } 12765 \\
\text { no: } 11609 \\
\text { yes: } 1156\end{array}$ & $\begin{array}{l}\text { obs: } 12765 \\
\text { no: } 10213 \\
\text { yes: } 2552\end{array}$ & $\begin{array}{l}\text { obs: } 13027 \\
\text { no: } 7252 \\
\text { yes: } 5775\end{array}$ \\
\hline $44 \mathrm{~g}$. Shower head flow rate & $\begin{array}{l}\text { obs: } 12885 \\
\text { no: } 11088 \\
\text { yes: } 1797\end{array}$ & $\begin{array}{l}\text { obs: } 12885 \\
\text { no: } 12162 \\
\text { yes: } 723\end{array}$ & $\begin{array}{l}\text { obs: } 12885 \\
\text { no: } 12009 \\
\text { yes: } 876\end{array}$ & $\begin{array}{l}\text { obs: } 13027 \\
\text { no: } 10808 \\
\text { yes: } 2219\end{array}$ \\
\hline 44h. Faucet flow rate & $\begin{array}{l}\text { obs: } 12932 \\
\text { no: } 11669 \\
\text { yes: } 1263\end{array}$ & $\begin{array}{l}\text { obs: } 12932 \\
\text { no: } 12342 \\
\text { yes: } 590\end{array}$ & $\begin{array}{l}\text { obs: } 12932 \\
\text { no: } 12257 \\
\text { yes: } 675\end{array}$ & $\begin{array}{l}\text { obs: } 13027 \\
\text { no: } 11397 \\
\text { yes: } 1630\end{array}$ \\
\hline 44i. Safety inspection & $\begin{array}{l}\text { obs: } 12751 \\
\text { no: } 4088 \\
\text { yes: } 8663\end{array}$ & $\begin{array}{l}\text { obs: } 12751 \\
\text { no: } 10212 \\
\text { yes: } 2539\end{array}$ & $\begin{array}{l}\text { obs: } 12751 \\
\text { no: } 7018 \\
\text { yes: } 5733\end{array}$ & $\begin{array}{l}\text { obs: } 13027 \\
\text { no: } 3675 \\
\text { yes: } 9352\end{array}$ \\
\hline \multicolumn{5}{|l|}{ Other CO measurements: } \\
\hline 45a. Cook stove & $\begin{array}{l}\text { obs: } 12788 \\
\text { no: } 8381 \\
\text { yes: } 4407\end{array}$ & $\begin{array}{l}\text { obs: } 12788 \\
\text { no: } 12018 \\
\text { yes: } 770\end{array}$ & $\begin{array}{l}\text { obs: } 12788 \\
\text { no: } 10296 \\
\text { yes: } 2492\end{array}$ & $\begin{array}{l}\text { obs: } 13027 \\
\text { no: } 8180 \\
\text { yes: } 4847\end{array}$ \\
\hline 45b. Kitchen & $\begin{array}{l}\text { obs: } 12844 \\
\text { no: } 9488 \\
\text { yes: } 3356\end{array}$ & $\begin{array}{l}\text { obs: } 12844 \\
\text { no: } 12181 \\
\text { yes: } 663\end{array}$ & $\begin{array}{l}\text { obs: } 12844 \\
\text { no: } 10694 \\
\text { yes: } 2150\end{array}$ & $\begin{array}{l}\text { obs: } 13027 \\
\text { no: } 9281 \\
\text { yes: } 3746\end{array}$ \\
\hline 45c. Main living area & $\begin{array}{l}\text { obs: } 12839 \\
\text { no: } 9478 \\
\text { yes: } 3361\end{array}$ & $\begin{array}{l}\text { obs: } 12839 \\
\text { no: } 12036 \\
\text { yes: } 8083\end{array}$ & $\begin{array}{l}\text { obs: } 12839 \\
\text { no: } 10406 \\
\text { yes: } 2433\end{array}$ & $\begin{array}{l}\text { obs: } 13027 \\
\text { no: } 9204 \\
\text { yes: } 3823\end{array}$ \\
\hline \multicolumn{5}{|l|}{ Other diagnostics and inspections: } \\
\hline 46a. Refrigerator energy use & $\begin{array}{l}\text { obs: } 12787 \\
\text { no: } 8109 \\
\text { yes: } 4678\end{array}$ & $\begin{array}{l}\text { obs: } 12787 \\
\text { no: } 12235 \\
\text { yes } 552:\end{array}$ & $\begin{array}{l}\text { obs: } 12787 \\
\text { no: } 12007 \\
\text { yes: } 780\end{array}$ & $\begin{array}{l}\text { obs: } 13027 \\
\text { no: } 7964 \\
\text { yes: } 5063\end{array}$ \\
\hline 46b. Exhaust fan air flow rate & $\begin{array}{l}\text { obs: } 12946 \\
\text { no: } 11524 \\
\text { yes: } 1422\end{array}$ & $\begin{array}{l}\text { obs: } 12946 \\
\text { no: } 12339 \\
\text { yes: } 607\end{array}$ & $\begin{array}{l}\text { obs: } 12946 \\
\text { no: } 11976 \\
\text { yes: } 970\end{array}$ & $\begin{array}{l}\text { obs: } 13027 \\
\text { no: } 11300 \\
\text { yes: } 1727\end{array}$ \\
\hline
\end{tabular}




\begin{tabular}{|c|c|c|c|c|}
\hline \multirow[t]{2}{*}{ Diagnostic measurement or inspection } & \multicolumn{3}{|c|}{ Diagnostic/inspection performed during: } & \multirow[b]{2}{*}{ Performed? } \\
\hline & $\begin{array}{c}\text { Audit/house } \\
\text { assessment }\end{array}$ & $\begin{array}{c}\text { Measure } \\
\text { installation }\end{array}$ & $\begin{array}{c}\text { Post- } \\
\text { inspection }\end{array}$ & \\
\hline 46c. Infrared scanning (camera) & $\begin{array}{l}\text { obs: } 12854 \\
\text { no: } 10586 \\
\text { yes: } 2268\end{array}$ & $\begin{array}{l}\text { obs: } 12854 \\
\text { no: } 12422 \\
\text { yes: } 432\end{array}$ & $\begin{array}{l}\text { obs: } 12854 \\
\text { no: } 10950 \\
\text { yes: } 1904\end{array}$ & $\begin{array}{l}\text { obs: } 13027 \\
\text { no: } 10301 \\
\text { yes: } 2726\end{array}$ \\
\hline 46d. Radon testing & $\begin{array}{l}\text { obs: } 13016 \\
\text { no: } 12948 \\
\text { yes: } 68\end{array}$ & $\begin{array}{l}\text { obs: } 13016 \\
\text { no: } 13012 \\
\text { yes: } 4\end{array}$ & $\begin{array}{l}\text { obs: } 13016 \\
\text { no: } 12977 \\
\text { yes: } 39\end{array}$ & $\begin{array}{l}\text { obs: } 13027 \\
\text { no: } 12946 \\
\text { yes: } 81\end{array}$ \\
\hline 46e. Other (specify: & $\begin{array}{l}\text { obs: } 12865 \\
\text { no: } 11821 \\
\text { yes: } 1044\end{array}$ & $\begin{array}{l}\text { obs: } 12865 \\
\text { no: } 12338 \\
\text { yes: } 527\end{array}$ & $\begin{array}{l}\text { obs: } 12865 \\
\text { no: } 12276 \\
\text { yes: } 589\end{array}$ & $\begin{array}{l}\text { obs: } 13027 \\
\text { no: } 11787 \\
\text { yes: } 1240\end{array}$ \\
\hline 46f. Other (specify: & $\begin{array}{l}\text { obs: } 13016 \\
\text { no: } 12685 \\
\text { yes: } 331\end{array}$ & $\begin{array}{l}\text { obs: } 13016 \\
\text { no: } 13001 \\
\text { yes: } 15\end{array}$ & $\begin{array}{l}\text { obs: } 13016 \\
\text { no: } 12944 \\
\text { yes: } 72\end{array}$ & $\begin{array}{l}\text { obs: } 13027 \\
\text { no: } 12687 \\
\text { yes: } 340\end{array}$ \\
\hline 46g. Other (specify: __ & $\begin{array}{l}\text { obs: } 13021 \\
\text { no: } 12858 \\
\text { yes: } 163\end{array}$ & $\begin{array}{l}\text { obs: } 13021 \\
\text { no: } 13012 \\
\text { yes: } 9\end{array}$ & $\begin{array}{l}\text { obs: } 13021 \\
\text { no: } 12957 \\
\text { yes: } 64\end{array}$ & $\begin{array}{l}\text { obs: } 13027 \\
\text { no: } 12858 \\
\text { yes: } 169\end{array}$ \\
\hline
\end{tabular}


Record the diagnostic measurements taken on THIS housing unit: (fill in all that were taken)

\begin{tabular}{|c|c|c|}
\hline Diagnostic measurement & Pre-weatherization & Post weatherization \\
\hline \multicolumn{3}{|l|}{ House air leakage (blower door measurement): } \\
\hline 47a. Air leakage rate $(\mathrm{cfm})$ & $\begin{array}{l}\text { obs: } 11239 \\
\text { min: } 0 \\
\text { max: } 104000 \\
\text { mean: } 3750.85 \\
\text { median: } 3108\end{array}$ & $\begin{array}{l}\text { obs: } 10947 \\
\text { min: }-50 \\
\text { max: } 187450 \\
\text { mean: } 3138.80 \\
\text { median: } 2100\end{array}$ \\
\hline 47b. House WRT outside pressure difference $(\mathrm{Pa})$ & $\begin{array}{l}\text { obs: } 8520 \\
\text { min: }-50.80 \\
\text { max: } 50164 \\
\text { mean: } 137.60 \\
\text { median: } 50 \\
\end{array}$ & $\begin{array}{l}\text { obs: } 8263 \\
\text { min: }-60 \\
\text { max: } 50145 \\
\text { mean: } 87.30 \\
\text { median: } 50 \\
\end{array}$ \\
\hline \multicolumn{3}{|l|}{ Duct leakage (pressure pan measurements): } \\
\hline 48a. Sum of pressure pan readings $(\mathrm{Pa})$ & $\begin{array}{l}\text { obs: } 1792 \\
\text { min: }-378 \\
\text { max: } 10118 \\
\text { mean: } 57.34 \\
\text { median: } 14.3\end{array}$ & $\begin{array}{l}\text { obs: } 1660 \\
\text { min: }-119.2 \\
\text { max: } 22256 \\
\text { mean: } 34.31 \\
\text { median: } 4\end{array}$ \\
\hline 48b. Number of registers included in sum & $\begin{array}{l}\text { obs: } 1706 \\
\text { min: } 0 \\
\text { max: } 2900 \\
\text { mean: } 8.45 \\
\text { median: } 7\end{array}$ & $\begin{array}{l}\text { obs: } 1556 \\
\text { min: } 0 \\
\text { max: } 525 \\
\text { mean: } 7.52 \\
\text { median: } 7\end{array}$ \\
\hline 48c. House WRT outside pressure difference $(\mathrm{Pa})$ & $\begin{array}{l}\text { obs: } 1264 \\
\text { min: }-50 \\
\text { max: } 2390 \\
\text { mean: } 32.99 \\
\text { median: } 50 \\
\end{array}$ & $\begin{array}{l}\text { obs: } 1216 \\
\text { min: }-50 \\
\text { max: } 3200 \\
\text { mean: } 34.18 \\
\text { median: } 50 \\
\end{array}$ \\
\hline \multicolumn{3}{|l|}{ Duct leakage (duct blower measurements): } \\
\hline 49a. Total duct leakage rate $(\mathrm{cfm})$ & $\begin{array}{l}\text { obs: } 414 \\
\text { min: }-50.30 \\
\text { max: } 18134 \\
\text { mean: } 823.61 \\
\text { median: } 145 \\
\end{array}$ & $\begin{array}{l}\text { obs: } 388 \\
\text { min: }-1.7 \\
\text { max: } 7500 \\
\text { mean: } 552.26 \\
\text { median: } 140\end{array}$ \\
\hline 49b. Duct leakage to the outside (cfm) & $\begin{array}{l}\text { obs: } 217 \\
\text { min: } 0 \\
\text { max: } 3646 \\
\text { mean: } 156.81 \\
\text { median: } 0\end{array}$ & $\begin{array}{l}\text { obs: } 197 \\
\text { min: } 0 \\
\text { max: } 3200 \\
\text { mean: } 128.79 \\
\text { median: } 0\end{array}$ \\
\hline 49c. Duct WRT outside pressure difference $(\mathrm{Pa})$ & $\begin{array}{l}\text { obs: } 310 \\
\text { min: }-50 \\
\text { max: } 300 \\
\text { mean: } 15.70 \\
\text { median: } 0\end{array}$ & $\begin{array}{l}\text { obs: } 294 \\
\text { min: }-50 \\
\text { max: } 300 \\
\text { mean: } 17.04 \\
\text { median: } 0\end{array}$ \\
\hline \multicolumn{3}{|l|}{ Steady-state efficiency (flue gas analysis): } \\
\hline 50a. Primary space-heating system (\%) & $\begin{array}{l}\text { obs: } 5881 \\
\text { min: } 0 \\
\text { max: } 61777 \\
\text { mean: } 81.92 \\
\text { median: } 80 \\
\end{array}$ & $\begin{array}{l}\text { obs: } 6208 \\
\text { min: } 0 \\
\text { max: } 9036 \\
\text { mean: } 77.40 \\
\text { median: } 82 \\
\end{array}$ \\
\hline 50b. Secondary space-heating system (\%) & $\begin{array}{l}\text { obs: } 230 \\
\text { min: } 0 \\
\text { max: } 100 \\
\text { mean: } 48.77 \\
\text { median: } 65\end{array}$ & $\begin{array}{l}\text { obs: } 221 \\
\text { min: } 0 \\
\text { max: } 100 \\
\text { mean: } 49.39 \\
\text { median: } 65\end{array}$ \\
\hline
\end{tabular}




\begin{tabular}{|c|c|c|}
\hline 50c. Hot water heater (\%) & $\begin{array}{l}\text { obs: } 1604 \\
\text { min: }-.02 \\
\text { max: } 160 \\
\text { mean: } 78.10 \\
\text { median: } 79.30\end{array}$ & $\begin{array}{l}\text { obs: } 1553 \\
\text { min: -.02 } \\
\text { max: } 136 \\
\text { mean: } 79.05 \\
\text { median: } 80\end{array}$ \\
\hline
\end{tabular}

F-23 


\section{MEASURES INSTALLED}

If you know whether in-house crew or a contractor installed a given measure, please check the appropriate box in the first two response columns. If a measure was installed but you do not know whether it was installed by in-house crew or a contractor, please check the box in the "Installed?" column.

\begin{tabular}{|c|c|c|c|}
\hline \multirow[t]{2}{*}{ Measure } & \multicolumn{2}{|c|}{ Installed by } & \multirow[b]{2}{*}{ Installed? } \\
\hline & $\begin{array}{l}\text { In-house } \\
\text { crew }\end{array}$ & Contractor & \\
\hline \multicolumn{4}{|l|}{ Air sealing work: } \\
\hline $\begin{array}{l}\text { 51a. General house caulking and weatherstripping } \\
\text { (e.g., doors, windows) }\end{array}$ & $\begin{array}{l}\text { obs: } 12782 \\
\text { no: } 7631 \\
\text { yes: } 5151\end{array}$ & $\begin{array}{l}\text { obs: } 12782 \\
\text { no: } 8827 \\
\text { yes: } 3955\end{array}$ & $\begin{array}{l}\text { obs: } 13035 \\
\text { no: } 3777 \\
\text { yes: } 9258\end{array}$ \\
\hline $\begin{array}{l}\text { 51b. House air sealing emphasizing bypasses (leaks } \\
\text { identified by auditor and/or crew without using a blower door) }\end{array}$ & $\begin{array}{l}\text { obs: } 12887 \\
\text { no: } 10862 \\
\text { yes: } 2025\end{array}$ & $\begin{array}{l}\text { obs: } 12887 \\
\text { no: } 11388 \\
\text { yes: } 1499\end{array}$ & $\begin{array}{l}\text { obs: } 13035 \\
\text { no: } 9375 \\
\text { yes: } 3660\end{array}$ \\
\hline $\begin{array}{l}\text { 51c. House air sealing emphasizing bypasses (leaks } \\
\text { identified by auditor and/or crew with aid of a blower door) }\end{array}$ & $\begin{array}{l}\text { obs: } 12638 \\
\text { no: } 7892 \\
\text { yes: } 4746\end{array}$ & $\begin{array}{l}\text { obs: } 12638 \\
\text { no: } 9858 \\
\text { yes: } 2780\end{array}$ & $\begin{array}{l}\text { obs: } 13035 \\
\text { no: } 5316 \\
\text { yes: } 7719\end{array}$ \\
\hline 51d. Air distribution system (duct) sealing and repair ${ }^{36}$ & $\begin{array}{l}\text { obs: } 12779 \\
\text { no: } 10254 \\
\text { yes: } 2525\end{array}$ & $\begin{array}{l}\text { obs: } 12779 \\
\text { no: } 11372 \\
\text { yes: } 1407\end{array}$ & $\begin{array}{l}\text { obs: } 13035 \\
\text { no: } 8886 \\
\text { yes: } 4149\end{array}$ \\
\hline 51e. Other non-window air sealing work (specify: & $\begin{array}{l}\text { obs: } 12991 \\
\text { no: } 12135 \\
\text { yes: } 856\end{array}$ & $\begin{array}{l}\text { obs: } 12991 \\
\text { no: } 12594 \\
\text { yes: } 397\end{array}$ & $\begin{array}{l}\text { obs: } 13035 \\
\text { no: } 11745 \\
\text { yes: } 1288\end{array}$ \\
\hline 51f. Other non-window air sealing work (specify: & $\begin{array}{l}\text { obs: } 13015 \\
\text { no: } 12909 \\
\text { yes: } 106\end{array}$ & $\begin{array}{l}\text { obs: } 13015 \\
\text { no: } 12907 \\
\text { yes: } 108\end{array}$ & $\begin{array}{l}\text { obs: } 13035 \\
\text { no: } 12802 \\
\text { yes: } 233\end{array}$ \\
\hline \multicolumn{4}{|l|}{ Insulation: } \\
\hline $\begin{array}{l}\text { 52a. Attic insulation (installed where there was no } \\
\text { existing insulation) }\end{array}$ & $\begin{array}{l}\text { obs: } 12781 \\
\text { no: } 11852 \\
\text { yes: } 929\end{array}$ & $\begin{array}{l}\text { obs: } 12781 \\
\text { no: } 11113 \\
\text { yes: } 1668\end{array}$ & $\begin{array}{l}\text { obs: } 13035 \\
\text { no: } 10204 \\
\text { yes: } 2831\end{array}$ \\
\hline 52b. Attic insulation (added to existing insulation) & $\begin{array}{l}\text { obs: } 12717 \\
\text { no: } 10123 \\
\text { yes: } 2594\end{array}$ & $\begin{array}{l}\text { obs: } 12717 \\
\text { no: } 10591 \\
\text { yes: } 2126\end{array}$ & $\begin{array}{l}\text { obs: } 13035 \\
\text { no: } 8028 \\
\text { yes: } 5007\end{array}$ \\
\hline $\begin{array}{l}\text { 52c. Attic insulation (existing insulation level not } \\
\text { recorded) }\end{array}$ & $\begin{array}{l}\text { obs: } 12976 \\
\text { no: } 12636 \\
\text { yes: } 340\end{array}$ & $\begin{array}{l}\text { obs: } 12976 \\
\text { no: } 12460 \\
\text { yes: } 516\end{array}$ & $\begin{array}{l}\text { obs: } 13035 \\
\text { no: } 12107 \\
\text { yes: } 928\end{array}$ \\
\hline $\begin{array}{l}52 \mathrm{~d} \text {. Wall insulation (normal density-two-hole } \\
\text { gravity blow technique) }\end{array}$ & $\begin{array}{l}\text { obs: } 12862 \\
\text { no: } 12656 \\
\text { yes: } 206\end{array}$ & $\begin{array}{l}\text { obs: } 12862 \\
\text { no: } 12458 \\
\text { yes: } 404\end{array}$ & $\begin{array}{l}\text { obs: } 13035 \\
\text { no: } 12253 \\
\text { yes: } 782\end{array}$ \\
\hline $\begin{array}{l}\text { 52e. Wall insulation (high density-one-hole tube-fill } \\
\text { technique) }\end{array}$ & $\begin{array}{l}\text { obs: } 12798 \\
\text { no: } 11778 \\
\text { yes: } 1020\end{array}$ & $\begin{array}{l}\text { obs: } 12798 \\
\text { no: } 11776 \\
\text { yes: } 1022\end{array}$ & $\begin{array}{l}\text { obs: } 13035 \\
\text { no: } 10779 \\
\text { yes: } 2256\end{array}$ \\
\hline 52f. Floor insulation & $\begin{array}{l}\text { obs: } 12909 \\
\text { no: } 11611 \\
\text { yes: } 1298\end{array}$ & $\begin{array}{l}\text { obs: } 12909 \\
\text { no: } 11898 \\
\text { yes: } 1011\end{array}$ & $\begin{array}{l}\text { obs: } 13035 \\
\text { no: } 10597 \\
\text { yes: } 2438\end{array}$ \\
\hline 52g. Rim or band joist insulation ${ }^{37}$ & $\begin{array}{l}\text { obs: } 12895 \\
\text { no: } 11846 \\
\text { yes: } 1049\end{array}$ & $\begin{array}{l}\text { obs: } 12895 \\
\text { no: } 12098 \\
\text { yes: } 797\end{array}$ & $\begin{array}{l}\text { obs: } 13035 \\
\text { no: } 11070 \\
\text { yes: } 1965\end{array}$ \\
\hline 52h. Foundation wall insulation & $\begin{array}{l}\text { obs: } 12946 \\
\text { no: } 12486 \\
\text { yes: } 460\end{array}$ & $\begin{array}{l}\text { obs: } 12946 \\
\text { no: } 12526 \\
\text { yes: } 420\end{array}$ & $\begin{array}{l}\text { obs: } 13035 \\
\text { no: } 12097 \\
\text { yes: } 938\end{array}$ \\
\hline
\end{tabular}

\footnotetext{
${ }^{36}$ Check 51d (Air distribution system (duct) sealing and repair) if duct sealing OR duct repair was performed. Check $55 \mathrm{~g}$ (Other space-heating system modification (specify: )) if NEW ductwork was installed. Check 58c (Duct vents, grills, or registers) if new vents, grills or registers were installed.

${ }^{37}$ Check $52 \mathrm{~g}$ (rim or band joist insulation) if sill box insulation was installed.
} 


\begin{tabular}{|c|c|c|c|}
\hline \multirow[t]{2}{*}{ Measure } & \multicolumn{2}{|c|}{ Installed by } & \multirow[b]{2}{*}{ Installed? } \\
\hline & $\begin{array}{c}\text { In-house } \\
\text { crew }\end{array}$ & Contractor & \\
\hline 52i. Duct insulation & $\begin{array}{l}\text { obs: } 12981 \\
\text { no: } 12342 \\
\text { yes: } 639\end{array}$ & $\begin{array}{l}\text { obs: } 12981 \\
\text { no: } 12538 \\
\text { yes: } 443\end{array}$ & $\begin{array}{l}\text { obs: } 13035 \\
\text { no: } 11916 \\
\text { yes: } 1119\end{array}$ \\
\hline $52 \mathrm{j}$. White roof coat & $\begin{array}{l}\text { obs: } 13025 \\
\text { no: } 12851 \\
\text { yes: } 174\end{array}$ & $\begin{array}{l}\text { obs: } 13025 \\
\text { no: } 12944 \\
\text { yes: } 81\end{array}$ & $\begin{array}{l}\text { obs: } 13035 \\
\text { no: } 12770 \\
\text { yes: } 265\end{array}$ \\
\hline 52k. Skirting & $\begin{array}{l}\text { obs: } 13029 \\
\text { no: } 13009 \\
\text { yes: } 20\end{array}$ & $\begin{array}{l}\text { obs: } 13029 \\
\text { no: } 12978 \\
\text { yes: } 51\end{array}$ & $\begin{array}{l}\text { obs: } 13035 \\
\text { no: } 12958 \\
\text { yes: } 77\end{array}$ \\
\hline 521. Other insulation (specify: & $\begin{array}{l}\text { obs: } 12809 \\
\text { no: } 11759 \\
\text { yes: } 1050\end{array}$ & $\begin{array}{l}\text { obs: } 12809 \\
\text { no: } 11917 \\
\text { yes: } 892\end{array}$ & $\begin{array}{l}\text { obs: } 13035 \\
\text { no: } 10879 \\
\text { yes: } 2156\end{array}$ \\
\hline $52 \mathrm{~m}$. Other insulation (specify: & $\begin{array}{l}\text { obs: } 12977 \\
\text { no: } 12895 \\
\text { yes: } 82\end{array}$ & $\begin{array}{l}\text { obs: } 12977 \\
\text { no: } 12860 \\
\text { yes: } 117 \\
\end{array}$ & $\begin{array}{l}\text { obs: } 13035 \\
\text { no: } 12779 \\
\text { yes: } 256\end{array}$ \\
\hline \multicolumn{4}{|l|}{ Windows: } \\
\hline 53a. New window (justified because cost effective) & $\begin{array}{l}\text { obs: } 13003 \\
\text { no: } 12126 \\
\text { yes: } 877\end{array}$ & $\begin{array}{l}\text { obs: } 13003 \\
\text { no: } 11811 \\
\text { yes: } 1192\end{array}$ & $\begin{array}{l}\text { obs: } 13035 \\
\text { no: } 10945 \\
\text { yes: } 2090\end{array}$ \\
\hline $\begin{array}{l}\text { 53b. New window (justified for reason other than cost } \\
\text { effectiveness) }\end{array}$ & $\begin{array}{l}\text { obs: } 12992 \\
\text { no: } 12720 \\
\text { yes: } 272\end{array}$ & $\begin{array}{l}\text { obs: } 12992 \\
\text { no: } 12377 \\
\text { yes: } 615\end{array}$ & $\begin{array}{l}\text { obs: } 13035 \\
\text { no: } 12098 \\
\text { yes: } 937\end{array}$ \\
\hline $53 c$. Window glazings ${ }^{38}$ & $\begin{array}{l}\text { obs: } 12790 \\
\text { no: } 12239 \\
\text { yes: } 551\end{array}$ & $\begin{array}{l}\text { obs: } 12790 \\
\text { no: } 12267 \\
\text { yes: } 523\end{array}$ & $\begin{array}{l}\text { obs: } 13035 \\
\text { no: } 11773 \\
\text { yes: } 1262\end{array}$ \\
\hline 53d. New window screen & $\begin{array}{l}\text { obs: } 13029 \\
\text { no: } 13015 \\
\text { yes: } 14\end{array}$ & $\begin{array}{l}\text { obs: } 13029 \\
\text { no: } 12890 \\
\text { yes: } 129\end{array}$ & $\begin{array}{l}\text { obs: } 13035 \\
\text { no: } 12880 \\
\text { yes: } 155\end{array}$ \\
\hline 53e. Window lock replacement & $\begin{array}{l}\text { obs: } 13006 \\
\text { no: } 12794 \\
\text { yes: } 212\end{array}$ & $\begin{array}{l}\text { obs: } 13006 \\
\text { no: } 12778 \\
\text { yes: } 228\end{array}$ & $\begin{array}{l}\text { obs: } 13035 \\
\text { no: } 12565 \\
\text { yes: } 470\end{array}$ \\
\hline 53f. Window screen repair & $\begin{array}{l}\text { obs: } 13032 \\
\text { no: } 13029 \\
\text { yes: } 3\end{array}$ & $\begin{array}{l}\text { obs: } 13032 \\
\text { no: } 13004 \\
\text { yes: } 28\end{array}$ & $\begin{array}{l}\text { obs: } 13035 \\
\text { no: } 13003 \\
\text { yes: } 32\end{array}$ \\
\hline 53g. Other window repair (e.g., sashes, frames) & $\begin{array}{l}\text { obs: } 12979 \\
\text { no: } 12523 \\
\text { yes: } 456\end{array}$ & $\begin{array}{l}\text { obs: } 12979 \\
\text { no: } 12470 \\
\text { yes: } 509\end{array}$ & $\begin{array}{l}\text { obs: } 13035 \\
\text { no: } 12020 \\
\text { yes: } 1015\end{array}$ \\
\hline 53h. Storm window & $\begin{array}{l}\text { obs: } 13016 \\
\text { no: } 12671 \\
\text { yes: } 345\end{array}$ & $\begin{array}{l}\text { obs: } 13016 \\
\text { no: } 12705 \\
\text { yes: } 311\end{array}$ & $\begin{array}{l}\text { obs: } 13035 \\
\text { no: } 12365 \\
\text { yes: } 670\end{array}$ \\
\hline 53i. Window shading (e.g., awning, film, sun screen) & $\begin{array}{l}\text { obs: } 13030 \\
\text { no: } 12980 \\
\text { yes: } 50\end{array}$ & $\begin{array}{l}\text { obs: } 13030 \\
\text { no: } 12920 \\
\text { yes: } 110\end{array}$ & $\begin{array}{l}\text { obs: } 13035 \\
\text { no: } 12871 \\
\text { yes: } 164\end{array}$ \\
\hline 53j. Other window treatments (specify: & $\begin{array}{l}\text { obs: } 12950 \\
\text { no: } 12596 \\
\text { yes: } 354\end{array}$ & $\begin{array}{l}\text { obs: } 12950 \\
\text { no: } 12501 \\
\text { yes: } 449\end{array}$ & $\begin{array}{l}\text { obs: } 13035 \\
\text { no: } 12154 \\
\text { yes: } 881\end{array}$ \\
\hline $\begin{array}{l}53 \mathrm{k} . \text { Other window treatments (specify: } \\
\text { ) }\end{array}$ & $\begin{array}{l}\text { obs: } 13007 \\
\text { no: } 12960 \\
\text { yes: } 47\end{array}$ & $\begin{array}{l}\text { obs: } 13007 \\
\text { no: } 12945 \\
\text { yes: } 62\end{array}$ & $\begin{array}{l}\text { obs: } 13035 \\
\text { no: } 12900 \\
\text { yes: } 135\end{array}$ \\
\hline \multicolumn{4}{|l|}{ Doors: } \\
\hline 54a. New door (justified because cost effective) & $\begin{array}{l}\text { obs: } 13000 \\
\text { no: } 12138 \\
\text { yes: } 862\end{array}$ & $\begin{array}{l}\text { obs: } 13000 \\
\text { no: } 12160 \\
\text { yes: } 840\end{array}$ & $\begin{array}{l}\text { obs: } 13035 \\
\text { no: } 11322 \\
\text { yes: } 1713\end{array}$ \\
\hline
\end{tabular}

\footnotetext{
${ }^{38}$ Check 53c (window glazings) for window glass replacement in cases where the window sashes and frame were not replaced.
} 


\begin{tabular}{|c|c|c|c|}
\hline \multirow[t]{2}{*}{ Measure } & \multicolumn{2}{|c|}{ Installed by } & \multirow[b]{2}{*}{ Installed? } \\
\hline & $\begin{array}{c}\text { In-house } \\
\text { crew }\end{array}$ & Contractor & \\
\hline $\begin{array}{l}54 \mathrm{~b} \text {. New door (justified for reason other than cost } \\
\text { effectiveness) }\end{array}$ & $\begin{array}{l}\text { obs : } 12954 \\
\text { no: } 12504 \\
\text { yes: } 450\end{array}$ & $\begin{array}{l}\text { obs: } 12954 \\
\text { no: } 12385 \\
\text { yes: } 569\end{array}$ & $\begin{array}{l}\text { obs: } 13035 \\
\text { no: } 11952 \\
\text { yes: } 1083\end{array}$ \\
\hline 54c. Door lock (new or replacement) & $\begin{array}{l}\text { obs: } 12973 \\
\text { no: } 12211 \\
\text { yes: } 762\end{array}$ & $\begin{array}{l}\text { obs: } 12973 \\
\text { no: } 12367 \\
\text { yes: } 606\end{array}$ & $\begin{array}{l}\text { obs: } 13035 \\
\text { no: } 11619 \\
\text { yes: } 1416\end{array}$ \\
\hline $54 \mathrm{~d}$. Door or door framing repair & $\begin{array}{l}\text { obs: } 13001 \\
\text { no: } 12359 \\
\text { yes: } 642\end{array}$ & $\begin{array}{l}\text { obs: } 13001 \\
\text { no: } 12345 \\
\text { yes: } 656\end{array}$ & $\begin{array}{l}\text { obs: } 13035 \\
\text { no: } 11707 \\
\text { yes: } 1328\end{array}$ \\
\hline 54e. Storm door & $\begin{array}{l}\text { obs: } 13023 \\
\text { no: } 12991 \\
\text { yes: } 32\end{array}$ & $\begin{array}{l}\text { obs: } 13023 \\
\text { no: } 12961 \\
\text { yes: } 62\end{array}$ & $\begin{array}{l}\text { obs: } 13035 \\
\text { no: } 12934 \\
\text { yes: } 102\end{array}$ \\
\hline 54f. Other door treatments (specify: & $\begin{array}{l}\text { obs: } 12939 \\
\text { no: } 12175 \\
\text { yes: } 764\end{array}$ & $\begin{array}{l}\text { obs: } 12939 \\
\text { no: } 12175 \\
\text { yes: } 764\end{array}$ & $\begin{array}{l}\text { obs: } 13035 \\
\text { no: } 11408 \\
\text { yes: } 1627\end{array}$ \\
\hline 54g. Other door treatments (specify: & $\begin{array}{l}\text { obs: } 13019 \\
\text { no: } 12911 \\
\text { yes: } 108\end{array}$ & $\begin{array}{l}\text { obs: } 13019 \\
\text { no: } 12841 \\
\text { yes: } 178\end{array}$ & $\begin{array}{l}\text { obs: } 13035 \\
\text { no: } 12736 \\
\text { yes: } 299 \\
\end{array}$ \\
\hline \multicolumn{4}{|l|}{ Space-heating systems: } \\
\hline $\begin{array}{l}\text { 55a. New space-heating system (justified because cost } \\
\text { effective) }\end{array}$ & $\begin{array}{l}\text { obs: } 12845 \\
\text { no: } 12576 \\
\text { yes: } 269\end{array}$ & $\begin{array}{l}\text { obs: } 12845 \\
\text { no: } 11170 \\
\text { yes: } 1675\end{array}$ & $\begin{array}{l}\text { obs: } 13035 \\
\text { no: } 10925 \\
\text { yes: } 2110\end{array}$ \\
\hline $\begin{array}{l}55 \mathrm{~b} \text {. New space-heating system (justified for reason } \\
\text { other than cost effectiveness) }\end{array}$ & $\begin{array}{l}\text { obs: } 12955 \\
\text { no: } 12576 \\
\text { yes: } 379\end{array}$ & $\begin{array}{l}\text { obs: } 12955 \\
\text { no: } 11987 \\
\text { yes: } 968\end{array}$ & $\begin{array}{l}\text { obs: } 13035 \\
\text { no: } 11632 \\
\text { yes: } 1403\end{array}$ \\
\hline $\begin{array}{l}\text { 55c. Space-heating system repair (e.g., controls, safety } \\
\text { items, flues) }\end{array}$ & $\begin{array}{l}\text { obs: } 12966 \\
\text { no: } 12613 \\
\text { yes: } 353\end{array}$ & $\begin{array}{l}\text { obs: } 12966 \\
\text { no: } 11742 \\
\text { yes: } 1224\end{array}$ & $\begin{array}{l}\text { obs: } 13035 \\
\text { no: } 11408 \\
\text { yes: } 1627\end{array}$ \\
\hline 55d. Space-heating system tune-up & $\begin{array}{l}\text { obs: } 12941 \\
\text { no: } 12318 \\
\text { yes: } 623\end{array}$ & $\begin{array}{l}\text { obs: } 12941 \\
\text { no: } 11171 \\
\text { yes: } 1770\end{array}$ & $\begin{array}{l}\text { obs: } 13035 \\
\text { no: } 10562 \\
\text { yes: } 2473\end{array}$ \\
\hline 55e. Vent damper & $\begin{array}{l}\text { obs: } 13028 \\
\text { no: } 12987 \\
\text { yes: } 41\end{array}$ & $\begin{array}{l}\text { obs: } 13028 \\
\text { no: } 12985 \\
\text { yes: } 43\end{array}$ & $\begin{array}{l}\text { obs: } 13035 \\
\text { no: } 12946 \\
\text { yes: } 89\end{array}$ \\
\hline 55f. Intermittent ignition device & $\begin{array}{l}\text { obs: } 13032 \\
\text { no: } 13029 \\
\text { yes: } 2\end{array}$ & $\begin{array}{l}\text { obs: } 13032 \\
\text { no: } 13015 \\
\text { yes: } 17\end{array}$ & $\begin{array}{l}\text { obs: } 13035 \\
\text { no: } 13013 \\
\text { yes: } 22\end{array}$ \\
\hline $\begin{array}{l}\text { 55g. Other space-heating system modification } \\
\text { (specify: }\end{array}$ & $\begin{array}{l}\text { obs: } 12897 \\
\text { no: } 12658 \\
\text { yes: } 239\end{array}$ & $\begin{array}{l}\text { obs: } 12897 \\
\text { no: } 11764 \\
\text { yes: } 1133\end{array}$ & $\begin{array}{l}\text { obs: } 13035 \\
\text { no: } 11551 \\
\text { yes: } 1484\end{array}$ \\
\hline $\begin{array}{l}\text { 55h. Other space-heating system modification } \\
\text { (specify: }\end{array}$ & $\begin{array}{l}\text { obs: } 13024 \\
\text { no: } 12983 \\
\text { yes: } 41\end{array}$ & $\begin{array}{l}\text { obs: } 13024 \\
\text { no: } 12911 \\
\text { yes: } 113\end{array}$ & $\begin{array}{l}\text { obs: } 13035 \\
\text { no: } 12865 \\
\text { yes: } 170\end{array}$ \\
\hline \multicolumn{4}{|l|}{ Air-conditioning systems: } \\
\hline $\begin{array}{l}\text { 56a. New air conditioner (justified because cost } \\
\text { effective) }\end{array}$ & $\begin{array}{l}\text { obs: } 13031 \\
\text { no: } 12999 \\
\text { yes: } 32\end{array}$ & $\begin{array}{l}\text { obs: } 13031 \\
\text { no: } 12903 \\
\text { yes: } 128\end{array}$ & $\begin{array}{l}\text { obs: } 13035 \\
\text { no: } 12869 \\
\text { yes: } 166\end{array}$ \\
\hline $\begin{array}{l}\text { 56b. New air conditioner (justified for reason other } \\
\text { than cost effectiveness) }\end{array}$ & $\begin{array}{l}\text { obs: } 13034 \\
\text { no: } 13006 \\
\text { yes: } 28\end{array}$ & $\begin{array}{l}\text { obs: } 13034 \\
\text { no: } 12960 \\
\text { yes: } 74\end{array}$ & $\begin{array}{l}\text { obs: } 13035 \\
\text { no: } 12932 \\
\text { yes: } 103\end{array}$ \\
\hline 56c. Air conditioner repair & $\begin{array}{l}\text { obs: } 13031 \\
\text { no: } 13020 \\
\text { yes: } 11\end{array}$ & $\begin{array}{l}\text { obs: } 13031 \\
\text { no: } 12942 \\
\text { yes: } 89\end{array}$ & $\begin{array}{l}\text { obs: } 13035 \\
\text { no: } 12930 \\
\text { yes: } 105\end{array}$ \\
\hline
\end{tabular}

${ }^{39}$ Check 51d (Air distribution system (duct) sealing and repair) if duct sealing OR duct repair was performed. Check $55 \mathrm{~g}$ (Other space-heating system modification (specify: )) if NEW ductwork was installed. Check 58c

(Duct vents, grills, or registers) if new vents, grills or registers were installed. 


\begin{tabular}{|c|c|c|c|}
\hline \multirow[t]{2}{*}{ Measure } & \multicolumn{2}{|c|}{ Installed by } & \multirow[b]{2}{*}{ Installed? } \\
\hline & $\begin{array}{c}\text { In-house } \\
\text { crew }\end{array}$ & Contractor & \\
\hline 56d. Air conditioner recharge/tune-up & $\begin{array}{l}\text { obs: } 13030 \\
\text { no: } 12990 \\
\text { yes: } 40\end{array}$ & $\begin{array}{l}\text { obs: } 13030 \\
\text { no: } 12844 \\
\text { yes: } 186\end{array}$ & $\begin{array}{l}\text { obs: } 13035 \\
\text { no: } 12804 \\
\text { yes: } 231\end{array}$ \\
\hline 56e. Ceiling or whole-house fans & $\begin{array}{l}\text { obs: } 13032 \\
\text { no: } 13018 \\
\text { yes: } 14\end{array}$ & $\begin{array}{l}\text { obs: } 13032 \\
\text { no: } 13002 \\
\text { yes: } 30\end{array}$ & $\begin{array}{l}\text { obs: } 13035 \\
\text { no: } 12989 \\
\text { yes: } 46\end{array}$ \\
\hline $\begin{array}{l}\text { 56f. Other air-conditioning system modification } \\
\text { (specify: }\end{array}$ & $\begin{array}{l}\text { obs: } 13013 \\
\text { no: } 12921 \\
\text { yes: } 92\end{array}$ & $\begin{array}{l}\text { obs: } 13013 \\
\text { no: } 12876 \\
\text { yes: } 137\end{array}$ & $\begin{array}{l}\text { obs: } 13035 \\
\text { no: } 12788 \\
\text { yes: } 247\end{array}$ \\
\hline $\begin{array}{l}\text { 56g. Other air-conditioning system modification } \\
\text { (specify: }\end{array}$ & $\begin{array}{l}\text { obs: } 13027 \\
\text { no: } 13022 \\
\text { yes: } 5\end{array}$ & $\begin{array}{l}\text { obs: } 13027 \\
\text { no: } 13022 \\
\text { yes:5 }\end{array}$ & $\begin{array}{l}\text { obs: } 13035 \\
\text { no: } 13016 \\
\text { yes: } 19\end{array}$ \\
\hline \multicolumn{4}{|l|}{ Ventilation: } \\
\hline 57a. Exhaust fan in bathroom (new) & $\begin{array}{l}\text { obs: } 12939 \\
\text { no: } 12607 \\
\text { yes: } 332\end{array}$ & $\begin{array}{l}\text { obs: } 12939 \\
\text { no: } 11685 \\
\text { yes: } 1254\end{array}$ & $\begin{array}{l}\text { obs: } 13035 \\
\text { no: } 11410 \\
\text { yes: } 1625\end{array}$ \\
\hline 57b. Exhaust fan in kitchen (new) & $\begin{array}{l}\text { obs: } 13003 \\
\text { no: } 12755 \\
\text { yes: } 248\end{array}$ & $\begin{array}{l}\text { obs: } 13003 \\
\text { no: } 12440 \\
\text { yes: } 563\end{array}$ & $\begin{array}{l}\text { obs: } 13035 \\
\text { no: } 12192 \\
\text { yes: } 843\end{array}$ \\
\hline 57c. Whole-house ventilation system & $\begin{array}{l}\text { obs: } 13033 \\
\text { no: } 13025 \\
\text { yes: } 8\end{array}$ & $\begin{array}{l}\text { obs: } 13033 \\
\text { no: } 12963 \\
\text { yes: } 70\end{array}$ & $\begin{array}{l}\text { obs: } 13035 \\
\text { no: } 12954 \\
\text { yes: } 81\end{array}$ \\
\hline $\begin{array}{l}\text { 57d. Other ventilation system improvements (specify: } \\
\text { ) }^{40}\end{array}$ & $\begin{array}{l}\text { obs: } 13007 \\
\text { no: } 12304 \\
\text { yes: } 703\end{array}$ & $\begin{array}{l}\text { obs: } 13007 \\
\text { no: } 12083 \\
\text { yes: } 924\end{array}$ & $\begin{array}{l}\text { obs: } 13035 \\
\text { no: } 11397 \\
\text { yes: } 1638\end{array}$ \\
\hline $\begin{array}{l}\text { 57e. Other ventilation system improvements (specify: } \\
\text { ) }\end{array}$ & $\begin{array}{l}\text { obs: } 13026 \\
\text { no: } 12878 \\
\text { yes: } 148\end{array}$ & $\begin{array}{l}\text { obs: } 13026 \\
\text { no: } 12860 \\
\text { yes: } 166\end{array}$ & $\begin{array}{l}\text { obs: } 13035 \\
\text { no: } 12715 \\
\text { yes: } 320\end{array}$ \\
\hline \multicolumn{4}{|l|}{ HVAC accessories: } \\
\hline 58a. New programmable (setback) thermostat & $\begin{array}{l}\text { obs: } 12860 \\
\text { no: } 12619 \\
\text { yes: } 241\end{array}$ & $\begin{array}{l}\text { obs: } 12860 \\
\text { no: } 11950 \\
\text { yes: } 910\end{array}$ & $\begin{array}{l}\text { obs: } 13035 \\
\text { no: } 11734 \\
\text { yes: } 1301\end{array}$ \\
\hline 58b. New standard thermostat & $\begin{array}{l}\text { obs: } 12987 \\
\text { no: } 12672 \\
\text { yes: } 315\end{array}$ & $\begin{array}{l}\text { obs: } 12987 \\
\text { no: } 12256 \\
\text { yes: } 731\end{array}$ & $\begin{array}{l}\text { obs: } 13035 \\
\text { no: } 11946 \\
\text { yes: } 1089\end{array}$ \\
\hline 58c. Duct vents, grills, or registers ${ }^{41}$ & $\begin{array}{l}\text { obs: } 13018 \\
\text { no: } 12167 \\
\text { yes: } 851\end{array}$ & $\begin{array}{l}\text { obs: } 13018 \\
\text { no: } 12488 \\
\text { yes: } 530\end{array}$ & $\begin{array}{l}\text { obs: } 13035 \\
\text { no: } 11642 \\
\text { yes: } 1393\end{array}$ \\
\hline 58d. Standard air filter & $\begin{array}{l}\text { obs: } 12975 \\
\text { no: } 11685 \\
\text { yes: } 1290\end{array}$ & $\begin{array}{l}\text { obs: } 12975 \\
\text { no: } 11088 \\
\text { yes: } 1887\end{array}$ & $\begin{array}{l}\text { obs: } 13035 \\
\text { no: } 9821 \\
\text { yes: } 3214\end{array}$ \\
\hline 58e. High efficiency particulate arresting (HEPA) air & $\begin{array}{l}\text { obs: } 13028 \\
\text { no: } 12998 \\
\text { yes: } 30\end{array}$ & $\begin{array}{l}\text { obs: } 13028 \\
\text { no: } 13012 \\
\text { yes: } 16\end{array}$ & $\begin{array}{l}\text { obs: } 13035 \\
\text { no: } 12981 \\
\text { yes: } 54\end{array}$ \\
\hline $\begin{array}{l}\text { 58f. Other HVAC accessories (specify: } \\
\text { ) }\end{array}$ & $\begin{array}{l}\text { obs: } 13007 \\
\text { no: } 12833 \\
\text { yes: } 174\end{array}$ & $\begin{array}{l}\text { obs: } 13007 \\
\text { no: } 12654 \\
\text { yes: } 353\end{array}$ & $\begin{array}{l}\text { obs: } 13035 \\
\text { no: } 12484 \\
\text { yes: } 551\end{array}$ \\
\hline $\begin{array}{l}\text { 58g. Other HVAC accessories (specify: } \\
\text { ) }\end{array}$ & $\begin{array}{l}\text { obs: } 13012 \\
\text { no: } 12995 \\
\text { yes: } 17\end{array}$ & $\begin{array}{l}\text { obs: } 13012 \\
\text { no: } 12956 \\
\text { yes: } 54\end{array}$ & $\begin{array}{l}\text { obs: } 13035 \\
\text { no: } 12941 \\
\text { yes: } 94\end{array}$ \\
\hline
\end{tabular}

${ }^{40}$ Check 57d (Other ventilation system improvements (specify: ) if repairs were made to existing bathroom/ kitchen fans.

${ }^{41}$ Check 51d (Air distribution system (duct) sealing and repair) if duct sealing OR duct repair was performed. Check $55 \mathrm{~g}$ (Other space-heating system modification (specify: )) if NEW ductwork was installed. Check 58c

(Duct vents, grills, or registers) if new vents, grills or registers were installed. 


\begin{tabular}{|c|c|c|c|}
\hline \multirow[t]{2}{*}{ Measure } & \multicolumn{2}{|c|}{ Installed by } & \multirow[b]{2}{*}{ Installed? } \\
\hline & $\begin{array}{l}\text { In-house } \\
\text { crew }\end{array}$ & Contractor & \\
\hline \multicolumn{4}{|l|}{ Water-heating system: } \\
\hline $\begin{array}{l}\text { 59a. New water heater (justified because cost } \\
\text { effective) }\end{array}$ & $\begin{array}{l}\text { obs: } 12999 \\
\text { no: } 12922 \\
\text { yes: } 77\end{array}$ & $\begin{array}{l}\text { obs: } 12999 \\
\text { no: } 12467 \\
\text { yes: } 532\end{array}$ & $\begin{array}{l}\text { obs: } 13035 \\
\text { no: } 12397 \\
\text { yes: } 638\end{array}$ \\
\hline $\begin{array}{l}59 \mathrm{~b} . \text { New water heater (justified for reason other than } \\
\text { cost effectiveness) }\end{array}$ & $\begin{array}{l}\text { obs: } 12938 \\
\text { no: } 12831 \\
\text { yes: } 107\end{array}$ & $\begin{array}{l}\text { obs: } 12938 \\
\text { no: } 12305 \\
\text { yes: } 633\end{array}$ & $\begin{array}{l}\text { obs: } 13035 \\
\text { no: } 12209 \\
\text { yes: } 826\end{array}$ \\
\hline 59c. Water-heating system repair & $\begin{array}{l}\text { obs: } 12937 \\
\text { no: } 12662 \\
\text { yes: } 275\end{array}$ & $\begin{array}{l}\text { obs: } 12937 \\
\text { no: } 12236 \\
\text { yes: } 701\end{array}$ & $\begin{array}{l}\text { obs: } 13035 \\
\text { no: } 11971 \\
\text { yes: } 1064\end{array}$ \\
\hline 59d. Water-heater tank insulation wrap & $\begin{array}{l}\text { obs: } 12830 \\
\text { no: } 11033 \\
\text { yes: } 1797\end{array}$ & $\begin{array}{l}\text { obs: } 12830 \\
\text { no: } 11887 \\
\text { yes: } 943\end{array}$ & $\begin{array}{l}\text { obs: } 13035 \\
\text { no: } 10099 \\
\text { yes: } 2936\end{array}$ \\
\hline 59e. Pipe insulation & $\begin{array}{l}\text { obs: } 12726 \\
\text { no: } 9743 \\
\text { yes: } 2983\end{array}$ & $\begin{array}{l}\text { obs: } 12726 \\
\text { no: } 10746 \\
\text { yes: } 1980\end{array}$ & $\begin{array}{l}\text { obs: } 13035 \\
\text { no: } 7796 \\
\text { yes: } 5239\end{array}$ \\
\hline 59f. Low-flow showerhead & $\begin{array}{l}\text { obs: } 12857 \\
\text { no: } 10256 \\
\text { yes: } 2601\end{array}$ & $\begin{array}{l}\text { obs: } 12857 \\
\text { no: } 11963 \\
\text { yes: } 894\end{array}$ & $\begin{array}{l}\text { obs: } 13035 \\
\text { no: } 9376 \\
\text { yes: } 3659\end{array}$ \\
\hline $59 \mathrm{~g}$. Faucet aerators ${ }^{42}$ & $\begin{array}{l}\text { obs: } 12843 \\
\text { no: } 10514 \\
\text { yes: } 2329\end{array}$ & $\begin{array}{l}\text { obs: } 12843 \\
\text { no: } 12162 \\
\text { yes: } 681\end{array}$ & $\begin{array}{l}\text { obs: } 13035 \\
\text { no: } 9857 \\
\text { yes: } 3178\end{array}$ \\
\hline 59h. Water heater temperature reduction & $\begin{array}{l}\text { obs: } 12966 \\
\text { no: } 12080 \\
\text { yes: } 886\end{array}$ & $\begin{array}{l}\text { obs: } 12966 \\
\text { no: } 12589 \\
\text { yes: } 377\end{array}$ & $\begin{array}{l}\text { obs: } 13035 \\
\text { no: } 11711 \\
\text { yes: } 1324\end{array}$ \\
\hline 59i. Other water heating system measure (specify: & $\begin{array}{l}\text { obs: } 12988 \\
\text { no: } 12703 \\
\text { yes: } 285\end{array}$ & $\begin{array}{l}\text { obs: } 12988 \\
\text { no: } 12559 \\
\text { yes: } 429\end{array}$ & $\begin{array}{l}\text { obs: } 13035 \\
\text { no: } 12285 \\
\text { yes: } 750\end{array}$ \\
\hline 59j. Other water heating system measure (specify: & $\begin{array}{l}\text { obs: } 13029 \\
\text { no: } 12998 \\
\text { yes: } 31\end{array}$ & $\begin{array}{l}\text { obs: } 13029 \\
\text { no: } 12961 \\
\text { yes: } 68\end{array}$ & $\begin{array}{l}\text { obs: } 13035 \\
\text { no: } 12935 \\
\text { yes: } 100\end{array}$ \\
\hline \multicolumn{4}{|l|}{ Other baseloads: } \\
\hline 60a. Indoor lighting ${ }^{43}$ & $\begin{array}{l}\text { obs: } 12587 \\
\text { no: } 7031 \\
\text { yes: } 5556\end{array}$ & $\begin{array}{l}\text { obs: } 12587 \\
\text { no: } 10316 \\
\text { yes: } 2271\end{array}$ & $\begin{array}{l}\text { obs: } 13035 \\
\text { no: } 5152 \\
\text { yes: } 7883\end{array}$ \\
\hline 60b. Outdoor lighting ${ }^{44}$ & $\begin{array}{l}\text { obs: } 13011 \\
\text { no: } 12484 \\
\text { yes: } 527\end{array}$ & $\begin{array}{l}\text { obs: } 13011 \\
\text { no: } 12618 \\
\text { yes: } 393\end{array}$ & $\begin{array}{l}\text { obs: } 13035 \\
\text { no: } 12087 \\
\text { yes: } 948\end{array}$ \\
\hline 60c. Lighting (indoor/outdoor location not recorded) & $\begin{array}{l}\text { obs: } 12939 \\
\text { no: } 12449 \\
\text { yes: } 490\end{array}$ & $\begin{array}{l}\text { obs: } 12939 \\
\text { no: } 12453 \\
\text { yes: } 486\end{array}$ & $\begin{array}{l}\text { obs: } 13035 \\
\text { no: } 12000 \\
\text { yes: } 1035\end{array}$ \\
\hline 60d. Refrigerator (justified because cost effective) & $\begin{array}{l}\text { obs: } 12907 \\
\text { no: } 12389 \\
\text { yes: } 518\end{array}$ & $\begin{array}{l}\text { obs: } 12907 \\
\text { no: } 11278 \\
\text { yes: } 1629\end{array}$ & $\begin{array}{l}\text { obs: } 13035 \\
\text { no: } 10769 \\
\text { yes: } 2266\end{array}$ \\
\hline $\begin{array}{l}\text { 60e. Refrigerator (justified for reason other than cost } \\
\text { effectiveness) }\end{array}$ & $\begin{array}{l}\text { obs: } 13010 \\
\text { no: } 12975 \\
\text { yes: } 35\end{array}$ & $\begin{array}{l}\text { obs: } 13010 \\
\text { no: } 12894 \\
\text { yes: } 116\end{array}$ & $\begin{array}{l}\text { obs: } 13035 \\
\text { no: } 12866 \\
\text { yes: } 169\end{array}$ \\
\hline $\begin{array}{l}\text { 60f. Other baseload measure (specify: } \\
\text { ) }\end{array}$ & $\begin{array}{l}\text { obs: } 12971 \\
\text { no: } 12690 \\
\text { yes: } 281\end{array}$ & $\begin{array}{l}\text { obs: } 12971 \\
\text { no: } 12744 \\
\text { yes: } 227\end{array}$ & $\begin{array}{l}\text { obs: } 13035 \\
\text { no: } 12475 \\
\text { yes: } 560\end{array}$ \\
\hline 60g. Other baseload measure (specify: & $\begin{array}{l}\text { obs: } 13031 \\
\text { no: } 13013 \\
\text { yes: } 18\end{array}$ & $\begin{array}{l}\text { obs: } 13031 \\
\text { no: } 12987 \\
\text { yes: } 44\end{array}$ & $\begin{array}{l}\text { obs: } 13035 \\
\text { no: } 12968 \\
\text { yes: } 67\end{array}$ \\
\hline
\end{tabular}

${ }^{42}$ Check 59g (faucet aerators) for low-flow devices installed on faucets.

${ }^{43}$ Check 60a (indoor lighting) for CFL bulbs or high-efficiency light fixtures installed inside the home.

${ }^{44}$ Check 60b (outdoor lighting) for CFL bulbs or high-efficiency light fixtures installed outside the home. 


\begin{tabular}{|c|c|c|c|}
\hline \multirow[t]{2}{*}{ Measure } & \multicolumn{2}{|c|}{ Installed by } & \multirow[b]{2}{*}{ Installed? } \\
\hline & $\begin{array}{c}\text { In-house } \\
\text { crew }\end{array}$ & Contractor & \\
\hline \multicolumn{4}{|l|}{ Client education: } \\
\hline 61a. Literature (e.g., brochures, booklets, manuals) & $\begin{array}{l}\text { obs: } 12766 \\
\text { no: } 4291 \\
\text { yes: } 8475\end{array}$ & $\begin{array}{l}\text { obs: } 12766 \\
\text { no: } 11312 \\
\text { yes: } 1454\end{array}$ & $\begin{array}{l}\text { obs: } 13035 \\
\text { no: } 3517 \\
\text { yes: } 9518\end{array}$ \\
\hline 61b. Videos, DVDs, or compact disks (CDs) & $\begin{array}{l}\text { obs: } 13023 \\
\text { no: } 12827 \\
\text { yes: } 196\end{array}$ & $\begin{array}{l}\text { obs: } 13023 \\
\text { no: } 12980 \\
\text { yes: } 43\end{array}$ & $\begin{array}{l}\text { obs: } 13035 \\
\text { no: } 12788 \\
\text { yes: } 247\end{array}$ \\
\hline $\begin{array}{l}\text { 61c. Hardware kit and/or kit of weatherization } \\
\text { materials }\end{array}$ & $\begin{array}{l}\text { obs: } 12940 \\
\text { no: } 12203 \\
\text { yes: } 737\end{array}$ & $\begin{array}{l}\text { obs: } 12940 \\
\text { no: } 12826 \\
\text { yes: } 114\end{array}$ & $\begin{array}{l}\text { obs: } 13035 \\
\text { no: } 12122 \\
\text { yes: } 913\end{array}$ \\
\hline $\begin{array}{l}\text { 61d. In-home education/discussion }{ }^{45} \text { conducted, but } \\
\text { time not recorded }\end{array}$ & $\begin{array}{l}\text { obs: } 12805 \\
\text { no: } 7120 \\
\text { yes: } 5685\end{array}$ & $\begin{array}{l}\text { obs: } 12805 \\
\text { no: } 12074 \\
\text { yes: } 731\end{array}$ & $\begin{array}{l}\text { obs: } 13035 \\
\text { no: } 6717 \\
\text { yes: } 6318\end{array}$ \\
\hline $\begin{array}{l}\text { 61e. }<15 \text { minute total in-home education/discussion } \\
\text { time }\end{array}$ & $\begin{array}{l}\text { obs: } 12942 \\
\text { no: } 11954 \\
\text { yes: } 988\end{array}$ & $\begin{array}{l}\text { obs: } 12942 \\
\text { no: } 12841 \\
\text { yes: } 101\end{array}$ & $\begin{array}{l}\text { obs: } 13035 \\
\text { no: } 11897 \\
\text { yes: } 1138\end{array}$ \\
\hline $\begin{array}{l}\text { time } \\
\text { tif } 15-29 \text { minute total in-home education/discussion }\end{array}$ & $\begin{array}{l}\text { obs: } 12952 \\
\text { no: } 11051 \\
\text { yes: } 1901\end{array}$ & $\begin{array}{l}\text { obs: } 12952 \\
\text { no: } 12540 \\
\text { yes: } 412\end{array}$ & $\begin{array}{l}\text { obs: } 13035 \\
\text { no: } 10835 \\
\text { yes: } 2200\end{array}$ \\
\hline $\begin{array}{l}\text { 61g. } 30 \text { minutes or more total in-home } \\
\text { education/discussion time }\end{array}$ & $\begin{array}{l}\text { obs: } 13003 \\
\text { no: } 12010 \\
\text { yes: } 993\end{array}$ & $\begin{array}{l}\text { obs: } 13003 \\
\text { no: } 12921 \\
\text { yes: } 82\end{array}$ & $\begin{array}{l}\text { obs: } 13035 \\
\text { no: } 11989 \\
\text { yes: } 1046\end{array}$ \\
\hline 61h. Classroom training & $\begin{array}{l}\text { obs: } 13027 \\
\text { no: } 12844 \\
\text { yes: } 183\end{array}$ & $\begin{array}{l}\text { obs: } 13027 \\
\text { no: } 12972 \\
\text { yes: } 55\end{array}$ & $\begin{array}{l}\text { obs: } 13035 \\
\text { no: } 12789 \\
\text { yes: } 246\end{array}$ \\
\hline 61i. Other client education approach (specify: & $\begin{array}{l}\text { obs: } 12849 \\
\text { no: } 12339 \\
\text { yes: } 510\end{array}$ & $\begin{array}{l}\text { obs: } 12849 \\
\text { no: } 12646 \\
\text { yes: } 203 \\
\end{array}$ & $\begin{array}{l}\text { obs: } 13035 \\
\text { no: } 12195 \\
\text { yes: } 840\end{array}$ \\
\hline 61j. Other client education approach (specify: & $\begin{array}{l}\text { obs: } 12919 \\
\text { no: } 12914 \\
\text { yes: } 5\end{array}$ & $\begin{array}{l}\text { obs: } 12919 \\
\text { no: } 12874 \\
\text { yes: } 45\end{array}$ & $\begin{array}{l}\text { obs: } 13035 \\
\text { no: } 12884 \\
\text { yes: } 151\end{array}$ \\
\hline \multicolumn{4}{|l|}{ Other health, safety, and repair: } \\
\hline 62a. Smoke alarm & $\begin{array}{l}\text { obs: } 12848 \\
\text { no: } 9721 \\
\text { yes: } 3127\end{array}$ & $\begin{array}{l}\text { obs: } 12848 \\
\text { no: } 10656 \\
\text { yes: } 2192\end{array}$ & $\begin{array}{l}\text { obs: } 13035 \\
\text { no: } 7568 \\
\text { yes: } 5467\end{array}$ \\
\hline 62b. CO monitor & $\begin{array}{l}\text { obs: } 12741 \\
\text { no: } 8331 \\
\text { yes: } 4410\end{array}$ & $\begin{array}{l}\text { obs: } 12741 \\
\text { no: } 10465 \\
\text { yes: } 2276\end{array}$ & $\begin{array}{l}\text { obs: } 13035 \\
\text { no: } 6095 \\
\text { yes: } 6940\end{array}$ \\
\hline 62c. Attic ventilation & $\begin{array}{l}\text { obs: } 12926 \\
\text { no: } 12096 \\
\text { yes: } 830\end{array}$ & $\begin{array}{l}\text { obs: } 12926 \\
\text { no: } 12227 \\
\text { yes: } 699\end{array}$ & $\begin{array}{l}\text { obs: } 13035 \\
\text { no: } 11411 \\
\text { yes: } 1624\end{array}$ \\
\hline $62 \mathrm{~d}$. Roof repair & $\begin{array}{l}\text { obs: } 12986 \\
\text { no: } 12749 \\
\text { yes: } 237\end{array}$ & $\begin{array}{l}\text { obs: } 12986 \\
\text { no: } 12645 \\
\text { yes: } 341\end{array}$ & $\begin{array}{l}\text { obs: } 13035 \\
\text { no: } 12419 \\
\text { yes: } 616\end{array}$ \\
\hline 62e. Ceiling repair & $\begin{array}{l}\text { obs: } 13013 \\
\text { no: } 12765 \\
\text { yes: } 248\end{array}$ & $\begin{array}{l}\text { obs: } 13013 \\
\text { no: } 12660 \\
\text { yes: } 353\end{array}$ & $\begin{array}{l}\text { obs: } 13035 \\
\text { no: } 12415 \\
\text { yes: } 620\end{array}$ \\
\hline 62f. Wall repair & $\begin{array}{l}\text { obs: } 13000 \\
\text { no: } 12594 \\
\text { yes: } 406\end{array}$ & $\begin{array}{l}\text { obs: } 13000 \\
\text { no: } 12628 \\
\text { yes: } 372\end{array}$ & $\begin{array}{l}\text { obs: } 13035 \\
\text { no: } 12228 \\
\text { yes: } 807\end{array}$ \\
\hline
\end{tabular}

\footnotetext{
${ }^{45}$ In-home education/discussion includes time spent talking directly to the WAP client about weatherization and/or energy use issues. It does not include time the WAP client spent observing inspection or weatherization activities being performed.
} 


\begin{tabular}{|c|c|c|c|}
\hline \multirow[t]{2}{*}{ Measure } & \multicolumn{2}{|c|}{ Installed by } & \multirow[b]{2}{*}{ Installed? } \\
\hline & $\begin{array}{c}\text { In-house } \\
\text { crew }\end{array}$ & Contractor & \\
\hline $62 \mathrm{~g}$. Floor repair & $\begin{array}{l}\text { obs: } 13012 \\
\text { no: } 12666 \\
\text { yes: } 346\end{array}$ & $\begin{array}{l}\text { obs: } 13012 \\
\text { no: } 12783 \\
\text { yes: } 229\end{array}$ & $\begin{array}{l}\text { obs: } 13035 \\
\text { no: } 12438 \\
\text { yes: } 597\end{array}$ \\
\hline $62 \mathrm{~h}$. Foundation repair & $\begin{array}{l}\text { obs: } 13029 \\
\text { no: } 12951 \\
\text { yes: } 78\end{array}$ & $\begin{array}{l}\text { obs: } 13029 \\
\text { no: } 12960 \\
\text { yes: } 69\end{array}$ & $\begin{array}{l}\text { obs: } 13035 \\
\text { no: } 12880 \\
\text { yes: } 155\end{array}$ \\
\hline 62i. Ground vapor barrier & $\begin{array}{l}\text { obs: } 13023 \\
\text { no: } 12189 \\
\text { yes: } 834\end{array}$ & $\begin{array}{l}\text { obs: } 13023 \\
\text { no: } 12258 \\
\text { yes: } 765\end{array}$ & $\begin{array}{l}\text { obs: } 13035 \\
\text { no: } 11428 \\
\text { yes: } 1607\end{array}$ \\
\hline $62 \mathrm{j}$. Gutter or downspout (installed or repaired) & $\begin{array}{l}\text { obs: } 13031 \\
\text { no: } 12970 \\
\text { yes: } 61\end{array}$ & $\begin{array}{l}\text { obs: } 13031 \\
\text { no: } 12932 \\
\text { yes: } 99\end{array}$ & $\begin{array}{l}\text { obs: } 13035 \\
\text { no: } 12871 \\
\text { yes: } 164\end{array}$ \\
\hline $62 \mathrm{k}$. Grading of lot & $\begin{array}{l}\text { obs: } 13033 \\
\text { no: } 13031 \\
\text { yes: } 2\end{array}$ & $\begin{array}{l}\text { obs: } 13033 \\
\text { no: } 13031 \\
\text { yes: } 2\end{array}$ & $\begin{array}{l}\text { obs: } 13035 \\
\text { no: } 13025 \\
\text { yes: } 10\end{array}$ \\
\hline 621. Plumbing repair & $\begin{array}{l}\text { obs: } 12996 \\
\text { no: } 12808 \\
\text { yes: } 188\end{array}$ & $\begin{array}{l}\text { obs: } 12996 \\
\text { no: } 12753 \\
\text { yes: } 243\end{array}$ & $\begin{array}{l}\text { obs: } 13035 \\
\text { no: } 12567 \\
\text { yes: } 468\end{array}$ \\
\hline $62 \mathrm{~m}$. Sewer repair & $\begin{array}{l}\text { obs: } 13031 \\
\text { no: } 13026 \\
\text { yes: } 5\end{array}$ & $\begin{array}{l}\text { obs: } 13031 \\
\text { no: } 13010 \\
\text { yes: } 21\end{array}$ & $\begin{array}{l}\text { obs: } 13035 \\
\text { no: } 13006 \\
\text { yes: } 29\end{array}$ \\
\hline 62n. Electrical repair & $\begin{array}{l}\text { obs: } 12995 \\
\text { no: } 12807 \\
\text { yes: } 188\end{array}$ & $\begin{array}{l}\text { obs: } 12995 \\
\text { no: } 12503 \\
\text { yes: } 492\end{array}$ & $\begin{array}{l}\text { obs: } 13035 \\
\text { no: } 12310 \\
\text { yes: } 725\end{array}$ \\
\hline 62o. Stair repair & $\begin{array}{l}\text { obs: } 13031 \\
\text { no: } 13026 \\
\text { yes: } 5\end{array}$ & $\begin{array}{l}\text { obs: } 13031 \\
\text { no: } 13019 \\
\text { yes: } 12\end{array}$ & $\begin{array}{l}\text { obs: } 13035 \\
\text { no: } 13015 \\
\text { yes: } 20\end{array}$ \\
\hline 62p. Install/repair non-skid material on stairs & $\begin{array}{l}\text { obs: } 13035 \\
\text { no: } 13035 \\
\text { yes: } 0\end{array}$ & $\begin{array}{l}\text { obs: } 13035 \\
\text { no: } 13034 \\
\text { yes: } 1\end{array}$ & $\begin{array}{l}\text { obs: } 13035 \\
\text { no: } 13034 \\
\text { yes: } 1\end{array}$ \\
\hline 62q. Install/repair safety gate at stairs & $\begin{array}{l}\text { obs: } 13035 \\
\text { no: } 13035 \\
\text { yes: } 0\end{array}$ & $\begin{array}{l}\text { obs: } 13035 \\
\text { no: } 12962 \\
\text { yes: } 73\end{array}$ & $\begin{array}{l}\text { obs: } 13035 \\
\text { no: } 12962 \\
\text { yes: } 73\end{array}$ \\
\hline 62r. Install/repair grab bar in bathroom & $\begin{array}{l}\text { obs: } 13034 \\
\text { no: } 13033 \\
\text { yes: } 1\end{array}$ & $\begin{array}{l}\text { obs: } 13034 \\
\text { no: } 13034 \\
\text { yes: } 0\end{array}$ & $\begin{array}{l}\text { obs: } 13035 \\
\text { no: } 13034 \\
\text { yes: } 1\end{array}$ \\
\hline 62s. Install/repair non-skid material in bathtub & $\begin{array}{l}\text { obs: } 13035 \\
\text { no: } 13035 \\
\text { yes: } 0\end{array}$ & $\begin{array}{l}\text { obs: } 13035 \\
\text { no: } 13033 \\
\text { yes: } 2\end{array}$ & $\begin{array}{l}\text { obs: } 13035 \\
\text { no: } 13033 \\
\text { yes: } 2\end{array}$ \\
\hline 62t. Install/repair metal chimney liner & $\begin{array}{l}\text { obs: } 12925 \\
\text { no: } 12877 \\
\text { yes: } 48\end{array}$ & $\begin{array}{l}\text { obs: } 12925 \\
\text { no: } 12648 \\
\text { yes: } 277\end{array}$ & $\begin{array}{l}\text { obs: } 13035 \\
\text { no: } 12610 \\
\text { yes: } 425\end{array}$ \\
\hline 62u. Lead abatement & $\begin{array}{l}\text { obs: } 13035 \\
\text { no: } 13015 \\
\text { yes: } 20\end{array}$ & $\begin{array}{l}\text { obs: } 13035 \\
\text { no: } 12991 \\
\text { yes: } 44\end{array}$ & $\begin{array}{l}\text { obs: } 13035 \\
\text { no: } 12971 \\
\text { yes: } 64\end{array}$ \\
\hline $62 \mathrm{v}$. Asbestos abatement & $\begin{array}{l}\text { obs: } 13026 \\
\text { no: } 13026 \\
\text { yes: } 0\end{array}$ & $\begin{array}{l}\text { obs: } 13026 \\
\text { no: } 12937 \\
\text { yes: } 89\end{array}$ & $\begin{array}{l}\text { obs: } 13035 \\
\text { no: } 12937 \\
\text { yes: } 96\end{array}$ \\
\hline $62 \mathrm{w}$. Removal or safe storage of household poisons & $\begin{array}{l}\text { obs: } 13035 \\
\text { no: } 13035 \\
\text { yes: } 0\end{array}$ & $\begin{array}{l}\text { obs: } 13035 \\
\text { no: } 13034 \\
\text { yes: } 1\end{array}$ & $\begin{array}{l}\text { obs: } 13035 \\
\text { no: } 13034 \\
\text { yes: } 1\end{array}$ \\
\hline $\begin{array}{c}62 x . \text { Other } H \& S^{46} \text { and repair items (specify: } \\
\text { ) }^{47}\end{array}$ & $\begin{array}{l}\text { obs: } 12750 \\
\text { no: } 11632 \\
\text { yes: } 1118\end{array}$ & $\begin{array}{l}\text { obs: } 12750 \\
\text { no: } 11233 \\
\text { yes: } 1517\end{array}$ & $\begin{array}{l}\text { obs: } 13035 \\
\text { no: } 10138 \\
\text { yes: } 2897\end{array}$ \\
\hline 62y. Other H\&S and repair items (specify: & $\begin{array}{l}\text { obs: } 12957 \\
\text { no: } 12573 \\
\text { yes: } 384\end{array}$ & $\begin{array}{l}\text { obs: } 12957 \\
\text { no: } 12157 \\
\text { yes: } 800\end{array}$ & $\begin{array}{l}\text { obs: } 13035 \\
\text { no: } 11820 \\
\text { yes: } 1215\end{array}$ \\
\hline
\end{tabular}

\footnotetext{
${ }^{46}$ Health and safety

${ }^{47}$ Check 62x (Other H\&S and repair items (specify: repaired or installed.
} 
63. If a new space-heating system was installed, indicate the primary fuel used to heat the unit during the winter after weatherization: (check only one)

Freq. Percent Cum.

\begin{tabular}{r|rrr} 
Natural gas | & $2,568.22$ & 21.81 & 21.81 \\
Propane | & 318.83 & 2.71 & 24.52 \\
Kerosene (fuel oil \#1) & 71.50 & 0.61 & 25.13 \\
Fuel oil (\#2) | & 253.22 & 2.15 & 27.28 \\
Electricity | & 448.59 & 3.81 & 31.09 \\
Wood | & 33.58 & 0.29 & 31.37 \\
Not applicable | 7,959.56 & 67.60 & 98.97 \\
Other | & 5.66 & 0.05 & 99.02 \\
DK | & 115.86 & 0.98 & 100.00 \\
- Total | & 11,775 & 100.00 &
\end{tabular}

64. If a new space-heating system was installed, indicate the type of primary space-heating system after weatherization: (check only one)

Freq. Percent Cum.

$\begin{array}{rrrr}\text { Central furnace } \mid & 2,259.25 & 18.93 & 18.94 \\ \text { Heat pump | } & 90.35 & 0.76 & 19.70 \\ \text { Electric, built-in | } & 22.28 & 0.19 & 19.89 \\ \text { Steam or hydronic | } & 784.01 & 6.57 & 26.46 \\ \text { Wall furnace | } & 169.27 & 1.42 & 27.88 \\ \text { Room space heater (non-portable) } \mid & 201.19 & 1.69 & 29.56 \\ \text { Portable space heater | } & 21.11 & 0.18 & 29.74 \\ \text { Cooking Stove | } & 6.66 & 0.06 & 29.79 \\ \text { None | } & 69.23 & 0.58 & 30.37 \\ \text { Not applicable | } & 6,662.92 & 55.84 & 86.22 \\ \text { Refused | } & 77.64 & 0.65 & 86.87 \\ \text { No Answer | } & 1,447.94 & 12.13 & 99.00 \\ \text { DK | } & 119.16 & 1.00 & 100.00 \\ \text { Total | } & 11,932 & 100.00 & \end{array}$

\section{Select "steam or hot water system" for homes heated with boilers.}

65. If a new space-heating system was installed and justified for reasons other than cost effectiveness, identify the reason it was replaced: (check all that apply)

- Cost of repair/retrofit exceeded 50\% of replacement cost

$\begin{array}{crr}\text { Freq. } & \text { Percent } & \text { Cum. } \\ \text { n/a } \mid 7,463.30 & 75.72 & 75.72 \\ \text { No } \mid 1,849.33 & 18.76 & 94.48 \\ \text { Yes } \mid 544.37 & 5.52 & 100.00 \\ - & 54 .--\end{array}$

- Existing heating system was not running

Freq. Percent Cum. 


\begin{tabular}{|c|c|c|c|}
\hline $\mathrm{n} / \mathrm{a}$ & $7,463.30$ & 75.72 & 75.72 \\
\hline No & $1,968.36$ & 19.97 & 95.68 \\
\hline Yes & 425.34 & 4.32 & 100.00 \\
\hline Total & 9,857 & 100.00 & \\
\hline
\end{tabular}

- Existing heating system was old (e.g., at end of life, too old to be repaired/adjusted) Freq. Percent Cum.

\begin{tabular}{|c|c|c|c|}
\hline $\mathrm{n} / \mathrm{a}$ & $7,463.30$ & 75.72 & 75.72 \\
\hline No & $1,666.7$ & 16.91 & 92.62 \\
\hline Yes | & 727.00 & 7.38 & 100.00 \\
\hline Total | & 9,857 & 100.00 & \\
\hline
\end{tabular}

- To switch fuel

\begin{tabular}{|c|c|c|c|}
\hline & Freq. & Percent & Cum. \\
\hline $\mathrm{n} / \mathrm{a}$ & $7,463.30$ & 75.72 & 75.72 \\
\hline No & $2,326.33$ & 23.60 & 99.32 \\
\hline Yes & 67.37 & 0.68 & 100.00 \\
\hline Total & 9,857 & 100.00 & \\
\hline
\end{tabular}

- To convert from a steam system to a hot water system

$\begin{array}{ccc}\text { Freq. } & \text { Percent } & \text { Cum. } \\ \text { n/a } \mid 7,463.30 & 75.72 & 75.72 \\ \text { No } \mid 2,393.70 & 24.28 & 100.00 \\ -------+--------------------------- \\ \text { Total } \quad 9,857 & 100.00\end{array}$


- Heat exchanger was cracked

$\begin{array}{crc}\text { Freq. } & \text { Percent } & \text { Cum. } \\ \text { n/a } \mid 7,463.30 & 75.72 & 75.72 \\ \text { No } \mid 1,769.68 & 17.95 & 93.67 \\ \text { Yes | } 624.02 & 6.33 & 100.00 \\ ---------- \\ \text { Total| } 9,857 & 100.00\end{array}$

- Boiler was leaking

\begin{tabular}{|c|c|c|c|}
\hline & Freq. & Percent & Cum. \\
\hline $\mathrm{n} / \mathrm{a}$ & |7,463.30 & 75.72 & 75.72 \\
\hline No & $2,333.54$ & 23.67 & 99.39 \\
\hline Yes & 60.16 & 0.61 & 100.00 \\
\hline Total & 9,857 & 100.00 & \\
\hline
\end{tabular}

- Safety switches/controls were not operational and could not be repaired

\begin{tabular}{|c|c|c|c|}
\hline & Freq. & Percent & Cum. \\
\hline $\mathrm{n} / \mathrm{a}$ & $7,463.30$ & 75.72 & 75.72 \\
\hline No & $2,355.17$ & 23.89 & 99.61 \\
\hline Yes & 38.53 & 0.39 & 100.00 \\
\hline Total & 9,857 & 100.00 & \\
\hline
\end{tabular}

- To replace unvented space heater(s)

\begin{tabular}{crr} 
Freq. & Percent & \multicolumn{1}{c}{ Cum. } \\
n/a $\mid 7,463.30$ & 75.72 & 75.72 \\
No $\mid 2,215.95$ & 22.48 & 98.20 \\
Yes $\mid 177.75$ & 1.80 & 100.00 \\
------+-------------- & 100.00
\end{tabular}

- Existing heating system was not safe to run for other reason (specify:

$\begin{array}{rrr}\text { Freq. } & \text { Percent } & \text { Cum. } \\ \text { n/a } \mid 7,463.30 & 75.72 & 75.72 \\ \text { No|2,242.82 } & 22.75 & 98.47 \\ \text { Yes | } 150.87 & 1.53 & 100.00 \\ ------+------------ & \end{array}$


- Other (specify:

$\begin{array}{crc}\text { Freq. } & \text { Percent } & \text { Cum. } \\ \text { n/a } \mid 7,463.30 & 75.72 & 75.72 \\ \text { No } \mid 2,216.85 & 22.49 & 98.21 \\ \text { Yes | } 176.84 & 1.79 & 100.00 \\ ---------- \\ \text { Total| } 9,857 & 100.00 & \end{array}$

66. Please identify any cost-effective energy-efficiency measures (not repair or health and safety measures) recommended by your energy audit procedures that you were unable to install in this housing unit because of insufficient funds: (check all that apply)

- $\quad$ Air sealing

$\begin{array}{crc}\text { Freq. } & \text { Percent } & \text { Cum. } \\ \text { No | 10,600.69 } & 97.68 & 97.68 \\ \text { Yes | } 252.31 & 2.32 & 100.00 \\ ------ \\ \text { Total | } 10,853 & 100.00 & \end{array}$

- Duct sealing

Freq. Percent Cum.

\begin{tabular}{|c|c|c|c|}
\hline No & $10,761.12$ & 99.15 & 99.15 \\
\hline Yes & 91.88 & 0.85 & 100.00 \\
\hline
\end{tabular}

- Attic insulation

\begin{tabular}{|c|c|c|c|}
\hline & Freq. & Percent & Cum. \\
\hline No & $10,621.59$ & 97.87 & 97.87 \\
\hline Yes & 231.41 & 2.13 & 100.00 \\
\hline Total & 10,853 & 100.00 & \\
\hline
\end{tabular}

- Wall insulation

\begin{tabular}{|c|c|c|c|}
\hline & Freq. & Percent & Cum. \\
\hline No & $10,750.39$ & 99.05 & 99.05 \\
\hline Yes & 102.61 & 0.95 & 100.00 \\
\hline Total & 10,853 & 100.00 & \\
\hline
\end{tabular}


- Floor/foundation insulation

Freq. Percent Cum.

\begin{tabular}{|c|c|c|c|}
\hline No & $10,741.89$ & 98.98 & 98.98 \\
\hline Yes & 111.11 & 1.02 & 100.00 \\
\hline Total & 10,853 & 100.00 & \\
\hline
\end{tabular}

- Duct insulation

\begin{tabular}{|c|c|c|c|}
\hline & Freq. & Percent & Cum. \\
\hline No & $10,782.11$ & 99.35 & 99.35 \\
\hline Yes & 70.89 & 0.65 & 100.00 \\
\hline $10 t a 1$ & 10,853 & 100.00 & \\
\hline
\end{tabular}

- New window(s)

Freq. Percent Cum.

\begin{tabular}{|c|c|c|c|}
\hline No & $10,765.45$ & 99.19 & 99.19 \\
\hline Yes & 87.55 & 0.81 & 100.00 \\
\hline Total & 10,853 & 100.00 & \\
\hline
\end{tabular}

- Storm windows(s)

\begin{tabular}{|c|c|c|c|}
\hline & Freq. & Percent & Cum. \\
\hline No & $10,770.09$ & 99.24 & 99.24 \\
\hline Yes & \begin{tabular}{|l}
82.91 \\
\end{tabular} & 0.76 & 100.00 \\
\hline Total & 10,853 & 100.00 & \\
\hline
\end{tabular}

- $\operatorname{Door}(\mathrm{s})$

\begin{tabular}{ccr} 
Freq. & Percent & \multicolumn{1}{c}{ Cum. } \\
No | $10,775.21$ & 99.28 & 99.28 \\
Yes | 77.79 & 0.72 & 100.00 \\
----------- \\
Total | 10,853 & 100.00
\end{tabular}

- Storm door(s)

$\begin{array}{crc}\text { Freq. } & \text { Percent } & \text { Cum. } \\ \text { No | } 10,799.70 & 99.51 & 99.51 \\ \text { Yes | } 53.30 & 0.49 & 100.00 \\ ------- & \end{array}$


- New space-heating system

Freq. Percent Cum.

\begin{tabular}{|c|c|c|c|}
\hline No & $10,807.34$ & 99.58 & 99.58 \\
\hline Yes & 45.66 & 0.42 & 100.00 \\
\hline Total & 10,853 & 100.00 & \\
\hline
\end{tabular}

- Space-heating system tune-up

Freq. Percent Cum.

\begin{tabular}{|c|c|c|c|}
\hline No & $10,800.33$ & 99.51 & 99.51 \\
\hline Yes & 52.67 & 0.49 & 100.00 \\
\hline Total & 10,853 & 100.00 & \\
\hline
\end{tabular}

- New air conditioner(s)

Freq. Percent Cum.

\begin{tabular}{|c|c|c|c|}
\hline No & $10,774.66$ & 99.28 & 99.28 \\
\hline Yes & 78.34 & 0.72 & 100.00 \\
\hline Total & 10,853 & 100.00 & \\
\hline
\end{tabular}

- Air conditioner tune-up(s)

Freq. Percent Cum.

\begin{tabular}{|c|c|c|c|}
\hline No & $10,798.11$ & 99.49 & 99.49 \\
\hline Yes & 54.89 & 0.51 & 100.00 \\
\hline Total | & 10,853 & 100.00 & \\
\hline
\end{tabular}

- HVAC thermostat

Freq. Percent Cum.

\begin{tabular}{|c|c|c|c|}
\hline No & $10,816.08$ & 99.66 & 99.66 \\
\hline Yes & 36.92 & 0.34 & 100.00 \\
\hline Total & 10,853 & 100.00 & \\
\hline
\end{tabular}

- New water heater

Freq. Percent Cum.

\begin{tabular}{|c|c|c|c|}
\hline \multicolumn{2}{|c|}{ No $\mid 10,808.48$} & \multirow{2}{*}{$\begin{array}{r}99.59 \\
0.41\end{array}$} & \multirow{2}{*}{$\begin{array}{r}99.59 \\
100.00\end{array}$} \\
\hline Yes & 44.52 & & \\
\hline Total & 10,853 & 100.00 & \\
\hline
\end{tabular}


- Water heater insulation wrap

\begin{tabular}{crr} 
Freq. & Percent & \multicolumn{1}{c}{ Cum. } \\
No | 10,760.34 & 99.15 & 99.15 \\
Yes | 92.66 & 0.85 & 100.00 \\
------- &
\end{tabular}

- Water flow devices (e.g., showerheads, faucet aerators) Freq. Percent Cum.

\begin{tabular}{|c|c|c|c|}
\hline No & $10,733.49$ & 98.90 & 98.90 \\
\hline Yes | & 119.51 & 1.10 & 100.00 \\
\hline Total & 10,853 & 100.00 & \\
\hline
\end{tabular}

- Lighting

$\begin{array}{ccc}\text { Freq. } & \text { Percent } & \text { Cum. } \\ \text { No |10,693.82 } & 98.53 & 98.53 \\ \text { Yes | } 159.18 & 1.47 & 100.00 \\ ------------- \\ \text { Total| } 10,853 & 100.00\end{array}$

- Refrigerator

\begin{tabular}{crr} 
Freq. & Percent & \multicolumn{1}{c}{ Cum. } \\
No | $10,708.47$ & 98.67 & 98.67 \\
Yes | 144.53 & 1.33 & 100.00 \\
------- &
\end{tabular}

- Other:

\begin{tabular}{|c|c|c|c|}
\hline & Freq. & Percent & Cum. \\
\hline No & $10,730.59$ & 98.87 & 98.87 \\
\hline Yes & 122.41 & 1.13 & 100.00 \\
\hline$t_{0}$ & 10,853 & 100.00 & \\
\hline
\end{tabular}

- None

\begin{tabular}{|c|c|c|c|}
\hline & Freq. & Percent & Cum. \\
\hline No & 624.13 & 5.75 & 5.75 \\
\hline Yes & $10,228.87$ & 94.25 & 100.00 \\
\hline Total & 10,853 & 100.00 & \\
\hline
\end{tabular}


67. If energy efficiency measures were checked in the previous question, provide a rough estimate of the cost for installing all the measures checked: $\$$

\begin{tabular}{|l|r|}
\hline observations: & 535 \\
\hline missing values: & 13950 \\
\hline mean: & 2744.67 \\
\hline standard deviation: & 5521.23 \\
\hline min: & 0 \\
\hline 10th percentile: & 40 \\
\hline 25th percentile: & 478 \\
\hline median: & 1467.31 \\
\hline 75th percentile: & 2745.66 \\
\hline 90th percentile: & 4800 \\
\hline max: & 35771 \\
\hline
\end{tabular}

68. Please identify any repair or health and safety measures recommended by your audit procedures that you were unable to install in this housing unit because of insufficient funds: (check all that apply)

- New window(s)

Freq. Percent Cum.

$\begin{array}{crr}\text { No | } 10,227.57 & 99.65 & 99.65 \\ \text { Yes | } 36.43 & 0.35 & 100.00 \\ ---------------- \\ \text { Total| } 10,264 & 100.00\end{array}$

- Window glazing(s)

Freq. Percent Cum.

\begin{tabular}{|c|c|c|c|}
\hline No & $10,253.76$ & 99.90 & 99.90 \\
\hline Yes & 10.24 & 0.10 & 100.00 \\
\hline Total & 10,264 & 100.00 & \\
\hline
\end{tabular}

- Window screen(s)

\begin{tabular}{|c|c|c|c|}
\hline & Freq. $\quad \mathrm{P}$ & cent & Cum. \\
\hline No & $10,256.41$ & 99.93 & 99.93 \\
\hline Yes & 7.59 & 0.07 & 100.00 \\
\hline Total & 10,264 & 100.00 & \\
\hline
\end{tabular}

- Window lock(s)

\begin{tabular}{|c|c|c|c|}
\hline & Freq. & Percent & Cum. \\
\hline No & $10,250.88$ & 99.87 & 99.87 \\
\hline Yes & 13.12 & 0.13 & 100.00 \\
\hline Total | & 10,264 & 100.00 & \\
\hline
\end{tabular}

- Window repair 
Freq. Percent Cum.

\begin{tabular}{|c|c|c|c|}
\hline No & $10,248.19$ & 99.85 & 99.85 \\
\hline Yes & 15.81 & 0.15 & 100.00 \\
\hline
\end{tabular}

- New door(s)

\begin{tabular}{crr} 
Freq. & Percent & \multicolumn{1}{c}{ Cum. } \\
No | 10,252.42 & 99.89 & 99.89 \\
Yes | 11.58 & 0.11 & 100.00 \\
------- &
\end{tabular}

- $\operatorname{Door} \operatorname{lock}(\mathrm{s})$

\begin{tabular}{|c|c|c|c|}
\hline & Freq. & Percent & Cum. \\
\hline No & $10,246.73$ & 99.83 & 99.83 \\
\hline Yes & 17.27 & 0.17 & 100.00 \\
\hline Total & 10,264 & 100.00 & \\
\hline
\end{tabular}

- Door repair

\begin{tabular}{crr} 
Freq. & Percent & \multicolumn{1}{c}{ Cum. } \\
No | $10,224.64$ & 99.62 & 99.62 \\
Yes | 39.36 & 0.38 & 100.00 \\
------- &
\end{tabular}

- New space-heating system

Freq. Percent Cum.

\begin{tabular}{|c|c|c|c|}
\hline No & 237.11 & 99.74 & 99.74 \\
\hline Yes & 26.89 & 0.26 & 100.00 \\
\hline Total | & 10,264 & 100.00 & \\
\hline
\end{tabular}

- Space-heating system repair

\begin{tabular}{|c|c|c|c|}
\hline & Freq. $\quad P$ & rcent & Cum. \\
\hline No & $10,256.16$ & 99.92 & 99.92 \\
\hline Yes & $\quad 7.85$ & 0.08 & 100.00 \\
\hline Total | & 10,264 & 100.00 & \\
\hline
\end{tabular}


- New air conditioner(s)

Freq. Percent Cum.

\begin{tabular}{|c|c|c|c|}
\hline No & $10,230.01$ & 99.67 & 99.67 \\
\hline Yes & 33.99 & 0.33 & 100.00 \\
\hline Total & 10,264 & 100.00 & \\
\hline
\end{tabular}

- Air conditioner repair

Freq. Percent Cum.

\begin{tabular}{|c|c|c|c|}
\hline No & $10,226.09$ & 99.63 & 99.63 \\
\hline Yes & 37.91 & 0.37 & 100.00 \\
\hline Total | & 10,264 & 100.00 & \\
\hline
\end{tabular}

- Ceiling or whole-house fan(s)

Freq. Percent Cum.

\begin{tabular}{|c|c|c|c|}
\hline No & $10,260.91$ & 99.97 & 99.97 \\
\hline Yes & \begin{tabular}{|l}
$\mid$ \\
\end{tabular} & 0.03 & 100.00 \\
\hline Total | & 10,264 & 100.00 & \\
\hline
\end{tabular}

- Exhaust fan(s) or ventilation system

\begin{tabular}{|c|c|c|c|}
\hline No & $10,214.59$ & 99.52 & 99.52 \\
\hline Yes & 49.42 & 0.48 & 100.00 \\
\hline Total & 10,264 & 100.00 & \\
\hline
\end{tabular}

- New water heater

Freq. Percent Cum.

\begin{tabular}{|c|c|c|c|}
\hline No & 221.34 & 99.58 & 99.58 \\
\hline Yes & 42.66 & 0.42 & 100.00 \\
\hline Total | & 10,264 & 100.00 & \\
\hline
\end{tabular}

- Water-heating system repair

Freq. Percent Cum.

\begin{tabular}{|c|c|c|c|}
\hline No & 250.12 & 99.86 & 99.86 \\
\hline Yes & 13.88 & 0.14 & 100.00 \\
\hline Total | & 10,264 & 100.00 & \\
\hline
\end{tabular}


- Refrigerator

\begin{tabular}{|c|c|c|c|}
\hline & Freq. & rcent & Cum. \\
\hline No & $10,215.56$ & 99.53 & 99.53 \\
\hline Yes & 48.44 & 0.47 & 100.00 \\
\hline Total & 10,264 & 100.00 & \\
\hline
\end{tabular}

- Smoke alarm

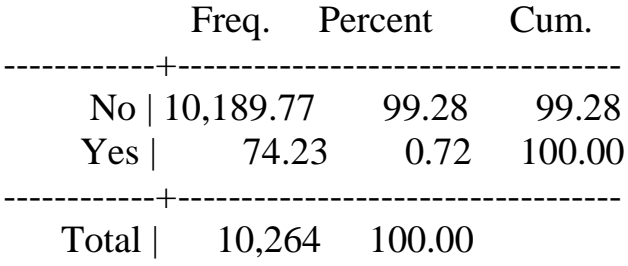

- $\mathrm{CO}$ monitor

Freq. Percent Cum.

\begin{tabular}{|c|c|c|c|}
\hline No & $10,185.51$ & 99.24 & 99.24 \\
\hline Yes & 78.49 & 0.76 & 100.00 \\
\hline Total & 10,264 & 100.00 & \\
\hline
\end{tabular}

- Attic ventilation

\begin{tabular}{|c|c|c|c|}
\hline & Freq. $\quad \mathrm{P}$ & Percent & Cum. \\
\hline No & $10,255.52$ & 99.92 & 99.92 \\
\hline Yes & 8.48 & 0.08 & 100.00 \\
\hline Total & 10,264 & 100.00 & \\
\hline
\end{tabular}

- $\quad$ Roof, wall, floor, or foundation repair Freq. Percent Cum.

\begin{tabular}{|c|c|c|c|}
\hline No & 243.65 & 99.80 & 99.80 \\
\hline Yes & 20.35 & 0.20 & 100.00 \\
\hline Total | & 10,264 & 100.00 & \\
\hline
\end{tabular}

- Plumbing/sewer repair Freq. Percent Cum.

\begin{tabular}{|c|c|c|c|}
\hline No & $10,249.93$ & 99.86 & 99.86 \\
\hline Yes & 14.07 & 0.14 & 100.00 \\
\hline Total & 10,264 & 100.00 & \\
\hline
\end{tabular}


- Electrical repair

Freq. Percent Cum.

\begin{tabular}{|c|c|c|c|}
\hline No & $10,255.62$ & 99.92 & 99.92 \\
\hline Yes & 8.38 & 0.08 & 100.00 \\
\hline Total & 10,264 & 100.00 & \\
\hline
\end{tabular}

- Other:

\begin{tabular}{|c|c|c|c|}
\hline & Freq. & Percent & Cum. \\
\hline No & $10,163.42$ & 99.02 & 99.02 \\
\hline Yes & 100.58 & 0.98 & 100.00 \\
\hline Total & 10,264 & 100.00 & \\
\hline
\end{tabular}

- None

Freq. Percent Cum.

\begin{tabular}{|c|c|c|c|}
\hline No & $\begin{array}{r}354.11 \\
990989\end{array}$ & $\begin{array}{r}3.45 \\
0655\end{array}$ & 3.45 \\
\hline Total & 10,264 & 100.00 & \\
\hline
\end{tabular}

69. If repair or health and safety measures were checked in the previous question, provide a rough estimate of the cost for installing all the measures checked: $\$$

\begin{tabular}{|l|c|}
\hline observations: & 416 \\
\hline missing values: & 14074 \\
\hline mean: & 1888.27 \\
\hline standard deviation: & 5181.53 \\
\hline min: & 0 \\
\hline 10th percentile: & 0 \\
\hline 25th percentile: & 50 \\
\hline median: & 286.75 \\
\hline 75th percentile: & 1300 \\
\hline 90th percentile: & 4373.85 \\
\hline max: & 39000 \\
\hline
\end{tabular}




\section{COSTS}

Provide the costs associated with installing the measures in THIS housing unit from all funding sources. Do NOT include any program management costs such as those associated with intake and eligibility determination, audits and house assessments, final inspections, contractor or crew management, and program administration. Also, do NOT include installation-related overhead costs such as those associated with vehicles, equipment, and training.

\begin{tabular}{|c|c|c|c|c|}
\hline & In-House Crew & Contractor & $\begin{array}{c}\text { Enter total if } \\
\text { crew/contractor } \\
\text { split is unknown }\end{array}$ & Total \\
\hline 70a. Material costs & $\begin{array}{l}\text { obs: } 5504 \\
\text { min: } 0 \\
\text { max: } 20170.5 \\
\text { mean: } 1196.49 \\
\text { median: } 607 \\
\end{array}$ & $\begin{array}{l}\text { obs: } 4432 \\
\text { min: } 0 \\
\text { max: } 139769 \\
\text { mean: } 2668.95 \\
\text { median: } 1331.53 \\
\end{array}$ & $\begin{array}{l}\text { obs: } 1963 \\
\text { min: } 0 \\
\text { max: } 198862 \\
\text { mean: } 12616.17 \\
\text { median: } 618.34 \\
\end{array}$ & \\
\hline 70b. Labor costs ${ }^{1}$ & $\begin{array}{l}\text { obs: } 4592 \\
\text { min: } 0 \\
\text { max: } 34350 \\
\text { mean: } 1627.16 \\
\text { median: } 664.20 \\
\end{array}$ & $\begin{array}{l}\text { obs: } 4246 \\
\text { min: } 0 \\
\text { max: } 92029 \\
\text { mean: } 1864.43 \\
\text { median: } 1144 \\
\end{array}$ & $\begin{array}{l}\text { obs: } 2271 \\
\text { min: } 0 \\
\text { max: } 87487 \\
\text { mean: } 5524.92 \\
\text { median: } 0 \\
\end{array}$ & \\
\hline 70c. Profit/overhead ${ }^{2}$ & $\begin{array}{l}\text { obs: } 1316 \\
\text { min: }-394.68 \\
\text { max: } 19194.32 \\
\text { mean: } 778.09 \\
\text { median: } 0 \\
\end{array}$ & $\begin{array}{l}\text { obs: } 881 \\
\text { min: } 0 \\
\text { max: } 7150 \\
\text { mean: } 20.65 \\
\text { median: } 0\end{array}$ & $\begin{array}{l}\text { obs: } 920 \\
\text { min: } 0 \\
\text { max: } 5226.80 \\
\text { mean: } 256.46 \\
\text { median: } 0\end{array}$ & \\
\hline $\begin{array}{l}\text { 70d. Enter total if } \\
\text { material/labor/profit/ } \\
\text { overhead split is } \\
\text { unknown }\end{array}$ & $\begin{array}{l}\text { obs: } 722 \\
\text { min: } 0 \\
\text { max: } 10283 \\
\text { mean: } 1522.52 \\
\text { median: } 656 \\
\end{array}$ & $\begin{array}{l}\text { obs: } 1789 \\
\text { min: } 0 \\
\text { max: } 799358.69 \\
\text { mean: } 27789.37 \\
\text { median: } 2745.2 \\
\end{array}$ & $\begin{array}{l}\text { obs: } 3333 \\
\text { min: } 0 \\
\text { max: } 1978573.6 \\
\text { mean: } 142968.32 \\
\text { median: } 6077.70 \\
\end{array}$ & \\
\hline \multicolumn{5}{|l|}{ 70e. Total } \\
\hline & \multicolumn{4}{|c|}{$\begin{array}{l}{ }^{1} \text { Crew-based labor costs should be based on the crew's fully loaded hourly rate } \\
\text { (rather than the crew's take-home pay rate) which may include costs associated } \\
\text { with medical and other insurance, workers compensation, vacations, and other } \\
\text { benefits. These labor costs should include the crew's time for traveling to and } \\
\text { from the job site. } \\
{ }^{2} \text { If contractor profit and overhead are included in the contractor's material and } \\
\text { labor costs, then leave these cells blank. }\end{array}$} \\
\hline
\end{tabular}


Divide the total costs spent on this housing unit (cell in lower right corner of above table) into the following expenditure categories:

\begin{tabular}{|c|c|}
\hline Expenditure category & Costs \\
\hline 71a. Cost effective energy-related measures (SIR > 1.0) & $\begin{array}{l}\text { obs: } 9100 \\
\text { min: } 0 \\
\text { max: } 1774073.6 \\
\text { mean: } 59591.39 \\
\text { median: } 2403 \\
\end{array}$ \\
\hline 71b. Incidental repairs & $\begin{array}{l}\text { obs: } 4365 \\
\text { min: } 0 \\
\text { max: } 652800 \\
\text { mean: } 2936.71 \\
\text { median: } 348\end{array}$ \\
\hline 71c. Health and safety and other non-cost effective measures & $\begin{array}{l}\text { obs: } 8750 \\
\text { min: } 0 \\
\text { max: } 204500 \\
\text { mean: } 6024.94 \\
\text { median: } 300\end{array}$ \\
\hline 71d. Enter total expenditures if above categories are not known & $\begin{array}{l}\text { obs: } 3240 \\
\text { min: } 0 \\
\text { max: } 319945 \\
\text { mean: } 4403.28 \\
\text { median: } 1753.16\end{array}$ \\
\hline 71e. Total & \\
\hline
\end{tabular}

Divide the total costs spent on this housing unit (cell in lower right corner of the two above tables) into the following funding sources:

\begin{tabular}{|l|l|}
\hline \multicolumn{1}{|c|}{ Funding source } & \multicolumn{1}{c|}{ Total funds } \\
\hline 72a. DOE funds & $\begin{array}{l}\text { obs: } 12858 \\
\text { min: }-693 \\
\text { max: } 696143.69 \\
\text { mean: } 21218.16 \\
\text { median: } 1400\end{array}$ \\
\hline & $\begin{array}{l}\text { obs: } 12725 \\
\text { min: } 0 \\
\text { max: } 1282430 \\
\text { mean: } 28270.03 \\
\text { median: } 684.96\end{array}$ \\
\hline 72b. Non-DOE funds & $\begin{array}{l}\text { obs: } 11515 \\
\text { min: } 0 \\
\text { max: } 326945 \\
\text { mean: } 709.34 \\
\text { median: } 0\end{array}$ \\
\hline 72c. Enter total expenditures if above categories are not known & \\
\hline 72d. Total & \\
\hline
\end{tabular}




\section{Housing Type Definitions}

Single Family Detached - House that provides living space for one family or household, is contained within walls that go from the basement (or the ground floor, if there is no basement) to the roof, and has no walls that are shared (or built in contact) with another household. A manufactured house assembled on site is a single family detached housing unit, not a mobile home.

Single Family Attached - House that provides living space for one household, is contained within walls that go from the basement (or the ground floor, if there is no basement) to the roof, has at least one wall that is shared (or built in contact) with an adjacent household, and has an independent outside entrance. An attached house does not have any other households living above or below, and does not share basement or attic space with other housing units. Also, an attached house does not share a heating or cooling system with any other housing units. Examples include row houses, townhouses, condominiums and side-by-side duplexes that do not have shared attics, basements or HVAC equipment.

Small Multi-family (2-4 units) - Building with two to four housing units (i.e., building that is divided into living quarters for two, three, or four families or households) in which one household lives above or beside another and does not meet the single family attached house definition. Includes houses originally intended for occupancy by one family (or for some other use) that have since been converted to separate dwellings for two to four families. Typical arrangements in these types of living quarters are separate apartments downstairs and upstairs or one apartment on each of three or four floors.

Large multifamily (5 or More Units per Building) - Building with five or more housing units (i.e., building that contains living quarters for five or more families or households) that does not meet the single family attached house definition.

Mobile Home - Home that is built on a movable chassis, is moved to the site, and may be placed on a permanent or temporary foundation. If rooms are added to the structure, it is considered a mobile home if the added floor area is less than the mobile home's original floor area; otherwise, it is a single family detached house. A manufactured house assembled on site is a single family detached house, not a mobile home.

Shelter - Structure whose principal purpose is to house individuals on a temporary basis who may or may not be related to one another and who are not living in nursing homes, prisons, or similar institutional care facilities. 